







\section{A MANUAL OF}

\section{S Y P H I L I S}

AND THF

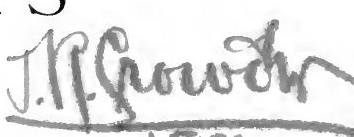

\section{VENEREAL DISEASES}

BY

JAMES NEVINS HYDE, A. M., M. I).

PROFESSOR OF SKIN AND VENEREAL DISEASES, RUSH MEDICAL COLLEGE; DERMA-

TOLOGIST TO THE PRESBYTERIAN, MHCHAL REESE, AND AUGUSTANA

HOSPITALS; AND CONSULTING PHYSICIAN TO THE HOSPITAL FOR WOMEN AND CHILDREN, CHICAGO;

ANI

FRANK H. MONTGONERY, M. D.

LECTURER ON DERMATOLOGY AND GENITO-URINARY DISEASES, AND CHIEF ASSISTANT

TO 1 HE CLINIC FOR SKIN AND VENEREAL DISEASES, RUSH MEDICAL

COLLEGE; ATTENDING PHYSICIAN FOR SKIN AND VENEREAL

DISEASES, ST. ELIZAEETH HOSPITAL, CHICAGU.

WITH 44 ILLUSTRATIONS IN THE TEXT

AND 8 FULL-PAGE PLATES IN COLORS AND TINTS.

\section{PHILADELPHIA}

W. B. SA UNDER S

925 WaLNUT STREET

I 896 . 
COPYright, I895, BY

W. P. SA C N D ER S. 


\section{nestest \\ $=$

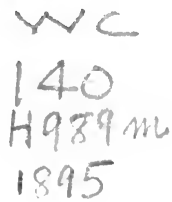 \\ PREFACE.}

This Manual has been prepared with the intent of meeting the special needs of the student and of the practitioner rather than of the expert. The aim has been to supply in a compendious form, and with detail, all practical facts connected with the study and the treatment of syphilis and the venereal diseases. Care has been taken to avoid all points in controversy and to exclude the data which are to be sought for in the more voluminous treatises on these subjects.

The authors are glad to express their special obligations to the classical works of Fournier, Jullien, and Mauriac; to Keyes' exceedingly practical treatise on G'nito-urinary Diseases, including Syphilis; to Morrow's valuable System of Genito-urinary Diseases, Syphilology, and Dermatology, in three volumes, and in especial to the carefully written chapters of that work on stricture of the urethra and syphilis of the eye and ear. The treatise of Bumstead and Taylor, which has so long and so well represented the advance of knowledge in venereal diseases, has frequently been consulted, as has also the standard monograph by Finger on Blennorrhoë, and the excellent handbook of Messrs. Culver and Hayden. 
The authors of this manual are also gratified in acknowledging in this public manner their appreciation of the courtesy of Messrs. William Wood \& Co. of New York, who kindly gave permission to reproduce the plates originally contributed by Dr. Hyde to their Reference Handbook of the Mcdical Sciences; to Dr. Petrini of Galatz, for permission to reproduce his fine plate showing the micro-organism of Ducrey; to Dr. John A. Fordyce of New York, for his kindness in supplying the micro-photograph of gonococci which has been employed as one of the illustrations; and to Messrs. Lea Bros. \& Co. of Philadelphia, for permission to make use of one of their original cuts.

The authors are also glad to express their acknowledgments to Dr. WV. F. Robinson for his efficient aid while these pages were passing through the press. 


\section{CONTENTS.}

PAGE

INTRODUCTION . . . . . . . . . . . . . . . . . I7

SYPHILIS . . . . . . . . . . . . . . . . . 27

Acquired Syphilis . . . . . . . . . . . . . 27

Syphilis of the Skin . . . . . . . . . . . . . . . 73

Syphilitic Affections of the Hair . . . . . . . . . . . . . . I33

Syphilitic Affections of the Nail . . . . . . . . . . I36

syphilis of the Mouth and the Tongue . . . . . . . . . . . 142

Syphilis of the Respiratory Tract . . . . . . . . . . . . . 152

Syphilis of the Bones . . . . . . . . . . . . . . I59

Syphilis of the Larger Joints . . . . . . . . . . . . . . I65

Syphilis of the Burse . . . . . . . . . . . . . I66

Syphilis of the Tendons and the Tendinous Sheaths . . . . . I66

Syphilis of the Aponeuroses . . . . . . . . . . . . . . I67

Syphilis of the Muscles . . . . . . . . . . . . . I67

Syphilis of the Heart . . . . . . . . . . . . . . I68

Syphilis of the Blood-vessels . . . . . . . . . . . . I70

Syphilis of the Lungs . . . . . . . . . . . . . . . I7 I

Syphilis of the Gastro-intestinal Tract . . . . . . . . . . I73

Syphilis of the Rectum and the Anus . . . . . . . . . . . . I75

Syphilis of the Genito-urinary Organs . . . . . . . . . . I 8 I

Syphilis of the Nervous System . . . . . . . . . . . . . I87

Syphilis of the Eye and Ocular Appendages . . . . . . . . . I94

Syphilis of the Ear . . . . . . . . . . . . . . . . . 203

Hereditary Syphilis . . . . . . . . . . . . . . . . . 205

Treatment of Syphilis . . . . . . . . . . . . . . 227

Acquired Infantile Syphilis . . . . . . . . . . . . . $2 \mathrm{~S}_{3}$

Syphilis in Relation with the Family and Society . . . . . . . $2 \mathrm{~S}_{4}$

Chancrold . . . . . . . . . . . . . . . . . . 293

DISORders nOt INVARIABly Venereat . . . . . . . . . 33 I

Balanitis and Balano-posthitis . . . . . . . . . . . . 33 I

Phimosis . . . . . . . . . . . . . . . . . 334

Paraphimosis . . . . . . . . . . . . . . . . 338 


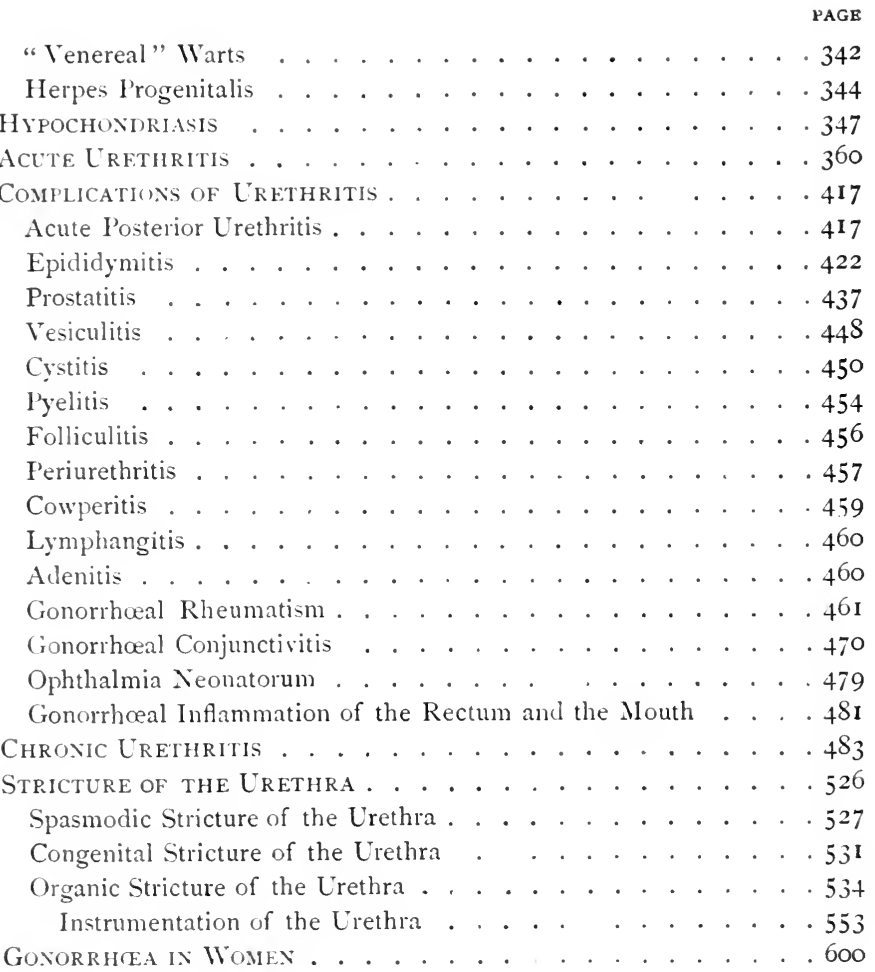




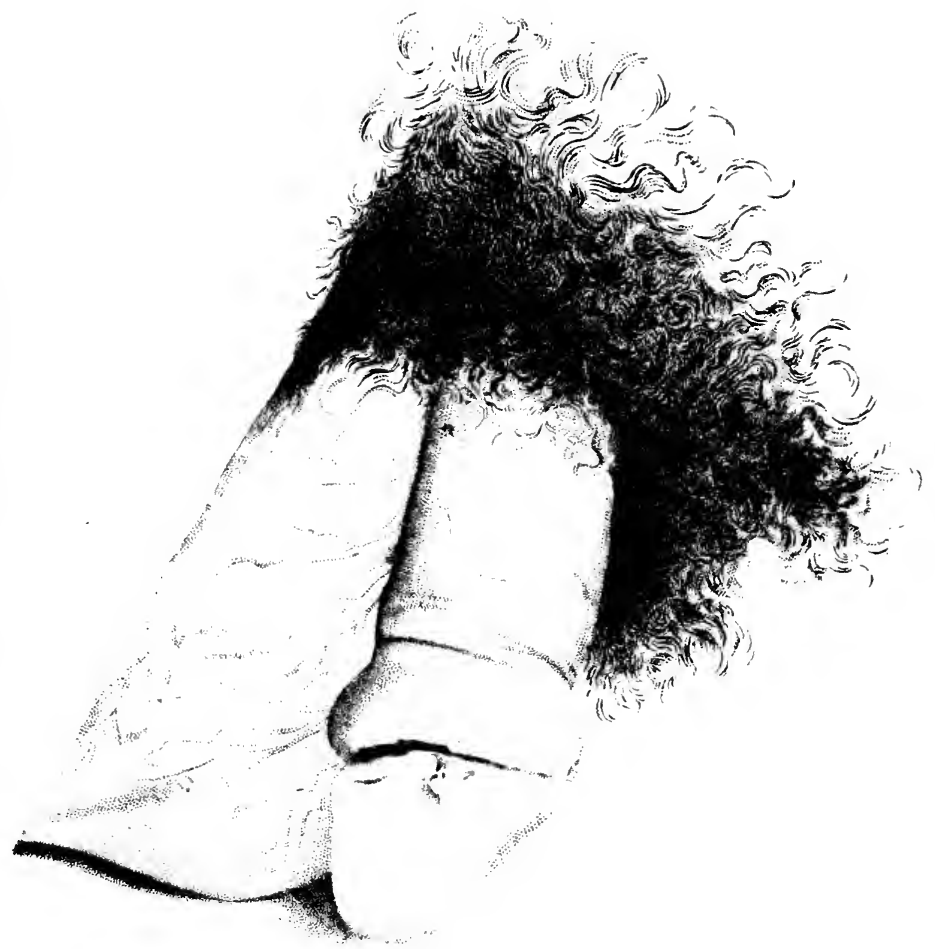





\section{LIST OF ILLUSTRATIONS.}

\section{FIGURES.}

FiG.

I. Palmar syphiloderm (after Keyes) . . . . . . . . . . . . 97

2. Moist papules (after Miller) . . . . . . . . . . . . . IOI

3. Large pustular syphiloderm (after Stelwagon) . . . . . . . IO7

4. Rupia (after Tilbury Fox) . . . . . . . . . . . . . IO9

5. Resolutive tubercular syphiloderm in groups . . . . . . . . . I I4

6. Serpiginous tubercular syphiloderm (after Stelwagon) . . . . . I I6

7. Ulcerative tubercular syphiloderm (after Keyes) . . . . . . . . II7

8. Gummata (after Jullien) . . . . . . . . . . . . I2I

9. Cicatrices resulting from extensive gummatous infiltration of the face . . . . . . . . . . . . . . . . I 24

I0. Sabre-blade deformity of the tibiæ in hereditary syphilis . . . . 216

I I. Hutchinson's teeth with osteo-periostitis and ulceration in inherited syphilis . . . . . . . . . . . . . . . 2 219

I2. Phimosis from gonorrhœa (Cullerier) . . . . . . . . . 335

I3. Paraphimosis (Cullerier) . . . . . . . . . . . . . 338

I4, 15. Reduction of paraphimosis . . . . . . . . . . . . 340

I6. Urethral syringe . . . . . . . . . . . . . . . . . . . . 41 I

I 7. Kiefer's urethral irrigation nozzle . . . . . . . . . . . . . . 497

I8. Klotz's endoscope . . . . . . . . . . . . . . . . . 500

19, 20. Urethral specula . . . . . . . . . . . . . . . . 50 I

2I. Brown's method of illuminating the urethra . . . . . . . . . 502

22. W. K. Otis's "perfected" urethroscope . . . . . . . . . . . 503

23. Winternitz's psychrophor . . . . . . . . . . . . . . . . 519

24. Keyes-Ultzmann syringe . . . . . . . . . . . . . . . . 519

25. Annular stricture (Dittel) . . . . . . . . . . . . 535

26. Tortuous stricture (Dittel) . . . . . . . . . . . . 536

27. The normal urethra (Thompson) . . . . . . . . . . 554

28. Proper and improper curves for unyielding male urethral instruments . . . . . . . . . . . . . . . 555

29, 30. Sounding of the urethra (Keyes) . . . . . . . . . . 557 
FIG

31. Sounding of the urethra (Keyes) . . . . . . . . . . $55^{8}$

32. Sounding of the urethra (Keyes) . . . . . . . . . . . 559

33. Relative pesitions of triangular ligament and lulb of urethra (Cul-

ver and Hayden) . . . . . . . . . . . . . . 56 1

34. Handerson gauge. . . . . . . . . . . . . . . . 563

35. Iinlhous bougie . . . . . . . . . . . . . . . . . . . 564

36. ()tis urethrometer . . . . . . . . . . . . . . 564

37. Olivary gum bougie. . . . . . . . . . . . . . . . 565

3s. Nercier ellowed catheter . . . . . . . . . . . . . . . 565

39. Gonley's whalebone bougies . . . . . . . . . . . . . 565

40. (jouley's catheter-staff . . . . . . . . . . . . . . . 567

4I. Cross's modification of Ciriale's urethrotome . . . . . . . . 590

42. Otis dilating urethrotome . . . . . . . . . . . . . . 591

43. Teevan's molification of Maisonneuve's urethrotome with guide $\cdot 592$

44. Suprapulic and rectal routes for the relief of retention of urine (redrawn from Holden) . . . . . . . . . . . . . . . . 594

\section{PLATES.}

PLATE

I. Initial sclerosis of the penis . . . . . . . . . Frontispiece.

2. Chancre and papillary growths of the tongue (Hutchin-

son)... . . . . . . . . . . . . facing page 35

3. Small papular syphiloderm (Stelwagon) . . . . . . . . . 92

4. Iustulo-ulcerative syphiloderm . . . . . . . . . " “ 108

5. Pustulo-ulcerative syphiloderm in a cachectic subject . " "

6. Syphilitic disease of the tongue (IJutchinson) . . . . " * $\quad$ I49

7. Fig. I. Itutchinsun's teeth. Fig. 2. Bacillus of Ducrey

(Petrini de ( ialatz). . . . . . . . . . . . . 296

S. Fig. I. (imococci in gonorrheal pus (Fordyce). Fig.

2. Ginucoccus (Bumm) . . . . . . . . . . . 4 362 


\section{S Y P H I L I S}

AND THE

\section{VENEREAL DISEASES.}

\section{INTRODUCTION.}

THE venereal diseases are for the most part transmitted from one individual to another in the contacts incident to the relations between the sexes. The day is long past, however, when a moral stigma could be affixed to the victim of such a malady by reason of the fact of infection. In the populous and crowded centres of modern civilization the innocent subjects of these disorders are numbered by hundreds and even thousands. They are in a special sense entitled to the encouraging assistance and the sympathetic service of the trained physician.

The great majority of the other victims are patients infected at a time of life when passion is most imperious, self-restraint less strenuously imposed, and the discipline which unfolds the deeper meanings of life is less understood and appreciated. The result is twofold: on the one hand are patients for the most part, fortunately, of an age and possessed of a vigor best capable of enduring without serious shock the perils of an intoxication 
of the system, and in a social state least likely to burden others, such as a wife or a child, with the consequences of disease; on the other hand are the subjects of these infections, who, without fixed habits, are obliged to conform to the rules of best living when actually suffering from their ailments, and who learn lessons which at their time of life are often indelible. The most radical of moral reforms with the best of ultimate results is constantly wrought by the several accidents described in the following pages.

In the early part of this century the prejudices of the people of most English-speaking countries and the odium connected with the acquisition and inheritance of venereal disease extended even to the professional men interested in their treatment. As a consequence, this department of medicine was largely relegated to the charlatan, who, under the control of ignorance and avarice, contributed to the exaggeration and confusion which still cloud the minds of many when they consider the subject.

To-day the change in these particulars is noteworthy. Science has solved some of the profoundest problems and achieved some of its most brilliant bacteriological and pathological victories in the territory once abandoned as a plague-spot. Some of the most cultivated, learned, and distinguished of the physicians of the last quarter of the nineteenth century have been content to labor and to glean in the field that was thus once neglected and abhorred.

It has been well for the race that these men could thus with untiring industry and interest investigate the diseases commonly described as "loathsome." But many of them have paid a price for their courage. It is 
impossible to give accurate statistics of the number of physicians innocently infected with the venereal diseases, and particularly with syphilis, when engaged in the practice of their profession as accoucheurs, surgeons, gynecologists, and those giving special attention to the affections of the genito-urinary organs of both sexes. Hundreds of them have been under our observation and care; thousands have thus suffered in every country. Only with the incessant precautions suggested by the later knowledge on the subject of the pathogenic microorganisms can a physician hope to be successful in the management of these disorders and himself escape their defilement. Nor in his attempts to compass this end can he with safety rely only upon the products of pharmacy and the skill of the chemist. He must be, in his person, his instruments, and his entourage, an embodiment of scrupulous cleanliness.

The disorders usually classed under the general title of "venereal" are syphilis, the several forms of infectious urethritis, and the soft (" simple" or "non-syphilitic") chancre. In a stricter sense of the term, and in the light of modern investigation, there are other disorders described in these pages capable of transmission in the sexual act. The more important, however, of the group are without question those here named. It is probable, though exact statistics are wanting, that infectious urethritis is the most frequent, soft chancre (certainly in particular classes of society) next, and syphilis, in all classes, last. Further comparison teaches that while gonorrhœea is most often a strictly venereal disorder, syphilis is with greater frequency an affection of the innocent; while, as respects a fatal issue, gonorrhœa, in its ultimate results upon the deep urethra, 
the bladder, and the kidneys, probably destroys more lives annually than does syphilis. Gonorrhœa more often than syphilis spares the subjects of tender age, and is further capable of indefinite recurrence in one subject; while for the immense majority of cases syphilis is a disease making but a single attack in the lifetime of the individual. The proposition once held cannot longer be sustained, that gonorrhœa and soft chancre are purely local diseases as contrasted with syphilis, which is admitted to have systemic effects. The generalized results of gonorrhœe are in many instances too striking to admit even of question; and in special cases the perpetuation of the soft chancre for years, with the damage resulting to rectum, vulva, abdominal wall, and thigh, with the production of marked cachexia, often renders that affection one even of greater severity than the milder cases of syphilis.

The questions relating to the history and antiquity of the venereal diseases have created a voluminous literature, with results not wholly satisfactory. The sacred literature of the Hebrews seems to bear record to the fact that blennorrhagic affections existed among the people of a remote antiquity, and that the gonorrhœal discharge was with them confounded with a seminal flow. Among the writings of Greek, Roman, African, and Spanish authors, both medical and literary, evidences are not wanting as to the existence of such a disease and its occasional confusion with other disorders of a venereal origin. Even as early as the beginning of the present century, English physicians confounded gonorrhœe, syphilis, and non-syphilitic chancre, and it was reserved for a comparatively recent date to distinguish definitely and finally between them. 
For the remote antiquity of syphilis there are not wanting authors who find in the sacred writings of the Hebrews, in the sarcastic, poetical, and historical literatures of Greece and Rome, in the ancient documents of Egypt, China, and Africa, and in the volumes written during the Middle Ages, records among the people of those periods of the existence of chancres and of the systemic results of certain genital lesions. The evidence of bones exhumed and supposed to belong to a prehistoric era is also adduced in support of the assumed antiquity of syphilis. On the other hand, there are many who believe that syphilis existed among the American aborigines before the advent in 1492 of Columbus and his companions to the American coast, and that the Spaniards, becoming infected by contact with the natives, brought the disease to Europe, where it appeared for the first time after their return. In the subsequent campaign of Charles VIII. of France against the kingdom of Naples the disease appeared and spread among the nations of Europe to an extent and with a severity before then unknown.

In reviewing the entire subject many of the arguments in favor of the historical antiquity of these diseases are, it must be admitted, weakened by the indefiniteness of the descriptions given. The bones alleged to be both prehistoric and syphilitic are either lacking in the display of unmistakable lesions of that disease or cannot conclusively be demonstrated to be both of prehistoric sepulture and since then wholly undisturbed by the hand of man. The arguments in favor of a prehistoric syphilis in America and of its subsequent deportation to Europe are weighty, but not without flaw. 
The conclusions are simple: the evidence of a remote antiquity for the venereal diseases in general is very strong. In the absence of the definite distinctions between them now established, and of a recognition of the pathological connection between the local manifestations of such diseases and their systemic effects, great confusion has existed in the past. Lastly, the venereal diseases have all, without question, been confused inextricably in the past, not merely with each other, but with a large number of dermatological affections, such as lepra, psoriasis, scabies, eczema, simple and venereal warts, and leucorrhœal and catarrhal discharges.

In the examination of patients affected with one or several of the disorders here considered, a systematic method is of as much value as in other departments of medicine. Besides ascertaining the name, age, residence, married state, occupation, and previous history of the patient, as well as the habits respecting the use of both alcohol and tobacco, it is desirable to know, for reasons that appear later, in the case of both man and woman, the record as respects living and dead children, miscarriages and abortions on the part of a wife, and the relative order of these, as well as the period in pregnancy when a series of abortions or miscarriages occurred. In securing the history of the family and of any antecedent disease it is necessary to record all facts respecting any prior disease of the skin, persistent headache, especially with nocturnal exacerbation, any attacks supposed to be rheumatic, and any persistent or ulcerative affection of the throat, eyes, scalp, or nails.

In the case of venereal disease it is important to know whether the patient can sleep at night without 
rising from the bed to empty the bladder; whether there is pain on micturition, and, in the latter event, whether the pain occurs before, during, or after the passage of the stream.

In the physical examination of patients the several bodily organs should be investigated with care, the surface of the body, when found practicable, being searched for traces of any existing or past exanthem, and particularly for scars, each of which may throw light on the conditions existing. The superficial glands of the body accessible to the fingers should be searched with a view to determining any enlargement or induration. The mouth, nostrils, eyes, and ears require minute observation of lesions present; and even in the absence of the latter the nails may exhibit markings indicative of the character, and at times of even the date, of prior nutritional changes.

In the case of male patients the entire surface of the body may often be exposed for examination, and the genital region then requires detailed inspection. By the fingers and the eye the physician can usually determine the existence of pediculi or nits in the pubic region, an eczema or a psoriasis of the cutaneous surface of the penis or the scrotum, mollusca of the latter region, or the evidence of scabies. By manipulation it will be discovered whether there is an inguinal hernia, a nondescended testis, a left- or more rarely a right-sided varicocele, a gumma of the body of the testicle, or traces of an ancient epididymitis involving the globus minor or major as a sequel of a preceding blennorrhagia. By the fingers alone it will often be practicable to recognize a urethral stricture, a periurethral phlegmon, an enlarged prostate, a syphilitic or a chancroidal bubo, a 
severe phimosis, a subpreputial sclerosis or other lesion, or a urethral chancre. In point of fact, a urethral sclerosis that cannot be recognized by the digit of the trained pliysician is among the greatest of rarities. Indeed, one might here enumerate the entire list of diseases of the ano-genital region, evidences of which the examining surgeon should not permit to escape his observant eye and trained touch.

In all classes of women the examination should be made with the special consideration to which the sex is entitled. A follicular or furuncular affection of the labia, a catarrhal discharge from the vulva, a sclerosis of the meatus or of the fourchette, or a stellate chancroid of the anal region, may often be determined by inspection alone. The physician must know to distinguish between a languette accompanying a syphilitic stricture of the rectum and hemorrhoidal tumor. He must be capable of recognizing the marked differences between a pruritus of the vulva, which is simply tormented by scratching, and an eczema of the same part. By carefully inspecting the dry and "sticky" mouth of a woman it can be determined with reasonable probability, before subjecting the urine to chemical analysis, that an "eczema" of the vulva is due simply to a glycosuria. The fingers should differentiate an inflammation of the vulvo-vaginal gland due to gonorrhoea from a syphiloma of the labium. Scabies of the genital region in a woman will usually be an echo of characteristic burrows about the axillæe or the breasts. By the touch one should be able to discover a hydrocele of the canal of Nuck, a varicocele, a carcinoma, an elephantiasis, a contracture of the vagsina, a laceration, an atresia of the hymen, or a vaginismus. 
Nor should it be concluded in either sex that a determination of the virgin state precludes the possibility of venereal disease. The physician should ever be on the alert to recognize a chancre of the tonsil, an infecting sclerosis of the lip in the child who has kissed a syphilitic nursling, a gonorrhœea affecting the vulva or the eyes of an infant, a paralysis in the middle period of life due rather to a pachymeningitis than to an apoplectic effusion.

Lastly, the physician entrusted with an intimate knowledge of the sources of diseases that are viewed with shame, loathing, and remorse, often imperilling the life of the individual, the safety of the uninfected, and the happiness of a home, has a part to perform which demands a high order of intelligence and sympathy. His it is to protect the innocent, to guard sacredly the secrets confided to his keeping, to conserve the family relation, and at the same time to bring the sufferer to a successful termination of the disease. It is difficult to decide that any one of these functions has a higher importance than another. It is only as the physician discharges his full duty in all points that he ultimately wins that trust and confidence which are the foundation of the largest professional success. 



\section{SY PHILIS.}

Synonyms.-Lues venerea; Morbus gallicus; Pox; "Bad disorder;" Frr. Vérole; Ital. Sifilide; Ger r. Lustseuche; Krankheiten der Französen; Span. Sifilis; Swed. Radezyge.

Syphilis is a general infectious disorder transmitted from one individual to another by both contact and inheritance, chronic in course, and displaying in a more or less determinate sequence symptoms involving one or several of the organs of the body. It is classed with the infectious granulomata, and it is due to the toxic effect of the invasion of the bodily tissues by a morbific germ. Though the identity and relations of the latter have not completely been established (as has been done in the case of the bacilli of tuberculosis and lepra), no doubt can be entertained as to its existence and potency.

\section{ACQUIRED SYPHILIS.}

Syphilis is said to be acquired when transmitted in another way than by inheritance. The term "contactsyphilis" has also been employed to distinguish the former from the latter.

Etiology.-The micro-organisms which are effective in the production of this disease have not yet been incontestably demonstrated. Donné, Hallier, Lostorfer, Klebs, Doutrelepont, Lustgarten, Fordyce, and many others have repeatedly, by difficult and delicate methods of staining, recognized bacilli in syphilitic tissue. The 
failure to distinguish the exact micro-organism whose toxine may be efficient as a cause of the disease is due partly to the fewness of the bacilli present in any one section, to the circumstance that the bacilli found in the smegma præputii are either identical with or very similar to the supposed syphilitic germ, and to a fact pointed out by Fordyce, that the general absence of giant-cells in syphilitic tissue forbids their use as a guide to the location of the bacilli.

But if the germ of the disorder has not yet been distinguished satisfactorily, no doubt exists as to the fact that a germ-carrying secretion or virus, which may be collected on the point of a lancet, is capable of transmitting the disease. This virus must be furnished by a person infected with syphilis.

The purveyors of this virus are usually in an early or active stage of the disease. They may furnish a pathological secretion, such as that supplied by a mucous patch, a chancre, a syphilitic pustule, or an ulcer. Such a secretion may be commingled with a physiological fluid (tears, saliva, milk), and be thus effective, however innocent to the view, though the physiological secretions of a syphilitic subject not thus mingled with a virus are rarely, if ever, noxious. The blood of such subjects is, however, capable of transmitting the disease. Pathological secretions of other character (gonorrhœal, leucorrhœal, vaccinal) may readily be commingled with the virus of syphilis, and thus be effective in its transmission.

The evidence as to the date when the syphilitic subject can no longer furnish an infectious virus is confusing. Up to a recent time it was believed that the late lesions of syphilis (so-called "tertiary") were incapable 
of furnishing such a virus. Instances are, however, on record disproving this; and, though the power to furnish a virus is gradually lost in every surviving subject of syphilis, it is safest to hold that any awakening of the morbid process at a late date may, however rarely, render such persons dangerous to the uninfected.

The modes of infection are both immediate and mediate. The direct contacts of the sexual act (including the perverted and unnatural imitations of the latter) and the opportunities of transmission afforded in kissing, biting, sucking, etc. are often the beginnings of syphilis. In the same category may be named all the accidental contacts which occur in the service of the physician, the nurse, and the midwife, and those where prisoners are manacled together.

The articles which have been mediately effective as virus-carriers are so many and so various as to forbid enumeration. The list includes a great number of household utensils (forks, cups, spoons), articles of domestic use (tooth-brushes, syringes, combs), articles employed in the professions (dentists' forceps, surgical instruments and appliances, razors, vaccinating needles, lancets), and, in brief, almost every substance brought into contact with the human body, from nursing-bottle to water-closet seat, and from the finger moistened in the mouth of the nurse and given to the nursling to the tools of the chiropodist.

Given an infective germ in its vehicle (the virus), furnished by an infected subject of syphilis (in a stage of that disease capable of transmissibility by contact), it remains to inquire whether the person inoculated with such a virus, mediately or immediately conveyed, will suffer from the disease. A categorical answer to this 
question cannot be given. There is reason to believe that all individuals are not equally susceptible to the action of the virus. These reasons are based on the accepted fact of repeated exposures of certain persons without evident results; of repeated exposures with results that are slight, or, if threatening at first, abortive as to any ultimate consequences; and of well-known analogies existing between this disease and others in which the proofs of susceptibility and non-susceptibility of individuals are irrefragable.

All such instances are, however, exceptions to a rule that is enforced by constant experience. The husband recently infected as a result of infidelity to his wife communicates his disease to the latter with almost unfailing regularity; the lover with a mucous patch upon his lip gives his disorder with an appalling certainty to the woman whom he kisses upon the mouth. For practical purposes it is best to assume that all men, women, and children are susceptible who have not been protected either by a previous attack of the disease or (a point to which attention is called later) by the experience of the mother who brings into the world a syphilitic child diseased by inheritance from the father, while she seems to escape.

\section{Chancre.}

Synonyms. - Syphilitic chancre; Initial lesion or sclerosis of syphilis; Hard chancre; Infecting chancre; Ger. Hartes Geschwür; Schanker; Fr. Chancre syphilitique.

The first evidence of a successful transmission of syphilis from an infected to a sound person is termed a "chancre," or, as this last term has often been errone- 
ously applied to non-syphilitic local venereal disorders, better the "initial lesion of syphilis."

The First Incubation.-After the successful introduction of the syphilitic virus into a sound body an interval occurs before the evolution of the initial lesion is appreciable to the eye. This interval is called the "period of the first incubation," a phrase suggestive of the ignorance of the earliest observers. It is almost certain that from the instant of a successful inoculation the subject is, however imperceptibly to human tests, syphilitic, and that there is, without pause or arrest, a multiplication of the effective germs of the disease to the point where the lesions produced by these germs become apparent to coarse methods of observation. This interval is by different observers made to extend over a period of time with singularly varying limits. The average is between twenty-one and twenty-six days, but the period has been claimed to be as brief as from one to two days and as extended as three months. The numerous chances of error in all these estimates need not be pointed out. Between ten and thirty days after infection the vast majority of all infecting chancres appear. The reverse is also true: on the first appearance of a chancre it may safely be estimated that infection occurred previously between ten and thirty days.

The chancre or syphilitic initial lesion appears at the site of inoculation. Its recognition, when first exhibited as the earliest indication of a serious disease, is a matter of the profoundest importance, seeing that the welfare of the individual, and often of others with whom he sustains intimate relations, may be conditioned upon its correct diagnosis.

The chief error committed by the practitioner and 
student anxious to master this problem lies in an effort to identify some particular chancre as a type of all others, and to base a diagnosis upon a comparison of others with this as a type. This is the familiar process by which men recognize in nature a flower or a bird, and in medicine a disease of so fixed a type as a corn or a carbuncle.

The sole constant characteristics of every chancre are - (a) an incubative period preceding its appearance; (b) a sclerosis, induration, or dense thickening of the base of the lesion, widely varying in grade and duration with different chancres; (c) a simultaneous enlargement and induration of the gland or glands in nearest anatomical relation with the chancre, constituting the "syphilitic bubo," or primary adenopathy. The first of these constant characteristics is an historical symptom, a knowledge of which may be withheld from the practitioner at the date of his examination. The last, though wellnigh constant of occurrence, may not have been declared fully at the date of the examination, or the glandular enlargement may be so slight or so deeply situated as to escape detection. It follows that in some cases it is possible that at a given moment the sclerosis may be the sole chancre-symptom present whereby the nature of the disorder may be declared. Yet there are several nonconstant symptoms which can usually be recognized without difficulty, and which leave the observer in little doubt as to the diagnosis. These symptoms are for the most part explained later.

A chancre is a modification of the sound or pathologically altered skin or mucous membrane, occurring after syphilitic infection, and displayed after an incubative period, characterized by a circumscribed sclerosis 
of tissue, and accompanied by an enlargement and induration of neighboring glands. Every chancre means a syphilis, mild or severe, that will follow. Every case of acquired syphilis points to a precedent chancre, recognized or unrecognized. Every chancre, further, is a symptom not merely of a syphilis that will follow, but of a syphilis actually present. The proof is found in the fact that infection of a sound individual from such a chancre is followed by the development not merely of a new chancre, but also of a new syphilis.

It is important to note at the outset, considering the definition given above, that a chancre may be either an isolated first lesion of syphilis or a modification of some symptom of another disease. Briefly, the study of chancres is the study less of lesions than of a series of singular modifications of lesions recognized in many other diseases, which, under the influence of syphilis, take on new aspects and undergo singular metamorphoses. Thus, the chancre may develop upon the sound skin of the arm as a consequence of intentional experimental inoculation, or upon the sound mucous membrane of the vulva as the result of infection in the sexual act. It may also originate as an untoward modification of a "cold sore" (herpes labialis) of the mucous membrane of the lip infected in the act of kissing, or be a significant change in the evolution of a vaccine vesicle, a blister on the finger, or an excoriated nipple.

Chancres may thus be represented at one time or another by every recognized lesion of the cutaneous surface, including the macule, papule, vesicle, pustule, bleb, tubercle, tumor, and ulcer. Only the most common types can here be enumerated conveniently.

Erosion (Superficial erosion).-This is the least con- 
spicuous, the oftenest ignored or misunderstood, and yet the commonest of chancre symptoms. It is recognized as a roundish, oval, or quite irregular macule or spot resting, soon after its evolution, upon a delicate bed of induration, giving to the touch the sensation of a thin sheet of parchment or of mica let into the underlying tissue. It is usually distinctly circumscribed, and exhibits a shallow or scarcely depressed erosion, centrally fixed or involving its entire face. In size it varies from a large pin-head to a bean, and may be many times larger. Its color is dull-reddish, grayish, or even whitish; it often resembles in hue a section of raw ham. It may be dry and glazed, or slightly moist and secreting a thin serum which glues to its surface any dressings that may have been applied to it. At times it has a grayish-white film over its face, and may even have a diphtheroid aspect. It may be uniformly level with the neighboring skin, or its edges may be raised and its centre slightly depressed. It very rarely suppurates freely or degenerates into a well-marked ulcer. These complications usually result from external irritation (caustics, mixed infection, urine flowing over the site, as in urethral chancre). The accidents of phagedena and sloughing are still rarer. When these chancres survive until general syphilis is declared, they are gradually transformed into symptoms of general syphilis, readily enlarging to elevated, granulating, rarely hemorrhagic masses smeared with a highly contagious puriform mucus and merging thus into the mucous patch and condyloma.

These erosions may be lifted away from their original sites by extensive underlying scleroses, and be thus greatly modified in appearance. They are then changed from flat macules to large-nut-sized and even larger 


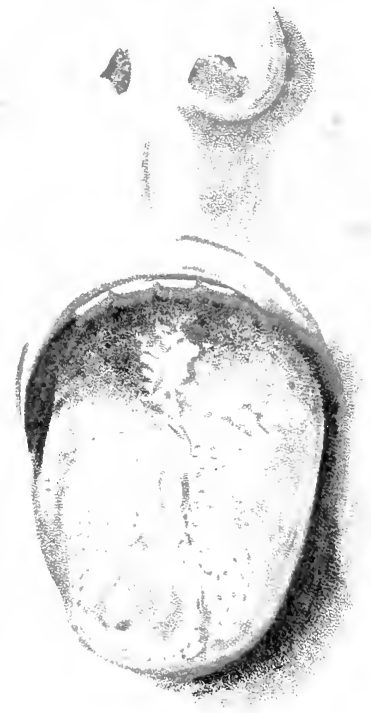

Chancre and papillary growths of the tongue (Hutchinson). 

irregularly outlined masses, ridges, and deformations of the lip, the vulva, or the preputial rim-favorite sites for their development. These odd-looking swellings, unlike each other and conspicuous chiefly for their irregular bulging, often as firm as ivory to the touch, are capped at one point or another by the smooth, shallow, dry and glazed or slightly secreting erosion described above. All are essentially giant-papules, undergoing a special evolution because of the pressureand friction-effects of their particular environment.

Papule (Dry scaling papule; Non-ulcerating, indurated papule).-This is the common result of inoculation of the skin as distinguished from that of the mucous surface. The chancre is here evolved as a pea- to a beansized papule or papulo-tubercle, indurated at the base, dry, scaling, and colored in various shades, according to its situation. It is occasionally seen upon the skin of the penis as the result of accidental infection of that part, and upon other cutaneous surfaces, as the thigh and the arm, as the result of accidental or experimental inoculation.

Ulcer.-Ulceration of the chancre is probably in every case the result of local irritation. This irritation may be accidental, as in the case where improper dressings or applications are made to the lesions, or intentional, as where savin cerate has been applied or horse-hairs have been passed through the base for the purpose of exciting suppuration with a view to supplying a virus for purposes of experimentation. Two types of ulceration may be recognized in chancres, the shallow and the deep. Both occur in beds of induration. Their causes have been discussed above; maceration (by mucus, by leucorrhøeal and blennorrhagic discharges), friction, improper 
treatment by local applications, filth, and neglect may all be cited as of consequence.

Shallow and superficial ulcers, scantily secreting serum, are usually imbedded like erosions in thin sheets of induration, but they may cap considerable elevations of tissue. Their edges are sloping, almost never clean-cut, punched out, or undermined; their floors rarely slough; their outline is irregular. At times they resemble shallow fissures, especially on the side of the frænum; at others they form at the bottom of a crevice between two walls of induration, as when the sclerosis involves the mucous membrane of both the corona glandis and the adjacent prepuce.

Decp ulccration of chancres invariably results from the action in excess of the causes suggested above, or from similar agencies. The "Hunterian chancre," so named because Mr. Hunter believed that it was the sole precursor of general syphilis, is a deep excavation in a large mass of induration. This crateriform ulcer is roundish, oval, or very irregularly shaped, often with a floor set in an angle, presenting thus the aspect of a deep fissure in a neoplasm. Its secretion is commonly scanty, though when profuse it may be hemorrhagic; its edges are sloping; its rim is densely indurated, capping a tumor-like mass varying in size from a hazelnut to that of a pullet's egg.

Mixed Chancre.-By this term is generally designated a venereal lesion which at the outset, usually a brief time after infection, exhibits all the characteristic features of the soft chancre ("chancroid," "chancrelle," etc.), but which, after a due incubative period has elapsed, becomes specifically indurated at the base, is acconpanied by syphilitic bubo, and later is followed by gen- 
eral syphilis. This accidental implantation of the virus of syphilis upon a soft chancre (or upon its site before the appearance of the latter) is analogous to the complication which ensues when a herpetic vesicle ("cold sore") of the lip or a cigarette-burn of the same region becomes infected with the virus of syphilis. In these cases it is the modification of the original process that announces the syphilitic complication.

The chancroid or "soft chancre" is essentially a pustular lesion, and its purulent secretion, whether from pustule or from suppurating abrasion or fissure, is indefinitely auto-inoculable, as distinguished from the secretion of the syphilitic initial lesion, which is scanty and non-auto-inoculable; hence all infecting chancres secreting an auto-inoculable pus are of the "mixed" type. The bubo, also, accompanying the soft chancre is usually inflammatory and has a tendency to suppurate, as distinguished from the dense multiple buboes of syphilis, which rarely suppurate and are often non-inflammatory in type. It follows, then, that the buboes of "mixed chancre" may exhibit the features of one or the other of the two disorders thus commingled. The important point to recognize is that syphilis may ensue after the occurrence of "mixed" chancre; and this possibility should never be forgotten in making the prognosis of any suspicious venereal sore. The individuals most often exhibiting these "mixed" chancres are of the pauper class frequenting public dispensaries and out-patient departments of hospitals-persons whose female associates are as uncleanly as they are vicious.

Another "mixed" variety, in the light of modern science, is the chancre of syphilitic origin that is also later infected with micro-organisms. This complication is 
more common than is generally supposed. All the pus cocci, several of the mucors, and a large number of foreign substances, usually inert, may often be recognized in chancres, especially in those of the filthy, but also of those who never previously suffered from venereal disease, and who, in ignorance or as the result of improper advice, suffer from neglect of cleanliness or from positive aggravation of the original disease.

Chancres of the Syphilized.-Persons infected with syphilis have usually but one attack in a lifetime. The exceptions to this rule are so rare as simply to enhance its value and importance. But the recent as well as the veteran victims of that disease expose themselves to it and to other venereal diseases with results which demand exact recognition.

Such persons, of course, may contract "soft chancres." But when exposed to fresh sources of syphilitic virus they occasionally exhibit, as a result, chancres of a formidable type and an obscure character, requiring some expertness for their proper recognition. Some of these results are (a) lesions like soft chancres, but atypical, less clean-cut at the edge, with much less purulent secretıon, and non-auto-inoculable; (b) slightly indurated chancres, strongly resembling the initial erosion chancre, without accompanying syphilitic bubo, and disappearing without leaving results of consequence; $(c)$ large indurations with deep central excavation, at times strongly resembling the "Hunterian" chancre, yet without bubo, and yielding completely to proper internal treatment. Some of all these are, without question, gummatous (socalled "tertiary") lesions of general syphilis, occurring with reawakened activity where, at the site of invasion, new bacilli have been introduced. Yet rarer are $(d)$ pea- 
sized and larger, exceedingly dense, circumscribed thickenings of the genital region, without erosion, ulcer, or hyperamia, and due to the causes named above.

Location of Chancres.-As distinguished from chancroids, which are very rarely extra-genital in site, syphilitic chancres may occur upon any exposed portion of the body-surface; very rarely indeed do they develop at long distances from the mucous orifices of the body (as, for example, in the bladder, osophagus, stomach, etc.). The genital region of the two sexes is most often involved merely because of the frequency of transmission in the ample opportunities of the sexual act. In this way the balano-preputial sulcus, the rim and inner face of the prepuce, the frenum, glans, and integument of the penis, the scrotum, the inner face of the thigh in contact with the latter, and the perineum become common sites. Urethral chancres are rarely. deeply situated, but they may commonly be recognized at the tip of the glans in men, where the indurated mass encroaching upon the limits of distensibility of what may be termed the "urethral nozzle" produces so much local irritation and consequent sero-purulent discharge that the symptoms are often mistaken for those of a blennorrhagia. When the glans in these cases is grasped firmly between the thumb and the finger, the induration may be felt, resembling a short section of a clay pipe let into the submucous tissue, and at the moment of pressure a characteristic whitening of the rim of the labia of the meatus urinarius bears witness to the extreme thickening of the initial lesion.

In women the labia majora and minora, the fourchette, the os uteri, the clitoris, the vestibule, the meatus urinarius, and, very rarely, the point of the superior 
commissure of the vulva are the usual sites of chancres. In these situations their transformation in situ to condylomata, mucous patches, and other secreting lesions of systemic disease is readily effected in consequence of the heat, moisture, and friction to which they are here exposed. In women the deformities of the genital region, venereal in origin, are commonly of exaggerated type, and, as a rule, in fetor, in abundance of secretion, and in volume they far exceed the corresponding lesions of the other sex.

Chancres of the vagina are rare; when they occur they usually escape observation. They are probably more common than is set down in the statistics of the malady. Chancres of the mucous envelope of the cervix are usually visible on its anterior limb. They are reddish or empurpled excoriations with an engorged areola; their face is often covered with a pultaceous and adherent film. In the genital chancres of women the inguinal glands usually escape involvement.

Extra-genital chancres are not of rare occurrence in the larger cities, and, as already pointed out, may be recognized in every region of the body. The most frequent sites are the lips, fingers, nipples, anus, tonsi's, tongue, nares, thighs, arms, and toes. They result from the contacts incidental to kissing, sucking, biting, vaccinating, the smoking of pipes, the nursing of children at the breast, the practices of sodomy, digital explorations and operations of the accoucheur, physician, and surgeon, and from many accidents of daily life. They belong, without exception, to the types of chancre already described, invariably following periods of incubation, occurring with well-marked induration, and accompanied by adenopathy of the glands in the vicinity 
of the infected part. Some are densely indurated fissures (nipple, anus, lip); some are indurated dry papules (as after vaccination, biting, tattooing); some are flattish plaques of a dull-red hue, or ulcers covered with an ashen paste (tonsils, tongue, uterus); some are irregularly shaped tumor-like masses (lips); some, finally, are simply symmetrical ovoid thickenings of normal tissue (finger, toe, hang-nail, etc.).

Number of Chancres.-The initial lesions of syphilis are seldom multiple; most often they are single. If dual in number or more numerous, they are, as a rule, multiple from the beginning. In these cases the inference is just that there has been a simultaneous accidental inoculation of all such points at a given moment. The non-auto-inoculability of the secretion of the initial lesion forbids its multiplication upon the person of an individual once infected, even as the result of an accident. The auto-inoculability of the pus of the "soft chancre," on the contrary, offers abundant opportunities for its spread from one point to another of the subject of the disease, and at the same time furnishes ample supplies for infection at any given moment in several points simultaneously. It follows that while in exceptional cases a patient may exhibit at one time two or three initial lesions of syphilis on his person, he never compares in multiplicity of chancres with, for example, a woman whose labial sores have supplied a pus streaming over the perineum where fifty, and even a hundred or more, soft chancres may at times be counted.

Induration of Chancres. - The specific induration of the initial lesion is one of its constant features. This sclerosis is recognized by the sense of touch in varying 
degrees as a distinctly defined thin plate or sheet of inelastic tissue let in beneath the excoriation, ulcer, etc., or as a dense mass with the hardness of ivory or cartilage, varying in size from a split pea to that of a pullet's egg, and even to masses still larger. At times the sclerosis is so dense as to suggest the hardness of marble. All these grades of induration are in part correlated to the degree of irritation to which, after its complete evolution, the chancre is subjected. The situation of the chancre is a factor determining in part the extent of the induration, as chancres of the vagina are proverbially less indurated, and those of the mucocutaneous borders (lips, preputial orifice, etc.) more conspicuously sclerotic, than others. The induration may precede or follow (much more often the latter) the evolution of the chancre, or it may first be observed at the moment of detection of the sore itself. The very late occurrence of induration in a chancre is usually a portent of good, as a delay of from twenty to thirty days after the appearance of a lesion supposed to be a precursor of syphilis usually negatives the expectation of that disease. The sclerosis may disappear before the healing of the chancre, or, what is quite common, may persist long after the involution of the latter, and even long after the occurrence of general symptoms. Occasionally one may recognize the pigmented, pigmentless, or sclerotic, keloid-like relics of induration six months after infection, and even after all symptoms of general syphilis have for the time disappeared. Sooner or later the induration always wholly disappears, and for the most part leaves behind it no traces of its existence, these facts seeming to bear no relation to the future of the patient. The so-called "relapsing indurations" are 
usually syphilomata, evidences of general syphilis, socalled "tertiary gummatous infarctions of the genital region."

The Portent of Chancres.-While it is true that every initial lesion of syphilis signifies that a syphilis, mild or grave, will ensue, it does not follow that from the number or the appearance of chancres a prognosis may be made as to the severity or the reverse of the ensuing disease. An exceedingly insignificant looking ham-colored spot in one individual may be followed by the most malignant form of the disease, and may lead to a syphilis of the second generation that may destroy in succession the fruits of a wife's pregnancies; while a group of three gigantic masses of sclerosis, each with excavations of an ulcerative type, may be followed by even meagre results. The reason for this disproportion may be found, as some allege, in the activity of the germs present, but it is more probably due to the kind of soil in which those germs are implanted.

Duration of Chancres.-Chancres may persist until the evolution of systemic syphilis. They may, however, be resolved and disappear almost wholly at an earlier date. When persisting still later, they are always changed to conform to the type of the general symptoms of the disease, and are in reality no longer chancres, but condylomata, granulating mucous patches, gummata, etc. When persisting to such a late stage, they usually announce the fact by significant changes, such as elevation of the surface, tumefaction of the mass, softening of the sclerosis wholly or in part, and hypersecretion.

Termination.-Chancres may terminate by complete resolution. However numerous and formidable in 
appearance, they rarely result in any mutilation of the part in which they have been seated. The simplest lingering traces of their existence are either moderately pigmented patches, such as occur on the skin of the penis in young subjects with very dark hair and eyes, or, as a sequence of such pigmentations in that class of individuals, even non-pigmented plaques as large as the original chancre, being, in fact, pigmented spots whence the pigment has slowly been removed. Chancres seldom leave scars, for the reason already given, namely, their indisposition to undergo ulceration. In this respect they are strongly distinguished from soft chancres, which, as a rule, suppurate and ulcerate, and often leave punched-out scars as relics of their ravages. IVhen syphilitic chancres actually leave scars, these are always the result of ulceration, and this ulceration is the fruit of some accidental complication of the local disease. Thus, the chancre of the urethra lies just where the stream of urine several times in the day necessarily passes over its entire face, and, this fluid being in a high degree irritating in consequence of the urinary salts it contains, the chancre often secretes quite freely, and may leave an odd-looking scar at the tip of the glans penis, this organ, after all is healed, looking as though it had lost its apex, while the external urinary meatus has for a distance of perhaps half an inch or more a "reamed-ont" aspect. Deeply-cauterized and filthy chancres, as well as those of "mixed" type, may leave small cicatrices. It follows that in making examinations for the army and navy and for life insurance, the non-discovery of scars upon the progenital region of men does not prove that they have not had a preceding syphilis, and the actual discovery of such scars in the 
progenital region is by no means conclusive that the subjects of the same have been syphilitic.

Diagnosis.-The diagnosis of the initial lesion of syphilis is made chiefly by a careful study of the symptoms already detailed. By the recognition of these special characters, rather than by the exclusion of the symptoms of other diseases, is the end best reached.

The chancroid or " soft chancre" is usually a pustular lesion, and is represented either by an unbroken pustule surmounting its characteristic sharply-cut ulcer, or, after the rupture of the pustule, by the pus-bathed ulcer itself, circular, oval, stellate, or linear in outline. However engorged its base, the latter is never indurated save in the "mixed" variety. There is no period of incubation, and, though at times single, the lesions are usually multiple and often exceedingly numerous, scores forming in extreme cases. The adenopathy of chancroid is represented usually by a single though occasionally by a double bubo. Rarely many buboes occur of a distinctly inflammatory type, with a tendency to suppuration and the production in the lips of the wound, when there is spontaneous bursting of the gland-abscess, of a chancroid by secondary infection. The purulent secretion of the chancroid is practically indefinitely autoinoculable- a fact accounting for the multiplicity of the lesions in many cases. Chancroids are usually genital in situation; rarely are they extra-genital, with the exception, particularly in filthy women, of the anus. The floor of the chancroidal ulcer is usually covered with a more or less tenacious slough resembling wet chamois-skin, and presenting in this particular a marked contrast with the shallow, scantily secreting, indurated, and sloping edges and floor of the initial lesion of 
syphilis. Lastly, the accidents of sloughing, phagedena, and enormous involvement of the skin and the subcutaneous tissues of the thigh in ulcerative and burrowing sinuses are almost unknown in syphilis of the cleanly, and are by no means of very rare occurrence in chancroids of all classes of patients.

The lesions of hurpes progenitalis are very readily differentiated from syphilitic chancres. The former are transitory, lasting at the longest for but a few days-a feature of prime importance in establishing a diagnosis, for any so-called "herpetic lesions" followed by ulcers lasting for ten days are probably not such, and should be viewed with great suspicion. Herpetic lesions in the progenital region are essentially vesicular, and are visible either as vesicles or as the relics of vesicles in the form of very superficial reddish plaques, where delicate and lightly-tinted crusts appear, or as slightly raw and tender, finger-nail-sized spots, furnishing a serum sufficient in quantity to moisten an applied bit of cotton. Their cause, further, may often be determined without great difficulty (venery, pollutions, gastro-intestinal derangements such as constipation, chills, gouty attacks, etc.).

Balanitis.-In this affection, as in herpes progenitalis, the disease, as distinguished from all varieties of chancre, is always short-lived and yields readily to treatment. In typical lesions the mucous membrane of the sac of the prepuce and of the glans penis becomes reddened, tumid, and in extreme cases of a deep purplish hue, with superficial excoriations of the external layer of the membrane in plate-like, finger-nail-sized plaques, which can be studied best in a well-marked case of blennorrhagia of the conjunctival membrane. There are distinct sensa- 
tions of itching and burning in the part, and the odor of the secretions is usually nauseous in consequence of the altered character, in this part, of the secretion from the glands of Tyson. There is no induration, no glandular complication, and never ulceration. The disorder is usually relieved, when not complicated, in the course of a few days by the application of a stimulating vinous lotion aided by astringents, a thin layer of absorbent cotton being interposed between the two folds of membrane in contact.

Verruca Acuminata ("Venereal warts," Moist warts, Condylomata, etc.).-Filiform, papilliform, single or multiple, often numerous, vegetations may develop, for the most part in the progenital region of the two sexes. These warty growths are usually pedunculated, but at times are flattened. They secrete a mucoid fluid of offensive odor; this fluid in syphilitic subjects is highly contagious. The growths vary in size from a pin-head to compound masses as large as the fist and even larger. As distinguished from chancres, they are never indurated, they rarely ulcerate, they are not accompanied by adenopathy, and they survive for periods of time far outlasting the life-history of even persistent initial lesions of syphilis. They may occur in virgins, but they are more common in the subjects of venereal disorders, as also in those suffering from leucorrhœal and other pathological fluids bathing the genital region. Rarely they have an extra-genital site, such as the face. In males they are apt to form in the sulcus behind the corona glandis, about the frænum, in the external orifice of the urethra, and over the scrotum; in women, chiefly about the fourchette and the labia. They are readily recognized by their resemblance to the comb of a cock, by the absence 
of ulceration and of induration of the base, and, when wiped clean, by their florid aspect and their readiness to bleed when scraped or cut away.

Epithelioma of the genital organs occurs most commonly after the middle periods of life in both sexesages when chancres are decidedly of less frequent occurrence than at others. In men the most frequent site of the disease is the glans penis, where a circumscribed, flattened papule, verrucous elevation, or shallow erosion may occur. The period of duration of these lesions is for most cases far greater than that of either chancre or gumma. The base of one or two of these primary growths may become indurated and the neighboring glands may enlarge; but the inactive, often slightly hemorrhagic or crusted papule or warty growth seated upon an infiltrated tissue, with an ulcer forming only after a long evolution of the primary symptom of the disease, is not to be mistaken for a chancrous lesion. When actually ulcerating, the resulting ulcer is of the type of the epitheliomata of the skin in general, with serous, scanty, or bloody secretion, everted edges, and excavated, often eroded, floor. For women the region of preference in the progenital forms is the clitoris, where the lesions above described may occur occasionally with striking deformity of the parts. The noninflammatory, often scarcely colored thickenings, erosions, warty growths, etc. of both labia and clitoris, in women past the menopause, are all to be separated from chancrous changes.

Molluscum epitheliale of the genital region in young persons, especially those of the male sex, is characterized by the occurrence, on the scrotum chiefly, of split-peasized, yellowish-white, waxy-looking, and imbedded or 
projecting bodies, usually exhibiting at one point or another of their globular surface a whitish or blackish punctum representing the occluded orifice of a sebaceous gland. They may be few in number, but often they are exceedingly numerous, studding the region affected with isolated but closely approximated lesions. They are never ulcerated, indurated, inflammatory, nor the seat of evidence of any acute process. It is impossible for the trained physician to mistake them for chancres, but the error is occasionally made by young and timid lay patients, who, having for good reasons become anxious about exposure to disease of the affected part, discover for the first time, on careful scrutiny, the molluscous bodies, and are filled with terror at the sight. There is never any glandular complication of these simple lesions, and in any doubtful case the expression of the cheesy mass from the orifice of the gland would establish the diagnosis.

Lichen planus of the genital region, particularly in the male sex, is at times liable to be mistaken for chancre. But the lesions are always papular, dry, and flattened at the apex, with a singularly characteristic polygonal outline, often very sharply defined. They are never seated on an indurated base, are not accompanied by glandular enlargement, are not eroded nor ulcerated, and are usually multiple, with at times marked invasion of the skin of the lower belly and the adjacent region of the thighs. An interesting feature of lichen planus of the genital region is the grouping of the lesions in lines, so that at times half a dozen or more of the small crimson, reddish, purplish, or dull leaden-hued papules stretch in a direct line from one point to another over the dorsum of the glans penis, in the skin of the organ. Angular as well as rectilinear figures, and even odd-looking 
cockades, may thus be formed. Lichen planus lesions of the genital region are often the seat of intense itching, and may be well scratched with the evidences of such traumatism upon and about them. They commonly persist for a period of time much longer than that limiting the continuance of chancres.

Psoriasis of the genital region is exposed in welldefined disks covered, as a rule, with large-sized laminated scales, the disks varying in size from a pin's head to that of a silver dollar and even larger. They occur upon the skin of the penis and the scrotum, with frequent involvement of the pubic region, lingering near the line of the hair and projecting beyond the latter upward and downward. The absence of secretion, of induration, of ulceration, and of glandular complication, and the frequent presence of the disease in other regions of the body, suffice to determine its character.

The late gummatous ("tertiary") lesions of general syphilis occurring in the genital region are exceedingly liable to be mistaken for chancres. Here the diagnosis rests upon the discovery, elsewhere upon the person, of the relics of a preceding syphilis, the frequently obtainable history of such a disease, the well-marked tendency of the late deposits of syphilis to ulcerate and spread by serpiginous destruction of the tissue involved (a rare complication of infecting chancres), and often upon a history of persistence of the gummatous thickening or ulceration for a time longer than that required for the fullest evolution of both chancre and general consecutive syphilis. The chancres of the syphilized, previously described, are often illustrations of this singular process, suggesting the origin of the mucous patch in the mouth of the tobacco-chewer, and in doubtful cases only the 
most careful study will suffice to distinguish between the two.'

Pathological Anatomy of Chancres.-As the syphilitic chancre is like and unlike all other cutaneous and mucous lesions, and as the eruptions of syphilis are like and unlike all cutaneous affections, so the minute anatony of chancres resembles that of many other pathological formations. Under the microscope one finds granulation-cells within reticulated fibrous meshes, and cell-infiltration partially or wholly blocking up the lumen of the vessels. Where erosions have occurred, naturally the epidermis is in various degrees removed, and the papillæ, with little or none of the rete left, are exposed or are even in great part removed. The characteristic induration of the chancre is due in part to new-formed connective tissue and in part to epidermal thickening. It is highly probable, however, that the lymph of the part is profoundly affected by a special ferment produced by the bacilli responsible for the disease, when the latter first multiply in exterior regions of the body. The absence of dense induration of chancres of the vagina and the cervix points conclusively to the fact that induration is a condition of site rather than of infarcted vessel or of multiplying connective-tissue fibres; and the extreme indurations seen at the muco-cutaneous margins of the body point equally to the action in those regions of a special influence upon the effective germs of the disease.

Treatment of Chancre.-Persistent efforts have been made from time to time to set aside the possibility of

${ }^{1}$ For a tabulated summary of the diagnostic differences between chancre and other genital lesions, consult the section devoted to the subject of Chancroid. 
syphilis following chancre by the radical destruction of the latter. The reasonableness of success in these efforts is on a priori grounds so great that in all probability they will never be abandoned wholly, but the actual results have thus far been disappointing and, for reasons that need not here be set forth, are enveloped in considerable doubt. The destruction of chancres by chemical agents and by the actual cautery has repeatedly failed not only to relieve the local symptoms, but also to prevent the occurrence of general symptoms. The same may be said of total excision of the primary lesion, and even of total excision of both primary sore and all the glands in the neighborhood involved in the disease. When the chancre, as is usual after cauterization, exhibits increased induration of its base, even though it may not be affirmed that the ensuing disease is the graver for the complication, it is certainly true that the chancre is less manageable than before. In some cases exceedingly grave destructive ulceration following gumnatous deposits has occurred in patients where these attempts have been made with all possible precautions to jugulate the disease in sound men. In this connection it must not be forgotten that even experts may be deceived in the recognition of both the chancres of syphilis and the lesions closely resembling the latter; and this possibility of error should not be ignored by the practitioner who is reasonably judicious. There is still a division among authorities on the question whether the initial sore is merely a local point from which, after sufficient multiplication, the microbe of the disease or its toxine is swept through the general economy, or whether the chancre is the local expression of an intoxication generalized at the outset. 
All chancres should be treated by strict observance of the requirements of hygiene. The affected part should be cleansed with warm water and soap, after which washings in hot borated solutions should be employed. In the event of tenderness or pain, as in the case of chancres of the pendulous portion of the penis, the part, when practicable, may be immersed in hot solutions of boric acid often each day, or even in extreme cases for hours at a time. After drying, the chancre should be well dusted with powder, such as boric acid (or boric acid and talc, I part to 4 , when the acid itself is at all irritating), europhen, aristol, hydronaphthol (I part to Ioo of fuller's earth), calomel (or I part of the latter to 3 or 4 parts of starch), or iodoform when the odor of the drug can be tolerated, and especially in the case of painful, intractable, or irritable chancres. When erosions form, having a raw, reddish, slightly secreting surface, and also when ulceration occurs, it is generally well to paint the surface of the sore, after the washing and before the application of the powder, with a solution containing from I to 2 grains of the bichloride of mercury in the tincture of benzoin. The drying, over the eroded surface, of the gum thus medicated is usually not unpleasant to the subject of the disease, and is also cleanly, protective, and efficient as a parasiticide. After all applications have been made, the surface, when practicable, should be guarded from contact with neighboring parts, not with a view to the prevention of autoinfection (which in these cases is not to be feared), but in order to set aside the possibility, always great, of irritation of the sore. In the instance of chancres which may be by this means sufficiently well dressed (sac of the prepuce, frænum, etc.), when practicable, it is well to 
draw the foreskin well over the interposed lint. As a rule, when the chancres are large and tender the male organ should be wrapped in a thick jacket of mercurialized wool and be brought up in the line of Poupart's ligament before the clothing is readjusted. Elastic or other ligatures should never be fastened about the penis.

In women the labia, when similarly affected, should be separated by antiseptic cotton, and for chancres of the cervix pledgets of lint, after dressing the part, should be pushed against the os with tampon supports. For these chancres the mercurialized benzoin lotion is an excellent application. Lotions often useful when chancres prove irritable under other treatment are the ordinary black wash, pure or diluted, and applied by the aid of moistened pledgets of lint; tannic acid and red wine, I part of the former to 30 of the latter; in some cases the lead-and-opium wash. As a rule, all salves and unguents are to be discarded in the management of chancres. The chief exception to the rule is furnished by lesions which secrete a fluid gluing the dressing so tightly to the part that when the lint which has been applied is removed no little pain is experienced and slight hemorrhage ensues. In this event, after the applications described above, the sore should be dusted, and then there should be applied lint on one face of which (that next the chancre) has been smeared carbolized vaseline.

In the management of chancres of the urethral orifice a bit of medicated lint may be introduced into the gaping orifice if required; but the most important of the measures to be followed is the immersion of the penis, whenever practicable, in a basin of warm water at the time of each urination, as by this means the urine is in a measure 
diluted at the time of its traversing the sore. Weak solutions of the nitrate of silver, employed not as a caustic agent, but with a view of making a satisfactory dressing of an eroded surface, may also at times be used with advantage.

The question whether the treatment of syphilis should be begun at the date of recognition of the chancre or at the time of the appearance of symptoms of general syphilis is considered elsewhere. Internal medication, however, of a patient affected with a chancre which has been recognized as indubitably an initial lesion of syphilis is by no means necessarily the treatment of that syphilis. Infecting chancres are peculiarly responsive to a properly-directed treatment by internal medicine, and the refusal to employ the latter is unwarranted when the diagnosis is practically assured and the lesions are either painful, portentous, or the source, as is often the case, of much mental distress to the patient. Mercury by the mouth is in these cases the one efficient remedy. It should not be ordered unless the diagnosis is satisfactorily established. In the majority of all cases coming under the management of experts the diagnosis is practically ensured from the first, either as the result of confrontation (discovery, in the person from whom infection was received, of lesions capable of conveying the disease) or of the recognition of classical features of an initial sclerosis in the person acquiring the sore.

It should not be forgotten, then, that the mercurial treatment of this period is a treatment directed to the chancre, and not to the as yet undeveloped disease to which the chancre points. If this be borne in mind, the end is readily reached. The metal should be used 
or disused, pushed to a higher dose or reduced in the quantity administered, according as resolution of the sclerosis is announced, the erosion begins to heal, and the affected part to assume its normal character as to size, color, and freedom from obvious lesions.

The preparations employed are those useful in the general management of syphilis, to which reference may be made. The protoiodide of mercury in doses of from $\frac{1}{10}$ to $\frac{1}{2}$ grain, calomel in doses of from $\frac{1}{10}$ to $\frac{1}{6}$ grain, gray powder, the bichloride (less preferable for this special purpose, as, in the doses ordinarily well tolerated, its operation is slower), and the biniodide may each be employed. In all cases of anæmic patients the hygienic and tonic remedies useful in the management of general syphilis should regularly be employed.

"Mixed" chancres do not call for destruction by cauterization. They are often tender and painful, and in these cases much trouble may be avoided by early and persistent use of the hot borated immersions and washings described above.

The buboes accompanying syphilitic chancre often require no treatment beyond that advised for the initial sore, whether local or by internal medication, as the progress of the one toward a favorable issue, or the reverse, is usually proportioned to the improvement or aggravation of the other. Often the glands are neither large, painful, nor tender, the patient scarcely appreciating the fact of their undue size and hardness. In other cases they are voluminous and are the source either of local distress or of discomfort experienced in the movement of neighboring parts (leg, thigh, arm, jaw, etc.). In this condition frequent ablution of the glands with water as hot as can be tolerated is the best, simplest, 
and most grateful method of treatment, followed, when needed, by a weak mercurial salve well rubbed into the skin covering the glands-ammoniated mercury, Io grains to the ounce of lanolin and vaseline; mercurial ointment, I part to Io of simple unguent. To either salve, when there is much pain and tenderness, a small quantity of the extract of belladonna or of the watery extract of opium may be added. These unguents should, however, be not too amply supplied with drugs of the narcotico-stimulant class, as, even in the strength of 10 grains to the ounce, systemic effects have been induced after application to the groin.

In the event of "mixed" bubo there commonly results abscess of the glandular contents and either spontaneous or artificial opening of the same. The treatment of this complication is that of the bubo of soft chancre.

With the healing of the chancre, when this is secured before the onset of general symptoms, ends a tolerably well-defined phase of syphilis, the period once called that of "primary syphilis," the period of lesions for the most part localized. Yet here, even at the outset of the evolution of the disease, it is made clear that no sharply-defined limits or periods are observed. For, as has already been shown, the chancre may at times persist long after general symptoms have been declared, and traces of it may be discernible even when grave complications of general intoxication have occurred.

The Evolution of Syphilis in Stages or ExcurSIONS.

In the early part of the present century Fernel and Hunter were followed by the late eminent Philip Ricord in establishing for the evolution of syphilis an artificial 
system based upon chronological data. This system commended itself to the medical men of the scientific world, and as a result it secured at an early date almost universal acceptance. According to this scheme, there were three "stages" of the disease: a "primary" stage, inclusive of the period of the infecting chancre and its accompanying adenopathy; a "secondary" stage, lasting from a few months to two or three years, in which appeared most of the syphilodermata and the disorders of the appendages of the skin and the mucous membranes; and a "tertiary" stage, lasting indefinitely from the close of the secondary period until a final result was reached either in one direction or the other, with the absolute cessation of the malady. In this latter stage occurred most of the affections of the deeper tissues, of the viscera, the bones, the testes, the nervous centres, and the fibrous and subcutaneous structures. The "secondary" was supposed to follow the "primary," and the "tertiary" was supposed to follow the "secondary" stage.

The objections to this chronological scheme have been multiplying for the last few years, until it has become needful either to abandon wholly its ingenious suggestiveness or to admit it and its conclusions only with exception and reserve. First among these objections may be named the implication that a classical syphilis should in its evolution persist throughout these three "stages;" the fact being that, as statistics clearly indicate, the largest number by far of all cases of syphilis never exhibit any signs of a "tertiary" stage. Second, the implication was made that in any given stage, especially in the so-called "secondary," the evolution of symptoms observed a definite order like 
that of the "stages," one crop, for example, of syphilodermata following another in a definite procession of symptoms; the facts being quite opposed to such a course, seeing that a syphilis thus regularly evolved, however conformed to the artificial time schedule of the schools, is clinically never seen. Third, there was overlooked or ignored a series of facts in which the chronological order of the scheme was violently reversed, so-called "tertiary" symptoms following "primary" without the evolution of any lesions which properly belonged to a "secondary" stage; while even the symptoms of the "secondary" period were at times found to succeed instead of preceding those described as "tertiary." Lastly, a fact of serious importance in the study of syphilis was to be considered-the fact that many, if not actually the larger number, of all cases of grave disease are thus grave from an early moment in the career of the malady, so-called "tertiary" symptoms developing with a degree of rapidity as startling as it is portentous. It was in recognition of these obvious and numerous violations of their chronological system that the French have been obliged to coin such explanatory" phrases as "precocious," "late," "tardy," "galloping," etc.-terms confessing the inadequacy of the time schedule, and yet employed not in the ordinary course, but in the grave crises of the malady-epochs when a fairly good working system should be ready and fitted for every emergency that may arise.

In order to grasp intelligently the facts of syphilis as they actually occur, it is well to make no attempt to force them into accordance with an artificial scheme, however cleverly arranged and readily understood, but rather to classify them in natural divisions. Thus 
studied, it will be seen that for the vast number of all cases of the disease there is no fixed line of demarcation between its consecutive phenomena, and no fixed period of time in which any given series of symptoms will be: begun or concluded. From the moment of infection to. any conclusion which the disease may acknowledge there is a regular progression, not.along one line, but along many lines, and these lines never alike or parallel, but divergent in a thousand directions. By classifying in certain groups these excursions along various routes a systematic knowledge of the evolution of syphilis may be obtained. Instead of a chronological schedule, one may more profitably, to use a different figure, employ the radii of a circle to represent to the mind the divergences of the different symptoms of syphilis from the fixed pathological centre represented by the initial lesion.

The most of syphilitic histories may be traced along the lines of advance represented by the four divisions hereinafter described. From the point of infection each of these lines of advance, or excursions, represents, it should be remembered, not a narrowly-bordered pathway of symptoms, but a general direction, with variations deflected on either hand to divergences from other directions-no single history, perhaps, following exactly the same course, but each trending near one or another of the excursions defined.

\section{Benignant Syphilis with Mild and Transitory} Symptoms.-Upon one extreme in this category are the cases in which typical initial scleroses with characteristic accompanying adenopathy are followed by symptoms which are either not at all appreciated by the subject of the disease or which barely suffice to awaken his or 
her attention. A slight efflorescence upon the abdominal surface, a few days of malaise, and the disease is at an end, irrespective of any treatment whatever. It is true that it has been claimed that grave syphilis eventually follows, but a sufficient number of these patients has been observed to substantiate the fact of a further complete immunity from all signs of the disease. Similar facts have been recognized in attempts at the production of the infectious granulomata in the lower animals, and even in the vaccination of heifers for the purpose of cultivating vaccine virus. There are simply some individuals who seem to be protected against the incursions of the disease by reason of an individual idiosyncrasy.

II. Benignant Syphilis with Relapsing or Persistent Superficial Symptoms.-This is the excursion observed, in all probability, in the larger number of all cases of syphilis occurring in the white races, and especially among those inhabiting the northern portions of the American and European Continents. In this category are to be recognized the patients in whom typical chancres are followed by characteristic so-called "early" manifestations of general syphilis. But all the lesions which result are superficial, and whether, as is often the case, they prove relapsing or persistent for long periods of time, involution is finally reached without the production of any permanent relics of the process. These histories are usually those of skin-symptoms (papules, scales, etc.) disappearing and reappearing -disappearing on the intervention of proper treatment, reappearing after neglect or discontinuance of the latter, or when the health, for any intercurrent reason, has been impaired, but never throughout producing a pro- 
found depression of the system nor inducing cachexia. The disease from first to last has been a serious annoyance rather than a formidable enemy, and if the cause were not known and the results had not been dreaded, but little anxiety would have been awakened by its encroachment.

It is these cases that furnish abundant proofs of the skill of the trained physician, and also of the tremendous energy exerted upon the health by its worst enemies, lack of proper hygiene, alcoholism, senility, debauchery, poverty, and prior wasting disease.

The cases included in this category may without warning, and often inexplicably, exhibit the symptoms enumerated in any of the other excursions described; but it is true that the majority of all cases under observation develop along the line here suggested-that of symptoms relapsing or persistent and superficial, and, however persistent, never ultimately followed by destructive results. In other words, patients of this large class, as a rule, entirely fail of exhibiting symptoms of the type described as " tertiary."

III. Malignant Syphilis with Relapsing or Persistent Profound Symptoms.-In this category are included the cases eminently of transitory type. They are speedily transferred by the best of management into the list of benign cases, or with and even without treatment are readily exchanged into the graver list of malignant cases catalogued in the fourth of the divisions here considered.

The malignancy of these cases is declared in the deterioration of the tone of the system, in the production of cachexia, and in some cases by the degeneration of lesions which in other patients are resolved without 
producing permanent relics of the process. Here at times develop in the viscera, nerves, bones, etc. gummata which resolve under appropriate therapy; at other times, when degeneration occurs, the repair is either satisfactorily good, or the damage resulting is so slight as not to interfere with the bodily health. The element of gravity is lacking in each case, however portentous at any one time may be the extent or the depth of the invasion. Often it is the fewness and depth, rather than the number and degeneration, of the lesions that justify the designation "malignant." It is in this class of patients, as in that just discussed, where the brilliant results of medicinal and hygienic treatment of the disease are most effectively exhibited.

IV. Malignant Syphilis with Relapsing or Profound Lesions that are Ultimately Destructive.In this division, represented probably by from 5 to 20 per cent., at the most, of all cases of syphilis, are catalogued the number of patients exhibiting signs of what may justly be described as grave syphilis. Here the disintegrating and ulcerating gumma destroys renal, nervous, hepatic, and osteoid cells, pierces through bone and cartilage with appalling rapidity, converts into one hideous chasm both the nasal and the oral cavities, produces the paralytic, the imbecile, the repulsively deformed, and at times pushes its destructive forces to a fatal result. For the most part, however, in acquired as distinguished from hereditary syphilis, even in grave cases a fatal result is not so much to be anticipated as is serious damage of the sort suggested. Syphilis in its worst manifestations and activities often mutilates, paralyzes, and cripples, but it rarely kills.

In this connection it is worthy of note that the fright- 
ful consequences which hedge about the track of the disease are not more conspicuous than the rapidity with which it traverses its path. Often before the last traces of the infecting sore have disappeared the hard palate is perforated, the body is covered with sloughing ulcers, or the liver is stuffed with ominous nodules. Here there has been no chronological order, no possible interval for the occurrence of a "secondary" stage, no pause in which even the best of treatment might have averted the conclusion. It is these cases that have necessitated on the part of the French-who still, for the most part, adhere to the chronological order of syphilitic manifestations-the adoption of such phrases as "galloping," "precocious," and "lightning." Indeed, of all cases of syphilis really entitled to be termed "malignant," it may be affirmed that the majority bear the impress of such malignancy in the rapidity of progress of the malady.

It is in this division also that the great triumphs of science may be achieved. Even in the worst phases of syphilis-those chiefly displayed in the fourth of the classes here enumerated-repair may be made to ensue when the destruction has been gravest and the systemic results are most profound.

Between these four radii most of the excursions of syphilis may be discerned. These lines are not all rectilinear; many lie along or near the main divisions, but pursue a tortuous course from chancre to complete relief of all srmptoms, the line now curving toward malignancy, now recurving to the other side. As a rule, the graver the case the straighter the excursion; the milder the symptoms the more numerous the 
deflections toward one side or the other, with no wide divergence to either. Rarely the course of syphilis is to be represented by a line wholly diverted from the first to the fourth of the main divisions of the circle here suggested.

The determining influences which result in these divergences are of the highest importance. First among all may be named the character of the soil in which the germ is implanted. The very young, the very old, those weakened by other maladies, by lack of food and of proper hygienic environment, the victims of drink, of debauchery, of poverty, of inherited weakness, -all suffer early and often from the added burden of syphilis. Second is to be named the early and effective intervention of proper treatment. Cases which have been neglected, those in which the disease has long been either ignored or treated inefficiently, are apt, before others, to display formidable symptoms. A third cause is described by authors as the complication resulting from the implantation of the germ of syphilis upon the system contaminated with tuberculosis, struma, and such cognate disorders as rickets, but these coincidences are much rarer than is generally supposed.

Fortunately for the future of the human race, the subjects of acquired syphilis are, as a rule, between the middle of the second and the conclusion of the third decade of life-a period when the system is best fitted to endure the severe ordeal to which, in this affection, it is reasonably sure to be subjected. With the ample opportunities for good treatment afforded in the Englishspeaking countries of the world, the majority of all victims of disease eventually escape payment of its severest penalties, marry, and beget healthy children. Though 
afterward they may in some degree be reminded of their old enemy, even as the victim of an ancient pneumonia or a broken thigh has reason at times to recall his former mishap, they go to their graves as do other men and women, with diseases of a different type, and with consequences unchanged by the infection wrought at an early period of life.

The Erolition of Syphilis Subsequent to the Chatcre.

It has been shown that chancres may persist to a point of time long after the exhibition of signs of general infection. Often, however, the period which may be described as the chancre-stage has been completed fully before such general symptoms appear. The term "primary syphilis," as has been shown, was once employed to designate this chancre-stage, and the next period of evolution of the disease was, as distinguished from that which preceded and that which followed, called the stage of "secondary syphilis." Between the two periods it was believed and taught that a distinct interval of pause or arrest occurred; this interval was given the title of "the second incubation," as subsequent in time to what was called "the first incubation" of the chancre. It is true that in many cases an apparent delay occurs after a distinct conclusion of the chancrestage before general symptoms of syphilis are declared, but it is equally certain that in other cases there is no appreciable delay, and that in yet others, where such delay or pause seems to occur, it is due to an apparent rather than a real incubation. Its features, when studied with the utmost care and skill, are declared both in the skin and elsewhere as symptoms of the gradual evolu- 
tion of the infective disorder, without any well-marked arrest. The wide range ascribed to this period of supposed incubation-from a few days to as many months -is a sufficient indication of the lack of precision involved in the use of the term. In general, it may be said that from the date of the appearance of a chancre to that of appreciable general syphilitic symptoms from forty to fifty days may elapse. This supposed period of incubation is without question shortened in malignant and rapidly-evolved cases, and is lengthened in those where an excellent constitution of the patient, exceptionally good treatment, or the mildness of the disorder has interposed a barrier to the extension of the malady.

It is wellnigh demonstrable, with the ample means at the disposal of the expert, that from the moment of the appearance of the chancre to the date of the onset of the earliest symptoms of generalized disease the signs of a gradual intoxication are with each day of its advance progressively apparent. These symptoms, often at first obscure, become usually much more obvious as the term of the supposed incubation draws to its further limit. Even, however, to the gross observation of the eye the victim of infection loses at the outset the usual appearance of health, and exhibits another which gradually acquires characteristic features-features by which, at times, the stadium of the disease may be recognized.

The skin, especially of the face, which is most often exposed to the eye of the observer, assumes a peculiar sallow or muddy hue varying from a yellowish shade to a deep reddish, somewhat empurpled tint. The facial expression may be one of dejection; there is often 
cephalalgia, anorexia, vague or very well marked rheumatoid pains, headache and backache, lassitude, neuralgia of various nerve-trunks, and, in cases, typical jaundice. By due exploration it may be discovered that transitory effusions have occurred beneath the periosteum of exposed bones: there may be retinal hyperæmia, hepatic and splenic enlargement, or albuminuria. The percentage of the oxyhamoglobin decreases with the number of the red blood-corpuscles, while the leucocytes increase. It is during this period also that there occur thermal changes which have been summed up rather loosely under the term "syphilitic fever."

The febrile symptoms recognized in an early or a late phase of syphilis occur neither with sufficient frequency nor with sufficiently characteristic features to justify the employment of a distinctive term. These thermal variations are most often of early occurrence, either before or soon after the exhibition of constitutional symptoms, and are in general due to the reaction of the system against the recently-introduced and multiplying toxine of the malady. Abnormal thermal variation may be wanting in more than 50 per cent. of all cases examined, or may be so slight as scarcely to attract attention; or it may be in a high degree pronounced, the physician, unaware of the precise cause of the disturbance, not infrequently assuming that the patient is suffering from a tertian or quotidian miasmatic fever. In well-marked and classical cases the temperature prior to the earliest eruptive phenomena rises to $103^{\circ}$ and even to $106^{\circ} \mathrm{F}$, and may then assume a continued or intermittent type with vespertine exacerbation. The fever is rather more often observed in the cachectic and weak than in the strong, and is often a precursor, when well marked, of a severe type of con- 
secutive symptoms. It is said to be more common in women than in men, but on this point there should be great reserve. Fevers occurring in other stages of the disease are usually symptomatic of destructive processes due to the disease; though it is to be noted that the subject of syphilis is often in a depressed condition, and furnishes a large field for the invasion of intercurrent disorders, such as la grippe and tonsillar and bronchial affections, several of which may excite febrile reactions not directly connected with the specific affection.

\section{Involvement of The LyMPhatic Glands.}

The syphilitic bubo, or specific induration and enlargement of certain glands in anatomical proximity to the site of infection, has already been described. It is at a period later in the evolution of the disease, and usually at or near the close of the so-called period of "second incubation," that the lymphatic system (the glands, more particularly, of the several accessible regions of the body) exhibits characteristic changes. In well-marked cases one, if not quite all, of the glands which may be appreciated by the touch of the observer becomes enlarged, engorged, soft, and voluminous, as distinguished from the densely-indurated buboes accompanying the chancre. This ganglionic engorgement is one of the most constant of the signs of systemic syphilis, and though at times it may escape observation or actually be absent, it is so conspicuous a feature of some cases that patients themselves often call attention to it. At a given moment it may be the sole appreciable symptom. It betokens, when well marked, a general intoxication, and, though not always proportioned to the intensity of the disease in any given case, it occurs at times as 
the result of an individual predisposition to lymphatic disorders.

The glands most often exhibiting these changes are the post-occipital, the chain extending along the posterior border of the sterno-cleido-mastoid muscles, the supraclavicular, the inguinal and axillary, the epitrochlear, the submaxillary, the submental, and the femoral. At times the lymphatic trunks leading to these glands exhibit similar changes.

The tumefied glands vary in size from that of a bean to that of a pullet's egg; they are usually rounded or oval in contour, smooth to the touch, and painless; at times, however, they are very tender, and, when not overlying bony tissue, movable. They have no tendency to degenerate, in this respect exhibiting a very noticeable difference from the syphiloma, or gummatous involvement, usually of a single gland, occurring early or late in cachectic subjects. The syphiloma has a uniform tendency to become at one point or another reddened and porky to the touch, and it soon breaks down into a characteristic abscess.

The voluminous and softish ganglia of early syphilis are found on examination to be constituted by a smallcelled infiltration of the lymph-channels and a succulent fulness of the tissue about the latter. Resolution is by the ordinary absorptive processes.

\section{Syphilitic Cachexia.}

The anæmia and leucocythæmia of syphilis occur either as a direct and sole result of systemic intoxication or as the indirect result of the latter in individuals predisposed to cachexia in consequence of an enfeebled condition of the system or of constitutional inheritance. This condi- 
tion is well seen in the infantile forms of the disease and in the victims of debauchery, drink, poverty, hospitalism, filth environment, and of other affections than syphilis. Tuberculosis, rickets, and scrofulosis are less frequently responsible for this condition than is generally believed. The symptoms of syphilitic cachexia may be declared early or late-in the former event usually toward the close of the pre-exanthematous stage of the disease; in the latter case at any time when the system exhibits signs of exhaustion in consequence of gummatous changes in bone, periosteum, nerve, or other important tissue of the body.

The signs of cachexia are a peculiar dull-tinted pallor of the skin, with vague pains, persistent weakness, flabbiness of the tissues, a distinct whiteness of the conjunctivæ, emaciation, and manifest disturbances of digestion, assimilation, and excretion. This condition, which may be produced solely by the disease and which may be relieved greatly by a properly-directed ferruginous and mercurial medication, may without question be induced or aggravated by the injudicious employment of mercury in the treatment of the disease.

Syphilis in Relation with Concident Injuries and Accidents.

It was at one time believed, largely on a priori grounds and after insufficient observation of cases, that syphilitic infection, if relatively recent, predisposed its subject to the exhibition of special lesions or special disturbances when exposed to traumatisms or to diseases of a different origin. These views have been changed radically since the date of a wider knowledge on the subject of the antagonism of toxines. 
As a matter of fact, the subject of recent syphilis exhibits a tendency to the production of lesions at sites of irritation (condylomata about the uncleansed anus; mucous patches of the mouth irritated by tobacco, smoked or chewed; paimar lesions of the hand-worker); but it is also tolerably clear that for the most part syphilitic subjects undergo surgical operations (cachexia and its complications aside) with very much the same results as in the non-infected. They also exhibit the classical signs of local irritation, not different from those seen in others (urticaria from the attacks of vermin; erythematous redness on the application of a sinapisn ; zoster after exposure of a nerve-trunk to the predisposing causes of that affection, etc.). It is now accepted that all pus-production in syphilis is the result of mixed infection, and that the staphylococci multiply in its subjects as at other times and in other persons.

On the supervention of other typical disorders in those under the influence of syphilis, the result is conditioned upon the proportionate activity of the one or the other malady. Recently-infected syphilitic subjects exposed to typhoid fever speedily lose all symptoms of the original and exhibit all classical features of the later disease, even to the date of a slow and apparently typical convalescence. On the re-establishment of the health the syphilitic affection, after an apparently absolute quiescence for weeks, resumes its former activity, and the progress of the infective process seens to be resumed at the point where it was temporarily interrupted.

Considering the number of both tuberculous and syphilitic subjects in large cities, it is a matter of great surprise that experts are so seldom confronted with the coincidence of the two affections in one indi- 
vidual; the same may be said of syphilis and of carcinoma, though the different ages of the patients liable to display early symptoms of these two affections may here exert some influence upon the statistics. An attack of erysipelas has often cleared the skin of syphilitic lesions, and, even when occurring in a patient whose luetic affection was grave, has emphasized the date of a recovery without further relapse. Indeed, of the larger number of all injuries and diseases occurring as accidents of the period when the subject of syphilis is displaying evidences of his disease, it may safely be asserted that they proceed to a conclusion which would have been anticipated if no systemic infection had existed.

\section{Syphilis of the Skin.}

In hereditary syphilis the bones or the viscera may first manifest the signs of the affection, since the new being is vitiated ab or'o. In acquired syphilis, on the contrary, the most obvious of the early lesions of the disease are perceptible in the skin and its underlying connective tissue and upon the mucous surfaces as well as in the superficial lymphatic glands and vessels.

"Syphiloderma" is a term used to include many of these superficial lesions, the early eruptive and late infiltrations and deposits being termed "syphilodermata," or, as the term has been anglicized since its first employment by the French, syphilides. The word "syphiloma" is generally restricted to (late) gummatous deposits in the several organs of the body, not merely in the skin, but also in the bones and the viscera.

The study of the eruptive symptoms in syphilis is of the very greatest importance not only for the expert, 
but also for him who aims to be an accurate diagnostician in any department of medicine. He who cannot properly interpret these significant symptoms is usually not merely an ignorant but an unsafe practitioner. The peace of families, the conservation of the marriage relation between husband and wife, the reputation of an innocent girl, and the health of uninfected men, women, and children may all be hazarded by the decision of a single case.

\section{General Features and Relations of the Syphiloder-} mata.-Syphilis may invade every organ of the body; it may also involve any portion of the skin. As the chancre may be situated on any part, so the syphiloderm may develop upon any given point of the bodily surface. As it has been seen that the chancre may be represented by every one of the several elementary and consecutive lesions of the skin, so the syphilodermata may develop in each of several forms-as a macule, a papule, a tubercle, a pustule, a bleb, or a tumor-and may betray such consecutive lesions as scales, crusts, ulcers, rhagades, fissures, and scars. A study of the syphilodermata is, in fact, a study of the changes impressed by the infective process upon the simple manifestations of all skin diseases. A syphiloderm may resemble an acne, a psoriasis, a seborrhcea, and even the skin-picture in variola. To determine with certainty that an eruption is syphilitic it is essential that the several modifications of lesions produced by syphilis of the skin be recognized fully. The actual result in any case is a composite of the ordinary pathological processes of congestion, inflammation, infarction of ressel, cellmultiplication, and secretory changes awakened in the tissues, which in all diseases resent these processes. 
Characteristics of the Syphilodermata.-Symmetry'. - Many disorders of the skin attended with eruptions exhibit symmetrically-arranged lesions, such as variola, the medicamentous rashes, and purpura. In syphilis the earlier cutaneous symptoms are usually symmetrical, but as the disease progresses the skin-lesions exhibit a greater tendency to asymmetry, until the latter becomes the rule rather than the exception. The macular syphiloderm of an early stage of syphilis usually displays this symmetrical arrangement in a marked degree.

Color.-Too much significance has been attributed to the supposed characteristic color of the syphilodermata, though often the hue displayed by such lesions is like none other. It is important to bear in mind the obvious fact that the color of an eruption in a blonde and in a brunette subject, in an infant and in an aged person, in a region such as the face and in another such as the inner and superior aspect of the thigh, exhibits the widest contrasts. It is also true that in every person affected with a cutaneous efflorescence the color varies from hour to hour with the degree of congestion of the integument.

In syphilis there is displayed no color which may not at times be recognized in non-syphilitic subjects; but the color with the other picture presented is usually highly suggestive. Its shades vary from a mixture of red, yellow, and brown to an empurpled hue, and they are rarely, if ever, commingled with the vivid and frank rosy tints of an erythema simplex occurring, for example, in a clear-skinned child, or the pure silverwhite of the scales seen in lichen planus. The terms "copper-colored" and "raw-ham tint" have been 
employed to designate the special hues of the syphilitic exanthem. The deepest shades of greenish-yellow, chocolate, and even black are often noted as sequences of the profound alterations occurring as the result of gummatous ulcers, particularly in the lower extremities.

Polymorphism (Multiformity).-The frequency of the coexistence of several lesions of different types in one person and at one time is a characteristic of syphilis shared by but few other maladies. It is not rare to find a subject of the affection first named exhibiting at a given moment condylomata about the anus, scaling patches in the palms, pustules upon the face, and papules of the thighs.

Configuration.-The arrangement of the syphilodermata in groups or, after coalescence, in figures having the outline of a circle, either complete or in segments, is highly distinctive. Thus are formed odd-looking and characteristic groups of lesions in figures suggesting the shape of a horse-shoe, a kidney; the letter $\mathrm{S}$, the figure 8 , and the arrangement of a brooch in oval or circular pattern with crescentic or circular "satellites" at its outer rim. The "serpiginous" feature of certain of the syphilodermata is the result of an evolution of lesions in similar lines spreading from one point of the skin to another in crescentic curves. This special configuration is probably associated with the distribution of the cutaneous nerves in definite areas.

Absence of Subjective Scnsations.-The absence of itching and pain in the great majority of syphilitic subjects displaying eruptive symptoms is a striking feature of the disease. The exceptions are, however, often well marked, a peculiarly sensitive individual suffering from pruritus even with macular lesions. It is 
also to be remembered that in a few special syphilitic lesions (particularly condylomata about the anus and the vulva, pustules upon the scalp, etc.) the itching may be extraordinarily severe, while the pain of a syphilitic ulcer may be excessive. It is none the less remarkable with how much toleration the average patient displays an abundant exanthem covering almost the entire surface of the body. Indeed, a careful physician is often the first to detect a syphilitic rash, the patient being wholly unconscious of its existence until informed of the fact.

Mode of Evolution.-The syphilodermata are developed with remarkable indolence, and in some cases, especially in those neglected, they have a tendency to recur in different types, to be succeeded by others of a different character, and to undergo extreme metamorphoses in sitû, so that, for example, a papule may enlarge, flatten, ulcerate, or disappear and be succeeded by others pursuing the same or another course. It may well be doubted, however, whether this is so much a mode of evolution of syphilis as a variation of its evolution due to the accidents of environment. Syphilis is a disease of relatively chronic type, and it is peculiarly subject to changes induced by improvement in the general health of the patient or the reverse, and in favorable cases by treatment.

Situation.-Every portion of the bodily surface may be the seat of a syphiloderm, but in different localities there is usually seen a different expression of these local manifestations. Those of pustular type are often seen upon the scalp and on the face; papules often appear over the neck and the brow; secreting lesions, about the mucous outlets of the body; scaling patches, on the palms of the hands, etc. 
Peculiaritics of Elementary and Consecutive Lesions.Papules are ever predominant lesions of an average syphilitic history. They are usually characteristically ham-colored, and in exposed situations they have a tendency to scale at the apex, to provide themselves around the border with a collarette of dirty-tinted scales, and in others to flatten into broad plaques, to crust, and even to ulcerate.

Tubercles are also common in syphilis, and they are usually grouped. Their color and their frequent tendency to ulcerate and crust distinguish them from the much more indolent tubercles of lupus and lepra.

The crusts of syphilis are usually bulky; they vary in color from a dirty greenish-brown to a dead black. When of rupioid type they are made up of laminated concretions like the shell of the oyster, this feature being produced by the concretion of pus and other inflammatory products upon a secreting ulcer, which, as it spreads beneath, furnishes continually a broader base for the conical crust with which it is capped.

Scales in syphilitic subjects are usually thin, are rarely very profuse or adherent, and are of a dirtywhitish hue. They never exhibit the nacreous shade of the psoriasic skin, nor, as heretofore shown, the silvery sheen of the scales in lichen planus. As distinguished from similar conditions in non-syphilitic disease, they are rarely the sole lesions present, but are more often complications or appendages of other lesions, as, for example, when they crown the apex of syphilitic papules or surround their base, or, as in the palm or the sole, when they furnish a ragged fringe encircling a dullred patch either ulcerating or threatening such destructive action. 
Ulcers in syphilis are usually characteristic. Their base is, as a rule, soft; their edges are steep or undermined and have a punched-out appearance; their floor is covered with a foul pultaceous slough; their secretion is purulent or hæmorrhagic; and their crusts are of the character described above. Often they are sursounded by an angry halo. Their outline commonly observes the several circular shapes already suggested, such as the arc of a circle, a horseshoe, a semilunar figure, etc.

Scars left as relics of ulcerative and degenerative lesions are in syphilis usually pigmented when recent, but when old the pigment gradually disappears from centre to circumference. In circular or oval contour they conform, for the most part, to the configuration of the ulcer or group of lesions that preceded their formation. When completely freed from their chocolate-tinted or violaceous pigmentation they are of a dead-white shade, not greatly differing in this respect from scars in general, but they are, as a rule, much smoother, more superficial, less attached, and more elegant in delicacy of surface wrinkling than most other cicatrices. Their site is often of striking importance: as in syphilis, they are apt to be situated on the anterior face of the lower extremities (the leg particularly), though they may form in any portion of the body (face, arms, scalp, wrists, etc.).

General Considerations relative to the Evolution, Involution, Variation in Type, and Accidental Features of the Syphilodermata.-The conception long held of the classical evolution of a syphilitic affection has to a great degree been modified by later observation and study. With reference to the syphilodermata, it was believed, and with some reason, that their evolution 
was by a series of successive eruptions, the one in due course following the other, those of a so-called "secondary" stage at first symmetrical and superficial, fading spontaneously and succeeded later by eruptions involving a deeper structure of the skin. Thus papules were thought to follow macules, pustules taking the place of papules, until a late or so-called "tertiary" stage was in proper course reached, when the syphilodermata, no longer multiple and superficial, became fewer, deeper, isolated, and in various degrees destructive to the underlying tissues.

Such was the classical ideal; but, as has been in part already shown, it was rather an artificial manikin for use in the schools than a pattern fashioned after observation of cases. If any such attack of syphilis has actually been observed, it was certainly an illustration of the very rare exception rather than of the rule.

There are many facts which lead to the conviction that an attack of syphilis in a sound young subject whose case is perfectly managed throughout, with no intercurrent accidents to change its features, is a syphilis exhibiting a single exanthem. This eruption would be of the type of the superficial and symmetrical macular syphiloderm, after the disappearance of which as a result of vigorous treatment no other skin-lesions would appear. Persistent, faithful, and skilful management of the case subsequently should permit no further manifestations of the malady. This is, it must be admitted, a rare event, yet it is one that can be studied as an objective fact, and, rare though it be, it certainly is not so rare as the ideal case exhibiting in turn and in due course each of the syphilodermata in an ordered succession.

The practical deductions from an acceptance of this 
new ideal are of importance. In the light of our present knowledge on the subject of micro-organisms and their rôle in the production of disease, it is clear that some of the syphilodermata are the result of mixed infection. Staphylococci are responsible for many, if not all, of the pustular lesions in syphilis. Again, it is capable of demonstration that many of the other syphilodermata are the fruit of local irritations, of errors in diet, in dress, in exposure, and in the habits of the patient. The impression that every eruption recognized in the subject of syphilis is due solely to that disease is so grossly misleading that it should carefully be excluded from all conceptions of the malady. The medicaments swallowed, the soaps employed, the articles of diet and drink consumed, play a significant part in many of the processes to be considered later.

Again, it has been believed that the profuseness of a syphilitic eruption of early development bears some relation to the severity of the disease and to the questions concerned in its prognosis. This is a conception based upon the old rather than upon the new ideal outlined above. As a matter of fact, the first frank expression of constitutional syphilis may be an abundant exanthem of macular type, extensively spread over the bodily surface, possibly sparing no area, and this may prove of better augury than one which feebly manifests itself and is too speedily followed by the symptoms of malignancy to be described later. Complete involution of an eruption of this character is often not followed by the evolution of a crop of small-or large-papular syphilodermata, nor, indeed, by any other eruption.

Classification of the Syphilodermata.-The skinlesions of syphilis are classified as follows: 
I. Macular.

(a) Pigmentary.

(b) Erythematous.

(c) Purpuric.

II. Papular, dry. (a) Miliary.

(b) Lenticular.

Papular, moist. (a) Mucous patches.

(b) Condylomata.

III. Pustular.

(a) Miliary.

(b) Lenticular.

IV. Tubercular.

V. Gummatous.

The compound adjectives "pustulo-crustaceous," "papulo-pustular," "gummato-ulcerative," and others are employed to express the frequent combinations of elementary and consecutive lesions to be recognized clinically in many cases of syphilis.

In these pages all such terms as "syphilitic psoriasis," "syphilitic lupus," etc. are discarded. Combinations of syphilis with other diseases, however rare, are certainly never expressed in dermatological lesions, for an eczema (which certainly may occur in a syphilitic subject) is not a "syphilitic eczema," but is an eczema of unmodified type; and a scaling syphiloderm is never by any possibility a "syphilitic psoriasis," but is a squamous skinlesion of the specific disorder present.

\section{Macular Siphilodernata.}

Pigmentary.-The pigmentary syphiloderm occurs without previous involvement of the skin, as a distinct network of pigmented, brownish, chocolate, or even blackish maculix, the hyper-pigmentation being conspicunus by reason of contrast with the white and unaltered skin about each discolored spot. Gradually, 
and very slowly as a rule, the pigment is diminished in the centre of each deposit, and there is formed a whitish central punctum from which the pigment is at last wholly removed. These colorations occur as uniform ill-defined shadings, as pea- to coin-sized spots, or as a reticular arrangement, one form often slowly passing into another as the pigment atrophy and hypertrophy progress side by side. The eruption is seen rather more often in women, and in them chiefly on the neck and shoulders, but it occurs also in men, and over the face, neck, and forearms.

This condition is decidedly more often seen in brunettes than in blondes, in this particular sharing the lot of most of the achronias of the skin. It especially affects in both sexes the Chinese, Indians, and negroes who have contracted the disease. It was once supposed to be rare, but without question is more common than was believed.

The eruption, if such it may be called, develops at any time after general syphilis is declared, but it is much more common in the earlier months of the malady. It is exceedingly indolent, persisting for months, and even in exceptional cases for years, being in but a slight degree amenable to specific treatment. Though thus persisting, the complete involution of the affection occurs without ulterior changes in the skin, which, as a result, does not become the seat of infiltration, of degeneration, nor of scaling. Indeed, it is probably more an indirect than a direct result of infection, and is peculiar in that it is decidedly more common not merely in those predisposed by individual characteristics to pigment anomalies, but also in the uncleanly and the neglected. Anatomically, it is found 
that a chronic endothelial inflammation of the smaller cutaneous capillaries occurs, under the influence of which the red corpuscles gradually lose their coloring matters, while eventually an obliterating endarteritis chokes the vascular channels. In the portions where the pigment has apparently been removed the normal quantity of coloring matter has at times been recognized; in other cases a true vitiliginous atrophy of the pigment has followed. It is highly probable that all these changes are under the immediate influence of the trophic nerves.

The pigmentary syphilide should not be confounded with tinea versicolor, which develops often on the neck and the breast, for in the disease last named not only is a fungus visible under the microscope, but the fawncolored patches are usually the seat of a fine furfuraceous desquamation, readily recognized when the fingernail is employed as a curette over the surface. The several chloasmata of other sources are, however, to be differentiated with care. Many of them appear on the face, and not elsewhere (the reverse of what is usual in the pigmentary syphiloderm). Vitiligo or leucoderma occurs often on the scalp as well as over the body and the face. Its disks are far more extensive than those of the syphiloderm, being often palm-sized and larger, and when occurring upon the scalp the hairs which spring from the achromatous patch are commonly white. In any doubtful case the symptoms of syphilis, usually declared by other signs in the event of a syphiloderm, should decide the diagnosis.

Circumscribed pigmentations of the skin in syphilis, and even of syphilitic lesions themselves, differ in a marked degree from the pigmentary syphiloderm, since all the former are, without exception, sequences of some 
other disturbance (relics of a papular or tubercular syphiloderm, ulceration and cicatrization of gummata, especially in the lower extremities, etc.).

The Erythematous Syphiloderm ("Syphilitic roseola," "Syphilitic erythema"). - It has already been shown that there are grounds for believing that syphilis in an ideal case, occurring in a strong and healthy young subject, well managed throughout the entire career of the disease, would probably have but one cutaneous expression. That expression would be the erythematous syphiloderm. If syphilis be in type a disease of but a single efflorescence, the eruption here designated represents that exanthem. It is the most common, the most frequent, the most benign, the earliest, and the most classical of the skin-symptoms of the disease, to be expected in the great majority of all patients, and rarely failing to appear when awaited and searched for by the eye of the trained physician. It is also in syphilis the exanthem most often overlooked, as it may be limited to regions covered by the clothing, and is for the most part unaccompanied by any subjective sensation such as itching. Women, especially those who are fleshy, when viewing its blush often suppose themselves to have been simply "overheated," and men, especially those inured to work in heavy flannels, look upon its lesions with no anxiety. It it often first demonstrated by the physician engaged in examining a patient for the detection of the character of a chancre.

The exanthem usually first appears between the sixth and the seventh week after the appearance of the chancre, and with exceedingly insidious onset, so that on the very first inspection only a few delicatelytinted spots occur on the surface of the belly; and in 
some cases, especially after indulging in a Turkish bath, a dance, or a generous dinner with wine, its lesions may be evolved with surprising rapidity.

The faintest expression of this syphiloderm can scarcely be described. It resembles to a degree the delicate marbling produced when the skin of a healthy person is exposed to cool air after immersion in a hot bath. When well defined, the spots appear as multiple, oval-shaped or rounded, irregularly-defined macules, neither elevated above nor depressed below the general level of the integument, having a diameter of from one-tenth to one-fourth of an inch. Their color varies in different skins and at different stages of evolution of the exanthem, being rarely of a pure rose or a vivid pink, but rather of a dull shade of yellowish-red. sometimes having an empurpled tint, at times so light as almost to. suggest a simple erythema. The color fades under pressure of the finger, but later persists, and when further development of the exanthem occurs the maculations furnish a slight elevation of the surface at each point of hyperæmia-a condition approximating that in which papular lesions appear. On complete involution, which often occurs without the sequel of another exanthem of the disease, there may be left transitory discolorations or lightly-pigmented macules persisting for several weeks. As a rule, under appropriate treatment the eruption fades, without the production of desquamation or other consecutive lesions, in the course of from a week to ten days, though occasionally it persists for several weeks.

The abdominal surface and the chest, both anterior and posterior, generally display the exanthem in greatest profusion, but it is also encountered in vivid efflorescence over the extremities, the face, the neck, and, 
indeed, over all the bodily surface. When distinctly evolved over the anterior surface of the belly and the back, it is often supposed by inexperienced observers to be strictly limited to these regions, but in almost all cases a careful search will reveal a faint mottling about the outer angles of the lips, in the palms of the hands, over the brows, and elsewhere. It is most brilliantly displayed on the abdominal surface when faintly seen elsewhere, chiefly because of the warmth and clothing of that portion of the body. In some cases it will be seen on close inspection that the arrangement of the macules is in generally circular outlines.

The eruption which represents the transition between that just described and the papular syphiloderm is termed the "maculo-papular." Its peculiarities are briefly those, in varying proportions, of the two primary lesions from which it has its name. The variations between these eruptive forms, macules and papules, are numerous and interesting.

In an exceedingly common variety the macular rash exhibits here and there, often with wide intervals of space, a few isolated papules, usually of the larger or lenticular type, scattered with seeming irregularity over the eruptive field, and springing usually from maculæ. They have a dull-reddish tint, and they often scale slightly over the flattish summit or at the base. These may be sparsely distributed over one region of the body; or when the trunk, for example, exhibits macules in wellnigh pure type, the lower extremities, where there has been some friction and usually also effects of gravitation, display these papules seated on an erythematous base. In yet other cases the papules are of miliary type and spring in large numbers directly from 
the erythematous spots, till each of the latter is thus surmounted apparently by a small elevation. Here again the circinate arrangement may be conspicuous. In other cases the mouths of the orifices of the pilosebaceous crypts are the seat of the disorder; in others the scalp becomes the site of a seborrhœal flux, the secretion drying into light crusts superimposed upon a macular exanthem, the color of the latter often being displayed beyond the border of the incrustation.

The macular syphiloderm may relapse under inefficient treatment in one or several efflorescences, but, as a rule, it appears in typical development but once in a syphilitic history. The evolution of what is often thought to be a late macular syphiloderm, occurring two and more years after infection, is an eruption which has erroneously been supposed to be due to syphilis. In these supposed "late" cases there is developed over the surface of the chest, and at times on the belly and elsewhere, multiple, usually coin-sized, oval, elliptical, superficial patches, scaling very slightly at the periphery, and with a clear centre. They are usually brownish-red or purplish-red in hue; they have been noted as rebellious to the treatment indicated by the disease present. Most of these are instances of pityriasis maculata et circinata, "pityriasis rosea" of authors. In the spring and the autumn many of the subjects of syphilis are peculiarly susceptible to this somewhat rare disorder, whose innocent lesions commonly disappear in a brief time under the influence of a tonic regimen, well combined with the use of the cinchona preparations and the salicylates.

Purpuric.-Hemorrhage into the several portions of the integument occasionally complicates not merely 
the erythematous but also other syphilodermata, such as papules and bullx. In these cases the occurrence of pin-head and larger purplish and mulberry-shaded spots that refuse to disappear under pressure indicates that the coloring matters of the blood have been effused through the tunics of the vessels. It is to be remembered, in all cases of syphilis where iodice of potassium has been administered for the relief of the disease, that this drug is capable of producing purpura of the skin, especially of the lower extremities. In some instances large disks and even wide areas of purpuric maculation are produced in both early and late periods of the disease. This symptom is, however, most commonly seen in the inherited forms of the disease, though it is not rare in adults. When due directly to the disease, and not to a drug administered for its relief, it should be viewed as a somewhat grave symptom. It accompanies several of the paraplegic and hemiplegic complications of nervous syphilis.

Anatomy.--Section of a macular lesion exhibits merely effusion between the component parts of the upper corium, with some displacement and elongation of the fibres of which it is composed. The capillaries are distended, and both within and without are encumbered with cells. The accessory portions of the skin lying in the upper part of the corium (sebaceous and pilary crypts) participate somewhat in the process, but the sweat-glands in the deeper portion are unaffected (Crocker, Neumann, Biesiadecki, and others).

Diagnosis.-The macular syphiloderm is distinguished from the eruptions accompanying exanthematous fevers by the features described above, as also by the temperature-changes perceptible in such ferers. In 
case of syphilitic fever other evidences of a systemic infection are commonly observed (adenopathy of the post-occipital and other glands; mucous patches of the mouth, anus, or vulva; alopecia; crusts upon the scalp, etc.). In the medicamentous rash due to copaiba there is commonly excessive itching; this and other rashes due to drug-ingestion promptly disappear on the withdrawal of the exciting cause. In tinea versicolor the presence of the vegetable parasite and the distinct limitation of the eruption to the regions covered by the clothing are important points of difference. The color of the eruption-a very distinct fawn shade or deeper tint-never has the reddish-brown hue of the syphiloderm. Pityriasis maculata et circinata is usually much less abundantly distributed, and its patches are always in ovals, commonly on the front and back of the chest and the shoulders, with scaling at the periphery of the clear centre, and displaying, when on the chest, an arrangement of patches with the long axis at right angles to the vertical line of the body.

The prognosis of the macular syphilodermata is in general favorable, and no gravity need be argued from either their profuseness or their deep shade of color.

\section{Papules.}

It has been shown that papules are among the most common of the syphilodermata. Their grouping, color, situation, and environment in many cases of syphilis are so characteristic as to be absolutely diagnostic of the disease. They may appear at any time from the third month to the conclusion of the first year, and even much later; they may develop in crops; they may immediately spring from a preceding macular exanthem, or succeed 
the latter after an interval; and they are usually symmetrical in the earlier and asymmetrical in the later of the periods named. They vary in size from a pin's head to that of a bean, and may be multiple or few, disseminate or grouped, generalized or limited to distinct regions of the body, conical or flat, dry or moist, in color shading from a light crimson to a dull copper. They may scale at the apex or be surrounded by a collarette of scales at the base.

Papules represent the syphilitic process in the skin and the mucous membranes, beginning with an indolent inflammatory process in the corium, inducing a thickening of the rete, some effusion of lymph-cells, and a breaking away of the horny layers of the epidermis from the summit of the circumscribed inflammatory product where the thickening of the skin occurs. As this change may involve different regions of the body, gross results are obtained, whose differences depend largely upon the site of each lesion. Papules upon the scalp, for example, are usually dry and scaly; when picked or scratched they often bleed and crust. Upon the exposed and dry surface of the skin, such as the extensor faces of the extremities, they are usually acuminate, dry, and squamous. On the brow, near the border of the hairs of the scalp, they often surround themselves with a delicate collarette of dirty scales, exposing a copper-tinted integument beneath and around the individual papules, the group being so characteristic as to have gained the title of the "corona veneris."

When papules form upon apposed surfaces, such as the skin covering the voluminous breasts falling over the thorax in women, or the folds of the nates in contact, or the scrotum lying next the integument of the thigh, papules 
enlarge, flatten, secrete, and in many cases produce a sensation of itching. Papules forming upon mucous sur faces also, by reason of the heat, moisture, and friction to which they are subjected, become flattened and secrete, forming thus the mucous patch. Papules developing upon or beneath the thick epidermis of the palms and the soles of adults are so bound down that they rarely rise above the general level, but the cracking of the scarf-skin at the level of the thickened subepidermic focus produces a characteristic scaling of the skin in the regions named.

Dry Papules.-(a) Miliary' Papules.-This abundant efflorescence is less frequently noted than other of the papular syphilodermata, for the reason that its very profuseness argues a neglected or ignored condition of the subject of the disease in its prior manifestations. Since these neglected and ignored patients are often women. the eruption is somewhat more often observed in them. The lesions are pin-head-sized, closely-commingled papules, symmetrically arranged, often widely dispersed, and even generalized, at times distinctly and even elegantly grouped in circles or segments of circles, light reddish to deep crimson in shade, the apex of each papule at times surmounted by a still finer vesicle containing a droplet of serum-an accident which usually points to a coincident febrile access. Involution occurs by fine scaling at the apex of each lesion and flattening of the papules to a dull, purplish-red maculation of the surface. In rare cases, chiefly of public patients, this eruption may be merely the preliminary stage of a diffuse pustular syphiloderm. At times it can be seen that the lesions are limited to the hair-follicles. There are few cases in which, when the eruption is at all well 
SIPHIIIS.

Plate 3.

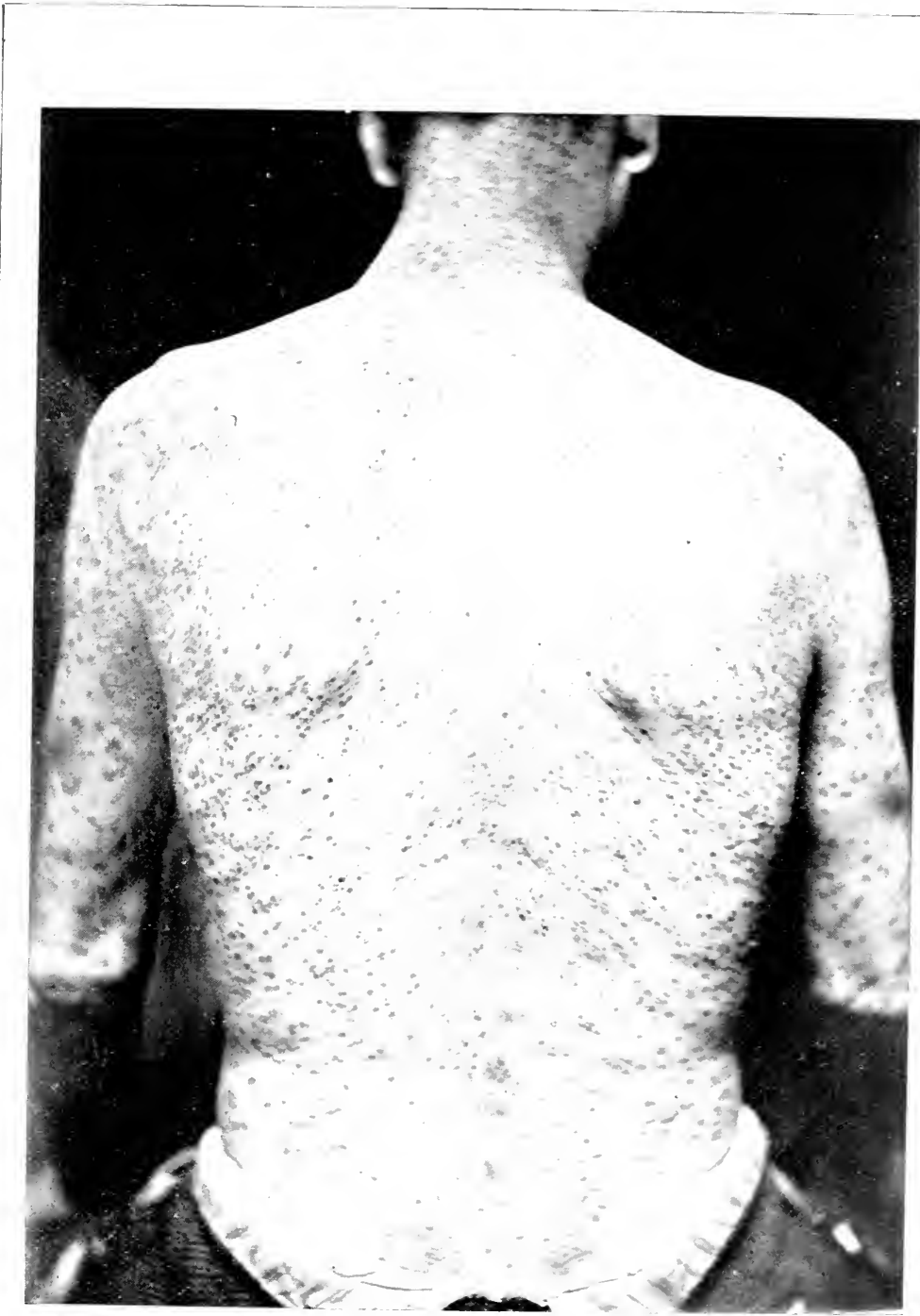

Small papular syphiloderm (Stelwagon). 

marked over the face, the neck, and the trunk, groups of much larger lesions, to be described below, may not be seen in other regions of the body.

Diagnosis.-The coincident symptoms (mucous patches, adenopathy, etc.) indicating the presence of a disease accompanied by other than skin-involvement usually suffice for the establishment of a diagnosis in these cases. Scabies and ringworm, the former due to an animal and the latter to a vegetable parasite, are distinguished by the presence of the exciting cause in each affection, the former being, as a rule, accompanied by an intense and characteristic pruritus, the latter by a circinate arrangement of the patches. The lesions of lichen planus are flattened at the summit and usually exhibit polygonal outlines, while the frequent linear and angular distribution of the papules is never seen in the syphiloderm. Psoriasis in some cases strongly resembles a scaling and well-developed papular syphiloderm, but the former disease is, as a rule, more extensive, and the scales are more abundant, more voluminous, and more lustrous. Keratosis pilaris in extreme expression over the limbs and the body is to be recognized by the obvious situation of each papular lesion at the orifice of the pilosebaceous crypt.

Prognosis. - The course of the eruption in healthy subjects, whether acutely or slowly pursued, is toward a favorable termination. At times the eruption proves intractable to treatment.

(b) Lenticular Papulcs.-The papules are here usually discrete, rounded or oval in contour, and vary in size from a pinhead to that of a large bean and even larger. They are rarely elevated to any extent above the level of the integument, and at times they are so flat as to be mis- 
taken for mere unsightly blotches of the surface. They vary in color from an exceedingly dull to a bright copper shade, and are usually remarkable for the fringe or collarette of dirty scales fraying away from their base, as described in connection with the "corona veneris." The eruption may appear in a few months after infection, and then disappear, or it may occur in crops lasting, with varying intervals, for one or two years after the onset of the disease. These papules are among the commonest of the syphilodermata, and, with variations of the sort described above as due to the accidents of site and environment, probably figure in a modified form in most of the lesions which are to be observed during the first two years after infection. The eruption spreads both by the outcropping of new lesions and by the enlargement of individual papules in situ, the latter being rather more common. As resolution occurs the papule flattens to the level of the skin, leaving merely a pigmented macule as a relic of its existence. These pigmented patches, especially over the face, are apt to be exceedingly rebellious to treatment and slow to disappear, much to the chagrin of the patient, who speedily comes to a realization of their peculiar significance.

The eruption may be quite general at the first, and later may limit itself to a favorite locality, such as the forehead, the back of the neck, the belly, the buttocks, the flexor aspects of the joints, the scrotum, and the outer face of the labia majora.

It is the modification by grouping and coalescence of the papular syphiloderm that produces the sub-varieties recognized by authors as "nummular" and "corymbiform." In the former the papules enlarge to flat disks of the size of large, and even of the largest, coins, cir- 
cumscribed, and with depressed crateriform centres, the contrast between the central area and the circumvallation of the smooth, copper-tinted ring being conspicuous. In the corymbiform arrangement satellite-like groups develop about the central disk. Other oddlooking forms are the result of different groupings of the coalesced or isolated papules, as in the shape of the letter S, of a kidney, etc.

Midway between papules and purely squamous lesions in syphilis stand the papulo-squamous syphilodermata, lesions in which the characteristically developed and situated papules of syphilis undergo a squamous transformation at the summit, where a little heap of dirty-looking, adherent, sometimes friable, but often corneous scales accumulates. This combination of scales and papules has been thought to resemble psoriasis, but the correspondence is rarely suggested to the trained eye, for the elevation of the lesions, the character of their scales, and the color of the dull-tinted papules on which they rest are significant. The circular outline of many of the confluent patches of the larger papulo-squamous disks and of psoriatic patches in generai is often confusing, and yet the bulkier and dirty-looking scales of the syphiloderm, the dull, ham-colored patch in the centre of the circinate group, often slightly infiltrated or thickened, offer a strong contrast to the more vivid hues of psoriasis. The clear-tinted and uniformly spread scales of the psoriatic patch, its centre either evenly thatched with such scales or, if quite clear, showing only a slightly shaded and non-infiltrated epidermis, are also to be considered in establishing a differential diagnosis. Over the face the papulo-squamous syphiloderm is often covered with a mealy or granular mass of scales of a dirty 
grayish hue, this character of the exuvium being due to admixture with a desiccated sebaceous product.

Diagnosis.-The differences between psoriasis and syphilitic papulo-squamous eruptions are of importance. It is only atypical manifestations of either disorder that are liable to be confounded. The reddish and bleeding surface left on removal of the scales from a psoriatic patch is never exactly reproduced in syphilis, and the localization of the former on the extensor surfaces of the extremities is never characteristic of the syphilitic exanthem. Seborrhoic affections, particularly of the face, resemble the scaling papular syphiloderm in the matter of the greasy crust with which they are covered and the generally dirty aspect of the patch, but the circinate contour of the syphilodern, never seen in the seborrhoic disorder (save in exceptional cases on the trunk), and the characteristic copper hue of the surface beneath the scales, sufficiently distinguish the syphilitic exanthem.

In almost all the syphilitic patches resembling those of either psoriasis or seborrhœa the infiltration of the body of the patch, with its higher wall of infiltration at the periphery, is evident on examination.

Palmar and Plantar Syphilodermata (Palmar and plantar "syphilitic psoriasis," etc.).-The papules of syphilis, when developing upon the palms and the soles, have, as already shown, not only a characteristic aspect and career, but are rarely to be confounded with other disorders. The peculiarity of the papule in this situation is that it is developed within and beneath the dense and roluminous corneous envelope of these regions, and hence fails to produce either a conical or flattened elevation above the surface; it produces instead a circumscribed thickening of the skin, (Fig. I), which in 
the epidermal portions scales, and in extreme cases induces an ulceration in the region of each papular thickening. These eruptive symptoms are often early to appear, and sometimes they linger after years have elapsed as almost the sole symptoms of the disease. They are much more common than is generally believed in the early periods of the malady - that is, within three months after infection-being usually recognized in some form by the expert when they escape the attention of all

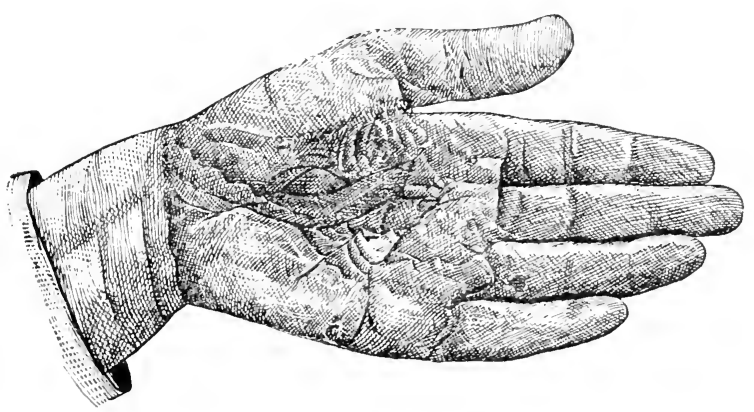

FIG. 1.-Palmar syphiloderm (after Keyes).

others, even of the patient. They occur usually symmetrically, involving both hands and feet, in the earlier manifestations, and asymmetrically in later stages, when either the feet alone or the hands alone, or even but one palm or one sole, is attacked. Instances are not very rare in which, with few other evidences of the disease, six and eight years after infection, a single palm exhibits a squamous syphiloderm, having displayed this symptom with slight variations for a series of years. In all the regions named the influence of the employment of the hands in labor is usually striking, the right hand being worse or solely involved in right-handed patients, and 
the feet worse in those who stand or walk much in the day; but marked exceptions occur.

In its simplest expression the epidermis of the region involved display's merely split-pea to lentil-sized discolorations productive of no sensation by which the patient is made conscious of their existence. The centre of the palm or the inner face of the instep is usually first affected, and the spots may be either discrete and without apparent order as to grouping, or develop in arcs of circles to be distinctly or dimly discerned. From these points they may spread to the dorsum of the hands and the feet, even over the dorsum of the digits, but in all such instances the extension from the palmar or plantar to the dorsal surface can be determined without effort. In this way the extension may be toward the interdigital spaces and the wrists and the ankles, the squamous process being in obvious relation with that first invading the palmar or the plantar area. When the digits alone are involved, the flexor aspect is always chiefly implicated, and here, as also in the palms and the soles, the natural folds and furrows of the skin furnish often a special territory for the incursions of the malady.

As the disease advances, both in time and in degree of involvement of the integument, the maculæ, of a hamred shade, furnish from the surface of each a slight exfoliation, which, as the disorder advances, becomes a true scaling, the epidermis being lifted away centrally, so as to produce about the morbid spot a dirty-looking, ragsed fringe of epidermis. An advanced stage of the discase is that where, usually in consequence of manual labor, friction, and exposure of the hands to soil, water, or chemicals, fissures result; these fissures make ineffectual attempts at healing, forming a new and tender 
epidermis which floors over the crack in the skin, only in turn to give way and be supplanted by succeeding fissures and new formations of epidermis until a palmar or plantar ulcer or an ulcerated fissure is excavated, bordered by successive plateaux of newer or older skin, the outer edge being represented by large, partly-detached, and ragged flakes of epidermis whose angular indentations or scallops roughly resemble the fracture of a pane of glass by a missile projected through its substance. Deeply ulcerated and exquisitely painful lesions of this class are more often palmar than plantar, by reason of the use of the hands in labor; but the feet of those who toil in sewer-digging, road-making, etc. suffer to a similar extent.

A variation of this eruption is termed the corncons syphilodirm, and its peculiarities are due to the accumulation at the site of each papule of a mass of horny cells, more or less friable, which may occasionally be dug out from their bed with the point of a pen-knife, or, being spontaneously thrown off, leave little shallow pits behind.

Diagnosis.-Eczema of the hands and the feet usually involves the dorsum, or, if the sole or the palm at all, only by extension to the latter from the former region. Eczema limited to the palms and the soles does, however, occur, but chiefly in adults whose organs are more or less continually immersed in water, especially water charged with mineral constituents. Patients of this class are usually dyers, laundresses, bar-keepers, or men engaged at soda-water fountains. The infiltrated areas of eczema are never well defined save in eczema marginatum of this region; the involvement of the skin is much more uniform; there is apt to be pustulation and 
vesiculation; there is never, under any circumstances, ulceration, even when the eczematous fissures are most painful; and the itching is apt to be well marked. Psoriasis is said to be in very rare cases limited to the palms and the soles, but these exceptions are so few as simply to prove the rule. In any doubtful case the discovery of psoriatic patches on the scalp, the sacrum, the elbows, or the knees would determine the question. It has been said that syphilis of the palms and the soles is ever accompanied by some unexpected lesion elsewhere, and it is often true that a mucous patch in the mouth or, in adranced cases, an undeveloped gumma of the leg will reward the careful explorer for his pains.

Moist Papules.-(a) Mucous patches (Mucous plaques; Ploques muqueuses). - The patch which is seated upon the mucous membranes in syphilis is pathologically identical with the mucous plaque or the moist papule of the skin. In both cases the papule-which in the palm or the sole fails to become elevated, but flattens to the point of exhibiting merely a scaling and plain macule-shows, in the regions of moisture, of friction or apposition of contiguous surfaces, and of heat, merely an oval or circular, scarcely elevated lesion. Its summit either furnishes a mucoid secretion or displays a thin pellicle more or less firmly attached, representing a sodden epidermal plate not as yet loosened from its underlying attachments.

Moist papules of the skin in syphilis occur in regions where the conditions are similar to those of mucous membranes with respect to heat, moisture, and the apposition of surfaces, as between the breasts of women, between the nates, in the axille and the groins of fleshy persons, and in the interdigital spaces. Here the lesions form flattened disks, slightly elevated above the general 
level, covered with a whitish or grayish pellicle, often slightly depressed in the centre, and looking not unlike one of the varieties of the soft corn. At times they have a reddish tint. They are generally moist, secreting a thin mucus which in warm weather and in the uncleanly has a fetid odor. These lesions are decidedly more common

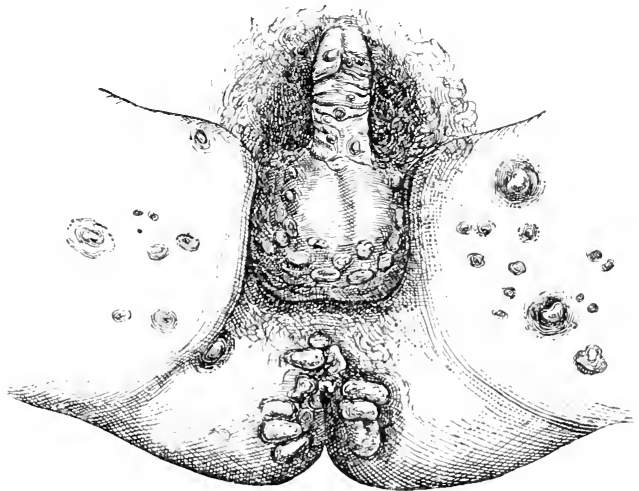

FI' 2 - Moist papules (after Miller).

in women than in men, and in the young adult rather than in the middle-aged. Occasionally they develop into large vegetating masses; at other times they ulcerate. Their secretion is highly contagious. There is no better illustration of the moist papule than the chancre of the mucous surface of the prepuce, which, having survived until general symptoms of systemic disease occur, undergoes a characteristic transformation in situ into a moist papule.

(b) Condylomata (Condylomata lata; Verruca acuminata; Moist wart; Venereal wart; Gcr. Spizen Warzen). -Condylomata are simply moist papules which undergo a hyperplastic metamorphosis in consequence of the 
extremely favorable circumstances under which they develop. Thus, a recently infected, young, fleshy prostitute of the filthy class is exceedingly apt to display lesions of this sort about the vulva and the perineum. There are two tolerably distinct types of this affection, namely, the flat condyloma and the pointed wart. Both occur in syphilitic subjects, but the former is seen only in that disease and is a distinct symptom of it; the latter is seen, when the conditions are favorable, not only in syphilis, but in other venereal diseases as well.

Condylomata are found in the regions favorable to the growth of all moist papules, but they are best seen about the anus, where they often encircle the anal orifice with broad flattened disks from the size of coins to that of the section of a large egg. They enlarge by the growth of the primary lesions and also by coalescence of the disks. They have a disgusting odor, they generally secrete a mucoid or even a puriform semi-fluid, and they are whitish both from this secretion and from the pellicle covering their broad surface. They are capable of self-multiplication, the lying of one disk against an exposed surface being at times sufficient to produce a similar lesion exactly at the point of contact. As distinguished from others of the syphilodermata, they are usually the seat of a tormenting pruritus.

The pointed a'art occurs in the subjects of syphilis and also in those whose parts are bathed with blennorrhagic, leucorrhœal, and other secretions not syphilitic. They are single, multiple, often exceedingly numerous, filiform, papilliform, or corymbiform, moist and pointed lesions varying in size from a pin-point to that of a fist, and even in extreme cases very much larger, the large-sized 
masses being always compounded of many primary warts, the septa between which can be recognized dividing the compound mass into separate lobes. They are often smeared with mucus, after the removal of which can be seen their vivid red color, each separate apex being provided with a delicate tuft. They are often compared in appearance to the comb of a cock. They rarely occur in virgins, but at times they develop in pregnancy, disappearing, as a rule, after delivery. Cocci and bacilli have frequently been recognized upon their surface. They bleed readily and freely when torn, scraped, or wounded by accident.

Pathologically, all moist papules are to be viewed as hyperplasias of the epidermis occurring under the influence of the syphilitic process; the pointed warts are not in all cases strictly defined syphilodermata, but are growths occurring under peculiarly favorable circumstances in the situations described. These growths are impressed with the syphilitic mode when occurring in the syphilitic subject. Anatomically they are found to be built up chiefly of thickened and enlarged rete-cells, the corium and the papillary layer exhibiting cellular infiltration, the papillæ reaching upward by elongations between the wide fields of the mucous pegs dipping between the papillary eminences.

Diagnosis.-Pemphigus vegetans (of Neumann) often occurs first about the vulva and the anus. Its lesions have frequently been mistaken for condylomata. Close study will, however, reveal that the vegetating masses in pemphigus spring from the sites of bulle, that they are, as a rule, more closely packed together, and that, instead of furnishing a mucoid secretion, they are bathed in a more profusely furnished fluid, which, as a rule, is desti- 
tute of any offensive odor. There is less flattening of the pemphigoid eminences; and when similar lesions occur in the mouth, the latter are to be distinguished from mucous patches by their extreme soreness and by the fact that in the latter situation they begin as blebs. In pemphigus vegetans there may be fever, and the signs of an exceedingly grave involvement of the system are usually present.

\section{Pustular Syphiloderiata.}

It has been seen that the type of the lesion of syphilis in the skin is to be recognized in the papule. This may spring from a macular lesion or be such ab initio, but whether the one or the other, or whether the further evolution be in the line of the squamous syphiloderm of the palm or of the moist condyloma of the vulva, in all these cases the career of the syphilitic affection may be described as pursued within natural parallels. When, however, vesicles, pustules, or blebs appear, it may in general be believed that some accident has intervened to divert that career into singular channels. These accidents are, briefly, first, medicaments employed for the treatment of the disease, productive of medicamentous rashes in the subject of syphilis; second, the invasion of the skin by staphylococci (staphylococci pyogenes albus et aureus); or, lastly, neglect or abuse of the skin, as in case of extreme filth, the application externally of injurious medicaments, and the attacks of animal parasites (fleas, bugs, lice, etc.). It is an interesting and noteworthy fact that the great number of all pustular eruptions in syphilis are observed in public and hospital patients. It is among the rarest of occurrences to find patients of the well-to-do class, properly treated, 
exhibiting these symptoms of the disease. These eruptions have, however, been so long classed with the exanthemata due exclusively to syphilis that some boldness is needed to relegate them strictly to the category in which they belong. They have, it is true, the syphilitic impress, but their exciting cause is an accidental, and not an essential, factor in the malady.

Under the title of the pustular syphilodermata are here included all fluid-containing lesions of the skin, such as vesicles, pustules, and bullae. Nany of these eruptive phenomena have been given unfortunate titles in the text-books, such as "varicella-form," "eczematous," "acne-form," etc. These names should all be obliterated from the nomenclature of syphilis; first, because it is unwise to describe one disease in terms of another with which it is liable to be confounded; second, because, to be of practical value in the way of description, a title should have a fixed meaning. The words "eczema," "impetigo," and "ecthyma," which have been used in this connection, no longer describe classically defined symptoms of any skin-anomaly, but mean, instead, ranges of widely differing symptoms due to various causes, and conveying to the eye no such fixed impression as these names are supposed to produce upon the mind.

Miliary Pustular Syphiloderm.-In this eruption pinhead-sized pustules, or, more properly, vesico-pustules, are evolved, each at the summit of a papule, and, as previously suggested, almost always as the result of a febrile state complicating the ordinary evolution of the disease. At times the cause, however, as in the other eruptive disorders of this class, is distinctly due to a secondary infection with the toxines of staphylococci. 
The lesions are pinhead-sized, but they may increase to the size of the larger pustules. At times they have a circinate grouping; at other times they are disseminated freely over the face, the trunk, and the flexor surfaces of the limbs. In exceedingly rare cases the eruption is generalized. The pustules may be surrounded by a characteristically tinted areola, and they may disappear by desiccation of the effused fluid into thin brownish or dark-colored crusts, or there may be coalescence of the pustules to the point of formation of a superficially suppurating surface. The firm, shot-like papules on the point of suppurating at the apex have at times been mistaken for the lesions of snall-pox, which they greatly resemble. This syphiloderm is frequently recognized within the first few months after infection.

It is somewhat difficult, and from a diagnostic point of view not highly important, to distinguish between the miliary and the lenticular pustules of syphilis, since the former are freely convertible into the latter, and the essential difference between all is merely the degree to which in each the minute abscess spreads in area and in depth. In the one class or the other are evolved the following clinical types:

I. Pustules situated at the orifice of the pilosebaceous crypt, occurring chiefly where those accessories of the skin are largest and most abundant (scalp, face, and upper chest). In these regions minute or even large beansized, acuminate, and conical or flattened pustules form; these pustules desiccate into thin crusts or furnish a superficial area of pustulation. The cicatrices left are rarely conspicuous or even permanent; more distressing to the patient is the brownish stain left. The lesions are often distinctly grouped. The general aspect of the 
region thus involved (lips, nose, forehead, etc.) is one of extreme dirtiness, even the regions of the skin not displaying eruptive symptoms being unwholesome in appearance and muddy in hue.

2. In a second clinical form the pustules are larger, usually flattish, and, after attaining the size of a pea or that of a large bean, surmount superficial, rarely very deep, circumscribed ulcers (Fig. 3). Here the pusformation is decidedly more abundant; the copper-

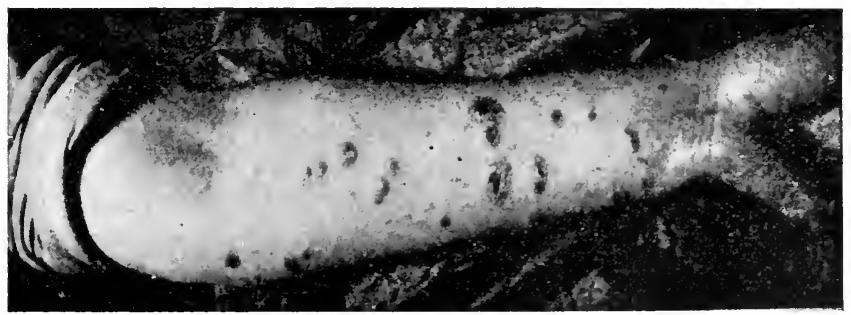

Fif. 3.-Large pustular syphiloderm (Stelwagon).

colored or even chocolate-tinted pigmentation left after involution is more marked, and the resulting scars are more often indelible.

3. In a confluent variety of the larger pustular lesions -as a rule, flattish and decidedly fewer in number than in other cases-there is distinct circular grouping of the pustules and the underlying ulcers. After fusion of the elements of the eruption an ulcerating ring forms, usually surmounted by a dirty-brownish crust, often with a ham-tinted stain at the outer border. Here healing may occur at one or several points, producing thus alternations of crusts and newly-formed epidermis in the ring or the parts of a ring surrounding either an integument unaffected centrally or a healed or healing ulcer. 
4. Another clinical form is to be recognized where the lesions are few and are not irregularly distributed over the entire surface, but where six or more form perhaps over the scalp, or a similar number along the alae of the nose, the extensor face of the elbow, or over the genital region, and perhaps none elsewhere.

\section{Pustulo-crustaceous or Pustulo-ulcerative Syphilo-} dermata. - These terms represent an artificial distinction preserved, as a matter of convenience merely, in classifying the pustular syphilodermata. The lesions thus designated represent a variation in the line of ulceration and consequent destruction of parts deeper than those affected in more superficial erosions (Pl. 4). The pustulo-crustaceous syphilodermata are all pathologically alike, differing chiefly in point of gravity. Each represents a secondary infection of the skin with cocci. Of the members of this group it may be said that the single or sparse lesions are commonly more destructive than those which are decidedly multiple; that in point of gravity a very great multiplicity of lesions betokens a gravity dependent upon the constitutional effect of the involvement of a large portion of the skin in an ulcerative process; and that generally the amelioration of the condition of the integument is proportioned to the improvement in the systemic state of the patient. They represent in general a somewhat late stage of syphilis, and one in which are found patients who are cachectic, poorly fed, or improperly treated or cared for (Pl. 5). Here the pustules tend to enlarge, to develop in more limited and circumscribed areas, to involve a greater depth of the corium and the subcutaneous tissue, and to be accompanicd by symptoms of malignancy. The area of each pustule or group of pustules assumes an angry look; 


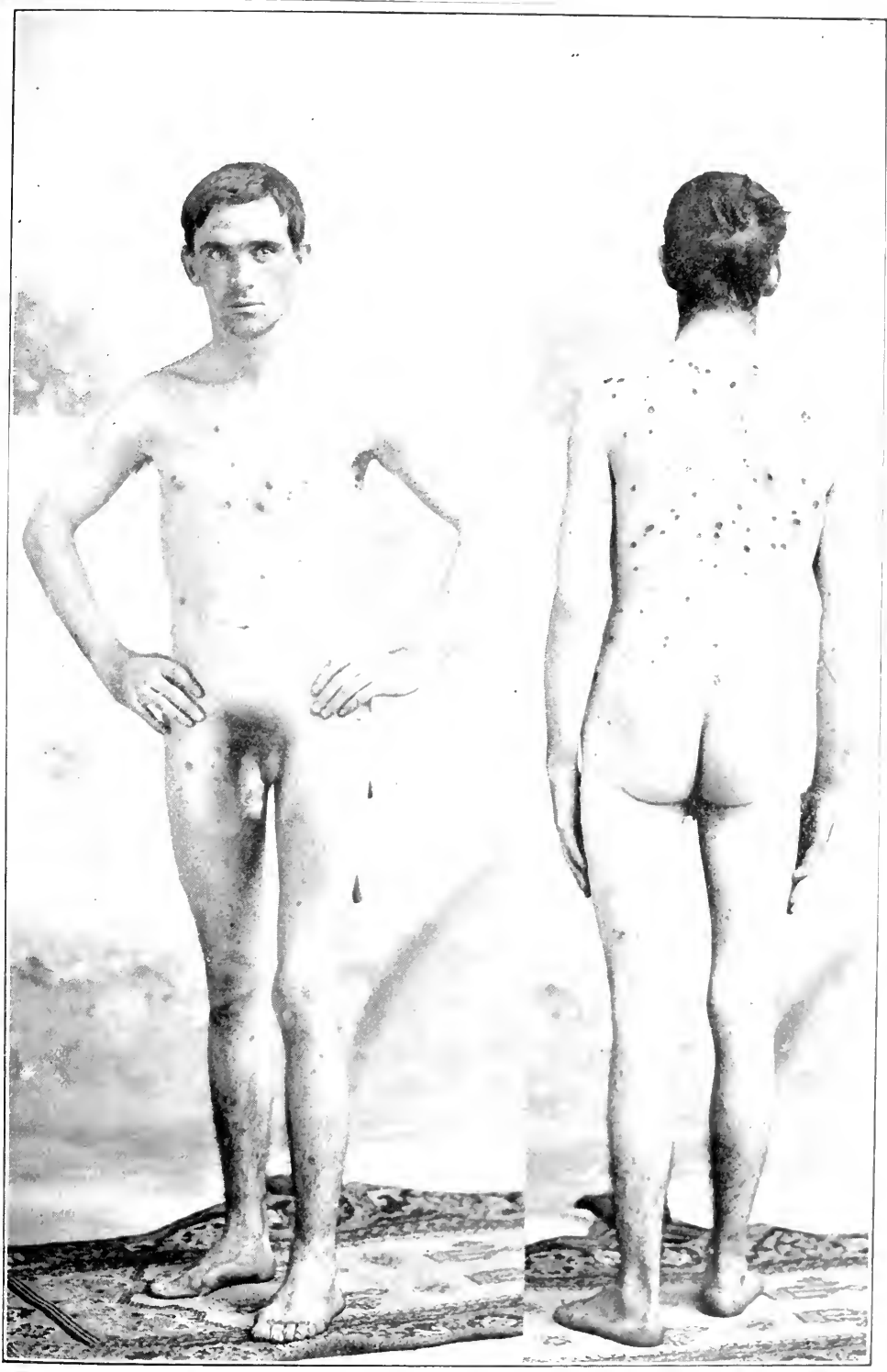

Pustulo-ulcerative syphiloderm, with survival of sclerosis of the penis. 

the pus formed is inspissated, hemorrhagic, and commingled with pultaceous sloughs; the resulting crusts are blackish, the scars are persistent, and the pigmentation is deforming and slow to disappear. The ulcers left by the largest and most formidable of these lesions are of the type of the syphilitic ulcer in general. They have clean-cut, punched-out edges, a floor covered with an adherent pus-bathed slough, an engorged base, and a roof at times constituted of the successive desiccations of pus formed from the spreading ulcer beneath, so that a stratified conical crust with limpetshell aspect is produced. Here, again, the circular, semicircular, horseshoe-shaped, and other combinations of the circle so oddly characteristic of the ulcers of syphilis are constantly encountered.

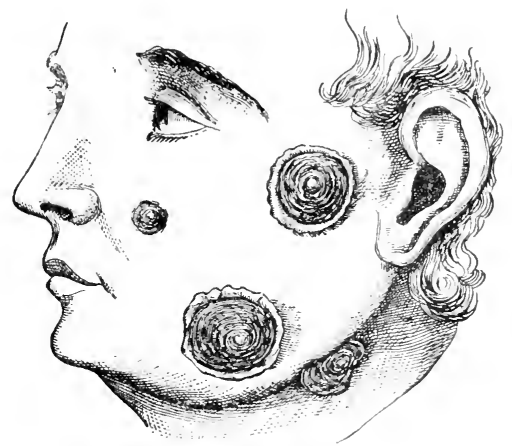

FIg. 4.-Rupia (after Tilbury Fox).

The oyster-shell-like crusts seen in various sizes in so many of the pustular syphilodermata, especially over large-sized lesions, were once supposed to be produced in other diseases, and the name rupia was given to the malady exhibiting these features. One of the axioms of the dermatology of to-day is that the symptoms of 
rupia are those of syphilis. Prior to the formation of these crusts there is always a history of, first, infection; second, of the evolution of pustular or bullous lesions with hemorrhagic or semi-purulent contents; third, of the bursting of the chambers containing these fluids; fourth, of their desiccation into greenish or greenishblack crusts, at first scarcely larger than a pea or a bean, later attaining the size even of a pullet's egg; and, lastly, of a series of elevations of the stratified and conical crusts by successive accumulations from a constantly widening area beneath, until the picture of the rupioid skin is complete. When repair ensues the crusts fall, the ulcers granulate and become simple and shallower, and cicatrization concludes the history. Here, as so often seen in other of the syphilodermata, the underlying ulcer may assume any of the circular outlines or the shapes of imperfect circles, the overspreading rupioid crust having a similar configuration. The exanthem is rarely generalized, though in extreme cases large areas of the trunk and the limbs may be involved, wide spaces of unaffected skin, however, usually intervening between the conspicuously contrasting crusts. The eruption occurs most frequently in the cachectic, the weak, and the victims of malnutrition, neglect, and poverty. In every instance, however, it indicates a secondary infection with cocci.

The other pustulo-ulcerative or pustulo-crustaceous syphilodermata are variants from the type represented in rupia, and most commonly in the direction of gravity. This is shown by such results as an increased depth of ulceration and more profound involvement and destruction of tissue. Some originate as single or multiple vesico-bullæ of apparently benign character; some as 

rapidly degenerate infiltrations which it is difficult to distinguish from gurnmata. All are apt to leave indelible cicatrices; yet, even after multiple ulcers of severe grade have riddled the integument in certain regions, the extent to which repair occurs and the evidences of damage are in the course of years smoothed away is, as a rule, surprising to those not familiar with these possibilities.

Diagnosis.-From the several lesions described above, varicella and variola, however much resemblance may be traced between the former and either of the latter, may usually be distinguished by the fever of invasion, by the relatively active rather than indolent evolution of their lesions, by the umbilication of the fully-formed variolous vesico-pustule, and by the multiplicity of lesions in severe variolous cases, in which the lesions usually far outnumber the pustules of even the best-developed syphiloderm of the same type. Signs of syphilis other than pustular eruptions may be recognized in most patients affected with that disease, such as mucous patches, glandular involvement, alopecia, etc. In acne the usual limitation of the eruption to the regions of preference of that disease (face, anterior and posterior aspects of the upper trunk) is generally suggestive, and the sprinkling of comedones among the pustules is significant. In syphilis, pustules of the face often appear in conjunction with similar lesions of the scalp; this condition is practically never seen in simple acne, the scalp in the latter affection being, when at all involved, the seat of either a seborrhœa or an alopecia furfuracea. Acne, however, is exceedingly common in syphilitic subjects, and it should always be recognized when complicating such cases. It occurs, first, as a result of ingested medicines, whether 


\section{2 SYPHILIS AND THE VEVEREAL DISEASES.}

properly or improperly administered for relief of the syphilis present (iodic or bromic acne); second, as the result of the causes efficient in the production of acne in the non-infected (alcoholism, dyspepsia, constipation, etc.). Hundreds of patients are annually treated for an ancient syphilis which has ceased to exhibit evidences of its existence and yet which is supposed to be in activity because of an unsightly acne.

Pathology.-Under the microscope, sections of a pustular syphiloderm resemble very greatly those made in variolous and other disorders having similar lesions. The usual rents in the epidermis are visible; its remaining strata are pus-infiltrated; the deeper rete is eroded, in parts exposing the corium; the individual elements of the latter are filled with lymph-cells; the bloodvessels are distended, and in places are choked. Chambers originally filled with pus and the detritus of tissue are readily recognized at different levels, according to the depth of involvement of the tissue. Stretched and torn rete and corneous cells are visible both in the cavities and in the walls of chambers formed by the exuded fluid. At times the site of the pustule is a hairfollicle, in which case its adnexa are also involved; at other times the pus-making process attacks the corium outside the pilary and sebaceous pouches. Not merely the entire corium, but the subcutaneous tissue as well, may be involved (Cornil, Kaposi).

\section{Tubercular Syphiloderiata.}

Pathologically there is little difference between the tubercles and the gummata of syphilis, and even clinically the distinction between the two cannot always be determined. In many cases, however, it is a matter of 
convenience to distinguish between the classical forms of these frequent lesions. As a rule, the tubercle is more superficial than the gumma, occurs in less grave forms of the disease, is more apt to resolve and less disposed to degenerate, develops at an earlier period, is much more often multiple and exceedingly numerous, and occurs in a larger number of patients in forms that are grouped.

Syphilitic tubercles may develop in the course of a few months after infection, but they are more common after the lapse of from two to ten years. They invade the face and the extremities, and in these situations and elsewhere (for they may be found in any region of the body) are pea-, split-pea- to bean-sized lesions, the smaller dimensions named being most frequently attained. They are firm, well-defined nodules, neither definitely flattened nor acuiminate at the surface, with a tendency to assume the globoid shape. In color they are reddish-brown or copper-tinted, the hue deepening to a dark empurpled shade in the extremities by reason of gravity, and in the face after great congestion or unusual exertion, such as dancing. Their grouping is distinctively and characteristically in circles and portions of circles, further extension of the eruption being by the formation of new and adjacent rings producing the figure 8 , the letter $\mathrm{S}$, the dumb-bell, and the "satellites," as in the arrangement of a jeweller's brooch. As they differ in respect to the mode of their involution, they furnish thus a basis for a useful distinction.

Resolutive (“Dry," “Atrophic”) Tubercular Syphiloderm.-In this class are placed tubercular lesions which degenerate not by ulceration, but by resolution through metamorphosis of the effused product 
II SYPHILIS AND THE VENEREAL DISEASES.

beneath an unbroken epidermis. The result is unique -namely, the formation of a cicatrix where there has been no loss of continuity in the outer layer of the skin. The tubercles are then effaced by a species of atrophic

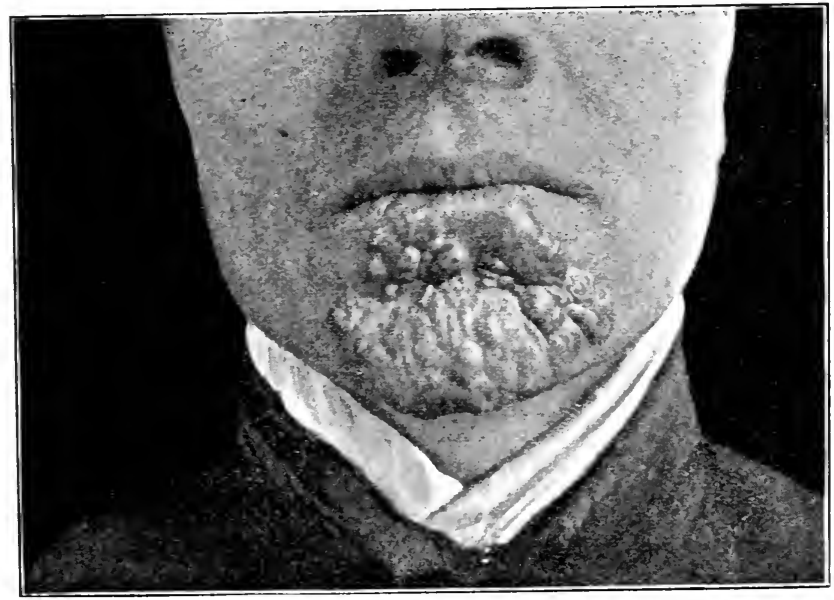

FIG. 5.-Resolutive tubercular syphiloderm in groups.

change, leaving a pigmented and cicatricial macule in the site of each tubercle, the pigment at a later date fading and leaving in its site conspicuous indented scars somewhat smaller than the original tubercle. These groups of scars, circumscribed and with their pigment but partially removed, forming portions of an imperfect circle, one arc of which is represented by tubercles as yet unresolved or but partially effaced, is one of the most striking of the pictures presented in syphilis, and one not imitated in any other disease. Upon the face (Fig. 5), about the knee, upon the elbow, or over the anterior aspect of the forearm in its lower third, these striking composite groups are always significant to the 
trained eye. Tubercles of this class upon the palms and the soles are exceedingly apt to scale in process of either evolution or involution, the scaling being at both the summits and the sides of the lesions. The tubercles are in no way distinguishable from those described below, save in the matter of their historical career.

Ulcerative Tubercular Syphiloderm.-In this artificial class the tubercles degenerate by ulceration, this change occurring in different cases as a modification either of the elementary lesion itself or of the underlying tissues to which the ulcer, originally linited to the tubercular mass, eventually extends. In the simplest form these tubercles soften at the summit, exhibiting at this point a more or less adherent, slightly sloughy crust. If this crust be removed with more or less force at an early period, it can readily be seen that an ulcerative process has begun to destroy the upper portion of the small tumor. All the stages of ulceration and repair that follow depend upon the general condition of the patient and the good or bad treatment and hygienic aid which he receives. When the ulcer spreads beyond the mass of the elementary tubercle, it passes into the category of gummatous lesions; but if the degeneration is limited to the original tubercle, the clinical picture is distinct. In these cases circlets, complete or partial, of crusted tubercles or of crust-covered and circumscribed ulcers surround an unaffected or infiltrated area of skin, the color of which is of the type seen in the resolutive groups described above. Upon the face, where these lesions are of special importance and of frequent occurrence because of the exposure of this region of the body to frictional, accidental, and atmospheric influences, the arrangement may be less distinctively in circles; 


\section{II6 SIPHILIS AND THE IENEREAL DISEASES.}

as, for example, over the sides of the nose, where crusted nodules may be indiscriminately sprinkled over one or both sides with as little order as the lesions of acne with which this syphiloderm has at times been confused.

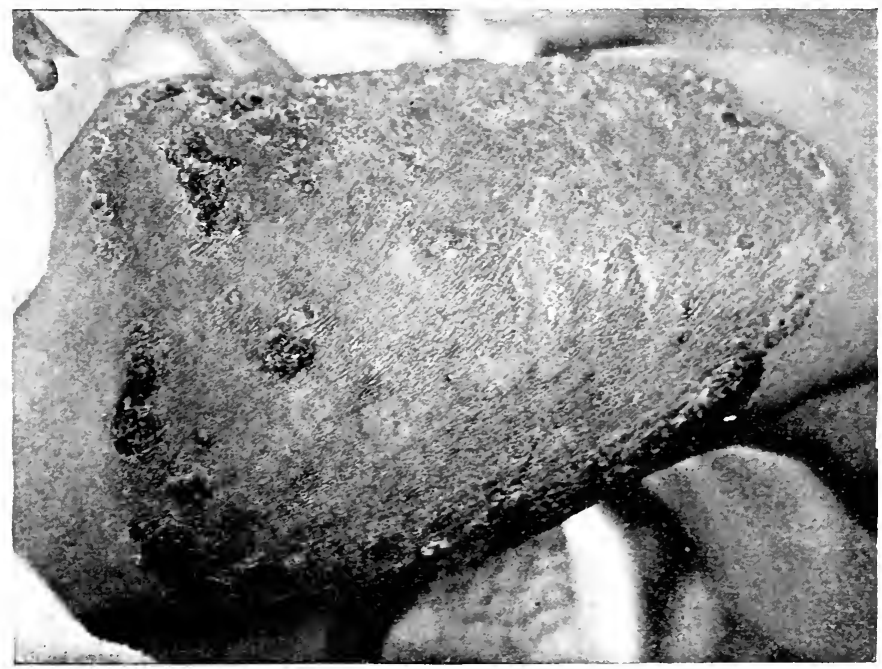

FIG. 6.-Serpiginous tubercular syphiloderm (after Stelwagon)

Upon the trunk and the limbs, however, the tubercles are often not merely grouped originally in circles or parts of circles, but they spread at times by serpiginous extension until wide areas have been swept over (Fig. $6)$, leaving, where the activity of the process was once declared, broad, palm-sized and even much larger cicatricial patches where the skin is thinned, and where one can recognize the pea-sized and smaller, depressed and circumscribed points, each representing the site of a former tubercle and ulcer. Often giant circles of involvement, affecting, for example, an entire buttock or a portion of the back, have in this way indolently progressed for 
years, the nature of the disorder being misunderstood for that period. Many patients thus afflicted have been treated for years for "lupus," "tuberculosis," and other affections, relief having speedily been effected after discovery of the exact nature of the malady.

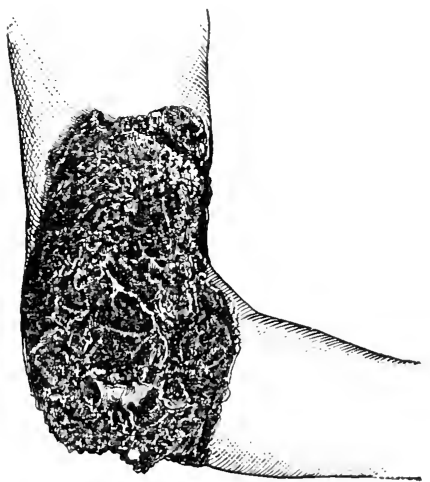

FIG. 7.-Ulcerative tubercular sy phıloderm (after Keyes).

When tubercles of this class coalesce and degenerate, it is at times difficult to recognize the elementary lesion present. In these cases, obscure only to the inexpert, the encircling series of small tubercles is replaced by a rim-like wall of elevated tissue, either broken down at several points by the ulcerative process or preparing to break down. The area enclosed may also be found to consist of an infiltrated disk with circles or segments of circles within the parent group, some ulcerating at the outer border, others wholly or partially cicatrized in ineffectual attempts to ensure repair. Typical tuberculoulcerative patches (Fig. 7) strongly resemble many of the ulcerations following degeneration of gummata, and it is to be remembered that the process in each is 
essentially the same, the differences being due solely to artificial classification. In all the grave and widelydiffused ulcerations springing from syphilitic tubercles, as a rule, the elements last named soon become inconspicuous features of the general process. Repair of the degenerative losses here described occurs by granulation of the ulcers, by effacement and resolution of those not yet having undergone degeneration, and by the eventual production of multiple cicatrices, which, being often arranged in groups of circles adjacent to or encompassed by others, furnish unmistakable evidence, years after the date of the development of the tubercles, of a syphilitic infection in the past.

Diagnosis.-The diagnosis of a tubercular syphiloderm, present or past, is of the very highest importance for the diagnostician, seeing that years may have elapsed after the date of infection before attention is attracted to the eruptive symptoms. Upon the diagnosis may rest a question of life or death, as, for example, when a man lies unconscious from a gummatous involvement of a portion of the meninges of the brain, and there is only a tell-tale scar on the buttock or the loin to indicate the original nature of his disorder.

The papular and tubercular forms of acne, especially in florid-faced male subjects of alcoholism, occasionally resemble a tubercular syphiloderm of the nasal region; but in syphilis there is usually complete failure of symmetry, one side of the nose being predominantly involved, though the exceptions are not rare. In acne the evident involvement of the sebaceous glands, the tortuous vessels visible about the lesions, and the general rosaceous appearance of the organ are characteristic. In syphilis, when at all advanced, there is either distinct 
crusting or superficial ulceration beneath the crusts, either of which signs suffices to distinguish the nature of the disease.

The several forms of eczema are all recognized by their inflammatory aspect, their catarrhal features, the intense pruritus they awaken, and the general absence of distinct contour. The scales and the absence of scarring and ulceration in psoriasis usually suffice for its determination. Lupus vulgaris, one of the forms of cutaneous tuberculosis, is perhaps more often confounded with syphilis, or the latter with the former, than are any confused diseases. The distinction is always a matter of great importance. It must be borne in mind, when confronted with any doubtful case, first, that lupus vulgaris is a disease most often beginning in the first or the early part of the second decade of life, syphilis usually dating either from the latter part of the second decade or from that which follows it; second, that lipus vulgaris is decidedly less common than tubercular syphilis; third, that the latter is usually presented, in any doubtful case, at a period from three to eight or more years after the date of infection; lastly, that, as regards chronicity, syphilis is a relatively rapid disease, producing in six months or less a destructive result which tuberculosis would require as many years to accomplish.

The nodules of lupus are readily perforated with a blunt-pointed needle; those of syphilis resist a firm impression. In lupus, even though a patch be formed, it distinctly lacks the ovoid or truly circular configuration assumed by groups of syphilitic tubercles, and it may be said never to produce the combinations of circles previously described. The same is true of the ulcers of lupus as distinguished from those of syphilis, the floors 
of syphilitic ulcers, further, being generally covered with a pultaceous slough surrounded by steep-walled edges, while the edges of the lupous ulcer are thin and stretch over softish, pulpy, jelly-like masses of indolent granulations. The degree of pain experienced is far greater in syphilis than in lupus. Over the face, lupus, whether destroying by absorption of the effused product or by ulceration, produces the characteristic "parrot's-beak" deformity of the nose or reduces it or the ear to a shrunken miniature of its former self; while syphilis boldly destroy's one ala and at the same time spreads in the nasal fossæ, attacking the bones of the nose and producing its special deformity by the sinking of the bridge. In tubercular syphilis of the face a circlet of lesions forming an infiltrated disk consisting of partly flattened and partly ulcerated tubercles is apt to attack one side of the brow near the root of the nose or to encircle one angle of the mouth; while a lupous patch will involve rather the centre of one or of both cheeks, and will display as many of its uniformly reddish-brown nodules in the enclosure as at the periphery of the patch.

Epitheliomatous ulcers, of the face especially, are more readily distinguished from those of syphilis. They are often surrounded by characteristic "pearls" of cancerous growth; they occur in a much older class of subjects; their floors are smooth and glazed, rarely sloughy; their edges are strongly everted; they are, as a rule, by no means painful; and they observe a far slower evolution, lasting for years without apparent change.

It is to be remembered also that in all forms of sycosis the hair-follicles are primarily involved, and the disease is strictly limited to the region of the male beard; that in leprosy the nodules of the face producing the leonine 
aspect are never arranged in circles, but in ridges and rows along the brows, and have a characteristically varnished appearance; that in rhinoscleroma-a disease reported in but a few isolated cases in America-there is a firm, ivory-like hardness to the portions of the nose involved that is not characteristic of syphilis; and that in zoster of the face it is rare indeed that both sides are involved, there being usually a strict limitation to one side of the face of firm nodules just ready to develop into vesicles.

\section{Gummatous Syphiloderi.}

Gummata of the skin are circumscribed firm nodules, usually involving either the subcutaneous or the submucous tissue, and often attacking later the underlying

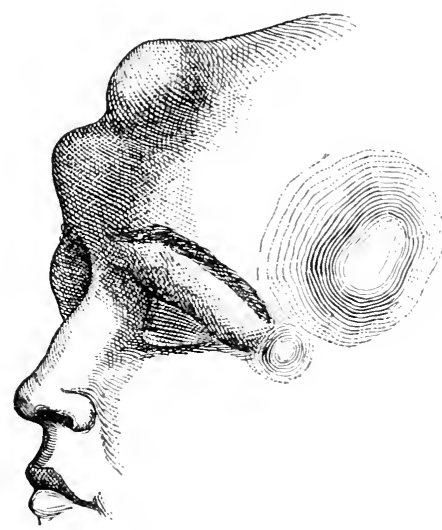

FIG. 8.-Gummata (after Jullien).

structures, such as fascia, periosteum, bone, cartilage, and tendon. They vary in size from that of a small nut to that of an orange, and they are at first uncolored elevations of the skin, but later, when degeneration is 
threatened, the integument over each nodule becomes purplish, livid, boggy, and thinned to a point where bursting of the contents of the gumma occurs through its connective-tissue envelope. Its name is derived from the gummy character of the product evacuated when bursting of the neoplasm ensues. When freely forming upon a level surface, gummata are usually globoid in contour, but they may be instead irregularly shaped and even flattened. They are rarely very numerous in one subject at a given time, many patients never exhibiting more than one, or at the most two or three, typical gummata (Fig. 8); in rare instances hundreds form at one time in the same person.

Gummata are usually counted as "late" syphilitic lesions, but they may develop within a few months after infection. As a rule, however, from two to five years or more elapse between the date of the appearance of the chancre and their evolution. They may be the final evidences of the syphilitic process, or they may again and again return in the neighborhood of the first site of their appearance, until the skin and the underlying tissues are seamed with scars commingled with healing and unhealed nodules, and connected by bridges of apparently sound skin beneath which run sinuous channels of ulceration.

At times the gummatous product is more or less widely diffused in a particular region of the body, such as the leg or the shoulder; in these cases, especially if the disease has existed for some time, the appearance is, however characteristic of late syphilis, not always suggestive to the eye of its real character. In these instances large areas as broad as the hand, often with tolerably distinct demarcation, present an exceedingly 
irregular and confused surface, seamed with ridges, oversprinkled with nodules and dense indurations, and perforated here and there with ulcers. In extreme cases, where the nature of the disease has been wholly unrecognized for years and where intercurrent eczematous and other affections have complicated the process, a singular variation of type occurs; and, especially where gravity has added its influence, as in the leg, there may be an elephantiasic result which requires careful scrutiny for an accurate diagnosis. In these severe cases the nature of the disease can generally be recognized after study of a small portion of the invaded area, where, wellnigh hidden in a mass of tumefaction, a telltale scar or a circular ulcer with typical edge and floor reveals the truth. In another extreme type the entire gummatous tissue breaks down into a gigantic ulcer as large as or larger than a platter, with an indolent and sloughy floor, a circular outline, and an engorged base. As a consequence of the force of gravity, it is most common to discover these ulcerations on the leg, but they are seen also on the shoulders and on the back. When in the former situation, however extensive, they rarely completely girdle the ankle or the lower third of the leg, as may other ulcerative processes in this region, but a considerable portion of the skin, usually posterior in position, remains unaffected. Upon the face the destruction, if less extensive, is usually more hideous (Fig. 9). Here a gigantic excavation may result from the breaking down of gummatous infiltrations whereby the nasal and oral cavities are converted into one gaping chasm, as in the severe grades of epithelioma. A large portion of the pinna of one ear may slough. The bones of the face, skull, and jaws frequently suffer, and ectro- 


\section{SIPHILIS ATD THE VEVEREAL DISEASES.}

pion, flattening of the nasal bridge, and extensive mutilation of the lips and the ears may ensue.

Not the least conspicuous among the distinctive features of these severe ravages of syphilis is the extraordi-

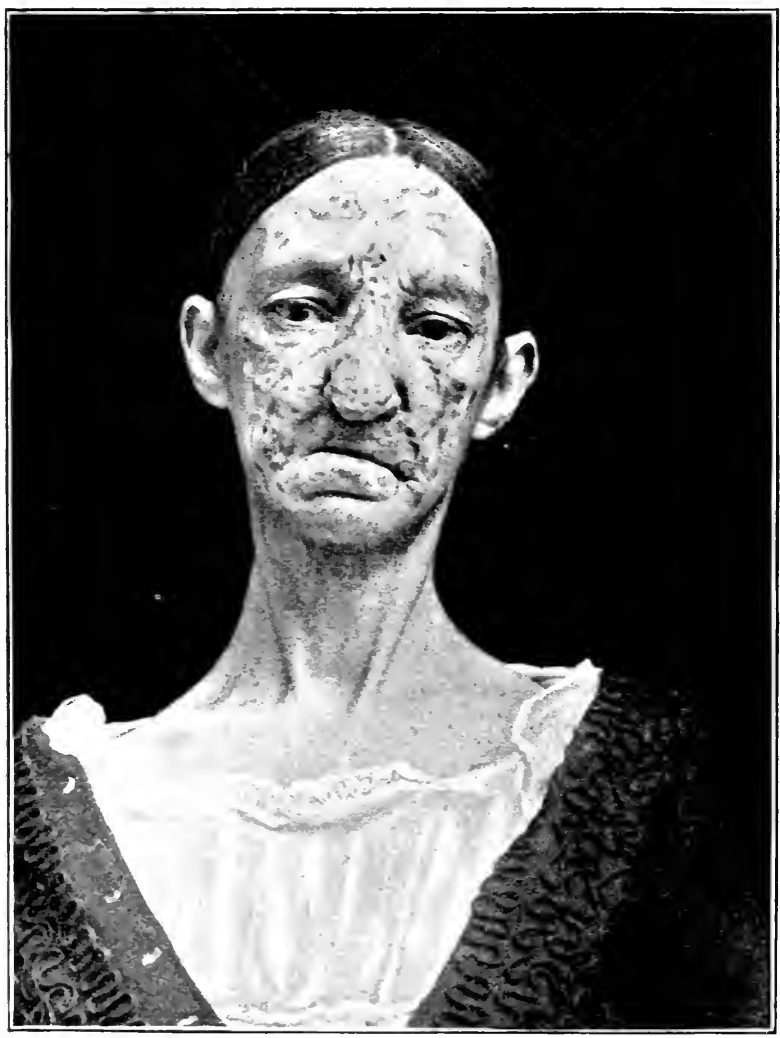

Fic, - Cicatrices resulting from extensive gummatous infiltration of the face.

nary extent to which, when properly treated, repair ensues. When the general cachectic condition (evident in almost all this class of patients) yields to proper hy- 
gienic and medicinal treatment, cicatrization follows after even the most extensive and mutilating damage; the deformity is slowly smoothed away so as to escape recognition save by the experienced eye, and the patient may enjoy a future life without return of the old trouble. In this way an obturator enables one man to close the gap between the mouth and the nasal cavity; another, who has an opening connecting the œesophagus and the larynx, can in certain postures and by the aid of special devices swallow food without its access to the respiratory tract; and even the most disfiguring scars of the face are slowly freed from pigment and diminished in circumference and irregularity until a degree of sightliness is produced (Fig. 9). It should not be forgotten, especially in relatively young patients, that even with the worst accidents the recovery, under anything like fair treatment, will surpass the hopes of the most sanguine. It is in this respect that syphilis draws a sharp and significant distinction between itself and all other diseases productive of destructive effects-a distinction of the highest value with respect to diagnosis.

Diagnosis. - The term "gumma" has lately been affixed to the somewhat similar cutaneous lesions of tuberculosis (lupoma, gomme scrofuleuse), and the resemblance between these and the gummata of syphilis is not slight. In the former the recognition of other tuberculous or scrofulous symptoms, their occurrence at an earlier period of life than most cases of syphilis, and the characteristic elevated longitudinal ribbons of empurpled and thinned skin, especially on the neck, enclosing depots of ill-conditioned pus, are common. When degenerating, there are formed linear or narrow ulcers with thinned edges and pulpy floors covered with soft granu- 
lations, the enlarged glands in the vicinity not yet being broken down.

Sarcomatous tumors are usually multiple, occur in conditions where cachexia is more marked, and are, as a rule, slower of evolution than syphilitic gummata, though at times undergoing rapid changes. They are rarer in the lower extremities than elsewhere-a distinguishing feature of gummata in syphilis.

Lipomata are readily differentiated from gummata by the softness to the touch of the former and by the "pillowy" feel of the growths, which, furthermore, are usually of far longer duration without change than syphilitic tumors. From a gumma, epithelioma is at times distinguished with ease, at others with very great difficulty. The following points are to be remembered: Cancer, as a rule, occurs at a later period of life, but at times the gummatous changes in syphilis occur at the same age. In epithelioma of the skin the "pearls" or waxy nodules, scarcely larger than pinheads of good size, are characteristic, and are never seen in syphilis. The course of an epithelioma of the skin is far slower than the career of a gumma, the latter rarely requiring more than a few months for its termination either by resolution or by disintegration, while a cancer of the skin may endure with less destruction for a decade of years. Multiplicity is true of the syphilitic more often than of the cancerous ulcer. The edges of the specific ulcer are steep or undermined; those of the epitheliomatous excavation are everted often to a very marked degree. In syphilitic ulcer the floor is sloughy or pus-bathed; in cancer it is, when typical, covered with a thin, varnish-like secretion which scarcely conceals the florid and irregularly excavated surface beneath. 
Gummata of the progenital region are at times liable to be confounded with initial scleroses and chancroids, but the accompanying adenopathy of the latter, their relatively rapid career, and the greater extent of the infiltration of the gumma usually indicate the difference. The chancroid is always more distinctly purulent and less indurated than the gumma.

Ulcers of the leg resulting from pressure-effects in the subjects of varicose veins of this region and of the thigh often present a strong resemblance to the ulcers of syphilis, but the distinction between the two can usually be made without difficulty. In the one case the enlarged veins, in the other the painful character of the trouble, the œdematous condition of the limb, the frequent coexistence of eczema, and the entire absence of a well-rounded scar or a deep circular ulcer, usually aid in the diagnosis. The picture in the non-specific disease is usually more serious than in the syphilitic disorder which it is sought to differentiate. The pigmentation in long-standing cases is far deeper and blacker in shade in the varicose condition, in consequence of the extravasation of blood. A tolerably clear outline to any given patch of diseased skin, and an absolutely unaffected integument in close proximity to an ulcerated or engorged patch, point always in the direction of syphilis.

Pathology.-Anatomically, the tubercle and the gumma of syphilis are practically identical. The process is essentially one of disintegration of the component parts of the nodule, with central fatty and purulent degeneration of fibres, cells, and nuclei, and peripheral proliferation with round cells commingled with few giant-cells surrounded by connective-tissue fibres. 
The zone of proliferation about the central depot of globules is evidently protective in character (Kaposi, Basset).

The groups of syphilodermata described above are classified in the artificial divisions, between which they can, for the most part, readily be separated. There are, however, a few manifestations of syphilis in the skin, the peculiar features of which justify special consideration. They are, in point of fact, modifications of the symptoms already described.

Serpiginous Syphiloderm.-The term "serpiginous" was originally employed, as its etymology suggests, to designate a lesion displaying "creeping" features, a slow and gradual extension from one point or from several points to others on the cutaneous surface. At present the word designates the peculiarities which may be assumed by one or another of the syphilodermata, rather than any special exanthem of syphilis. In a serpiginous eruption there is extension of the disease, either by ulceration or by retrograde metamorphosis, at the periphery of an involved patch, while the central portion is the seat of partial or complete cicatrization. While this effect may not rarely be noticed in any of the ulcerating or resolving syphilodermata in groups, the term "serpiginous" is applied specifically to those cases in which this peripheral extension and centric involution are decidedly more pronounced than other features of the disease at any time present.

The serpiginous feature may be assumed, as has been seen, by a group either of pustules, tubercles, papules, or gummata. Beginning with one or a group of several of such lesions, the process may be either superficial or 
deep. As a rule, the most ambulant and erratic of these serpiginous patches belong to the former rather than to the latter class.

On the earliest recognition of a serpiginous tendency in any patch of disease, it can be seen that the clearlydefined peripheral wall is spreading either in equal radiations from a central point (artificially placed) or, rather more commonly, more actively in one direction than in any other. The peripheral wall may be built up either of confluent papules or tubercles or any crusted lesions of the types named, or by sequelæ of any of the latter in the form of a shallow ulcer, circular in outline, resembling a moat about an enclosed field. The central area may then be made up of infiltrated integument, pigmented or otherwise discolored, or by small coinsized cicatrices, or by partly-healed ulcers of smaller dimensions than the mother-lesion within which they are confined. As the environing circle with its wall and open or crusted ulcer widens, the central area proceeds to a more complete involution, leaving at last broad spaces often converted into a smooth scar-tissue, or a field in which the delicate creases and punctate markings suggest the action of the tool of the engraver on the surface. This odd-looking involvement of the integument may be in progress for months and years, spreading from one or more primary points and gradually migrating over an entire thigh or abdomen, the patient meantime often displaying in other respects a marked degree of general health and vigor.

When the action is deeper, the ulceration, invading the subcutaneous and even the deeper structures, usually begins with the disintegration of tubercle or gumma and spreads by extension, more often downward and deeply 
rather than peripherally and at the superficies. In this way there are digged in the muscles, the fascia, and between the tendons, gigantic excavations each having usually the circular outline and the sloughy floor of the syphilitic ulcer, and exhibiting a marked tendency toward phagedena. Both superficial and deep forms of serpiginous ulceration occur for the most part in persons of a broken-down constitution; they are, however, encountered among the fleshy, the consumers of alcohol in excess, and those given to modes of life which in non-syphilitic subjects are specially favorable to the production of gout. They are exceedingly rare in young and sturdy subjects of the disease.

Diagnosis.-The disease most likely to be confounded with serpiginous forms of syphilis of the skin is a variety of chancroid to which, at times, the title "serpiginous chancroid" has been applied. In the latter affection the ulceration is most often subcutaneous in situation, spreading for years, at times insidiously, beneath bridles and bridges of apparently unaltered skin, often with enlarged glands in the vicinity, generally upward over the belly or downward over the inside of the thigh, almost never elsewhere. The history usually gives some clue to the solution of the problem.

The vegetating forms of epithelioma are often exceedingly like the serpiginous syphiloderm, extending from a central area in verrucous growths at the periphery of a patch which has at times a well-rounded outline. In this event the advanced age of the patient, the absence of enclosed minor ulcers and cicatrices within the encompassing ring, the extreme slowness of the process as contrasted with the extension of the syphilitic affection, and the distinctiy verrucous character of the growth 
at the circumference of the patch, aid in establishing a diagnosis. Again, carcinomatous disease is decidedly more frequent on the face than elsewhere, while the largest of the syphilitic lesions are usually visible on the trunk or on the limbs.

Tuberculosis of the skin (lupus vulgaris and other forms) is so very rarely encountered with truly serpiginous characters that one views with some distrust a diagnosis of "serpiginous lupus." In any such rare case the history of the disease, the age of the patient when first attacked, the characters of the ulceration, and the other distinguishing features of the lupoid ulcer given above, should suffice for the determination of its nature.

The vegetating syphiloderm is another of the titles given, not to a special cutaneous lesion of syphilis, but to a feature which may be assumed by one or more of such lesions. In these cases there is a tendency to assume the papillomatous type, with hypertrophy of the epidermis and of the deeper portions of the skin. These hypertrophies usually occur as complications of the moist rather than of the dry lesions of syphilis in the skin, and they are of more frequent occurrence in regions where there is both unusual heat and moisture. They are also much more apt to develop in young and fleshy subjects of the disease, and particularly in young and fleshy women.

In these cases wart-like and papillomatous vegetations develop from either plane macules and irritated surfaces or from papules, pustules, condylomata, or ulcerating points. They vary in size from lenticular growths to masses as large as an orange and even much larger, usually secreting a foul-smelling puriform mucus 
from the side, base, or summit of the vegetation. These growths are particularly liable to occur in the region of the scalp, about the folds of the axille, the groins, and the nates, and about the anus. The rounded, flattened, acuminate, or tufted summits of these excrescences are usually covered with crusts due to the desiccation of the puriform secretion with which they are smeared, and on the removal of which the dull-reddish or florid surface of the masses can be distinguished. When removed artificially or spontaneously the superficial character of the process is readily determined.

The diagnosis is from lupus, pemphigus vegetans, frambosia, yaws, and all the simple papillomatous and warty growths. The distinction between the skinlesions of syphilis and the two diseases first enumerated has already been given. In frambesia there is always an absence of the other symptoms usually shown in patients with vegetating syphilodermata; the subjects of the disease, further, are chiefly those either living on or recently leaving the coasts of Africa. The creamy secretion, the acid reaction, and the shorter career of yaws are all significant. The warty growths found on the scalp and elsewhere of persons not infected with syphilis often present features strongly resembling the vegetating lesions here described, and the distinction between them all is to be looked for in the peculiar characters of the syphiloderm. In the latter, the size of the single or the abundance of the frequently multiple growths, the fetor of the secretion, and the accompanying symptoms of an infective disease are chiefly to be relied upon in the establishment of a diagnosis. 


\section{Syphilitic Affections of the Hair.}

The most important of the changes produced by syphilis in the hairs is an alopecia, important both because of the disfigurement it produces and because of the aid it furnishes in establishing a diagnosis of the disease. Syphilis, however, involves the nutrition of the hair often without production of an alopecia, working in many subjects of the disease a special dryness and other symptoms of malnutrition without fall of the hair sufficient to be conspicuous. There are two welldifferentiated forms of syphilitic alopecia: in the first form the loss of hair is due simply to the action of the virus of the disease, presumably upon the nerves of the scalp; in a second form the alopecia is directly induced by changes in the scalp.

Syphilitic Alopecia without Obvious Structural Change in the Integument.-This is decidedly the most common form of the affection, exhibiting conspicuous features in many patients, and probably occurring in an unobtrusive form in the great majority of all welldeveloped cases. It may coexist in the same person with an alopecia due to structural changes; it may be partial or general, though the latter is of exceedingly rare occurrence; and, as a rule, it develops among the earliest symptoms of systemic intoxication. At times only the hair of the scalp is affected; at other times the scalp, brows, lids, axillæ, and extremities are, in one or several regions, made partially bald. Usually the hairs of other regions are lost only when the scalp is involved, but at times when the scalp is unaffected the hairs of the brows or of the beard may fall.

All grades of loss are perceptible, from that escaping 
casual observation to that in which the scalp is laid bare over wide areas, the hairs falling in large numbers, slight traction upon any filaments selected at once bringing them painlessly from their pouches. As a rule, the resulting deformity is manifested to the eye in a characteristic "raggedness" of the hirsute covering, bare patches being particularly noticeable over the temples and the occiput, as distinguished from the pre-senile losses often seen where the thinning is largely limited to the vertex. When, however, the scalp is shaved or the hairs are clipped close, it is clear that the loss occurs chiefly in finger-nail-sized areas, often closely set together, never producing the palm-sized, completely bald patches of most non-syphilitic diseases accompanied by alopecia. Upon the eyebrows the loss is often highly conspicuous by reason of its lack of symmetry, the hairs of one brow, for example, being removed when the other is intact, or one-half of the hairs falling from one or the other extremity of the brow of one side. The conspicuousness of these forms of alopecia makes them most offensive to the conscious subjects of the disease. When the loss involves the region of the male beard, the patches are usually similar to those seen on the shaven scalp; but occasionally a baldness of the bearded face occurs in large patches which it is difficult to distinguish from alopecia areata.

Syphilitic Alopecia due to Structural Changes in the Integument.-In these cases the alopecia is consecutive to the evolution of a syphiloderm in the region of the skin affected with the hair-loss. The pre-existing lesion in the best-marked cases is then of an ulcerative type, resulting in a destruction of tissue limited to the area where the loss of hair occurs; in this event even 
the baldness is often a minor symptom when compared with the graver metamorphoses of the skin in which the hairs were once implanted. Other syphilodermata may, however, be responsible for the alopecia, such as macular, papular, papulo-pustular, and tubercular lesions, as also gummata, the latter involving also the subcutaneous structure. Most of these lesions are effective by actual destruction of the hair-follicle by either ulcerative or resolutive changes following the syphilitic deposit. As compared with the simpler form of alopecia previously described, it is noticeable that the alopecia due to structural change in the skin is often remediless, while the former is almost invariably followed by a return of the hairs; that the tissue-change is most often circumscribed and limited to a single region of the body, particularly the scalp; and that the alopecias of early syphilis, which are often multiple and unaccompanied by destructive changes, differ widely in every feature save the hair-loss from the invasions of the hair-sacs by the late, usually gummatous, deposits of the disease. When the milder forms of consecutive alopecia occur, they often result from a species of syphilitic involvement of the sebaceous glands of the scalp and of other regions, finger-nailsized patches of the part involved being covered with fine, often greasy scales, the integument being manifestly hyperæmic and tinted in the dull-reddish hues of the syphilitic macule.

Diagnosis.-In almost all forms of syphilitic alopecia the diagnosis is established by the discovery of other symptoms of the disease, which, as a rule, may be discovered if sought for with special care. It is, however, true that in exceptional cases the force of the first intoxication of the system seems to expend itself wholly 
upon the hirsute covering of the body, and in these losses it may be a matter of difficulty to discover the site of the original chancre and its possibly persistent underlying sclerosis.

Alopecia areata most strongly resembles the syphilitic form of baldness, but in the former the patches are usually large, the skin denuded of hairs is smooth and white, the line of demarcation after the few hairs that are loosened at the periphery have been epilated is much more distinctly outlined by vigorous filaments, and, seeing that children are not rarely affected, the subjects of the disease are at times much younger than those suffering from acquired syphilis.

The congenital, pre-senile, and senile losses of hair are usually symmetrical and permanent; they occur at epochs of life which commonly contrast with the average age of acquisition of syphilis, the exception occurring in pre-senile forms of baldness, where there is usually a definite history of preceding seborrhœic trouble. The simpler varieties of baldness are, however, of much longer duration than the common forms of syphilitic alopecia. In ringworm of the scalp the presence of the parasite and the tender age of the subject of the disease are significant. In psoriasis of the scalp the highly characteristic scale-accumulation, often extending beyond the confines of the scalp at the brow is a diagnostic feature.

\section{Syphilitic Affections of the Nail.}

It is usual to distinguish between two different forms of syphilitic invasion of the nail and its peripheral tissues, the term paronychia being employed to designate the changes in the nail-substance which are consecutive to 
those occurring in the tissues about the nail; while the term onychia is limited to changes occurring primarily, as regards obvious symptoms, in the nail itself. The two conditions may coexist. The distinction is, however, though useful for clinical purposes, scarcely based upon pathological facts, seeing that it is highly probable that no changes whatever occur in the nail proper prior to disturbances in the nervous or other structures with which it is in relation.

Changes in the Tissues Surrounding the Nail, with or without Consecutive Lesions of the Latter (Paronychia syphilitica).-In the more superficial variety of this disorder the epidermis and often the deeper portions of the skin in a circumscribed patch, usually at one extremity of the nail-groove, thicken and assume a warty aspect. This local thickening may be resolved in successive exfoliations with some resulting tenderness, or there may be superficial excoriations, fissures, or even resulting ulcers. One or several digits may be involved, the fingers more often than the toes, on account of the exposure of the former in the occupations of life. Sometimes the integument of one or several joints of the digit is implicated in the process. This complication occurs within a few months after infection, or it may be delayed to one or two years after-rarely the latter. It is most often contemporaneous with maculo-papular and papulosquamous lesions of other regions. The consecutive changes in the nail, when such occur, are of the milder types elsewhere described.

In a deeper form of involvement of the tissues about the nail, a nodule, dull ham-tinted and tender, varying in size from that of a pea to that of a bean, forms either in the nail-fold, the nail-groove, or the matrix, usually 
upon one side. Occasionally the more prominent skinsymptom is a deep and ill-defined infiltration. The cracking and exfoliation seen in the superficial form may be conspicuous in the deeper form of the disease, the infiltration undergoing in favorable cases complete resolution under appropriate treatment, though its course is commonly indolent. In other cases ulceration ensues, the part becomes tender, at times exceedingly painful, and the pus which may be discharged gives no such relief as in the "run-around," the course of which is much more brief. The affection persistently lingers when the toes are involved. The odor of the secretions furnished, especially by the great toe, which on account of its prominence often suffers, is, as usual in this region, often highly offensive.

Uiceration, whether resulting from the superficial or the deeper involvement of the parts about the nail, occurs as a complication of both processes in various grades. The course of such complications is always modified by treatment. As usual, the fingers and the great toe, for reasons already explained, suffer more than the other digits.

The ulcer, whether starting from nail-fold or matrix, assumes, as a rule, with startling rapidity its formidable features. The edges of the ulcer are raised, often undermined; the floor is covered with an unhealthy, partly purulent slough, usually well attached, with dull-colored granulations springing from its mass. The color of the whole is characteristically empurpled and unhealthy. The prominent club-shaped aspect of the distal phalanx, swollen to two or three times its usual volume, presents a vivid contrast with the adjacent and unaffected phalanx, which seems in comparison to be 
shrunken or atrophied. Viewed at a distance, the deformity often seems to be produced by a pushing of the nail-substance, whether involved or not, far to one side of the longitudinal axis of the digit, the reason for this being the bulk of the swollen and inflamed tissues on one side of the phalanx. The nail may be lost or partially destroyed in the process. The new-formed nail may be misshapen or well formed. As a rule, the repair procured by the best treatment is surprisingly good in view of the marked deformity and the threatening character of the lesions, especially when, as may be the case, many of the fingers are simultaneously attacked.

This complication, usually occurring in the first two years after infection, is often a portent of grave syphilis; it is apt to occur in middle-aged patients with brokendown constitutions.

Changes in the Nail, with and without Involvement of the Adjacent Tissue (Onychia syphilitica).-( $(a)$ Atrophic Changes in the Nail.-Every grade of atrophy of the nail may occur in syphilis, and the milder forms are much more common than is generally supposed. They are often detected by the expert in his examinations when they escape the attention of the patient. In the simpler manifestations the nail-substance loses its lustre, acquires a dirty-yellowish hue, and slowly covers itself with various striations, markings, dots, and spots, often presenting a characteristic "worm-eaten" appearance. The friability of the nail is increased to a perceptible extent, and its broken or nicked free edge is seen in many, if not all, the digits, especially those of the fingers (onyxis craquélé of the French).

(b) Hypertrophic Changes in the Nail (Syphilitic onychauxis).-In this form, which may coexist with the 
atrophic changes described above, and which is rarer than all others, portions only of the nail may be perceptibly thickened by increased growth, or the entire nail may be enormously increased in bulk, changed in color, and marked by the pinhead-sized dots or depressions, sharply cut in outline, where small circumscribed atrophic changes have occurred.

(c) Separation of the Nail from Matrix, Bed, or Fold. -This change, an exceedingly common one in syphilis, may involve one or all the nails of the hands and the feet. As a rule, several of the digits are affected, the hands by preference. The detachment may be partial or total.

Among partial detachments, much more common than all others, the mildest is seen in early periods after infection. The separation usually occurs first at the distal extremity of the nail where it is attached to the side of the nail-bed, and is visible beneath the nail-substance as a delicate linear or ribbon-like stripe, parallel with the long axis of the nail, resembling a serous exudation beneath the nail-substance; or the line of separation is whitish in hue, and the separation occurs at the bottom of the nail-groove or across the entire width of the nail. One-half or more of the nail may thus be detached from its connections, the separated substance undergoing the usual changes in color and polish.

When the separation is complete, it may result from changes beginning as in the partial forms described above, or with changes in the matrix, the latter being more common. Usually the latter ceases to provide for the further growth of the nail-substance, and the nail which is to be shed is simply slid along its nail-fold until it is exfoliated, undergoing meanwhile the atrophic 
changes already described, in markings, striations, etc. upon its surface. When the nail-bed is left bare it is speedily covered with a substance which, in all favorable cases, eventually furnishes a new nail.

Variations from this type are furnished by defective and imperfect attempts, instead of by total cessation of effort, of the matrix to furnish the nail-substance. In the former event ridges of mingled atrophic and hypertrophic nail-substance mark the boundaries between the diseased plate, newly formed, and the healthier nail produced prior to the date of the infective process. As a result the nail is shed, and its successor is formed after a lapse of time in which the nail-bed is in part exposed and beset by imperfectly formed, thinned, irregular, or "worm-eaten" fragments of horny substance.

Diagnosis.-In general, the nature of the disorder of the nails is readily established, as there is usually a history, and in almost every instance other symptoms, of infection. The indolent course of the disease, the tendency to ulceration of the soft parts about the nail, and the deformity resulting in the production of a bulbous or club-shaped distal phalanx, are all significant. Ingrowing toe-nail, chiefly of the large toe, presents an obvious explanation for the tumefaction and pain. Chancres seated in the site of a "hang-nail," especially among physicians infected in the practice of their profession, are commonly associated with enlargement and induration of the epitrochlear gland of the limb involved. Tuberculous affections from inoculation of the manual digits are rarely situated at the nail-border, and they are usually of verrucous rather than of papular type. 


\section{Syphilis of the Mouth and the Tongue.}

The study of syphilis as it affects the mouth is of great importance because of the frequent implication of this cavity, because of the persistence and significance of the symptoms presented, and because of the possibilities of transmission of the disease amply afforded. In such a region as this is well illustrated the tendency of the disease to exhibit its symptoms at sites of special irritation. The chewing and smoking of tobacco, the holding of pipes, cigars, and cigar-holders in the mouth, and even the practice of chewing a toothpick after meals, are fruitful sources of lesions in this region of the body. The drinking of very hot or iced fluids and the use of highly-spiced, acetous, or salted foods have a similar tendency.

Chancres occurring upon the tongue, the lips, and the tonsils have been considered elsewhere. It is needful here to recall the fact that lesions suggesting in appearance mucous patches of the tonsils, with an ashen surface and deep engorgement, deep indurations of the anterior segment of the tongue capped with a superficial abrasion or ulcer, and circumscribed scleroses with much tumefaction of the inside of the cheek or the gum, if associated with dense induration of the glands anatomically connected with these parts, should not hastily be taken for symptoms of consecutive syphilis.

The lesions of systemic syphilis in the oral cavity are, when more or less speedily succeeding the appearance of the chancre, usually superficial, multiple, and wellnigh symmetrical, as distinguished from those occurring later in the disease, which are often single and deep as well as destructive. 
These lesions correspond strictly with those already studied as of occurrence in the skin, being of the type of macules, papules, tubercles, warts, scales, pustules, gummata, and ulcers. Each type, however, acknowledges a modification due to the peculiarities of site, the mouth being habitually moistened with mucus and saliva, and being exposed to friction of contiguous surfaces and of articles of ingested food and drink, which, as already shown, add the effect of heat, cold, and chemical agents to the other effective causes of disease in this region. Again, the pressure upon the tongue and the inner face of the cheeks of carious and even sound teeth having projecting edges, not appreciated in conditions of health, is capable of inducing or modifying the symptoms here presented. As a rule, however, the syphilitic lesions of the mouth are of moist rather than of dry type, with the result that the mucous patch is probably of greater frequency as a syphilitic symptom than any other lesion exhibited in the course of the disease, particulariy in male patients using tobacco.

Macular Syphilis of the Mouth.-Well-defined broad areas of vivid or dusky redness may often be seen over the arch of the soft palate, upon the tongue and the pillars of the fauces, and along the gingivo-labial furrows soon after general syphilis is declared. At times the redness is limited to finger-nail-sized plaques, or even punctate spots of heightened color, upon the mucous membrane. These spots may disappear on proper treatment, or they may persist and furnish a basis for the evolution of one or more of the other lesions to be mentioned.

The chief complication of patches of this type is the assumption of an erosive and superficial or ulcerative 
and deep action, due, as a rule, to the irritant effect of the agencies already described. In milder expression the epithelium loses its attachment to the underlying tissue in consequence of a macerative effect upon the weakened membrane, and the reddish pellicle first seen on examination disappears, leaving a raw-looking and tender spot the size of the original macule. In other cases a sharply-cut ulcer results, with floor more or less speedily extending to the depth of the mucosa, assuming a linear shape with its long axis at right angles to the lines of traction (along the width of the tongue, parallel with the groove of the gingivo-labial junction, etc.).

The papular syphiloderm is represented on mucous surfaces by the mucous patch (plaque muqucuse, mucous tubercle, moist papule, etc.). These lesions are singie or more commonly multiple, usually very well defined patches, which, being at first, and usually but for a brief time, reddened macules, speedily acquire an opaline hue over their flat surface, suggesting the action of nitrate of silver upon mucous membrane. They vary in size from a split pea to that of a bean, but they are often of greater size, involving a space as large as a penny or as extensive as the inner face of the lip or the arch of the palate. They are roundish, oval, or very irregular in contour, and they are often perceptibly raised above the general level. They are usually painful, and they are often seen $c n$ face on membranes in contact, such as the inner face of the cheek and the gum, and the two halves of the angular crevice behind the last molar teeth. A variation of this lesion is seen when a diphtheroid and bulkier film extends over the face of the patch or patches.

The most common complication of this lesion is the 
superficial erosion which succeeds it, and which becomes visible as a vivid or dull-reddish, moist and shining or dry and glazed sequel of the removal, by friction or other agency, of the pellicle of epithelium covering the typically developed mucous patch. The elevation of these plaques by infiltration is not uncommon, and a further but rarer complication is furnished when this hypertrophic effect is exaggerated. In the latter event a well-elevated roundish disk, obviously thickened, and often with a distinctly elevated rim, rises, especially within the labial angles, but also elsewhere; this disk is usually less painful than the simplest expression of the mucous patch, and is annoying chiefly by reason of its interference with the motions of the mouth. Often it is traversed by one or more fissures, which are then painful and apt to bleed when the tissue is unusually stretched.

Papules of moist type-seen on mucous membranes, and much more rarely in the mouth than about the vulva or the anus-also at times assume a verrucous aspect, and are represented by growths resembling the pointed wart or the venereal wart, already described. They are usually smaller in the mouth than in the other regions where they occur, they do not furnish an offensive secretion, and they are more amenable to local treatment. The so-called "toad's-back" appearance of the tongue is produced by the confluence of a number of flattish and aggregated papules, each retaining its individual outlines, and producing thus an effect resembling the markings on the carapace of a terrapin.

Ulcerative complications of the papules of syphilis in the mouth (nucous patches, etc.) are of the type already described, superficial and often exquisitely painful in the earlier and less irritative stages, deeper and reaching to 
the corium and beyond in the greatly irritative and later lesions of the disease. Ulcers here have a marked tendency to creep along the lines traced by the angles of adjacent surfaces, but they also occur as formidable circular lesions in the crypts of the tonsil, on the posterior wall of the pharynx, and on the inside of the lips.

Tubercular lesions of mucous membranes are simply enlarged papules developing with complications of the types described above. Pustules occurring in the syphilitic mouth are results of secondary infection with staphylococci; they are usually seen only after rupture of the roof of the lesion, when the floor of the original chamber is to be recognized as an erosive or ulcerative patch.

The fissures which form as a result of syphilis of the mouth may be complications of one or several of the lesions described above, or may be the direct results of local irritation at certain special sites. These sites are the outer angles of the lips, often involving both the mucous and cutaneous surfaces, the margins and dorsum of the tongue, and the muco-cutaneous borders of the centre of the lips; but they also develop elsewhere. Care is required to recognize even deep longitudinal fissures of the tongue, the walls of the crevices, when the organ is protruded, often falling together and wholly concealing a crack extending deeply beneath the mucous membrane.

The squamous lesions of syphilis in the mouth are of the type usually described as "dry," the failure of secretion at the involved points being usually conspicuous. They appear, rather more rarely than mucous patches, on the borders of the tongue, on the inner aspect of the lips, on the lingual tonsil, and along the line of the inner faces of the cheeks corresponding with the junction 
of the teeth of the upper and lower jaws. They are dry, infiltrated, and usually circumscribed patches, rarely as uniformly rounded or oval in contour as mucous patches, and linear in shape or in ribbon-like bands. Their color is grayish or bluish-white, occasionally almost silver-white with a lustrous aspect. Often, when seated upon the tongue, the affected organ has a shaven appearance, the French from this circumstance giving to this condition the term glossite tonsurante. Livid, opaline, bluish-white, slate-tinted, and otherwise colored patches of thickened and scaling epidermal tissue are often seen in the mouths of syphilitic patients, especially of men who have been chewers, and more frequently smokers, of tobacco. These conditions may be observed in the first, second, or any subsequent year after the date of infection. They are at times amenable to treatment, but they are often refractory.

Leucoplasia of the Mouth (Leucokeratosis lingux; Leucoma buccæ; Psoriasis linguæ; Leucoplakia buccalis; "Smoker's patches of the mouth," etc.).-It is impossible to study the scaly patches of the mouth occurring in syphilis without considering a series of phenomena exhibited in this region, the pathological and clinical position of which, with respect to syphilitic and other disease, is not yet completely established. By no distinctive features can these symptoms be in each case assigned with certainty to one category or another. They stand in different cases in some relation to syphilis, to epithelioma, and to lichen planus. What is definitely known can be summarized as follows: In male patients, almost exclusively in smokers, but also in others, appear patches, striæ, spots, plaques, fanshaped lesions, and bands of a dull-whitish, opaline, 
lead-white and silver-white tint, smooth and shining or roughened and beset with milium-sized nodules, which are consecutive to mucous patches or which occur in the mouths of syphilitic patients where such lesions have existed. They occur along the line of the jaws, on the gums, at the commissure of the maxillæ, in the folds between the lips and the gums, on the sides and dorsum of the tongue, and elsewhere. They may be the seat of fissures or may result in ulceration. In rare cases they exfoliate; still more rarely there may occur a highly exaggerated hypertrophy of the implicated tissue, in which a stripe of dead-white, thickened, and exceedingly dry tissue covers the dorsum of the tongue or one of the other regions named above, this tissue being so bulky as seriously to interfere with the necessary movements of the mouth. Epithelioma, not only in those of advanced years but in men of middle age, is liable to result from the long-continued irritation of the part. In other cases the disease is without question a lichen planus of the mouth, not to be distinguished as to etiology from the other patches here described, seeing that lichen planus of the integument often responds to a very marked extent to the agents by which the involved tissue is irritated.

It is practically impossible in many cases to draw a distinction, merely from the clinical appearance, between these several symptoms, nor is the fact greatly to be regretted. The leucoplasic condition is, in fact, not a disease, but a symptom common to several diseases. As pigment settles about the syphilitic and eczemato-varicose ulcer of the leg, and as the elephantiasic affection of the same organ occurs as a complication of syphilis, lymphangitis, erysipelas, and other maladies, so the scaling patches of 

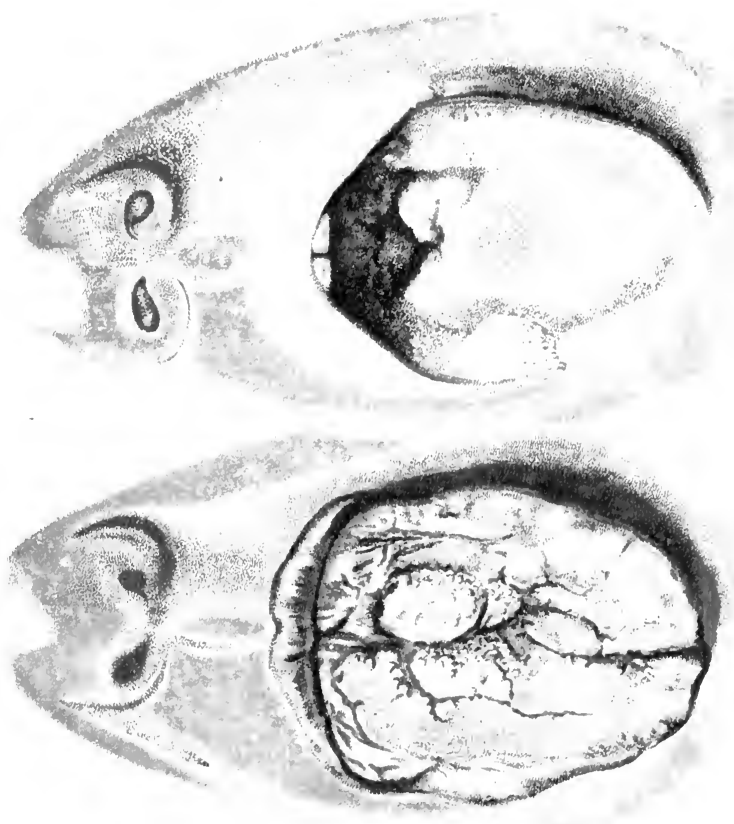

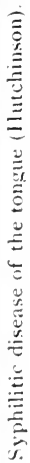



the tongue irritated by tobacco-smoke, carious teeth, neglect, and bad treatment form in both the syphilitic and the non-syphilitic patient, in the victim of lichen planus and in the patient who eventually succumbs to a grave cancerous affection of the mouth. It is safe, in all cases admitting of any doubt, either carefully to exclude the possibility of syphilitic infection or to treat the patient for that disorder.

Gummata of the Mouth.-Gummata occur in all the regions of the mouth as circumscribed or diffuse infiltrations, but they are most often encountered in the mass of the tongue, usually on one side, with well-defined limitations. They begin as insidiously evolved pin-head to small-egg-sized masses, usually single, at times multiple, though rarely numerous, breaking down into ragged ulcers with a rapidity and a facility not noted in the course of similar lesions of the derma. They occur, as a rule, several years after infection, but in obtinate users of tobacco, especially in chewers (as distinguished from smokers, who suffer from mucous and scaly patches), they have been seen as early as during the first year of infection.

When the hard palate is involved, it is common to discover in nearly the centre of the palatine vault a lentil-sized firm mass, which with astonishing rapidity softens until it exhibits a central orifice through which a probe can detect dead bone. In other cases a painless or possibly slightly tender gummatous nodule of the hard palate may persist for months without softening, attention being called to it by a persevering explorer of the case of a patient suffering from some other obscure symptom of the disease, upon which important light is shed by the discovery of the oral lesion. When very large 
gummata form and rapidly disintegrate, the result in grave cases is only equalled by the severe ravages of cancer in the same region. The oral and nasal cavities are in these cases rapidly fused until they expose to view a single gaping chasm, with the possibility of performance, imperfect yet surprisingly satisfactory, of necessary function. Here, as so often in the history of destructive syphilis, the repair wrought by skilful treatment is extraordinarily happy in results. The huge clefts and cavities of the tongue close, with the production of a sound scar-tissue which may resist disease for the remainder of life, and from which one can scarcely estimate the degree of the original damage. The use of an obturator, after all ulcers have been healed, by cutting off the nasal from the oral cavity may restore to the voice its natural timbre. All these grave changes occur in broken-down subjects of disease, or in those from some cause specially predisposed to complications of this character.

Diagnosis.-The distinction between scaly patches, lichen planus, and smoker's patches has already been considered. Cases are of frequent occurrence where a differential diagnosis is impossible, for the reason already given-that the conditions described under these terms are often symptoms common to several diseases. Mercurial stomatitis with ulceration is readily distinguished by the fetor of the breath, the symmetrically swollen condition of the tongue, the indentation of its sides by the teeth, and the line at the border of the gums. The condition known as exfoliatio areata lingua (a phrase describing symptoms rather than a disease) is characterized by the occurrence, especially in subjects of a tender age, though adults also suffer, of a well-defined elevated 
patch spreading in circular outline over the tongue in areas as large as a penny and larger, leaving the tissue where it has extended smooth and varying in color, in different cases, from a light rosy shade to an empurpled hue. Often the patch dips down over the tip or the sides of the tongue. The area is commonly unilateral in site, not often symmetrically involving the two sides of the organ. However much these areas may suggest syphilis, they are, as a matter of fact, rarely seen in that disease. In some cases they are, without question, the result of grinding the tongue between the teeth in the sleep of young patients with digestive disorders.

Epitheliomatous changes in the mouth are often difficult to distinguish from syphilis of the same region. In cancer the process is slower than in syphilis; the patient, as a rule, is older; the pain is commonly greater; the floor of the resulting ulcer is more florid; the lesion in advanced cases is larger and bulkier; in less advanced cases there is a decided tendency to assume a verrucous or fungiform aspect; the edges of the ulcerated patch are everted; and the disturbance of function is decidedly greater. In any advanced case the degree of cachexia produced is practically the same in the two affections.

In tubcrulosis of the mouth the lesions are slow of evolution, are usually at first superficial, and are not often limited in the tongue; the induration is slight; the ulceration is superficial and is studded with puncta of caseous degeneration; and systemic sympathy is marked. 'In all these diseases glandular enlargement may accompany the mouth-lesions, but in carcinoma the adenopathy of typical cases is more constant; it is less frequently noted in tuberculosis; and in syphilis it chiefly complicates chancre of this region. 
Pempligus a'egetans and other forms of pemphigus and herpes in many cases exhibit mouth-symptoms. The mucous membrane of the mouth is then usually raw, red macules representing the floors of bullæ whose roof-wall has been ruptured. In these patients there are pain, exquisite sensitiveness of the mouth, and in bad cases extreme dysphagia; but the presence of bullous lesions elsewhere, the temperature record of the patient, and the relative acuity of symptoms are all significant.

Pathology.-Anatomical study of sections of tissues in most of the complications described above indicates that the inflammatory, hyperplastic, sclerous, gummatous, and degenerative processes in the mucous and submucous tissues are in all respects analogous to those recognized in the skin and in the subcutaneous tissues. Smali-celled infiltration, interstitial hyperplasia, epidermal hypertrophy, elongation and thickening of the papillæ of the corium, endarteritis, and increase in the number of rete-cells, often with smaller cells within the limits of the original protoplasmic envelope, are to be recognized in most processes. The presence of giantcells in numbers, as well as of bacilli, distinguishes tuberculous disorders of the mouth; while nests of cells in the corium are characteristic of the epitheliomatous changes to be recognized as complications of leucoplasic patches.

\section{Syphilis of the Respiratory Tract.}

Syphilis of the Nasal Passages.-The frequency of involvement of the nose and the nasal passages in syphilis is due to the exposure of these regions in so many cases to climatic and other influences, as well as to the anatomical peculiarities of the parts. 
Chancres within the borders of the nares are exceedingly rare. An indurated lesion following, after a proper interval, the employment of instruments for the treatment or observation of any disorder of this region, if accompanied by enlargement and induration of the neighboring glands, should be regarded as highly suspicious. In the early periods of syphilis the more common affections of the nose are'acute and chronic rhinitis, macular and mucous patches, and circumscribed and diffuse gummatous infiltration of tissue. In these cases the chief symptoms are local thickenings, a seropurulent discharge from the nares, and sensations of pain and fulness of the part. Gummatous changes may occur in any portion of the nasal cavity, beginning with the mucous and submucous tissue, and spreading thence, often with destructive violence, to periosteum and bone. So delicate are the osseous and other structures of this region that their involvement may be followed by degenerative results in an incredibly brief time. A patient complaining of nasal symptoms may even in the course of a few days suffer a perforation of the septum or exhibit bony sequestra exfoliated and thrown off in a fetid discharge. At times the turbinated bodies enlarge and exhibit traces of fibroid degeneration. The term owena was formerly given to the catarrhal symptoms common to these patients, a disgusting odor being imparted to the breath by the destructive changes going on in periosteum and bone, accompanied by discharge of a purulent, hemorrhagic, or serous fluid often mingled with detritus of bone. The highly offensive odor of this secretion is often as disagreeable to the patient as to those with whom there is personal contact. As a result of the several changes indicated, the bridge of the nose 


\section{I54 SIPHILIS AND THE VENEREAL DISEASES.}

may be destroyed, producing thus a saddle-shaped flattening, with at times a tilting upward of its tip-a deformity as characteristic of syphilis as the "parrot'sbeak" shape and the subsequent destruction of the tip are peculiar to lupus of the same organ. It is by these processes that the arch of the palate is perforated and at times practically destroyed. Other sequelæ of this disorder are the production of bridles and bands stretched from one side to another of the nasal cavity; the obliteration of the passages by cicatricial occlusion; and in grave cases, when serere osseous changes have taken place, the extension of the disease to the meninges of the brain with resulting convulsions and a fatal issue.

Syphilis of the Pharynx.-Chancre of the tonsil has already been described, its erosion being commonly situated on the inner face of the tonsillar mass, which is then enlarged, painful, and apt to be covered with an ashylooking pultaceous slough, the glands beneath the jaw suggesting the nature of the difficulty. The opposite tonsil often sympathizes with the disorder, being engorged and at times eroded.

The posterior wall of the pharynx is often the seat of circumscribed and diffuse inflammatory thickening (due to syphilis) and of mucous patches and gummata. There is in these cases a very characteristic smearing of the fauces with a tenacious mucus, frequent efforts being made by the patient in hawking to rid himself of the resulting discomfort. Fibroid thickening and gummatous ulceration are not rarely encountered, a characteristic ulcer resulting from these changes being recognized as a circular, well-defined excavation, with clean-cut edges and sloughy floor, visible chiefly on depression 
of the base of the tongue. Grave destructive results in extreme cases extend to the bone and to the large vessels lying near the pharynx on cither side. Vegetations and verrucous growths are rare in this region.

Syphilis of the Larynx. - The morbid changes in the larynx due to syphilis occur in early and late periods of the disease, and in both circumscribed and diffuse manifestations. These changes may result from others occurring in the upper portion of the respiratory tract (nares and pharynx), or they may develop primarily in the larynx itself.

Macular lesions with transient or persistent erythema are not rarely encountered on the mucous surface; as a result, the submucous tissues may be involved in deepseated infiltration. The complications are erosions and superficial ulcers seated on an engorged base, or more rarely on a surface not changed in hue from the normal, visible, on laryngoscopic examination, over the epiglottis, the vocal cords, the ventricular bands, and other parts. Symmetrical, multiple, shallow ulcerations, involving, with relative acuity several portions of the larynx at one time, are peculiar to syphilis. In some cases the plane macular surface changes to one that is decidedly elevated, exhibiting a grayish and reddish tint suggestive of mucous patches in the mouth, though it is to be observed that typically developed mucous patches are rarely seen in the larynx, on account of its relative protection from many of the effective causes of these lesions in the mouth and the nose.

Later, deeper and more serious accidents to the larynx result from gummatous changes. The deposit is in the form of single or multiple, milium-sized nodules or diffuse infiltrations involving the submucous tissues of 
either the epiglottis, the interarytenoid space, the vocal cords (particularly their free border), or the subglottic folds, these growths being sufficient in extreme cases seriously to interfere with the functions of the larynx. The mucous envelope of the gummata may at first be intensely hyperæmic and even covered with a vascularized membrane of a vivid red color, or the hue may be yellowish, grayish, or even scarcely altered from that of the surrounding part. The result may be complete involution without further change-an occurrence by no means rare in healthy subjects under proper treatment; or, exceptionally, ulceration may ensue, and that to a degree of gravity inducing partial destruction of perichondrium, cartilage, or bone. At times fibroid tumors resembling gummata in external form spring from irritated patches where macular lesions or erosions have existed, inducing as much suffering and exposing the patient to as much danger as other new growths of this region. Again, membranoid bridges, bridles, and bands stretch from one side to another of the laryngeal cavity, occluding its lumen and producing subjective symptoms not differing from those resulting from the presence of tumors. Verrucous growths also develop about the ventricular folds, proving formidab!e by their interference with the movements of the organ. There may result from any of these changes characteristic ulcers, single or multiple, usually the latter in late syphilis, with defined elevated and hyperæmic margins, often surrounded by a zone of inflammation, covered with a pultaceous slough. These ulcers when healed leave cicatrices which, as they contract, may either prove harmless or may draw together the walls or folds of the larynx, or fasten the epiglottis to the tongue or to the pharyngeal wall. Suppuration 
of one or more recesses of the organ, deep-seated abscess, ankylosis, paralysis, hemorrhage, sudden and dangerous œdema, and the presence of a necrotic cartilage in the larynx acting as a foreign body, are all complications of severe types of the disease.

The chief symptoms recognized without laryngoscopic examination of the patient are a characteristically hoarse and raucous voice, cough, dyspnoea, and cephalic symptoms due to imperfect aeration of the blood. These symptoms vary from the mildest to the severest distress, the dyspnœea in extreme cases requiring tracheotomy. A middle-aged man with a voice reduced to a faint whisper, full inflation of the lungs being effected by deep inspirations at long intervals, should invariably be studied with a view at least, before all else, to setting aside the diagnosis of syphilis.

It is to be noted carefully that while experts in laryngoscopy often find in doubtful cases of this category products of simple inflammmation, and even foreign particles, choking the chink of the larynx, the onset of these troubles is generally to be ascribed to localized syphilitic manifestations interfering with the normal action of the glottis. As it is the syphilitic mouth which early and late acknowledges the unfavorable influence of tobacco, so the syphilitic larnyx is exposed to irritation by the unfavorable influences of dust, smoke, and an insalubrious atmosphere.

Diagnosis.-In tuberculosis of the larynx the existence of pulmonary symptoms of disease, the general physical aspect, condition, family history, and age of the patient, and the discovery of bacilli in the sputa, usually suffice to determine the nature of the disorder. With respect to age, it is interesting to note that tuberculosis of the 
larynx generally occurs at an earlier period of life than does syphilis of that organ with grave complications. In tuberculosis, as a rule, the affected membrane is lighter in color, the process is slower, the ulceration is more shallow, the damage in extreme cases is far less serious, the dysphagia and the constitutional effect are far more pronounced, and the fatal issue is more probable and imminent than is the case in syphilis. On the whole, it may be said that a striking feature of syphilis of the larynx is that recognized in syphilitic involvement of many other organs-namely, a singular toleration on the part of the patient of even a serious mutilation or destruction.

Carcinomatous, as distinguished from syphilitic, involvement of the larynx is a disease of later life, develops in much slower course, and is often accompanied by hemorrhage, which is relatively rare in syphilis.

The prognosis in the great majority of cases is favorable. After wellnigh complete aphonia for months and even for years, restoration of the voice has been secured.

Syphilis of the Trachea and the Bronchi.-Lesions of the trachea and the bronchi due to syphilis are far rarer than those of the upper air-passages, or, if occurring more frequently than is believed, they for the most part escape observation. In general, it may be said of syphilis of the air-passages that its invasions are from without inward, and in the matter of frequency and multiplicity are conspicuous the shorter the excursion from the lips and the nares. The more deeply, however, syphilitic lesions spread toward the bronchi and the lungs, the greater, as a rule, is the gravity.

The changes noted in the trachea and the bronchi are practically those studied in the larynx, with differences 
due to the changed anatomical situation. The lesions may be consecutive to those occurring in the larynx, or they may be developed d'emblée. Circumscribed and diffuse patches of inflammation, fibroid changes, gummata, erosions, and ulcerations are the chief lesions in the course of which the perichondrium and cartilages may be involved. Membranoid occlusion of the trachea and of one bronchus, extreme stenosis, cicatricial stricture produced by bridles and bands, and fistulous sinuses connected with abscesses of one or another region, usually the lower, are sequels of different cases. The entire trachea has been converted into a contracted and distorted tube as a result of a slowly spreading serpiginous ulcer.

\section{Syphilis of the Bones.}

Periosteum and bone may be involved in both early and late syphilis, these complications occurring from a few months to a score or more of years after infection. The bones most frequently involved are those of the skull and the face, the palate, the tibia, the sternum, the clavicle, the ribs, and the scapula. One or several bones may be simultaneously or successively affected; rarely there is symmetrical involvement, as when both tibiæe or radii are coincidently attacked.

Most of the changes in these organs are due to circumscribed or diffuse gummatous deposits either in the periosteum, between it and the osseous tissue, within the bone-substance, or in the medulla. These gummatous deposits by pressure upon contiguous structures may seriously impair the function of other important organs, as when the deposits spring from the inner tables of the skull. 
Gummata of periosteum and bone are circumscribed, commonly multiple, grayish or yellowish-gray rodular masses, occasionally in diffused patches. The periosteum is usually first attacked. In regions accessible to the touch, as over the anterior face of the tibia, a well-defined swelling may then be recognized, covered with normal integument displaying symmetrical tumefaction, though at times beset with irregular and jagged projections. These tumors vary in size from a bean to that of a large egg; they are usually tender and exceedingly painful even when not impressed with the contact of a foreign body, the pain being characteristically heightened at night by the warmth engendered beneath the bed-clothing. The nocturnal pains of periostitis and osteo-periostitis are, indeed, so uniformly aggravated at night that they are generally considered diagnostic, and they are justly regarded with special suspicion in any case where syphilis had not been before suggested if they occur with quotidian regularity. They vary in character, being either boring, hammering, splitting, or crushing. When intense and characteristic, the patient is, as a rule, wholly unable to remain at rest, though he may secure transient relief by constant motion of the affected part, as when the legs are drawn upward and downward in bed-a series of movements highly suggestive of bone-syphilis. The pains are in part, without question, due to compression of inflammatory and other products between the tense and inclastic periosteum and the unyielding mass of the osseous tissuc. These symptoms may in some cases be of purely inflammatory type, but, however acute, it is probable that in all cases the gummatous process is chiefly responsible for the result. 
When the bony tissue actually participates in this disorder, the result is a node- $\mathrm{a}$ firm and more or less sensitive tumor, usually smooth and fairly well defined in outline, either globoid or exhibiting a longitudinal elevation like the "splint" of a horse, its length parallel with the long axis of the limb. The pains are usually of the sort experienced in periostitis. The course of the node may be either complete involution, which usually occurs under treatment, or persistence as a less painful and tender, even wholly insensitive, bony growth, or degeneration by softening, the tumor breaking at the centre and leaving a typical syphilitic tertiary ulcer with exposed bone at the base, eventually healing after exfoliation of the sequestrum, with scar-tissue implicating both bone and integument.

The resulting defornity depends upon the region involved; that occurring after destruction of the bones of the nose has been described in the pages devoted to syphilis of that organ. The deformity resulting when the extremities, the skull, and the spine are attacked is far less significant in acquired than in inherited disease.

Pathology.-Minute gummata of periosteum and bone are small-celled new growths tending to central degeneration by breaking up of their molecular elements in a characteristic atrophy. The cells of the outlying portions are larger, and often are in communication with a new growth of fine vessels. The medullary substance of the bone is at first increased in thickness. The term rarefying osteitis has been given to that process in which, while the marrow enlarges and the enlarging Haversian canals are stuffed with new cells, there is thinning and eventual absorption of the osseous trabeculæ, forming thus spaces in which the gum- 
matous deposit is made, with the effect of producing a weakening of the actual osseous structure. Either the length or the thickness of a bone may be thus to gross appearances greatiy increased, while its substance is actually reduced.

The term formatize osteitis is given to that condition in which new bone is formed during the metamorphic changes described above, by the production of trabeculæ originating in the embryonal cells in the medullary spaces, these cells commingled with corpuscles from the originally involved bony tissue. The new growths may develop between periosteum and bone or from the surface of bone denuded of its covering. In this way the cavities produced may be filled with new bony tissue. In a more advanced stage the new osseous formation may undergo a sclerotic hardening, the induration becoming as dense as ivory. Condensing ostcitis, or cburnation, produces a new growth which encroaches upon the medullary cavity or, pushing externally, may produce an annular, node-like, or splint-like appendage to the bone involved. These processes of rarefaction, boneformation, and even bone-degeneration to the point of production of a sequestrum through an ulcerative opening, may occur simultaneously in different parts of one bone or side by side, one lamella thickening while that adjacent softens. This multiformity of processes is a characteristic feature of bone-syphilis.

When the gummatous process involves the medulla, an osteo-myelitis may result, with degeneration and the bursting of an abscess externally, which is rare; or a formative osteitis with encroachment on the lumen of the medulla, the latter condition being the more common sequel. 
Diagnosis.-Bone-syphilis in acquired disease is usually recognized without difficulty, since the history of the patient and the character of the pains produced are suggestive. It is to be noted, however, that temporary swellings along the axes of the tibia occur in erythema nodosum, in which event there is usually, with tenderness of the node-like masses, marked redness of the integument covering the swelling. The acuity of symptoms is also suggestively different from the slowness of career of both syphilitic, tuberculous, and rheumatic nodes of the same part.

Secondary infection may occur in both periostitis and osteitis, and in such instances purulent foci result commonly in abscess. In examination of bones with a view to determination of probable cause of death, the existence of "worm-eaten" cavities, of irregular thickenings, and of perforations of entire plates of bone is indicative of syphilis.

Syphilitic Dactylitis (Syphilitic panaris; "Syphilitic finger").-This affection, first described in a classical essay by Dr. Taylor of New York, requires special description on account of its characteristic features.

This disorder is one involving the articular and periarticular tissues of the digits, more particularly of the fingers; it occurs in both inherited and acquired disease. In a first variety the subcutaneous, fibrous, and connective tissues concerned in the formation of the joint are primarily involved, one or more phalanges exhibiting changes, chiefly on the dorsal aspect, slowly or (more rarely) rapidly, and with remissions or continuously. The process is essentially a gummatous infiltration of the structures concerned in the articulation. The digit is either over-flexed or over-extended, swollen, and cov- 


\section{I64 SYPHILIS AND THE IENEREAL DISEASES.}

ered with an empurpled integument; its motions are impaired; and distinct crepitus is perceptible on palpation, due to erosion of the cartilages composing the joint. Ankylosis, abscess, destruction of the capsule and the entire joint, or simple impairment of the function of the articulation, with repair, may ensue.

In a second form the process is first instituted in the osseous, periosteal, or medullary structures, which become the seat of gummatous changes resulting in thickening of the two involved parts. The process may result, as shown above, in either rarefying, formative, or eburnating osteitis, so that the digit may be increased or decreased in size, or become softish and cheesy when handled, or as firm as ivory. Ulceration and abscess bursting through the stretched and empurpled skin may lead to the formation of fistulous tracts communicating with bone that is either carious or in process of slow repair. An oval, symmetrical tumor limited to a single phalanx of one or more digital or metacarpal bones, crepitating under firm pressure and painful and tender, is wellnigh characteristic of syphilis. The atrophy of a proximal or middle phalanx as a result of the processes here described, whereby a distal is made to fall upon a proximal phalanx, or the distal and middle phalanges upon the adjacent metacarpal bone, is highly suggestive of the same specific process.

Care should be had to recognize the distinction between these deformities and those due to tuberculosis, paronychia, and gouty or rheumatic affections of the digits. Lepra, the "melanotic whitlow" of Hutchinson, and the lesions of syringomyelia are all to be differentiated. 


\section{Syphilis OF THE LARGER JoINTS.}

Pains in the joints as well as in the bones and the muscles are not rare in early syphilis. These sensations do not necessarily imply the existence of a localized lesion of these organs, but they often point to neuralgic conditions due to the circulation of intoxicated blood. At times, without doubt, they are due to the action of mercury administered for the relief of that intoxication in persons peculiarly subject to the action of the metal.

Synovitis and arthritis in syphilis may involve one or several of the larger joints simultaneously, in which case the symptoms por se are scarcely to be differentiated from the same symptoms in the subjects of other diseases. The articulations are tumid, tender, painful, and hot to the touch, with limitations in flexion and extension, and evident fluctuation when synovium is effused in a fluid form. Patients thus affected may exhibit pyrexic symptoms; rarely have they been in good health prior to the date of syphilitic infection. As a rule, when examined they are pallid and weak. The termination of the arthritic complication may be by resolution without sequelæ, by ankylosis, or by destruction of important structures in and about the articulation affected.

Pathology.-The synovial membrane is usually in these cases the seat of gummatous infiltration, with wellmarked tufts springing from its surface; or the subsynovial structures, the ligaments, the capsule of the joint, the cartilage, and the subchondroid tissue may be involved, with the result of producing eventually thickening, degeneration, or the bursting of an abscess externally, and the formation of sinuses connecting with the joint-cavity. 
The diagnosis of syphilitic arthritis is made chiefly by consideration of other symptoms of the disease usually present, as well as by the history of the patient. Strumous, tuberculous, and other systemic affections exhibiting arthritic symptoms may in general be recognized by the earlier age of the patient. In syphilis the knee and the sterno-clavicular and scapulo-clavicular joints are chiefly involved. The hip is very rarely attacked in syphilis. Adult male patients are liable to display these symptoms usually from two to four years after infection.

\section{Syphilis of the Burste.}

The bursæ may be acutely inflamed, with symptoms of tumefaction, tenderness, pain, sensations of heat, and redness or an unchanged color of the skin over the part; but this complication is rare. Nore often an insidiously deposited gummatous material accumulates within or about the bursa. In practice the distinction is often well made between a gummatous degeneration of the tissue about a large bursa, later involving and opening into the latter, and a specific primary inrolvement of the sac. The subsequent career of the lesion, whether after resolution or after degeneration, is practically that of the same process in the skin. When the prepatellar bursa or that over the tuberosity of the tibia is implicated, the disorder has been termed "tertiary syphilitic housemaid's knee."

\section{Syphilis of the Tendons and the Tendinous Sheaths.}

The tendons and the tendinous sheaths may be acutely or slowly involved in gummatous processes beginning 
either in the teno-synovial sheath or in any of the contiguous parts. (The process is usually accompanied by pain, swelling, and disturbance of function. The issue, even after extensive hydrops, is usually complete resolution, but more or less persistent thickening, ulceration, or agglutination of the tendon to its sheath may follow.

\section{Syphilis of the Aponeuroses.}

The aponeuroses may be involved in the processes of syphilis, usually by extension of gummatous infiltrations from adjacent tissues. The significance of this lies chiefly in the consequences to the structures with which such aponeuroses are in anatomical connection.

\section{Syphilis of the Muscles.}

Myositis occurs in syphilitic subjects in differing forms. It has been supposed that the muscular pains experienced soon after infection in any subject proceed from an "irritative myositis," but, as has been shown, it is probable that these pains are due either to the nerves supplying the muscles, which acknowledge the presence of a special toxine without change in the tissues, or to the special sensitiveness of some patients to the early action of mercury administered with a view to the relief of the disease. In any event, the muscle-lesions in such cases are not yet demonstrated.

In chronic interstitial forms of myositis it has been demonstrated with sufficient clearness that a gummatous infiltration, diffused or in distinct foci, may involve the muscle-bundles, resulting in compression of the latter, with consequent pain, distortion, and even permanent contracture. The ultimate issue as regards the infiltrate is either fatty degeneration and coagulation-necrosis, 
ulceration and fistulous connection with the outer integument, or complete resolution with restoration of function.

Progressive ossifying myositis is a rare complication of formative and eburnating osteitis, though it is claimed to have resulted from changes in the central nervous system.

Atrophy of muscles in syphilitic subjects, especially in those who have been its victims for years, is more common than is usually believed to be the case. It may result from $(a)$ gummatous involvement of the nerves, the ganglia, or the tissues about the same; (b) from gummatous deposits in the muscles themselves; or $(c)$ from disuse of the limbs and the body in syphilitic subjects as a result of disease of other organs involving long-continued decubitus, or of life in a wheeled chair (grave ulceration of feet and legs, severe ulceration opening into the knee-joint, etc.).

\section{Syphilis of the Heart.}

Pericarditis is a rare complication of syphilis; it results from gummatous deposits in the fibrous tissue or from implication of the pericardia by the extension thither of a degenerative process originating in neighboring organs.

Gummata in the form of distinct yellowish circumscribed nodules may be found post-mortem in the septa and the substance of the heart, usually accompanied by hypertrophy and thrombus. On section these gummata are seen to be non-vascular and composed of a capsule of connective tissue within which lies centrally a sclerotic mass. In the tissue where these gummata have been implanted the muscles are replaced by fibrous bands. 
The fibrous myocarditis of syphilis is clue, according to Councilman, to an encroaching endocarditis affecting the coronary arteries, as a consequence of which the heart-muscles undergo various degenerations. The subendothelial tissue of the heart may be responsible for changes which have been described as a syphilitic endocarditis, in which whitish nodules have been detected along the free edges of the valves, with thickening and induration of the pericardium, shortening of the chordæ, and thrombi of the free surface.

Aneurysm of the Heart.-In a few instances saccular dilatations of the ventricular space, with walls indurated in part and in part thinned, have been recognized postmortem in the ventricles, one or several of such dilatations being visible in a single subject.

Among all the lesions recognized after death in the heart and the vessels of the subjects of undoubted syphilis, it is difficult to determine which should be described as directly due to that disease, and which to the indirect results of cachexia and to the presence of a chemical toxine engendered by the mutusl play of micro-organism and invaded tissue. Without question, some of the conditions described above are tre indirect results of specific infection, the direct attack of which has been pursued along different lines.

The symptoms of many of the lesions suggested above are not readily differentiated from those occurring in non-syphilitic subjects. They are for the most part betrayed in disturbances of respiratior, precordial distress, angina, asthma, palpitation of the heart, and other symptoms accompanied by nocturnal aggravation. In the simpler syphilitic affections of the heart the distress is usually paroxysmal, and the general condition 
of the patient is one of weakness occurring simultaneously with the cardiac disturbance. Complete relief may ensue under treatment, but fatal results are recorded in a proportion of recorded cases.

\section{Syphilis OF THE Blood-vessels.}

Arterio-sclerosis.-There are two forms of disease to which the title artirio-sclerosis has been given. These are the diffuse and the circumscribed (or nodular) forms. Both are due to a primary fatty metamorphosis of the muscular walls, with consequent dilatation of the lumen and compensatory increase of the intima of the vessel, which, as also the muscular overgrowth, may subsequently undergo hyaline or atheromatous degeneration. From these changes aneurysmal pouches may form; and the modern view that all aneurysms not originating in trauma should be suspected to be syphilitic, is in part due to the fact that iodide of potassium has proved of value in so many instances.

Endarteritis Obliterans.-In this special affection there is proliferation on the part of the endothelium of the ressel, resulting in a thickening which eventually involves all the tunics of the vessel, and in an encroachment upon its calibre tending to obliteration. The process is differentiated from the arterio-sc!erosis described above chiefly in the production of a neoplastic as distinguished from the purely hypertrophic thickening of arterio-sclerosis. A gummatous periarteritis in which the adventitia and the media are involved has also been observed in both the circumscribed and diffuse forms. The lyaline and amyloid degenerations of the smallsized arteries, as well as the primary changes described above, are encountered as well in non-syphilitic disease. 
Here, as in syphilis of the skin, the mode of involvement rather than the lesion is characteristic of syphilis. It is the recognition of several necrotic points with restriction of the lumen of the vessel by thickening of the intima that suggests the nature of the process in any given case.

\section{Syphilis of the Lungs.}

The great difficulty in discriminating between gummata of the lung and tubercles of the same organ has up to the present obscured the characteristic features of syphilitic disease. Gummata occur as firm, often quite dense, whitish, grayish, or reddish-gray nodules, set in consolidated lung-tissue, and varying in size from a split-pea to that of a small egg. They are built up of granulation-tissue; they degenerate rapilly by caseation, fatty metamorphosis, and central necrosis. Fibrous trabeculæ pass from the outer envelope of the mass toward its centre, as if to produce lobulation. These lesions are found in the posterior and lower lobes of the lung oftener than in its apices, furnishing thus a valuable diagnostic difference between syphilis of the lung and the apical disorders of early pulmonary tuberculosis (Spitzenkatarh). Diffuse infiltration of gummatous material in the lungs is characterized by the consolidation of a smaller or larger area, as the result of accumulation in the alveoli of an epithelio-fibrinous exudate, or from a new growth of connective tissue. On section the lung closely resembles the condition seen in simple pneumonia, its substance being firm and in color grayish and reddish. Under the microscope the connective tissue is seen to extend from the blood-vessels into the thickened alveolar parietes, almost obliterating the alve- 
oli or changing them into narrow clefts with epithelial linings. The absence of leucocytes is conspicuous. Councilman, who amply illustrated this subject, describes this condition as a "true syphilitic pneumonia."

Gummatous Fibrosis of the Lung.-In this condition the tissue about the bronchi and the arteries undergoes a fibrinous metamorphosis to the point of production of thick, cord-like radiations spreading from the root of the lung toward the pleura, inducing later, by contracture, both emphysematous and atrophic states of the constricted pulmonary tissue. Along these fibrous bands are set gummata of usual type which may degenerate by ulceration. Irregularly alternating points of constriction and dilatation of the bronchi produce the symptoms of bronchitis of non-specific type-evolution of pus-cells with thickening and erosions of the mucous surface.

Ulceration in the lungs, with the consecutive formation of carities, as in pulmonary tuberculosis, has been both affirmed and denied as of occurrence in syphilis. There is good reason, however, to believe trustworthy the recorded cases in which cavities have been found, communicating or not with bronchi, surrounded by firm cicatricial tissue, and associated with other symptoms of that disease in unquestioned subjects of syphilis.

Diugnosis. - The discovery of tubercle bacilli in any case is of the greatest value in establishing a distinction batween syphilis and tuberculosis of the lungs. The physical signs of consolidation, dyspnoea, and cough are in the two usually similar. We have seen severe hemorrhage, eren to the point of fainting, with perfect recovery. The chief important points are the localization of the disease in syphilis (as already shown); an ap- 
parent limitation of all symptoms, in certain cases, to the chest; the remarkably good thoracic development and general physique of the subjects of the disease; the frequent absence of fever; and the marked dyspncea of some of the affected.

\section{Syphilis of the Gastro-intestinal Tract.}

Syphilitic lesions of the oesophagus are known only in the report of a few isolated cases, upon which some doubt rests in consequence of their great rarity. Of cases in which the stomach is reported to have been involved, though the recorded instances are somewhat more numerous than of osophageal invasion, but little is known of any characteristic symptoms. Gummatous infiltration of the mucous and submucous tissue is supposed to be responsible for areas of definite outline where at one or more points thickening and subsequent ulceration have occurred. Syphilis of the intestinal canal is rarely encountered save in the anorectal pouch. Its lesions are due to gummatous deposits, either diffuse or in localized points, the latter often corresponding with the sites of the agminate glands. The results are seen in fibrous thickenings and dense infiltrations, with ulceration at one or several points. Often there is coincident peritoneal adhesion and serous effusion.

Syphilis of the Liver.-Gummata are not rarely found in the liver of the subjects of syphilis, where they appear as few or numerous grayish-red and grayish-yellow nodules lying near the capsule or deeply set in the substance of the organ. When lying near the superficies they usually induce contracture of the hepatic capsule, which is also often thickened and attached to the 
adjacent organs. The nodules are composed of connective tissue, which undergoes a metamorphosis into dense cicatricial bands appearing, when they are fully developed, to divide the hepatic mass into lobules. Centrally the nodules undergo softening and necrosis, due to obliteration of the vessels which supply them. Most observers agree with Virchow, that there is also a fibrosis affecting the syphilitic liver, not due to gummatous deposits. In these cases fibrous bands stretch from the capsule in many directions, compressing the hepatic substance between the divisions thus artificially produced, which are further intersected by lesser striations of fibres passing from the larger bands. The effect is very like the shrunken condition of the gland occurring in cirrhosis. As a sequence of this and also of the other changes noted above, amyloid degeneration both of the walls of the hepatic vessels and of the liver-cells themselves may occur. Calcareous metamorphosis is rarely seen, and ulceration is of rare occurrence. $\mathrm{IVe}$ have noted a single case only in which an adult within the first year of infection died apparently as the sole consequence of syphilis of the liver. This organ was stuffed with gummata to an extent interfering seriously with the performance of its function.

During life it is rare that any symptoms are displayed sufficiently distinct to point unmistakably to hepatic involvement. Icterus is by no means rare in syphilis, especially in its early months; there can be little question, however, but that the symptoms may be wholly due to functional derangement of the liver. Pain and tenderness in the hepatic region, and ascites, may or may not be present. There are no signs absolutely diagnostic of hepatic discase in syphilis. 
Syphilis of the spleen and of the pancreas is exceedingly rare. When unmistakably involved, the spleen may be large and soft, as in non-syphilitic affections, or enlarged and indurated from fibrosis, or affected with diffuse, yet more rarely circumscribed, gummatous deposit. As usual in splenic enlargements, when voluminous as a consequence of syphilis, the organ is usually many times its normal size.

When the pancreas is attacked, the lesions of syphilis are usually found in and about the head of the gland, which, like the spleen, may be either enlarged or dense and contracted. In the latter event the acini are firmly compressed, as in the case of the hepatic cells of the liver, by an interstitial overgrowth, corresponding with the condition of fibrosis found in the spleen. Circumscribed gummata of this gland are rare, but they have been noted in both large and miliary-sized nodules.

Gummatous changes of the suprarenal glands have been reported in a few instances. The affection may be said, however, in consequence of its great rarity, to be a pathological curiosity.

\section{Syphilis of the ReCtum and the Anus.}

Chancres of the anal region are apt to be ignored in consequence of the fact that physician and patient do not usually suspect the nature of the trouble. In our experience these lesions, as distinguished from the soft chancres of the anal region occurring in women, are more common in men, and result usually from practices against nature. These initial scleroses are often supposed to be "piles," of which complaint is usually made. Split-pea-sized and firm papules are then visible, usually one only, just beyond the anal verge, and the 
bubo of the vicinity is distinguishable in the inguinal region or elsewhere. Other scleroses of this part are erosions and ulcers. The star-shaped ulcer of the soft chancre of the anus is never imitated by the syphilitic sclerosis, by reason of the failure of auto-inoculability. Chancres within the verge of the anus are rarely. seen.

The early perianal lesions of systemic syphilis are usually, and especially in the case of young adults, flat papules, springing or not from macular lesions. These may be discrete or confluent, in the latter event producing a perianal zone of infiltration with a dull redness that might lead the inexpert to suppose the case to be one of eczema, especially when, as is often the case, the lesions of this region are the seat of a considerable pruritus.

In consequence of heat, moisture, and friction, these papules have a uniform tendency to flatten and to furnish a secretion. In this way miliary and (more often) lenticular papules, condylomata, elevated mucous patches and mucous plaques, verrucous growths, and other hypertrophic lesions develop about the anal orifice. As a consequence of their softness they readily break down into fissures radiating from the anus, and even into formidable ulcers. The secretion they furnish is commonly cxceedingly foul. Many of the widely variant hypertrophies once known under the misleading title of "lupus of the vulva" (esthiomenc) are papillomatous growths about the anus as large as an egg and larger, beginning in an overgrowth of flat moist papules of this region. As these lesions are rapidly developed, so in favorable cases and with the best of treatment they can be made to disappear speedily. 
More minute ulcerations occur at the verge of the anus, usually multiple, reddish or grayish in hue, oval and elongated, rarely circular, not very painful, and discovered perhaps by the physician engaged in making a careful search for lesions. With reference to some of these, a doubt exists as to their exclusive origin from the infectious disease present. They are seen in persons who have never been infected, and they are discovered with surprising frequency, by practitioners who habitually make examinations of the anal region, in all classes of all subjects after middle life. A line of demarcation is drawn between these and the other ulcers of syphilis, in the fact that with exceedingly few exceptions simple ulcers never produce the formidable ravages to which almost every syphilitic loss of tissue at times succumbs. The really serious destructions of tissue about the anus are produced chiefly by the chancroid.

Tuberculous ulcers of the anal region, to which for a long period the title "tuberculosis of the skin" was practically limited, are wholly different from the minute lesions described above. The tuberculous losses resemble rents or tears of the tissue; they have sharply cut walls, deep floors looking like clefts, and are as irregularly outlined as if cut at random. Syphilitic ulcers of this region are circular in outline and have undermined walls and pultaceous floors. Multiple tuberculous ulceration of the rectum always occurs in connection with other symptoms of tuberculous disease.

Gummata of the Rectum. ("Ano-rectal syphiloma;" Syphilitic stricture of the rectum).-Several processes have been described in connection with gummatous changes in the rectum, and there have been given to the resulting deformities of this organ names which distin- 
guish merely different phases of one disorder. The simplest consideration of the subject is that which traces the career of a single process in these several manifestations.

Gummata develop in the rectum as smooth, circumscribed bodies set in the mucous or submucous tissue. They may be single, multiple or exceedingly numerous, or diffuse in two significant directions. In the one the area of development occupies a district more or less parallel with the long axis of the gut. In this event contracture of the infiltrated tissue does not involve coarctation of the rectal walls. In the other case the gummatous involvement occurs in an annular form, encircling the rectal pouch usually between two and three inches from the anus. In the latter event contracture of the gummatous mass acts in the same manner and direction as a sphincter muscle, and induces coarctation of the walls of the rectum. All the phenomena of stricture of the rectum may result from this annular gummatous change in the intestine, and the "ano-rectal syphiloma" of certain French authors is thus produced.

The questions arise whether every stricture of the rectum is consequent upon gummatous changes, and also whether every stricture of the rectum, as has been believed, is due to syphilis.

With respect to the first question, it is clear that while every syphilitic stricture of the rectum is practically due to gummatous infiltration of the rectal walls, it by no means follows that the beginning of the mischief lay in gummatous change. Early in the history of most cases there is a record of uneasiness at stool and perhaps of blood-smeared fæces, indicating that some local lesions, possibly erosions or superficial ulcers, had ex- 
isted before the more serious change occurred. The unfortunate part of such histories is the rarity with which the expert explores the rectal pouch before gummatous infiltration can be demonstrated. The second question can be dismissed with some certainty, even in the face of dogmatic assertions to the contrary. Syphilis is the cause of the majority of all cases of stricture of the rectum. But this serious disorder may also result from the contraction induced by chancroids of the same part, and it is probable that it may also result from tuberculosis and other changes in the same organ. A few traumatic cases are on record.

When an annular gummatous band constricts the rectum, it produces a fibrinous change in the wall of the gut, the contracture of which, whether there be or not antecedent changes in the mucous membrane, sets up a proctitis liable to result in such changes. It has been seen that in certain organs, notably the liver, an unquestioned gummatous deposit may result in a very firm and contractile fibrosis. This is what happens in the rectum. In some of these gummatous involvements the fibrous metamorphosis of the walls of the rectum is so completely annular in its direction that a steadily increasing contraction occurs in the grasp of the ring, encroaching more and more upon the calibre of the gut. By interference with the excretion of the intestinal contents, and by inducing a catarrhal condition of the bowel above the coarctation set up by such interference, one of the gravest and most menacing of the complications of syphilis in the human body is eventually established.

On digital exploration the milder cases suggest to the touch that the mucous surface is merely thickened: at times both increase in thickness and roughening of the 
inelastic surface can be appreciated. Later the finger encounters an annular and sensitive band, dense in structure, unyielding, and varying with respect to the size of the usually central aperture which it surrounds, the latter being at times sufficiently pervious to admit the tip or the entire thickness of the digit; or the gut may be so occluded as to furnish no perceptible opening. The free edge of this strictured portion is usually sharp to the touch. The commonest complications are papillomatous and other growths, with ulceration of the mucous surface of the rectum and dilatation of the pouch above the stricture. Very constant of occurrence are peculiar lobulated or tongue-like growths about the anus (languettes), in many cases wholly external to the gut, usually numerous, and due to congestion of the parts below the site of constriction.) These growths are almost pathognomonic of the disease. Hemorrhage, prolapse of the fundus of the bladder, and constant dribbling of urine are also symptoms of extreme distress in women, that sex furnishing by far the largest number of all patients. There is usually a steadily increasing sense of weight in the pelvis, and after ulceration painful defecation, with either flattened stools or liquid evacuations, the sole relief of the intestinal obstruction occurring as the result of a diarrhœa.

The diagnosis is to be made between the lesions of the rectum produced by syphilis, chancroid, carcinoma, and tuberculosis. For the most part, the history of the patient and microscopical examination are required in order to ascertain the facts.

"Proliferating syphilitic rectitis" (rectitc proliférante syphilitique of the French) is a term used to designate the form of rectal disease in syphilis characterized by 
unusual hypertrophic growths in the form of vegetations and nodules on the rectal membrane.

\section{Syphilis of the Genito-Urinary Organs.}

In Men.-The penis is the frequent seat of the initial scleroses of syphilis, of all consecutive lesions of the same disease, and of gummata which ulcerate and at times produce extensive ravages of both cutaneous and subcutaneous tissue. These lesions have heretofore been described in these pages, as have also the chancres of the infected occurring after exposure to fresh sources of disease. When gummata develop in the corpora cavernosa, they are represented by pea- to larger-sized nodules, interfering with perfect erection of the organ. Very rarely annular bands form about the pendulous portion of the penis, distinctly circumscribed, and suggesting by their firmness the presence of a metal ring. The chancre situated at the tip of the urethra, accompanied by a sero-purulent discharge and liable to be mistaken for a blennorrhagia, has also been described. Deeper gummatous deposits in the urethra and at the basc of the penis are quite rare. Syphilis of the prostate gland and of the seminal vesicles is said to occur, but in the few rare cases reported no positive knowledge is had respecting the characters of the disorder.

Gummatous deposits in the epididymis and the cord are decidedly more common than is generally supposed. Both early and late in the disease the globus major (much more rarely the globus minor) of the epididymis becomes indurated, inelastic, and at times somewhat tender. When thus affected, the nodule has been compared by an English writer to the condition which might be recognized if an iron nut were screwed fast over the 
upper part of the testicle. One or both testicles simultaneously may be involved, the distinctly circumscribed firm mass being readily recognized on palpation. A pachyvaginalitis also occurs with serous effusion in the sac of the tunica, exactly simulating the hydrocele of simple cases. Blood, pus, or serum may be found on exploratory puncture, and the indurated mass of the gummatous area may be discovered behind. Gummatous changes in the cord, circumscribed and diffuse, also occur where the epididymis has been, in whole or in part, the seat of the same trouble.

Syphilitic orchitis is among the frequent complications of late syphilis, the gummatous change occurring very insidiously, often without any knowledge whatever of the change on the part of the patient. This condition is so frequently discovered for the first time by the physician in his examination of the patient that it is wise in all cases of gummatous changes recognized elsewhere (bones, subcutaneous tissue, nervous system) to examine with a special view to the recognition of disease of the testicle.

When the body of the testis proper is attacked, fibrosis (as of the liver, already explained) or gummatous infiltration may ensue, and the latter in either circumscribed or diffuse form. A part or the whole of one or of both glands may be involved; often the nodular elevations of the surface of the gland may be recognized by palpation. In other cases the dense induration of the testicle may be determined with accuracy by the touch and by its well-defined limitations, but the tissue is quite smooth and has the feeling of marble. The gland may be unaltered in size or more voluminous than normal, attaining in extreme cases the size of the largest orange. 
The apparent increase in size may be due to an accompanying hydrocele. When resolution occurs, the gland may slowly diminish in size by the absorption of the sclerotic or gummatous mass, and, as the deposit has usually squeezed the secreting cells of the organ to the point of destruction, the ultimate result is the shrivelling of the testicle to a diminutive miniature of its former self, as after the occurrence of mumps of the same gland. In other cases the gumma degenerates, attachments form between the gland and the scrotal envelopes, softening occurs at a central point, and the gumma bursts with the subsequent production of ulceration and fistulous connection of the testicular mass with the integument of the scrotum. At times, as a consequence of the contractility of the muscular and other parts not affected, the parenchymatous tissue is forced through the scrotal opening until " benign fungus of the testicle" results-a condition until lately not well understood.

In the matter of diagnosis gonorrhœal epididymitis so commonly affects the globus minor that a distinction between it and a syphilitic change is usually readily established; but it is not to be forgotten that in both disorders the location of the lesion may be different. Tuberculosis of the testicle commonly begins with involvement of the prostate, and it is a malady wellnigh invariably of those who are not victims of venereal disease. In syphilitic affections of the scrotum the lesions are those of the general surface of the integument, changes in their aspect being due to friction, motility, heat, and other accidents of the location.

In Women.-In the genital region of women, as well as in that of the male sex, the initial scleroses 
and consecutive lesions of syphilis are common. Chancres of women are not often recognized, by reason of their hidden position within the vulvar portal. The late gummatous lesions of this part should be distinguished from the condition long termed "lupus of the vulva" (csthiomenc of Huguier). Under this title have been described gummatous lesions of the vulva, in which category are to be classed both circumscribed and diffuse indurations, hypertrophic growths (as in strictures of the rectum and due to the same cause, tongue-like langucttes, and otherwise shaped papillomatous masses), and ulcerations with ragged edges destroying in whole or in part the ostium vagina and invading the region of the perineum and the anus. The frequent firm œdema of the vulva is supposed to be due to changes apart from the syphilitic process. Cancer of this region, especially of the clitoris, is to be excluded in establishing a diagnosis, as is also Breisky's "kraurosis of the vulva," a rare disease accompanied by contraction of the parts. Tuberculosis of the vulva is exceedingly rare, and it probably occurs with even greater rarity dissociated from vaginal lesions.

Syphilis of the vagina, if not rare of occurrence, is rarely observed. Chancres and consecutive lesions are inapt to form in the vaginal walls, and even when these are implicated in gummatous changes the morbicl process usually spreads to this mucous surface from others in the vicinity. The urethra of women may be the site of chancres and early and late lesions of the disease; in very rare cases stricture results from gummatous involvement of the submucous tissue, especially in long-standing cases of syphilitic stricture of the rectum. The mucous surface of the cervix and of the os uteri is the seat of 
both chancre and consecutive lesions more often than is generally supposed; the former have previously been described. Mucous patches and other consecutive lesions of syphilis in this region, in their appearance and evolution, scarcely differ from those seen within the oral cavity. Care should be observed, in formulating a diagnosis, not to confound epithelioma of the os, polypus, and chancroid with the lesions of syphilis. The affections of the womb, ligaments, tubes, and ovaries due to syphilis are rare, and careful investigation of the subject is wanting.

The bladder is rarely the seat of either early or late syphilitic lesions. Proksch is almost alone in his researches on the subject of gummatous changes in the vesical walls, with ulceration and the formation of a sinus connecting the gummatous nodule with the vesical cavity. Two cases have been observed by us; in one case a papillomatous growth occurred as a result of syphilitic changes in the wall of the bladder (revealed by suprapubic cystotomy); in the other, a man sixtytwo years of age, there had been hypertrophy of the prostate before infection, and a gummatous mass developed within the gland, reaching into the fundus of the bladder.

Syphilis of the Kidney.-The early changes in the kidney due to syphilis may occur within a few months after infection, the symptoms being those of an acute nephritis with slowly or more rapidly developing œedema of the face and the limbs, dysuria, frequency in voiding the urine, headache, backache, and profound asthenia. Albumin, blood, epithelium, blood-corpuscles, and casts may all be present in the urine. Under vigorous treatment these patients almost universally recover, even 
when the danger seems extreme. The organ is found enlarged in most cases, the cortical portion is increased in relative size, and the tubules are blocked with epithelial débris and colloid masses. The glomeruli examined with the microscope may exhibit the same catarrhal state or be normal in appearance.

In the late lesions of the kidney there is found, as in the liver, a species of fibrosis ("interstitial inflammation") with resulting contracture and pressure-effects upon the glomeruli, or gummatous deposits, circumscribed or diffuse, the latter rather more rarely. As a consequence of either process amyloid or fatty degeneration may occur, in rare cases, simultaneously in the same organ. The lardaceous kidney of syphilis is large and white and unilateral or bilateral. At times good recovery ensues where but one organ was probably involved. The same is true of gummatous changes. In both conditions the urine may contain albumin, blood, casts, epithelium, and even pus-cells. Usually the cortical and pyramidal portions of the kidney are involved. There is strong reason to believe that gummatous changes in the kidney in syphilis are of greater frequency than is suspected, many patients recovering from even severe renal symptoms without grave results. It is to be remembered also that many of the renal changes minutely described in the treatises on pathology are supposed by modern authors to be indirectly due to syphilis. The prognosis is grave when both organs are involved and amyloid degeneration has taken place; syphilitic changes in one kidney or in a portion only of one are to be regarded with greater hopefulness. WVe have watched for fifteen years, after grave syphilitic involvement of the kidney, patients who suffered from no 
return of renal symptoms. Surgical removal of a single kidney found to be affected with syphilitic changes has been followed by recovery.

\section{Syphilis of the Nervous System.}

Syphilis both early and late in its career affects the nervous system, the earlier manifestations being, for the most part, reactive, without appreciable lesion, and due chiefly to the circulation in the system of intoxicated blood. Late lesions of the nervous system may occur from a few months to several years after infection, and may result from syphilis of the osseous system, producing indirectly pressure or other injurious effects upon the nerves or the nervous centres in anatomical relation with the bones; or from syphilis of the meningeal coverings of the nerves, with effects not widely different from those exhibited when the bones are involved; or from syphilis of the nervous cells and fibres, or from syphilis of the larger vessels furnishing nutrient material to the nerves. Gummatous deposits may be responsible for the symptoms present in any of the several complications named, the evolution and subsequent history of the neoplasm having already been described. In one or another of these several forms syphilis of the nervous system occurs more often in male than in female patients, for the reason commonly accepted-that men are, as a rule, more than women subject to mental care and physical fatigue in business and toil. By some authors the nervous system is credited with the larger number of all the so-called "late" or "gummatous" changes noted in syphilis-a proportion, however, that is chiefly conspicuous in the statistics of experts in nervous maladies. Certain it is 
that women as well as men suffer severely from the nervous complications of the malady; and, inherited disease excepted, it is probably true that a fatal issue in syphilis can more often be ascribed to the nervous system than to any other. The importance of the recognition of nervous syphilis and the pressing need of its appropriate therapy can scarcely be exaggerated.

Syphilis of the Brain and of the Cranial Meninges. -In brain-syphilis the effective lesion may be related to any one of the conditions noted above. The commonest localization is in the cortical portion of the. brain, a gummatous deposit, either circumscribed or diffuse, directly or indirectly implicating the meninges. Meningo-encephalitis involving extensively one or both hemispheres, or a portion only of the nervous structure at one or several points, may result in varying grades of resulting damage. When an endarteritis obliterans (or, more rarely, a mesarteritis or a periarteritis) occurs, the injury is by thrombosis and subsequent occlusion, or by the formation of small aneurysms as in syphilis of the blood-ressels, or by dislodgement of one or more fragments of an embolus and their later transference in the blood-current to points at a distance from a forming neoplasm. For the localization of the nervous lesion by the aid of the symptoms in any case presented, the student is referred to the results of the admirable studies of this theme presented in the works on general pathology. Collectively, the symptoms may be described as, first and most common, headache, usually characteristically severe, of a boring, hammering, constricting, or grinding character, generally with very distinct nocturnal exacerbation, accompanied or not by vomiting, and at times terminating in relief in the most capricious man- 
ner. This pain may be aggravated by percussion or pressure over certain points of the cranium, and often is marked along the lines traced by the distribution of the trigeminus. A striking feature of all these disorders is the multiformity of the symptoms present and their capriciousness as to grave or insignificant results. Thus, symptoms of coma or of paralysis may appear or disappear in a way utterly impossible without grave sequence in any non-syphilitic disease. The multiformity so characteristic of the surface symptoms of the disease is often striking when the nervous system is attacked. Mental hebetude, stupor, coma of insidious beginning, convulsions, or a seizure simulating that of epilepsy, but different from it in that the average patient does not wholly lose consciousness, may each be significant. Of equal importance may be named hemianopsia, motor or sensory aphasia, disturbances of olfaction or of taste, persistent dilatation of one pupil, or paralysis of a single muscle or of a capriciously selected group of muscles within the orbit.

When syphilis affects the larger ganglia, the gummatous deposit is less likely to be implanted in the nervous tissue proper than in the walls of the larger vessels, especially those of the middle cerebral artery, the complete or even partial occlusion of which by an obliterating arteritis is apt to be followed by a monoplegic or hemiplegic attack, the consequences of which may be serious. Here the onset of the disease may be insidious and unaccompanied by the chain of symptoms of brainsyphilis; or all these may be present, with severe headache, mental hebetude, and even coma. As a rule, however, the patient suffering from a syphilitic hemiplegia is entirely conscious, and, though for weeks 
previous the victim of an agonizing cephalalgia, is relieved of most of the cranial distress when motor paralysis is established. The reflexes of the wrist, of the elbow, of the knee, and of the ankle are usually exaggerated in the paralyzed extremities both after and before the seizure. It will be remembered that in consequence of decussation of fibres, the gummatous changes of one side of the brain are for the most part responsible for paralytic phenomena of the other. Recovery may be relatively rapid in the course of a few weeks, or it may require years for its completion. In some cases the damage done is irreparable, and contractures result in both upper and lower extremities; the speech becomes mumbling, and the patient, while life is yet conserved, reaches in almost every function of the body one of the lower levels of physical degradation.

Lesions of the crus are apt to be betrayed in oculomotor paralyses associated with hemiplegia of the other side of the body, while those of the pons are liable to be followed by facial paralysis in which the arm and the leg of the opposite side are involved. In the case of affection of the medulla there is often a similar association of paralyses - a hemiplegia of one side and an involvement on the other of the vagus, glosso-pharyngeal, hypoglossal, or other nerves whose nuclei have a medullar site. There may be also a bilateral palsy of the four extremities, the result depending upon the extent of gummatous change in the meninges.

Paralyses of the oculo-motorius are so frequent in syphilis that their occurrence always leads to special inquiries on the part of the careful diagnostician respecting a possible syphilitic origin. The third, sixth, and fourth nerves (most commonly the two first named) may, when 
affected, produce ptosis, paralysis of the superior oblique, external and internal recti, and failure of accommodation to light. The capriciousness with which one or more of the muscles innervated by these trunks are selected for attack is highly characteristic of syphilis.

Syphilis of the Cord and of the Meninges.-The symptoms of syphilis of the cord and its coverings are spastic paralysis of both lower extremities, involuntary action of the rectum and the bladder, exaggeration of some or all of the tendon reflexes, contractures of muscles, particularly of the adductors of the thighs, more or less anæsthesia, a tendency to the formation of bed-sores, and, in cases, pains of a severe character in the loins and the lower limbs. These changes may result from gummatous deposits in the vertebræ or in the meninges of the cord, or from a distinct specific myelitis or meningomyelitis occurring in the cervical, dorsal, or lumbar region, one or all. Other symptoms which may be present in exceptional cases are changes in the ocular system (for example, persistent dilatation of one pupil), in the genital system (increased or diminished sexual desire and vigor), and paralysis limited to wrist-drop of both upper extremities, to cephalalgia, to aphasia, etc.

The etiological relation of syphilis to tabes has been the fertile source of a controversy which has at last been settled by an overwhelming preponderance of testimony, derived, for the most part, from evidence furnished not so much by syphilologists as by the statistics of insane asylums. Syphilis is without question a precedent fact in more than 90 per cent. of all cases of tabes. Patients of this class are, however, in the category of those little benefited by treatment for specific disease. Here, as in other ailments following infection, it seems that the 
result is less directly due to the toxic agents of the malady than to some chain of factors set in operation by the syphilitic germ.

The symptoms of tabes dorsalis in syphilis and in a presumably small minority where syphilis has at least not been proven are the same, and for a description of these symptoms the reader is referred to treatises on general medicine. Care should always be taken, in establishing a diagnosis, to avoid setting down as symptoms of syphilis those due to other changes in the cord; as, for example, when there is a loss of one or more bones of the digits of the feet, or when from the same cause the nails are exfoliated.

Cerebro-spinal syphilis (multiple cerebro-spinal syphilis) is a term employed to indicate those cases in which there is simultaneous involvement of both brain and cord. The number of these cases is larger than is commonly believed.

Dementia Paralytica and other Mental States due to Syphilis. - It is exceedingly difficult to ascribe to the proper cause the many singular and diverse mental states recognized in syphilitic subjects. It is to be remembered that of a thousand victims of infection a certain proportion were before the accident predisposed strongly, from other influences (heredity, accident, etc.), to nervous disease, and that many others, during the long course of treatment required for the relief of syphilis, are exposed to numerous influences tending to induce mental states of a morbid character (business reverses, affliction, accidents). It is not very rare to find grave states of hypochondria leading to self-destruction in a certain class of young subjects of both sexes after infection; and transient dementia is occasionally en- 
countered, with and without hallucinations and stupor. In these cases, as Sachs has shown, very remarkable intermissions and recovery stamp the disorder as due only to lues. The persistent dementias which prove complete are, fortunately, rare in syphilis. They usually follow the graver lesions of the nervous centres.

Dementia paralytica of the alienists (délire des grandeurs, general paresis), with epileptiform and apoplectic seizures, mental hebetude leading to failure of almost all the mental faculties, pupillary inequalities, tremor in articulating (lips and tongue), with singular changes in the moral qualities of the individual, is, like tabes dorsalis, amply delineated in the descriptions to be found in the best works on nervous diseases. To-day there is no question in the minds of the experts of large experience, chiefly those engaged in institutions for the insane, that syphilis is a precedent fact in a great majority of all cases. As in the instance of tabes, the infective process seems to be rather an indirect than a direct cause of the issue. The frequent relation of tabes with dementia paralytica would alone suggest the syphilitic origin of the last-named disorder, even if statistics were not at hand to confirm the fact.

Syphilis of the Peripheral Nerves.-The cephalalgias of nervous syphilis are, without question, at times represented by neuralgias due to specific involvement of the peripheral nerves. "Syphilitic sciatica" is as distinctly a symptom of a condition recognized in the subject of lues as is nocturnal cephalalgia; and cases are recorded in which gummata of bone and of other tissues in the tract of a nerve-trunk have by compression or other accidents induced serious changes in the nerves themselves. 


\section{Syphilis of the Eye and Ocular Appendages.}

The lachrymal gland is rarely involved either in a primary gummatous infiltration or secondarily as a result of implication of other organs in the orbit. The same is true of the lachrymal cormucle, which may become tumid, engorged, dense, and eventually the site of ulceration in the rare cases in which it has been found diseased. The canaliculi, the functa, the sac, and the nasal duct may be involved in any one of the early or late manifestations of syphilis when the eye is involved, usually as the result of some lesions in the vicinity, as, for example, chancres, papules, tubercles, ulcers of the edges of the lids, iritis with its frequent accompaniment of conjunctivitis, and the panophthalmias seen in filthy and destitute charity patients in dispensary practice. Again, many of the syphilitic lesions of the nasal passages lead indirectly to catarrhal and purulent inflammatory affections of the sac. Periostitis of the bones forming the nasal cavity is a frequent source of these purulent catarrhs. Stricture and eventual obliteration of the duct may result either from gummatous deposits in the mucous or submucous tissue or from osteo-periosteal changes in the channel. The external symptoms of these affections are epiphora, a swelling of the part, and tenderness with a sense of fulness. There is commonly evacuation of a sero-purulent fluid when pressure is exerted over the tumor. Eventually there may be abscess and ulceration at the point of bursting. The osteoplastic metamorphosis of the bony walls of the canal, described heretofore as eburnation (one of the varieties of formative osteitis), occasionally occludes the duct by the formation of a growth which chokes its calibre; but more often 
the bony changes here are in the line of caries and necrosis, relieved by spontaneous or artificial removal of segments of bone.

Syphilis of the eyelids may be exhibited in chancres or in the syphilodermata of systemic disease, such lesions being located either on the edge, on the conjunctival surface, or on the cutaneous covering of the lid. In the case of chancre the diagnosis is readily made when consideration is had of the induration of the lesion and its bubo, the enlarged gland being usually the pre-auricular of the involved side. Eyelid-chancre has the usual characteristics of chancres seen elsewhere and previously described, the chief peculiarities of the site being an enormous tumefaction of the lid that occasionally (not invariably) results, and the consequent epiphora and photophobia. When the initial sclerosis ulcerates, the excavation is shallow and oval, with elevated edges, densely sclerosed base, and a floor secreting rather more freely than chancres in other situations, on account of the irritation to which it is subjected.

Syphilodermata are more frequently found on the cutaneous surface of the eyelid; rarely an isolated lesion or several lesions may be discovered on the conjunctival surface; but in our experience these accidents result most commonly from special causes inciting the mucous membrane to morbid activity (traumatism, iodism, foreign bodies beneath the lid). Gummata, when present, form nearer the free border of the lids than elsewhere; they may be single or multiple in this region, and their ulcers, when they degenerate, are characteristic. When the tarsus is infiltrated with a gummatous deposit, a firm tumor results, implicating the entire lid (usually the upper) or but a portion of it, with 
and without involvement of the cutaneous surface. Here, as in syphilis of the testis and the liver, after complete absorption of the neoplasm has been effected the tarsus may lose its original texture and elasticity.

The conjunctiva may be the seat of any one of the processes previously described in connection with syphilis of the mucous membrane, save that the limitations of the area involved and the large portion of it that is protected by apposition of contiguous surfaces save it from many of the sources of disease to which the lining membrane of the mouth and of the nares is especially subject. Chancres of the conjunctiva have been reported without implication of the lid, but they are exceedingly rare. Papules, tubercles, pustules, and ulcers occur upon the conjunctiva as elsewhere, and are readily recognized by the symptoms heretofore described. Ulceration of the conjunctiva, whether from breaking down of a gumma or as the result of pustulation of the surface, produces one or many points where superficial losses of tissue occur, circumscribed, with uneven base, covered usually with a more or less adherent yellowish-white film, beneath which the surface is eroded. On the free edge of the lid the ulceration often assumes a linear shape and spreads along the entire edge, excavating its thickness. Cases are recorded in which gummata of the ocular conjunctiva produced an annular infiltration. surrounding the cornea, which, after degeneration of the former, has undergone necrosis.

Syphilis of the cornea occurs either in the form of an interstitial keratitis, with points of opacity usually at first centrally situated, spreading thence outward and involving the deeper layers, which may become vascularized; or the opacity spreads from the periphery to the centre, 
and eventually produces the characteristic "groundglass" appearance of the cornea. In another form there are definite points of opacity, the puncta being pin-point to pin-head in size, usually not numerous though multiple, the transparency of the unaffected portions of the cornea being unaltered. Gummatous deposits in the cornea, of the type of the gumma of other regions, have occasionally been observed.

Syphilis of the sclerotic is betrayed in superficial and parenchymatous forms of scleritis, some authors describing a gummatous form as distinct from the latter, the difference in all being, however, one chiefly of external appearance of the lesion. In the milder cases darktinted, even empurpled patches occur, of congestive aspect, with thickening of the tissues and obvious involvement of the overlying conjunctiva. These macula may be single or multiple; they are rarely very numerous, and are said never to form a pericorneal zone, though extreme cases occur where the deep congestion involves a large part of the exposed sclera. There is usually some pain, although at times none is experienced. Iritis is rarely present. In the parenchymatous form all the symptoms above described are exaggerated and complications are more common. The disorder is really a diffuse gummatous change, as distinguished from the circumscribed forms of gummatous deposit, in which elevated or flattened nodules, usually developing on the temporal side of the globe, exhibit telangiectases overlying the conjunctiva.

Syphilis of the iris is the most common of all luetic affections of the eye. Iritis, acute, subacute, or chronic, occurs both early and late after infection, much more often seen after the involution of the chancre. Usually 
but one eye is affected, rarely both. Recurrences are apt to be limited to the organ originally involved. In our experience there is usually an exciting cause even when syphilis is present, determining the onset of the affection and even its selection of a weak eye. For example, there are few experts in the cities of the North who have not noted an increase in the number of cases of iritis in a group of syphilitic patients treated after the streets have suddenly been covered with snow. Forms of plastic, serous, and gummatous iritis are described by authors, the three forms being distinguished merely by a preponderance of one or more symptoms present in any given case. The chief symptoms regularly noted on the part of the patient are photophobia, lachrymation, deep-seated pain, and imperfect vision; while the physician recognizes tumefaction and a change of color in the affected iris; irregularity of the pupillary opening, due, as a rule, to posterior synechiæ, giving an oval, at times even a jagged, outline to the pupil; marked sluggishness of the iris when light is suddenly admitted to it; and deep ciliary injection, distinguished by radii of straight pinkish vessels forming a halo about the cornea and contrasting vividly with the longer, more tortuous, and brick-colored vessels set superficially, deeply engorged, and belonging to the conjunctiva. In the forms of serous iritis of authors the aqueous humor is turbid, the tension of the eyeball is increased, and the field of the pupil, especially near the margin of the iris and the posterior face of the cornea and of the iris, becomes the seat of exudative deposits. The term "gummatous iritis" is by some authors limited to the distinct formation of nodes, papules, or reddish-yellow tubercles on the surface of the iris; but it is probable that all syphilitic 
forms of iritis are due chiefly to gummatous deposits even when no circumscribed nodules appear on the anterior face of the curtain.

The prognosis of all forms of iritis is good. The chief danger arises from adhesion of the iris to the capsule of the lens as a consequence of posterior synechia-a complication which may usually be set aside by the production of extreme mydriasis. Glaucoma results in a very small proportion of cases.

Syphilis of the Ciliary Body.-Serous, plastic, and gummatous forms of cyclitis are recognized, the chief difference between them being the mode of gummatous infiltration, all being due to deposit of gummatous material either in diffuse or in circumscribed form. When the ciliary body is implicated the symptoms are the following: visual disturbance in various grades; usually, not invariably, diminished tension; ciliary injection; and an exudation varying in amount and character within the posterior chamber, and at times also involving the vitreous. Often the symptoms of iritis and of choroiditis are present, and the disease is then properly described as "irido-choroiditis." In well-marked cases the attached portions of the iris are pushed forward by the vis a tergo of the exudate, blocking up the pupil and distending the posterior chamber, while its free border is more or less fixed by posterior synechix. Glaucoma or softening of the globe may result, and the issue is, in general, grave. The gummatous material, whether deposited in points on the membrane of Descemet or spreading to the ciliary body from nodules on the face of the iris, undergoes changes, either by resolution or by disintegration, not different from those recognized in other portions of the globe. 
Syphilis of the choroid is more common than any luetic affection of the eye save iritis ; in point of seriousness, while not so grave in the majority of cases as cyclitis, the affection may result in irreparable damage. The symptoms are, in general, clouding of the vitreous humor by reason of exudates forming fixed or floating specks, fibrils, threads, membranes, or even, in extreme cases, semi-solid masses of irregular form occupying either the anterior or the posterior half of the choroid, and accompanied or not by retinitis and disturbance of vision in various degrees. Iritis is a complication when the anterior portion of the choroid is chiefly involved, retinitis when the posterior segment is affected. There occur rapid diminution of ocular tension, deep-seated pain, and amaurosis in various degrees of severity. The remote results of these serious changes are the formation of staphyloma, cataract, detachment of the vitreous, and ultimate atrophy and shrinkage of all the constituent coats of the eye. By the aid of the ophthalmoscope in well-marked choroiditis whitish-yellow or reddish-yellow patches, fairly well circumscribed, can be recognized about the posterior pole of the ocular axis, often with a distinctly pigmented halo and with a tendency to atrophy of the tissue in which they have developed.

The crystalline lens and the vitreous humor, when attacked in syphilis, always exhibit nutritional changes secondary to morbid processes in the uveal tract.

Syphilis of the retina furnishes a list of grave disorders as respects vision. Chorio-retinitis is practically a complication of choroiditis, as already described. When the retina is distinctly involved, a membranous film appears to be stretched between it and the observer. There is also scotoma and deficient central vision. The 
forms of pure retinitis where the choroid is not involved are rare. By the aid of the ophthalmoscope it can be seen that the fundus of the eye is misty, the papilla is obscured, and the disk, which may be engorged, is encircled by a grayish retina. The symptoms are hemeralopia, lachrymation, photophobia, diminution of central vision, and the appearance to the patient of bright circles or patches which revolve about the point on which the eye is fixed. When a distinct exudation occurs the inner layers of the retina are involved and indistinctly circumscribed elevations occur chiefly about the posterior pole of the eye. When, as a result of these or of the other changes in syphilitic disease of the retina, hemorrhages occur, the symptoms and appearance are those of similar complications in non-specific disease. The "central recurrent retinitis" of Von Graefe is exhibited in opacities about the macula, which disappear at the time of the improvement of the vision, but which may return with the production of characteristic streaks radiating from the disk along the lines of the vessels.

The optic nerve may be affected by syphilis either within the cerebral tissue, within the orbit, or between the orbit and the brain, and as a result either of morbid changes in the adjacent tissues (bones of the orbit or foramen) or of primary involvement of the nerve-tissue.

Papillitis (inflammation of the intraocular extremity of the nerve) is betrayed by tumefaction of the disk (with its outline obscured by surrounding œedema), venous stasis, and arterial stenosis. When both eyes are thus affected, and they exhibit signs of choked disk, the diagnosis is of an intracranial lesion (gumma of bone, vessel, meninges). When but a single eye is involved, the source of the trouble may be wholly within the orbit. 
There may be amblyopia, hemianopsia, or more or less complete amaurosis. Preservation of fairly good visual power with symptoms of choked disk is supposed to be due to the integrity of the layer of cones and rods in the retina.

In neuritis descendens of one side the lesion obviously has existed between the chiasm and the orbit; when both sides are involved, the lesion is situated posterior to the chiasm. In these cases the change occurs primarily in the tissues outside the nerve-sheath, the latter being secondarily involved, as are also the nervous fibrillæ within the sheath. The most common causes are arteritis, mesarteritis, endarteritis, meningitis, and gummata of the encephalic nervous tissue.

Atrophy of the optic nere' may result from any of the changes described above, or from encephalic or spinal disease. The differences between the inflammatory, cerebral, and spinal forms, as distinguished by the ophthalmoscope, are chiefly color-changes in the optic disk from a grayish-blue to a bluish-green shade, and the various degrees of reduction in size of the arteries and the veins, the picture being more or less hidden by an obscuring mist. In some cases, however, no ophthalmoscopic changes can be recognized, and the location of the site of the effective lesion must be inferred from other symptoms. In hemiopia fugax (flittering scotoma) and true hemianopsia the lesion, without visible ophthalmoscopic changes, is probably seated in one optic tract.

Syphilis of the ocular muscles has already been described in connection with the subject of nervous lesions. It is merely needful to repeat here that the great majority of all cases of disturbance of function of these muscles is due not to a specific myositis, but to 
intracranial lesions (pachymeningitis, obliterating arterial disease, etc.).

Syphilis of the bony walls of the orbit is exhibited in osseous changes of the types already described in connection with bone-syphilis, periostitis, osteo-periostitis, hyperostosis, exostosis, caries, and necrosis, these conditions representing a series of changes due to the evolution of a gummatous product, its absorption or degradation, and the formative processes (by fibrosis, eburnation, etc.) already studied. As a consequence of these changes in the orbital bones, exophthalmos (protrusion of the eyeball outward, along its axis, or, as is not uncommon, to one side) may result, with secondary consequences due to stretching and traumatism of the optic nerve. In other cases the nerve is injured by pressure, and atrocious neuralgias may follow. In yet other cases abscesses form and burst externally, at times with resulting exfoliation of osseous sequestra, at others with the formation of fistulous tracts leading to carious bone. When exostosis occurs from the walls of the orbit, the tumor usually forms on the inner wall and projects toward the central axis; but it may also develop near the apex and produce exophthalmos or grave pressureeffects. These growths as a result of syphilis are extremely rare.

\section{Syphilis of the EAR.}

The auricle may be the seat of chancre or of any of the cutaneous lesions of systemic syphilis-macules, papules, pustules, tubercles, gummata, ulcers, etc.

The meatus may also be found affected with any of the lesions occurring upon the auricle. Exceedingly intractable ulcerations occasionally progress just within 
the meatus, at the junction of cartilage and bone. These ulcers greatly resemble the ill-conditioned ulcers often visible at the same time and in the same patient just within the nares. Condylomata are not rare within the meatus, where they may often be recognized as circumscribed, scaling elevations of the surface, furnishing an admixture of pus and cerumen, in extreme cases eventually inducing by their presence a typical otitis externa. Blocking of the canal may ensue, and in severe cases ulceration with scarring. Rarely there results permanent contraction of the meatus.

The membrana tympani is rarely the seat of syphilitic lesions. Luetic changes have, however, been recognized in this situation, and ulceration has at times resulted from degeneration of minute gummata situated upon the drum.

The diseases of the tympanum due to syphilis are obscured by reason of the difficulty experienced in precisely locating any lesions capable of producing the symptoms exhibited in any given case, and by the further fact that the symptoms presented are so nearly alike in the victims of both syphilitic and non-syphilitic aural disease.

Catarrhal inflammatory affections of the middle ear occur, resulting in hypersecretion, pus-formation, or the formation of plastic products, the distinction between these affections being established by symptoms rather than by any recognized lesions. Most of these troubles are associated with or spring directly from disorders of the naso-pharynx, which is so frequently involved in systemic syphilis; others arise from changes in the osseous walls of the Eustachian tube or from periostitis of the tympanum. The symptoms of these diseases of 
the middle ear are chiefly deafness in varying degrees, pain, serous or purulent discharges, tumefaction to the point of obstruction of the Eustachian tube, and râles on its insufflation.

The changes in the labyrinth due to syphilis are as yet little understood. The ossicles may be ankylosed, and all the tissues composing the labyrinth may be thickened either primarily or as a result of extension of disease from the tympanum. The symptoms are found in a series of widely differing subjective sensations of a morbid character, associated with imperfect audition, diminution of bone-conduction, and vertigo often resembling that occurring ab aure laso.

\section{HEREDITARY SYPHILIS.}

Syphilis may be transmitted from progenitor to offspring as a strictly inherited disease. The term "congenital" has been somewhat loosely applied by different writers either to inherited syphilis or to syphilis acquired at birth of an infant and due to infection from recently developed chancres of the maternal passages. In these pages the term "hereditary syphilis" is employed to designate exclusively the disease acquired by inheritance. The term "congenital," as liable to beget confusion, should be dropped from the nomenclature.

A vast amount of discussion has been elicited by questions concerning the etiology of inherited syphilis. It is sufficient here merely to state that, for most cases, the fact of a syphilitic child points to inheritance from the mother. When the father is without question syphilitic, and children are born syphilitic, the mother, free from all evidences of the disease, has probably been infected. She betrays no evidences of this infection 
either because at the date of observation syphilitic symptoms previously exhibited have disappeared, or because the proofs of her morbid state are to be sought exclusively in the fruit of her several pregnancies and in the striking fact that she is incapable of infection by her syphilitic offspring, according to the law of Colles, given on page 207.

The apparently healthy mothers who give a history of a succession of abortions, miscarriages, and birth of infected children due to syphilis can to-day be grouped in a class well recognized by every expert. It cannot always be determined whether such women have been infected with syphilis directly from the husband, as has been claimed, or indirectly from the syphilitic contents of the uterus. These mothers may even be in generally poor health, anæmic, weak, and debilitated, or they may exhibit every evidence of sound health, being vigorous, brawny, red-cheeked, and with all their functions duly performed. In these instances, without question, the syphilitic symptoms consist of the fruit of a series of conceptions. Just as syphilis of the healthy adult in some of its stages limits itself definitely to a persistent or recurrent patch of tubercles on the buttock or on a hand, so in these apparently healthy women the disease limits itself absolutely to the symptoms exhibited -a diseased ovum, fœetus, or infant, frequently several of each in a single history. When, however, the mother is without question exempt from the suspicion of syphilis, and the father is as surely syphilitic, the child invariably escapes. Cases of such absolute cxemption on the part of the mother are those where, the father being assuredly the subject of the disease, the mother has never had an abortion, a miscarriage, a diseased child, 
or any other symptom of the disease, and exhibits all the evidences of sound health.

Colles's law, first formulated in 1837 by Abraham Colles of Dublin, embodies the well-known fact that, although it is admitted that the mouth of a syphilitic infant is infectious for every other person, no mother of such a child was ever given syphilis by her own offspring. No woman ever had a chancre of the nipple result from frequent contacts with the infectious secretions of her child's mouth. This law, the reported exceptions to which are so few and so poorly established as to be worth nothing in the way of refutation, points conclusively to the fact above stated, that syphilis of the child always points to syphilis of the mother, either present or past, revealed by unquestioned symptoms or by a series of syphilitic conceptions betrayed as such by the occurrence of abortions, miscarriages, stillbirths, and infected offspring.

The period of pregnancy beyond which the mother cannot, even if infected, transmit her disease to her unborn child is not fixed. It is probable that with different patients the period changes, the differences being due to the general health of the mother and to her aptitude or inaptitude for furnishing favorable ground for the action of the toxines of the disease. After the sixth month the child probably escapes; but even so late as the seventh or eighth month, if the mother be infected, there is risk to the fœetus.

There have arisen in connection with this subject a few unimportant questions, the responses to which are by no means trustworthy. No man, in fact, can study the literature of the etiology of inherited syphilis, and have had experience of the disease in both public and private 
practice, without realizing the possibilities of error, in any given case, respecting the fact of parental disease and of error arising from the infective accidents common in the modern social life of large towns. Especially is this the case since the date of recognition of the practically innumerable opportunities for accidental infection afforded by the medium of utensils, instruments, and the contacts of professional and domestic service. Thus, a child (born of a syphilitic woman) reported to be after birth the victim of an initial sclerosis, followed by signs of acquired disease, has almost certainly been infected by some accident after birth, and was free from inherited disease when born. The question also whether inherited syphilis can be transmitted to a third generation can for the immense majority of all cases be answered in the negative, the exceptions reported being not always thoroughly purged of the suspicion of accidental infection. No expert fails to observe at intervals cases of supposed "inherited disease" where a rigid and carefully conducted examination demonstrates that the disease was, as a matter of fact, acquired; and the children of such parents, really suffering from acquired syphilis, and not from an inherited malady, would readily be assumed to represent a transmission of hereditary lues to a second generation. The question respecting acquisition of syphilis later in life by the subject of inherited disease is to be answered with the same caution and reserve. Certain it is that syphilis in a few cases, after it is acquired, does not wholly protect its victim from a second attack of the same disease. We have already seen that these subjects of infection will occasionally, after exposure to initial scleroses, suffer from singular and occasionally severe local ulcerations, simulat- 
ing new initial scleroses, even at times with adenopathy of the neighboring glands, the prior infection, though preventing a new syphilis, being apparently insufficicnt to protect against local reinfection. The cases here considered are not, of course, of the class where gummatous deposits occur in the genital region of the infected at the site of merely local irritation. This reasoning applies with special force to the rare cases reported in which a syphilis derived from progenitors whose virus has been attenuated, presumably, in a score or more of years, loses at last, in the second generation, its power to furnish immunity against reinfection.

Syphilis of the Placenta.-The placenta may exhibit signs of syphilis after the futus has become the subject of unmistakable symptoms of the same disease. Many of the placental changes recorded by authors are undoubtedly confused with those occurring in non-syphilitic processes, and there is no basis for assuming of this viscus that it differs from others in that its lesions in the subject of syphilis are wholly due to the infectious disease present.

In some cases of undoubted syphilis of the offspring the placenta has been found wholly free from morbid symptoms, while at other times an endometritis placentaris occurs, in which dense nodules are found with a whitish fibrous capsule and a central mass composed either of clusters of spindle-cells or of degencrating softish masses of a yellowish-white hue. With these gummata are masses representing transformed villihyperplastic, compressed, thickened, agglutinated, or even wholly destroyed. Atheromatous and other metamorphoses involve the vessels of the umbilical cord, with resulting thickening of the intima, thrombosis, and even 
vascular obliteration. A result fatal to the contents of the uterus succeeds an involvement in these changes of the larger portion of the placenta. When handled, the placenta in well-marked cases is in parts firmly indurated, is heavier than usual, and its lobulations disappear. In some cases circumscribed gummata may be detected by palpation of the mass, which, when incised, discloses grayish-yellow nodules with a fattily changed centre. Efforts to diagnosticate syphilis as derived from either the father or the mother by recognition of changes in the placenta are apt to lead to untrustworthy conclusions.

It is a matter of importance to note that the liquor amnii of the woman bearing a syphilitic fotus is capable of communicating the disease to an accoucheur.

Symptoms of Hereditary Syphilis. - One of the earliest and most frequent symptoms of syphilis in the product of conception is death of the ovum or foetus; and in a number of consecutive conceptions these symptoms often become conspicuous in a series of accidents of the same character. Thus, a woman infected by her husband soon after marriage may have a series of preg. nancies, covering a number of years, in which abortions occur, first at an earlier and later at a more advanced stage of gestation, these succeeded by one or more miscarriages, and the latter by the birth of a mature child surviving but a few hours. Eventually a child may be born apparently healthy at birth, but developing before the fourth month symptoms of inherited syphilis. Even after a series of such pregnancies there may at last be brought into the world a healthy child who never exhibits signs of constitutional disease. The mortality in these cases falls between 60 and 90 per cent. 
About seven-eighths of diseased infants exhibit symptoms of the inherited malady before the termination of the third month. Of the remaining eighth a certain proportion have actually exhibited symptoms either ignored or misunderstood. A small but unknown proportion betray evidences of transmitted disease at a date between the fourth month and the close of the first year of life. Cases were once reported of so-called "late" inherited syphilis in which the symptoms of the disease were supposed to be first displayed at or about the puberal epoch; but there are few physicians who do not look with suspicion on such reports. It is believed, not without good reason, that the most of such patients really betrayed evidences of syphilis in infancy, but, as occurs so often in acquired cases, such symptoms were overlooked or were assigned to the indefinite category, often misinterpreted, of "children's disorders." Cases of bone disease in adults known to have syphilitic parents, the osseous lesions first appearing in the second generation after the twenty-fifth, the thirtieth, the fortieth, and even. as reported in one case, after the sixtieth year, are in general to be accepted with great reserve.

Cutaneous Lesions of Hereditary Syphilis.-When miscarriages occur as a result of inherited disease, the fotus has often perished some days before its expulsion, and its skin is then usually macerated and, in consequence of the feeble union between the epidermis and the corium, raised here and there in bullæ, usually flaccid and filled with an ill-conditioned serum. This condition is often improperly termed "syphilitic pemphigus." In other cases there is born a viable child with a specific exanthem either affecting one region (for example, the palms and the soles) or extensively and 
even generally evolved. In yet other cases the newborn infant may present at birth all the evidences of sound health, and at a later date, before the close of the third month, may develop insidiously the symptoms of cutaneous disease. Every practitioner is suspicious of an infant born into the world, even though living, considerably under the average weight, weazened, yellowtinted, and snuffling, with the appearance of a "little old man" or a "little old woman," and exhibiting one or several "blisters" on the fingers or the toes. The appearance of premature senility in these weazened and speckled infants, with a flaccid skin which may be gathered between the fingers like that of some of the lower animals, with a circlet of papuies about the anus or the mouth, with a feeble stridulous cry, and with obvious weakness, is often sufficient to enable one to establish a diagnosis at a glance.

A macular syphilodirm in these infants has, in general, the shade observed in acquired cases, the difference being chiefly the larger size of the individual spots, their more pronounced shade, varying from a dull red to an empurpled hue, and their tendency to desquamate and secrete in regions of friction, pressure, and moisture. The color in some feeble and weazened children is a characteristically dirty brown, rarely imitated in any non-syphilitic infant. This exanthem may disappear or recur or be followed by others of a graver type.

The papular syphiloderm of inherited disease is rarely as generalized, as dry, or constituted of as small-sized individual lesions as the corresponding eruption of acquired disease. In the infant, papules are apt to be grouped about portions of the face, of the trunk, or of the limbs; are often seated upon a hyperæmic base; are 
in general distinctly grouped; and usually tend to coalesce and become flattened, scaling, or, in regions of moisture, friction, and pressure (as about the anus and the vulva), to secrete freely. In point of fact, the necessity of constantly applying napkins over the anogenital region of infants, and the frequency with which (in the case of syphilitic infants especially) the accumulation of faces and urine on these articles of clothing is permitted, make this region one in which the lesions of the disease are apt to be displayed not only often but in largest evolution. It is always incumbent upon the cautious practitioner to inspect the anal region of infants exhibiting an exanthem about which any suspicion is entertained.

Often the circular outlines of groups of papules in hereditary disease is exceedingly distinct, the central portions of the enclosed area being apparently unaffected. These rings or portions of rings may be seen clustered about one angle of the mouth, where cracks may form in the angle itself, or about the buttock, or over the palmar and plantar regions.

Bullous lesions in inherited syphilis are not rare, and commonly betoken grave conditions of the system. They may exist at the moment of birth of the dead or the living child, or they may afterward develop as pinhead-to bean-sized and larger elevations of the epidermis, filled, as a rule, with an ill-conditioned sero-pus or blood, having an inflammatory areola of dirty hue, and followed, after bursting and release of their contents, with blackish, greenish, and dirty-yellowish crusts. The palms and the soles, as also the digits of either hands or feet or both, may be the seat of these lesions, which may be followed by ill-conditioned ulcers. 
Tubercles in inherited syphilis are usually multiple, deeply seated, and grouped, and they soon undergo degeneration. They often precede a condition in which form greenish-black sloughs, ulcers spreading deeply beneath.

The forms of hemorrhagic syplitilis described by authors include those in which severe umbilical hemorrhage occurs at or soon after birth, as also the cases in which bullous lesions become filled with blood, and those in which distinctly purpuric blotches spread sparsely or in large numbers over the integument. Some of these forms are undoubtedly not to be distinguished from hæmophilia.

The mucous mimbranes, in inherited as in acquired disease, display bullous lesions, papules, tubercles, mucous patches, and even pustules. These several lesions in the profound dyscrasic state of weakly infants often rapidly degenerate into the most formidable ulcerations. By the presence of the secretions which are abundantly furnished in children, and desiccated readily by the currents of air when the mouth is kept open habitually in the weak state of the child, the nares become blocked by an obstructive rhinitis, and the respiratory tract, particularly of the larynx and of the trachea, is greatly encumbered. In this way arise the catarrhal symptomsthe peculiar "snuffles" of the syphilitic baby, its feeble and stridulous voice, the necessity of abandoning its grasp of the mother's nipple in order to breathe and to cry,-all marked characteristics of the disease in the new-born of the second generation. In advanced stages of involvement of the mucous surfaces the respiration becomes seriously impeded when there is unusual effort of any sort. When no special effort is made the child 
lies listless in its mother's lap or arms, with pinched, sallow features, its limbs flaccid and extended, and its cxpression indescribably apathetic. At any time ciuring the evolution of the symptoms here described an intercurrent disorder (pneumonia of a low grade, an incoercible diarrhœa, or a progressive marasmus) may bring on a fatal termination. The respiratory tract as far as the bronchi is much more readily involved in infantile than in acquired disease.

The nails are involved in inherited disease both primarily and as a result of changes in the nail-fold, the matrix, or the bed. When primarily affected, any one of the lesions named above may appear and be followed by suppuration or ulceration, with shedding of the nail ; or the nail-plate may become dry, fissured, "worneaten," yellowish, crumbling, or in various degrees distorted, or it may sucldenly be shed.

The hairs in hereditary syphilis fall as in acquired disease, and this either before or after the birth of the infant. The loss may be partial or complete, and if partial may consist either of a thinning of the hairs in one region or of their removal $c n$ masse from definite areas, circular, irregularly shaped, or in ribbon-like stripes. Usually the scalp is chiefly involved.

The lympluatic aessels and glands exhibit changes due to inherited syphilis, notably by signs of engorgement, infiltration, and enlargement. The thymus is chiefly involved, but all the thoracic and abdominal glands may be implicated, including the parotid, the inguinal, and the axillary.

The genital organs of both sexes may be attackerl, the penis and the testicles of male infants (epididymis and testes) especially. In both sexes these organs may be 
undeveloped, approaching in appearance the rudimentary type.

The bones are more often involved in inherited than in acquired disease, the percentage of cases in which there is osseous change being over one-third. Many lesions of bone in children are unrecognized in consequence of the greater gravity of other symptoms present. The bones most frequently involved are the tibia, the ulna, the radius, and other bones of the extremities, the clavicles, and the bones composing the skull. The special lesions recognized are those described under the title of

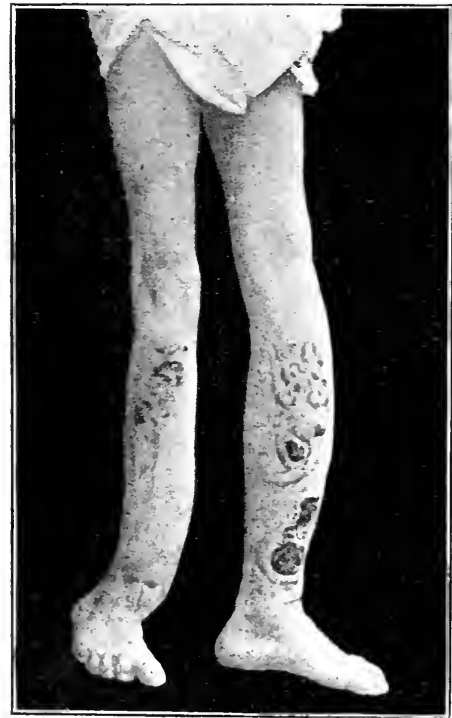

Fig. 10.-Sabre-blade deformity of the tibia in hereditary syphilis osseous lesions in acquired disease-namely, circumscribed gummata of outer plates and medullary canal, periostitis, and rarefying or formative osteitis. Caries, necrosis, and the induction of both ulceration and osteophytic growths are not rare. These growths at times induce premature closure of the fontanelles, resulting eventually in microcephalic idiocy. These lesions, with the others named, when affecting bones in contact with important nervous cells or trunks, may induce all the phenomena of nervous syphilis, cephalalgia with nocturnal distress, paralytic symptoms, 
epileptiform seizures, remediless surdity, and even imbecility.

The hyperostoses of the tibia (occasionally of both tibia) produce at times a highly characteristic change in the contour of the legs. Marked anterior convexity results from an osteophytic growth along the crest, which has been termed by the French "sabre-blade deformity" (laml de sabre) (Fig. IO). Frightful ravages occur also in the face, which may be converted into a wide area of destructive processes, the orbits half distended with shrunlien and sightless globes, the upper lip and the maxilla absent and furnishing the orifice of a chasm composed of the oral and nasal cavities studded with partly healed ulcers and fungotis masses.

The pscudo-paralysis of hereditary syphilis produces helplessness of a single member, due to separation of the epiphysis from the diaphysis of one of the long bones. When unrelieved for some time, the ultimate sequence may be atrophy of the muscles. This epiphyseal separation is usually induced by an osteo-myelitis-a condition to be distinguished from that in which pseudoankylosis results from decubitus and posture-fixation in consequence of grave disease of other organs (for example, a lower extremity after long-continued ulceration of a gummatous tumor seated upon the adductor muscles of the thigh).

Fractures in bone-syphilis of infants are not rare, but it is to be noted that in these cases repair commonly ensues as after fractures of the non-infected.

Care should be observed, in the diagnosis of bone disease in hereditary syphilis, not to confound the lesions. with those of osteomalacia or rickets, though it has been held that the symptoms of the latter are actually those 
of syphilis in the second generation. In rickets the bones are thinned, and not enlarged as in syphilis, and do not show the characteristic bosses or nodes visible in the skulls of many syphilitic infants. In rickets also the fontanelles are open, rather than closed prematurely by osteophytic growths; the ribs are beset with ridges or nodes; and the characteristic symptoms of the "knock-knee" and the protuberant belly are in every well-marked case conspicuous. In tubercular and other bone diseases of children there is, of course, exclusion of a history of syphilis in the progenitors, and of abortions and still-born children; absence of other symptoms of syphilis; a frequent limitation of the disease to a single bone; a tendency to the production of what is known as "cold abscess;" abnormal thermal variations; and, in the instance of tuberculosis, the possibility of the discovery of tubercle bacilli.

The dactylitis syplilitica of inherited syphilis scarcely differs from the symptoms of the same affection in acquired disease. One or several digits may be involved, the changes occurring in the early years of life. The fall of the distal phalanges upon the head of the corresponding metacarpal bone is usually highly significant when the proximal phalanx has been removed by absorption of its mass; and the same is true of the oval tumors representing symmetrical involvement of all tissues surrounding a single phalanx, with fistulous sinuses leading to carious bone or cartilage concerned in an adjacent joint. In some cases the disease begins in the joint, with symptoms of subacute inflammation and exudation; or the capsules, the tendinous sheaths, or the fibrous tissues may be involved, with the result of producing a synovitis of more insidious development. 
In yet other cases the synovial membrane thickens and becomes the seat of overgrowths simultaneously with the thickening of the periarticular tissues.

The nasal passages in inherited syphilis are chiefly affected with a variety of syphilitic rhinitis of purulent type, whose secretion, flowing over the lips, produces

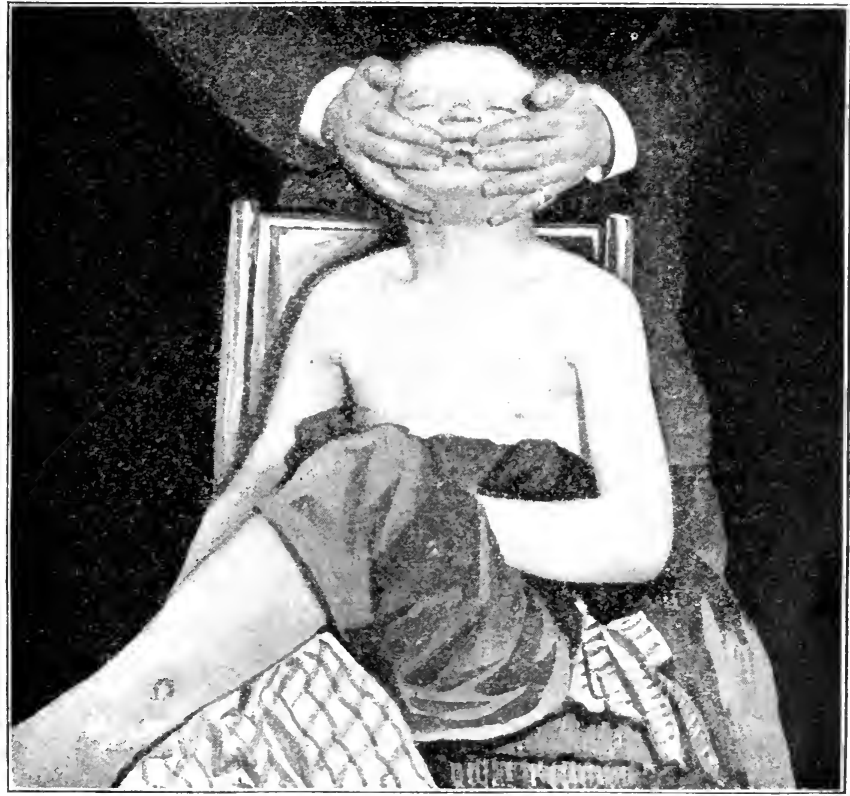

FIG. II.-Ifutchinsun's teeth with osteo-periostitis and ulceration in inherited syphilis

by excoriation a characteristic dermatitis. Infiltration of the mucous membrane lining the passage results, as also the obvious condition of "snuffles" already described. Sucking, respiration, and phonation are seriously impeded, and in late cases destructive effects upon the nasal and palatine bones may result. 
The ticth in inherited syphilis are characteristically altered, being, when affected, retarded in evolution and imperfectly formed in the first dentition, and in the second distorted. Hutchinson in I 863 described changes in the permanent teeth that are not invariably, but generally, found in syphilitic children reaching an age when the eruption of these teeth has been accomplished. The central upper incisors are chiefly involved, showing semilunar notches at the free or cutting edge, these teeth and others being often also pegged and changed in color to a yellowish hue (Fig. I I). Often minute pits can be detected in the enamel. These changes in the teeth, when associated with parenchymatous keratitis and the scars of former fissures at the angles of the mouth, are justly regarded by most physicians as pathognomonic of inherited syphilis.

The pharynx in hereditary syphilis may be the seat of mucous patches, erythematous blotches, or circumscribed or diffuse infiltration. In exceptional cases verrucous vegetations form on the membrane; the tonsils become voluminous; ulceration of a superficial or a deep character may involve the submucous tissue; or grave forms of stomatitis supervene, the membrane of the mouth exhibiting on exposure an ashen look. In patients of unusual debility hemorhagic effusions occur. When the bones are attacked the hard palate may be involved, and, especially in inherited disease which has existed for some years, the oral and nasal cavities are fused by ulcerative and destructive processes into a single formidable chasm. Often the anterior portion of the nares, the upper lip, and the hard palate in front are merged in a common ulcerative fossa. These destructive results may originate in either one of the 
two cavities, or in a grave gummatous involvement of the skin of the face followed by severe sloughing.

The lary'nx, the tracinca, and the bronchi may each be the seat of changes in inherited syphilis-infiltrations, circumscribed or diffuse, of the mucous or submucous tissues, followed or not by ulceration which may destroy the perichondrium or the cartilages. Here, as from other of the mucous surfaces affected in the disease, polypiform and verrucous excrescences may spring from the membrane, and when situated in the larynx or the trachea produce severe dyspncea and disorders of phonation. The ulcers of this region differ but little from those exhibited in acquired disease, being single or multiple, and situated centrally or on one or both sides of the larynx. The lesions of the trachea and the bronchi are rare and are of the same general character as those of the larynx.

Attention has already been directed to the clinical symptoms dependent upon the changes here noted-namely, the husky or stridulous cry of the infant, often progressively hoarse until wellnigh complete aphonia results. The impairment of respiration, the frequent raucous cough, evidently productive of pain and taxing to the utmost the strength of many of these feeble, wailing infants, and the symptoms of dyspncea, laryngeal spasm, and œedema of the glottis, are all significant.

The lungs, when involved in inherited discase, exhibit changes in the line of either definitely formed gummatous deposits or of "syphilitic pneumonia," the process then diffusely involving a large area of a single lobe or an entire lobe of one lung. The tissue is firm on section, sinks in water, is grayish in hue, and its alveoli are distended with swollen epithelium. Gummata of the 
lungs are commonly miliary or lenticular in size, with central necrosis proceeding to fatty degeneration. These nodules have a grayish hue, and they are set in dense pulmonary infiltrations of inflammatory type.

Syphilis of the asophagus, the stomach, and the intestinal tract is rather less rare than in acquired disease. In the intestines, especially, both definitely circumscribed and diffuse gummatous infiltrations have been recognized, large and single or numerous small ulcers resulting. The peritoneum may in some cases participate in the inflammatory processes present. Whether the symptoms be recognized before death (by palpation of the abdominal walls or by a catarrhal condition of the bowel) or post-mortem, agglutination of the intestinal coils usually has occurred.

The liver, when involved in inherited syphilis, may be the seat of circumscribed or diffuse gummata set in a dense hepatic mass, with obliteration of many, if not all, of the hepatic capillaries as a result of arteritis. There is commonly an odd-looking marbling or mottling of the hepatic surface. At times the portal vein exhibits enormous overgrowth of its connective tissue, choking its lumen; at other times the liver-cells seem to be compressed by a small-celled infiltrate squeezing the parenchyma. The gummata may be miliary in size or as large as filberts, both types having a characteristic grayish hue. This color is not to be confounded with that of the minute, semi-transparent granules supposed to represent unaltered hepatic tissue. The surface of the organ is either perfectly smooth or dotted with punctiform depressions, probable sites of localized hepatitis induced by the presence of gummata. A portion only or the whole of the liver may be affected, and the 
changes in its volume, the degree of its scirrhous hardness, and its shade of color are due to differences in the stages of its involvement.

The spleen is enlarged in from one-fifth to onc-fourth of all cases of inherited disease, being in cases many times more voluminous than in health. There is commonly a hyperplasia productive of a densely indurated mass, or circumscribed or diffuse gummata, these changes often coexisting with hepatic lesions of the disease.

The rectum and the amus of infants affected with hereditary syphilis are involved as in acquired disease, stricture of the rectum, however, rarely resulting. In infants not properly cleansed the production of condylomata, moist papules, and secreting tubercles about the anus and the vulva is greatly favored by the accumulation of fæces and urine on the napkins of the child.

The kidneys, when involved in syphilis of the second generation, may present evidences of interstitial inflammation, lardaceous degeneration, and alterations in the epithelium of the convoluted tubes not characteristic of this special malady.

The nervous systcm in hereditary syphilis may suffer from changes in the brain, the cord, or the peripheral nerves, in their membranous or osseous envelopes, and in the tissues with which the latter are in intimate relation.

The cranial bones are in cases characteristically changed by circumscribed or diffuse atrophic osteitis of gelatiniform type (the bones becoming softened to the consistency of jelly, or presenting a "worm-eaten " appearance), or by osteophytic osteitis, as a consequence of which bosses (nodes, exostoses, hyperostoses) form in 
special regions, producing in the skull of the infant a highly characteristic deformity. In one type the cranial bones are merely symmetrically changed and fixed or floating; in another they bulge as in hydrocephalus; in another the forehead pushes forward above, producing the effect of massiveness; in another each frontal protuberance is symmetrically studded with circumscribed rounded bosses or prominences; in yet another the brow edges forward in the mesian line, producing a keel-like aspect. Microcephalus may result either from formative osteitis making a cruciform or other shaped bridge over the fontanelles, thus interfering with the development of the skull, or from simple failure of evolution, the stunting being dependent upon general rather than upon local causes. Whether or not this condition and that of rachitis be due in some cases to syphilis indirectly, and in others to different morbid states not well understood, is not fully determined. The general belief among experts is that these and other evidences of failure of development are sympioms of cachectic states which may be induced by syphilis and other affections.

Subacute and chronic types of leptomeningitis, in which the dura mater or the pia mater may be involved primarily or secondarily, are not rare among syphilitic children; they require to be compared closely with the other signs of inherited lues, in order to be differentiated from tubercular affections of the meninges. Hemorrhages are occasional complications of these attacks, provoked by the presence of gummata in the cranial bones or in the pericranium. The cerebrum, the cerebellum, the pons, and the medulla may each be the seat of changes produced by any of the forms of arteritis studied in connection with the brain-syphilis of 
acquired disease, the ultimatc results being seen in the formation of aneurysmal pouches, irregular distribution of blood to the nervous tissuc, thrombosis, embolism, and hemorrhages. Gummata, as in acquired disease, may develop in the nervous substance; and encephalitis, cerebral sclerosis, ependymitis, ecchymosis, and softening may ultimately result.

The clinical symptoms springing from these organic changes, slight or severe, vary from feeble-mindedness and mental states suggestive of complete idiocy to insanity and epileptiform attacks, though the latter are rarer in congenital than in acquired disease. Single or multiple paralyses of centric origin, hemiplegias and paraplegias, with the usual accompaniment of severe, continued, or recurrent headache, are common results of these intracranial lesions. Recurrence of nervous phenomena of a severe grade, a distorted cranium, and an idiotic mental state point to inherited syphilis of the child even in the absence of any history of infection of one or both parents. The evidences of inherited disease, in the rare instances in which the cord and the peripheral nerves are involved, are obscure. Cases are rare in which opportunity is offered for their investigation. The oculo-motor paralyses of acquired disease are here occasionally noted.

The $c y e$ in inherited syphilis is subject to many of the disorders observed in acquired disease. Parenchymatous keratitis (chronic interstitial keratitis) is often found associated with the teeth described by Hutchinson, already noted, the combination of the two affections practically establishing a diagnosis of inherited syphilis. The cornea in these cases first becomes cloudy in punctate lesions recognized on close inspection as seated in 
the parenchymatous tissue. Gradually, in the course of a few weeks or less, the entire cornea presents a characteristic "ground-glass" appearance, in consequence of the multiplication and fusion of these points of opacity, with a pericorneal zone of injection, one or both eyes being attacked, and the two organs simultaneously or successively. The issue is either a gradual clearing up of the opaque condition, which at its fuliest evolution practically occludes the entrance of light, or a more or less intense injection and vascularization of the corneal surface. Iritis is rarer in inherited than in acquired disease, but it occurs in one or both eyes and before or after birth, plastic effusions in the worst cases gluing the iris to the capsule of the lens. Choroiditis and retinitis also occur in inherited disease, with the iris and the pupil unchanged, and dust-like particles in the vitreous humor originating in patches of infiltration of the choroid. Retinitis and optic neuritis in children have rarely been observed.

The Ear.-The persistent deafness of many subjects of inherited syphilis arise from changes in the tympanum and the middle ear. The lesions correspond, for the most part, with those heretofore described in connection with acquired syphilis. The deafness is due, in general, to a labyrinthitis characterized by a cellular infiltration of the membranous labyrinth and to a serous effusion into the endolymph, with eventual increase of connective tissue which may later undergo a species of cicatricial contracture. Prominent subjective symptoms are the usual morbid aberrations of audition (roaring, blowing, ringing, singing, and other sensations), more or less rapidly changing to absolute surdity. There may be, as in adults, coincident vertigo, cephalalgia, and febrile phenomena. 


\section{TREATMENT OF SYPHILIS.}

No treatment of syphilis may be regarded as worth the name that excludes early and persistent attention to the general health of the patient. This hygienic care, as contrasted with the medicinal measures employed, by far outweighs the latter in importance, and practically decides for many cases the question of the gravity of the issue or the reverse. The worst errors committed in the management of syphilis are due to trusting exclusively in the efficacy of drugs for relief of the disease.

Hygienic Considerations.-The patient affected with syphilis should always be given a sufficiently ample dietary, the food to be simple and digestible. For the gouty the food should not be that allowed the cachectic and the anremic. Allowance being made for these extremes, it may be said in general that the syphilitic patient requires an ample supply of nutritious and digestible food, seeing that, even in the case of the subject of the disease who is at the outset well fed and well nourished, it cannot always be known when the toxines of his malady so change the systemic condition that at a date not far distant the picture may be altered for the worse.

Alcoholic beverages may be used in the treatment of syphilis with wise discretion. In the case of the enfeebled the weaker stimulants, such as white wines, beer, ale, porter, and the malt extracts, may often be employed with great advantage to the patient; while the use of such articles as spirits, champagne, and Burgundy or Port, if drunk freely and in persons of a gouty state, 
may be positively injurious, and may actually prolong the period during which the malady requires treatment. Here, as in so many questions arising in medicine, the judgment of the practitioner, instead of a fixed rule, must finally decide.

Bathing is of importance in all cases. The very hot baths largely employed in the various health-resorts and springs of all countries are without question often harmful, and are to be ordered for the average patient only after due consideration. The skin of the body, however, should, when practicable, be sponged daily, exception being made for the menstrual period in women. Persons of a delicate constitution should simply moisten a handful of warmed salt with hot water and rub this over the skin-surface, using afterward a coarse towel or a flesh-brush to ensure a vivid reaction. For stronger patients, especially vigorous young men, daily cold sponging of the entire body, from the neck to the feet, with water to which salt has been added in the strength of one-quarter of a pound to the gallon is of high value. Hot baths and hot applications of all sorts for a skin liable to exhibit a syphilitic exanthem are decidedly objectionable. By keeping the surface well polished and in a high degree of tone the liability to pustular and other syphilodermata is practically set aside.

The use of tobacco, either by smoking, by chewing, or as snuff, is harmful to the mouth and to the nares of syphilitic subjects, inviting as it does the occurrence in these parts of mucous patches and other lesions. It is decidedly the wisest course in every case to interdict absolutely these practices from first to last. In the same connection it is well to remember that male patients deprived of tobacco are apt to hold cigars or a tooth- 
pick in the mouth, or even to chew gum for hours at a time, in order to allay the craving for tobacco. Each of these practices is harmful, and has repeatedly produced the most painful and persistent fissures of the commissures of the lips, and even obstinate ulcers.

It is well to bear in mincl the measures recognized as efficient in the management of other disorders producing deterioration of the general health. Diversion of the mind, abstraction from the fatigue and anxiety of business and professional work, foreign and domestic travel, the invigorating influences of a sea-voyage or a sojourn in the mountains, out-door living and open-air amusements,-all these have a distinct value in appropriate cases.

The recently infected subjects of syphilis, and often those who have suffered longer, should in general exclude the possibility of a determination of the activities of the disease to any one region of the body by setting aside, so far as practicable, all local sources of irritation. Carious teeth should be removed or their cavities be stopped; projecting edges of teeth in contact with the tongue should be removed by the dentist's file; a weak eye (particularly if employed out of doors with snow on the ground) should be protected; and a ponderous varicocele, a scrotal hernia, or a hemorrhoidal tumor should receive proper attention.

Time for beginning the Systemic Treatment of Syphilis.-It has already been shown that treatment of the chancre, whether by internal or external medication, is not the treatment of the disease which follows. Attempts to abort syphilis at the onset are usually as futile as similar efforts to jugulate the other maladies with which man may be affected. In any case in which, 
whether from the local phenomena (initial sclerosis, syphilitic bubo) or from special conditions aside from the local symptoms, it is deemed prudent to begin the treatment of syphilis before the establishment of an absolute certainty respecting its diagnosis, general treatment may properly be instituted, with the distinct understanding that such treatment will neither assuredly abort nor mitigate the symptoms which are to be expected later. The reverse is also true-namely, that delay in instituting systemic treatment of syphilis until the fullest recognition of the disease has been established in no wise jeopards the future of the patient nor his amenability to the later management of his malady. It has already been set forth in these pages that in a sound young patient free from signs of other trouble, infected with syphilis and properly treated thereafter, there would probably result but a single exanthem (the macular syphiloderm), upon the disappearance of which, when all the other hygienic and therapeutic conditions were absolutely fulfilled, no other symptom of the disease should follow. However impossible the attainment of such an ideal, its practical realization in selected cases points with clearness to the clinical fact that some expression of the disease, early or late, is in the nature of the affection to be expected. After such complete expression subsequent processes may be in the line of involution rather than of evolution. A very abundant macular syphiloderm not uncommonly disposes of the major part of all symptoms of systemic syphilis, and if this first exanthem be aborted, suppressed, or greatly influenced by energetic treatment (which is certainly in some cases effected), the future of the patient is to a degree clouded. One early vivid and generalized efflo- 
rescence is an augury for good in an otherwise healthy subject. No treatment is superior in results to that directed with energy, system, and skill to a disease permitted a first frank evolution.

The question, often formulated, "How long should the treatment of syphilis be continued?" is best answered by stating the length of time during which the disease may persist. For some patients the disease and the treatment, as has already been seen, are alike ended long before other infected subjects have ceased to exhibit symptoms or to be treated for their relief. The treatment of rebellious syphilis in the unfavorable class of patients already described is, indeed, a tedious matter. For the average of subjects of the disease, healthy before infection and managed skilfully, it is not difficult to fix the duration of treatment. Most of such patients after two and one-half or three years have passed are practically well. There are few sound persons thus cared for who may not suspend medication for weeks at a time after the conclusion of the second year.

Systemic Treatment of Syphilis.-Medicinal treatment of syphilis is conducted by the aid of remedies both ingested and externally applied. The former method is usually termed "internal," as distinguished from "external" treatment. Both methods have been employed at different periods of time, either separately or in conjunction, with favorable results. These modes of medicinal treatment have been by some writers made to conform to certain systems; as, for example, the so-called "tonic" method, in accordance with which a dose ascertained to be effective in the case of a single individual is continuously administered for a given length of time-a number of 
consecutive months or years. Another system is the " interrupted," in accordance with which the patient is submitted to treatment of the disease by special medicaments for a period of time, followed by a longer or shorter suspension of the remedy. Of the so-called " expectant" method of treating syphilis it is sufficient to say that few modern practitioners would dare to subject themselves to the charge of leaving a patient affected with the disease to such grave possibilities of dangerous and even fatal results. In these pages the effort is made to set forth the treatment of syphilis on a rational basis, and wholly independent of any system whatever. Indeed, the shilful physician will ever free himself from the shackles of conventional rules, and will learn by experience to employ with advantage for his patients the method which in each single case is most clearly indicated and best adapted not merely to relieve for the time being the symptoms of the disease, but also to set aside its possibilities of damage in the remote future.

Mcrcury. - In the face of vast opposition, and despite the fact that a large number of the lesions of syphilis have been attributed where they do not belong-to the assumed toxic effect of the metal which is of chief value in securing its relief-mercury to-day stands pre-eminent throughout the civilized world among drugs esteemed efficient both for the relief of the symptoms and for the radical cure of the disease. Like most agents that are both energetic and efficient, it is not a proper use, but an abuse, of its compounds that has brought upon it so much odium.

It has long been regarded as axiomatic that mercury is chiefly valuable in the early, and the preparations of iodine in the later, periods of syphilis. In general this 
may be admitted to be true; but the exceptions to the rule are so many that it may often be violated with the greatest possible advantage to the patient.

The preparations of mercury administered by the mouth are, in the order of their value in the management of syphilis, the protoiodide, the bichloricle, the biniodide, the tannate, blue pill, calomel, and the gray powder. Among American and French physicians the protoiodicle has, and we think justiy, a decided preference.

On the supervention of the first symptoms of general syphilis, it is well, when the method of treatment by the mouth with digestion of drugs in the stomach is selected, to begin with a mercurial course by the aid of the protoiodide. This preparation is to be exhibited steadily until all obvious symptoms of the disease are remored, and afterward to an extent hereafter to be discussed. It is well to begin with an average dose of the metallic salt, and to increase or decrease this dose as indications may be furnished by the patient. Whether one or another article be selected for use, that medication only can be regarded as both efficient and desirable which is not intolerable to the system, under the influence of which the patient gains in weight, and which enables him to digest food with appetite and profit as regards nutrition. The following are practicable formulie for the purposes named:

$\begin{array}{ll}\text { R. Hydrarg. iodid. virid., } & \text { gr. xij ; } \\ \text { Mas. ferri carb., } & \quad 5 \mathrm{j} .-\mathrm{M} \text {. }\end{array}$

Ft. pil. No. lx.

Sig. One or two pills after each meal.

From $\frac{1}{10}$ to $\frac{1}{3}$ grain may thus be given after each meal 
to a patient of adult years and average weight. The dose may be reduced or increased from day to day as required-diminished especially if there be looseness of the bowels, which to an extent is guarded against by the use of the ferruginous preparation named. For Vallet's mass the citrate of iron and quinine may be substituted in doses of from 1 to 3 grains.

When looseness of the bowels or colic is induced, the dose should be diminished or the habits of the patient with respect to food and drink should be controlled more carefully. The drinking of iced water, the eating of icecream, and the free use of fruits and of certain kinds of fish are often responsible for the excessive action of the bowels and for the pain induced by the drug.

Instead of in pills, the combination given may be administered in capsules, the preparations for this purpose lately placed upon the market being readily digested and more soluble even in the fluids of the mouth than a coated pill which has been desiccated by time and by hot weather. The tablet triturates of the same metal are often used, but are open to the disadvantage of disintegrating when carried about in the pocket. The centigram granules of Messrs. Garnier \& Lamoureux, which have long been esteemed highly in America and abroad in the management of the disease, are certainly of great value, as the pill is elegantly made and is efficiently preservative of its contents. Upon comparison with pills of the same dose of American manufacture, made, for the most part, by shilful precipitation of the green iodide, the latter will in general be found to be superior. The French pill, however, has an actual advantage in the greater impurity of its constituents. For therapeutic purposes the dose of the yellow pro- 
toiodide in pill form, made by American chemists, should be nearly one-half that prepared by the French in the pill named.

It is very rarely necessary to give an opiate in combination with mercurials in the treatment of syphilis, with a view to the introduction of a larger quantity of the metal into the system, or to relieve the diarrhœa produced by even small doses. This practice is a remnant of crude attempts at treatment instituted before the days of modern refinement in diagnosis and methods. It is to be regarded as a last resort for cases of extreme urgency and of very unusual irritability of the intestinal canal. Not merely may the combination beget the opium-habit in a person thus habituated to the drug (and frequently such habits have been acquired during treatment for syphilis), but harm is wrought by interference with digestion. He who hopes to be brilliantly successful in managing syphilis will ever be jealous of any impairment of the digestive functions of his patient.

"A chronic disease," as a great syphilographer has written, "requires a chronic remedy." Without adhering to any system, if it be found that, by steadily pursuing a mercurial course with the aid of the protoiodide, the symptoms of the disease disappear, and afterward the patient can still take the drug to advantage in doses that enable him to gain in weight or to hold it at nearly the maximum while attending to his or her usual vocation, meantime enjoying every sign of good health, a desirable and satisfactory end has been obtained. Certainly no change, under these circumstances, is specially desired or required. It is well, when this fortunate issue is reached, to have the patient kept under more or less careful observation, the practitioner being at all times 
ready to change the treatment, general or local, as may from day to day be suggested by any accidents that arise.

At any time, however, when such a course seems desired, any one of the other preparations of the metal named may be substituted for the protoiodide. These preparations are the bichloride, in doses of from $\frac{1}{60}$ to $\frac{1}{12}$ grain; the biniodide, in doses of from $\frac{1}{50}$ to $\frac{1}{16}$ grain ; the tannate, in doses of from $\frac{1}{2}$ to I grain; blue pill, in doses of from $\frac{1}{t}$ to I grain; calomel, in doses of from $\frac{1}{10}$ to $\frac{1}{4}$ grain; and the gray powder, in doses of from I to 5 grains. Of these preparations, the biniodide, the tannate, blue pill, calomel, and hydrargyrum cum cretâ may be given in pill form; the bichloride and the biniodide preferably in solution; and calomel and the gray powder in the form of either powders or pills. It is to be remembered that in giving mercury it is not so mportant to discover how large a dose a patient may take with impunity at one time for the relief of his disease as to know how large a dose may be taken for long periods of time with the same end in view. It is rarely necessary to give more than 3 grains of the protoiodide by the mouth daily, nor more than $\frac{1}{3}$ or $\frac{1}{4}$ grain of the sublimate, nor more of the other compounds named than can be regarded as an average rather than as a large dose.

Upon the slightest evidence, however, even when any of these doses is being pushed to a proper maximum, that the disease or its symptoms is not properly yielding, there can be but little question that the proper course is to change the preparation selected. In syphilis the infective cells become later less amenable than at first to the antagonizing remedy, and each group of these cells 
may become a focus from which, by the well-known processes of cell-multiplication and the production of toxines, the morbid process may be relighted to activity. Even the most trivial of lesions is to be combated energetically in this early stage; and when resolution is not visibly progressing, not only is the remedy for internal use to be exchanged for another, but the topical treatment of any lesions present, as explained elsewhere, should either be modified or be more energetically and persistently pushed.

The season for the happiest results from the management of syphilis is the first semester of its career. If any treatment may justly be described as "abortive," it is not that which seeks to jugulate the malady in its chancre-stage, but that which vigorously and efficiently obliterates all symptoms in the period of early evolution.

We believe that when all progresses satisfactorily, the patient who secures complete immunity from symptoms of his disease in the first half year does better when no recognized antisyphilitic remedy is administered save mercury. Many of the best treated patients have never swallowed the compounds of iodine except in combination with mercury. Other things being equal, he who has secured complete relief from syphilis without using the iodide of potassium has usually had either a mild or an exceedingly tractable form of the disease.

Iron is administered with decided advantage to the great majority of all patients affected with syphilis, and it is well to order.it in all cases where it is not contraindicated. A convenient method is to prescribe a ferruginous tonic before the first and the last meal of each day, while the mercurial in pill or other form is taken after 
each meal. Iron does its best work for most patients when ingested in a fluid form. The following is a convenient formula :

R. Ferri et quin. citrat., $\overline{\tilde{s}} \mathrm{sS}$;

Limon. syrup., Aq. destill.,

$\mathrm{f} \overline{\mathrm{s}} \mathrm{ij}$; ad $f_{\overline{5}}$ viij. $-M$.

Sig. A teaspoonful in a wineglassful of water before the first and the last meal of the day.

Iron may also be given in pill form or be combined with the bichloride in formulas of which the following may be taken as a sample:

R. Hydrarg. chlorid. corros., gr. j-ij ;

Ferri tinct. muriat., Acid muriat. dilut., Syr. aurant. flor., Aq. dest.,

à̃. fojij-iv;

$\mathrm{f} z \mathrm{ij}$; ad f zviij.-M.

Sig. A teaspoonful in a wineglassful of water after each meal.

When there is constipation, patients often find it of advantage to take some such formula as that given above, in proper doses after the first and the last meal of each day, and one or two pills or tablets of the protoiodide after the middle meal of the day.

Inunction of mercury, or its systematic introduction by the skin, is one of the superior methods of treating syphilis. This practice has the excellent recommendation of sparing the stomach, which may then be reserved for food and drink, for tonics, and for whatever else in the way of adjuvant ingesta may at any time be required. The disadvantages of inunction are its relative uncleanliness and the need of more or less skill and time in its employment. Few patients of the better class like to resort 
to it for more than brief periods of time. There are few, however, who may not reap substantial benefits from smearing mercury even for short periods. It is wise to employ inunction, first, in all grave cases; second, in all cases of emergency; third, whenever the stomach proves intractable to drug-ingestion ; and fourth, whenever, even after generally favorable results from medication by the mouth, there are persistent lesions refusing to yield to general and local treatment.

Mercurial ointment of the United States Pharmacopœia, in 50 per cent. strength, still heads the list of mercurial preparations available for inunctions. It cannot be employed with equal advantage in combination with any drug. Mercurial plasters have in some cases a decidedly beneficial result as local applications; and the 5, 10, and 20 per cent. oleates may also at times be used with advantage when there is no special urgency. No such reliance, however, can be placed upon them as upon the officinal blue ointment.

When inunction is to be employed, the dose of the ointment should be varied according to the weight and the general condition of the patient, from $\mathrm{I}$ to 3 scruples being ordered to be well rubbed in at a single sitting. The smaller may often with advantage in any given case be increased to the larger dose named. The skin should usually have a preliminary cleansing in tepid water with soap, and often also a washing with borated water or with alcohol in order to ensure an aseptic state of the skin. Professional rubbers generally do better service than untrained servants or the subjects of the disease themselves; but often the last named-especially women who are anxious not to betray to others the nature of their malady-learn to practise inunction with 
excellent results. The ointment should be rubbed in until it has practically disappeared, with a gentle kneading motion of the hand, or, better, the two handspractically the movement used in massage. The hand of an assistant, if such be employed, need not be protected by gloves, seeing that a talc paste such as the modified Lassar may practically be made protective. The rubbings are preferably made at night, after which the patient retires to bed in clothing which is suffered to be soiled by contact with the salve. Diaphoresis induced by the drinking of hot fluids afterward (milk, spirits, etc.), recommended by some authors, is unnecessary in order to secure the best results of the treatment. In hospitals it is customary to make the patients rub each other, usually on the back and simultaneously, the ward nurse having then to anoint but a single patient in the line. It is usual to order from one to four or five scores of rubbings, to be given on successive or alternate nights or at intervals of several days. Often it is desirable to give a course of twenty inunctions, after which the rubbing may be suspended while other treatment is pursued, the inunctions being renewed until the entire number advised is completed. When required, for special reasons, a useful method of inunction is to order the skin well rubbed with the salve, the part anointed being then covered by the customary clothing. which is worn afterward for a series of days. In this way stockings impregnated with the ointment may be kept in contact with the feet, while flannel undergarments may be employed with the same end in view. There are, however, few patients who relish the dirtiness and messincss of this practice, which is, in general, to be reserved for special cases, such as those where 
neither the patient nor professional masseurs can do the work.

When giving inunctions, the rule enunciated with respect to mercury by the mouth should not be forgotten. The ferruginous tonics are to be administered systematically while the patient is under the influence of the metal. He should also be given a generous diet and should have out-door air and exercise.

The regions selected for inunction of the body are of some importance, as it is desirable that the ointment be rubbed into those parts where the skin is provided with glands through whose excretory orifices the metal may gain access to the economy. It is also desirable, in view of the readiness with which mercurial inunctions induce an artificial dermatitis in the region of application, that a new area of inunction be selected on successive days. This area secured, however, the refinements of authors respecting the selection of special regions of the body for inunction have little foundation in the way of attaining practical results. Inunctions of the thick sole of the foot are often as efficient as those on the sensitive and thinner integument of the groin or over the subclavian regions.

We are in the habit of ordering inunctions first for regions of the upper segment of the body in succession, and later for those of the lower half successively, for reasons connected chiefly with the garment worn after the completion of the process. In this way, on one night the thighs and groins, on another the legs and soles, are anointed, and after this the drawers or pajamas worn are washed; on other nights the surface of the belly and the breast, the arms and the axillæe, the back, or the neck and the head may be smeared. It is to be 
borne in mind, further, that when the inunction is not employed with a view to acting directly upon lesions present upon the integument, the value of the inunction as regards the general system is as great when applied in one region as another; and it is a matter of importance to encourage patients, especially those in private practice, to continue with their inunctions for the longest period advised. For the most part, they will consent to rubbings of the lower portions of the body for a longer time than to inunctions of the upper regions. The penis, scrotum, anus, face, ears, and the vulva and breasts of women are in general to be spared.

There are few patients, whether informed or not, who remain ignorant of the nature of the treatment when inunctions are employed. As a consequence, they are at times exceedingly anxious about "catching cold," using acids in their dietary, etc., for fear of toxic results. While due prudence must guide the practitioner in advising patients on these points, it is rare, with a properly advised course of inunctions and with the subject of the disease kept under the observation of an intelligent physician (as should always be done throughout the treatment of syphilis), that accidents happen, even after imprudent behavior on the part of the patient.

When inunction is practised with a view to direct medication of the skin (for example, in case of palmar or plantar syphilodermata), care should be observed to make the applications so far as practicable over all persistent lesions present. It is the skilful obliteration of all foci where infective cells, micro-organisms, or toxines may be present that preserves against the lighting up of morbid processes in these centres of undiminished activity. 
When a dermatitis is induced by the frictions incidental to the inunction process or as a consequence of the mercurial application, the results are rarely serious. Discontinuance of further rubbings over the region which thus expresses its resentment will usually suffice, in the course of a few days, to relieve the symptoms. In any case where treatment really seems needed, the application of a simple dusting-powder or of Lassar paste usually suffices to allay the itching and the local irritation.

The modified Lassar paste is made by adding 2 to 4 drachms each of talc and zinc oxide to $1 / 2$ ounce of white vaseline, with from 5 to 20 grains of salicylic acid, the whole rubbed together until a smooth and impalpable paste results. It is more or less adherent to the skin, and, apart from the value of its medicinal constituents, has the advantage of protecting the surface to which it is applied.

Fumigation.-The treatment of syphilis by the aid of the mercurial vapor-bath is both efficient and speedy. In any emergency it is capable of producing more rapid effects in a given time than any of the methods thus far described. In the large cities it is customary to send patients to bath establishments, where, by the aid of somewhat elaborate apparatus, aided by steam-supply and by special devices for exposure of the head without necessitating inhalation of the vapor, the patient is fumigated by the aid of trained assistants. However, with the Lee or the Maury apparatus, and an extemporized chamber, constructed either of bed-blankets or of ticking, which can be fashioned by any seamstress so as to encompass the patient's body as high as the neck, the same results can be attained with trifling trouble and expense. 
Indeed, without any special apparatus, an ordinary tinsmith can construct a pan for holding the metallic salt to be vaporized over a spirit-lanp, which, with a kettle of boiling hot water by its side, furnishes all required accessories.

Calomel or cinnabar is usually selected for vaporization, and often the two in combination-about one-fourth more of the latter than of the former when the two are commingled. From I to 3 scruples of the single salt or of the two may be employed at a sitting, the quantity being estimated not merely from the condition of the patient but from the size of the chamber to which steam is admitted, since much more may be used in the large receptacles of the bath-houses than in the extemporized blanket or tiching tent which may be employed by a country physician at the bedside of his patient. The exposure should last for about half an hour-less if the patient becomes faint during the steaming. As a rule, the subject of the disease should be fasting at the bathhour, which is preferably that preceding his or her accustomed hour of sleep. As it is by no means rare for the subject to become faint, it is well to have a stimulant at hand, and, even in sending patients to the bathhouses, to advise the carrying with them of a small flask of sherry or brandy. A bath every third or even every second day is sufficient save in cases of emergencyas when there has been ignorance of the nature of the disorder before the first consultation, and the patient has a highly disfiguring facial exanthem forbidding his or her customary association with family or friends. In such event, and for brief periods of time, a bath may be taken daily; but in these cases, as well as in the others, it is needful to remember that the patient is often debilitated 
by the steaming, even when vastly improving in the matter of the removal of the lesions which place his case in the emergency class. These emergency cases, furthermore, are often those of patients suffering from febrile reactionary symptoms (syphilitic fever), and the need of ferruginous tonics, of quinine in ample doses, and even of a generous glass of wine with the dinner, should not be forgotten. Local mercurial fumigation by means of the apparatus sold in the shops is of value in many cases for direct application of the vapor both to the skin and the mucous cavities. IVe have, however, practically limited our use of this method to the nasal passages, where its value is without question. In country practice, where apparatus of the desired sort is not immediately at hand, a hot flat-iron and a paper cone answer admirably for directing mercurial fumes into the nasal passages. The dose of calomel or of cinnabar selected for local fumigation must be reduced considerably from that used in the general bath. For the nose from 2 to 5 grains of calomel may be vaporized; for the face a somewhat larger dose may be used.

Hypodomatic Injcction.- This method of introducing mercury into the system is properly described after the others, since, as a matter of practical experience, it is not only employed far less often than others, but promises to be reserved at no distant date for use only in special cases. By it the metal, pure or in combination, is injected directly beneath the integument.

The advantages of this method are rapidity of effect, the sparing of the digestive tract (a feature which it shares with both fumigation and inunction), its simplicity and cleanliness as contrasted with the two methods named, and its surrender of the dosage into the hands 
of the practitioner, and of him only-a feature of importance. Other advantages claimed, but not yet demonstrated to the satisfaction of experts, are the speed with which it effects a radical cure, the failure of relapses in the cases thus treated, and the exclusion of the gummatous phases of the disease. The objections to the method are great: it has often proved dangerous, and in a few instances fatal; it is liable to produce furuncles, nodes, abscesses, sloughing, and other lesions at the site of injection; it is likely to beget an overweening confidence on the part of both physician and patient that the disease is in course of radical treatment, while precious time is lost that might have been employed in protecting the victim of the malady from its ravages at a future epoch.

If, nevertheless, a hypodermatic mercurial treatment be selected, too much care cannot be taken in the preparation of the skin and the instruments before the operation. Only sterilized solutions should be employed, and the skin over the region of introduction should first be cleansed thoroughly with warm water and soap, then dried, then washed with alcohol and dried, and then moistened with a I: I000 solution of the sublimate. The needle employed should be of steel, gold, or silver, somewhat longer than that of the ordinary instrument, and in an aseptic state-as also, needless to add, should be the hands of the operator. The region most often selected for injection is the post-trochanteric, with the patient reclining on his belly and the muscles completely relaxed. The needle, with syringe attached and charged, taken from a 5 per cent. carbolized bath in a tray, should be pushed slowly down to the region where the salt is to be deposited, the physician avoiding always, first, 
entrance to a vein (known by the ease with which the syringe begins to discharge its contents, as contrasted with the obstruction encountered in muscle), next, regions of unusual pressure or friction, and, lastly, the inferior portion of the derma or very near the panniculus adiposus, where severe sloughing may follow.

The damage resulting from hypodermatic injections of mercury may be the formation of nodes, abscesses, erythematous patches, and sloughing at the site of the deposit; alarming cardiac and pulmonary symptoms after injection within a vein; sudden death; exhaustion coming on slowly after the operation; considerable pain, at times agonizing, at the site of the puncture; and salivation, with other systemic signs of the toxic action of the medicament. In well-managed cases, however, it is to be admitted that, with a properly constituted solution and with due precautions, hundreds of injections have been given with no untoward consequences.

A great amount of literature exists on the subject of hypodermatic injections of mercury for relief of syphilis, and the list given of selected articles employed for the purpose is intended to serve chiefly as an index.

Soluble Salts of Mercury. - Corrosive sublimate is employed for hypodermatic injections in the strength of from $\frac{1}{12}$ to $\frac{1}{10}$ grain, dissolved in a few minims of water suspended in olive oil, or emulsified, as with vaseline or mucilage. The injections may be made as often as once every second or third day. The following are practicable formulæ:

P. Hydrarg. chlor. corros., Glycerin., Aq. dest., gr. j ; $\bar{a} \bar{a} . \mathrm{f} z \mathrm{j} \cdot-\mathrm{M}$.

Sig. Inject 10 minims. 
injection), the formamide in I per cent. solutions, the alaninate, and the bcnsoate, each of which has its partisans, and none of which has succeeded in achieving a large usage at the hands of experts.

Insoluble Salts of Mercury.-Calomel, $\frac{1}{2}$ to 3 grains suspended in a chloride-of-sodium solution, in mucilage, in glycerin, or in oil, has been injected every five to ten days, as well as metallic mercury, from 5 to 20 grains in a similar vehicle.

Olcum cincreum (gray oil) is made by emulsifying lanolin and chloroform and adding metallic mercury in double the quantity of the unguent: 20,30 , and 50 per cent. ointments are compounded with this basis, by the addition of olive oil. From $\frac{1}{2}$ to 1 grain of the 50 per cent. solution has been injected once or twice weekly, with progressively increasing intervals between the injections. Ycllow oxide of morcury has been added to mucilage or olive oil and injected so that from I to 2 grains have been used at a single dose. The black oxide of morcury' is employed in 10 per cent. oil, in glycerin, and in gum emulsions; and cinnabar, in the strength of I grain suspended in oil.

The conclusions which it is safest to accept, after reviewing the subject of hypodermatic injections in syphilis, have been well summarized by Dr. White of Philadelphia, who took pains to collate the opinions of a number of American experts on this question. The method has not as yet shown results which warrant its adoption as a means of routine treatment to the exclusion of, or in preference to, others; it has, on the contrary, some apparently insuperable disadvantages and even dangers, which render it improbable that it will ever be generally adopted. 
The Toxic Effects of Mercury (Hydrargyrism; Salivation; Mercurial Pains, etc.).-Like most medicinal agents of well-marked efficacy, mercury, when improperly administered or when administered to peculiarly susceptible subjects, may produce toxic effects. Some of these effects ensue rapidly (so-called "acute" symptoms), others more slowly (the "chronic"). One of the most common and umpleasant of these results is salivation, an accident displayed in many grades. In the slightest grade there is moderate fetor of the breath; slight inspissation of the saliva; some tenderness of the teeth, more particularly of the molars when brought together; a sponginess of the gums, which bleed readily when pressed upon; a metallic taste in the mouth; and a peculiar pasty aspect of the dorsum of the tongue. All these symptoms may be exaggerated in various grades to the point where the parotid and submaxillary glands become tender and tumid, the saliva flows in a full stream from the mouth, the teeth are loosened and fall, the mucous membrane of the mouth becomes swollen and often eroded in patches, the tongue is swollen, protruded, and ulcerated, and the bones of the jaw are nccrosed. The breath in all cases has an unmistakable and nauseous odor, and the patient is also generally in a depressed condition of mind and disturbed in most of the bodily functions.

Among the results that develop more slowly may be named many of the evidences of gastro-intestinal dyspepsia (inappetence, eructations, heartburn), progressive adynamia and anæmia, pains in the joints, occasionally limited to one of the larger joints and associated with temporary immobility from pain, and symptoms simulating those of muscular rheumatism. While there is a 
large list of ailments, not here set down, popularly accredited to the toxic effects of mercury, it may be said of most of such symptoms that they are due either to syphilis or to some other cause, and are wrongly imputed to the action of the metal. We have never been able to persuade ourselves that for any reasonable period after the ingestion of mercury had been suspended (months at the longest) any general effects of it are perceptible in a previously healthy subject; and the records of the physicians in charge of the laborers in mercury-mines attest the same fact. There the toxic effects are distinct and often grave, severe salivation being more or less rapidly followed in all grave cases by osseous necrosis. Nothing is found in these records corresponding with the "chronic rheumatic disorders," "eruptions upon the skin," and other ailments popularly charged to a continued use of mercury, and occurring years after its suspension.

Let it be noted further that in the few cases where, early in a syphilitic career, mild salivation has accidentally occurred (the writers have seen but few cases in many years), the issue is not altogether without its bright side. As a matter of fact, the few salivated patients have in the results obtained compared most favorably with others; and in one specially dangerous and extreme case, where salivation was intentionally produced, the issue was in the highest degree satisfactory, as a valuable life seemed thereby to have been saved.

When mercurial stomatitis supervenes, with symptoms of salivation, the metallic dose, if the case is not exceedingly urgent, should at once be suspended and the diet be limited to nutritious foods in a liquid or a semiliquid state (broths, cream, soft-boiled eggs, etc.). The fluids used for drinking should be neither hot nor cold, 
and all salted, spiced, and acetous articles of diet should be forbidden. The mouth should be washed frequently with bland lotions of flaxseed tea or borated or carbolated fluids, always diluted, such as:

$$
\begin{aligned}
& \text { R. Potass. chlorat., } \quad 3 \mathrm{j} \text {; } \\
& \text { Mel. despum., } \\
& \text { ilyrrh. tinct., } \quad \bar{a} \bar{a} \text {. f } \tilde{\tilde{s}} \mathrm{ss} \text {; } \\
& \text { Aq. dest., } \quad \text { ad f } \tilde{\tilde{s}} \text { viii.-M. }
\end{aligned}
$$

Sig. To be used as a mouth-wash and gargle, diluted with tepid water until grateful to the surface.

Often cluring the day the gums should be rubbed gently but thoroughly, within and without the circle of the teeth, with a tepid myrrh-and-cinchona wash (equal parts of the tincture of each suspended mechanically by shaking in water) applied by means of a soft piece of linen wrapped about the forefinger. We invariably order iron internally in these cases, and if any specific medication is employed before the toxic effects subside, we employ in small doses one of the salts of iodide. Recovery under good treatment is in any well-managed case rapid and complete.

Iodine and its Compounds. - With relation to the therapy of syphilis, iodine and its compounds stand next after mercury in popular estimation; and if just reserve be made, they certainly stand in this relation when properly employed in selected cases. There are two axioms that still very largely influence the minds of professional men on this question: one is that while mercury is most valuable in carly periods of syphilis, the compound.s of iodine are chiefly valuable in the late or gummatous periods; another is that while mercury cures 
the disease, the compounds of the other metal relieve without curing. Both axioms are imperfect generalizations of a wide experience, which must, however, not be permitted to warp the judgment of the practitioner in any case where the one or the other drug is chiefly indicated.

The following are conditions in which it is common, and in general wise, to employ the iodine compounds:

First: In all attempts to resolve gummatous lesions promptly, the iodides are wellnigh unequalled in the armamentarium of the expert. Here (as in gummata of the brain, the testis, the liver, the spleen, or the kidney) life may be saved by their efficient employment, and in other cases (gummata of the periosteum, the meninges, etc.) a great amount of suffering may be spared. Second: The iodides are often in the highest degree valuable in any stage of syphilis when the patient either is intolerant of mercury or, if sufficiently tolerant, cannot be made, when ingesting it, to gain in weight, in appetite, and in the proper performance of his functions. Third: When it is desired to produce a profound impression on a syphilitic lesion, and the patient is being subjected to the action of mercury by any of the methods of its external emplorment (fumigation, inunction, ctc.). Fourth: The iodides are in some cases, not as a routine treatment, valuable as furnishing an alternate medication of patients long subjected to the action of mercury.

It is exceedingly doubtful if, as was once thought, the iodide of potassium aids in the elimination of mercury previously introduced into the system. The speedy effects of the salt are well known, and its rapid appearance in the urine after ingestion (as shown by starch and other tests) is readily demonstrable. When the patient 
is under its influence the application of starch to the tongue produces there a blue color, and the nitrate of silver a yellowish tinge, as a consequence of the resulting double decomposition.

The brilliant results which the iodides in selected cases are capable of accomplishing, and the dread of producing ill effects by the action of mercury, have buttressed the great popularity of the former with the general practitioner and with the public. Iodine and its compounds are represented in almost all the proprietary preparations sold in the shops for the relief of syphilis. Iodine is the one remedy earliest and most often resorted to by the ignorant, and it is the one which is last used, and then most effectively, by the expert. Given the patient who has been relieved of syphilis without a resort to iodine, and his case has probably been managed better. and is more secure as to its future, than that of another in which a large use has been made of the iodine salts.

The best known preparations are the iodides of potassium, sodium, lithium, strontium, starch, and rubidium; but the iodide of potassium has long held its own at the head of the list, and it gives promise of doing so for years to come. While given often in combination with other drugs, it is by no means settled that these combinations (save with mercury, as described later, possess any value over the simpler solutions. Of these solutions, none is better than that made by adding an ounce of the iodide of potassium to a fluidounce of distilled water; but as this combination often deposits the salt by precipitation about the cork of the vial after a brief interval, and as it is at times somewhat difficult of digestion, the iodide is often administered in drop doses from a solution made by adding half an ounce of the 
potassic salt to an ounce of the essence of pepsin (Fairchild's or another). The first formula has the advantage that it may be given, when that article of food does not disagree with the patient, in milk as well as in water.

The dosage of the iodide depends almost wholly on the emergency presented in any case where it is thought best to employ it. In uncomplicated cases it may be administered in teaspoonful doses of a solution containing 5 , 10 , or 20 grains to the drachm; but in cases where an emergency has arisen it is customary with experts to order one of the stronger solutions named above, and, beginning with drop doses, to increase gradually either one drop per diem, or one or even two or more drops each dose, until much larger doses are reached than are usually employed in cases where no emergency exists. In these cases large, and even extremely large, doses are slowly reached and for long periods of time steadily maintained. In this way $300,400,600$ grains, and even more, of the iodide of potassium have been given in twenty-four hours, and with favorable results as respects the object in view. In reaching the larger doses the following rules should be observed: $(a)$ The dose should not be increased after the occurrence of any of the serious symptoms of iodism, described later; (b) nor if constipation of a marked character occurs; $(c)$ nor if any decided indication of trouble occurs in the urine (albuminuria, etc.); $(d)$ nor if the immediate effect in view is secured (relief of a dangerous cephalalgia in syphilitic meningitis; relief of torturing pain at night from an osteoperiostitis, etc.).

The articles added to solutions of the iodide of potassium with a view to improve its efficiency or to prevent iodism are yearly accorded less and less favor, though 
once held in high esteem. Thus the chloride of ammonium in doses of $;$ grains and the carbonate of ammonium in doses of 10 grains were thought to increase the efficiency of the iodine salt, while arsenic and arsenious acid were added with a view to the prevention of iodic acne.

A great number of remedies, mostly from the vegetable kingdom, have been extolled for years as not merely of themselves sufficient to "cure" the disease, but, failing that, to add to the value of the iodide of potassium by assisting in its assimilation and by giving tone to the stomach. It would be a waste of space to enumerate these remedies, beginning with sarsaparilla, which even at this late day still holds sway over the minds of the credulous, and ending with the "McDade" formula:

R. Smil. sarsaparilla, fl. ext.,

Stilling. sylvat., fl. ext.,

Kappæ minor., fl. ext.,

Phytolacc. decand., fl. ext., $\bar{a} \bar{a} . \mathrm{f} \tilde{\tilde{z}} \mathrm{ij}$;

Xanthoxy1. Carolin., tinct., $\quad$ fzj.-M.

Sig. Teaspoonful to tablespoonful doses in water before food.

None of these "vegetable" remedies can be demonstrated to have any curative effect upon syphilis, apart from the metallic salts with which it is usual to compound them. They are never ordered by the writers, for, if merely a tonic and carminative effect be desired, much more valuable remedies are available. Strychnia, quinine, and iron or the mineral acids, the latter often in conjunction with a mild mercurial, are superior from every point of view to all of the "vegetable infusions and decoctions." 
The fluid extract of coca, much praised by Taylor, stands on a different footing, since it is not claimed in any sense to be a specific for the disease, but only a valuable agent in exerting a tonic effect upon the nervous, vascular, and lymphatic systems. We have used the coca in many cases with great advantage both in the form of the wine (Mariani, Metcalf) and in that of the fluid extract, as follows:

R. Erythrox. coc., fl. ext.,

$\mathrm{f} \tilde{\mathrm{s}} \mathrm{ij}$;

Gentian., tinct. co.,

Cinchon., tinct. co.,

Elix. calisayæ,

$\bar{a} \bar{a} . \mathrm{f} \tilde{\jmath} \mathrm{j} ;$

fร̄iv.

Sig. A tablespoonful in water after food.

R. Erythrox. coc., fl. ext.,

$\mathrm{f} \tilde{\mathrm{s}} \mathrm{ij} ;$

Cinchon., tinct. co.,

Gentian., tinct. co.

$\bar{a} \bar{a} . \mathrm{f} z \bar{j} \mathrm{j}$.

Sig. Two teaspoonfuls in water after food.

Toxic Effects of the Iodine Compounds.-Iodism, or the symptoms of toxic effects after the ingestion of the compounds of iodine, is much more frequent than the production of toxic effects by mercury, on account of the rapidity with which iodine affects the system, and also on account of the far larger number of persons susceptible to such effects. In discussing this subject it may for practical purposes be assumed that the iodide of potassium is the remedy selected, seeing that it is the most commonly ingested of all the iodine compounds employed in the treatment of syphilis.

Like all drugs exerting in any degree a toxic effect, the iodide of potassium will often display its mischievous energy after but a small dose has been administered- 
even so small as I or 2 grains. In other cases the toxic results are declared only when very large doses are reached. It is the popular and semi-professional belief that some persons have a special idiosyncrasy forbidding them ever to make use of the drug, but that in many patients, by care and a skilful adjustment of the dose, there can be established a "toleration" which will enable the vast majority to ingest even the largest quantities. There is truth, probably, on both sides of this question, though its final determination is difficult. With the employment of the graduated dose, with the bowels freed from irritating contents, with the habits of life regulated as carefully as they always should be in the subject of syphilis, the end sought can generally be attained. The toxic symptoms of iodism nay be of the very slightest grade (the occurrence of one or two irritable nodules over the face), or they may have grave sequences which may be in the highest degree alarming.

It has been said of the iodide of potassium that it is capable of producing upon the skin a picture resembling that of every cutaneous affection, and this statement is certainly suggestive of an interesting series of facts. The lesions produced in the skin are usually of the acne type, and limited to the face, the shoulders, the neck, and the upper portion of the trunk-the regions chiefly affected by the disease named. Classified, they range between macules and papulo-pustules, tubercles, nodes, bulle, and phlegmonous, purpuric, and ulcerative lesions.

Other symptoms are the production of a metallic taste in the mouth, salivation, coryza of a persistent type (often with an exceedingly abundant serous discharge from the nares), and several forms of urticaria, verging in extreme cases to the type of angioneurotic oedema, with 
spasm of the glottis when the swellings occur in this region, dyspnœa, and involvement of the joints. In other cases all the symptoms of peritonitis are present, with tumefaction of the belly, intense pains, constipation, and fever. Other toxic effects are slower of evolution, and are exhibited in a progressive anorexia, weakness, decolorization of the skin (of anæmic type), and decided loss of sexual desire and vigor. As a rule, the toxic effects of the iodide of potassium speedily disappear when the exhibition of the remedy is suspended and one of the tonic methods of treatment is substituted. When the anæmia from long-continued use of the iodide is added to the cachexia of syphilis, the result is especially unfortunate, and only the clear eye of an experienced physician can precisely discriminate between the two.

Mixed Treatment.-The term "mixed treatment" has been employed to designate the method by which mercury and the iodine salts are employed in combination in the treatment of syphilis. It is obvious that they may be administered simultaneously when mercury is introduced either by inunction, by fumigation, or by hypodermatic injection, and the iodides are at the same time given by the mouth; also when at one time in the day a mercurial and at another an iodide dose is ordered; and, lastly, when mercury and a salt of iodine are administered at the same time in a single dose. It is for the combination last named that the title "mixed treatment" has been especially reserved.

The following are a few of the "mixed" formulæ most often employed toward the end of the first year of syphilitic treatment, or earlier when there is a special indication for the use of the iodides, as when gumma- 
tous, tubercular, nodular, or threatening lesions persist upon the skin or over the mucous membranes, refusing to yield to mercury alone:

R. Hydrarg. biniodid., grs. j-iij ; Potass, iodid., jss-ij ;

Syr. aurant. cort., f 亏̈iij;

Aq. dest., ad $\mathrm{f} \tilde{\bar{z}} \mathrm{vj}-\mathrm{M}$.

Sig. A teaspoonful in a wineglassful of water after food. At times the bitter tinctures are added to formula of this character, in order to produce a tonic effect; but, as already pointed out, it is in general better in syphilis to administer tonics separately and before meals, seeing that the dose of the tonic remedy is commonly fixed, while it is often of service to administer the mercurial in a dose that can be changed so as to increase or decrease, when required, the amount of the metal ingested.

The decomposition of the mercuric bichloride by the iodide is often practised in compounds of the "mixed" class, but as there is some uncertainty about the result in different solutions, it is better to order the biniodide. Bichloride combinations with iodide of potassium are usually made as follows :

R. Ilydrarg. bichlorid.,

gr. j-iij ;

Potass. iodid., डss $-i j$;

Glycyrrhiz., syr.,

$\mathrm{f}$ 亏ij ;

Aq. dest.,

ad $\mathrm{f} \tilde{\tilde{z}} \mathrm{vj} .-\mathrm{M}$.

Sig. A teaspoonful in water after food.

The Treatment of Syphilitic Lesions as they are Presented in the several Organs of the Body.-The treatment of clancri has been discussed in the pages devoted to that subject (Pp. j I-57). 
Syplitis of the skin is amenable to the treatment appropriate to the systemic state, but at times, especially when the lesions are localized and asymmetrical, local treatment is of especial value.

The erythematous and papular syphilodermata, when persisting on the exposed surfaces of the body (face, hands, etc.), may be sponged with warm water at night and afterward anointed with-

R. Hydrarg. ammon. (vel. chlorid. mit.), gr. v-xx;

Bals. Peruv.,

$m x$;

Unguent. aq. ros.,

亏̈j. $-\mathrm{M}$.

Sig. External use.

Or, when a lotion is preferred, the parts may be sponged with a solution of the bichloride in rose-water, $\frac{1}{2}$ to I grain to the ounce; or the following may be used:

R. Hydrarg. chlor. corros., gr. ij ;

Vin. rect., spt.,

Benzoin., tinct.,

Tolutan., tinct.,

Glycerin.,

$\bar{a} \bar{c} . \mathrm{f} j \mathrm{j} ;$

Aq. ros.,

$f 3 j$;

ad f $\tilde{z} \mathrm{vj} .-\mathrm{M}$.

Sig. Shake. External use.

When seborrhœic crusts form about the forehead, nose, lips, ears, etc., the following may be employed with advantage:

R. Hydrarg. sulph. rub., gr. j-ij ; Sulph. precip., $\quad 3 j$;

Bals. Peruv., $\quad m x$;

Unguent. aq. ros., $\quad$ 亏ुj.-M.

Sig. External use.

Vaseline should be ordered as a salve-basis when the unguent is to be applied over a hairy region. Resorcin 
is useful, in the strength of from a scruple to a drachm of ointment.

For the papular and scaling lesions, which are often obstinate, especially over the palms and the soles, nothing is better than mercurial ointment in full strength, or, in regions where the skin is tender, in the strength of one-half, one-quarter, or less. Lesions of the palms and the soles, when persistent, require preliminary softening with warm water and green soap, the parts being well shampooed, especially at night, before the application of the salve, the latter being kept in contact with the skin during the hours of sleep. The ointment of the nitrate of mercury in the strength of 2 drachms to the ounce may often be added with good effect to the Wilkinson salve ${ }^{1}$ or to an unguent compounded by adding to an ounce of lard from $\frac{1}{2}$ to I drachm of one of the tars (oleum rusci, oleum cadini, oleum picis) and from I to 2 drachms of precipitated chalk, the whole being compounded secundum artem by boiling, sifting, and stirring. The articles found useful in non-syphilitic papular and scaling lesions (chrysarobin, salicylic acid, and ichthyol) may here also often be used with profit. The soothing salves (diachylon ointment, oleate of bismuth, and benzoinated zinc ointment freshly prepared)

${ }^{1} \mathrm{R}$. Sulphuris sublimat.,

Ol. rusci,

$\bar{a} \bar{a} \cdot \bar{s}^{\mathrm{ij}}$;

Sapon. virid,

Adipis,

Cretæ præparat.,

$\bar{a} \bar{a} . \bar{\jmath} \mathrm{ss}$;

gr. $\operatorname{lxxv}-\mathrm{M}$.

Sig. External use.

The best oleum rusci should be used in preparing this ointment; but if the best cannot be obtained, either the oleum picis or the oleum cadini may be substituted for it. If possible, use the oleum rusci having the peculiar odor of Russian leather. 
may also be used, adding, to the latter particularly, the mercurial specially indicated.

Condylomata, secreting papules, warts, vegetations, etc. about the ano-genital parts and over the axillæ, the groins, and elsewhere are readily relieved. They are all foul-smelling, and they require deodorizing solutions of chlorinated soda or of boric acid, after which they are to be dusted thoroughly with equal parts of calomel and starch or with boric acid. The apposed surfaces are to be separated by the interposition of antiseptic lint. For the dusting-powder named may be substituted europhen (which is usually very comforting in its relief of the odor and the pruritic condition), hydronaphthol, or iodol. Where the warty growths are exuberant they may be painted with solutions of the bichloride in flexile collodion, $1 / 2$ scruple to the ounce, or with a modification of the well-known wart-cure formula :

$$
\begin{array}{ll}
\text { R. Acid. salicylic., } & \exists \mathrm{j}-3 \mathrm{j} ; \\
\text { Extr. cannabis indic., } & \exists \mathrm{ss}-\mathrm{j} ; \\
\text { Collodion. flexil., } & \overline{\tilde{3} j} .-\mathrm{M} .
\end{array}
$$

Sig. To be painted over the part.

By thorough treatment with these and similar solutions it will very rarely be necessary to employ severer measures. The actual cautery, the curette, and the several caustics may, indeed, be employed, but in general this course indicates a lack of skill on the part of the physician in his employment of the simpler measures.

Pustular lesions, crusted or ulcerated, especially over the face, require careful attention, since the production of scars in this region may mark the patient for life. It is well to remember, in the management of all such emergencies, that they represent a mixed infection, and that 
the staphylococci are to be combated. All crusts should be removed by repeated hot borated washings, after which the surface, if thoroughly cleansed, may first be wiped with alcohol or touched with a solution of the following sort:

$\begin{array}{ll}\mathrm{R} . \text { Hydrarg. chlor. corros., } & \text { gr. } \mathrm{j}-\mathrm{ij} \text {; } \\ \text { Benzoin., tinct., } & \mathrm{f} \tilde{\mathrm{z}} \mathrm{j} . \mathrm{M} \text {. }\end{array}$

Sig. For external use only.

One of two courses may then be pursued: The skin-surface may be dusted with calomel, europhen, boric acid, or hydronaphthol, and over all there may be applied a delicate film of cotton fastened at the edges to the surface with a light layer of flexible collodion; or faceplaster may be superimposed over the cotton; or a film of collodion may be applied over the powder directly. An alternate course is to apply as a dressing one of the mercurialized pastes: a little practice will suggest from day to day, to both practitioner and patient, which paste best serves the special indications. From I to 20 grains of ammoniated mercury or calomel may be added to the Lassar paste (the formula for which has already been given), or I or 2 drachms of mercurial ointment, each in place of the salicylic acid usually ordered in the combination named.

When pustular or gummatous lesions of either the skin or the subcutaneous tissue break down to form a syphilitic ulcer, the treatment in each event is the same. All crusts are to be removed thoroughly-if need be, by soaking and washing in hot borated water as described above-all sloughs are to be plucked away or curetted, and the surface is to be made as clean as possible. The floor and the edges of the ulcer are then freely and 
deeply cauterized either with the nitrate of silver in stick or in solution or with fuming nitric acid. Delay should be made in the after-dressing until all oozing has ceased, after which one of the powders is to be applied, followed by an antiseptic compress and, when practicable, a bandage. The latter is chiefly valuable over the lower extremities, where support is as imperatively required for most syphilitic ulcers as for those of eczemato-varicose type. The flannel bandage cut on the bias answers well for patients unable to apply skilfully the common roller bandage over leg or arm; and for those able to afford the slightly greater expense the silk elastic stocking is both convenient and in a high degree useful in the way of support. In this event we are in the habit of ordering for use next the skin, by both sexes, a long white cotton stocking such as is usually worn by women, the elastic silk support being drawn over the stocking.

In all indolent ulcerations of the extremities support is readily effected, after dusting with an appropriate powder, by dressing with superimposed strips of rubber or of adhesive plaster encircling two-thirds of the circumference of the limb. Internally the treatment of these ulcers is best conducted by the "mixed" method, the iodide being usually demanded in the fullest doses, and the mercurial at the same time by inunction or by addition to the iodide by the mouth in one of the "mixed" formulæ.

In the early forms of syphilitic alopecia not obviously due to changes in the scalp, it is well to clip or cut the hair short and to shampoo the scalp three or four times in the week with the tincture of green soap flavored with lavender-water or cologne-water. After such shampooing, and also, at times, when none is employed, 
the following lotion may be well rubbed into the surface:

R. Cantharid., tinct., Hydrarg. chlor. corros., Capsici, tinct., Ol. sesami, Spts, vin. rectif., Aq. ros., $\mathrm{f} \tilde{\mathrm{s} j}$; gr. j-iij ; $\bar{a} \bar{a} . \mathrm{f} \tilde{\bar{s} s s} ;$ $\mathrm{f} \overline{\mathrm{sij}}$; ad f $\tilde{\tilde{s}}$ viij.-M.

Sig. External use over the scalp.

When crusts form upon the scalp, they should be removed by the shampoo; a salve may often afterward be employed, such as ammoniated mercury (or calomel), 5 grains to the ounce of vaseline, or precipitated sulphur I drachm and cinnabar I grain to the ounce of the same salve-basis.

In the management of syphilitic lesions of the mouth it has already been shown that prophylaxis is of prime necessity. The exclusion of tobacco in every form, the stopping of all carious teeth, and the removal of those requiring extraction should be secured at the outset. The chewing of sum, the constant holding in the mouth of a toothpick which is practically chewed, and even the sharp edge of a sound tooth against which the tongue plays, may each be responsible for a deeply extended ulcer. All mucous patches should be touched daily, or twice daily when practicable, with either a 5-, a IO-, or a 20-grain solution of the silver nitrate, or by the solid stick, which can be used freely in the hand of an expert. It is, however, quite unsafe, as a rule, to entrust silver solutions and caustic to patients themselves or to their friends, as the abuse or the misuse of this valuable agent follows in a great percentage of cases. 
For individual use the milder washes, lotions, and gargles may be ordered, such as-
R. Potass. chlorat.,
$3 \mathrm{j}$
Mel. despum.,
Myrrh., tinct.,
Aq. dest.,
$\bar{a} \bar{a} . \mathrm{f} \bar{s} s s ;$
ad f $\tilde{\jmath} \mathrm{vj} .-\mathrm{M}$.

Sig. To be used, diluted with water as required, in the throat.

A teaspoonful of this solution in a third of a tumblerful of pure water will usually be found grateful. For it may be substituted $\mathrm{I}$ drachm of the potassic chlorate in 6 ounces of peppermint-water, or a few drops of the following modification of Bellamy's iodized phenol:

R. Acid. carbolic.,

Iodin., tinct.,

Glycerin.,

Spts. vin. rect.,

Aq. dest.,
$3 \mathrm{j}$;

f3ss;

$\bar{a} \bar{a} . \mathrm{f} 3 \mathrm{ij}$;

ad $\mathrm{f} \ddot{\mathrm{z} j}$. - M.

Sig. Ten to fifteen drops in large dilution as a mouthwash and a gargle.

"Listerine," a proprietary preparation, is popular with many patients (it is chiefly a weak distillate of eucalyptus), diluted as required. Mercurial washes are also advised from I to 4 grains of the sublimate being added to the half pint of water, with tincture of myrrh and honey in the proportions already given. The objection to these washes is the possibility of the solution being accidentally swallowed, and for that reason other local remedies are to be preferred. The compressed tablets containing 5 grains each of the potassic chlorate may also be used, a half dozen or more, in case of need, being dissolved in the mouth and swallowed daily. In all in- 
dolent cases, especially where the drug is indicated internally, we are in the habit of administering quinine, a few grains each day being laid upon the tongue, the local bitter effect of the alkaloid speedily diffusing itself through the mouth. Gummatous lesions require the use of the stronger caustics and call for the iodide of potassium internally in ample doses. In extreme cases only should the galvano-cautery be used. When the soft or the hard palate is involved, the medication of the patient should be prompt, as remediless damage may be inflicted in a single day. The iodide of potassium may be pushed rapidly to the largest tolerated dose, a mercurial is usually employed by inunction, and caustics are used freely in order to stimulate the engorged tissue to a healthier activity. In these cases, as in those of the milder mucous and scaling patches of the mouth, all very hot, very cold, acetous, salted, and highly-seasoned articles of food and drink should sedulously be excluded from the mouth.

Nasal lesions of syphilis require the internal treatment indicated in each particular case, but the local management is of importance. The solutions of nitrate of silver and the application of the crayon itself are in the first rank of local importance, the strength of a solution and the severity of the application being determined by the physician on the basis of the grade of the lesions prescnt. Local fumigations with mercury, as already shown, are of great value in all cases of severity; and unguents of the yellow oxide or of ammoniated mercury, I grain to the ounce of cold-cream salve, may be applied after the use of a caustic solution. Douches are required in all cases of ozæna; they are to be prepared with chloride of sodium, I drachm to the pint of distilled 
water, one or more drops of Bellany's iodized phenol to a few ounces of the same, or a weak borated wash. The following vapor may advantagcously be snuffed into the nostrils in cases of ozæna:
R. Acid. carbolic.,
Iodin., tinct.,
$3 j$;
Aq. ammon.,
$\mathrm{f} 3 \mathrm{ss}$;
Aq. cologniens.,
$\mathrm{f} 3 \mathrm{ij}$;
ad $\mathrm{f} \overline{\mathrm{j} j}$.

Place in a two-ounce glass-stoppered vial, half filled with cotton, and designate: "Vapor for snuffing through the nostrils."

All sequestra of bone require removal by accepted surgical measures, care being taken lest forcible removal before the pieces of bone are entirely separated from their connections result in severe shock or, as has happened, in fatal hemorrhage. The snare and the electro-cauteric apparatus may be needed in special cases.

Pharyngeal lesions resulting in stenosis or in adhesions are to be treated like similar sequelæ in the larynx-with blunt or cutting dilators, the knife, or the galvanocautery. Excellent results may be obtained in all mild cases by albolene sprays medicated with menthol (I to 2 grains to the ounce), with carbolic acid, with pinus Canadensis, or with iodized phenol solution (20 drops to the ounce of albolene). Caustics (nitrate of silver, chromic acid) are required for touching papillomatous and other growths. In syphilis of the larynx and of the trachea much good often results from mercurial inunction of the overlying skin. In general, the iodide of potassium is indicated internally; in mild cases, however, or in those not threatening, the "mixed" treatment answers well. 
The nails, when involved in syphilitic changes, require special attention. Besides the constitutional treatment required in all cases, the digits should usually be protected from injurious contacts by cots. In gummatous lesions the iodide or the "mixed" treatment is indicated internally, and in non-ulcerative forms a weak mercurial salve may be applied. In ulcers of the soft parts about the nail caustic applications followed by dusting with europhen or calomel are needed. However great the apparent deformity, and however exquisitely painful the ulceration, after immersion in warm borated water followed by dusting-powders, and the gentle but firm compression of the parts with antiseptic cotton between bandage and ulcer, the dressing may be made both efficient and comfortable. In gouty and cachectic states special treatment is required to obviate these conditions.

Syphilis of the bones and the periosteum calls in general for treatment by iodide of potassium, to relieve the osteocopic pains and tumefaction, while mercury is needed to ensure against trouble in the future. In many of these cases the "mixed" treatment answers well. Externally, when the osseous tissue involved is within the reach of such treatment, mercury is of the highest value, unguents and oleates of mercury being chiefly employed. Mercurial plaster is often serviceable when cut to the proper shape and more or less continuously worn over any accessible nodes or tumors. It is rarely, if ever, necessary to incise local gummatous deposits; and any sequestra formed as a result of syphilitic caries or necrosis should be removed surgically only when completcly separated. The familiar surgical procedure of making long incisions the length of a bony "splint" along the tibia is, in syphilitic cases, for the most part 
wholly unnecessary and without value. Surgical interference is on rare occasions required when there is pressure upon a nerve-trunk by an osseous or osteo-periosteal tumor. All abscesses require proper surgical opening and drainage. Separation of an epiphysis demands correction of any resulting deformity, and immobilization of the limb by the aid of a splint.

Plastic operations are often of decided value in correcting the most hideous of the facial deformities produced by bone-syphilis; and in grave cases an artificial nose may be worn. Sinking of the nasal bridge is well corrected by platinum and other supports. In dactylitis syphilitica amputation should almost never be practised. Great relief can be obtained by medical treatment (iodide of potassium internally), by mercurial frictions where there is no degeneration of tissue, by incision of all suppurating tissue (in lines parallel with the long axis of the limb in the effort to avoid wounding tendons), and by strict observance of antiseptic precautions.

The viscera, when the seat of the lesions of syphilis, require, for the most part, internal treatment, which is to be pursued according to the indications in each case. It has already been noticed that the suggestion that syphilis is a possible cause of aneurysm was originally due to the recognized value of iodide of potassium in that affection. In syphilis of the heart, lungs, liver, kidney, and spleen the iodide of potassium, occasionally in combination with mercury, is indicated, and in many cases is of great service. In other cases, especially when degeneration of cells has been wrought, the treatment, while not always curative in its results, exhibits a marked power in arrest of the malady. In all cases of visceral syphilis, however, there is urgent need of systemic treat- 
ment beyond that which might strictly be called "antisyphilitic," patients being often in a cachectic or anæmic state. Special complications also arise (ascites, albuminuria, cough, hæmoptysis, jaundice, etc.), requiring the particular treatment recognized in general medicine as appropriate to each.

Syphilitic lesions of the rectum and the anus always demand special attention. By the aid of nitrate of silver in solution (from 5 to Io grains to the ounce) all fissures of the anal folds may be stimulated, and they may be dressed subsequently with iodol or europhen powders with superimposed lint. Before each stool, the rectum, as far as can readily be reached with the finger, is to be well smeared with the following:

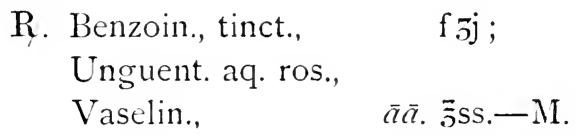

Sig. For external application with the finger as directed. Enemata of warm water must be ordered if the bowels are impacted.

All gummatous lesions call for iodide of potassium in the largest doses required to secure involution, mercury being at the same time carefully and judiciously employed. Strictures of the rectum are to be treated at first with dilatation by rubber bougies, care being taken not to rupture the gut and induce a peritonitis. When, as is too often the case, the result is a mere temporary benefit, the last resorts are posterior proctotomy, division of stricture by the galvano-cautery, inguinal colotomy, and complete excision of the neoplasm, as in case of carcinoma. In emergencies requiring surgical interference we have found inguinal 
colotomy most serviceable. In a few instances, after the irritation produced by the passage of feces over the rectum has been removed, the latter organ has under treatment returned to a condition permitting of closure of the artificial anus in the groin. As most of the subjects of this disorder are women, the pain attending subsequent menstruation often requires attention.

Syphilitic lesions of the epididymis and body of the testicle are usually amenable to treatment with iodide of potassium internally and mercury externally, the latter employed in the form of either the oleate or the ointment. Many of these cases call for prompt and energetic treatment to avert aspermatism, which too often ensues even if atrophy does not follow absorption of the gummatous mass. An accompanying hydrocele usually requires tapping. Even after implication of both testes it is often difficult to persuade patients of the need of absolute disuse of the sexual organs.

Lesions of the nervous system, whether of the brain, the meninges, or the cord, usually require internal treatment of similar character in each complication. It has been customary to employ in these cases the largest tolerated doses of iodide of sodium or iodide of potassium; and the result in favorable cases is as brilliant as it is satisfactory. One of these salts, or both in combination, may be administered in a saturated solution in drop doses, the vehicle being milk, alkaline water, or an essence of pepsin taken before or after meals, with another dose at bed-time. In all emergency cases it is well to disregard the hours of meals, and to administer the remedy every four hours during the wakeful periods of the day and night. By the addition of $\mathrm{I}$ or 2 drops of a saturated solution either to each or to each third or 
fourth dose, very large quantities of iodide of potassium have been taken with favorable results, an ounce and even two ounces and more having been thus ingested within twenty-four hours. For the caution requisite in the attainment of and persistence in these large doses the reader is referred to preceding pages devoted to the subject of the employment of the iodides.

Inunctions of mercury at the same time with the medication may generally be practised with the best results; while the "mixed" treatment is to be reserved for cases exhibiting no signals of danger and calling for no specially energetic management.

The belief, however, is now gaining ground that too much stress has in the past been laid upon the treatment of nervous syphilis with the iodine salts, and that the great value of mercury has needlessly been ignored in these grave complications of the disease. We are in the habit, in all severe cases, of carefully testing the condition of the patient by giving $\frac{1}{10}$ grain of calomel, or even more, every hour until a decided effect has been produced; and certainly the gravest types of pachymeningitis have thus been relieved. Tonics are demanded in all cases of nervous syphilis where the crisis of the disease has been combated successfully, and often before such an event. The diet should be in a high degree nutritious, the feet are to be kept warm, and tobacco and alcohol are rigidly to be excluded. For the majority of patients we are opposed to the employment of hot baths in nervous syphilis, preferring, in general, the daily sponging of the body-surface with a strong solution of salt and water followed by frictions with the flesh-brush. Fumigations also, valuable though they may be in other syphilitic states, are, equally with the hot bath, to be 
avoided, as in various degrees are apt to induce congestion of the nervous centres. In syphilitic patients convulsive seizures of a severe grade have been precipitated both in the fumigation chamber and in Turkish and Russian baths.

Syphilis of the eye and its appendages is to be treated internally on the principles already formulated. Mild astringent lotions locally answer well for most of the simpler inflammations of the canaliculi and the sac; in rare cases only is division for stricture or the introduction of the probang required. When the sclerotic is involved, instillations of atropia, combined, if there be pain, with cocaine, are useful. In iritis of all forms repeated applications of hot water slightly impregnated with boric acid are to be made over the closed lids, and solutions of atropia (from I to 4 grains to the ounce) instilled sufficiently often to ensure persistent dilatation of the pupil and to tear loose any adhesions between the iris and the capsular envelope of the lens. Persistence in these efforts is rewarded with success in cases which often look desperate, when blood is effused into the anterior chamber, and there are apparently unyielding attachments of the free border of the iris. Opium for relief of pain is rarely required in well-managed cases, and is contraindicated by the effect of the drug on the pupil; hence, if it be used in an emergency, the atropia must be employed in doses sufficient to counteract fully the pupillary action of the narcotic. Leeches may be ordered to the temple in plethoric subjects or in case of emergency. Mercury is, as a rule, best employed by inunction, and the iodide by the mouth.

Seclusion of the affected eye from the light must be secured, and must be continued for some time after relief 
is obtained, in order to avoid recurrence, which is not rare. Posterior synechiæ, as liable to result eventually in glaucoma, call for operative interference only when persistent. Iridectomy is a last resort in cases which by good management should have had a more favorable issue. In all affections of the choroid the ciliary muscle should be paralyzed with atropia, and when the optic nerve is involved strychnia is indicated. In all bony affections of the orbit the iodide is to be pushed to the fullest doses tolerable, as in gummatous lesions of the liver, brain, and testis.

Syphilitic lesions of the external ear demand semiliquid unguents containing mercury, carbolic acid, or boric acid, applied by means of a toothpick wrapped in cotton or on pledgets of lint. Weak mercuric oleate $(5$ per cent.) mixed with oil of benne may also be applied. In the interest of antisepis, the meatus should be cleansed daily with warm borated douches; regetations should be snipped away with fine scissors, and their bases cauterized with the nitrate of silver, care being taken to avoid the drum. Too violent treatment is likely to occlude the canal by a consequent swelling. When this swelling occurs, the walls are to be prevented from adhesion by the interposition of pledgets of lint. Warm sublimate lotions, I : I0,000, are also valuable when operative treatment is not demanded.

In syphilis of the tympanum the naso-pharynx always requires attention. Inflation of the Eustachian tube with iodinized vapor is in this region distinctly beneficial. The constitutional management is of importance, and especially the care of the feet, which should be kept dry and warm and be dusted nightly with either salicylic or boric acid. Suppuration of the middle ear is a grave 
complication which may terminate fatally; for details of its strictly antiseptic management the reader is referred to special text-books on aural disease. Syphilitic involvement of the labyrinth can be treated only by internal medication.

Hereditary Syphilis.-A woman known to be syphilitic and pregnant should have prompt and energetic antisyphilitic treatment, in the interest not only of herself but also of her unborn child. Genital lesions require frequent and careful applications with a view to asepsis. Warm borated lotions, or solutions of the permanganate of potassium, I grain to 2 ounces, should be used-with caution, however, when employed as vaginal lotions, since a stream of warm water directed against the cervix of a pregnant uterus has brought on labor.

The special treatment of the pregnant woman is by mercurial inunctions pushed within the limits of a decided effect upon the gums, and suspended for periods during which she is to be subjected to "mixed" treatment. The mercuric protoiodide, blue pill, and other pilular vehicles of the metal are less serviceable than the method named. In advanced syphilis the iodide in full doses is of unquestioned value and has saved the lives of many children.

In the management of the syphilitic infant at the breast the mercurial and other treatment of the mother is not to be neglected. Whether the very small amount of mercury detected in the milk is of value, or whether the improvement which has been noted on the part of the child is due to the enrichment of the quality of the milk of the mother whose health is benefited by the treatment, it is not necessary to determine. In administering iodide of potassium to a nursing mother it should 
be remembered that at times the remedy has a very decided influence in inducing suppression of the milkan accident of serious import when a syphilitic child is at the breast.

The direct treatment of the syphilitic infant is a matter of the greatest moment. Only upon very strong evidence should treatment of a syphilitic infant be begun before it has betrayed symptoms of inherited disease, since, even after the birth of "intensely" syphilitic fcetuses and a series of abortions, there are brought into the world children who never exhibit signs of the disease even when both parents have recently been infected. This note of warning should be heeded, as some physicians are ready to pronounce a sickly child syphilitic simply because they have knowledge of the venereal accidents of one or both parents occurring a brief time before pregnancy.

Seeing that a syphilitic infant does not infect the breast of its mother, the child should always, when practicable, be thus nourished, and should never be suffered to take the breast of a sound woman. If breast-milk cannot be had, goat's milk may be employed as a substitute, or sterilized cow's milk, or cream and warm water. A healthy wet-nurse should at all hazards be prevented by the physician from exposing herself to the dangers of infection. A syphilitic wet-nurse is unfit for service. Where the utmost care is requisite in the cleanliness of the mouth, nose, anus, vulva, umbilicus, etc., the woman who is herself suffering from the accidents of infection is liable to be a carrier, not of a new syphilis, but of the germs of a secondary infection from pyogenic cocci.

When exhibiting snuffles and the exanthemata of 
hereditary disease, the child may be given internally calomel rubbed up with sugar of milk-from $\frac{1}{20}$ to $\frac{1}{10}$ grain to the weak; to those who are stronger, from $\frac{1}{3}$ to $\frac{1}{2}$ grain three times in the day. The crushed tablettriturates of this salt administered in milk serve a useful purpose. An accompanying opiate to relieve diarrhœa, advised by some authors, is rarely needed if the dosage be adjusted accurately to the requirements of each case.

Tonics are as necessary for the infant as for the adult affected with syphilis. A few drops of a solution of citrate of iron and quinine, a drachm to the ounce, may be given in syrup; or Monti's formula:

R. Ferri lact,

Hydrarg. chlor. mit.,

Sacch. lactis, gr. v;

gr. iss ;

gr. $\times x \times .-M$.

Ft. chart. No. $\mathrm{x}$ :

Sig. One to be given after taking the breast.

The gray powder, once highly commended, is uncertain, in consequence of its liability to the production of the bichloride of mercury. It is given in doses of from $\frac{1}{10}$ to $\frac{1}{2}$ grain, according to the weight of the child. We believe these preparations to be preferable to the others named below, which should, on the whole, be reserved for cases where there is decided intractability under the dosage of the mild chloride or the gray powder. But the protoiodide is given in combination with lactate of iron in doses of from $\frac{1}{10}$ to $\frac{1}{4}$ grain rubbed up with the sugar of milk; black oxide of mercury, in doses of a similar size; and corrosive sublimate, in doses of from $\frac{1}{100}$ to $\frac{1}{50}$ grain.

Iodide of potassium in solution may be administered to young children in doses of from $\frac{1}{3}$ grain to 4 grains. 
This remedy, however, in infants and children is exceedingly liable to produce a severe grade of medicamentous dermatitis and the other accidents of iodism; furthermore, it is not so often as in adults productive of brilliant therapeutic effects. It is chiefly indicated when there are osseous lesions and those involving the brain, the viscera, the testes, the eye, and the ear. The mixed treatment advised for adults can often be used, however, with advantage in cases where no emergency exists, as, for example:

$$
\begin{array}{lc}
\text { R. Hydrarg. biniodid., } & \text { gr. j; } \\
\text { Potass. iodid., } & \bar{z} \text { iij ; } \\
\text { Syr. simpl., } & \\
\text { Aq. menth. piperit., } & \bar{a} \bar{a} \text {. f } \bar{z} \text { iss.-M. }
\end{array}
$$

Sig. From 3 to Io drops to be swallowed in large dilution, after taking food, three times a day.

Mercurial inunctions, advised by some authors only after the child has attained a certain age and degree of strength, we employ with great advantage as soon as indicated in the earliest periods of life. It is not the method, but the skill directing the method, that renders this procedure possible. In very young infants the skin is exquisitely sensitive and unable to endure mercurial frictions. In such cases the mercurial ointment is combined with I, 2, or more parts of pure white vaseline, and the swathing band is well anointed with the mixture, care being observed that the bandage be fastened so that it does not turn, and also that one part of it only be anointed. In this way the constant motions of the child produce a gentle inunction, which may be pushed to any desired cxtent by increasing the quantity of the mercurial in the unguent until a scruple or more is rubbed 
in daily. During all these applications the child should be watched carefully, and on the slightest evidence of debility, anæmia, or increased restlessness the remedy should be suspended. The same course should be pursued when it is observed that the skin over which the ointment has been applied is the seat of a mild dermatitis; in which event, if the general condition warrant, the inunctions may be practised over another region, as over the feet or the shoulders. In point of fact, a syphilitic infant furnishes a ready and constant indication of the value of the treatment instituted for its relief, inasmuch as its increase in weight, its improvement in color, and its capacity for eating and sleeping are promptly changed for the better or the worse according as such treatment is or is not rightly directed. In the event of irritation of the skin being produced in any region where a mercurial has been applied, the inunctions should at once be suspended and the integument of that part dusted with a soothing powder such as talc or starch, or, in case of need, anointed with freshly made benzoinated zinc ointment.

What good can be wrought by inunction is within the range of either mercurial ointment, pure or reduced, or the several mercuric oleates. The red precipitate in the strength of a I per cent. ointment, the white precipitate in the strength of I part to $\mathrm{IO}$ of lanolin or vaseline, and mercurial plaster for regions of limited area, have all been praised by authors, and may be regarded as of value when a change is thought desirable. Hypodermatic injections and fumigations have been employed in hereditary as in acquired syphilis, but no urgent reason for their use can be adduced. In the event of their selection, the dose should be reduced somewhat 
from that employed in adults, according to the age of the child. From $\frac{1}{10}$ to $\frac{1}{40}$ grain of the sublimate can be injected between the first and fourth years, the smaller doses only in the first twelvemonth of life. The sublimate baths recommended by Elsenberg contain about a grain of the metal, an equal quantity of the ammonium chloride being added, to the gallon of warm water. The local applications found useful in acquired syphilis may be employed when needed in the case of syphilitic infants. The addition of ammoniated mercury, calomel, or yellow oxide to the Lassar paste already described, in the strength of from 2 to 30 grains to the ounce, will be found available in many of the syphilodermata. Tumors and nodes should rarely be opened surgically, as they can commonly be made to disappear under an appropriate therapy. When the lids are affected, warm borated lotions, or those containing the bichloride, I part in I0,000, may be employed, followed by a weak salve containing a grain of the yellow oxide of mercury. Atropine should be instilled, as often as required, both in keratitis and in iritis, in the strength of from $\frac{1}{10}$ to $\frac{1}{4}$ grain to the ounce. Leeches may be required over the mastoid process to relieve the severe deafness of inherited disease, which, if not energetically treated, may result in deaf-mutism. No applications are better for the special rhinitis of hereditary syphilis than those containing nitrate of silver, from $\frac{1}{2}$ to $I$ grain to the ounce being injected or wiped over the surface, and followed by an albolene spray. In some cases this spray answers well, employed alone or after the addition to it of a few drops of carbolic acid and a single drop each of the tincture of iodine and glycerin. The mouth should be cleansed thoroughly and repeatedly with solutions of 
boric acid and honey, usually best applied by dipping in the solution a soft rag or a handkerchief which is wound about the finger of the nurse and then applied to every part of the child's mouth. The anus should be kept scrupulously clean, and should frequently be dusted with boric acid or boric acid and talc in equal parts; if condylomata form, these should be deodorized with liquor sodæ chlorinatæ, and after drying should be dusted with calomel and talc, I part of the former to 4 parts of the latter. Mercurial plasters are useful applications to tumors and swellings over bone, digit, joint, or muscle, and can also be wrapped neatly about an involved testis.

\section{Acquired Infantrle Syphilis.}

The acquired syphilis of infants differs from the inherited form chiefly in the important particulars that its evolution is on the lines observed by the acquired disease, and that the patient does not start life with lesions of the viscera, of the bones, or of other important organs. As a rule, under proper care the issue in these cases is fairly favorable. The acquired syphilis of infancy is chiefly remarkable for its display of moist and secreting lesions and for its failure to relapse in cycles as does acquired disease of adults. The first-named feature is due to the soft character of the infant's tissues; the lastnamed, to the constant control to which the child is subjected when the disease is duly recognized and properly cared for. But in unrecognized or neglected acquired syphilis of infancy the results may be as mutilating and as disfiguring as in the worst phases of acquired disease of later years. 


\section{SYPHILIS IN RELATION WITH THE FAMILY AND SOCIETY.}

It is obvious that if every infected individual were restrained from communicating syphilis to another, the extension of the disease would speedily be checked. Unfortunately, the barriers to such an advance in the improvement of the public health seem at present to be insuperable. The duty of the physician, however, is none the less clear and urgent. The victim of the disease should be impressed with the fact that he is a possible source of danger for the uninfected, and should be shown the methods by which he is to protect those with whom he must necessarily come in contact. To the father of a family and to the unmarried of both sexes it is, in general, proper to state the nature of the disease recognized, if this be unknown before the date of consultation, and also to point out the danger of transmission and the methods by which such accident may be prevented. In the presence of a syphilitic wife ignorant of her condition the physician is placed in a position of peculiar delicacy. As a rule, these innocent victims of the disease have been infected by a guilty husband. The physician then discharges his task if he insists upon a personal interview with the master of the household, declaring the facts to the latter and insisting upon the need of informing his partner at once of the nature of the disorder, that she may take such measures as will ensure her safety. In such cases it is plainly the office of the husband rather than of the physician to perform this disagreeable duty. An infected wife separated from her husband by death, divorce, or mutual 
arrangement should, as a rule, learn the truth directly from her physician. It is her right to understand the nature of her disorder, that in any emergency she may have a clue to the treatment which may then be demanded urgently. Women have actually lost their lives in consequence of ignorance on this point. Before, however, any communications of this sort are made by the physician to the patient, the former should be absolutely certain of the correctness of the diagnosis. As the peace of a family is often at stake, an error here is unpardonable. In any case of doubt further advice should be sought.

If the husband or the wife be infected from an extramarital source, it is the obvious duty of the diseased to inform the sound partner of the fact, that he or she may take precautions sufficient to ensure safety. Here, too, it is plainly the duty of the guilty to inform the innocent, and the physician has a right to insist upon the performance of this duty, to save not merely the uninfected, but also his own personal reputation. Otherwise, when the facts are eventually discovered (and they usually are) he may be held to have been a party to a plot to conceal the truth to the damage of the infected. If there be a positive refusal of the patient to discharge this duty, there are two ways in which the physician may proceed: One is that suggested by Fournier: The physician may send a written letter to the guilty party, insisting upon the need of telling the truth, and retain a copy of this letter for later justification. The other way is for the physician to decline further connection with the case.

As a matter of fact, in the great majority of all cases the infected consort cohabiting with the non-infected 
person who is ignorant of the facts sooner or later trans. mits the disease, notwithstanding all protestations and precautions. As a matter of fact also, the "confessed" cases are those where transmission almost never occurs. There is sufficient popular dread of the disease to ensure the forewarned against the incurrence of risk. Exceptions may possibly be made in the instance of long separation of husband and wife, or of long-continued illness of either, rendering the performance of the sexual act impossible or remotely improbable; and also in cases where each of the married couple habitually occupies a separate chamber and bed. In some of these cases the temptation to indulge in the sexual act, from a sudden and scarcely resisted impulse, at a time when objective symptoms of the malady seem to be for the moment removed, is to a large extent set aside.

It is a remarkable evidence of the tenacity with which the marriage tie unites even those who have disregarded its sacredness, that but a small proportion of the men who confess to their wives their fall and their infection by that fact alone break up their families. It is an offence against a woman, usually unpardoned, if her husband, after violating his marriage vow, afterward inflicts upon her a venereal disorder through a cowardly dread of confessing the truth. The courts fully recognize this, and give her, when she asks it, speedy and just redress. In daily practice, however, a man who, unfaithful to his wife, has been, as a consequence, infected, and who confesses to her his story rather than contaminate her in his embrace, usually wins her sympathy and often retains her love. She respects his courage, and if, as often proves to be the case, the husband has committed his offence when under the influence 
of alcoholic stimulants, she often forgives. The conscientious physician cannot be too strongly urged to conserve the health and the peace of families threatened by the advent of an infectious disease by exerting all his influence in the direction of securing a confession to the wife by the husband, who in the great majority of all cases is the one at fault.

It need not here be set down that the unmarried infected with syphilis should not indulge in the sexual act. When under an engagement to marry at the date of infection, both parties to the contract should earnestly be advised to cancel the engagement. In the intimacy between two such persons made possible in most classes of society, a kiss upon the lips has often served to transmit the disease, and to convert an affectionate regard into a feeling of detestation and horror. For most of these people a period of three years at the least is likely to elapse before the physician can consent to a union, and it is unjust to expect a young woman to bind herself for that period to any man capable of acquiring syphilis by the usual methods of its transmission. The course which will in the end save the most mental and physical misery for both persons, and which will in the future furnish the least anxiety to the physician, is disruption of the bond.

With respect to the marriage of the veteran of syphilis the decision may be different. There is no time in the life of the infected when, for any reasons known to science, it may positively be affirmed, without possibility of disproof, that he or she can become the progenitor of healthy children and not infect a partner in marriage. This is, in effect, a proposition that the infected should never marry; and, as thousands of men 
annually do marry and have sound children and never transmit syphilis to their wives, it follows that there must be some rule which, if not absolutely safe, will furnish in its application a maximum of practical and satisfactory results.

It is wholly unfair, when considering the question of marriage from the point of view of medical science, to set the patient who has made a satisfactory progress toward the termination of syphilis in a category apart from the tuberculous, from those having a record of recurring insanity in their family histories, and from those affected with infirmities tolerably certain to terminate life within a brief period of time. All these classes annually marry and intermarry, with disastrous results to themselves and to society. The reterans of syphilis make a far better statistical showing.

It is impossible to lay down rules for all cases, but the following limits are fairly well established in practice: A previously healthy young man or woman, skilfully treated for between three and four years after infection, and free for the last year from any but the most insignificant symptoms, will in the large majority of cases fail to infect a married partner or transmit syphilis by inheritance.

No man should marry, whatever time may have elapsed after infection, who has not had a long interval -at the very least six months-of absolute freedom from symptoms; and the reverse is true, that no man should marry, however remote the date of his infection, who bears upon his person active symptoms of his disease. There are subjects of syphilis who should never marry, though these are few. In them the disease has induced a cachexia permitting an evolution of the malady to the 
point where the systemic infection is too profound and too persistent to permit a return to a normal standard of health.

When syphilis has actually been transmitted from husband to wife, or the reverse, and the two, after a reasonable abstinence, again cohabit, a problem of some gravity is presented to the physician. As a rule, sexual indulgence between such consorts should be postponed to the utmost limit, seeing that in case of offspring the chances of inheritance of the parental disease are doubled by reason of the infection of both father and mother. Even here, so provident is nature for its wellbeing, the child may completely escape; but the peril is very great. In this case husband and wife should be conjured to take every precaution against the occurrence of pregnancy; and the only safe and justifiable precaution is total abstinence from sexual indulgence. Nor is this conscientious denial of the bodily appetites the utopian dream of a social reform for the future. Every physician of experience has had knowledge of husbands and wives who, impelled by a high sense of duty to themselves, to their families, and to the world, have lived for years in asexual companionship, waiting for the time when their physical union would not be shadowed by the possibility of bringing a reproach upon themselves and a curse upon their offspring.

With respect to the question, frequently raised, as to the insurability of the infected in life-assurance socictics, the companies who accept risks in the United States are not as yet agreed in their practice. The physician, however, who examines the applicant can, when the exact facts of the syphilitic history are obtained, make a reasonable forecast of the longevity prospects. With a 
history of mild syphilis, and one terminating without appreciable results six months or a year before the date of the examination, the forecast is decidedly good. There is not here a question as to the danger of transmission of the disease, but solely one of longerity. The longevity prospects of the average of the infected are better than the companies themselves probably believe. The number of the infected subsequently dying of tuberculosis or of carcinoma is exceedingly small; and this immunity, as the later acquisitions of science suggest, is related to the inevitable war waged between pathogenic micro-organisms. With evidences of a recent or grave syphilis the examiner may well be cautious; but even here there is little prospect that life will be shortened save by the occurrence of some of the nervous complications of the disease.

The regulation of pubiic prostitution by law with a view to the extermination of syphilis has long been practised in France, Belgium, and other countries, either generally or with defined limitations. This regulation has for the most part included surveillance and periodical examination of the persons of public women, with segregation of all the infected by the aid of enforced hospitalism. The results have been, from a scientific point of view, in a high degree unsatisfactory. It is a significant fact that the country that has longest regulated prostitution by law has also furnished the most voluminous literature, and until a recent period the most authoritative writers, on the subject of syphilis. The scheme of sanctioning prostitution in any way has always been repugnant to the commonwealths inheriting the traditions of the Anglo-Saxon race, and, now that such sanction is recognized as practically valueless, it is 
in the highest degree improbable that the United States will ever, in the effort to solve this problem, imitate the practice of the Old World.

The proper view of this question, as of most of the questions connected with the sexual relation, must surely include both men and women. The law which demands a periodical examination of the female should also require a periodical examination of the male prostitute. Every expert to-day recognizes the fact that the syphilitic male is as liable to disseminate his disease as his companion of the other sex. If one must exhibit a certificate of health before sexual congress is permitted, so should the other. If one, in order to escape the penalties of the law, is to secure an official license, so should the other. In these closing years of the nineteenth century, when women of the highest character and intelligence are interesting themselves in this subject, no sensible person can doubt that if any regulation whatever be ordered, it will, assuredly in America, bear equally upon both sexes.

But, all said and done, the representatives of advance in social science should clearly recognize the fact that syphilis is not, as has been claimed by a class of hysterical writers in many lands, a scourge threatening, above all other maladies, the devastation of the human family. Tuberculosis annually destroys many more victims. It would not be unjust to demand that the State shall ensure the fullest security to life for the residents of large cities, in the way of provision for pure water, milk, ice, food, and freedom from accidents, before it attempts to police the houses that are visited only by those leading immoral lives. The proportion of syphilitic to other diseases in no part of the world exceeds a variation of between 2 and 5 per cent. when both sexes are estimated 
in the statistical returns. Most of the published tables, unfortunately, include figures obtained from army and navy hospitals, where men only are sheltered.

The great safeguard against syphilis is sexual morality, without which no safeguards are worthy of the name. It is held by writers that for young men this is too lofty an ideal ; but such objectors have no practical knowledge of the moral standard upheld by many of the wisest thinkers and realized by thousands of self-denying youths in every community. The physician who does not exert his influence in the interest of this standard, by which men and women alike not merely protect themselves from these maladies, but ensure also the safety of the community in which they live, has yet to learn the alphabet of sound health. 


\section{CHA NCROID.}

Synonyms.-Soft chancre; Simple chancre; Noninfecting chancre; Fr. Chancre mou; Chancrelle (Diday); Ger. Einfacher Schanker; Weicher Schanker.

Chancroid is a contagious venereal disease characterized by the occurrence, chiefly in the genital region, of one or more, often several, suppurating and ulcerative lesions, due to the presence of micro-organisms, and not ultimately productive of specific constitutional symptoms. The secretions of a chancroid lesion, when unmingled with those of syphilis, are never succeeded by the symptoms of the last-named disease. It is, however, to be noted that both the virus of syphilis and that of chancroid may be implanted at one moment upon the same susceptible point, and from such a point the phenomena of the two diseases may afterward be evolved.

The establishment of an absolute distinction between chancroid and syphilis has been reserved for the latter half of the present century. For a long time after the distinctive differences between the two affections were recognized and classified, the scientific world discussed with energy the questions respecting "the unicity or duality of the chancrous virus." No one, however, at present holds that there is a duality of the syphilitic virus or of chancre. The unicity of each, to employ an outworn phrase, is unquestioned. But it is certain that 
there is a contagious venereal disease, local in its effects, communicable at the same time with syphilis, the features of which may be confused with those of the initial sclerosis of that disease.

To demonstrate without possibility of error that an individual may be the subject of even a grave ulcerative lesion which is never followed by syphilis, incurred in sexual exposure of the genital region, may be named as one of the achievements of modern science; but a grievous price has been paid for this knowledge in the errors which have resulted on the part of both physician and patient. Thousands of initial scleroses of syphilis are annually mistaken for chancroids; and even the onset of unmistakable signs of systemic syphilis, after such blunders have been conmitted, has been for a time ignored or misconstrued. The false security engendered by over-confidence, ignorance, and folly furnishes the background for a historical warning which no man can afford to ignore. It is well, at the very outset of a study of soft, non-infecting chancres, or chancroids, to realize the great danger of confusing them with the initial scleroses of a disease whose impress may last for a half-century, and whose symptoms may actually be intermingled with the most classically developed of chancroid ulcers.

Etiology.-There is little doubt in the mind of any modern observer as to the existence of a specific microorganism which is the effective agent in the production of the chancroidal ulcer. At the present writing the identification and the recognition of the etiological value of such a micro-organism are not established. The proof of existence of such a germ rests practically upon the same basis as that generally assumed for the agent effect- 
tive in the production of syphilis. That the discovery of the one will throw a flood of light upon the etiological importance of the other cannot be doubted.

At the present time, however, there are not wanting those who assert that the effective micro-organisms of chancroid are simply the staphylococci and the streptococci which are concerned in the production of pus in general. In support of this view it is claimed that the peculiarities of the chancroid are due chiefly to the anatomico-physiological characters of the soil in which it chiefly thrives-namely, that of the ano-genital region of the cachectic and the filthy; that the recognized pusorganisms are found in all cases of chancroid; that, in spite of exhaustive bacteriological research, no other organisms have yet been demonstrated as capable of producing the disease; that the results of inoculation of the skin of the ano-genital region with simple pus are not distinguishable from chancroid; and that the secretion of such artificial lesions is capable, like that from the chancroid, of repeated auto-inoculation.

But, per contra, it is to be noted that chancroids at times occur in those who are neither filthy nor cachectic, and that the worst results may be exhibited in individuals of a healthy class; further, that while inoculations of simple pus (for example, that from an acne pustule) have produced lesions scarcely distinguishable from those of chancroid, yet that pus even from these sources is by no means always of the "simple" character claimed, since tubercle bacilli and other micro-organisms little suspected as present have been distinguished in pus taken from supposedly innocuous sources.

The clinical argument against the position described above is very strong. A periurethral phlegmon may 
burst through the integument of the penis; an abscess of the vulvo-vaginal gland complicating a gonorrhœa in women may open through the vaginal wall near the vulvar orifice; a large pustule of the skin of the penis may be produced by the presence of the acarus scabiei; a suppurating balanitis in phimosis complicated by a tight stenosis of the preputial orifice may result in the practical imprisonment of an exceedingly foul purulent product; and in uncomplicated cases, neither in any of these nor in similar accidents of the same region that might be cited will there be the slightest approach to the formation of a chancroid. Such an occurrence, if well authenticated, would at once revolutionize all the accepted doctrines in this field; and, to push the possibilities no further, were simple pus sufficient to induce a chancroid in a filthy and cachectic subject, the surgeons of ships' crews, at a distance both in time and space from port, their sick afflicted with scurvy and attacked with vermin, might expect an outbreak of lesions which, as a matter of fact, are never seen except when sailors have been recently in contact with public women in some haven of entry.

The number of observers claiming to have identified the micro-organism of soft chancre is large. At the beginning of the list are the names of Salisbury and Didier, and these names are followed by those of Luca, 1)ucrey, Welander, Krefting, Strauss, Jullien, and Unna. Most of the later authors have busied themselves with the micro-organism of Ducrey, a short and thick bacillus with rounded extremities, occurring in groups and chains between and in the bodics of the cells themselves. It is readily stained with alcoholic solutions of fuchsine, methyl-violet, and gentian-violet. Krefting used as a 


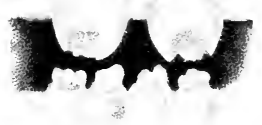

1

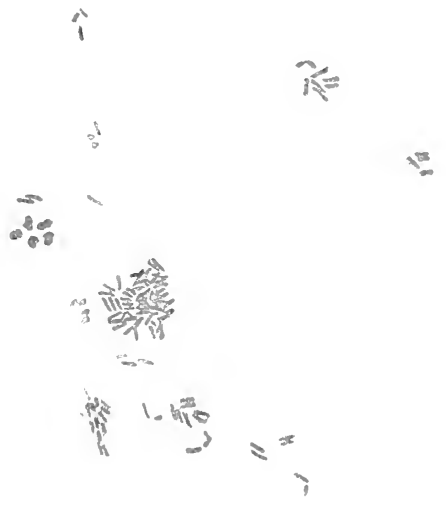



staining solution 16 grams of a 5 per cent. borax solution, 20 grams of a saturated aqueous solution of methylviolet, and 24 grams of distilled water. Streptococci and staphylococci were found in the first generation only of cultures, rarely in the fifth or the sixth. In all cases the streptobacillus of Ducrey was recognized by these observers, with the exception of Jullien and Strauss, who were unable to discover it in their examinations. The determination of the problem is for the time being relegated to further investigation.

The Lesions of Chancroid.-The clinical symptoms of chancroid depend largely upon accidental circumstances, the important factors being, first, contact with neighboring parts (friction, maceration, etc.), and second, the site of infection. The typical chancroid develops where the site is such that the lesion can progress symmetrically and at the same time be uninjured by traumatism. With these conditions fulfilled (as after intentional inoculation) the earliest symptom is the production of-

The Pustular Lesion.--This lesion develops at the site of infection, first as a minute hyperæmic macule which is evolved in twenty-four hours after inoculation, a pinpoint-sized vesico-pustule appearing within forty-eight hours after and being surrounded by a reddish halo. Day after day, progressively, this lesion, when protected, enlarges and changes to a pustule of the type once described as "ecthymatous," attaining the size of a small coin.

When the roof-wall of this pustule is broken and its purulent contents are carefully removed by wiping, the floor of the original chamber may be recognized as an ulcer, corresponding in circular outline and dimensions 
with the original pustule. The floor of this ulcer is covered at first with a pultaceous and sloughy deposit; later, as repair ensues, it assumes at first a violaceous and velvety aspect, and still later presents the features of a healthy granulating surface. The circular walls are steep and abrupt, as if produced by a sharp punch. The base in uncomplicated cases is invariably soft and supple, never in the least suggesting the stony hardness of a typical sclerosis of syphilis. There is usually a more or less angry-looking areola spreading to a variable distance away from the centre. The suppuration, at first abundant, becomes decidedly more scanty as the stage of repair approaches. In general, the condition is one of inflammation accompanied by more or less soreness and pain of the part. In this respect also the lesion differs from the commonly painless induration of certain scleroses of syphilis.

The Erosive Lesion. - Here the modification results from the early removal of the roof-wall of the pustule by an accident (softening by maceration with mucus, friction of contiguous parts, etc.), or from infection of a site where, for any reason, the pus produced does not become chambered. At such points there is infection of an open surface, such as the mouth of a follicle, the site of a ruptured herpetic vesicle, or the seat of a slight trauma (about the verge of the over-stretched anus, over a torn frænum, etc.). In these cases the lesion is ab initio a suppurating ulcer. Its contour is rounded, oval, or conforms to the accidents of site to be named later. The pus is thick, creamy-yellow in hue, and when removed discloses an empurpled floor or one covered with the peculiar wash-leather-like slough, resembling nothing so nearly as the floor of a typical 
gumma after bursting. Occasionally these sores, after exhibition of "open" symptoms, cover themselves with an adherent crust which increases in size as the ulcer spreads beneath, so that lesions as large as a silver dollar and even larger may thus be formed. The character of some of these developing and crusted chancroids may be misapprehended by both patient and inexpert physician, who may be applying unguents, powders, or other dressings to the outer surface of a large crust of this character. The removal, however, of such a crust may disclose an abscess as large as the section of a hen's egg, with characteristic chancroidal ulcer for the floor.

Variations of chancroid are from each of the types described above. The shape, for example, may be altered greatly by the infection of a wound or of a point situated between two abruptly elevated mucous sufaces. In the former event the lesion may be linear (chancroid of the anus or frænum) or dumb-bell-shaped (as when the sore begins in the sulcus back of the corona glandis of the penis and spreads in a double circle over the prepuce and glans).

Number.-The chancroid may be single; but it is usually multiple, and this multiplication may be enormous. Usually no more than from four to six lesions are seen at one time upon a single individual; but in exceptional cases hundreds may be counted, as when, in women, the secretion from a few lesions on the upper portion of the vulva flows over not only the lower portion but the entire perineum and anus.

Multiplicity in the number of chancroids depends chiefly upon auto-inoculability of the secretion. The secretion of the initial sclerosis of syphilis is non-auto- 
inoculable save in those cases where there is mixed infection (with chancroid), or irritation of the lesion by accidental agencies, causing suppuration. The abundant pus of the chancroid, however, furnishes the amplest material for ensuring multiplicity of lesions, not merely (as constantly happens) at the moment of infection, but also after infection, to the point of production of two or more chancroids which proceed promptly to multiply when contact with adjacent parts is not prevented.

Size.-The majority of chancroids do not exceed in size the section of a large bean; but great variation exists between the extremes of the minute, pin-pointsized lesions, scarcely attaining average dimensions, and the largest ulcers, which may considerably exceed in size a platter, covering, for example, a broad area of the skin of the belly and spreading downward over the inner face of the thigh.

Duration.-The persistency with which a chancroid, even after extensive and thorough cauterization, unfailingly pursues its career of evolution and involution is one of its distinguishing features. It outlives, as a rule, all the tissue-destruction produced by an ordinary abscess of the region where it occurs, and in one form or another it commonly consumes a definite time before its last traces are removed. From three to six weeks may be said to be the average duration of a simple and wholly uncomplicated case. All the complications of the disease, however, may prolong its term.

The chronic ulcers occurring chiefly about the genital region of the lower class of prostitutes, but seen also in men, persisting for many months and even for years, belong to a special category which will be described later. Here the unusual cluration of the disease is due, 
not to any inherent tendency of the affection to prolong itself indefinitely, but to accidents of the process.

Incubation.-Properly speaking, there is no period of incubation for the chancroid. As a rule, by exceedingly careful observation with a lens the infectious process is made evident within a few hours, twenty-four at most, after the introduction of the virus. In the average of loosely observed clinical cases patients declare that their infection became evident a few days after exposure. It is rare that chancroids appear later than the tenth day after infection.

Cases, however, are not wanting where the first symptoms of the disease become apparent two or three weeks after contact. In this event it is generally believed that the virus was simply deposited on the surface, not encountering a follicle whereby access was obtained to the deeper tissues, and that later by its presence the virulent secretion excited an irritation which eventually opened up a portal to the lymphatic system. The ease, however, with which chancroids reproduce themselves after mere contact should throw discredit upon such an hypothesis.

By far the most acceptable explanation of apparently long "incubative" periods is the ignorance of the patient, for these periods of time, of the existence of the disorder. Incredible though it may appear, there are few experts who, after recognizing threatening inguinal adenopathy, are not guided by this condition to exploration of the genital region, with the result of discovering and first pointing out to the patient a previously unsuspected chancroid. Often a minute, pin-point-sized lesion is thus found lurking in one of the pockets by the side of the frænum, or hidden immediately behind it, or in 
another unobtrusive region. It should be remembered in this connection that many, but by no means all, of the patients displaying these symptoms are of the filthy class, with associates of similar social grade. In men of this type it is not rare to discover a remarkable toleration of the uneasiness produced by a long-continued accumulation of the smegma præputii, and the sensations produced by chancroids are mistaken by such patients for the pruritic symptoms induced by the mild balanitis which retention of the smegma often excites.

Subjective Sensations.-From what precedes it will be seen that the chancroid, as distinguished from an infecting sclerosis, may be the source of subjective symptoms. These symptoms may be merely pruritic or may be of the grade of severe pain. Exceptions in this particular are noteworthy. At times the infecting chancre of syphilis is painful and the chancroid is unproductive of sensations of a morbid character; but for the majority of cases the chancroid is distinguished by its inflammatory character and by the tenderness and pain associated with it. These symptoms are more pronounced when the lesion is rapidly progressing as an ulcer, or when-a rare accident in modern practicethere are complicating accidents of the order of gangrene or phagedena.

Absence of Specific Induration.-The base of the typical chancroid, however large-sized and deeply ulcerated, is invariably pliable, softish, and non-indurated. It never exhibits, save in mixed forms, the characteristic ivory-like hardness and density of an equally typical initial sore of syphilis. There may be inflammatory engorgement, and, after extensive cauterization, a marked 
thickening of the tissues on which the ulcer rests, but a truly characteristic hardness is never produced by these means.

While this is true, the fact remains that only a skilled touch, and even that in doubtful cases only after repeated examination, can decide accurately upon the nature of the disease. It is not a wholly safe procedure to base a decision as to the character of a venereal sore upon the test of its induration at a given moment under the finger and thumb of an expert. There is a decided difference between a voluminous mass of infiltration at the base of a simple chancre which has been inflamed by any of the accidents to which it has been exposed, and the exceedingly delicate, parchment-like induration of the syphilitic chancre in some of its least pretentious types. In brief, upon the presence or absence at any one moment of induration, or what seems to be induration, an exact diagnosis cannot invariably be based.

Auto-inoculability of the Secretion.-The purulent secretion furnished by a typical chancroid is indefinitely auto-inoculable on the person of the affected individual -a fact repeatedly demonstrated by the occurrence clinically of lesions in regions in close proximity to chancroids. Thus, a sore situated on one labium is tolerably sure to infect a corresponding point on the other side; a chancroid upon the outer face of the scrotum, the portion of the thigh naturally in close contact. In this way it happens that in cases scores and even hundreds of chancroids are found in filthy and neglected subjects where the indefinite auto-inoculability of the sores has been in no way inhibited. That the pus-corpuscles are chiefly responsible for this virulence would be suspected on a priori grounds, even had it not 
been demonstrated that the secretion, when deprived of its pus-cells by filtration, is either non-inoculable or produces, when any results at all are obtained, an atypical lesion.

In this connection it is needless to do more than set down the fact that in the early part of the last half of the present century the practice of so-called "syphilization," enthusiastically lauded in Sweden, was based on an erroneous interpretation of the auto-inoculability of the chancroid. By practically exhausting the power of the skin to react against a great number of artificial inoculations with chancroidal pus it was thought that syphilis was eradicated. The doctrines then held have long since been abandoned, and the practice has properly been relegated to a place among the curiosities of medicine.

Location.-Chancroids are said to occur, like the initial scleroses of syphilis, upon any portion of the integument and the adjacent mucous surfaces; but such statements cannot be accepted without reserve. Certainly there is no proportion whatever between the frequency of extra-genital infecting scleroses and chancroids, the former being in large centres of population scarcely a curiosity, and the latter being one of the rarest of all experiences. The most frequent site of chancroids is, with overwhelming preponderance, the genital region; and the aphorism still holds, that chancroid is the most truly venereal of all the diseases classed under that title.

In men the most frequent sites of chancroids are the frænum, the prepuce, the glans, the sheath of the penis, and the tip of the urethra. In the last-named region, however, infecting chancres are much more common. In 
women the sites of common occurrence of chancroids are the labia majora and minora, the vestibule, and the mucous membrane of the vagina near the ostium. Anal and perianal chancroids are far more common in women than in men, by reason of the readiness with which the auto-inoculable secretion flows over the perineum to the sensitive and readily eroded mucous orifice of the anus.

Extra-genital chancroids are chiefly found upon the mouth, the eyelids, the lips, and other parts of the face. They are among the rarest of all venereal lesions.

Complications.- Hixed Chancre.-The subject of chancroid may exhibit, in the course of a few days after exposure, several typical lesions the result of simultaneous infection or consecutive auto-inoculation. All these lesions, in the course of a fortnight, may be progressing toward complete involution, when one of them (rarely more) may begin to assume the characteristics of an initial sclerosis of syphilis, general symptoms of the disease following in due course. These cases are illustrations of coincident infection with the virus of the soft chancre and of syphilis, the resulting sore being of the type commonly termed " mixed." Here two diseases coexist, precisely as when patients of the lower class present themselves at the public charities, suffering at the same moment from local evidences of syphilis, blennorrhagia, and infection of the skin or of the mucous membranes with pyogenic cocci. For details of the mixed chancre the reader is referred to the pages of this work devoted to the subject of syphilis.

Vegetations and other Lesions of the Skin and the Mucous Membranes.-Venereal warts, herpetic vesicles or patches of membrane affected with balanitis and pos- 
thitis may coexist with chancroids, and at times disguise their features to a marked extent.

Phimosis and Paraphimosis.-These affections are frequent complications of chancroid, and when of severe grade often produce excessive pain and distress; but the results are, however threatening, commonly not serious. In some cases one or several chancroids are imprisoned beneath an irreducible, enormously swollen, purplish-hued prepuce, its orifice discharging a foul and purulent fluid which may by auto-inoculation serve to identify the character of the imprisoned lesions, inaccessible save after operative interference. There are often one or two small, tell-tale chancroids on the verge of the preputial orifice, indicating the character of the lesions within the pouch. At times the distress is so great that the glands in the vicinity enlarge by sympathy. The "mixed" chancre in this situation speedily betrays itself by a sclerosis which may often be detected with the thumb and finger through the tissue of the odematous and empurpled prepuce. Often, too, in public practice these conditions are complicated with gonorrhœea, the pus of which escapes, with that from the sores, through the stenosed preputial orifice. Sloughing may ensue in unrelieved cases, but it is an exceedingly rare result, and need never be feared in any properly treated cleanly patient. In a severe type of complicated disease the glans penis pushes its way through the sloughing upper limb of the foreskin, whose tumid and dependent lower limb presents the odd appearance of a second glans at the extremity of the penile organ.

In paraphimosis the result is different, though the tumefaction may be fully as great, and the destructive action in grave cases may be as formidable. In severe 
phimosis the line of ulceration, forming in an effort to relieve the tension, spreads at right angles to the shaft of the penis, in the sulcus behind the proximal roll of œdematous tissue. When chancroids are present, it is rarely the case that this line of ulceration, intended to afford relief, does not suffer infection. In serious states the ulceration spreads upward over the integument of the organ, fusing several of the chancroids present into a single gigantic ulcer. The "subpreputial frill" of writers is the lower limb of an œdematous prepuce, in these cases often retracted and beset with chancroidal ulcers.

Phagedena.-This complication may coincide with or be succeeded by sloughing and gangrene-accidents exceedingly rare in the evolution of the initial scleroses of syphilis, and, as a matter of fact, in the practice of modern medicine rare even in chancroids. When phagedena occurs, however, it is a serious disorder and is usually difficult to manage.

In these cases the result may be due to improper local or general treatment (violent cauterization; subjection of the patient to the action of mercury, under the impression that the disease is syphilitic in nature, etc.), filth, cachexia, struma, and the other causes of local or general deterioration of vigor. There may be extension of the ulcer in one or in all directions by a serpiginous process wherein the disease spreads by virtue of its auto-inoculable virus, and at the same time destroys the tissue in its path. This may be a superficial or deeply spreading process; it may be relatively rapid or, what is more common, exceeding slow and painful, scarcely giving rise to much distress. As the disease spreads in one direction it may heal in another; or it may result 
in the production of a large area of ulceration, with uneven and irregular floor covered by a sloughy, pultaceous, and adherent mass; softish base; scanty or semipurulent secretion; abruptly steep, occasionally undermined, edges; always without sclerotic induration of the base, and, however long its duration, never followed by the signs of systemic syphilis.

One well-marked and fortunately rare type of serpiginous or chronic chancroid has been found so rebellious to treatment and so persistent in its course that it has been regarded by some writers as a modification of true chancroid in the direction of lupus. Some of the forms of so-called esthiomene unquestionably belong to this category. In obstinate cases the chancroid persists for a year or more, very slowly spreading over the abdominal surface upward, or downward over the inner or outer face of the thigh. The ulceration may spread superficially or deeply, and in the latter case may even be subcutaneous, burrowing immediately beneath the skin or the fascia, undermining the tissues in areas of the size of a large platter, with fistuious tracts uniting its lines of subcutaneous excavation, the latter here and there communicating with the surface by irregularly set ulcerating openings, suggesting the "man-holes" of a system of sewerage. Here an empurpled integument covers the ramifications of the burrows, ridges, and open ulcers, a thin, virulent secretion destroying slowly what it touches. These features together furnish a characteristic picture. This severe complication of chancroid occurs chicfly in women, particularly among filthy prostitutes, but it is also seen in men and among those debilitated by alcoholism, venery, poverty, hospitalism, and cachexia. 
Gangrene, when it complicates chancroid, is usually so promptly destructive of all parts affected that it often serves at once to end the specifically morbid process. Here the accident may be rapid or slow of occurrence, and the gangrene may be superficial, removing merely the sore itself and the tissue on which it rests; or the process may be deep, the slough embracing the glans or the entire body of the male organ, even the testicles being laid bare in the scrotum. Here a blackish or greenish-black slough is seen, involving the whole or a large part of the sore and the tissue upon which it rests. This complication of chancroid is distinguished from phagedena in that it more often attacks filthy men than women. At the outset there is usually a coincident inflammation of the surrounding parts, which often assume an erysipelatous appearance. These severe accidents of chancroid are very much rarer in modern practice than in the days preceding the modern methods of asepsis.

\section{Lymphangitis and Lymphadenitis (Bubo; Chan-} croidal bubo; Chancroid adenopathy; vernacular, "Blue-ball;" Gor. Virulenter Bubo; Fr. Boubon).-Inflammation of the lymphatic vessels and the perivascular tissue is a complication of chancroid, as also of the other venereal diseases. It is rather rare as compared with other accidents of the disease. When the inflammation is well marked the lymphatic trunks may be recognized as tender, indurated, and painful cords, of the thickness of a wheat-stalk or of the little finger, stretching away from the site of the lesion toward the inguinal, pubic, or crural regions. At times the overlying integument is unchanged in color, at others it is of an erythematous hue, and in extreme cases it may even threaten 
to burst, as in the case of the glandular disease accompanying the same process.

The lymphadenitis, or bubo, of chancroid differs from that of syphilis chiefly in its inflammatory character and its marked tendency to the production of an abscess terminating by bursting. Primary syphilitic adenopathy, it will be remembered, is characterized by the involvement of several glands, occasionally of but one, in anatomical connection with the region of infection. When the chancre has a genital site, a chain (" pleiad") of densely indurated glands, each of nearly the size and firmness of a marble, neither painful nor tender, can be recognized in one or both groins. Save in the case of " mixed" infection none of these glands exhibits a tendency to inflammatory softening or degeneration, as in the case of the lymphadenitis of chancroid.

When the bubo accompanying chancroid threatens to burst, one or more tender and painful points can be discovered, usually in the inguinal region, representing the sites of as many glands. With greater or lesser rapidity, often in the course of but a few days, all these points, but more often one predominantly, enlarge until the glandular character of the tumor becomes evident, with aggravation of the pain and tenderness, both with and without motion of the muscles in the vicinity. In typical cases the gland, at first merely voluminous and movable, becomes fixed, and the overlying skin is involved in the periglandular inflammation, being then dusky, empurpled, and gradually thinned, precisely as in the case of the integument covering a syphilitic gumma about to burst. Then follow, in course, fluctuation, softening, rupture of the capsule of the gland, which becomes converted into an abscess, and escape of the contents either 
into the neighboring subcutaneous tissue or externally through a rent in the skin. When several glands coincidently suppurate a single enormous abscess may result from their fusion. The pus evacuated spontaneously or by surgical procedures is foul, thick, hemorrhagic, and at times auto-inoculable, as in the case of the pus furnished by the original chancroid. The abscess-cavity, when examined after spontaneous rupture, exhibits undermining pockets, grayish, pus-soaked walls, detritus of tissue, and encroachment on the cavity by other glands in the vicinity, either threatening suppuration or only incidentally and less seriously involved.

In some cases of spontaneous rupture of the abscess the lips of the rent speedily become inoculated, and the resulting sore exhibits all the evidences of an enormous inguinal chancroid, its long axis lying irregularly parallel with the line of Poupart's ligament, its edges steep or undermined, its floor pus-and slough-covered, its secretion foul, its ragged lips teased with every motion of the thigh. Many of the enormous chronic chancroids already described as supposed varieties of lupus originate in gigantic ulcerations of this character. Under favorable circumstances, however, with patients of a sound constitution and properly treated, the phases of repair ensue even after exhibition of serious symptoms, and the result is eventual cicatrization with the production of an indelible scar, whether surgical interference be or be not employed.

It is probable, though not wholly demonstrable, that in some cases threatening buboes accompanying chancroids undergo a species of abortion by resorption. Certain it is that the glands in these instances may enlarge and become both painful and tender, with the 
result of an eventual resolution short of pursuing the career just sketched. Whether in all these cases the buboes were of the type commonly denominated "virulent," or were merely inflammatory and sympathetic phenomena accompanying the original venereal lesion, cannot be determined.

The symptoms of bubo and of lymphangitis are as distinctly marked in women as in men, but the rarity of these complications among women is remarked by all observers.

In men buboes occur in from 10 to 30 per cent. of cases of chancroid, the figures changing according to the class from which the author collects his statistics. Hospital patients are much less liable to exhibit these complications than the filthy class of dispensary outpatients in large cities. In private practice typical bubo in the more cleanly classes is decidedly rare, and may occur soon after the first appearance of the chancroid, or may succeed complete cicatrization of the inguinal sore. Occasionally the bubo develops with typical features when there has been no suspicion of chancroid, this lesion being discovered later lying behind or near the franum. At times the bubo, in consequence of decussation of the lymphatics, forms on the side opposite that on which the sore originally appeared.

The etiology of bubo is in part obscure. Exciting causes are, assuredly, weakness of the constitution, filth, over-exertion, improper treatment of the original sore, neglect of the implicated region aside from lack of cleanliness, and enormous multiplicity of lesions. "Virulent" bubo may, however, occur when none of these supposed causes has been in operation, though, fortunately, this event is rare. 
With respect to the presence of micro-organisms of an etiological significance in the pus of a chancroid bubo, and the possibility of reproduction by auto-inoculation. opinions differ. By some writers it is held that typical chancroid of the groin caused by the bursting of a bubo in that region results from inoculation of the lips of the wound, not with the pus originally contained in the abscess-cavity, but with that furnished by the yet unhealed chancroid. The results of experimentation are not conclusive. At times the bubo, however, as already seen, develops only after the sore has healed; in such cases, of course, the possibility of infection of the bubo from the chancroid is set aside. In these cases, as also in those where total excision of the chancroid has been practised before inoculation-experiments, and where the pus employed in such experiments has been withdrawn by aspiration from an unopened inguinal abscess, the results are not satisfactory. It has already been shown that the micro-organism of chancroid has not yet been so definitely demonstrated that its presence or absence can be trusted in the determination of the character of any lesion. Of the micro-organisms of Ducrey and Unna, which are unquestionably identical, it may be said that they are most often not demonstrable in the pus furnished by a chancroidal bubo. It has hence been inferred that bubo was caused by some ptomaine resulting from the invasion of a strepto-bacillus, but all this as yet lacks proof. Whether, then, the micro-organisms themselves or their toxines are conveyed from the site of infection to the gland or glands which suffer as a consequence, it is merely certain that the germs and their products are originally related to the infective process, and that in no other disease do inflammation and sup- 
puration of the lymphatic glands present precisely the same picture as in the bubo accompanying chancroid.

Diagnosis. - The indications of importance in the diagnosis of chancroid are, first, when practicable, to exclude positively the presence of syphilis, either in initial sclerosis or in later manifestations of systemic disease; second, to remember that the possibility of mixed chancre clouds every case until the longest period of incubation of syphilitic chancre has elapsed without symptoms of the disease; third, to recall the most significant characteristics of the chancroid, which are its occurrence without an incubative interval, its lack of induration, its continuously purulent character, its multiplicity, its autoinoculability, its inflammatory symptoms, and its bubo. In all doubtful cases the decision should be reserved until a definite period has elapsed. Periurethral phlegmon is distinguishable by its defined outline and inflammatory character, its frequent complication of a previously existing urethritis, and its situation, which is usually near the distal extremity of the male organ and in the body of the corpus spongiosum.

In distinguishing between venereal lesions (including chancroid) and non-venereal disorders of the genital region, the age, occupation, character, habits, and antecedents of the patient should be considered. An epithelioma of the penis or of the clitoris is rare in youth, while a majority of all the affections acquired in the sexual act originate in the second, third, and fourth decades. Patients in middle life with no venereal antecedents are in a different catcgory from those who have suffered from repeated attacks of urethritis or " chancre." Commercial travellers, women having public occupations, and residents of large cities are more exposed to the 
accidents of genital infection than are those who live in the country and those who are surrounded by the safeguards of a home.

The most striking differences between chancroid, syphilitic chancre, herpes progenitalis, and a few of the more common affections of the muco-cutaneous surfaces of the genital region are exhibited in the appended table. 


\section{CHANCROID.}

History

Etiology

Incubative stage

Lesion :

site

character

number

color

contour

Occurs in subjects of syphilis and others; prior sexual exposure.

Infection, accidental or intentional, by medium of pus from chancroid or chancroidal bubo; usually in or near the ano-genital region.

None after actual access of virus to lymphatic channels. Lesion rarely later than one week after infection.

Nost commonly genital; rarely extra-genital. Pustulo-ulcerative lesion throughout; few exceptions.

Multiple, as a rule, both at the outset and by subsequent auto-infection. Rarely, though at times, unique; occasionally very numerous.

Pustule yellowish; ulcer, when wiped clean, florid; crusts greenish and blackish.

Round, oval, and, when fused, circular.

Subjective sen- Pain, tenderness, soreness, occasionally great pain. sations

If ulcerating, base Engorged, soft, supple when not cauterized, rarely indurated.

floor Pus-soaked, slough-covered; showing ragged tags.

elges Abrupt, steep, punched-out: at times undermined.

secretion Foul, purulent, hemorrhagic, often offensive in odor.

crust Bulky, blackish-greenish; often concavo-convex, forming roof of a pus-chamber.

Inoculability and Auto-inoculable indefinitely; with difficulty transmitted auto-inocula- to animals. Infection of genital region commonest. bility

Induration

Base, as a rule, non-indurated; supple; if inflamed, boggy, indeterminate in outline, non-elastic, attached to adjacent tissue; if deeply cauterized or irritated, at times indurated, sinulating sclerosis of syphilis.

Career

Usually in uncomplicated cases a cycle, from initial pustule to cicatrization, of from six to eight weeks. Resulting indelible scar. 


\section{ChaNCRE.}

History Follows infection at any point of the body, usually in non-syphilitic subjects.

Etiology Infection with syphilitic virus (sexually or by accidental or intentional inoculation, as in tattooing, vaccination, nursing upon the nipple, etc.).

Incubative stage Usually between ten and thirty days; average, twenty. one days.

Lesion :

site

Any infected region of the body.

character Minute plane lesion with erosive surface, dry or moist papule, or large tubercle.

number Usually single, rarely multiple; if the latter, multiple at the outset, and not by later auto-inoculation.

color Raw-ham; dull-reddish; scales at times changing the hue.

contour Highly irregular, observing chiefly the peculiarities of anatomical site; when on a free plane surface, usually rounded or oval.

Subjective sen- Often entirely wanting; at times somewhat painful. sations

If ulcerating, base Thin, circumscribed, or enormous and well-defined.

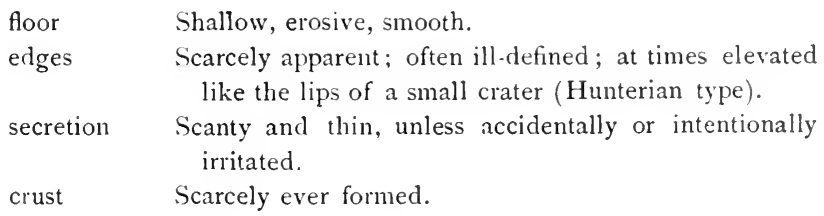

Inoculability and Non-auto-inoculable save in " mixed" infection. auto - inoculability Induration

Characteristic; marked; thin and papery; or dense, ivory-like, non-adherent, movable, insensitive to pressure, defined.

Career

May persist for months after general symptoms appear, or may practically disappear within six weeks; usually, in uncomplicated cases, no scar resulting. 


\section{Accidents :}

\section{Chanckoli).}

lymphangitis May occur, but rare.

bubo

In one-tenth to one-third of male cases.

phagedena

In neglected and ill-treated cases not rare.

gangrene

Systemic results

Influence of

treatment In exceptional cases severe and even grave.

In protracted cases deterioration of general health.

Systemic treatment worthless; local treatment of highest value.

\section{$B u b u$.}

Date of appear- At any time, even soon after healing of lesion. ance

Symmetry

Usually monolateral, with involvement of several glands, one predominantly; occasionally bilateral.

Frequency

One-tenth to one-third of all cases in men.

Number of involved glands

Size

Often one gland only, rarely more than two, typically involved.

From large nut to goose's egg; at times as large as small cocoanut.

Inflammation,

Classically developed, with involvement of overlying glandular and periglandular

Induration integument, heat, pain, redness, and swelling.

\section{Non-indurated.}

Career

Resolution; or, mole commonly, suppuration, with indelible scar resulting.

Infectiveness of In cases auto-infection from pus; in others non-autopus

Diagnostic value of treatment

Lymphangitis

Situation inoculability.

General treatment unavailing; local treatment imperative.

Rare, but of occurrence.

Usually in males, along dorsum and toward root of penis.

Color of overly- Inflammatory hue. ing integument

Pain and tender- Often well marked. ness

Career

Proceeds to resolution, more rarely to suppuration. 


\section{Accidents :}

\section{CHANCRE.}

lymphangitis Occasionally noticed; then painful.

bubo Characteristic and constant.

phagedena Almost never occurs.

gangrene Very rare; occurs only in cachectic patients.

Systemic results Occur in various grades of severity in all cases.

Influence of Effective at an early stage.

treatment

\section{Bubo.}

Date of appear- Within a fortnight after development of initial sclerosis. ance

Symmetry

Bilateral as a rule; at times symmetrical.

\section{Frequency}

Constant. At least one gland is affected in every case.

Number of in- Usually several glands, one or both sides of the body. volved glands

Size

Uniformly moderate, cherry- to large-marble-sized.

Inflammation, None in uncomplicated cases. glandular and periglandular

Induration

Career

Firmly and densely indurated.

Termination by resolution; scars rarely result.

Infectiveness of No pus, save in mixed cases. pus

Diagnostic value General treatment effective. of treatment

Lymphangitis

Situation

Rare.

Usually in lines proceeding from site of sclerosis.

Color of overly- Rarely, though occasionally, congested. ing integument

Pain and tender- May be absent or as severe as in chancroid. ness

Career

Rarely suppuration; usually resolution. 
Herpes Progenitalis.

II istory

I'revious recurrence at irregular intervals after digestive disturbances, venery, uncleanliness, and other sources of general or local irritation.

Etiology

Incubation

Lesion :

character Vesicles and sequels of vesicles.

number

color

contour

subjective sensations

If ulcerating

base

floor

edges

secretion

crust

Inoculability and auto-inoculability

Induration oses.

None.

Separate lesions rounded.

Tingling, pricking, itching. superficial erosions occur.

Imperceptible.

smooth, at times florid.

Scarcely appreciable.

A thin, colorless serum.
All local irritations, chemical, mechanical, and physiological, and the general factors producing the neur-

Multiple, as a rule; rarely very numerous, often grouped, occasionally confluent.

Floors of broken lesions slightly florid.

No true ulcer forms. When ulceration occurs, there is invariably mixed infection; at times exceedingly

Very superficial and thin, like a delicate scale.

Only in cases of mixed infection.

Absolutely none; pseudo-induration produced by caus. tics, etc. injudiciously or improperly applied. 


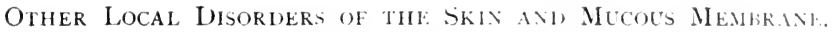

History

Etiology

lncubation

Lesion :

charactet

number

color

contour

subjective sensation:

If ulcerating

$\begin{array}{ll}\text { base } & \text { Insignificant. } \\ \text { floor } & \text { Insignificant. } \\ \text { edges } & \text { Insignificant. } \\ \text { secretion } & \text { Insignificant. } \\ \text { crust } & \text { Insignificant. }\end{array}$

Inoculability and auto-inoculability Induration verruca.

\section{None.}

In lalanitis, the same local irritations as in herpes; in verruca, unually precedent gonorrhcea; in psoriasis and eczema, lesions of other regions; in epithelioma, persistence for long period before examination.

Local irritations in eczena and balanitis; infection in scabies; irritating secretions (gonorrhceal, etc.) in

Superficial multiple and confluent excoriations in balanitis; pustules in eczema and scabies; scales in eczensa and psoriasis; warty papules or plaques in epithelioma; warty growths in verruca.

Usually multiple in all; occasionally but one pustule in scabies, and a single verruca; often patches in eczema and psoriasis.

Whitish in psoriasis; yellowish in scabies, with blackish cuniculus; reddish, crimson, and purplish in balanitis; florid or smeared with whitish mucus in verruca; dull-reddish in epithelioma.

Irregularly rounded excoriations in balanitis; defined patches in psoriasis and some of the eczemas; usually pedunculated warts; poor definition in epithelioma.

Itching in scabies and eczema; occasionally burning in epithelioma.

Ulcer only in late stages of epithelioma and in mixed infection of balanitis.

Only in cases of mixed infection. Scabies transmitted by acari; a few of the eczema forms are infective from the presence of trichophyton, mucors, etc.

None save in well-developed epitheliomatous wart. 


\section{Herpes Progenitalis.}

Accidents :

lymphangitis None save in mixed cases.

bubo

None save in mixed cases.

phagedena None save in mixed cases.

gangrene None.

systemic result, None.

Influence of Local treatment effective in two or three days; general treatment treatment required only for neurotic and gouty states in recurrent cases.

Treatment.-The management of the general condition of the subject of chancroid is usually simple. The recumbent position is required in all severe or threatening cases, especially when complications exist. In the simpler cases no internal remedies are indicated; in others a tonic regimen is urgently required, including a generous diet and the use of the ferruginous tonics, among which, in this connection, the potassio-tartrate of iron has long been especially esteemed. Mercury and the compounds of iodine are in unmixed cases actually harmful, and should not be employed.

The local treatment of chancroid is usually pursued on one of two lines, the one occasionally supplementing the other: The first is by antiseptic dressings; the second aims at obliteration of the lesion by surgical or chemical measures.

Antiseptic treatment is always indicated, and in the end is probably the most satisfactory for the majority of all cases. By this method the sore, as soon as its character is fully determined, is washed frequently with lotions containing either boric acid, carbolic acid, or corrosive sublimate-the first in saturated solution, the second in the strength of I part to 50 , the third in the 
Other Local Disoriders of the Skin and Mucous Membrane. Accidents :

lymphangitis None save in epithelioma of advanced grade.

bubo Occurs in severe scabies and eczema (adenopathy of sympathy).

phagedena (only in grave epithelioma.

gangrene ()nly in complicated and grave epithelioma.

Systemic results None save cachexia in grave epithelioma.

Influence of

treatment

I.ocal treatment usually effective promptly in balanitis, verruca, some of the eczemas, and some of the epitheliomata; psoriasis often obstinate.

strength of I part to 1000 or 2000 . The first named is preferred, and, when it is possible to immerse the entire organ, should be employed as a continuous local hot bath of the temperature most grateful to the patient. When employed intermittently, the immersions or washings should be made as often and for a space of time as long as practicable. Mercurial and carbolized fluids are better employed as lotions.

At the outset all crusts should be removed and the pus washed away in warm water with the aid of soap, after which, so far as possible, the pultaceous floor of the ulcer should be cleansed. The surface may then be sprayed with peroxide of hydrogen, or with water to which has been added, in the cup of the atomizer, from IO to I 5 drops of iodized phenol:

$$
\begin{array}{lc}
\text { R. Acid. carbolic., } & \overline{3 \mathrm{j} ;} \\
\text { Iodin. tinct., } & \mathrm{f} . \overline{\mathrm{ss}} ; \\
\text { Glycerin., } & \\
\text { Spts. vin. rect., } & \bar{a} \bar{a} . \mathrm{f} z \mathrm{ij} ; \\
\text { Aq. dest., } & \mathrm{ad} \overline{\tilde{j} \mathrm{j}}-\mathrm{M} .
\end{array}
$$

Sig. For external use only, diluted.

After the washing either a dry or a wet dressing may 
be employed. The former answers well for most cases, the ulcer, when dried, being well dusted with either europhen, iodol, aristol, boric or salicylic acid, or hydronaphthol. The three first usually answer well; the fourth and fifth named are sometimes productive of pain when applied over a very sensitive sore, and hence should often be reduced with starch, talc, or bismuth. Hydronaphthol is usually mixed with fuller's earth I part of the former to 50 or 100 parts of the latter. Iodoform is chiefly valuable as a local narcotic, but it is highly objectionable on account of its odor. It may be ordered for patients confined to the room for a brief time, when it is not necessary to conceal the character of the disorder from others visiting the apartment. The deodorized preparations of iodoform are not preferable to the other powders named above, which have no specially disagreeable odor. Calomel, pure or mixed with equal parts of the subnitrate of bismuth, is useful as a resort where other preparations do not answer well. In fact, many patients exhibit an idiosyncrasy with respect to these local applications. After the antiseptic treatment of the sore wet dressings are employed by laying a pledget of lint moistened with antiseptic astringent, sedative, or even stimulating solutions. To the class of preparations first named belong those employed in the lotions already described; to the second class belong solutions of sulphate of copper and sulphate of zinc, IO to 20 per cent. strength; to the third class, solutions of cocaine, morphia, and lead (often added to ingredients suggested for other lotions), as well as the black and yellow washes; in the class last named are included alcoholic lotions, embracing the aromatic wine, popular with the French. 
The destruction of the chancroid is wrought by the aid of the actual cautery (Pacquelin knife, galvanocauterization apparatus, hot iron), and by chemical agents, including nitric and pure carbolic acid, zinc chloride, caustic potash, cupric sulphate, and the nitrate of silver. The last is, however, ineffective for complete destructive action, and is chiefly useful as a stimulating application to sluggish iesions, for which purpose it is admirably adapted. Gaylord recently advised a 40 per cent. formaline solution. All destruction of chancroids by these methods should be accomplished with strict antiseptic precautions.

The operative procedures by the instruments of the surgeon are curetting the sore itself and the neighboring tissue, and excision of the part, with attempts at securing union-such immediate union as is possible after the surgical excision of simple lesions of moderate size. Both methods require the strictest observance of antiseptic precautions, and both, in the best of hands, have been followed by infection of the resulting wound, as also by the development of syphilis in cases where the diagnosis was not well established.

The objections to the destruction of chancroids by each and all of these severe measures are not to be lightly set aside. They may briefly be summarized as follows: (I) These destructive procedures obscure and aggravate the existing local disorder. It is in many cases difficult, if not impossible, at the date when destruction is practised, to make certain that the case is not one of mixed infection; and, without question, initial scleroses thus treated are apt to exhibit excessive induration at the base of the sore. No practitioner can be assured that a chancroid will not be complicated with syphilis 
until about one month has elapsed after the first appearance of the lesion; hence all destructive procedures undertaken during the first month of the existence of a chancroid may be disastrous to an on-coming syphilis. (2) Many of these operations, even when performed with the utmost care and repeated, utterly fail of accomplishing the end in view. The sore, instead of becoming converted into a simple ulcer, the desired issue in all attempts of this class, becomes an ugly and intractable lesion, persisting unaccountably, often without exhibition of classical symptoms of chancroid, the despair of the inexperienced and the horror of the patient after the suffering undergone in the heroic and ineffectual treatment to which he has been subjected.

On a careful survey of the field, it seems probable that destructive treatment of chancroids will before long follow in the wake of the now practically abandoned attempts to annihilate the syphilitic chancre.

Continuous Immorsion.-There is no treatment of the threatening or actually destructive chancroid comparable in value with the local or general continuous water-bath. Its value depends upon the fact that the pathogenic microbes of the disease lose their vitality at a high temperature which is tolerable by the body. The patient, when the part upon which the sore is seated can be immersed, spends the greater part of the wakeful hours of the day with his ulcer wholly submerged in water as hot as can be tolerated; at times boric acid may with advantage be added to the bath. In other cases it is much easier to employ the sitz-bath, in which the patient partially reclines with the entire ano-genital region immersed, the water of all such baths being maintained at a high temperature by the aid of supplies 
from a source of heat. The patient leaves the bath only for the purpose of evacuating the bladder and bowel. In all grave cases both the night and the day are spent in the water, the patient being, of course, under the constant supervision of a trained nurse. The most formidable of the phagedenic, sloughing, and gangrenous lesions with destruction of tissue are thus readily and even brilliantly converted into healthy ulcers which speedily assume the phases of repair, if effective constitutional treatment of the patient, with proper food and tonics, be at the same time secured.

All ointments are contraindicated in chancroids in a toxic condition, seeing that the germ of the disease finds a suitable culture-field in these greasy applications even when they are medicated. There are but a few indications for their employment; one is when the lint overlying the dry or wet dressing adheres so firmly to the part, in consequence of the discharges which leak through, that when the dressing is removed there is bleeding from the edge or floor of the ulcer. Another exception relates to chancroids of the urethra: in these cases the lint may be spread with carbolated vaseline-not in contact with the sore itself, but merely to facilitate removal of the dressings.

Treatment of Complications.-Urethral chancroids may generally be exposed by the aid of an ear-speculum or a pair of urethral forceps, after which the treatment of the sores may be practised as in the case of those existing in other regions. Pledgets of lint smeared with petroleum jelly and medicated with one of the powders already named may be inserted in the urethra. Urinating with the penis immersed in hot water is of great service in relieving the pain of micturition, and aids in 
securing repair of the ulcer. The black and yellow washes, pure or dilute, may subsequently be applied.

Chancroids complicatid aith phimosis, the sore being so imprisoned that it cannot be exposed within the sac of the prepuce, are usually the source of alarm to the patient and anxiety to the physician, but the real danger in any case is much less than is generally believed. With the aid of careful syringing of the sac through the preputial orifice, sufficient cleanliness of the surface of the sore may usually be secured to ensure repair for most cases, especially as lotions may be employed about the integument of the tumid and often reddened and empurpled prepuce, serving to still further reduce the inflammatory symptoms. In other cases, however, the chancroid becomes threatening, and exposes the patient to the danger of a slough forming, after the fall of which the glans escapes through a species of buttonhole through the swollen and distorted prepuce-a rare accident. In these and other severe cases resort must be had to operative procedures. A serious objection, however, is the danger of auto-infection of the wound inflicted by the surgeon. As a rule, therefore, it is wise to reserve operative interference for cases of emergency:

Circumcision of the prepuce, or incision either over the dorsum or on one side or another of the prepuce (as advocated by Taylor), may be practised in these emergency cases; but the surgeon will always do well to remember that in the best of hands and with every precaution infection in these cases has occurred repeatedly.

The operative treatment of the bubo of chancroid is sradually receding into the class of reservations advocated in the management of the sore itself. Early surgical treatment of these complications, once indiscrimi- 
nately advocated for all cases, has at last given place to a more judicial waiting for the evolution of the malady to the point where intervention is inevitable.

The abortive treatment of the bubo includes rest in the recumbent position (which is perhaps the best of all measures having this end in view); hot fomentations with boric acid; cathartics and a restricted diet; the local application of leeches; and applications with a view to a resolvent effect, such as the tincture of iodine, mercuriai ointment ( I part to Io of lanolin), belladonna ointment, and salves containing the salts of iodine, as, for example, the compound iodine ointment. Pressure by a spica bandage and by the application of bags filled with hot shot is also of value. The common treatment by painting with tincture of iodine is by most experts practically abandoned as useless. The internal remedies employed, such as sulphide of calcium and mercury, are of little, if any, value.

Injection of chancroidal buboes has been practised with hot solutions of boric acid, bichloride and benzoate of mercury, and carbolic acid. Dangerous results have followed some of these injections, and those containing the mercuric benzoate have in cases been found ineffective.

The operative treatment of bubo is by free incision, all antiseptic precautions being strictly observed, with excision of all glands wholly or partially implicated in the morbid process, subsequent curetting of the surface, and careful washing with hot borated solutions. The subsequent dressings are with iodoform gauze. These operations, when carefully practised, are followed by exceedingly satisfactory results, the bubo being speedily converted into a healthy ulcer. 
As the resulting scar is, however, both deep and indelible, and ever afterward points unmistakably to the nature of the original disorder, efforts are constantly being made to rob these procedures of some of their surgical severity. With special care many surgeons in private practice now succeed in penetrating the abscesscavity of the gland with a fine bistoury or a large aspirator needle. The evacuation of the contents by squeezing is followed by injection of a hot borated solution or, as White suggests, of iodoform ointment. Aspiration of the abscess with subsequent injection of hot borated water often suffices, without the production of a serious scar. 


\section{DISORDERS NOT INVARIABLY VENEREAL.}

\section{BALANITIS AND BALANO-POSTHITIS.}

BALANitis is an inflammation of the mucous membrane covering the glans penis. It is usually accompanied by more or less inflammation of the prepuce (posthitis).

Etiology.-Balanitis frequently complicates gonorrhoea and chancre, but it may occur independently of these affections, and may be non-venereal in origin. It is caused by mechanical or chemical irritation of the mucous membrane, and it most frequently results from retention beneath a long prepuce of gonorrhœal or other pus or of irritating vaginal or other secretions. Neglect to cleanse the parts, permitting the normal secretions to decompose and become irritating, may be a sufficient cause.

Symptoms.-In the beginning of the inflammation the surface of the glans is slightly reddened and is covered with a thin, creamy layer of mucus and pus. The redness rapidly becomes more intense, the discharge thicker and more profuse. As a result of maceration the epithelium is destroyed in patches, leaving irregularly outlined excoriations; these excoriations are usualiy superficial, but they may become quite deep and simulate the early stage of chancroid. The inner surface of the prepuce usually participates in the process, thus 
producing a balano-posthitis. The entire body of the prepuce may be inflamed, with slight or extensive œedema and tumefaction. Inflammatory phimosis-or, more rarely, paraphimosis-may result. The inguinal glands may become somewhat enlarged and tender, but they rarely suppurate. The subjective sensations are usually those of slight itching and pricking, most marked in the sulcus back of the corona; but in severe cases the glans becomes very sensitive, so that walking and other movements of the body are painful unless the penis be carefully supported and protected. Scalding on urination is usual, especially if phimosis be present.

With a long, tight prepuce balanitis may become chronic; the surface is then red and velvety, showing granular or even warty elevations.

Diagnosis.-If the prepuce can be retracted, the diagnosis can usually be made without difficulty. The excoriations of herpes are preceded by distinct vesicles, and other portions of the glans are not inflamed. When balanitis follows herpes, the history of the disease furnishes the only means of determining its origin. Syphilitic chancre and chancroid are too distinct in their characteristics to be confounded unless they are complicated with balanitis. Careful examination will detect the induration of an initial sclerosis, even in the rare diffuse forms. The ulcers of chancroid are much deeper than the excoriations of balanitis, and the pus is autoinoculable. In severe cases of balanitis it is not wise to exclude the possibility of an underlying chancre or chancroid until a few day's treatment has reduced the redness, swelling, and infiltration of the parts. In gonorrhcea, when the prepuce is long, and especially if the preputial orifice be filled with cotton to catch the dis- 
charge, the pus works backward and covers the glans, producing an appearance that may be mistaken for balanitis. Cleansing and inspection of the parts will readily reveal the source of the discharge.

When balanitis is complicated by phimosis, an accurate diagnosis of the underlying conditions is more difficult (see Plimosis).

Treatment.-The treatment of balanitis without phimosis is simple. The indications are to keep the parts clean and free from pus, and the inflamed surfaces dry and separated from each other. The prepuce should be retracted and the parts be cleansed in simple warm water from two to four times a day. From 3 to 4 per cent. of boric acid or I per cent. of carbolic acid may be added to the water, but soap or other irritating substances should not be used. After washing, the parts should be dried gently by patting with antiseptic cotton or with a soft cloth, and covered with a fine dusting-powder. Over the powder is laid a thin film of the cotton or a piece of lint cut to a shape and size that will just cover the glans and leave the meatus free. The prepuce is now pulled forward to cover all, and the dressing is complete. For mild cases a powder containing I part of boric acid and from 2 to 4 parts of refined talc is sufficient. Of equal service are calomel, bismuth, or zinc oxide, each alone, or in combination with one of the others, or reduced with talc. In severe cases, when the surfaces are very sensitive, iodoform is excellent and gives relief. Before applying the powder the surface may be wiped gently with a solution of nitrate of silver (gr. $\mathrm{xx}$ to $3 \mathrm{j}$ in $\overline{3 j}$ ), and deep excoriations may be touched lightly with the solid stick.

If the powders are not productive of comfort, the 
cotton or lint may be moistened with a mildly astringent and soothing solution before it is applied over the powder, or the latter may be omitted altogether. Solutions of carbolic acid ( $\mathrm{I}$ per cent.), boric acid (2 to $\mathbf{5}$ per cent.), dilute lead-water, red wine ( $\bar{j}$ to $\bar{z}$ ss in $\bar{z} \mathrm{j}$ ), or the following may be used:

$$
\begin{array}{ll}
\text { R. Zinci sulphat., } & \text { gr. j-ij ; } \\
\text { Morph. sulphat., } & \text { gr. ss.; } \\
\text { Atropin. sulphat., } & \text { gr. } \frac{1}{ \pm} ; \\
\text { Aqua, } & \text { 3j.-M. }
\end{array}
$$

Sig. For external use.

As the condition improves, powders will be more serviceable. For some time after recovery the parts must be cleansed daily and the surface of the glans and the prepuce be separated by a film of cotton.

In men who are subject to frequent recurrences of balanitis, the mucous membrane may be rendered less sensitive and less liable to inflammation by the longcontinued use of a powder containing from Io grains to $\frac{1}{2}$ ounce of tannic acid to the ounce of talc, starch, or lycopodium.

\section{PHIMOSIS.}

In severe forms of balano-posthitis-usually when secondary to chancre, chancroid, or gonorrhœa-the swelling and infiltration of the parts may be sufficient to prevent retraction of the prepuce back of the glans, thus producing an inflammatory phimosis. If a nian with congenital phimosis more or less complete acquire a venereal disease, inflammation of the tissues is almost sure to follow, since cleansing of the parts is very dif- 
ficult, and irritating discharges are retained in contact with the membrane.

Symptoms.-The swelling, œdema, and inflammatory symptoms may be slight, giving the patient little inconvenience, or they may be severe and very painful. The distended glans is then covered by a reddened, sensitive, and greatly swollen prepuce, increasing the distal extremity of the penis to several times its normal size and giving the organ a distorted or club shape (Fig. I2).

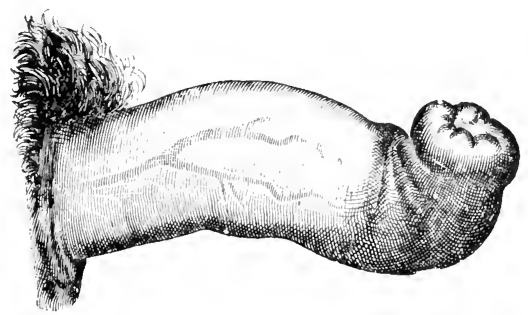

Fig. 12.-Phimosis from gonorrhœa (Cullerıer).

The discharge escapes from the narrow opening of the prepuce, and may dry in bulky crusts around the thickened edges. Sometimes there is excessive œdema and swelling, with but slight inflammation and discharge. Pressure may interfere with circulation and result in gangrene of portions of the prepuce or rarely of the glans. Gangrene first appears upon the inner surface of the prepuce, and is preceded on the outer surface by evidences of interrupted circulation-namely, a dark-red, livid, or bluish color of the skin. If left to itself, the gangrene usually destroys enough of the prepuce to release the glans and relieve the pressure; the circulation being thus restored, the slough is thrown off and the surfaces cicatrize, leaving an irregular, ragged prepuce. As a result of repeated inflammations the prepuce 
may become permanently thickened and hardened, so that its retraction is impossible. When a series of soft chancres have been located at the orifice of the prepuce, the resulting scars may contract and produce phimosis.

Diagnosis.-An accurate diagnosis of the underlying conditions in inflammatory phimosis is difficult and often impossible. When syphilitic chancre is present and sufficiently developed, its induration may be felt through the prepuce, and typical enlargement of the inguinal glands may be detected. A hidden chancroid is frequently followed by one or more chancroids at the orifice of the prepuce, the result of auto-inoculation; and, since the retention beneath the prepuce of infectious pus favors absorption, such a chancre is apt to be followed by an inflammatory or a virulent bubo. In doubtful cases the history may be of value; or a few days of treatment may reduce the swelling and inflammation and render an accurate diagnosis possible. If gonorrhœe is present, it can be detected even when the meatus cannot be exposed. The preputial sac is cleansed by inserting between the glans and the prepuce the tip of a syringe or an irrigator and injecting an aseptic solution until the fluid comes away clear. The patient then urinates in one or two glasses. The presence of pus in the urine indicates gonorrhcea.

Treatment.-The preputial sac should be irrigated three or four times daily with warm water, which may contain I per cent. of carbolic acid or 3 per cent. of boric acid. The nozzle of the syringe or irrigator, gently inserted between the glans and the prepuce, should be directed in turn to every part of the sac, and sufficient fluid should be used to cleanse the sac thoroughly of all 
pus and other accumulated matter. The flat nozzles made for this purpose are excellent, and their use excludes the possibility of injecting the urethra-a mistake that should carefully be avoided. The cleansing of the parts may be accomplished less perfectly by wiping out the sac with bits of cotton wrapped on the ends of wooden toothpicks. After cleansing the surfaces one of the soothing or astringent lotions recommended for balanitis may be injected into the sac. In the large majority of cases a few days of this treatment suffice to reduce the inflammation and to render retraction of the prepuce possible. If swelling and redema are extensive and inflammatory symptons are severe, the penis should be immersed for twenty minutes or more, several times daily, in hot saturated solutions of boric acid, and during the rest of the twenty-four hours should be supported by dressings that will hold it in the groin or over the pubes, in order that position may favor return circulation. If gangrene is feared, the patient should lie on his back, with the penis supported and constantly wrapped in boric-acid fomentations as hot as can be tolerated. Tonics should be given when indicated by the general condition of the patient. Circumcision is rarely necessary, and when performed upon an inflamed prepuce the operation gives unsatisfactory results. If gangrene is imminent, calling for immediate relief of pressure, or if it be necessary to cxpose a phagedenic chancre of the glans, it is well to slit up the dorsum of the prepuce. If soft chancre be present, any freshly cut surfaces are cortain to become infected. If gangrene has begun in any part, pressure should be relieved by incisions, hot fomentations should be applied, and the patient should be kept quict in bed. Quinine 
and tonics should be given to meet the indications of each case.

In adults, congenital phimosis and phimosis due to thickening of the tissues or to cicatricial contraction should be treated by circumcision.

\section{PARAPHIMOSIS.}

When a short prepuce becomes inflamed and œedematous, it is apt to roll back of the glans, where it rapidly becomes more swollen and infiltrated until it cannot be returned to its normal position. This form of paraphimosis is usually mild in type. A more serious form often occurs where a longer prepuce with a narrow opening, especially if rendered yet narrower by inflammation, is slipped back of the glans. This may occur in coitus or in cleansing the glans, but is most frequently

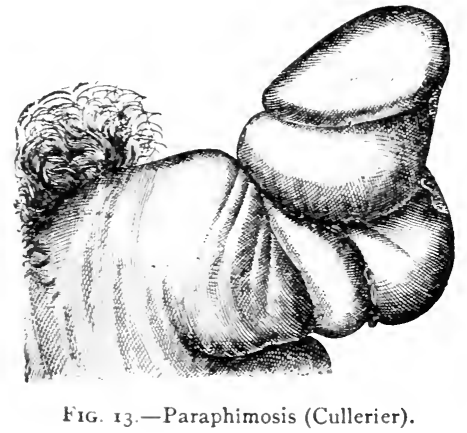

found complicating hard or soft chancres or other local disorder. The retraction may be the result of excessive swelling, of accident, or of failure to return the prepuce over the glans after local treatment and dressing.

Symptoms.--In spontaneous paraphimosis due to inflammation of a short prepuce the most prominent 
symptom is the mass of swollen and œedematous tissue back of the glans. The swelling is often irregular in form, larger below than above, and is usually soft, puffy, or doughy. The glans is but slightly if at all affected, and subjective symptoms are wanting. If the condition is allowed to persist, the infiltration of the tissues may increase until the swelling becomes tense, white, and glistening. In such cases the glans becomes more or less swollen and darkened in hue, showing an interference with circulation. Strangulation rarely follows.

When a long prepuce with a tight opening is retracted and allowed to remain, it produces constriction of the penis back of the glans, which soon becomes turgid and livid. The soft tissues of the prepuce in a few hours become greatly inflamed and swollen. Just back of the glans is a large roll of tense, glistening œdematous tissue, which may be white or of a reddish color (Fig. 13). Back of this roll, and often concealed by it, is a deep furrow produced by the constricting band or ring. Behind this furrow is usually another smaller fold of swollen and odematous tissue. At first these swollen, œdematous folds are soft though tense, but after a few days plastic infiltration makes them thicker and firmer, and may even cause adhesions.

Strangulation occurs when the constricting band shuts off all circulation from the parts in front. The glans is then even more swollen, is darker in color, becoming purplish or almost black, and is cold and insensitive to touch. If this condition be untreated, it is rapidly followed by gangrene of the prepuce or of the glans. Fortunately, in the large majority of cases gangrene destroys the constricting band in time to save the glans.

Treatment.-In all recent cases an attempt should be 
made to return the glans back of the constricting ring. If reduction is not accomplished, and there is no immediate danger of strangulation, rest, elevation of the penis, and the constant application of boric-acid fomentations will promote absorption of the infiltration, and will in almost all cases render further operation unnecessary. In addition to fomentations, astringent lotions may be used. The affected part should be watched closely to prevent the possibility of strangulation and gangrene.

If strangulation occur, immediate reduction or opera. tion is imperative. If an even pressure be exerted with the fingers or with a

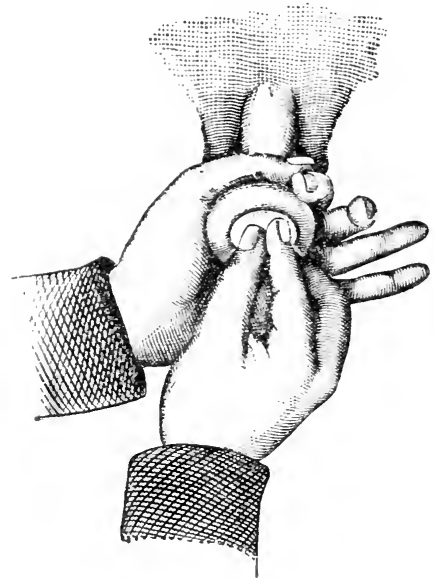
narrow bandage, the glans may be reduced sufficiently to enable it to pass through the constricting ring. In

FIGS. 14, 15.-Reduction of paraphimosis.

acldition to pressure, ice or iced water may be applied, and the odematous fold in front of the ring may be scarified in order to allow the serum to escape. If these measures fail, the patient should be put under the influence of an anæsthetic, when the resulting relaxation 
of the tissues greatly aids in reducing the paraphimosis. The corona and the adjacent portion of the prepuce should be oiled, and, when possible, some of the oil should be worked under the constricting band. With the thumb and the forefinger of the left hand encircling the body of the penis just back of the stricture, the glans is seized with the thumb, index, and middle fingers of the right hand, and by them squeezed into the smallest possible compass (Fig. I4). Pressure should be exerted laterally, in order so to reduce the diameter of the glans that the left thumb and forefinger may fetch over the preputial constriction. Sometimes the finger-nails may be worked under the constriction, and thus aid in the reduction. Keyes recommends seizing the penis behind the strictured prepuce in the fork of the index and middle fingers of both hands, one placed on each side. This method gives more even pressure forward. The glans is thus compressed between the two thumbs (Fig. I5). The rounded end of a hair-pin or a blunt-pointed director may be inserted under the constriction on each side, and the glans compressed between the digits while the prepuce is slipping forward.

With the patient under ether reduction can be accomplished in most cases if sufficient care and patience be exercised; but if all attempts fail, it is necessary to divide the constricting band of tissue. A tenotomy-knife or a blunt-pointed bistoury with the blade flat is inserted under the band. When possible, the blade is brought to the median line above before the knife is turned so as to bring the edge upward, and the ring is divided from within outward. After reduction the case may be treated as one of inflammatory phimosis.

In reducing paraphimosis so much manipulation and 
handling of the parts are necessary that, in case a contagious ulcer is present, the surgeon is in danger of becoming infected unless the epidermis of his hands is sound.

In the older cases of paraphimosis in which odema is the chief, if not the only, symptom, rest, position, and warm dressings may be supplemented by the application of pressure and of strong astringent lotions. For such cases Keyes recommends the free use of collodion.

\section{VENEREAL WARTS.}

This title is applied to vegetations appearing upon the genitals and the genital region. The term is not strictly accurate, for, while these warts are commonly associated with venereal diseases, and are almost always the result of exposure of a delicate membrane to venereal secretions (gonorrhceal, syphilitic, leucorrhceal, etc.), the lesions may spring from other causes (uncleanliness, warmth, and moisture). In pregnant women they are sometimes found bordering the vulva, where they are doubtless produced by irritating discharges. It is possible that these vegetations possess a distinct though feeble contagious element, but this contagiousness has never been demonstrated. In men the favorite location of venereal warts is in the sulcus back of the glans penis, but they are found over all parts of the glans and the prepuce, and occasionally within the urethra. They may also appear on any portion of the penis, scrotum, perineum, and inner surface of the thighs, and about the anus. In women they are commonly found over and about the vulva, over the perineum and anus, and sometimes within the vagina. They may be single, but they are usually multiple, and they vary in size from a single 
filiform projection to a close aggregation of filiform or papillary elevations forming a mass as large as a hen's egg or even larger. Individual papillæ are usually acuminate, but may be rounded, club-shaped, or flattened. Instead of becoming aggregated in larger masses, they may appear as smaller but more numerous elevations; at times hundreds coexist upon the genitals and the nerghboring regions. They may so fill the preputial sac as to cause phimosis, paraphimosis, or, rarely, gangrene. When situated on a free surface, where they are dry, they are firmer and have the color of the normal skin, but when protected and moistened they are softer, are pinkish or bright red in color, and are covered with a whitish or yellowish puriform mucus having a very offensive odor. The larger masses may be pedunculated or sessile, and form irregular-shaped vegetations resembling in appearance cauliflower or the comb of a cock. Under the influence of warmth and moisture they grow luxuriantly and rapidly by peripheral extension. When larger and flattened they may be mistaken for condylomata. The latter are broader and flatter than venereal warts, are not made up of so many small projections, and are found in connection with other evidences, or with a history, of syphilis. Papillary epithelioma may be distinguished from a venereal wart by the indurated base and border of the cancerous growth, its slower development, its tendency to degenerate and to form typical deep ulcers, and the infrequency with which it appears before the fortieth year.

Treatment.-Cleanliness is first in importance. In many cases, if the parts be kept clean and covered with a simple dusting-powder, the venereal growths gradually shrivel and disappear. The treatment recommended for 
balanitis is often efficient; if necessary, the lotions and powders employed in that affection may be increased in strength. When persistent, pedunculated masses and small vegetations may be removed with the scissors or the curctte, and the base cauterized with nitric or acetic acid, or with nitrate of silver in stick. After such treatment the surfaces should be kept clean and covered with iodoform, aristol, or similar powder. The large growths with a broad base will usually shrivel under the application of tannic acid or other astringent powder; if they persist, nitric or acetic acid may be applied once a week until the base is destroyed, a powder being used during the intervals. If the growth can be kept dry, bichloride of mercury in collodion ( $\mathbf{j} \mathrm{j}: \overline{\mathbf{z}} \mathrm{j})$ may be applied every second or third day. In using this preparation care must be taken to prevent its contact with other surfaces than those for which it is intended, and the possibility of balanitis following its use should always be borne in mind.

\section{HERPES PROGENITALIS.}

This disorder is not always venereal in its origin, but in many cases it follows local irritation or inflammation caused by venereal diseases, contact with irritating secretions, excessive venery, or sexual excitement. A long, tight prepuce and a gouty or rheumatic diathesis may act as predisposing causes. Neurotic individuals seem to be unusually susceptible to the disease.

Symptoms.-The disorder, which appears in the form of one or more groups of minute, pin-head-sized vesicles on an inflamed base, may affect any portion of the genital organs and the surrounding integument in both sexes, though it is much more common in men. In the 
male sex it is most frequently found upon the inner surface of the prepuce, in the sulcus, or on the glans; in women, on the hood of the clitoris, on the labia minora, and on the inner face of the labia majora.

The eruption is accompanied, and may be preceded, by sensations of burning, pricking, or itching. There is usually but one group of vesicles, which contain a clear fluid. On the mucous membrane these vesicles rupture in a few hours, leaving sharply defined circular excoriations which, if unirritated, heal in two or three days without other treatment than cleanliness. The whole process lasts a week or less. When the vesicles are situated on the integument, the contents dry and form small crusts, which remain for a few days until healing is complete. Sometimes the first group of vesicles is followed by others, and the disorder is thus continued through several weeks. If the lesions be irritated (by coitus, severe treatment, etc.), balanitis may follow, and in rare cases there may result ulcerations simulating soft chancre, with inflammation of the inguinal glands. One attack of this affection predisposes to others, so that it is commonly recurrent, appearing periodically or at irregular intervals for months or for years. The persistency with which this simple disorder recurs is not only annoying but also peculiarly distressing when in consequence patients believe themselves to be subjects of syphilis. In the hands of the ignorant or the unscrupulous these deluded victims often undergo specific treatment for long periods.

Diagnosis.-The excoriations of herpes can usually be distinguished from those of balanitis by the circular outline of the herpetic lesions, which is rarely entirely lost even when the vesicles have coalesced, and by the 


\section{SYPHILIS AND THE VENEREAL DISEASES.}

absence of more extended inflammation. When the excoriations suppurate and form superficial ulcers, it may be impossible to distinguish them from beginning chancroid, but by cleansing the parts and keeping them covered with iodoform or aristol herpetic ulcers will at once begin to improve, and in a few days will be entirely healed. An initial sclerosis may appear as a small excoriation, but underlying induration can be detected; the sore is indolent, and is soon accompanied by characteristic enlargement of the inguinal glands. In making a prognosis it must be remembered that chancre may follow in the site of an herpetic lesion if the longest period of incubation of syphilitic chancre has not elapsed between the date of exposure and that of the examination.

Treatment.-The local treatment of herpes progenitalis is that of balanitis. Recurrences may often be avoided by improvement of the general health and by hygienic living, including abstinence from alcohol, tobacco, and highly seasoned food. In obstinate cases the surface of the glans and prepuce may be hardened by the long-continued use of tannic acid in powder or in solution of water and alcohol. 


\section{HYPOCHONDRIASIS.}

THE morbid mental states produced by real or fancied venereal disease are numerous, and are equalled only by the hypochondriasis springing from ignorance and perversion of the physiological functions of the sexual organs. These morbid mental conditions are of occurrence in both sexes, far more frequently in men than in women, the subjects being generally near the puberal epoch. The symptoms presented differ in grade of severity, and when of marked character they may result in more physical distress than the maladies themselves, of which there is either slight evidence or a mere dread. For practical purposes the sexual hypochondriac and the patient in terror of a venereal disease, actual, possible, or wholly imaginary, may be considered in the same category.

It is a matter of common remark that the physiology of the generative organs of the male sex is less understood by the average physician than that of the corresponding functions in women. In the management of the youths, commonly unmarried, who are sufferers from the mental states here considered, it is important to recall the following facts:

Among the mammalia of the lower animals the sexual propensity is in general gratified with impunity in promiscuous relations, the young males copulating with in- 
dividuals of the other sex freely on the earliest impulse, when not restrained by stronger adult males and by females who are not ready to accept approaches. From this erent dates the sexual life of the animal, the male, when no longer capable of performing the sexual act, being often killed by stronger and more ambitious rivals or being abandoned to die apart from the group of breeding animals. Throughout this sexual life the male animal has, in the state of nature, no seminal emissions and no perversion of controlling sexual instinct. As a rule, he refuses to solicit the female of his kind when she is pregnant. In many cases the period of activity of the sexual impulse of the female corresponds with that of the other sex, and beyond that season the two often exist together in a harmonious asexual life. Obviously, this arrangement is designed solely with the view of reproducing the species.

The female, as a rule impregnated at the earliest ovulation, begins with this event a sexual life which thereafter consists of a series of pregnancies and sucklings of offspring until she is incapable of further conception, when she also either dies or is killed, having fulfilled her part in the struggle for existence. There is no history of menstruation-a function which, with its important accessory phenomena in generations of transmitted tendencies, distinguishes the female of man alone in the animal creation.

When man is studied in his artificial social surroundings, he is seen at once to be amply fitted for the part he is to play in life of a broader scope and deeper intent than that of the brutes. The young human male is required by the written and unwritten laws of most civilized countries to deny himself the grati- 
fication of his sexual appetite until he is capable of union with one woman and of providing for the support of a family. During the time which intervenes between the attainment of puberty and marriage there is a period of unrest, and in many cases even of physiological storms which ever and again disturb the tenor of his days. If he happens to be among the large number of lads who early in life have practised masturbation, a perusal of the advertisements of the charlatan, with their record of horrors, may awaken in his breast a dread of a frightful future. He comes to his physician or friend (well for him if the former be also the latter) with a tale of involuntary seminal emissions at night, with and without erection; an escape of semen when straining at stool ; "lost manhood;" defective memory; spots dancing before the eyes; "impotence;" sexual debility; general weakness; and disinclination for society.

With the trained physician it is scarcely necessary to discuss this group of "symptoms." Involuntary nocturnal discharges of semen occurring in a young man several times during one night, or even on successive nights, are "unnatural " simply because the human male animal is not living in the state of nature briefly sketched above. These losses are of similar import whether occurring with erection and accompanied by a lascivious dream, or in the total unconsciousness of sleep. They are truly physiological, and amount to the price paid by the youth who is attempting to lead a correct life and who refuses to lower his moral standard. The frequency of these discharges has little bearing upon any question of disease, since in perfectly sound youths seminal losses may be even often repeated without detriment to the general 
health. A frequency at one time is usually compensated for by a relative absence at another. The emissions may be followed on the succeeding day by a feeling of lassitude, slight frontal headache, and mental dulness, but the balance is always struck by nature, as there is usually afforded a respite from the sexual fever (if such it may be called), when the discharge or the series of successive discharges is at length for the time being made to cease. These losses wholly correspond in physiological function and meaning with the menstruation of the young woman, whose monthly flux in a sense represents the price paid by her for virginity, clean living, and a delay of the performance of the sexual function until she is solicited in marriage by an acceptable suitor. It is true that after marriage menstruation may occur periodically, but, as a rule, it is suspended during pregnancy. Married men also, temperately indulging in sexual relations, occasionally have periodical involuntary seminal losses. It is well known that the comeliness of a young girl is almost proportioned to the regularity and character of her monthly periods. None the less is it certain that the attractiveness of a youth leading a clean lifethat which makes the eye of man and woman dwell on his person with a sense of delight, the promise of manhood writ large on his features and figure-is to a degree proportioned to his involuntary losses at night.

Nor is the periodicity observed in menstruation not perceptible in the corresponding function of the other sex, seeing that not only are married men at certain seasons of the month specially disposed to the gratification of sexual desire, but that the unmarried also, at certain times in the month more than at others, have an 
access of similar import. There is, in fact, a prostatic no less than a uterine ebb and flow of sexual congestion, and the reverse, that is responsible for many phenomena of health and disease perceptible in the two sexes and often wretchedly misinterpreted. Many, indeed, of the "pollutions" of young men at night (suggesting the menstruation of women not accompanied by ovulation) are discharges largely made up of the prostatic fluid rather than of semen.

Much the same explanation can be given of the "losses at stool" on straining which are such a bugbear to the uninitiated. These losses, too, are largely made up of the fluids of the prostatic sinuses, expressed by pressure upon the gland exerted by a scybalous mass in the rectum. There is no evidence whatever that this "loss at stool" is, in the life of any continent man, a sign of disease. Men differ greatly in the quantity of prostatic secretion they furnish, precisely as they differ in the amount of salivary fluid supplied during mastication. There is no fixed standard for all men, as there is none for the food they daily eat and for the amount of excreta they void as a consequence of the assimilation of portions of that food. Indeed, many married men, regularly and temperately gratified sexually, find a notable quantity of the same glycerin-like secretion at the meatus urinarius after stool when the general health is absolutely unimpaired and their organs are in a normal state. The milky fluid seen by the sexual hypochondriac when actually engaged in voiding urine is, as is well known, not seminal in character, but is due to the presence of the alkaline phosphates, and originates in conditions wholly apart from the sexual organs. The clearing up of this cloudiness by the addition of a small 
quantity of acid in the presence of the sufferer is usually of value in restoring his mental equilibrium.

At times the microscopical examination of the prostatic fluid exuded at stool, and even of the urine of the hypochondriac, reveals the presence of spermatozoa. Even here no evidence is presented that the person is the victim of disease. The spinal and other symptoms of true spermatorrhœa need not here be considered. As a morbid state it is unquestionably rare, and is not to be classed with the functional derangements of the puberal epoch and its sexual alternations of storm and calm.

Masturbation may be prolific of serious physical mischief; but many of its results are exaggerated, and for the great majority of youths who discover the nastiness and indecency of the habit, and who abandon it, no serious consequences ensue. This is the verdict of conservative and experienced physicians the world over. The worst of its results, for the majority of men, are mental-the sense of unmanliness, the loss of selfrespect, and the dread of the future which it begets. The best recourse in this morbid mental state is a timely confession to a wise physician, the acceptance of some explanation of the physiological function of the sexual organs, and the receipt of a good deal of encouragement for the future. The youth should be informed clearly that during a young man's period of sexual probation he has a constant conflict to wage between his passions and his better self, from which conflict he should emerge a victor-if not wholly unscathed, at least like the soldier who has made a brave fight and has conquered, not without some resulting scars, the enemy that sought to vanquish him. The best of fathers and husbands are veterans of such conflicts. 
"Impotency" is a condition of which a great number of men know nothing, however cloquently they may bewail its occurrence. Having suspected in themselves some weakness, they often attempt to perform the sexual act, chiefly with a view to experiment, and the novelty of the situation, the dread of failure, or the fear of contracting some disease has left them powerless; or, possibly, when engaged in fondling and caressing i female companion they have experienced a flow cither of the prostatic or of the seminal fluid itself, which has persuaded them beyond peradventure of a "sexual weakness" with which they are afflicted.

The point of view for all questions of this order is that which regards equally the two sexes. The inexperienced youth should be like the inexperienced girl in the first approaches after marriage-shrinking, trembling, timid, and unprepared. It is estimated that from 60 to 70 per cent. of young husbands fail in the first sexual attempts after marriage, and whatever figures may here represent the truth must surely be applicable to the other sex. The truth is, that while the young of the lower animals are early taught by experience to perform the sexual act without dread and, in the wild state, for the most part without preference of individual, men and women reared in civilization and surrounded by the usual safeguards of social order require to mutually educate each other in the matter of physical union. Never is the sexual act so vigorously and effectively accomplished as when the strong emotion of love unites two human beings and elevates the performance of the brute to the level of a pure morality. Hence the complaints of "premature ejaculation of semen " and of "failure of erection" on the part of the hypochondriac 
have no meaning when interpreted in the light of science.

The "lost manhood" of these fond youths is an echo from the outgivings of the parasites of the profession. Manhood in its best sense bears small proportion to the vigor and capabilities of the sexual organs. In so far as man is distinguishable from the brutes is he removed from their sexual habits and powers. The lower the individual in the scale of civilization, the more conspicuous, as a rule, is his sexual power and the number of resulting progeny. The negro, the Indian, and the halfbreed are in this point widely removed from the highest types of the Anglo-Saxon. Even when in the grasp of disease, the tuberculous, the syphilitic, the leprous, the idiotic, and those burdened with the inherited and acquired maladies of the pauper fetch into the world their superfluous brood to be a burden to society and a reproach to civilization; while men and women far above them in the social scale, and superior alike in point of physical endurance and mental energy, perform the sexual act with far less inclination, frequency, and readiness. For some of the very noblest types of manhood and womanhood, indeed, indulgence in the sexual act is notably infrequent, the resulting offspring few, and the marriage state often unsought.

The listlessness, loss of memory, muscæ volitantes, and lack of physical energy cited by the hypochondriac as evidences of his condition are obvious misinterpretations of the changes from day to day incidental to all active lives. The fatigue of nervous anxicty is not necessarily morbid. The memory of some of the insane is remarkably good; it is a faculty conspicuously wanting among some of the greatest men of history, and in most 
persons is largely the result of their education and environment.

"Impotence" is a word that for the expert has no longer a definite meaning. Every healthy adult male is, in the sense in which that word is popularly employed, both potent and impotent. Some men, like the negro, are capable of committing a rape as often as the occasion offers, merely to gratify lust ; fortunately, they are few and usually meet with a violent end. Fortunately, also, the best type of man, living a clean life, is wholly unable to perform the sexual act save with the one woman in the world whose life is devoted to him alone. For the men within these two extremes a thousand accidents-disgust, anger, excessive bodily or mental fatigue, recent evacuation of the seminal vesicles, and who can say what else-daily render them "impotent" in the sense in which this word is often used.

Nor is the man who, living a clean life, chances to possess unusual sexual vigor, for this reason to be set down as a type of superb manhood. With infinite forecasting of the needs of the race, it is ordered that the sexual propensity be most eager, most energetic, and most effective in exercise at an early period of life, when the real vigor of a man, mental and physical, is actually immature. Obviously, all is planned with a view, first, to the perpetuation of the race, and, after that, to provision for the young of the family under the shelter of the roof reared by the strong hand and provided with sustenance by the experienced brain of the maturer man.

When the sexual hypochondriac oversteps the limits here set-those, namely, within which a fairly healthy youth passes through his period of sexual probation, 
with a heart saddened and perhaps affrighted by the ghosts that beset his pathway-then he enters a pathological field which cannot be named without a sense of disgust. The unnatural practices which the records of history teach are as old as the Saturnalia of the Roman Empire, prevail only to a limited extent in our own day. Alienists, chiefly those connected with state institutions for the care of the insane, have probed the depths of this vileness, and with scientific precision have analyzed the symptoms of sexual psychopathy as they are betrayed in masochism, sadism, tribadism, sapphism, pederasty, sodomy, bestiality, erotomania, and satyriasis.

To the credit of humanity it may be added that these habits are often manifestations of insanity due to lesions of the nervous centres. The victims of these disorders are the frequent subjects of epilepsy, imbecility, or dementia, and often exhibit microcephalic, asymmetrical, or scar-compressed crania.

Syphilophobia is a term used to describe the condition in which patients become morbidly terror-stricken over the possibility of having incurred syphilis. The term may be used, if not with accuracy, at least usefully. to include those who are morbidly anxious lest any of the venereal diseases other than syphilis, such as gonorrhoea, have been incurred. It matters not what be the object of terror, the mental state is practically the same.

It is seldom that in the venereal diseases any more wretched complexus of symptoms is presented than in a well-marked case of syphilophobia. The weird of the thing seizes alike on men and women, and while it endures, life is usually embittered, the happiness of a home often blighted, and the body wasted under the ner- 
vous strain. Sleep, digestion, nutrition, and peace of mind vanish; the tongue becomes dry, the eyes haggard, the person neglected. From this extreme there is every gradation to the other, where there is simply a shortlived and happily-ended anxiety: Only an abounding selfishness can impel men to these conditions, but many patients claim that their unending terror is based on apprehensions for another-a wife, a betrothed, a daughter, a sister, a husband, or a friend. This condition may endure for but a few days or weeks, or it may last for years. It may even be for a long while shared in full measure by a consort. In a few cases we have seen men and women go insane under the burden of the anxiety. In these extreme instances it may well be believed that the insanity was lingering unrecognized until the accident suggesting fear of venereal disease became the immediate and exciting cause of the disaster.

The bases of the suspicions of these people are widely different. Some individuals exhibit tangible lesions of the surface, which they choose to misinterpret, after a real or fancied exposure to venereal disease. Thus a facial acne, a keratosis pilaris of the outer faces of the thighs and arms, a telangiectasis ("spider cancer") of the surface of the chest, some innocent mollusca of the scrotum, or a few aphthous ulcers of the mouth due to indigestion. serve as unmistakable signs of syphilis. Often in their restless anxiety these victims swallow medicaments with a view to eradicating the malady with which they are convinced they are infected, and these drugs, by the production of a medicamentous rash, add to the supposed evidences of disease.

When no lesions are present on which to build these anxieties, a basis is readily discovered in the anatomical 
peculiarities of the body. In this way the bluish tinge at the rim of the corona glandis is taken to be a sign of "gangrene;" the fungiform papillæ of the tongue are named as "mucous patches;" the reddish hue of the meatus externus urinarius indicates "inflammation." In some cases the testicles are too closely drawn up to the body; in others they are too lax; in yet others the penis is shrinking; in women the vulva is beset with "papules" when its follicles are unusually conspicuous. If these unfortunates once become possessed of works on medicine, they are usually worse distraught. It is not at all surprising that even classical illustrations of this singular craze are furnished in the persons of physicians themselves. The subject, even though it seem to have a ludicrous side, is not without its tragic aspects. We have known men to take their lives in despondency over such fancied disorder. The trained physician must be ready to appreciate every phase of the madness.

The treatment of the sereral forms of hypochondriasis described requires the utmost skill, prudence, and good judgment on the part of the physician. The chief remedy at hand is a knowledge of the truth. This alone is often sufficient. A common-sense explanation and a little encouragement often suffice to remove a persistent cloud of shame and dread. Unfortunately. as the history of the world clearly proves, men and women will not always listen to the truth. Sympathy loes good at times; at others it is worse than useless, and ridicule, even scolding, may answer the end better. For the obstinate subjects who refuse all aid of this character and persist in retailing their long list of symptoms to the car of the physician, he may cren make shift to accomplish his end by wholesome threats. It 
may be wise in cases to point to the idiotic condition that occasionally crowns a labor of the sort upon which the hypochondriac is engaged, or even, as a last resort, to make it appear that, since the patient will listen to neither truth nor reason, his friends must be made acquainted with the facts in his case. The last is often an effective argument. It is a pitiable fact that some of these states are utterly hopeless. The patients either belong to the insane class or are of those of whom it was long since written that even brayed in a mortar their folly will not depart from them.

For the young male sexual hypochondriac important advice touches his moral surroundings; but over and above this he should be made to abandon tobacco and alcohol, to employ the flesh-brush after his cool morning bath, to avoid hot and Turkish baths, and even cold bathing at night, which is apt to be followed by undue stimulation when he is well warmed in bed. His food should be nutritious and simple. It is a common error for these young persons to attempt living on a slim diet to avoid stimulating the sexual propensity. All medicines of the sort commonly given to allay nervous excitability, such as bromide of potassium, are rigidly to be excluded. For these subjects they are vile remedies, and they exert an injurious effect upon the mental tone of the person who relies upon them. From a scientific point of view, they are given with a wholly false conception of the end to be attained. The bowels should be evacuated daily, and there should be open-air living and physical exercise. The dance, theatrical performances, club life, and the perusal of certain kinds of literature are equally harmful. To persons of this class medical books are to be especially prohibited. 


\section{ACUTE LRETHRITIS.}

THE term "urethritis" includes all forms of urethral inflammation. By far the most common of these inflammations is gonorrhoea. Cases of urethritis originating without the influence, direct or indirect, of gonorrhoeal infection are rare. Other forms of urethritis, arising from constitutional defects or from mechanical or chemical injury to the urethral membrane, occur, but they are chiefly interesting from the standpoint of etiology, since in symptoms and treatment they correspond with some of the stages of the more common disorder. In the following pages, except where mention is made of other forms of urethritis or of the disease as it occurs in women, the subject under consideration is gonorrhoea in the male.

Etiology.-From an etiological standpoint, the best classification is that of Lustgarten, who recognizes two classes-infectious urethritis (including gonorrhœa, pseudo-gonorrhcea, syphilitic urethritis, and urethritis tuberculosa) and non-infectious urethritis.

I. Infectious Urethritis. - 1. Gonorlaa (jovir, semen, and $p=\tilde{c}_{2}$, to flow).-Synonyms: Urethritis; Clap; Blennorrhagia: Blennorrhcea; Gleet; Gcrm. Tripper; Schleimfluss; Unreiner Fluss; Gonorrhoë; Fr. Blennorhagic: Gonorrhée; Chaudepisse; Span. and Ital. Gonorrea.

The term "gonorrhoea" is a misnomer, but it has 360 
been so long employed in a specific sense that it must be retained for the present rather than be supplanted by one more scientific. In these pages its use is restricted to indicate that acute, infectious form of urethritis which follows a period of incubation, pursues a more or less definite course, and produces a discharge which contains gonococci and is capable of causing a like form of inflammation in the mucous membrane of the healthy urethra of another individual. Of all diseases, gonorrhoea is the most correctly termed venereal, for it most frequently results from sexual intercourse, and is rarely acquired in any other way. Simple contact of a gonorrhœal discharge with the mucous membrane is sufficient in the large majority of cases to communicate the disease, though the vagina is less easily infected than the urethra, and some persons, particularly under special circumstances, are less susceptible than others. The general condition of the individual, the state of the mucous membrane exposed to infection, and the circumstances of the exposure, other than the presence of a gonorrhœal discharge, play a much less important part than in the other venereal disorders. A man who exposes his urethra to a gonorrhœal discharge has few chances of escaping infection. On the other hand, since the source of contagion lies solely in the discharge from the diseased membrane, and does not exist in the blood and in special secretions of the body, as in syphilis, the opportunities for acquiring gonorrhoa outside the sexual act are rare. It must not be forgotten, however, that gonorrhœa may be innocently acquired, and that it is possible for the contagion to be conveyed by means of a towel, the seat of a public water-closet, and other media. 
The Gonococcus.-It is now generally conceded that the active factor in the production of gonorrhœa is the gonococcus of Neisser. This micro-organism is found in the purulent discharge of acute urethritis, known as gonorrhcea; in the muco-purulent discharge and threads (tritper fadcn) of certain chronic and subacute forms of urethritis; in the gonorrhœal discharges from the vagina, uterus, conjunctiva, and rectum; and a few cases have been recorded in which gonococci were found in the secretion of the mucous membrane of the mouth and the nose. This micro-organism has also been found in joints affected with gonorrhceal rheumatism, in periurethral folliculitis and abscesses, and in suppurating vulvo-vaginal glands. Bumm, Wertheim, and others have repeatedly succeeded in cultivating the gonococcus, and by inoculating the healthy urethra with these cultures have produced an acute urethritis having an incubation period of from two to five days and a duration of five or six weeks, the discharge containing gonococci. In one instance the twentieth generation of a pure culture was thus successfully employed. These experiments seem to demonstrate fully the pathogenic character of this micro-organism. It should be remembered, however, that even the normal urethra may be inhabited by one or more species of diplococci, which so closely resemble the gonococcus in all particulars, including staining properties, that the most expert cannot always differentiate them with certainty.

(a) Preparation and Examination of the Specimen.-In selecting gonorrhceal pus for examination it is well to avoid that found at the meatus, as this pus is more liable to contain other organisms that might render the examination complicated and confusing. It is better to 
G. Gonococci in gonorrhoeal pus; $\times 500$ (from a photomicrograph by Dr. John A. Fordyce). 2. Gonococcus (after Bumm): a, from a pure culture: $h$, secretion from a g norrhole conjunc-
tivitis, showing an epithelitl cell partial contain gonococci; and a group of three gonococci. goncocel; three pus-cell-, wo of which (schematic). 

obtain pus that may be squeezed out of a deeper portion of the urethra. A small drop of this pus is thinly spread on a slide or a cover-glass, by means of a platinum wire or by pressing the drop between two coverglasses and then slipping them apart. The thin film is allowed to dry in the air, and is then fastened to the glass by slowly passing it three times through the tip of the flame of an alcohol lamp or a Bunsen burner, the pus-covered side being upward. The film is then covered with a few drops of the staining fluid, or the cover-glass is floated, film side down, on the liquid. The preparation should remain in the stain from one to five minutes, depending upon the strengtl of the solution, after which the surplus stain is gently washed off with a jet of cold water. The specimen can now be examined in water or in slycerin, or, what is better, it can be dried carefully with soft blotting-paper and mounted in Canada balsam.

The stain employed may be almost any of the basic aniline dyes, as methyl-blue, Victoria blue, methyl-violet, gentian-violet, or fuchsin. These dyes may be used in aqueous solutions of varying strength, but they do not keep well, and it is best to prepare the fluid each time it is wanted. This may easily be done by kecping on hand a saturated alcoholic solution of the stain, a very small quantity of which can be added, drop by drop, to a watch-glassful of distilled water until the latter is of the required strength and color. The following is a rapid and satisfactory method: A solution of methylblue is prepared by dropping a saturated alcoholic solution of the stain into a watch-glassful of distilled water or into a solution of potassium hydrate (I : IO,O00) until the liquid has a dark-blue color. The cover-glass, prepared 
in accordance with the above directions, is floated on this liquid, pus side down, for from one to two minutes; it is then taken out and the surplus stain is washed off. It may now be placed at once, without drying, upon a slide and examined, or it may be carefully dried and mounted on a slide with Canada balsam. In a specimen thus prepared the gonococci appear dark blue, while the cells show a very pale blue protoplasm and grayish-blue nuclei.

The gonococci are readily decolorized by acids or by alcohol as in Gram's method. In doubtful cases the last-named method is claimed to be capable of making the diagnosis a certainty, but it has been pretty well demonstrated that a few other diplococci lose their stain in exactly the same manner as do the gonococci, and that even this test cannot always be relied upon. The method is as follows:

I. Prepare cover-glass as above described.

2. Stain for from two to five minutes in a saturated solution of gentian-violet in aniline-water. (Anilinewater is prepared by adding 5 parts of aniline oil to Ioo parts of water and shaking thoroughly. The milky fluid thus produced is filtered until it comes through clear and transparent To this fluid a saturated alcoholic solution of the stain is added, drop by drop, until the liquid loses its transparency and a distinct opalescence results.)

3. Place the preparation for from one to one and a half minutes in iodine solution (iodine I part, potassium iodide 2 parts, water 300 parts). In this solution the preparation becomes quite black.

4. Place in alcohol, and allow to remain until no more color is extracted. 
5. Wash in distilled water.

6. Stain for half a minute in a weak solution of Bismarck brown.

7. Wash in distilled water.

8. Dry and mount in Canada balsam.

The gonococci and a few other diplococci lose their blue stain by this method and take a brown stain. Such diplococci as retain the blue stain are not gonococci.

For a satisfactory examination of the preparation, good lenses, with a magnifying power of at least 500 diameters, and a substage condenser should be employed, an immersion lens being always desirable, and in doubtful cases usually necessary.

(b) Characters. - Each single gonococcus has a shape much like that of a kidney or a coffee-bean; but, like other diplococci, these organisms appear in pairs. Each pair is thus made up of two individuals so placed that the flattened surfaces face each other, but are separated by a narrow space, which in the stained specimen appears as a clear (unstained) line (P1.8). In the process of reproduction each individual divides into two, the dividing-line being at right angles to the flattened surface. The result is two pairs instead of one, and the usual grouping of the gonococcus is explained, for it is found rarely in chains, but usually in clumps of four or multiples of four. The micro-organism is secn not only between and upon the epithelial cells and pus-cells, but also within the latter. This position in the pus-cell is characteristic of the gonococcus, and possibly of a few other rarer forms of diplococci, and is determined by observing that the micro-organisms are in focus at the same time with the nucleus and outlines of the cell, and 
by noting that, though a pus-cell may be filled with gonococci even to its border, they rarely, if ever, project beyond it, as would frequently be the case if they were simply lying on the surface. A cell may contain a single group, or so many as to conceal the nucleus or finally to burst the cell-wall, allowing the groups of gonococci to escape.

Until recently, culture of the gonococcus has been unsuccessful except on human blood-serum. Wertheim used culture-plates according to Koch's method, made with I part of human blood-serum and I or 2 parts of peptone, agar, or gelatin solution. His cultures were easily made and grew rapidly. Finger recommends a culture-medium composed of urine and agar, I part of the former to 2 of the latter. He finds that the gonococci do not grow well in a strongly alkaline medium, and that a temperature of $40^{\circ} \mathrm{C}$. $\left(\mathrm{IO}^{\circ} \mathrm{F}\right.$.) will destroy them if continued a number of hours.

(c) Value in Diagnosis. - The characteristics of the gonococci that distinguish them from other diplococci are their shape, their grouping in fours or multiples of four, their position in, as well as on and outside of, the cell, and their staining properties, including especially the readiness with which they lose their stain when treated with alcohol. When diplococci possessing these characteristics are present in large numbers, there is no doubt that they are gonococci; but there have becn found in urethral discharges diplococci which possess all these characteristics, but which yet are not capable of producing a gonorrhoea. They are, however, not common, are never present in large numbers, as is the rule with gonococci, and are usually associated with a larger number of other micro-organisms common to the urethra. They are 
probably present, and liable to be a source of error in diagnosis, in about 5 per cent. of all cases. Some believe this percentage should be much larger. The question is plainly a most difficult one to decide, since the only method yet found of making the differential diagnosis with absolute certainty lies in culture- and inoculation-experiments. These methods must have a narrow range of application, since the cultivation of the gonococcus is too laborious and difficult to be carried out in every case or by anyone but an expert; while inoculation is practically out of the question, since man is the only animal yet clearly proven to be susceptible to the disease.

The three following facts have been demonstrated clearly: I. Gonorrhœeal pus, or a pure culture of gonococci from such pus, produces gonorrhœe. 2. Pus free from gonococci, or a pure culture of micro-organisms found in such pus, does not produce gonorrhcea. 3. A slight subacute or chronic discharge containing a small number of gonococci may produce a true gonorrhœa; but a discharge of apparently the same nature, and containing diplococci that cannot be differentiated from gonococci, often fails to produce an infection of any kind.

In all cases of acute urethritis it is evident that the microscope is of great value in making a diagnosis, for the discovery of gonococci in the discharge at once decides it to be a gonorrhœe and infectious in nature (its immediate origin is not necessarily infectious; see Bastard Gonorrhaca), while an acute discharge that after repeated and thorough examinations on several successive days fails to show gonococci may safely be pronounced non-gonorrhœal. In a large class of subacute 
and chronic forms of urethritis the microscope renders valuable service, but often by its use alone the diagnosis cannot exactly be determined, and one must then rely largely on clinical conditions and experience to decide upon the infectious or non-infectious nature of the case in hand.

2. Pscudo-gonorrhara-A few cases are reported in which micro-organisms other than gonococci were apparently responsible for urethritis closely resembling a gonorrhœa, and in which culture- and inoculation-experiments were made by competent observers. These cases are rare, and not yet sufficiently understood to call for special consideration by the general practitioner.

3. Syphilitic Urethritis.-During the early stages of syphilis mucous patches may form in the urethra and be the source of a scarcely noticeable discharge. In a patient under treatment for recognized syphilis such a discharge is of little importance and calls for no special treatment or precautions, as it is assumed that no physician will allow a patient whom he is treating for syphilis to indulge in sexual intercourse. In unrecognized cases such a discharge might prove a source of infection. Further, a urethral chancre may furnish a discharge closely resembling that of gonorrhoea, and this mistake in diagnosis is frequently made as the result of careless examination. Manipulation of the parts should reveal the presence of the sclerosis.

4. Urethritis Tubcrculosa.-Primary tuberculosis of the urethra has been reported in rare instances. It occasionally occurs in the form of ulceration in generalized tuberculosis of the genito-urinary tract. The discharge from the urethra should show tubercle bacilli. The examination must be conducted with great care, 
and special methods must be employed, otherwise the smegma bacilli and other micro-organisms. found in the urethra and in the urine will lead to confusion and error.

II. Non-infectious Urethritis.-A mild or even a violent urethritis may follow mechanical or chemical violence to the urethra. It is difficult to decide how often the cause of these apparently frequent cases lies solely in the urethral injury, since other causes, on careful investigation, are often disclosed. The commonest source of error lies in the fact that a urethra damaged by a previous gonorrhœa may show no evidence of its diseased condition until it becomes the seat of urethritis as the result of an irritation that would have no effect upon a perfectly sound urethra. Some individuals of a strumous or gouty diathesis seem prone to urethritis on comparatively slight provocation, such as the passage of highly acrid urine, indulgence in beer or alcohol in any form, or ungratified sexual excitement. In the majority of these cases, however, there is a history of true gonorrhœe at some time in the past. Somewhere in such a urethra there probably has existed an area of congestion or thickening or a forming stricture. Those forms of urethritis due to syphilis and to tuberculosis have already been mentioned. But while a diathesis is frequently responsible for the readiness with which some men acquire the disease, and while the systemic condition often exerts a most important influence upon the course and duration of the local process, in the vast majority of cases the production of urethral inflammation requires the presence of an irritant acting locally.

Mechanical violence may produce an inflammation of a urethra that has previously been sound. This form of urethritis follows the improper use of sounds and 
other instruments by unskilled or careless operators, the introduction by the patient of foreign bodies into the urethra or the bladder, and occasionally the passage of fragments of calculi from the bladder. This form of urethritis usually develops within a few hours after the violence is done; its intensity is in direct proportion to the amount of mechanical damage inflicted upon the mucous membrane, and it generally disappears promptly on removing the cause, without further treatment than is necessary to keep the urine bland and unirritating to the injured surface.

Chemical aiolence, resulting from the use of too strong injections, from irritating vaginal secretions, from the internal use of cantharides, and possibly from too great concentration of the urine, may produce urethritis in varying degrees of severity. Here, again, the symptoms come on promptly without any period of incubation, and, as a rule, the cause having been removed, disappear rapidly under very simple treatment.

With these forms of urethral inflammation should be considered the cases of urethritis caused by irritating vaginal secretions and discharges in women in whom no trace of gonorrhœa can be recognized on examination. That some men under favorable circumstances do thus acquire a urethritis is unquestionably true. On the other hand, a married man who has had no previous disease of the urethra, who is otherwise well, and who is indulging in no sexual excesses, rarely acquires a urethritis from his wife, even though she have a leucorrhœal or menstrual flux. Even the discharges from a carcinoma of the uterine neck or from a tuberculous ulcer usually fail to injure the urethra of the husband. It would seem that these discharges are 
capable of causing urethritis only when there is a previously diseased urethra, a strumous diathesis, a debilitated condition of the body, prolonged sexual excitement, excesses in coitus, or, as most frequently happens, a combination of several of the conditions named.

Clinical experience shows that a woman, wife or mistress, may be incapable of giving urethritis to husband or lover, while a stranger may promptly acquire the disease in a single coitus with her. This is cited as proof of the so-called "acclimatization" of which Ricord and other writers speak. Not infrequently a newly-married man consults his physician for a mild or even violent urethritis, in great alarm lest his really innocent wife be not pure. If his urethra was previously sound (in the majority of such cases the man has had a gonorrhœa at some previous time), it is probable that his wife has leucorrhœa, or both have disregarded the beginning or the end of the menstrual flux, besides indulging to excess, possibly after partaking of alcoholic or other stimulating articles of food and drink. Such a urethritis subsides in a few days under simple treatment, and if in the future their sexual relations are properly regulated, the husband will in all probability remain free from any future attack.

It is in this class of cases that the tact as well as the skill of the physician is severely tested. He will be asked innumerable questions, and upon his answers may depend the integrity and future domestic relations of an entire household. It is far better to let the guilty escape, or to permit a patient to think that a successful imposition has been practised upon his physician, than falsely to accuse the innocent. With this end in view he will bear in mind the following facts: 
I. A healthy man with a sound urethra in all probability cannot acquire a urethritis from a healthy woman, even if he indulge to excess.

2. A healthy man with a sound urethra does not, as a rule, acquire a urethritis from a woman with vaginal secretions or discharges which are not gonorrhceal. To this rule, however, clinical experience furnishes some undoubted, and many apparent, exceptions.

3. There are instances in which a man of strumous, gouty, or tubercular diathesis, or with a debilitated general system, may acquire a mild form of urethritis from a healthy woman, particularly if sexual excesses with her be accompanied by other excesses, as at table. If the woman, instead of being healthy, has a leucorrhoea, urethritis follows more frequently and may be severe in type.

4. There are many men with a slightly damaged urethra, the only evidence of which may be a drop or two of muco-purulent discharge at the meatus in the morning, or the presence of small threads of pus in the urine, or, indeed, with no apparent symptoms, who are ready to light up anew a urethritis as a result of any of the causes above named. Slight disturbances of the general economy (such as bronchitis, constipation, or diarrhœe), the excessive use of tobacco, beer, or alcohol, violent exercise, and great fatigue sometimes suffice without sexual excitement for such relapses; while sexual indulgences of any nature are almost certain to be followed by a reappearance of the old trouble. These are cases of so-called bastard gonorrha. They are generally subacute in type from beginning to end, and may present no other symptoms than a slight discharge, with possibly moderate itching at the meatus. They 
usually run a short course, but they may be protracted, and if the irritation be sufficiently violent they may present any degree of inflammation up to that found in gonorrhœa. These cases are non-infectious in their immediate origin; but if, as is often true, gonococci were still present in such a damaged urethra, they may multiply under the added inflammation, and a true gonorrhœa, generally subacute, may result.

5. A woman may have a true gonorrhœa which the examining physician is unable to detect. This is especially true if the disease be limited to the urethra, and the acute stage, with swelling and redness of the parts, has passed; or, again, if there be left only a chronic and subacute inflammation of the vulvo-vaginal glands. Noeggerath and others have proved beyond dispute that the Fallopian tubes, the uterus, and the cervix uteri may be the seat of a true gonorrhoal inflammation and yet present no evidence that can be detected in a most careful examination of the external genitals and vagina. In the face of such evidence it is surprising that some physicians are willing, after examining public women, to give them a certificate to the effect that they have no venereal disease. Ninety per cent. of all pelvic diseases in women liave their origin, it is claimed, in gonorrhcea. From coitus with a woman having one of these unrecognized forms of gonorrheea some men will cscape, while others will acquire the disease.

6. The non-infectious forms of urethritis have no period of incubation, as in gonorrhœea, but promptly follow the operation of the exciting cause. They tend to a rapid recovery upon the removal of that cause, and are usually mild in type as compared with gonorrhoea, though they may exhibit a severe grade of inflamma- 


\section{SYPHILIS AND THE VEIEREAL DISEASES.}

tion, and, rarely, be protracted in course and clinically indistinguishable from true gonorrhœa. The microscope shows no gonococci.

Symptoms.-As a matter of convenience in description, the course of gonorrhoea is here studied in successive stages; these stages are not, however, always definite in duration or sharply defined one from the other.

I. Stage of Incubation.-At the time of infection the virus is conveyed to the sound membrane in a very small quantity-too small to cause immediately a perceptible irritation-and it is not until the gonococci have developed and multiplied that they or their products, or both, produce a visible disturbance in their new habitat. The time required for this development varies from one to fourteen days, but in fully two-thirds of all cases it is from five to seven days. This wide variation is undoubtedly due in part to the degree of virulence of the particular virus, to the character of the soil upon which it is implanted, and to other circumstances attending its inoculation; but the characteristics of the individual also play an important part. A highly sensitive man, and, in particular, one who is fearing and carefully watching for the result of an exposure, will detect the earliest slight symptoms, which would pass unnoticed by the average man; while among the careless and uncleanly the discharge may become pronounced before attention is directed to it.

When unmistakable symptoms appear before the third day, careful inquiry will usually elicit a history of more or less recent gonorrhcea from which the patient has really never fully recovered, though he may have supposed himself well. The case is then one of bastard 
gonorrhœa. In simple urethritis the period of incubation is wanting or is of only a few hours' duration.

2. Beginning or Prodromal Stage.-The stage of incubation may be said to end, and the prodromal stage to begin, when the patient first notices, especially on urinating, a slight teasing, pricking, ticliling, or uneasy sensation at the meatus urinarius. On examination the lips of the meatus may be slightly red, or, if the patient has not urinated for some time, they may be slightly stuck together by a drop of viscid mucus resembling in appearance the white of an egg. Without the aid of the microscope this fluid cannot be distinguished from the mucus secreted by the urethra and prostate during sexual excitement; the redness is no more than could come from accidental chafing or friction of the parts with the clothing; and in the majority of cases the diagnosis must be withheld for a day or two until the beginning of the active or increasing stage. If an early diagnosis is important, a drop of mucus should be squeezed out of the urethra and be examined for gonococci. If they are found, the diagnosis is established at once; but failure to find them will not warrant the exclusion of gonorrhoea until several slides have been examined on successive days.

3. Increasing Stage.-The itching or other sensation at the meatus now becomes one of slight burning or smarting on urinating, and gradually increases in severity. The secretion increases in quantity until it is sufficient to slightly stain the linen, and becomes thicker and opaque. The microscope shows pus-cells which increase in number each day. The lips of the meatus become more sensitive, red, swollen, and ererted, and as the disease progresses the entire glans and prepuce may 


\section{SYPHILIS AND THE IENEREAL DISEASES.}

gradually share in the inflammatory process. In an untreated case these symptoms gradually increase in severity for from eight to fifteen days after the beginning of this stage.

The discharge becomes purulent, at first milky, and then thicker and creamy in consistence. It grows darker in color until of a greenish yellow, the tint being due to the presence of a small amount of blood. If the mucous membrane becomes cracked, the blood may appear in the discharge in streaks, or there may be considerable hemorrhage. The discharge is often excessive; sometimes several drops appear at the meatus, and fall, if not wiped away, during the few moments occupied by the physician in examining the patient, to whom this constant dripping is a source of inconvenience and frequently of great mental distress.

The pain on urinating, which at first was slight, increases and may become agonizing in severity, causing the patient to retain his urine as long as possible. Often, when he does urinate he is afraid to permit the urine to pass in a full stream, since this would stretch the swollen and sensitive mucous membrane and thus cause more pain. Then, too, the calibre of the urethra is smaller than normal, in consequence of the thickening of its mucous membrane, and the stream of urine is thus diminished in diameter, divided, twisted, forked, and frequently interrupted. The urine may come only in drops, or there may be complete retention. This last is rare except in the case of an individual who had a stricture previous to his present attack. The pain, at first located near the meatus, now extends along the entire pendulous portion of the urethra, though it is usually most intense near the meatus, the fossa na- 
vicularis, or at the root of the penis. It is not limited to the time of urination, but is more or less constant. and it often radiates from the penis to the testicles, cord, perineum, groins, thighs, and back. There is also in these regions a feeling of weight and tension.

During this stage there is usually more or less sexual irritation, with painful erections and with seminal emissions which may be mixed with blood. The inflamed and thickened nucous membrane of the urethra is not capable of distention, and, moreover, the inflammation may extend to a part or all of the corpus spongiosum, filling up the meshes of its structure with plastic lymph and rendering them also incapable of distending to meet the demands of the elongated corpora cavernosa during erection. These two bodies above, which usually escape the inflammation, become distended with blood, elongated, and pull upon the inflamed and sensitive but now inelastic tissues of the urethra and the corpus spongiosum. The result is a torturing pain and a curving of the penis forward and downward, forming a bow of which the urethra is the taut string. At such a time the lips of the meatus may be seen drawn in, forming a funnel. This is chordic, and is especially apt to come on at night under the influence of the warmth of the bed. The sufferer will rise and by the application of heat or cold reduce the chordee, only to have it return when again he is warmed in bed. In this manner his rest at night is broken. Sometimes, becoming desperate, he attempts forcibly to "break" the cord by resting the penis on some hard substance and striking it with his fist. The usual result is sudden hemorrhage, and later the formation of traumatic stricture.

The inflammation, at first limited to the lips of the 
meatus, extends until the entire glans is swollen, turgid, red, or even highly inflamed and excoriated. The prepuce becomes œedematous, and balanitis of mild or severe grade may be present. As a result phimosis and paraphimosis are frequent complications. The lymphatics of the penis may become inflamed, and may be felt as hard cords, usually about the size of a knitting-needle, but at times much larger, extending to the symphysis pubis. They are usually painful and sensitive, their course being marked by a red line. Occasionally these firm cords act upon the erect penis as the urethra does in chordee, producing a curvature in various directions. The inflammation of the lymphatics usually readily terminates in resolution and does not often call for serious consideration. The inguinal glands may be involved and become slightly swollen and tender, but suppuration is rare.

Constitutional symptoms are wanting except as they result from loss of sleep and from mental distress. The latter is often excessive, rendering a man totally unfit for his usual vocation.

4. Stationary Stage.-With good hygienic management the inflammation, after reaching its height, remains stationary for about a week, though under unfarorable surroundings and in unhealthy individuals this stage may be protracted for several weeks. Usually, at the end of the third week from the first appearance of the discharge the stage of decline begins.

5. Stage of Decline.-During this stage the symptoms gradually subside; the discharge grows less, until, at about the end of the fourth or fifth week, it is again represented by only a few drops daily of a sticky mucous discharge, which in the course of another week 
or two disappears entirely, and the patient is well except for a sensitive condition of the urethra that will probably persist for some weeks.

The foregoing description applies to an untreated, uncomplicated case of gonorrhoea in an otherwise healthy man, living under good hygienic conditions, who is not subjected to too much physical exertion, who is indulging in no excesses in the way of food, drink, or tobacco, and who avoids all sexual excitement. In such a case the duration of gonorrhøea, from beginning to end, is usually from five to eight weeks; but even in uncomplicated and typical cases the disease varies greatly in its duration and intensity. The increasing stage of the disease, usually about twenty days, may be prolonged for weeks; or after the inflammation has reached its height it may remain stationary for some weeks instead of a few days. Most frequently protracted, however, is the stage of decline. Instead of steadily and uniformly progressing to recovery, the disease may improve for a time and then remain unchanged, or the process may be lighted up afresh and recovery be delayed by a series of relapses.

Frequently, some indiscretion on the part of the patient after he considers himself practically well causes a return of acute symptoms, though they are rarely so severe in type as in the early stages of the disease. Each succeeding relapse is usually less severe than that preceding, but the prolongation of the inflammation increases the natural tendency of the disease to localize itself in a chronic form in the fossa navicularis, in the region of the bulb, or in any part of the urethra that has happened to suffer most severely during the acute process. Thus are left circumscribed areas of granulation or 
thickening of tissue which may result in chronic gleet and may finally go on to the formation of stricture.

The disease varies as widely in the intensity of its symptoms as in its duration. In very mild cases there may be, from beginning to end, almost no pain or other evidence of inflammation aside from the discharge, which may nevertheless be profuse. In the majority of skilfully treated cases of gonorrhoea the other symptoms rapidly subside, and the discharge remains the chief, if not the sole, source of trouble to the patient. In very severe cases, on the contrary, the constant and severe pain, increased by great sexual irritation, chordee, bloody seminal emissions, hemorrhages, and discomfort in urination, together with mental distress and loss of sleep, tell forcibly on the general health. If the disease extends to the posterior urethra-as it does in many cases-one or more serious complications (posterior urethritis, epididymitis, prostatitis, vesiculitis, or cystitis) may result.

The causes of this wide variation in the course of gonorrhce lie partly in the peculiarities of the individual, but largely in his hygenic surroundings. Syphilis, tuberculosis, scrofula, gout, malnutrition from any cause, and great fatigue, all tend to retard the recovery of the disease; while sexual excitement of any kind, the use of tobacco, alcohol, or stimulating foods, and too much or too vigorous physical exertion, as in walking, dancing, or riding, all tend to prolong and increase the severity of the inflammation. If a patient with gonorrhoea acquire a febrile disease, his urethral symptoms usually subside while the fever lasts, but return with the disappearance of the fever. Indigestion, constipation or diarrhcea, and other minor disturbances of the general health, as a common cold, usually delay and aggravate the 
course of gonorrhea. The first attack, particularly of a young man, is, as a rule, the most severe, but it is also most likely to terminate in complete recovery. Successive attacks may be less severe, but they are generally more protracted and obstinate and exhibit greater tendency to become chronic.

The symptoms of non-infectious urethritis may be nothing more than a few drops of muco-pus, possibly so small in amount as to cause no more than a slight gluing together of the lips of the meatus. Men of strumous or lithæmic diathesis, and particularly those having a slightly damaged urethra, present these subacute cases after excesses of any kind. From this low type of inflammation there are all gradations to that described as the acute stage of gonorrhcea, though severe cases are rare, and are usually the immediate result of chemical or mechanical violence to the urethra. Too frequently they result from local treatment for imaginary ills. The duration of acute symptoms is bricf, varying from a few hours to a few days.

Bastard gonorrla'a is usually subacute, presenting the symptoms found in the declining stage of gonorrhœa. Under simple treatment the urethra thus affected generally returns to its former condition in about ten days or two weeks. If, under the influence of the irritation, the gonococci which may have been lying quiescent in the urethra multiply sufficiently, the inflammation may be more acute in type, slow in reduction, and in no way different from a mild attack of gonorrhcea, except that the inflammation reaches its height more rapidly and the period of incubation is brief.

Pathology.-Our knowledge of the pathology of gonorrhœea is very imperfect, owing to the fact that in 


\section{S2 SYPHILIS AND THE IENEREAL DISEASES.}

this disease few opportunities are afforded for making histological and post-mortem examinations. Finger gives the following description of the gross appearances: "Urethritis constitutes an inflammation of the mucous membrane and submucous tissue, with all its characteristics, such as redness and swelling and secretion of a mucous, muco-purulent, or purulent discharge. The intensity of the inflammation will vary, and hence the mucous membrane presents different appearances. Sometimes the swelling will be slight and the injection dendritic; sometimes the redness and swelling will be very marked. The glands and follicles always appear to be affected early and intensely. They become swollen; their openings gape in the shape of a funnel. The inflammation also extends to the lumen of the glands, and even the parenchyma takes part in the inflammation and in the production of morbid secretion. If the lumen of the gland or follicle is now occluded by a firm plug of mucus or pus, retention of pus and the formation of cysts result. Desquamation of the epithelium and superficial losses of substance also take place at the mouth of the follicle, and if the process is severe may lead to small ulcerations (clap-ulcers). Deeper ulcers may also develop, perhaps, from the rupture of one or more cysts due to occlusion of the gland-openings. This early and intense implication of the glands explains the obstinacy of clap and its tendency to relapse. The latter is due to the persistence of the process, which has died out on the surface, in one or more glands, where the virus increases and may then be discharged upon the surface; perhaps because the secretion of the virus is increased by local and general irritating influences, such as coitus and excesses in Baccho." 
The histological changes have been studied chiefly in gonorrhœal inflammation of the conjunctiva and the rectum ; but, reasoning from analogy, it is probable that soon after a secretion containing gonococci is deposited upon the mucous membrane of the urethra, these micro-organisms find their way between the superficial cells to the deeper epithelial elements and to the upper layers of the connective tissue. Here they proliferate, their presence causing an irritation which produces an active hyperrmia with dilatation of the vessels and exudation of serum. The hyperæmia rapidly becomes an inflammation, with the appearance of large numbers of leucocytes and round cells in the tissues. The leucocytes make their way to the surface, carrying with them large numbers of gonococci. This they continue to do throughout the entire course of the disease, until the invading microorganisms are entirely removed. The round-cell infiltration and other evidences of inflammation now disappear, new epithelial cells take the place of those that have been destroyed and exfoliated, and the process is at an end.

It is not usual for the mucous membrane in all parts of the urethra to recover at once. The inflammation and the gonococci may linger for months or years in one or more glands, in the fossa navicularis, in the culde-sac of the bulb, or in some other portion that has happened to suffer more severely than others.

Diagnosis.-Examination of the Paticnt.-No physician will succeed in the treatment of venereal diseases who does not habitually make thorough and careful examinations. With this end in view he should obtain a fairly good knowledge of his patient's general condition and history-the more complete the better-before coming to the consideration of the local trouble. Other- 
wise this information will be acquired, if at all, in unsatisfactory and detached fragments, and quite probably so late as to necessitate changes in instructions and treatment already given. This not only is a waste of time, but it leads to confusion in the mind of the patient and does not increase his confidence in his physician. Besides learning the general state of the health, the physician should be informed regarding the habits of eating, drinking, tobacco-usage, sleep, exercise, and all hygienic surroundings. Exact history of any previous venereal diseases should be obtained.

In the local examination thoroughness is imperative. No intelligent practitioner or student of medicine will order treatment for a man on the strength of the statement that he has nothing but a discharge or a "running" from the urethra. Nor is it sufficient merely to look at the discharge. The clothing should be removed, and in the majority of cases the fingers of the examiner should first seek the inguinal region. If he finds indurated, insensitive glands on one or both sides, he will search for the initial lesion of syphilis, which he may find in the form of a urethral chancre or of a sclerosis so trifling that it would pass unnoticed but for the information obtained by the fingers in the groin. If he find a single gland enlarged and sensitive, he will suspect the presence of chancroid, of a lymphangitis, or of some other complication not frequent in gonorrhoea. The fingers will next explore the testicles, where the presence of inclurated nodules in the epididymis may tell of previous attacks of gonorrheea or of syphilis which have been denied by the patient and which call for considcration during the treatment of the present disorder.

such an examination of the groins and testicles, if 
made at the outset, requires but a few scconds, but if left until the fingers are soiled-as they are quite liable to be -in the examination of the penis, these regions may be neglected. The discharge has led the examiner to make a diagnosis of gonorrhøea or urethritis ; the patient complains of no pain in the groins or in the testicles, and their exploration calls for the time and trouble involved in an extra washing of the hands. An initial sclerosis, acquired at the same time with the gonorrhœa or independently of it, may thus be overlooked, or the patient may return in a few days with a violent epididymitis which might have been prevented had the evidence of former attacks been noted and proper precautions been taken. If the prepuce be long or in a state of phimosis, the physician will make every effort-cleansing the parts, if necessary - to determine that the discharge comes from the urethra, and not solely from the preputial sac affected with balanitis; it may come from both. He will also, by manipulating the parts, satisfy himself, as far as possible, regarding the nature of any other complications that may be present.

Differential Diagnosis.-A urethral inflammation is probably one of three conditions-gonorrhoea, bastard gonorrhœe, or simple urethritis. The first is the most common, constituting a large majority of all cases; the second is of frequent occurrence; while non-infectious urethritis pure and simple, occurring in a man whose urethra was previously sound, is unusual. It is evident that the diagnosis between bastard gonorrhoea and simple urethritis will often be made with great difficulty. If repeated examinations fail to find gonococci in the discharge, the diagnosis must rest upon the history of the patient. If he has had former urethral discharges, 
it is safe to assume that his urethra had never fully recovered from the earlier attacks. In these cases an accurate diagnosis, so far as the immediate treatment is concerned, is not essential, for in either case the discharge usually subsides in a few days under simple treatment; the diagnosis, however, is of importance in the matter of prognosis and in determining the proper course to be pursued in the future.

The average patient can with difficulty wait for the conclusion of the examination before asking to be informed definitely if he has true gonorrhœa or if his discharge results from what is popularly known as "a strain" or from contact with innocent vaginal discharges. In almost the same breath he will probably ask how long a time is required for "curing" him. These questions can often be answered at once, but frequently they call for the exercise of much tact, good judgment, and good sense on the part of the physician. The man who indulges in illegitimate sexual relations with a mistress or with a woman generally supposed to be respectable is loath to believe that he is infected. He is often positive in his declarations that he has not been exposed to a venereal disease. Egotism and ignorance give him faith in a woman he personally knows to be immoral. The man, further, who indulges in promiscuous sexual relations, relying for his safety upon artificial methods and derices for avoiding disease, is slow to realize that his precautions have failed to protect him. Such measures are certain to fail sooner or later.

On the contrary, the young practitioner is inclined to be too incredulous regarding urethral discharges declared to have been innocently acquired, and rarely properly appreciates the fact that a large proportion 
(possibly one-third) of the seemingly acute and subacute cases are in reality exacerbations, wrought by some of the causes already discussed, of a pre-existing chronic disease. While in the majority of cases the diagnosis may be made with precision on the first examination, especially if the microscope be used, there are times when the prudent physician will postpone his decision for a few days, and will consider carefully the individuality and surroundings of his patient before answering the questions put to him. His aim must be to relieve the suffering and to protect the innocent.

The diagnosis practically lies between gonorrhoea, bastard gonorrhœa, and a simple urethritis. Their causes, characteristics, and symptoms need but brief review here. For convenience they are set forth in tabular form.

\section{Gonorrhar.}

History of former attacks not necessary.

The patient's general condition has no bearing on the origin of the disease, but may exert a marked influence on its course.

Urethra may have been sound at the time of infection.

Cause of disease lies solely in exposure to a gonor. rhœal discharge.
Bastard Gonorrhar.

The patient has had former attacks.

Enfeebled constitution is often a factor in the production of the disease, and frequently exerts a decided influence on its course.

Damaged condition of urethra necessary. Generally evidenced by gluing of lips of meatus in the morning, or by shreds in the urine. Patient may have seen no evidence of disease for months, and believed himself well.

Immediate cause usually found in sexual indulgence, with or without other excesses. Occasionally other excesses are alone sufficient.

\section{Non-infectious L rethritis.}

History of former attack not necessary.

Enfeebled constitution is sometimes the chief factor in the production of the disease, and may even be the sole cause of it. continuance.

Damaged condition of urethra not necessary, but probably present in many cases.

Cause found in direct mechanical or chemical violence to the urethra, often the result of too energetic treatment. In the cachectic and debilitated, sexual and other excesses may suffice. 
Gonorrata.

Period of incubation ranges from one to fourteen days, rarely less than three, and usually from five to seven.

Begins with slight symp. toms, which gradually increase in severity for from ten to fourteen days, when the inflammation is usually of a very high grade.

Duration is usually from five to eight weeks. Acute stage lasts three or four weeks.

Discharge contains gonococci in large number.

Bastard Gonorrha. Won-infectious L rethritis.

Period of incubation usually one or two day's.

Usually subacute throughout. When more acute, the symptoms increase in severity more rapidly, but rarely become so severe as in gonorrhcea.

Duration very indefiniteperhaps one or many weeks. Acute stage usually lasts a week or ten day's.

Gonococci usually present in small number.
No period of incubation. Symptoms usually appear in a few hours.

Inflammation reaches its height in a few hours. If resulting frum marked violence to the urethra, symptoms may be very severe; otherwise it is usually subacute in type.

Actute forms usually recover in a few days, as do the subacute cases, though the latter may continue in chronic form.

No gonococci.

The microscopic examination should be conducted with great care, all instruments, preparations, slides, and cover-glasses being scrupulously clean. If the first slide examined shows typical gonococci in abundance, and all the other evidence points to a gonorrhoea, there can be no doubt of the diagnosis; but if the first slide fails to show gonococci, at least two or three others must be properly prepared and examined before excluding gonorrhcea. In very early stages, while the discharge is yet muco-purulent, the number of gonococci present is much smaller, and several slides should be examined on successive days before deciding that gonorrhoea is not present. In the declining stage it is even more difficult to find gonococci, as they are present in smaller number and are not infrequently associated with other micro-organisms which lead to error in diagnosis. Even during the purulent stage the gonococci are not evenly distributed through the discharge, but are usually most abundant in those drops which come from a portion of 
the surface most recently inflamed; consequently it is always best, for purposes of examination, to squeeze out a drop from the deeper portions of the urethra, instead of taking that which happens to be at the meatus. In a true gonorrhcea, however, the gonococci are usually present in sufficient number to render their demonstration comparatively easy, and the discovery of only a few diplococci in an acute urethritis does not warrant a diagnosis of gonorrhcea. The dried gonorrhocal discharge found on clothes or the underwear of a patient, if moistened, removed, and properly stained, may show gonococci.

Besides the gonococci and the pus-cells containing them, the microscope shows, in the earlier discharge, many flat epithelial cells. These cells gradually disappear, and the field is completely filled with pus-cells, which in turn become less numerous as the discharge decreases, and, instead of the flat epithelium of the early stage, cells of transition epithelium are seen, often in considerable numbers.

In the examination of any urethral discharge the possible presence of posterior urethritis should always be kept in mind. If the urine be given a careful examination and the two-glass method be employed, the diagnosis will not be difficult.

Treatment.-Prophylaris.-American and Fnglish writers have been criticized for neglecting to discuss prophylaxis in their works on gonorrhoea. At first thought such a discussion would seem superfluous, but there undoubtedly exists among the laity, and, unfortunately, among some physicians, the belief that there are means by which a man may protect himself from venereal diseases while indulging in promiscuous sexual 
relations. Of the various devices and methods recommended and employed, there are none that can be trusted to ensure the desired end. The large majority of men who resort to these means in illicit sexual relations sooner or later become infected with disease. The only prophylaxis a right-minded physician can advise is clean living.

Goncral Considerations.-In the entire range of medicine there are few diseases in which so many remedies have been tried, and in which so many methods of treatment have been advocated, as in gonorrhœa. An attempt to describe them all would be useless. In these pages space is given only to such methods of treatment as experience has proved to be of value in mitigating the violence or in shortening the duration of the disease. These methods, unless otherwise indicated, relate to the - treatment of true gonorrhcea. The discovery of gonococci in a discharge makes it possible to predict, within certain bounds, the intensity and duration of the disorder, demonstrates its contagious nature, and calls for much care and caution not necessary in the management of a non-infectious urethritis. From a therapeutic point of view, however, no important distinction is made between the different forms, an inflammation of a given intensity calling for a given treatment. The duration of any one stage in the non-infectious forms is, of course, brief as compared with a corresponding stage of gonorrhea, and much treatment other than hygienic is rarely called for. Usually, removal of the cause, rest, and perhaps an alkali to ensure a bland condition of the urine, are all that are necessary. In bastard gonorrhœa the symptoms are usually subacute, though they may be severe. The treatment is that recommended for cor- 
responding stages and degrees of inflammation in gonorrhœa.

Abortive Treatment.-Many abortive measures for the treatment of gonorrhœa have been recommended by reputable physicians, and "rapid cures" are regularly advertised in the public press. But these novel methods that promise quick results are not unattended by danger. The practitioner who is strongly tempted to try the last highly recommended local treatment in the hope of benefiting his patient should recognize these dangers and should remember that there is a great difference between checking a discharge and curing the disease.

Since the recognition by Neisser of the gonococcus as the essential cause of gonorrhoea, numerous efforts have been made to discover some means of destroying the micro-organism and of thus preventing the further extension and continuation of the disease. Many agents are capable of at once destroying the gonococci when brought in contact with them, but they also either prove so destructive to tissue or so aggravate the existing inflammation that the resulting damage to the urethra is greater than that produced by the gonococcus. Injections of strong solutions of nitrate of silver or of bichloride of mercury or of other preparations have in some instances destroyed the micro-organism, and after causing an intense inflammation of the mucous membrane have seemed to shorten the duration of the disease. More frequently such treatment has failed to destroy all the gonococci, and has resulted in complications which prolong the disease and add to its severity.

The investigations of Bumm and others have shown that the gonococci rapidly find their way beneath the epithelium to the papillary body and to the lymph-spaces 
of the upper connective-tissue layers. Here they multiply and are brought to the surface by the leucocytes. It is not strange, then, that abortive measures fail, since parasiticides cannot be brought in contact with the more deeply seated gonococci without first destroying the epithelium. A local application that will meet the three requirements of Neisser-that is, a remedy that will kill the gonococcus, leave the mucous membrane uninjured, and not increase the inflammation-is yet to be discovered. As a consequence, up to the present time all attempts at abortive treatment have been failures. The cases reported to have been aborted by local treatment were possibly of the class of non-infectious urethritis or bastard gonorrhœe, that would in any event have run a brief course. Others were perhaps cases of true gonorrhcea, dismissed and reported as cured as soon as the discharge ceased. It is now known that the urethra may not return to its normal condition for weeks after the discharge disappears, and that often such cessation of the discharge proves to be only temporary.

Local Treatmont in the Early. Stages of Gonorrhaca.-Regarding the propriety of using local treatment in the acute stage of gonorrhoea authorities widely differ. The treatment of gonorrhøea will at some future date, perhaps, be purely local, but there has not yet been found a method of local treatment in the acute stages of gonorrhoea that is completely efficacious, or even wholly safe, in the hands of any but the expert.

The method by irrigation, which once promised so much and which had so many enthusiastic adrocates, is rapidly becoming a thing of the past. When properly pursued, and with mild solutions, it served admirably in cleansing the urethra, in subduing to some extent the in- 
flammation, and in modifying the symptoms. The discharge frequently ceased after two weeks of treatment; but that irrigation restores the urethra to a healthy condition sooner than other forms of treatment has not been proved. It is a difficult method to pursue, except where the patient is under complete control, as in a hospital, and in any but the most skilful hands it often results in complications (epididymitis, prostatitis, posterior urethritis, etc.) necessitating the cessation of treatment. Recently, Finger found that pure cultures of the gonococcus could be exposed for two minutes to the action of bichloride of mercury (I : 5000), carbolic acid (I : 1000), potassium permanganate ( $1: 1000)$, or nitrate of silver (I:I000), and yet grow when transferred to plate-cultures. This being true, it is apparent that irrigation as usually employed would not destroy the gonococci in the urethra, but would simply remove such as were on the surface.

Powders and soluble suppositories are not to be considered. They almost invariably do harm. Mild astringent and cleansing injections are, however, still largely used, even in the early stages of gonorrhoea. They very rapidly lessen the amount of discharge; clinical experience, however, shows that they fail to shorten the duration of the disease, but rather tend to prolong it in a subacute stage. Certain it is that disagreeable and serious complications occur much more frequently in cases treated by injections from the start than in cases in which no injection is used until the most active stage of inflammation has passed. The substances injected are either antiparasitic or astringent, or both. The parasiticide may destroy some of the gonococci, but it cannot reach many of them during the early stages; the astringent may; and probably does, lessen the intensity of the inflammation, 
but it is questionable if it is best to push this effect too far, as inflammation and suppuration are nature's methods of removing the offending micro-organism, which as yet cannot be destroyed by any more rapid process. One cannot hope to eradicate the disease before its cause has been removed. If Metschnikoff's theory of inflammation be accepted, and the leucocytes be viewed as phagocytes, it certainly is not desirable by constringing the vessels to limit the number of such leucocytes, whose duty it is to devour and remove the gonococci. Metschnikoff's theory is both interesting and plausible; applied to urethral inflammation, it is sustained by abundant clinical evidence showing that when the inflammation and discharge are suppressed by the use of injections in the early stages of gonorrhcea, the disease runs a mild but protracted course, and the last lingering discharge shows gonococci for a longer period than in cases which have had the usual two or three weeks of acute inflammation followed by a period of steady but more rapid decline.

The danger in this direction, however, is possibly not so great or so frequent as that resulting from the use of irritating injections which increase the inflammation to such an extent that posterior urethritis, epididymitis, prostatitis, cystitis, or vesiculitis results. These complications are often very painful, necessitating the suspension of all treatment for the gonorrhœa, and frequently compelling the patient to remain in bed for days or weeks. Unless the physician has had large experience in this class of cases, and has his patient under his immediate control, local treatment of gonorrhcea should not be begun until the active has passed into the subacute stage; and many patients will make a complete and satisfactory recovery without receiving at any time local treatment. 
Hygienic Management.-There is practical unanimity of opinion on at least one point in the treatment of gonorrhœa-namely, that proper hygienic surroundings are of great importance. There is no better treatment for the large majority of all first attacks of gonorrhœa than rest in bed for three or four weeks, with absolute quiet, freedom from sexual excitement, a light and simple diet, a daily movement of the bowels, and a proper performance of the other bodily functions. Unfortunately, it is rare that such a course can be pursued. Few men with a gonorrhœe are willing, unless compelled to do so, to abandon their usual vocation, and many, through fear of disclosing their condition, will continue their work notwithstanding most painful complications and contrary to their physician's orders. Through fear of betraying his secret to his companions or to members of his household a man will often fail to obey the instructions given him, and refuse to change his habits to meet the requirements of his case.

One of the first points, therefore, to be secured in the management of a case of gonorrhœea is a complete understanding on the part of a patient that it is necessary for him to carry out faithfully the instructions given, and that he is thus responsible, to a great extent, for the result. This is not usually an easy matter, as the idea is often firmly fixed in the mind of the patient that an injection will accomplish all that is necessary, with little or no effort on his part. The practitioner who takes the time and pains to point out the truth will have far better results, and meet with much less annoyance, than if he devote his time to making trial of the last highly recommended and best-advertised injections. The management of gonorrhœa cannot be made a matter of routine, 
but requires always some consideration of the individual and his surroundings. Directions should be given clearly and in detail.

(a) Rest.-The patient should spend all the time possible in the recumbent position. This is of special service during the early acute stages, since, by removing the pressure of blood from above, it lessens the congestion of the parts. All violent exercise, such as gymnastics, running, dancing, and horseback- or bicycle-riding, should not be practised, and even walking or much standing is harmful.

(b) Food.-The more nearly the patient can restrict himself to bread and water or bread and milk, the better; but if he must continue his usual work or a portion of it, or if he be already poorly nourished, a more nutritious diet is necessary. Such he may find in fish, the lighter meats, soft-boiled eggs, and cooked vegetables. As a rule, he should avoid all articles difficult of digestion, all rich or highly seasoned food, and all acids, sweets, and especially fats.

(c) Beierages.-Alcohol in all forms, and especially in the form of malt liquors, is prohibited. Coffee, chocolate, and cocoa are injurious, but tea in moderation may be allowed. Nilk is of value, and may be given freely if it agrees with the individual. Pure water does excellent service if drunk in quantities sufficient to keep the urine bland and unirritating. Smaller amounts of Vichy, seltzer, or other alkaline waters answer the same purpose. If there be much burning on urinating, and frequent desire to pass urine, a thin, strained, flaxsced-tea (made from the whole seed and rendered palatable by the addition of a small quantity of lemon-peel) will often give relief if drunk in quantities of a quart or 
more daily. Lemonade is usually agrecable to the patient, and, like some other vegetable acids, is occasionally of service in rendering the urine alkaline, the more so if a small quantity of bicarbonate of soda be added; but it does not answer equally well in all cases, and sometimes proves decidedly irritating.

(d) Tobacco in all forms should be avoided. Smoking is especially bad.

(e) All sexual relations and stimulation of the sexual organs are harmful, and the patient should avoid company, books, pictures, thoughts, and circumstances that might result in sexual excitement. He must determine to do this at once, since the congestion and inflammation of the parts, due to his gonorrhœea, are alone sufficient to keep them in a state of irritation. For the purpose of lessening the tendency to congestion of the genitals, there should be a daily evacuation of the bowels by the aid of saline laxatives. The patient should sleep in a cool room, on a hard mattress, and without too much covering; the married man should not occupy the same bed with his wife. The immersion of the genitals in water as hot as can be tolerated, and for a few seconds only, just before retiring, often reduces the frequency of annoying and painful erections. A longer use of cold water may answer the same purpose, and both means are valuable in reducing erections which awaken the patient during the night. On the contrary, prolonged hot or warm baths are harmful in that they encourage the local congestion. Urinating while the penis is immersed in hot water usually renders that act less painful.

(f) Dressing of the Parts.-Cleanliness is of first importance; the parts should be washed daily in warm 
water, using soap on all but the inflamed surfaces, and, of course, keeping it out of the urethra. The patient should wash his hands after each dressing, and should be instructed regarding the great danger attending the contact of the smallest amount of the discharge with the eyes. To catch the discharge and to protect the patient's clothing, the penis may be carried in a light muslin bag containing in the bottom a small quantity of cotton, the bag being fastened by means of tape to a suspensory bandage or to a band about the waist. A simple and very satisfactory method is to take two thicknesses of ordinary muslin, about a foot square, and so pin them to the inner surface of the undershirt that the penis can be gathered up in their folds. These cloths are cheap, and can easily and quickly be removed, burned, and replaced by fresh pieces-if necessary, several times during the day. If desired, a pair of swimming-drawers can be worn beneath the other underwear, and will serve to hold the muslin in better position. Keyes recommends wrapping the penis in two sheets of ordinary toilet paper and twisting the free end, forming a paper bag in which to catch the discharge.

Rubber and oiled-silk bags, and all heavy wrappings of the penis, are decidedly harmful, in that they tend to keep the organ hot and congested. Dressings should never be tied to or about the penis, since they interfere with the circulation and usually cause a troublesome oelema of the prepuce. Nor, for catching the discharge, are pieces of cotton or other dressings held in place by a long prepuce to be recommended, since they imprison the pus within the urethra, and also keep it in contact with the sensitive mucous membrane of the glans and prepuce, thus often exciting a balanitis. 
If the patient is required to be active or on his feet much of the time, and particularly if with a former gonorrhœe he has had an epididymitis, he should wear a well-fitting suspensory bandage. The first object of a suspensory is to support and elevate the scrotum and the testicles, relieving the tension on the cord, at the same time slightly lessening the blood-pressure and aiding the return circulation. This object can be accomplished by a single bag of proper depth and width to fit the parts, suspended only from a waistband. The bag must not be too deep, or it will not furnish support; while if too shallow it will exert uneven pressure and will slip off, not retaining the parts. Frequently a bag that does not fit well can be made to do so by lining and filling out some parts of it with antiseptic wool. Cotton is not so good, as it soon mats and becomes hard.

This simple suspension of the genitals may be sufficient in many cases, but if there be the slighest tendency to involvement of the cord or the testicles, another object is to be accomplished-the subjection of the testicles to as little motion as possible in walking or during other movements. For this purpose the suspensory bandage described above will need, in addition, some perineal or thigh-straps that will hold the organs snugly against the symphysis. In order to do good, and not harm, a suspensory must fit snugly, but must not be too tight, nor should it press upon any portion of the urethra. It is usually most serviceable when it is most comfortable to the patient. Instead of a suspensory bandage a jockstrap may be used, though it is not so convenient and is usually more difficult to fit properly. Tight-fitting swimming-drawers of firm material often answer every purpose. An excellent substitute for any of the pre- 
ceding may easily be made as follows: Pass a bandage, of such material and width as will be comfortable, around the waist for a belt; take a strip of soft linen or several thicknesses of cheese-cloth or mull, about eight inches wide, and fasten one end of this to the middle of the belt behind with safety-pins; bring it between the buttocks, over the perineum, and up to the belt in front, where, by fastening at several points, it can be made to fit the genitals, elevating them and holding them close against the body.

Intcrnal Treatment.-In the early stages hygienic management is of first importance. Fournier and other French authorities advocate no other treatment internally or locally until the stage of decline. This is the socalled "expectant plan." While the use of large doses of the balsams with a view to aborting the disease is not to be recommended, its early stages may be rendered much less severe, and the entire course of gonorrhœa be shortened, by the proper use of internal remedies.

Alkalies and diurctics are of decided value in keeping the urine bland and unirritating, and should be used in sufficient doses throughout the disease to accomplish that end, unless the fluids daily ingested prove sufficient for the purpose. Excellent results are obtained from the use of bicarbonate of soda or of citrate of potash in doses of from 5 to 20 grains three or four times daily; the latter acts also as a diuretic, and is in some cases to be preferred. The quantity required will vary from day to day, enough being given to keep the urine alkaline. If taken about two hours after eating, the effect upon the urine is more pronounced than if given at any other time, and interference with digestion is not so marked as when taken immediately after eating. Consequently it is often well to prescribe these remedies in the form 
of compressed tablets of 5 grains each, that can casily be carried in the pocket and swallowed at any time with a glass of water. They may also be given in simple solution, in peppermint-water, or with any flavoring desired.

Balsam of copaiba, if easily digested by the patient, is one of the most valuable of drugs, but there are unquestionably certain individuals who cannot digest it. It should never be ordered if it disturbs digestion and nauseates so that the patient cannot eat, though many who at first had some difficulty in its digestion soon manage very well if it be taken in small but gradually increasing doses an hour or two after eating (occasionally some other hour will be found to be better), or if some preparation of pepsin be taken with it. Occasionally a few doses of the drug cause the appearance of an exanthem known as the copaiba rash. This is a bright red eruption of macules or maculo-papules over the abdomen and the extremities, or even over the entire skin-surface. The eruption appears rapidly and is accompanied by pruritus. It is a trifling complication, disappearing promptly on withdrawal of the copaiba, but it is a source of annoyance to the patient and necessitates the abandonment of the drug.

If copaiba is digested reasonably well, it is usually of great service and may be given from the beginning, though if digested with some difficulty its use should be postponed until the stationary stage, at which time it is of pronounced value. Many object to the use of the balsamic preparations in the early stages of gonorrhœa, on the ground that they are stimulating to the mucous membrane. Clinical experience, however, teaches that copaiba very markedly lessens not only the discharge, but also the painful and distressing symptoms 
of the acute stages, and, moreover, shortens the duration of the disease. Finger states that he believes copaiba taken internally acts in the urine as a parasiticide, destroying some of the gonococci. The action of copaiba (and allied drugs, such as sandalwood and cubebs) is undoubtedly local, and is due either to the drug itself or to the products of its metamorphosis present in the urine as it passes over the mucous membrane of the urethra. This fact was demonstrated by Ricord and Roquette in patients with urethral fistulæ who acquired gonorrhœa. While taking copaiba internally the portion of the urethra, back of the fistula, that was washed by the urine showed marked improvement, while no change was apparent in the part anterior to the fistula, and through which no urine passed. The patient continuing the internal use of the balsam, some of his urine was injected into this anterior portion of his urethra, the result being a lessening of the inflammation.

In examining the urine of a patient who is taking copaiba it must be remembered that the mineral acids produce with it a flocculent precipitate which can easily be mistaken for albumin. This precipitate is soluble in an excess of acid or in alcohol. Copaiba is best given in the form of the balsam, either in capsules of 10 minims each or in one of the following preparations:

R. Balsam. copaibæ, 亏ेss-ij ; Syr. tolutani, $\overline{3} \mathrm{j}-\mathrm{ij}$;

Acacia pulvis, Sacchar. albi, $\bar{a} \bar{a}$. q. s. ad ft. emuls. Lavand. spirit., 3j ; Aqua destill., q.s. ad $\overline{\tilde{s}} \mathrm{vj} .-\mathrm{M}$.

Ft. emulsio.

Sig. Teaspoonful dose. 
R. Potassæ citratis,

Balsam. copaibre,

Extr. fl. hyoscyami,

Syr. acacix,

Aqua menth. pip., q. s. ad ziij.-M.

Sig. Shake. Teaspoonful in water.
$5 i j-v j$;

5iij-vj ;

5ss-zij ;

亏̈iss ;

The last preparation is recommended by Keyes, who adds that "The mixture may be largely modified by substituting sandalwood oil for copaiba, leaving out the hyoscyamus when not required, substituting bicarbonate of soda for the citrate of potash if the diuretic effect is not desired, and wintergreen-water for mint-water, or even adding licorice, according to taste." Oil of sandalwood or oil of cubebs may be added to either of the above emulsions if desired.

The remarks made with reference to copaiba apply in the main to the oil of sandalwood. The latter is, however, more easily digested and does not produce an exanthem, though it may cause congestion of the kidneys with resulting characteristic pains in the back and the loins. In a majority of cases, owing to the uncertainty of obtaining the pure drug, less uniform results are obtained from the oil of sandalwood than from copaiba; but while it is not so efficacious in subduing the painful symptoms of the acute stages, it often seems more effective in reducing a subacute discharge. It should be tried in the acute stages instead of copaiba when the latter is not tolerated. It is best given in capsules each of Io minims; or it may be dropped on lump-sugar, in which case an ounce of sandalwood oil may be flavored by adding to it 10 or 15 drops of the oil of wintergreen or the oil of peppermint; or it may be substituted for 
copaiba in either of the emulsions for which formulas have been given.

Preparations of cubcbs are, as a rule, too stimulating to be given while the inflammatory symptoms are at all marked. They are of great service in checking the last drops of a lingering discharge, either at the close of an acute case or in subacute and clironic cases. They usually cause no disturbance in digestion, and they frequently act as tonics to the stomach. The best preparation, if fresh, is the powder. It should be given in doses of from Io grains to 2 drachms. When desired by the patient, it nray be given in capsules or be administered in some syrupy or mucilaginous drink. The oleoresin in Io-minim capsules, from one to three at a dose, is perhaps as good as the powder; while the fluid extract in from Io-minim to drachm doses often gives good results. In stubborn cases of subacute and torpid type it is advisable to make occasional changes in the preparations given. Good results are often obtained from a combination of cubebs and sandalwood, with the addition sometimes of copaiba.

In prescribing any one or all of these preparations several rules should be observed. A dose on retiring for the night should be given, in addition to that after each meal, thus keeping the urine constantly under the influence of the drug. The doses should be small at first, particularly in the early stages, and should gradually be increased up to the full tolerance of the patient's stomach, or until the desired effect is produced; for if this can be accomplished in smaller, it will, of course, be worse than useless to give larger doses. If, after a week or two of full doses, no benefit has been derived from the chug (provided always that the patient has had proper hygienic 
management and surroundings), or if the patient's stomach begin to rebel, a change to one of the other preparations should be made. No one of these remedies, particularly copaiba, should be given continually through too long a period. If, at the end of one, two, three, or four weeks, symptoms of gastric disturbance begin to appear, or if, in the case of sandalwood, there are indications of congestion of the kidneys, such as pain, or a sense of burning and oppression in the loins, another drug should at once be substituted.

Treatment of Successive Stages of Gonorrhad.-Attention to all the details of hygienic management are of the greatest importance in all the stages of gonorrhoea, and have been fully discussed in the preceding pages.

(a) Prodromal Stage.--It is not often that the physician has a chance to observe this stage of the disease, which usually lasts but a day or two, and passes unnoticed by the average man unless he is watching himself in fear of the possible result of a suspicious intercourse. If there is a clear history of exposure followed by a period of incubation, and certainly if there can be expressed a drop of mucus in which there are a few gonococci, gonorrhoea will undoubtedly follow, and the patient should be put under hygienic treatment at once, with the addition of an alkali internally. If circumstances point rather to the presence of a non-infectious urethritis, the same treatment will do no harm, though it may not be necessary to interfere to the same extent with the patient's habits of living, removal of the cause if discovered, the administration of an alkali, and rest being in the majority of cases all the treatment that is necessary. The general condition of the patient may call for consideration. 
(b) Increasing Stage.-During this stage the patient with a well-managed case of gonorrhœea usually finds his chief source of anxiety in the urethral discharge, and looks upon its daily increasing amount with much apprehension. It is then that the physician is tempted to satisfy his patient by using methods that will promptly check the discharge, and in this course there may be danger. Often, by too vigorous local treatment the modification of the discharge is followed by complications so painful and distressing that the sufferer would gladly welcome a return to the discharge if he could be relieved from his new and serious symptoms. The treatment in this stage should therefore be directed more to the alleviation and, if possible, prevention of painful symptoms and complications, and to the general condition of the individual, than to the suppression of the discharge.

The pain and burning on urinating-ardor urinceshould be controlled, if possible, by drinking large quantities of fluid to dilute the urine, and, as already described, by the use of alkalies. This object will nearly or wholly be accomplished when the urine is kept alkaline. Some preparation of copaiba or of sandalwood may then be given in small doses. In cases where the pain is very great, much relief is afforded by immersing the penis in lot water during the act of urinating. If these means are found in any case to be impracticable, the fluid extract of hyoscyamus may be given with the alkali several times a day, in doses of from $I$ to 5 minims. The injection of a weak solution of cocaine just before urinating is recommended by some authors, but its use does not to any great extent lessen the amount of pain, and it must be remembered that deaths have been reported from the use of cocaine in the urethra. 
In cases of complete retcution or where the urine will pass in drops only, a good plan is to place the patient in a hot-water bath-a sitz-bath will answer-and allow him to stay there quietly for a few minutes, an hour if necessary, until the urine passes. This is a most effectual means of emptying the bladder without distress to the patient, and it usually enables him to urinate with much less difficulty the next time the act is attempted. Occasionally this procedure will not suffice, and a catheter must be used; this should be soft and small, in size about No. I2 or I4 of the French scale, and should be introduced with great care, after first injecting the urethra with warm olive oil, that its inflamed membrane may be damaged as little as possible. The operation is made still easier if, in addition to the above precautions, the catheter is passed while the patient reclines in the bath. A careless use of the catheter will damage the mucous membrane, add to its swollen condition, and therefore increase the urethral obstruction. If other means fail, ice in the rectum may be tried. It should be pulverized and put in a suitably shaped flannel bag. Finally, aspiration may be necessary. Complete retention is, however, unusual, unless the patient before acquiring his gonorrhœea had a stricture.

The treatment of chordce and other forms of sexual irritation attending gonorrhœe must be chiefly hygienic, and has already been considered, the requirements being absence of all sexual excitement, a light diet, regular daily evacuation of the bowels, sleeping in a cool room on a hard mattress without too much covering, and the immersion of the penis for a few seconds in hot water or for a longer period in cold water, just before retiring, to be repeated during the night if necessary to reduce pain- 
ful erections. If the patient is in the habit of sleeping on his back, he may lessen the congestion of the genitals by lying on his side. He can force himself to do this by tying a towel about his waist with the knot resting on his spine. If all these details are faithfully carried out, it is rare that camphor, belladonna, lupulin, bromide of potassium, and other so-called "anaphrodisiacs" will be needed, or, if tried, will be found beneficial. They are all unsatisfactory, and frequently disturb the functions of other organs of the body. Lupulin is probably the best of the list, and bromide of potassium the next, but to produce more than a moral effect they must be given in large doses.

The treatment of the complications that may occur during these and the succeeding stages of gonorrhoa is considered elsewhere.

(c) Stationary Stagc-When the inflammation has reached its acme it tends to persist unchanged for about a week or even longer. The treatment is that of the preceding stage, except that at this time the amount of copaiba or of sandalwood taken may be increased. Four doses a day should be ingested, beginning with about Io minims of the balsam of copaiba, or, if this is not well borne, Io minims of the oil of sandalwood, and the dose should gradually but steadily be increased until the symptoms improve or until the stomach will tolerate no more. Few can digest at a dose more than 20 or 30 minims of either of these preparations. When such quantities have been given for a week without producing favorable results, it is wise to change or to try a combination of the two; or, if the discharge continue and the inflammatory symptoms be not high, some preparation of cubebs may be added. Local treat ment is not yet indicated. 
(d) Stage of Decline.-If the discharge rapidly subsides, it is not wise to tax the stomach too severely with copaiba or sandalwood, for fear of having to suspend their use altogether; but if one of them has not already been pushed to the limit, now is the time to do so. As the discharge diminishes in amount and becomes less purulent and more mucous in character the copaiba may be dropped, and sandalwood oil or cubebs, or both, be substituted. If under this treatment the discharge entirely ceases, the remedy in use at the time should be continued for another ten days, gradually decreasing the quantity until at the end of the time the patient may be taking only a small dose at night. All medication may now be stopped, and if at the end of another two weeks there is no discharge or other symptoms, and the drop of mucus squeezed from the deep urethra on several successive days show no gonococci, the patient may be allowed to return gradually to his accustomed habits of living. He must, however, be cautious in beginning the use of alcohol and tobacco, and for the unmarried sexual intercourse promises an added danger for some time to come, while the married must be careful not to indulge to excess.

Injections. - The stage of decline is that which is most frequently prolonged, and if the discharge does not continue to diminish under hygienic management and internal treatment, injections may be used to advantage. Many habitually begin their use at the outset of this stage, but, as a rule, it is better to postpone the use of an injection until other treatment proves insufficient. While symptoms of inflammation persist, injections must be used, if at all, with the greatest caution, the strength of the liquid used being always in inverse proportion to the acuteness of the symptoms, otherwise there is danger of 
producing an exacerbation of the existing trouble. Better a slow but steady disappearance of the discharge than a series of relapses from too energetic treatment. Injections and other forms of local treatment should always be reserved as a last resort for a patient who has had a posterior urethritis, an epididymitis, a prostatitis, or a cystitis complicating his gonorrhcea at any time during its course. The appearance of one of these complications during such treatment calls for its immediate suspension.

Injections are used to greatest advantage when the discharge has been reduced to a thin muco-pus, or possibly to a few drops of mucus that appear only in the morning, but which refuse to disappear under other treatment. They are also indicated in cases of urethritis, of whatever origin, which run a subacute course and which do not respond to internal treatment and proper hygienic management. These statements do not sanction the use of an injection in every case in which the lips of the meatus are adherent in the morning, or its prolonged use in cases where a morning drop or two at the meatus will not disappear after an injection has been used for a fortnight, or in cases which are not being otherwise properly managed. Frequently one injection after another is tried, the treatment being persevered in for weeks with the hope of removing the last traces of a discharge. It is well to remember that in these cases the local treatment may keep up the irritation, and that many patients recover as the result of merely stopping the injections.

To inject the urethra properly calls for the right kind of a syringe and some skill, easily acquired by the majority, on the part of the patient. The syringe should hold from 2 to 4 drachms, though the urethra usually will hold less than two. The piston should fit tightly, to permit 
no leaking, but at the same time should move freely and easily within the barrel, and should have a ring at the end to receive the index finger. The nozzle should be a blunt cone, so that the tip, which should be perfectly smooth, will barely enter the meatus, and will not project into the urethra to irritate or damage the mucous membrane (Fig. 16). The syringe having a barrel of

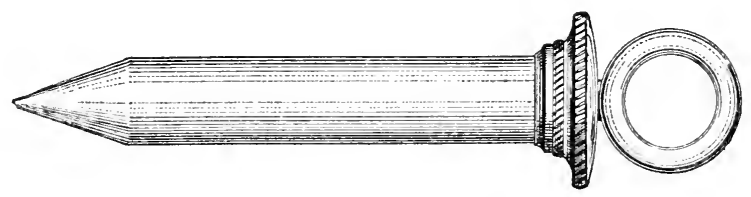

FIG. 16.-Urethral syringe.

hard rubber with a soft-rubber tip fulfils these requirements admirably. It is perhaps needless to add that the syringe should be scrupulously clean.

To inject successfully, the patient should encircle the penis, just back of the corona, with the thumb and the forefinger of the left hand, exerting no more pressure than is necessary to enable him to extend the organ to its full length as the fluid is forced in. The syringe should be held in the right hand, with the tip of the index finger in the ring at the end of the piston, while the barrel is firmly held between the other three fingers and the thumb. With the penis gently drawn out to its full length, and with the tip of the syringe pressing into the meatus with just sufficient firmness to prevent leakage, the fluid is slowly and steadily forced in until a slight ballooning or a feeling of fulness and tension in the urethra informs the patient that the urethra will hold no more. As the syringe is withdrawn the lips of the meatus are gently held together, retaining the medicament in the 


\section{I2 SYPHILIS AND THE IENEREAL DISEASES.}

urethra for about a minute before allowing it to escape. The whole procedure should be gentle; any forcing of the fluid back of the compressor urethræ muscle into the deep urethra and the bladder is to be avoided. Slight pressure upon the piston is not dangerous, and no more is necessary. Injections may be used once, twice, or even three times a day, the last on retiring for the night; but, should an injection cause much burning or pain, it must be allowed to escape at once, and before it is employed again the fluid must be diluted largely. The patient should always first urinate, thus cleansing the urethra before each injection.

Substances almost innumerable have been recommended and employed for urethral injections, but the skilful physician will select a few, usually not more than two or three, and learn to use them well. He becomes thoroughly familiar with their effects upon the varying stages and conditions of urethral inflammation, and accompishes better and more definite results if he uses these few remedies, in varying strength and frequency of application, than if he resort to others excellent in themselves, but with which he is less familiar. The following are among the best:

$\begin{array}{ll}\text { R. Liq. plumbi subacetatis dil., } & \bar{j} \mathrm{j} ; \\ \text { Morphize acetatis, } & \text { gr. j.-M. }\end{array}$

This formula is recommended by Keyes for use in lesser stages of inflammation. In subacute cases the following are good:

$\mathrm{R}$. Potassii permanganat.,

gr. ss $-\mathrm{j}$;

Aq. destill.,

亏j.-M. 
Or,

Or,

R. Zinci sulphat., Aq. destill.,

R. Acid. tannic., Aq. destill., gr. $\mathrm{j}-\mathrm{ij}$;

zjj. - M.

gr. ij-vj ;

亏ुj. $-\mathrm{M}$.

In ordering an injection it is well to advise at first a dilution with two or three times its bulk of water; if this be ineffectual, and yet produce no irritation, the strength may gradually be increased. On the contrary, no injection should be continued which causes more than a slight smarting sensation while in the urethra and possibly for a few minutes afterward. The idea of "cauterizing" the urethra or "burning out" the disease has long since been abandoned. Injections are further considered in connection with the treatment of Chronic Uretluritis.

Summary of Treatment in Stagcs.-(a) Prodromal Stagc.-Hygiene; alkali; guarded prognosis.

(b) Increasing Stage.-Hygiene; proper local dressing; proper use of local hot and cold baths; alkalies; balsam of copaiba if well digested; if not, sandalwood oil, the dose of either being from 5 to Io minims four times a day.

(c) Stationary Stage.-Same as preceding, except that the dose of copaiba should steadily be increased to the point of improvement in symptoms or until the stomach begins to show signs of rebeilion. If copaiba be not tolerated or be ineffectual, sandalwood oil is employed; if both are well digested, but when given singly are ineffectual, they are to be combined. When the inflammatory symptoms are mild, some preparation of cubebs may be added or substituted. 
(d) Stage of Declinc.--Same as preceding, except that as the discharge subsides copaiba is abandoned and sandalwood oil and cubeb preparations are substituted. If under this treatment the discharge persists in a subacute form, injections are indicated. Mild solutions are at first employed; later, if necessary, the solutions may be increased in strength.

The practitioner should never forget his patient while treating his patient's urethra. A feeble or cachectic subject should not have a restricted diet that may still further reduce his strength and vitality. A man with a weak stomach should not swallow medicaments that induce marked digestive disturbances. No physician should persist in directing his efforts solely to the urethral discharge when the general condition of the patient calls for tonic or specific treatment. Sometimes a subacute urethritis in a feeble or cachectic individual refuses to subside under treatment ordinarily indicated in a urethritis of the same grade and character, but improves rapidly and disappears after the administration of iron, quinine, strychnine, cod-liver oil, or malt, together with fresh air and sunshine-in short, under such treatment as is called for by the general condition.

Prognosis.-That gonorrhœa does not often directly threaten life is true; but that it is as harmless as many young men, and even not a few inexperienced physicians, believe is far from true. Cases have occurred in which, as the result of poor hygienic surroundings or bad treatment, a high grade of inflammation has been followed by gangrene, septic infection, and death. Much more frequently death follows some of the complications of gonorrhœa, such as a prostatitis with prostatic or periprostatic abcess; peritonitis following inflammation of 
the seminal vesicles; epididymitis; cystitis; pyelonephritis; or, more remotely, gonorrhoal rhcumatism, endocarditis, or pericarditis. While the inmediate danger to life is not so great, there is great danger that there will be left some form of chronic urethritis, stricture, chronic prostatitis, cystitis, vesiculitis, an ankylosed knee-joint, or an indurated epididymis which will render the affected testicle incapable of performing its function: if both epididymes have been involved, the man may for ever be denied the privilege of having children of his own. Or there may result some of the so-called "functional" and "nervous" disturbances of the genitourinary system which so frequently undermine the tone of the entire nervous system, resulting in neurasthenia, hypochondriasis, and kindred obscure disturbances.

The first attack of gonorrhœe, under proper management and in a healthy man, has a tendency to run a definite course toward recovery. It is quite unusual, however, for a patient with gonorrhœa to be so situated that all his surroundings are favorable to his complete recovery. Such a situation is difficult to obtain for any but those who for some other reason are compelled to remain in bed during the course of the disease; and, as each successive attack cxhibits a greater tendency to become chronic or to leave some portion of the urethra permanently damaged, the consequence is that but few urethras once infected with gonorrhœa ever return fully to their normal condition. Noeggerath believes that a man never fully recovers from his first gonorrhœa, and claims that nine-tenths of all women married to men who have ever had gonorrhœa eventually become sufferers from some form of pelvic inflammation. This is an extreme view, and one not yet fully accepted. The 
question is further considered in connection with the subject of prognosis in Chronic Urethritis.

The duration of a gonorrhœa under proper treatment depends so much upon the individual, his habits, his surroundings, and the previous state of his urethra, that it is impossible to make definite statements applicable to all cases. The prognosis must always be guarded. Usually the first attack of gonorrhœa, under favorable circumstances and with good treatment, lasts from five to eight weeks. The discharge may disappear or be suppressed by local treatment much earlier, but this fact by no means proves that the urethra is in a healthy condition or that slight irritation may not induce a discharge showing gonococci in abundance and proving highly infectious. Successive attacks, though less acute, are generally of longer duration. In general, an attack following a short period of incubation, in which the symptoms rapidly reach a climax of intensity, terminates in recovery earlier than another with a longer period of incubation in which the symptoms are subacute. 


\section{COMPLICATIONS OF URETHRITIS.}

\section{ACUTE POSTERIOR URETHRITIS.}

THE term "acute posterior urethritis" is applied to inflammation of the membranous and prostatic portions of the urethra. The compressor urethræ muscle forms the dividing-line between the two anatomical divisions of the urethra, known as the anterior and posterior (or deep) urethra, or the pars anterior and the pars posterior. The pars anterior includes the bulbous and pendulous portions of the urethra; the pars posterior, the membranous and prostatic portions. The division is of importance from a pathological point of view, because of the close anatomical relations between the posterior urethra and the epididymis, prostate, bladder, and seminal vesicles. Inflammation of any of these organs is liable to occur with or after posterior urethritis, and when one of them is implicated the presence of posterior urethritis may be taken for granted.

Etiology.-A posterior urethritis may appear any time after the third week of gonorrhcea, or before the third week in cases subjected to improper local treatment or which have not had proper hygienic management, or in cachectic and debilitated individuals. At any time during the course of gonorrhoa the extension of the inflammation to the pars posterior is favored by any mode of living or treatment that tends to congest or irritate this portion of the urethra. As a result of 
accident, of injury from instruments, of deep injections, of highly acid urine, or of fragments of calculi, posterior urethritis may appear independently of inflammation of the pars anterior. ${ }^{1}$

Symptoms.-About the end of the third week of gonorrhœa the inflammation, which in favorable cases has been limited to the pars anterior and should now begin to decline, may involve the pars posterior. This condition is usually announced by a more or less sudden increase in the frequency of urination, the patient sometimes being compelled to urinate every few minutes. In severe cases the inflamed mucous membrane of the prostatic urethra becomes so sensitive and irritable that it will not tolerate the presence of the smallest amount of urine in the bladder, and but a few drops are required to excite an uncontrollable desire to urinate, the tenesmus often being excruciatingly painful.

A few drops of blood may appear at the close of each urination, or the hemorrhage may be considerable-sometimes sufficient to pass backward into the bladder and to color all the urine. The perineum may be the seat of burning or cutting pains which may radiate to the end of the penis, to the testicles, the groins, or the back. In the majority of cases the symptoms are not so violent, and the patient complains only of a feeling of pressure and discomfort, with possibly burning, tickling, or slight pains in the perineal region, together with a more or less increased

1 Many authorities now claim that posterior urethritis is not a complication, but a natural sequence, of gonorrhce; that it is present in the large majority of acute cases; and that it usually appears during the first week - as a rule, without marked symptoms and independently of local treatment. The questions of etiology, diagnosis, and treatment are at present the subject of active investigation and discussion. 
frequency in micturition. There is usually considerable irritation of the sexual organs, manifested in prolonged and painful erections at night, and in frequent seminal emissions, which may be mixed with blood.

When the inflammation involves the posterior urethra the process in the anterior portion often subsides to a great extent, with marked diminution in the discharge from the meatus; and though the reverse may be true, yet the sudden cessation of a gonorrhoal discharge should always lead one to suspect this complication. The acute symptoms in a posterior urethritis usually last but a few days, but the process is generally prolonged in a subacute form and shows a decided tendency to become chronic.

Diagnosis.-The occurrence, during gonorrhœe, of frequency of urination, tenesmus, hemorrhage, or the sudden cessation of the discharge, should lead one to examine for posterior urethritis. The finger in the rectum finds the prostatic and membranous portions of the urethra sensitive; slight pressure increases the pain and tenesmus, but the prostate is not enlarged. Examination with instruments in the urethra is contraindicated, but the urine should be examined carefully by Thompson's two-glass test. This test is based on the supposition that pus secreted in the prostatic urethra cannot pass the compressor urethræ muscle and find its way out through the pendulous portion; but, on the contrary, if more pus collects than the prostatic urethra can hold, it will pass back into the bladder and mingle with the urine, rendering the latter cloudy. If the patient passes the contents of his bladder in two glasses, the first glass will contain urine plus the washings of the urethra, while the second will contain the urine as it exists in the 
bladder. If this second portion is clouded by the presence of pus, the latter evidently comes from some portion of the genito-urinary tract back of the compressor urethræ muscle.

The exact localization of the source of pus in the bladdier is often difficult and calls for careful microscopical examination, but the presence, during the course of gonorrhœa, of pus in the second glass, together with the occurrence of the above-described symptoms, will point strongly to posterior urethritis. If the urine is passed frequently, there may be times when no more pus will accumulate than the prostatic urethra can hold (when it will all be washed out with the first urine), so that the urine in the bladder will remain clear and will appear so in the second glass. This occasional appearance of clear urine in the second glass will exclude cystitis. In less acute cases, since the amount of pus produced is small, the urine in the second glass may always be clear unless the urine has been retained in the bladder three or four hours. It is important, consequently, that the morning urine-also that passed at the time of the visit-be examined. The degree of cloudiness and the amount of pus in the urine of the second glass give some indication of the intensity of the inflammation. ${ }^{1}$

Treatment.-The gencral hygienic management is that of gonorrhœa, except that rest is of still greater

1 The only cloudiness of urine considered in these pages is that produced ly pus and mucus. The nature of the sediment in any specmen of turbid urine should be determmed by the usual methods of urinalysis or microscopical examination. Gentle heat clears a turbidity due to the presence of urates: acetic acid, that caused by phosphates or carbonates; bacteria and pus can be removed only ly filtration. 
importance, and in severe cases with much tenesmus or hemorrhage rest in bed, or at least in the recumbent position, is absolutely necessary. Iarge quantities of bland fluids, such as flaxseed or slippery-elm tea, should be drunk, and the urine should be rendered sterile by the use of boric acid, salol, or salicylate of sodium, in doses of from 5 to 10 grains four times a day. Since marked alkalinity of the urine would favor ammoniacal decomposition in the bladder, it is best to keep the urine neutral, and the dose of alkalies, if given at all, should be small. Copaiba and sandalwood are valuable in most cases, but they may prove irritating, and should then be stopped. If the urine is markedly alkaline and contain pus, it may be adrisable to give benzoate of ammonium in small doses sufficient to keep the urine neutral, but if given too freely it will prove a source of irritation.

For the purpose of controlling the pain and tenesmus, suppositories containing morphine ( $\mathrm{gr} . \mathrm{I} / 4$ ) and atropine (gr. $\frac{1}{60}$ ) may be used in the rectum, or from $I$ to 10 minims of the fluid extract of hyoscyamus may be given every few hours. The use of a catheter is to be aroided if possible, and is rarely necessary if the directions given for the treatment of retention of urine in gonorrhoea be faithfully followed. Allowing the patient to urinate while sitting in a tub of hot water will rarely fail to give better results than the catheter. If the posterior urethritis has come on during the declining stage of gonorrhoea, or if for any reason local treatment of the anterior urethra has been instituted, such treatment must be suspended at once. If local treatment is to be tried, it should be in the form of a direct application to the deep urethra. The methods are described under treatment of Chronic Urethritis. 


\section{EPIDIDYMITIS.}

With the exception of posterior urethritis, epididymitis is the most frequent complication of gonorrhœa. It occurs in from 6 to 15 per cent. of all cases of acute gonorrhœea, and it usually makes its appearance during the third or fourth week of that disease. It frequently begins as late as the eighth week, and it may occur much later, though most of the cases appearing some months or years after an attack of gonorrhcea are undoubtedly due to an exacerbation of a chronic urethritis, to stricture, or to other causes. It is also found as early as the second week, and cases are reported as beginning during the first week, of gonorrhcea.

The epididymitis is usually single, the left testicle being involved somewhat more frequently than the right. When both testicles are implicated, the second follows several days or weeks after the first, simultaneous epididymitis of both testicles being very rare. The first attack is usually acute, and predisposes the patient to the disease, which may thus become chronic. Occasionally, in cachectic subjects or when due to stricture or chronic urethritis, it may be subacute from its origin. It is usually accompanied by inflammation of the tunica vacinalis, and less frequently by orchitis.

Etiology.-Epididymitis occurs during acute gonorrhcea without other apparent cause. It is more frequent in neglected and poorly-treated cases than in those treated in accordance with the hygienic and other rules given for the treatment of gonorrhcea. Any of the causes mentioned as capable of producing an exacerbation of the urethritis or irritation of the urethra may increase the danger of epididymitis. It is probably 
always preceded by inflammation of the pars posterior, from which position the inflammation travels readily and continuously through the ejaculatory ducts and the vas deferens to the epididymis: evidences of inflammation of these intermediate parts are often wanting, and it is possible that in some cases the lymphatics convey the infection directly from the deep urethra to the epididymis. Some writers speak of reflected irritation as a sufficient cause. Some individuals are very susceptible to the disease, while others seem proof against it, despite neglect, reckless living, and poor treatment. One attack always predisposes to another. It occurs in subacute and chronic (also acute) forms in chronic gonorrhœea and stricture, especially when these conditions have been aggravated by improper treatment, disordered living, violent exercise, etc. Finally, it may occur independently of urethral disease, as a result of traumatism, or possibly from prolonged sexual excitement or exposure to cold.

Symptoms.-In observant and sensitive patients, and especially if the previous subjective symptoms of gonorrhøea have been slight, the inflammation of the epidiclymis is usually preceded by prodromal symptoms in the way of slight chills, fever, and malaise, with vague, uneasy sensations or slight pain in the groin and radiating to the kidneys and the testicle. Occasionally the inguinal pain is severe, and the cord is tender and feels as though it were suspending a heavy weight; or there may be a sensation of pressure in the perineum, with vesical tenesmus and difficulty in urination. Less frequently, inflammation and swelling of the cord are recognized for several days preceding an epididymitis; and in rare instances the process is limited to the cord. 
In unobservant individuals and in those already suffering considerable inconvenience and distress from gonorrhcea the prodromal symptoms frequently pass unnoticed. In such cases the first recognized evidence of the complication is usually a sudden decided pain in the affected testicle. If examined at this time, some portion of the epididymis, usually the globus minor or major, is found to be slightly swollen and very tender. During the next twenty-four hours the pain and swelling increase rapidly; the entire epididymis soon becomes inrolved, and can be felt as an irregular, well-defined, moderately firm, half-moon-shaped tumor enclosing the superior, posterior, and inferior borders of the testicle. It is very painful, especially when the testicle is allowed to hang without support, and is exceedingly tender to the touch. Under favorable circumstances or with good treatment the disease may progress no further, and after a few days the symptoms will begin to subside: this result is not common even with the best treatment.

Nore frequently the inflammation extends from the epididymis to the tunica raginalis, which becomes more or less distended with fluid, thus adding greatly to the swelling and pain and partially or wholly obscuring the outline of the epididymis and testicle. The testicle proper becomes engorged and distended with blood, and occasionally true orchitis (which terminates in resolution), with its intense and characteristic pain, may be present. The loose tissues of the scrotum become inflamed, œedematous, and swollen, sometimes forming irregular, thickened tumors that may be carelessly taken for the inflamed testicle itself. The testicle with its epididymis and their coverings thus form an irregular or oval tumor that may become larger than a man's fist, reddened, hot, exceed- 
ingly painful, and tender. The cord may become swollen and very painful, often drawing the testicle up toward the groin; in rare instances it becomes partly strangulated in the inguinal canal, resulting in intense pain, collapse, and all the symptoms common to strangulation with inflammation.

The intensity of the symptoms, however, varies greatly in different cases. The swelling may be limited to a part or all of the epididymis, which is more or less indurated and tender, or it may be increased by fluid in the tunica vaginalis. This fluid may be scanty in quantity and may serve merely to form a fluctuating tumor which but partially obscures the outline of the testicle and epididymis, or it may be sufficient to forcibly distend the cavity, forming a tense, exceedingly painful tumor which conceals entirely all traces of the enclosed structures. Swelling and infiltration of the scrotal tissues may be slight, but they are usually marked and are often sufficient to make an examination of the deeper parts impossible. As a result of ill-fitting dressings, swelling of the scrotum may be pronounced in cases that are otherwise mild.

The pain in epididymitis varies greatly, but in acute cases it is usually intense. The organ is very sensitive, and the slightest pressure upon it causes the patient to feel nauseated and faint. Without proper support for the testicle walking is often impossible. Absolute rest and support of the scrotum in one groin or wer the pubis lessens, but does not entirely remove, the pain. If there be much inflammation of the testicle proper or strangulation of the cord-both uncommon occurrences-position has little influence on the pain, which is even more intense than in epididymitis, and it may be compared to that of renal colic. A similar but less severe grade of 
pain is produced when the tunica vaginalis is greatly distended, but usually the most tender part is the epididymis, which can thus be located by palpation even through a swollen and odematous scrotum.

The course of the disease varies considerably, being, as a rule, much shorter and more even when the parts are put at rest and given proper treatment than in cases in which such rest and good management cannot be obtained. It is further influenced by the idiosyncrasies and general health of the patient. In an acute case the symptoms usually increase rapidly in severity for three or four days or a week, remain stationary for a few days more, and then decline, so that at the end of ten days or two weeks from the beginning the pain is practically gone and the swelling is limited chiefly to the epididymis, some portions of which are still indurated and tender. An uncomfortable sense of weight and soreness may remain for some time.

From the beginning, if the patient be kept on his back and the scrotum be well supported, the pain usually subsides rapidly, and it may become slight before the swelling has begun to disappear; but if he sit or stand and allow the testicle to depend, the pain promptly returns. As the pain subsides the patient often thinks himself able to get up and return to his business, but a few hours or a day of ordinary activity may send him to bed with the pain and swelling nearly as severe as before. Even at the end of two weeks, when he seems to be practically well, if he is very active and fails properly to support the testicle, a relapse may be expected. Relapses are not uncommon, and, while less severe than the first attack, may prolong the disorder indefinitely and result in a permanent induration of the 
globus minor or major. As a rule, the globus minor, or less commonly the globus major, remains more or less swollen, indurated, and tender for some weeks; while the last traces of induration disappear gradually. in the course of months or years. or persist permanently in the form of a hard, insensitive nodule.

For a few days during the height of the attack there is usually some ferer with its attending symptoms: such constitutional disturbances are usually mild, but occasionally they are quite severe. The gonorrhoal discharge, which commonly diminishes with or just previous to the appearance of the swelling. may disappear cntirely, but it recurns when the swelling subsides.

Subacute attachs occasionally complicate stricture or seet. The symptoms come on more slowly, are much less severe than in the acute form, and are usually confined to the epididymis or some portion of it and to the cord. The testicle, the tunica raginalis, and the scrotum are not at all or but slightiy inrolved, and constitutional symptoms are wanting. The epididymis, and frequenty. the cord, is somewhat swollen. tender, and sensitive, but a well-fitting suspensory or other support usually enables the patient to attend to his usual work without much discomtort. though violent exercise should be aroided. As in gonorhoea, the gleety discharge disappears during the swelling. to return as the latter subsides.

As a result of repeated subacute attachs or of relapses in acute epididymitis, the inflammation may become chronic. Portions of the epididymis are then constanty swollen, thichened, and tender, simulating tuberculosis of the organ, except that the nodular enlargements are smoother and less irregular in outline, and that slight causes suffice to produce a subacute inflammation of the 
entire body of the epididymis. The cord and the connective tissue about it may also be swollen, infiltrated, and sensitive, and may be the seat of neuralgic pains. Exceptionally there is chronic suppuration in portions of the inflamed tissues.

Diagnosis.-The characteristic symptoms appearing during the course of a gonorrhoea usually render the diagnosis easy. Orchitis, the only other disorder for which it might be mistaken, is rare and is not associated with urethral inflammation, but is caused by injuries, mumps, cold, and constitutional disorders. The swelling in orchitis involves the testicle proper, comes on more slowly, and forms a smaller tumor which is oval, smooth, peculiarly hard and tender, and not obscured by fluid in the tunica vaginalis. The pain is more intense and unbearable than in epididymitis, and is not influenced by position. Its course is slower, and it may result in destruction of portions or of all of the testicle through atrophy, suppuration, or gangrene.

Cases have been reported in which epididymitis of an undescended or abnormally situated testicle has been mistaken for strangulated hernia, etc. Such an error can be avoided by an examination of the scrotum, which would show the absence of one testicle. There have also been reported cases in which the inflammation has been limited to the vas deferens, with the formation of a rounded painful tumor extending from the ring to the epididymis. There are on record a few cases in which an ordinary epididymitis has been preceded by partial strangulation of the cord, with symptoms suggesting strangulated hernia or obstruction of the bowel with peritonitis.

Treatment.-Since epididymitis is almost invariably a 
complication of urethral inflammation, its prophylactic treatment lies in th proper hygienic and other management of the primary disorder. If an individual has had a previous epididymitis, he should wear, during the course of his gonorrhcea, a well-fitting suspensory bandage, live as quietly as possible, and avoid active exercise (especially lifting, jumping, dancing, etc.) and all irritation of the sexual organs, following faithfully the hygienic rules given for the treatment of gonorrhœe. Epididymitis can sometimes be prevented if, upon the first appearance of pain or uncomfortable sensations in the testicle or the groin, the patient lie on his back, with the scrotum elevated and covered for a few hours with hot applications.

In acute cases of epididymitis the objects of treatment are to lessen the inflammation and pain and to promote resolution. The essential requirements are complete rest, elevation and support of the testicle and scrotum, and the application of heat. A light diet and simple laxatives to produce free evacuation from the bowels constitute the only internal treatment, unless the condition of the individual calls for special medication. All treatment for gonorrhœea, except that necessary to keep the urine bland, should be suspended. The patient should rest quietly on his back. The scrotum should be covered completely with fomentations or poultices, and should be supported carefully, by means of a sling or a bandage, in a comfortable position over the symphysis or in one groin. The most satisfactory bandage for this purpose is the last of those recommended for dressing the organs during gonorrhoea, as it can easily be made to fit a testicle and its dressing of any size or shape. Another simple device is found in a large handkerchief or napkin folded once to form a triangle; the middle of the long (folded) side is 
placed under the scrotum, and an end (acute angle) is fastened on each side to a belt made of any convenient and comfortable material. The free (right) angle is brought up over the genitals and dressings and fastened to the belt in front. To keep the handkerchief from slipping upward it may be necessary to sew to its posterior border a narrow band that can be pinned to the belt behind.

Heat is best applied by means of fomentations, which, when skilfully employed, are more effective than poultices, and, being light, are often more comforting to the patient, for in a severe case the testicle may be so sensitive that the weight of a poultice cannot be tolerated. They may be made of a number of layers of gauze, or of from one to four thicknesses of a light white flannel faced with a piece of gauze, linen, silk, or cotton, which will be less irritating to the skin than the flannel. They should be large enough to more than cover the scrotum completely, and they should be covered in turn by a larger piece of oiled silk or of rubber tissue, which will serve to retain the heat and to keep the clothing dry. They should be applied as hot as the patient can tolerate them with confort, and should be changed often enough to keep them hot (from once in half an hour to once in two hours). Two sets of cloths are necessary, that one may be hot and ready for immediate application when the other is removed, as much harm may be done by having the parts exposed to a lower temperature while preparing the fomentation. It is equally important that in making changes the testicle be moved or disturbed as little as possible. The cloths may be wrung out of simple hot water, but it is better to add a teaspoonful of boric acid to each pint of water.

When the patient has neither the assistance nor the con- 
veniences necessary for the frequent application of fomentations, an ordinary flaxseed poultice may be substituted. This poultice should be from a quarter to half an inch thick, faced with a thin soft cloth to keep the wet meal from adhering to the scrotum, and the whole should be covered with oiled silk. Poultices retain the heat longer than fomentations, and need not be changed so frequently (from once in four hours to once in eight hours).

In the majority of cases a few hours of the above treatment will make the patient comfortable while he remains quiet. If these measures do not give relief, from $\frac{1}{2}$ ounce to I ounce of fine-cut tobacco should be stirred thoroughly in a pint of the boiling water which is to be used for fomentations or poultices. This is a very effective anodyne, but it may produce nausea. Instead of tobacco, ro grains or more of powdered opium to the pint of water may be used. Sometimes sprinkling the surface of the fomentation or the poultice with fine-cut tobacco or with laudanum gives good results. Other anodynes may be applied, under the poultice, in the form of powder, liquid, or ointment, but they are rarely needed. If these measures are not sufficient, and if the pain be due to extreme distention of the tunica vaginalis, puncture will allow of escape of the fluid and will give immediate relief. In exceptionally acute cases, with strangulation of the cord and extreme pain which is not relieved by the usual treatment, ten or more leeches may be applied above the groin, along the course of the cord, followed by the use of hot water to encourage bleeding: the effect on the pain is often prompt and decided.

In all cases of epididymitis, when the patient can afford the time, rest in the horizontal position and elevation of the scrotum should be continued for ten days or two 
weeks, or until all symptoms have disappeared except a small, tender, inclurated swelling of the globus minor. The fomentations or poultices hasten absorption of the inflammatory products, and should be continued when practicable, though after the first few day's, when the symptoms have begun to subside, they may be replaced by a more convenient and nearly as efficient dry dressing formed by wrapping the scrotum in a layer of wool and covering all closely with an impervious covering of oiled silk or of rubber tissue. The heat and moisture natural to the parts are thus retained, forming what is known as a " dry poultice."

When a patient with acute epididymitis refuses to go to bed for a few days, other methods may be tried; but he should first understand that his recovery will be slower and that a permanent induration of the globus minor and obstruction of the vas deferens will probably follow. The scrotum over the affected testicles may be smeared lightly with an opium-and-belladonna ointment, covered with a "dry poultice," and the whole supported and made as immovable as possible with the wide bandage already recommended, or-what is often more effective when the patient is on his feet-with the HorandLanglebert suspensory, which may be obtained from the makers of surgical appliances. By avoiding sudden and rapid movements the patient is often enabled to move about with comparatively little discomfort. For these cases Dr. W. S. Halstead and others touch the surface of the scrotum lightly in several places with the point of the cautery at a white heat; iodoform ointment is then applied, and the testicle is properly supported. This method frequently relieves the pain and allows the patient to remain up and to move about. Tincture of 
iodine and strong solutions of nitrate of silver have been used to paint the scrotum and to produce counter-irritation, but they usually fail to do much good, and they often cause a severe dermatitis of the scrotum.

When possible, every patient with epididymitis should be kept on his back until the pain has subsided and the swelling has been reduced somewhat (from three to eight days); but if he is then unwilling to spend more time in bed, he may be allowed to rise and go about if his testicle is first properly strapped. To determine if a testicle is ready for strapping, the organ is taken in the hand and gently manipulated for several minutes, gradually bringing the testicle to the bottom of the scrotum, which is encircled, just above the testicle, by the thumb and forefinger, forming a ring which gentle pressure is making gradually narrower. These manipulations will probably cause some pain in the testicle or in the groin, but this pain will usually disappear without relaxing the pressure if the operating hand be held motionless for a few seconds. If the pain is but slight when the ring formed by the thumb and the finger is too small for the testicle to escape through it upward, and the testicle is thus secured in a smooth, tense, and shining pouch of the scrotum, strapping is proper.

For strapping the testicle rubber adhesive plaster or lead-plaster may be used, in strips half an inch wide. The hairs should be cut from the scrotum, to prevent their being pulled by the plaster on its removal. The most difficult and most important part of the whole procedure lies in applying the first strip of plaster, which must be made to take the place of the thumb and the finger in forming a ring to hold the testicle in the position described above. The strip should be half an inch 


\section{SIPHILIS AVD THE VENEREAL DISEASES.}

wide and three or four inches longer than necessary to encircle the testicle. To its under (adhesive) surface is fastened a cotton bandage an inch and a half wide and enough shorter than the plaster to leave one end of the latter uncovered for two or three inches. The bandage is used to prevent the edge of the plaster from cutting the scrotum.

The patient stands, or sits on the edge of a chair, in front of the operator, who has taken the precaution to have his strips of plaster, bandage, scissors, etc. ready and within easy reach. When the left hand has once more secured the testicle in the desired position, the pressure may be relaxed without changing the position of the hand, and the prepared adhesive strip is placed around the scrotum in the position just vacated by the thumb and the finger. The thumb and the finger again encircle the scrotum above and outside of the adhesive strip, holding the end covered by the bandage in position and forcing the testicle down, while the right hand brings around the free end of the plaster and fastens it to the back of the fixed end. If properly done, the testicle is secured in a smooth, tense, purplish pouch of the scrotum, the adhesive strip forming a ring too small to allow the escape upward of the testicle. After waiting two or three minutes to allow the pain to subside (as it will do if the plaster be not drawn too tight), the operator applies several more circular strips parallel with the first, each strip overlapping the one last applied by about half its width. When strips applied in this direction will no longer fit the surface, others may be fastened to the first strip on one side, carried over the testicle, and fastened to the first strip on the opposite side, until the entire surface below the ring is firmly and 
completely covered. A long circular strip should finally be applied to cover and hold the ends of the strips last applied. When finished the covering should exert even pressure upon the entire surface, thus encouraging absorption and preventing the possibility of a return of the swelling.

It is always best to have the patient rest quietly for half an hour or more after strapping the testicle, until the pain caused by the manipulations has disappeared, when he can support the scrotum with a suspensory bandage (made to fit by lining it with cotton), and go about his business in comfort and without fear of a relapse. If the dressing remains painful after an hour, or if it becomes so at any time, it should be removed either by cutting the separate strips or by immersing the whole in hot water until it can be slipped off. Pain will follow strapping if the testicle is not ready. for it, if the ring formed by the first strap be too tight, or, as frequently happens, if the ring be so large that the testicle is forced partially into it by the other straps. At the end of from twenty-four to forty-eight hours the swelling will have been so reduced in size that the dressing no longer exerts pressure upon the testicle, which sometimes escapes through the ring, and a new strapping is necessary. The procedure is repeated four or five times until all that remains of the swelling is the indurated globus minor.

For several weeks, until all swelling is reduced to a painless induration, a well-fitting suspensory should be worn. This is done to prevent a relapse and to hasten absorption, a process that may be aided by daily inunction over the nodule with oleate of mercury (2 to 10 per cent.), and by lining the suspensory with oiled silk 
and a thin layer of wool to form a light "dry poultice." Too early suspension of treatment, and especially of support for the parts, may lead to chronic inflammation of the epididymis and the cord.

The treatment of subacute and chronic epididymitis is that of the declining stages of the acute process. In addition, all predisposing and exciting causes should be removed.

Prognosis.-Epididymitis almost always terminates in resolution; suppuration is very rare except in the uncommon chronic cases. Absorption of inflammatory products is rapid at first, and at the end of a few weeks of good treatment all pain and tenderness have disappeared, and there remains only some swelling and induration of the globus minor or major. This remaining induration may require months or years for its final absorption, and frequently persists permanently in the form of a hard nodule found to be composed of inflammatory deposits in and surrounding the seminal canals, which are thus completely occluded.

Permanent induration is most common in the globus minor, and, as this body is composed of the convolutions of a single tube, the blocking of any portion of it prevents the passage of the semen from the testicle proper to the vas deferens. Even when all apparent induration has disappeared, and this portion of the epididymis again feels normal to the palpating finger, the canal may be filled and obstructed at some point. Complete absorption of the deposit and reopening of the canal in the globus minor can be expected in but a small minority of cases. In the globus major complete absorption is more common, and even if it does not occur, some of the tubules may escape 
obstruction. It follows that the large majority of men who have had epididymitis on both sides are sterile. The cause of their sterility lies solely in mechanical obstruction to the passage of semen, since the testicle does not atrophy nor is the man impotent. He retains his sexual appetite and power, and ejaculates a fluid resembling semen except that it contains no spermatozoa.

In a tubercular or syphilitic patient epididymitis may be followed by the appearance of the constitutional disease in the epididymis.

Subacute and chronic cases of epididymitis terminate favorably when proper treatment is continued for a sufficient period.

\section{PROSTATITIS.}

I. Acute Prostatitis.- IVhen gonorrhœal inflammation reaches the posterior urethra, it frequently includes the superficial glands and follicles of the prostate, and it may readily involve the entire structure of the organ. Prostatitis commonly appears after the third week of gonorrhœa, its symptoms following or appearing simultaneously with, and possibly obscuring, those of posterior urethritis. It occurs also with chronic urethritis and with stricture. The exciting causes are practically those of posterior urethritis and epididymitis-namely, coitus; prolonged or intense sexual excitement; violent exercise; excessive use of alcohol, tobacco, or highly seasoned foods; exposure to cold; and mechanical or chemical injury due to the use of instruments or injections, or possibly to a concentrated and irritating urine. It is possible that these causes may produce prostatitis independently of gonorrhœa. 
Symptoms.-Follicular Prostatitis.-If the inflammation be limited to a few follicles, the symptoms will be those of posterior urethritis, with the probable addition of sharp, sticking pains most noticeable at the close of urination. The finger in the rectum may find one or more firm, tender nodules in the substance of the prostate, which is possibly somewhat congested and slightly swollen, but not inflamed or very sensitive. These in. flammations may undergo resolution, the symptoms disappearing with those of the posterior urethritis; or they may extend to the rest of the prostate; or, finally, they may linger indefinitely in the form of a chronic folliculitis.

Diffuse or Parcncleymatous Prostatitis.-In this form of prostatitis the symptoms are much more pronounced and characteristic. The prostate swells rapidly, notwithstanding the fact that it is surrounded by a firm, fibrous capsule, and the resulting pressure to which the inflamed organ is subjected produces violent pains and interferes greatly with the urinary and sexual functions. There is frequency of urination, tenesmus, and the patient experiences a fecling of fulness and warnth in the rectum, producing an almost constant desire to empty the bowel, and leading him to make frequent and often violent efforts to expel what he thinks is a mass of fæces, but which is really the swollen prostate protruding into the rectum. Defecation is painful, and there may be tenesmus of the bowel. Urination is also painful, especially at the close of the act, when the patient may experience violent sharp pains due to the squeezing of the tender prostate by the sphincter vesica muscle, and the last irops of urine may be mixed with blood. The stream of urine is often reduced in size, and the pressure upon 
the prostatic urethra may be sufficient to cause complete retention.

In addition to the subjective sensations already described, the patient complains of fulness, pressure, weight, and pain in the perineum, which may be hot and so tender that the sitting posture or crossing of the legs cannot be endured. The pain is variously described by patients as sharp, lancinating, shooting, boring, or throbbing in character, and radiates from the prostate and perineum to the urethra, testicles, thighs, and back. With the finger in the rectum the prostate is outlined as a firm, hot, pulsating, more or less irregular tumor, which is exceedingly sensitive to pressure, and which in severe cases may become almost as large as a man's fist, and may entirely occlude the rectum.

The disease is usually accompanied from the beginning with some fever and constitutional disturbance, and by diminution or cessation of the urethral discharge during the swelling of the prostate. A marked feature of prostatitis, and one for which the inexperienced practitioner is rarely prepared, is the mental attitude of the patient, whose restlessness, fears, and anxiety are out of all proportion to the severity of the process. As Keyes well says: "The patient is irritable, despondent, and suspicious, often, in fact, wild to an extent amounting to mild acute mania." He is inclined to be dissatisfied with all that is done for him-in short, is usually a very unsatisfactory patient to treat during the acute process, unless he can have a constant attendant to watch over him and properly to carry out the physician's orders.

The course of the disease, when it ends, as it com-. monly does, in resolution, is short; the symptoms appear more or less suddenly, rapidly increase to the 
highest point, and almost as rapidly subside, so that the acute stage varies in duration from four to ten days, and final recovery follows in another week or two. As the symptoms subside and the urethral discharge reappears, the latter may at times be changed in character by admixture with a thick, viscid mucus and pus from the prostatic follicles; and if the seminal vesicles have been involved, the discharge may contain a few spermatozoa.

Instead of undergoing resolution, the inflammation may go on to suppuration involving portions or all of the prostate. The formation of pus is usually announced by a decided chill and a marked increase in temperature, and the constitutional disturbance may be considerable. The feeling of tension in the perineum is usually diminished, and the pains may lose their intense, boring character and become cutting and throbbing. Retention of urine commonly results. Fluctuation can sometimes be felt through the rectum. If untreated, these abscesses rupture into the urethra, the rectum, or the perineum, the order of frequency being that given. Exceptionally, they extend beyond the limits of the prostate, burrow extensively between the layers of the pelvic fascia, and open into the ischio-rectal fossa, the inguinal region, or even into the peritoneum, and may cause, death from sepsis or from peritonitis. Occasionally an abscess will discharge into both urethra and rectum or into the urethra and some other region, as the perineum, and result in urinary fistula. Rupture of the abscess brings immediate relief from pain; if the abscess be a small one, opening into the urethra, it will usually fill with granulations and slowly heal. An opening into the rectum is unfavorable, since the cavity is more liable to infection and can be kept clean only with great difficulty. 
Occasionally during gonorrhœa, stricture, or consequent inflammation of the seminal vesicles or of the vas deferens, suppuration may occur in the tissues surrounding the prostate (periprostatic abscess). As these abscesses are situated in looser tissues, their symptoms are less acute than in prostatic abscess, and the finger in the rectum locates them outside the capsule of the prostate; but in other respects their course is practically that of prostatic abscess.

Treatment.-First and most important is rest, which in severe cases should be made as nearly absolute as possible. The patient must resist his constant desire to urinate and to empty the bowel, and must refrain from straining at stool or in urinating if he would avoid the dangers of prostatic abscess. Rest in bed with the hips elevated, the application of fomentations large enough to cover anus, perineum, and hypogastrium, hot sitz-baths or hot enemata given two or three times daily, the internal administration of alkalies and bland fluids in quantities sufficient to keep the urine unirritating (see hygiene of Gonorrha a), and the use of anodynes to control pain and tenesmus, constitute the best treatment of most cases; if begun early and faithfully continued, this treatment will usually render the attack a mild one.

Anodynes are best given in the form of opium-andbelladonna suppositories, and in quantities sufficient to allay the irritation of the bladder and rectum and to keep the patient quiet. As in posterior urethritis, hyoscyamus is often valuable in relieving tenesmus. Mustard or turpentine may be added to the fomentations to produce counter-irritation, and in severe cases ten or fifteen leeches may be applied to the perineum.

The patient should be put on a light diet, and his 
bowels should be moved with enemata, cathartics being generally contraindicated, though a brisk calomel purge at the beginning of treatment, or $\frac{1}{10}$ to $\frac{1}{6}$ grain of calomel given every hour until the bowels move, is often productive of excellent results. Sometimes it is necessary to give bromides and chloral to quiet the mental excitement, produce sleep, and allow the patient rest, but usually they are not as beneficial as the presence of a well-trained attendant who will properly execute the physician's orders, keep the patient under control, and add to his comfort and rest by daily sponging or skilful rubbing of the body, etc. All treatment of gonorrhca should be suspended with the first symptoms of prostatitis, and the prostate should not be teased by too frequent examinations through the rectum. Retention of urine should be relieved by the use of the hot bath when possible, but if this fails a small soft catheter may be used gently after first injecting the urethra full of warm oil.

Finger and other German surgeons highly recommend the use of cold, instead of hot, local applications. If besun early enough, the course of the disease may be cut short by using the cold rectal sound. This instrument is a hollow sound with two longitudinal compartments connected at the end, through which water may flow in a constant stream. The sound is well oiled, is gently introduced into the rectum until it comes in contact with the prostate, and cold water-even ice cold-is allowed to flow through it for half an hour or an hour, once, twice, or three times a day.

When an abscess forms the treatment is surgical, a perineal opening being always the most desirable. If fluctuation can be felt through the rectum, the abscess may be 
aspirated or be punctured with a trocar, but an opening into the rectum is to be avoided when possible, since some of the contents of the intestine are certain to get into the cavity and to interfere with healing. When a small abscess bursts into the urethra, boric acid or salol and bland fluids internally, to keep the urine aseptic and unirritating, constitute the only treatment required unless further symptoms appear. Abscesses which open in other directions should be treated on surgical principles-with irrigations and astringent injections.

II. Chronic Prostatitis.-Chronic prostatitis may follow an acute attack, may occur in subacute form during chronic urethritis or with stricture, or may arise from any cause that produces prolonged congestion or irritation of the prostatic urethra. The inflammation may be limited to a few of the superficial follicles and glands opening into the urethra, and be very mild, simply catarrhal in type, or it may involve the entire glandular structures, together with more or less, or even all, of the parenchyma of the prostate.

Symptoms. - In the mild forms, commonly known as chronic follicular prostatitis, or frostatorrace, in which the superficial glands and follicles are alone affected, the chief symptom is the discharge from the meatus of a thick, sticky, bluish or milky-looking fluid composed of a mixture of thick, glairy mucus from the prostatic follicles, usually some pus, and more or less mucus from other portions of the urethra. This discharge is intermittent, appearing most frequently at the close of urination, at stool, or after an erection, or it can be pressed out of the prostate by the finger in the rectum. The urine in the second glass is usually cloudy; it may contain comma-like shreds even when that in the first glass is 
clear, since the mucus, pus, and shreds are pressed out of the prostatic follicles by the sphincter vesica and the muscular fibres of the prostate in the act of expelling the last drops of urine. Under the microscope the discharge is seen to contain pus-cells, polygonal and cylindrical epithelium, amorphous and fatty matter, and the needleshaped and whetstone-shaped "sperma-crystals." If, by pressure on the prostate, a drop of the prostatic secretion be obtained free from urine, and to it be added a drop of a I per cent. solution of ammonia phosphate, and the mixture be allowed to dry slowly under a cover-glass, these crystals can easily be demonstrated. Spermatozoa are not present unless the seminal vesicles are inflamed.

The patient usually describes these discharges as seminal losses, and believes himself the subject of spermatorrhoea. In consequence he is often despondent and hypochondriacal and inclined to exaggerate greatly the severity of his subjective discomforts, which are usually confined to some vague and uneasy sensations in the perineum, with possibly some increased frequency in urination and some irritability of the sexual organs.

Extension of the inflammation to the deeper glands and parenchyma of the prostate produces a graver form of the disease, known as chronic parcnchymatous prostatitis. The symptoms of this form vary greatly, depending upon the extent and severity of the process. In addition to the discharge, there may be tenesmus and increased frequency of urination, with pain and possibly slight hemorrhage at the close of the act. There are burning, heavy, uneasy sensations in the perineal region, with pains radiating to the urethra, testicles, groins, thighs, and back. These sensations are increased on urination, defecation, or sexual intercourse, and the pains 
may even be neuralgic in character, bcing often described as neuralgia of the urethra, testicle, and bladder. There are often teasing, tickling sensations of the prepuce and the meatus. In severe cases the pain is greatly increased by jolting, crossing the thighs, walking, or even by the sitting posture.

The deep urethra and the vesical neck are often exceedingly sensitive, and spasmodic contractions of the sphincter muscles may cause a sudden stopping of the stream near the close of urination. There is usually irritation of the sexual organs, with frequent emissions, which may be bloody; in severe cases, though emissions and prolonged, often painful, erections are common, sexual desire and gratification may be diminished or entirely absent. Spermatozoa are present in the semen, but, owing to the absence of normal prostatic secretions, they are inactive, and sterility results.

The patient is usually mentally depressed, irritable, and melancholy. Other constitutional disturbances are slight at first, and may remain so for months, notwithstanding severe local symptoms and a hypochondriacal state of mind that is most deplorable; but unless the local disorder is relieved and the mental condition is improved, there follows, sooner or later, a long series of morbid nervous phenomena constituting the unfortunate state generally known as "neurasthenia." These symptoms are vague, varied, and numerous. At first they refer chiefly to the genito-urinary system, but later they may apply to any or every organ in the body. Among the earliest and most common complaints of these patients, besides those already given, may be mentioned constant weariness and weakness, aching pain in the back and legs, a heavy, dull feeling in the head, headache, loss 
of memory and inability to concentrate the mind upon work, sleeplessness, hot and cold flushes or numbness with tingling sensations, and any or all of the symptoms that may result from impaired functional activity of the various organs of the body. The patient may complain of many of these symptoms while still appearing well nourished, but in most cases that fail to improve, malnutrition, loss of weight, and impaiment of the general health sooner or later result. The skill, patience, and tact of the physician are often taxed to the utmost in his efforts to determine if it be the local disease or the hypochondriasis that is chiefly responsible for the neurasthenic symptoms.

Dr. W. T. Belfield has called attention to the fact that chronic inflammation of the prostate is usually not limited to that organ, but invades portions or all of the seminal vesicle, vas deferens, and epididymis, together with the surrounding connective tissue; he states that chronic suppuration is a frequent feature of such inflammation, the small abscesses being usually unrecognized until they rupture into the urethra, rectum, or pelvic tissues, or until revealed by autopsy. The extent and nature of this more diffuse inflammation may be resporisible for some of the varied symptoms usually ascribed solely to chronic prostatitis.

Treatment.-For the milder cases the use of the cold-steel sound, as recommended for chronic urethritis, is the most valuable remedy. Deep injections of nitrate of silver or lanolin ointments may be used in connection with the sound. Correct hygiene and the general health of the patient are of greatest importance, and sometimes the best results are obtained from a trip to the sea-shore or to the mountains combined with other measures 
adapted to the needs of the individual. The mental state frequently calls for as much treatment as does the local condition, since it may be impossible to obtain rest and proper hygiene for the affected parts until the fears and anxieties of the patient have in some measure becn removed. The cessation of local treatment to allow the organs a period of rest is often necessary; and it must not be forgotten that too continuous or too energetic measures may aggravate the existing disorder and produce a severer type of the disease. Hot $\left(1 \mathrm{IO}^{\circ}\right.$ to $\mathrm{I} 2 \mathrm{O}^{\circ} \mathrm{F}$.) irrigation of the deep urethra is sometimes beneficial.

In the more severe forms blistering the perineum is the most effective treatment. Mustard, turpentine, or cantharides may be used, but cantharidal collodion is perhaps the most convenient. The perineum is slaved, the scrotum and the margin of the anus are protected by a simple thick ointment, and the collodion is applied to one-half of the perineum. The scrotum is then protected and kept out of the way by the use of a suspensory, and the perineum is covered with a light dressing of absorbent cotton. When the resulting blister has healed and the soreness diminishes, the other half of the perineum may be treated in the same way, and the process may be repeated often enough to keep one side or the other of the perineal surface constantly irritated for a number of weeks, until the symptoms of prostatitis have been largely relieved. During this treatment the patient must be kept quiet and in bed as much of the time as possible, while the state of his general health should be carefully studied and improved in every way possible. Tonics, mild laxatives or enemata, alkalies, and diluent drinks are necessary in most cases. 
Prognosis.-Recent cases recover under good management; older cases are less favorable, but with time, patience, hygiene, and good treatment they are greatly relieved and usually recover. The general health commonly remains good unless disturbed by loss of rest and long-continued mental depression. Suppuration very rarely results fatally.

\section{VESICULITIS.}

Inflammation of the seminal vesicles may occur during or after the third week of gonorrhœa, as the result of a direct extension of the process from the posterior urethra. Acute vesiculitis, however, is less common than the chronic form, which may follow the former, but which more frequently appears in subacute form during chronic urethritis. It may also be due to stricture or to prolonged congestion, irritation, and inflammation of the posterior urethra from any cause. It is more common in neurotic persons. Unrecognized acute or chronic vesiculitis is undoubtedly present in many cases of complicated urethritis.

Symptoms.-In the acute form the symptoms are practically those found in acute posterior urethritis and acute prostatitis, with which disorders vesiculitis is often associated. The differential diagnosis is frequently difficult. In addition to tenesmus, frequent micturition, painful and disagreeable sensations in the perineum, etc., there are usually characteristic disturbances of the sexual functions. Sexual desire is stimulated and may be almost constant, and there are frequent emissions occurring on the slightest provocation, and prolonged, sometimes painful, erections. Ejaculation is usually precipitate, unaccompanied by pleasure or relief, and 
extremely painful. The seminal discharge contains pus, dead spermatozoa, and frequently blood.

In subacute and chronic vesiculitis the above symptoms may be present in lesser degree, or there may be great diminution of sexual desire, with, however, frequent and more or less painful emissions. The discharge contains less pus and blood and fewer spermatozoa, but is thicker and more gelatinous than normal, and, if obtained pure by expression with the finger, shows a tendency to coagulate. There are often neurasthenic and other symptoms described in connection with chronic prostatitis.

If the bladder be full and counter-pressure be made above the pubes, the finger in the rectum will reach, just above the prostate and to either side, a considerable portion of the seminal vesicle, which in acute cases is swollen, hot, throbbing, and sensitive, and in subacute cases is distended with abnormally thickened secretion and is tender on pressure. (The normal vesicle can rarely be recognized by the finger in the rectum.) The condition of the prostate should be determined before concluding the examination. Under favorable circumstances acute vesiculitis subsides in a week or less, and usually terminates in resolution; but it may result in abscess or be followed by chronic inflammation.

Treatment.-In acute vesiculitis the treatment is that of acute prostatitis-namely, absolute rest, hot applications, anodynes, and general treatment of the patient. Much pressure upon the vesicle or frequent local interference should be avoided, for fear of rupturing the vesicle or encouraging suppuration. If abscess forms, the treatment is surgical, as in prostatic and periprostatic abscess. 
In chronic vesiculitis hygiene and constitutional treatment have proven of greater service than local measures. Irrigation of the rectum with hot water may help in some cases. "Stripping" the vesicle, as recommended by Dr. Eugene Fuller, 'sometimes gives good results. The following is a brief outline of the method: The patient stands with his back to the operator, bends (at the hips only) forward, and rests his hands upon a chair. With one finger in the rectum and with the other hand making counter-pressure over the bladder, which should be full, the operator gently presses upon such portions of the distended vesicle as he can reach, and empties it of its contents. The secretion may appear at once at the meatus or be washed out later by the urine. The frequency with which the operation may be repeated varies greatly in different cases, but averages about once a week. Too frequent or rough manipulations aggravate the existing disorder and may result in acute vesiculitis.

\section{CYSTITIS.}

If, during gonorrhœe or grleet, the posterior urethra is involved, the inflammation may readily extend to the adjacent mucous membrane of the bladder. Such a cystitis, though it may include all the vesical membrane, is usually limited to the surface about the urethral orifice, the region commonly known as the neck of the bladder. It is probably due to simple extension of the inflammation from the urethra or to pus-infection, and not to invasion of the membrane by gonococci, since cystitis accompanying gonorrhoea is usually much more amenable to treatment than is the primary disease. It is

"Jounal of Cutuncous and Ginito-urinary. Diseases, Sept., I $\$_{93}$, June and July, I 894. 
often classed as a mixed infection, though gonococci have never been demonstrated in the tissues of the bladder. The exciting causes of this form of cystitis are those of posterior urethritis, and they are found chiefly in such acts, surroundings, treatment, ctc. as tend to congest or irritate the deep urethra or to convey pus from the urethra to the bladder.

Symptoms.-The symptoms are essentially those of posterior urethritis. They may be very mild and scarcely noticeable, or so severe that there is a constant, almost irresistible, desire to urinate, with violent tenesmus and the expulsion every few minutes of a few drops of urine mixed with pus and blood. Between these two extremes the symptoms may vary greatly.

There is frequently a feeling of weight and discomfort in the perineum, with pains which radiate to the penis, testicles, groins, and back. The pubic and hypogastric regions are often tender and sensitive. In severe cases, in those of long duration, and especially in those complicated by the presence of stricture or enlarged prostate, there may be atony of the bladder-walls and partial retention of urine. If unrelieved, this condition is usually followed by ammoniacal decomposition of the urine, which then has a strongly fetid and ammoniacal odor, and contains a quantity of thick, viscid sediment that adheres to the bottom and sides of the vessel in which the urine is voided. Such urine is necessarily irritating to the mucous membrane of the bladder, and increases the severity of the inflammation.

Constitutional symptoms are usually slight; but if large areas of the vesical mucous membrane be involved, there may be chills, fever, and other systemic disturbances. Such symptoms, however, appearing somewhat 
suddenly during the course of cystitis, should always lead the physician to suspect the added presence of pyelitis. There is often more or less of the mental distress common to inflammatory disorders of the genitourinary tract. As in other complications of urethritis, the urethral discharge diminishes or disappears entirely during the course of the new disorder. Cystitis may vary in duration from a few days to several weeks or months, and may terminate in complete resolution or in some of the chronic forms of the disease.

Diagnosis. - The subjective symptoms in cystitis, prostatitis, and posterior urethritis are so much alike that they should never be relied upon for a diagnosis. Rectal examination will determine the presence or absence of prostatitis, and careful examinations of the urine should decide if the inflammation has extended from the pars posterior to the bladder, for gonorrhœal cystitis without posterior urethritis rarely, if ever, exists.

Thompson's two-glass method, as described in connection with Acutc Postirior Urethritis, should be used, though it is often desirable to have the urine passed in three separate glasses. If the inflammation be limited to the posterior urethra, there will be times-when the urine has been retained in the bladder for but a few minutes, possibly half an hour or an hour-when the first portion of urine alone will contain pus, the other portions being clear. When this occurs, cystitis may be excluded. If the vesical neck be also involved, the first glass will contain most of the pus, but all the urine will be more or less clouded. If the cystitis be more extensive, the (juantity of pus will be greater, and during the intervals of urination it will settle to the base of the bladder. In this case the amount of mucus and pus in the first glass 
depends on the activity of the inflammation in the posterior urethra and at the vesical neck; the second glass contains the more or less clouded urine from the upper part of the bladder; while the urine in the third glass contains the mucus and pus that has collected at the base of the bladder, and is therefore more heavily clouded than that in either of the other two glasses.

If in cystitis the urine be acid in reaction, as it may be in the early stages, and be allowed to stand in a glass for a few minutes, two layers of precipitate will form. The first layer, that at the bottom, is composed chiefly of pus, is white, more or less dense, and crumbly in appearance. Above this is a looser, flocculent or cloudy layer of mucus and muco-pus. This upper layer forms more slowly, but soon settles sufficiently to leave a clear layer of urine at the top. In the more severe as well as in the older forms of cystitis the urine is usually neutral or alkaline in reaction. As the degree of alkalinity increases the pus and mucus form a thicker, glairy, stringy substance which adheres to the membrane of the bladder and to the vessel in which it is placed. It cannot be dropped from one test-tube to another, but goes over in stringy masses. This appearance is most marked in ammoniacal decomposition of the urine, which then not only has a foul odor and contains pus, but also shows under the microscope large numbers of micro-organisms, amorphous phosphates, and coffin-lid, triple-phosphate crystals. This condition of the urine is not found unless cystitis is present.

During the course of cystitis, pyelitis may occur without additional symptoms, so that in every case in which the urine constantly contains pus the possible presence of pyelitis should be considered. 
Treatment.-The details of treatment are practically those of acute prostatitis. The chief indications are met by rest in bed, large dilution of the urine with bland drinks, hot local applications and hot baths, and anodynes to relieve pain and tenesmus. It is not desirable to render the urine alkaline, as that would favor ammoniacal decomposition. Balsam of copaiba-less frequently' oil of sandalwood-is often highly efficacious in reducing the acute symptoms. Boric acid or salol in doses of 5 or Io grains every three or four hours is valuable in sterilizing the urine and preventing decomposition. Citrate or acetate of potash in IO- to 20-grain doses three or four times a day is sometimes of service as a diuretic.

If the inflammation continues and becomes chronic, the treatment is that of chronic or catarrhal cystitis from other causes.

\section{PYELITIS.}

Inflammation of the pelvis and calices of the kidney occasionally follows gonorrhœal cystitis. Its occurrence is favored by the presence of stricture, enlarged prostate, or any interference with the free outward flow of urine. Cachexia, bad hygiene, and alcoholic excesses may also favor its development.

Symptoms. - This complication may develop insidiously, and until severe enough to affect the general health it may present no symptoms in addition to those of cystitis. In the majority of cases, however, there is pain, of a dull, burning character, in the back and the loins, extending possibly to the bladder, testicles, perineum, and thighs. The pain is increased by pressure over the kidneys or by active exercise. There is usually some fever accompanied by chills, that may recur with a regularity suggesting malaria. 
Diagnosis.-The sudden appearance, during cystitis, of constitutional disturbances should suggest pyelitis. Other symptoms are of value, but the diagnosis rests chiefly upon examinations of the urine. In pyelitis the urine is decidedly acid unless modified after reaching the bladder. The pus is intimately mixed with the urine, and on standing settles to the bottom in a greenish, compact, creamy or oily-looking layer. If decomposition in the bladder is prevented, the urine is not only acid, but on standing remains so for several days, and bacteria do not readily develop in it, as they do in urine from cystitis.

Albumin is present in larger amount than would be furnished by the pus alone. Under the microscope the urine shows, besides pus and mucus, cylindrical masses of pus-cells, occasional hyaline or granular casts, some red blood-corpuscles, and epithelial cells that in some cases may be recognized as peculiar to the kidney. Later in the disease there are sudden changes from day to day in the amount of pus present in the urine. Finally, in doubtful cases the bladder may be washed out thoroughly and the urine allowed to collect for fifteen or twenty minutes, when it is drawn from the bladder with a catheter; if pus is evenly mixed with the urinc, it undoubtedly comes from the kidney.

Treatment.-The treatment of cystitis should be continued in the form of rest in bed, diluent drinks, diuretics, anodynes, and the avoidance of all stimulating articles of food and drink. Hot baths, with hot fomentations or cupping over the region of the kidney, often aid in relieving pain. Copaiba, boric acid, or salol can often be used to advantage. Under such treatment, and with the removal of the cause, this form of pyelitis usually termi- 
nates in prompt recovery, though it may progress to graver stages of the disease or may become chronic.

\section{FOLLICULITIS.}

In most cases of gonorrhœa inflammation extends to some of the follicles and glands opening into the urethra. This complication may occur in any part of the canal, but is most frequent in the fossa navicularis, bulb, or prostatic portion, where the glands are large and numerous If the inflammation is mild in type, the follicle becomes slightly swollen and tender and discharges pus into the urethra. This condition may be present in a number of glands during an acute gonorrhœa without adding appreciably to the urethral symptoms, and therefore without being recognized, but it can easily be demonstrated when the follicles at the orifice of the urethra are involved. The lips of the meatus are then red and swollen; if their surfaces be cleansed and slight pressure be made upon them, pus will be seen escaping from the narrow openings of the follicles.

If the inflammation is more severe, the duct may become occluded by the swelling of the mucous membrane, and the follicle or gland becomes a cyst filled with pus. At first no larger than a pin-head, and slightly, if at all, painful, it may remain stationary for some time as a small firm nodule; or it may grow slowly, remain inactive for months or years, and finally. be absorbed; or it may increase more rapidly, become sensitive, soften, and discharge externally, leaving a fistula which may persist indefinitely. When, as occasionally happens, one of these cysts opens into the urethra, it may discharge its contents and refill repeatedly, or may become irritated by the urine and result in peri- 
folliculitis. Folliculitis may persist in subacute form and furnish a urethral discharge long after the rest of the urethral membrane has recovered.

Treatment.-If the inflammatory symptoms are acute, rest and hot applications should be employed. When fluctuation is detected, an incision should be made, to allow the escape of the pus externally and to prevent an opening into the urethra. Later the cyst may be enucleated entirely or the sac may be injected with a drop of pure carbolic acid or a strong solution of nitrate of silver. When a cyst opens into the urethra, the cautious use of the full-sized steel sound is of service in keeping the sac empty. In indolent cases absorption may be promoted by the external use of oleate of mercury in strength varying from 2 to 10 per cent.

\section{PERIURETHRITIS.}

As an unusual result of folliculitis the inflammation extends beyond the limits of the follicle or gland and invades the surrounding cellular tissue. The process may then terminate in a circumscribed abscess or, very rarely, in the suppuration and destruction of large portions of the spongy and cavernous bodies of the penis. The commonest sites of periurethritis are in the fossa navicularis, where the abscess generally forms on one side of the frænum, and in the bulb, where it usually develops in the central line. Beginning in the bulb, the inflammation may involve the root of the penis and the scrotum, or rarely the entire perineum and the tissues about the anus. The abscess usually opens externally, but it may discharge into the urethra, and may result in urinary fistula or in infiltration of urine through large portions of the surrounding tissues, causing suppuration 
and destruction of these parts. The conditions favorable to infiltration and abscess-formation are found just back of a stricture, where the urethral walls are damaged and weakened. The abscesses are more or less painful, and may interfere by pressure upon the urethra with the passage of urine. When suppuration is extensive the constitutional symptoms may be marked. The cicatrices which are left after healing of such abscesses may be slight, or so extensive and deforming that an erection of the penis is attended by crooking or bending of the organ, and possibly by pain.

Treatment.-Absolute rest of the genital organs, and, when possible, of the entire body, is of the greatest importance. To this end a light diet, gentle evacuation of the bowel, absence of all sexual excitement, and horizontal position of the body are necessary. If the inflammation be recognized early, it may be aborted by cold compresses and inunctions of the oleate of mercury. If suppuration begin, boric-acid fomentations should be constantly applied, and an early, often a deep, incision is necessary to evacuate the pus and to prevent an opening into the urethra. It is neither wise nor safe to wait for fluctuation before using the knife. If there are other evidences of suppuration, or if the inflammation has been in progress for a week, it is better to make a free incision than to run the risk of allowing the abscess to open into the urethra. This is especially true of an abscess situated in the bulb, where an internal opening is liable to be followed by extensive infiltration of urine and by perineal abscess. After discharging, the cavity should be cleaned daily and packed loosely with iodoform gauze until healthy granulations are obtained. All manipulations should be gently and carefully performed, lest 
communication between the gland and the urethra be reestablished and a urinary fistula be formed.

When an abscess ruptures into the urethra, the treatment consists in rest, fomentations, and a position that will favor drainage. The case must be watched carefully, and as soon as local swelling, pain, interference with urination, or fever indicates extravasation of urine and further suppuration, an external opening should at once be made. The further treatment is that of urinary fistula.

Resolution may be incomplete, leaving a small, indolent nodule which persists for months. Such a condition will usually disappear under inunctions of oleate of mercury.

\section{COWPERITIS.}

Inflammation of Cowper's glands is a rare complication of gonorrhœa. It may occur after the second week, but it usually begins between the third and fourth weeks. The patient complains of a sticking pain, of tension, or of tenderness in the perineum on pressure (as in sitting). On examination a deep-seated, round or oval, tender nodule, about the size of a bean, is discovered midway between the anus and the posterior border of the scrotum and at one side of the raphé. It is sometimes pear-shaped, in which case the larger end is toward the anus. The tumor usually grows rapidly in size, and by pressure upon the urethra may interfere with micturition. The surrounding tissue becomes involved, so that the tumor loses its sharp outline, becomes doughy or boggy to the touch, and may extend somewhat beyond the median line. Suppuration is attended by local throbbing pain and by chills and fever. Many mild cases undergo resolution, but a large abscess may form and may open ex- 
ternally or internally. In the latter case there is great danger of infiltration of urine and deep perineal abscess.

Treatment.-The treatment is that of periurethral inflammation and abscess.

\section{LYMPHANGITIS.}

In severe cases of gonorrhœa more or less lymphangitis is common. In its simplest and mildest form there are no subjective sensations, but one or more lymphatics, usually on the dorsum of the penis, can be felt beneath the skin as small indurated cords. Occasionally such a cord may be traced to the groin. If the inflammation runs a little higher, perilymphangitis results and is manifested by reddish streaks along the course of the lymphatics, which may be knotted and tender. There may be a more diffuse redness of the skin, which is then œdematous, swollen, hot, and sensitive. The inguinal glands may become swollen, tense, and painful.

Treatment.-Rest, elevation of the penis, and boricacid fomentations are usually all the treatment necessary. As a rule, the inflammation terminates in resolution. If pus forms, it should be evacuated early to prevent burrowing in the loose tissues, as a considerable portion, or even all, of the skin and the subcutaneous tissue of the penis could be thus destroyed.

\section{ADENITIS.}

During a severe case of gonorrhœa it is not uncommon for one or more of the inguinal glands to become slightly swollen and tender; but suppuration is quite unusual, and when it does occur the abscess heals kindly after discharging the pus, which is not autoinoculable. 
Treatment for the adenitis, aside from that given for the gonorrhœa, is usually unnecessary. When the gland first begins to swell and become sensitive, it may be well to paint the overlying skin with tincture of iodine or to apply a 2 per cent. oleate of mercury. If pain and swelling become pronounced, rest, hygiene, and boric-acid fomentations give relief. If pus forms, the abscess should be opened and treated on surgical principles. Scraping is rarely necessary, since the cavity usually heals kindly and rapidly under daily cleansing and packing with iodoform gauze.

\section{GONORRHGAL RHEUMATISM.}

In certain individuals gonorrhceal infection is always attended by a complication known as "gonorrhoeal rheumatism." These individuals are not, as a rule, subject to other forms of rheumatism.

Etiology.-Various theories are offered to explain the cause of this disease and its relation to gonorrhoeal infection, but none of them have yet been proven. In some cases there seems to be no doubt that the gonococci are carried through the blood to the joints, for, although these micro-organisms have not been discovered in the blood, they have been found in the fluids in the affected joints, and their identity has been fairly well established through their behavior with stains and through culture-experiments. Successful inoculations from these cultures are, however, wanting. In other cases the joint disease seems to be essentially pyæmic, and to be due to infection with the pus coccus, which gains admittance to the circulation through the urethral or other epithelium that has been damaged or destroyed 
by the gonococci. It is believed that some cases are due to ptomaine-poisoning.

The disease does not occur with non-infectious urethritis, but it has resulted from gonorrhceal infection of the conjunctiva or vagina, and therefore cannot be due simply to urethral irritation. It occurs in about 2 per cent. of all cases of gonorrhoea, and it is much more frequent in men than in women. A rheumatic diathesis and the usual causes of the commoner articular rheumatism have no apparent influence in the production of gonorrhoeal rheumatism; while an individual who has once had this complication of gonorrhœa rarely escapes in future infections, even when every precaution is taken to prevent its occurrence.

Symptoms.-The period at which the disease appears varies, but in about three-fourths of all cases it occurs during the third or fourth week of gonorrhoea. It may be much later, and has been reported as early as the fifth day. It does not have the effect-as do most of the complications of gonorrhœea-of diminishing the urethral discharge; on the contrary, changes in the degree and intensity of the urethral inflammation are usually promptly followed by similar changes in the rheumatic symptoms.

In about 40 per cent. of all cases the disease is limited to a single joint, and when polyarticular it is usually confined to two or three joints, which it attacks in succession, and not simultaneously. Finger collected statistics showing the joints affected in 376 cases as follows: Knee, I 36 ; tibio-tarsal, 59; wrist, 43; finger, 35 ; elbow, 25; shoulder, 24; hip, I 8 ; maxillary, I4; metatarsus, 7 ; sacro-iliac, 4 ; sterno-clavicular, 4 ; chondro-costal, 2; intervertebral, 2; crico-arytenoid, 2; 
peroneo-tibial, I. Besides the joints, synovial bursæ and the synovial sheaths of tendons and muscles may be involved.

All attempts to classify the widely varying symptoms of gonorrhœal rheumatism in distinct types have proven unsatisfactory. The classification here followed is substantially that given by Finger:

I. Acute Monarticular Gonorhaal Rheumatism.-This is the most frequent form of the disease, and usually appears in one of the large joints, most commonly the knee. The local disorder may be preceded by slight constitutional disturbance and by tenderness of several articulations, or the first symptoms may be pain and swelling of the affected joint. The tumefaction usually increases rapidly, with sufficient exudation to produce considerable tension. Pain is usually moderate, but may be mild or violent. Fluctuation is alvays distinct. Fever-which may be as high as $103^{\circ} \mathrm{F}$.- -and other systemic disturbances are present for a few days, but they rapidly subside. The swelling and the exudate remain and interfere with motion of the joint.

The exudate may be wholly absorbed in the course of a few weeks, complete recovery following, or there may be a relapse of the acute symptoms-usually following exacerbations of the urethritis-which greatly increases the natural tendency of the disease to become chronic and to terminate in hydrarthrosis. In rare instances the disease terminates in suppuration, which is announced by its usual symptoms-namely, chills and fever, an increase of the local swelling, pain which becomes throbbing in character, and an intense redness of the skin covering the parts. The pus bursts through the capsule and burrows between the tendons and mus- 
cles to the surface. The usual result of this process is pyæmia and death. Recovery with ankylosis is possible.

2. Acutc Polyarticular Gonorrheral Rheumatism.-The symptoms are those of the preceding variety, except that the disease involves two or more joints and that the constitutional disturbances are usually more pronounced. The latter may be acute and severe for a few days, but they do not last long. They may, howerer, recur a number of times as new joints are affected or following exacerbations of the urethritis. The fever does not often go above $103^{\circ} \mathrm{F}$. The pericardium and the endocardium are rarely implicated. The mild character and the brief duration of all the general symptoms, as compared with the severity of the local disorder, form a striking feature of the disease.

The affection may be limited to two-often symmetrical-joints, or it may pass in succession to three or four: the implication of a larger number of joints is exceptional. Simultaneous invasion of two or more joints is unusual, and the disease does not travel rapidly fromi one joint to another, nor does the involvement of a second joint hasten recovery in the first. Absorption of the effusion is slow, and, as in the monarticular variety, the disease may terminate in recovery, in chronic hydrarthrosis, or in pyæmia.

This variety of the disorder resembles more than do the others simple inflammatory rheumatism.

3. Subacute Polyarticular Gonorrharal Rhcumatism.This form of the malady is identical with the preceding, except that the fever is never higher than $\mathrm{IOI}^{\circ} \mathrm{F}$. and that the subjective symptoms are very slight. It is evident that a sharp dividing-line cannot be drawn 
between the two forms. Finger considers this variety of the disease second in order of frequency.

4. Chronic Gonorrhal Rhcumatism; Hydrarthrosis.This is a common form of the clisease, is usually monarticular, and is commonly found in the knee, the ankle, or the elbow. It may follow the acute or the subacute form, or it may appear independently. In the latter case it often develops insidiously, and it may not be noticed by the patient until the effusion is sufficient to interfere with motion; or the effusion may take place rapidly, and may be accompanied by some pain which is increased on walking or on other movements of the joint. Fluctuation may be the sole evidence of the disease. If the effusion be considerable, motion in the joint is more or less limited; if the exudate be cxcessive, there may be abnormal mobility of the joint, due, undoubtedly, to loosening of the ligaments. Absorption is occasionally rapid, but usually it is very slow and the fluid may be many months in disappearing. Adhesions and other deformities may leave an impaired joint.

Fournier's classification recognizes a form of gonorrhœal rheumatism in which neither structural nor functional evidences of disease are observed, and which may present no symptoms other than vague, wandering or persistent pains in some of the larger joints. These pains are very similar to those sometimes found in the early stages of syphilis, and they are often very rebellious to treatment.

The synovial sheaths of tendons connected with the affected joints may be involved, and, occasionally they are affected independently of the joints. There is redness of the skin, with a doughy, painful swelling that may extend some distance along the tendon. Motion of 
the muscle is prevented by the pain, which may persist after the swelling disappears. The bursa in front of the tendo Achillis and that beneath the inferior tuberosity of the os calcis are frequently involved. Other bursa are occasionally implicated. Rheumatic symptoms are sometimes present in the muscles, more especially in those connected with the affected joints.

Ophthalmic symptoms are not infrequently present. They are most common in connection with the polyarticular form of rheumatism. In rare instances they appear without rheumatic symptoms in other parts of the body. The parts of the eye that may be affected are the iris, the membrane of Descemet, and the conjunctiva. The iritis presents symptoms similar to those occurring when the affection results from other causes: the conjunctiva is reddened; the subconjunctival capillaries are injected, and can be seen radiating outward from the margin of the cornea; the iris is clouded and discolored, and its movements are sluggish; vision is somewhat impaired; posterior synechiæ may form; pain may be severe or absent, but there is usually photophobia and lachrymation.

In inflammation of the membrane of Descemet (aquocapsulitis, serous iritis) the symptoms are less acute. The fluid in the anterior chamber is clouded by the presence of a plastic exudate and epithelial cells from the iris; vision is thus impaired. Flocculi may be deposited on the membrane of Descemet, giving this part of the cornea a punctate appearance. The iris may be slow in its movements, and exceptionally synechire may form, but other symptoms of iritis are wanting. This is the commonest form of ophthalmia associated with gonorrhoeal rheumatism. 
The conjunctival form of gonorrhacal rheumatic ophthalmia, described by Fournier, is very rare, and is simply a mild form of conjunctivitis having a scanty, muco-purulent discharge. The prognosis is entirely favorable. This form of conjunctivitis must not be confused with the infectious gonorrhœal form (see table of diagnosis in Gonorrhacal Ophthalmia).

These forms of ophthalmia usually follow the course of the gonorrhœa on which they are dependent. As a rule, they affect both eyes, but not at the same time. The prognosis is favorable, and the only results to be feared are the adhesions which may follow iritis, or, rarely, inflammation of the membrane of Descemet.

Among the occasional and unusual complications of gonorrhœal rheumatism are pericarditis and endocarditis. Neuralgia of the sciatic and other nerves has been reported, as well as indefinite spinal symptoms consisting of disturbances of sensory and motor functions of the nerves. Purpura, erythema multiforme, and other eruptions on the skin may occur in connection with the disease, but in most cases the eruption is probably due to some drug given to relieve the rheumatism.

Pathology.-Regarding the pathology of the disease little is known. Post-mortem examinations have been made on a few fatal pyæmic cases. In some of these cases there were erosions of the cartilages and bones, and even complete destruction of the cartilages, with dissection of the periosteum from several inches of the femur. Gonococci were found in some instances. In more favorable cases puncture or incision of the joint has disclosed a serous, sero-fibrinous, or sero-purulent fluid, which in the majority of cases contained cocci that were apparently identical with gonococci. 
Diagnosis. - When the disease complicates successive gonorrhœal infections, or when it is limited to a single joint, the diagnosis is usually made without difficulty. The following is a table of differential diagnosis as given by Fournier, with some modifications:

Gonorhaul Rhenmatism.

Cause.-Gonorrhoeal infection.

Cold and rheumatic diathesis without influence.

Rare in women.

Fever and systemic symptoms usually mild and often absent. Acute symptoms, when present, are of brief duration.

Often limited to one joint, never involves many.

When polyarticular, the joints are affected consecutively, and not simultaneously:

Moves from one joint to another less quickly. No delitescence; no real jumping from one joint to another.

Local pain less intense and shorter in duration. More relief from rest and position. Pain often slight or even absent.

Secondary hydrarthrosis common.

No sweating.

Lrine not modified.

Blood does not furnish marked buffy-coat.

Cardiac complications very exceptional.

\section{Simple Rheumatism.}

No relation to gonorrhœe. Habitual causes are cold, inheritance, rheumatic diathesis, etc.

Common in the female, though less frequent than in the male.

Fever and other systemic symptoms are almost always present and are much more severe and more prolonged.

Rarely limited to less than two or three joints; may involve nearly all.

Simultaneous involvement of several joints is the rule.

Movable, ambulatory fluxions; rapid delitescence, jumping from one joint to another.

Pains are usually intense, sometimes excessive, last much longer, and are but partially relieved by rest and position.

Secondary hydrarthrosis rare.

Abundant sweats, usually acid, are characteristic.

Urine specially modified.

Blood forms a firm, concave clot with buffy-coat.

Cardiac complications frequent. 
Gonorhaul Rheumatism.

Frequently complicated by a special ophthalmia, synovitis of tendionsheaths, burse, etc. The latter localities may be exclutively implicated.

Relapses are frequent, and are usually dependent on exacerbation of urethritis. Recurs almost invariably with succeeding gonorhoeal infections.
Simple Rheumatism.

Eye not affected; the burse escape, as do usually the sheaths of the tendons.

Relapses frequent, but independent of state of urethra. Can often be traced to exposure to cold, weather-changes, etc.

Treatment.-No satisfactory treatment of gonorrhœal rheumatism has yet been found. In the acute and subacute stages salicylate of sodium, salol, and occasionally phenacetin, are of some value. Taylor recommends oil of gaultheria in capsules each of from 10 to 20 minims three or four times a day. Rest, a light diet, tonics, and hygrienic measures are always indicated. The urine should be kept unirritating by the use of bland drinks and, when necessary, alkalies.. Copaiba, sandalwood, and cubebs are of decided benefit when they lessen the urethral inflammation, since changes for the better or the worse in the rheumatic symptoms usually closely follow similar changes in the urethritis. For this reason local treatment of the urethra and all possible sources of urethral irritation should carefully be avoided.

The most satisfactory results usually come from local treatment of the joint. During the acute stages absolute rest, with the constant application of fomentations or poultices as hot as can be tolerated, will usually give prompt relief. In unusual cases, when the pain is excessive, tobacco or opium and belladonna may be added to the fomentations. Blistering of the surface and frequent applications of the Paquelin cautery are excellent 
methods in both acute and subacute cases. To promote absorption in subacute and chronic cases, the surface over the joint may be painted with iodine or rubbed with oleate of mercury in lanolin (2 to Io per cent.); or massage, friction, and electricity may be found of benefit. All forms of local treatment should be supplemented by firm, even pressure secured by a properly applied roller or elastic bandage or by an elastic cap or splint made to fit the joint. In chronic and persistent cases it may be necessary to immobilize the joint in a plaster cast. Aspiration of the joint followed by irrigation with a 2 per cent. solution of carbolic acid may prove effective when other measures fail. In the rare cases in which suppuration occurs the treatment is entirely surgical.

The ophthalmic symptoms call for little special treatment. In iritis a solution of atropine ( $\mathrm{gr}$. ij to $\tilde{\tilde{j} \mathrm{j}}$ ) should be used to keep the pupil dilated and to prevent adhesions. All other treatment is chiefly symptomatic. A light diet, rest, correct hygiene, and proper treatment of the urethritis are always in order.

Prognosis.-With thorough and persistent treatment most cases recover. Fatal cases are few, but the disease has a decided tendency to continue as a chronic hydrarthrosis. The prognosis should therefore be guarded.

\section{GONORRHGAL CONJUNCTIVITIS.}

Synonyms.-Gonorrhceal ophthalmia; Blennorrhagic ophthalmia; Purulent ophthalmia; Blennorrhagic conjunctivitis; Purulent conjunctivitis.

In the adult gonorrhoeal conjunctivitis is fortunately rare, but when it does occur it is an exceedingly grave disease, since it usually results in impairment or destruction of rision in the affected eye. 
Etiology.-Direct infection of the conjunctival membrane with pus containing gonococci is apparently the sole cause. The smallest particle, however, of such pus, even after it has been dried for some days, is sufficient to cause infection. Successful inoculation of the urethra has been accomplished with gonorrhœal pus diluted to one part in a thousand. These statements apply only to gonorrhœal conjunctivitis, and not to other, usually milder, forms of purulent conjunctivitis. In most cases the disease of the conjunctiva is found in individuals suffering from gonorrhœa, and who have conveyed some of the discharge from the genitals to the eye; but pus from any form of gonorrhœal inflammation may be carried by means of fingers, handkerchiefs, towels, etc., and produce the disease in any eye with which they come in contact. In this way the eyres of the physician, nurse, or companion are occasionally infected.

Symptoms.-The period of incubation, or the time which elapses between the infection and the first apparent symptoms, varies from a few hours to two or three days. Usually the duration of this period cannot be determined, since the infection is rarely recognized at the time of its occurrence.

The symptoms begin as a mild conjunctivitis, with lachrymation and itching, burning, or irritation of the conjunctiva, which is more or less reddened and injected. These symptoms, however, rapidly increase in severity; the discharge often becomes purulent in a few hours, and the inflammation reaches its greatest intensity on the second or third day. The lids are then œdematous and swollen, usually hard and tense, with a dusky-red, glistening surface, and to the touch are hot and painful. The upper lid often overhangs the lower, and the patient is 
usually unable to open the eye. The discharge is thin, creamy, and abundant, and escapes between the edges of the lids, flowing over the cheek, where it may dry in crusts and excoriate the skin; later it is thicker and less abundant.

On carefully separating the lids a quantity of the retained discharge will escape, and the conjunctiva is scen to be intensely red, swollen, rough and spongy, and often dotted with hemorrhagic points. As the lids are opened the pent-up secretion sometimes escapes in quite a jet, and the examiner should be very careful to keep his own eyes at a safe distance. The swollen and congested ocular conjunctiva is lifted up from the globe by the exudate, overlaps the margin of the cornea, and forms a circular wall around it. The cornea thus forms the bottom of a depression filled with pus, and cannot be seen until the latter is removed. A plastic exudate may cover portions of the conjunctiva, the removal of which exudate is followed by hemorrhage.

Pain in the eye and in the orbital region is often intense. The local temperature is increased, but general fever is mild or absent. Systemic disturbances are usually limited to those caused by the pain, anxiety, and mental distress.

The great danger lies in extension of the inflammation to the cornea-a process that is encouraged by the irritating effects of the pus retained in contact with the surface of the cornea, and by the interference in the corneal circulation resulting from the pressure produced by the chemosis and the tensely swollen, heavy lids. Cloudiness of the cornea may be present; this cloudiness may disappear under treatment, leaving no permanent defects. Ulceration of the cornea, however, is to be 
dreaded; it begins as superficial losses of tissue, usually near the margin, but it may first appear at the centre. Such ulceration may progress rapidly and destroy large portions or all of the cornea, resulting in staphyloma, prolapse of the iris, escape of the entire contents of the eye, or even purulent panophthalmitis. In less severe cases the ulceration may be arrested by prompt treatment, and the vision may be but partially lost. The duration of the disease is from four to twelve weeks. In favorable cases, with no involvement of the cornea, complete recovery occurs in five or six weeks, or there is left a chronic conjunctivitis which disappears under appropriate treatment.

Pathology.-There is inflammation of the conjunctiva and of the subconjunctival tissues. The point of special interest is the presence and location in the tissues of the gonococci: they rapidly penetrate to the upper layers of the subepithelial tissues, where their presence is soon followed by the phenomena of inflammation. Just where they chiefly proliferate is an undecided question, but they are most numerous in the epithelium and in the secretion.

Diagnosis.-The symptoms are usually so pronounced that the diagnosis is not difficult. Purulent conjunctivitis from other causes presents symptoms of the same type, but less severe, and the secretion does not contain gonococci. Treatment is the same. To avoid confusing the two distinct types of ophthalmic disease that may complicate gonorrhøa, the following table of Fournier's is given: 
Gonorharal Conjunctivitis.

Essential cause is inoculation of the conjunctiva with gonorrhceal pus.

A rare affection.

May affect subjects not suffering from gonorrhoea.

Lsually but one eye involved.

The symptoms are those of the gravest forms of purulent conjunctivitis; they affect the conjunctiva primarily.

Symptoms fixed, not going from one eye to the other.

No tendency to relapse in subsequent gonorrhceas.

No coincidence with rheumatic manifestations.

Prognosis excessively grave; often loss of eye.

Eye is saved only by most energetic treatment.

Gonococci in the discharge.

\section{Gonorrhacal (Rheumatic)}

Ophthalmia.

Not contagious; develops under the influence of an internal cause, the nature of which is unknown.

An infrequent complication of gonorrhrea. More common than gonorrhceal conjunctivitis-I $4: \mathbf{I}$.

Only attacks patients already suffering from gonorrhcea.

Commonly both eyes.

symptoms are those of inflammation of the membrane of Descemet, of an iritis, or of a mild conjunctivitis.

Symptoms may be mobile, passing from one eye to the other.

Frequent relapses in the course of subsequent gonorrhceas.

Occurs with gonorrhoeal rheumatism, rarely without.

Prognosis without gravity.

Expectation, or the simplest treatment, sufficient for a cure.

No gonococci.

Treatment.-The treatment must be prompt and thorough. A few hours' delay may cause the loss of the eye. The patient should go to bed in a darkened room, and should have a trained nurse in constant attendance, to keep the surfaces cleansed and properly dressed and to protect the sound eye from infection. In some cases it is best to protect the sound eye by sealing it hermetically with a layer of light rubber tissue covered with a thin layer of cotton or gauze, which, with the rubber, is fastened by means of collodion to the skin surrounding the orbit. An opening for ventilation may 
be left at the outer side. Rubber plaster may be used instead of collodion, while for those who can obtain it promptly Buller's shield is the best device.

In the beginning, if the patient be strong and robust, several ounces of blood may be abstracted from the temple by means of leeches or cups, and a brisk cathartic may be administered. This treatment may be followed for several days by laxatives and a light diet. In a less vigorous patient these measures would be too severe, since it is very important that the general strength and the recuperative powers be maintained fully. In cachectic or debilitated subjects or in those with poor hygienic surroundings the task of trying to save the cornea is exceedingly difficult.

The objects of local treatment are (I) to keep the surfaces clean and to prevent the accumulation of secretion beneath the lids; (2) to reduce congestion by the constant application of cold; (3) to relieve pressure; and (4) in all but mild cases to combat the process with applications of astringent or caustic solutions. The accumulation of pus about the edges of the lids and under them should be wiped away gently with lint or with bits of cotton wrapped on the ends of toothpicks. Such lint, cotton, and toothpicks should promptly be burned. No dressing of any description should be used a second time. Safety for the patient's sound eye and for the eyes of the physician and the attendants demands that every piece of cloth or other dressing that has once come in contact with the smallest particle of the discharge should immediately be destroyed by burning.

After the first cleansing further accumulation of pus beneath the lids should be prevented by frequent washing with a 3 per cent. solution of boric acid in distilled or 
boiled water. A solution of bichloride of mercury ( 1 : 20,000) may be used instead. The lids are gently separated, and the liquid is allowed to flow over the surfaces until all secretion is removed. The solution is best applied by sqeezing it out of pieces of cotton or sponge; a bulbsyringe may be used, but the ordinary irrigating syringe is liable to spatter and to endanger the other eye. The patient's head should be turned slightly to the side of the affected eye, to prevent any possibility of the solution reaching the opposite side, and a pus-basin or wads of cotton should be held in position to catch the discharge. This irrigation of the inflamed surfaces should be repeated every five, ten, or fifteen minutes, both day and night, during the acute stage, and less frequently as the discharge becomes less abundant. The object is to keep the surfaces, and especially the cornea, free from pus.

During the intervals between the washings cold is applied by means of pieces of soft linen, large enough to cover the eye, taken out of ice-water or from the surface of a block of ice. These pieces of linen are removed and burned, and are replaced by fresh ones every minute or two. This constant dressing and handling of the eye must be done with the utmost care and gentleness, and should be made to interfere as little as possible with that physiological rest which is so greatly to be desired in any acute inflammation. The fingers should not come in contact with the globe, or, if possible to avoid it, with the edges of the lids. Pressure (of heavy dressings, etc.) should be prevented. If the upper lid is thick and tense and difficult to evert, thus preventing proper cleansing of the eye and producing pressure upon the cornea, canthoplasty should be performed freely. The fingers or a 
wire speculum hold the lids apart and thoroughly stretch the skin over the outer canthus; one blade of a pair of sharp, strong scissors is passed under the lid, and the point is carried to the bottom of the cul-de-sac; a single sharp cut, which should be exactly horizontal, divides the tissues to the margin of the orbit. Pressure is thus relieved and free irrigation of the eye is made possible. If the parts heal too rapidly, it may be necessary to repeat the operation.

In mild cases frequent cleansing and the constant application of cold may be all the local treatment necessary. In most cases, however, when the conjunctiva becomes greatly swollen and the discharge profuse and purulent, a $\mathrm{I}$ or 2 per cent. solution of nitrate of silver should be used. If the cornea is clear, 2 or 3 drops of such a solution may be dropped between the lids; but a better method is to evert the lids and apply a 2 (occasionally a 3 or 4 ) per cent. solution to the conjunctiva by means of a bit of cotton twisted on the end of a toothpick. After a few seconds the surfaces may be gently wiped dry or washed with a common salt-solution. By allowing the nitrate solution to remain on the conjunctiva a longer or shorter time the duration and extent of the caustic action, indicated by the whitening of the surfaces, can be controlled, and danger of irritating the cornea by contact with the fluid is avoided. In ulceration of the cornea the avoidance of such irritation is a matter of special importance.

Following such an application the discharge is greatly lessened, though the swelling continues, and pain is temporarily increased. If the iced cloths do not relieve the pain, solutions of atropine or of cocaine may be used. After a few hours the discharge reappears and contains 
fine shreds of the eschar resulting from the caustic. Frequent washings and another application of the silversolution are then in order. In the early stages of the affection solutions of the nitrate should be used but once in twenty-four hours; later they may be used every six, eight, twelve, or twenty-four hours, depending upon the rapidity with which the conjunctiva recovers from the application. Dr. Joseph A. Andrews has well emphasized the fact that the caustic solution should not be used until the eschar produced by the previous application has disappeared entirely. The use of the nitrate of silver solution should always be preceded by a thorough washing of the surfaces, and may be followed at intervals of an hour or two by the free use in the eye of a pure vaseline.

The cornea should be watched carefully. If it becomes cloudy or ulcerates centrally, a solution of atropine ( $\mathrm{gr}$. ij to $\overline{\mathrm{j}} \mathrm{j}$ ) should be dropped in the eye often enough to keep the pupil well dilated. If ulceration begins at the margin, sulphate of eserine (gr. $j$ to $\overline{\tilde{s}} \mathrm{j}$ ) should be used instead of atropine, and with sufficient frequency to keep the pupil tightly contracted, thus lessening the danger of prolapse of the iris in case of perforation. In exceptional cases, with pus in the anterior chamber and with bulging of the cornea, puncture (paracentesis) is advisable; and occasionally it is necessary to relieve the pressure upon the cornea by free incisions into the chemotic conjunctiva. Such incisions should be made after, never before, application of the caustic solution.

As the inflammatory symptoms subside and the disease progresses toward recovery the treatment is less active, but careful watch of the eye must be maintained for fear of a relapse. In the declining stage, if the cornea 
is clouded, absorption may be hastened by the use, for ten or fifteen minutes several times a day, of hot fomentations or irrigations; in the intervals the use of cold cloths is continued. When the discharge has become slight the cold cloths may be given up and the surfaces may be brushed lightly every day or two with a I per cent. solution of nitrate of silver or of sulphate of zinc.

Prognosis.-The prognosis is always grave, but it is least favorable in the cachectic or the feeble and in those who have had previous disease of the eyes. Noyes gives the result in 40 cases as follows: In Io the cornea escaped injury and recovery was complete; of the other 30 with involvement of the cornea, 5 retained useful vision, 9 retained some vision, and 16 lost all vision, in the affected eye. The chronic (granular) conjunctivitis which often results usually yields to appropriate treatment.

\section{OPHTHALMIA NEONATORUM (BLENNORRHEA NEONATORUM).}

The term "ophthalmia neonatorum" is applied to purulent conjunctivitis appearing in children a day or two, or occasionally a few weeks, after birth. It is much more common than gonorrhøeal conjunctivitis in the adult, and is therefore of greater importance, but it is of interest chiefly to the obstetrician and the ophthalmologist. The condition is the result of direct or indirect infection of the child's eyes with secretions from the mother's vagina. This infection may occur during birth, but in most cases it probably occurs during the first washing of the child, or from accidental contact with sponges, napkins, handkerchiefs, etc. used by the mother. Indirect infection by some means is undoubtedly the cause of the disease when it appears after a few days. The source of infection is 
not necessarily gonorrhoeal ; other pus or irritating secretions may produce in the child a conjunctivitis differing but slightly, if at all, from that produced by gonorrhcea.

Symptoms.- The symptoms are essentially those of gonorrhceal conjunctivitis in the adult; but the disease may be even more acute in its course, and loss of sight is possibly more frequent.

Prophylaxis.-In case the mother have a suspicious vaginal discharge, her vagina should be cleansed with an antiseptic solution before the child is delivered. Inmediately after birth the child's eyes should be washed thoroughly with a 3 per cent. solution of boric acid, and a drop of a 2 per cent. solution of nitrate of silier should be dropped in each eye (Credé's method). If the resulting inflammation be too severe, it may be limited by the application of cold. Every precaution should be taken lest the child's eyes be infected later, through the careless use of handkerchiefs, towels, etc.

Treatment.-The treatment is practically that of the disease in the adult, except that the nitrate of silver should be used with greater caution and in weaker solution (one-half per cent.), and be limited in its application to such cases as fail to improve under the use of frequent washing and iced cloths. It is even more important than in the adult that caustic solutions should be kept from the cornea. Canthoplasty is rarely required in the infant. Cachexia, debility, and lack of derelopment (premature birth) predispose to unfavorable results.

Many children for a few days after birth have a mild form of conjunctivitis which gives the lids a red and sticky appearance. These cases call simply for occasional bathing in simple borax-water or alum-water, and should not be confounded with the purulent form of the disease. 


\section{GONORRHGAL INFLAMMATION OF THE REC- TUM AND THE MOUTH.}

A few well-authenticated cases are reported in which the mucous membrane of the anus, of the rectum, or of the mouth has been involved in a gonorrhoeal inflammation. Such cases are, however, so rare that they may be classed among the curiosities of medical and surgical practice. The gonococcus does not readily invade these membranes, but when the disease does occur in these localities, it is undoubtedly due to local infection with gonorrhceal pus, and not, as has been suggested, to metastasis. Infection may be the result of accident or uncleanliness in those suffering from gonorrhoea, or it may be due to unnatural coitus.

Occurring in the anus and the rectum, the disease begins with itching and burning sensations which rapidly increase in intensity until, in a few days, pain is constant and greatly increased on defecation. The membranes become intensely red, hot, congested, and swollen, and secrete at first a thin, creamy discharge, which soon becomes thicker, darker, and profuse. The inflammation is usually limited to the anus or to the membrane below the internal sphincter. The diagnosis between gonorrhoeal and other forms of proctitis will rest chiefly upon the discovery of gonococci in the discharge and upon the history. In women the cause may be found in a gonorrhoea of the vagina or the urethra, the discharge from which has been allowed to run down over the anus and infect the membrane. When the disease occurs in one who has practised sodomy for some time, the sphincters are relaxed, the anal folds are wanting, and the anus may be more or less funnel-shaped. 
If limited to the anus, the disease should be treated simply with cleansing and astringent lotions and powders, and the surfaces should be separated with soft dressings, the principles and details of treatment being essentially those recommended for balanitis. Excoriations, fissures, and superficial fistulæ may be touched with solutions (or the solid stick) of nitrate of silver. If the disease extends into the rectum, care should be taken to secure a regular daily evacuation from the bowel, and the rectum should be irrigated thoroughly once or twice daily with a warm saturated solution of boric acid. For this purpose the rectal irrigator devised by Dr. James P. Tuttle is desirable. It may be necessary to dilate the sphincters and to apply a solution of nitrate of silver to excoriations and superficial ulcers that may be present.

Gonorrhœal inflammation of the mucous membrane of the mouth has been reported in very few instances, the largest number of cases being in new-born infants undoubtedly infected during birth by vaginal discharges. The symptoms are those of a severe stomatitis. The diagnosis is made from the history and by the finding of gonococci in the discharge. In new-born children the disease appears much earlier than do other forms of stomatitis. The treatment consists in frequent washing of the mouth with warm saturated solutions of boric acid (the addition of slippery elm or of flaxseed to the solution is sometimes very grateful), and in the application to the surface of astringent solutions. Nitrate of silver in strength varied to meet the indications of each case is the best preparation.

Gonorrhceal inflammation of the nose has been mentioned by several writers, but an unquestionable case has not been reported. 


\section{CHRONIC URETHRITIS.}

Synonyms.-Chronic gonorrhœea; Gleet.

Before terminating in complete recovery every cave of acute urethritis passes through a subacute stage with a muco-purulent and finally a mucous discharge. Following a first attack of gonorrhoea, in a healthy man under favorable hygienic surroundings, this muco-purulent stage tends to recovery without local treatment; but when following repeated infections, or an infection in an unhealthy individual or in one subjected to improper treatment or other injurious influences, this subacute stage may be prolonged indefinitely, and is known as "chronic urethritis " or " gleet."

Etiology.-In the cachectic, chronic urethritis may occur independently of an acute attack; but almost all cases originate in gonorrhœea.

The influences which interfere with the proper recovery of gonorrhœa and which tend to prolong the disease in chronic form are numerous and vary widely in different individuals. The general health of the patient is an important factor. In gouty, rheumatic, strumous, syphilitic, tubercular, anæmic, or debilitated persons it is not unusual for gonorrhœa to be followed by chronic urethritis. It occurs frequently as a result of repeated infections, or after a first infection in which there have been a series of relapses. 
Probably the chief factors in the production of chronic urethritis lie in the failure of the patient to observe a proper sexual hygiene during and after an attack of gonorrhœa; and the most persistent and intractable cases are found in men who, in spite of their disease, are indulging in promiscuous sexual relations, or who are subjecting themselves to other forms of sexual excitement, or who, in their efforts to get well, are constantly irritating the urethra by improper or excessive treatment. Mention has already been made of the fact that the cessation of a urethral discharge does not necessarily mean that the urethra has returned to a normal condition. It is difficult to make the average patient understand this fact, and realize the necessity of his living hygienically for some weeks or months after his gonorrhœa is apparently cured. Offenders of one class resume sexual relations as soon as the discharge becomes invisible. Such men often incur fresh infection, and have within a few months a series of gonorrhœeas some one of which will surely terminate in the chronic form; or, if fortunate enough to escape fresh gonorrhœal infection, nevertheless the recently inflamed mucous membrane of the urethra becomes the seat of small areas of chronic congestion and infiltration, resulting in a gleety discharge. Others, without sexual intercourse, indulge in sexual excitement, physical or mental, which aggravates the congestion of the parts and does not permit the rest necessary for a complete recovery.

Alcohol and tobacco are irritants to the mucous membrane of the urethra, and if used during the course of gonorrhœa or urethritis tend to prolong the disease. The resumption of their use too soon after the apparent cure of an inflammation of the urethra is many times responsi- 
ble for a return of the discharge; while many subacute cases refuse to get well until the patient abandons his habits in this particular. Similar in effect to the use of alcohol and tobacco, though in lesser degree, are excesses in eating, especially of nitrogenous and highly seasoned foods. Imperfect digestion, followed by incomplete assimilation and metabolism, necessitates the elimination through the urine of products foreign to normal urine, and therefore irritating to the urethral mucous membrane. Imperfect functional activity of the skin and the bowels, by adding to the quantity of solids in the urine, and therefore to its irritating qualities, may retard the recovery of a urethritis. Excessive physical exercise, while not so harmful as in acute urethritis, sometimes exerts a deleterious influence upon a chronic discharge.

A frequent source of chronic urethritis lies in excessive and ill-advised treatment, the patient trying one injection after another, using sounds, soluble bougies, and other forms of local and internal treatment, in hopes of removing the last trace of his discharge. These cases are most frequently found in unmarried men who are greatly worried about themselves and who drift from one physician to another. They try the "infallible" prescriptions recommended by their friends, and in the course of their wanderings they sooner or later fall into the hands of charlatans, and submit to all sorts of operations and treatment. If properly advised in time, before operative procedures have damaged the tissues, many of these cases recover completely as a result of simply suspending all treatment and living hygienically.

When the mucous or muco-purulent stage has lasted for some weeks, or when there has been a series of relapses, the disease usually becomes localized. The 
greater portion of the urethral mucous membrane recovers its normal condition, but certain circumscribed areas become the seat of chronic congestion and infiltration, or some of the complications which have arisen become persistent. The causes of this localization lie largely in the anatomical structure of the different parts of the genito-urinary tract. The inflammatory process naturally becomes more firmly seated in those portions well supplied with follicles, glands, and ressels. For this reason the prostatic, if once infected, and bulbous portions of the urethra and the fossa navicularis are especially liable to the persistent forms of inflammation and congestion. In most cases of chronic urethritis some portion of the anterior urethra is involved.

Among the local conditions which may exert an unfavorable influence upon chronic urethritis or be responsible for its persistence are areas of congestion and infiltration in the urethra, stricture, hypertrophy of the prostate, prostatitis, vesiculitis, lacunal inflammation, Cowperitis, folliculitis, periurethral abscess, fistulæ with internal openings, mucous patches in the urethra, and local tuberculosis.

The role of the gonococcus in the etiology of chronic urethritis is not yet definitely determined. These microorganisms can be demonstrated in many cases, but they are present in small numbers, and often can be found only after repeated examinations; while in quite a percentage of cases repeated careful examinations fail to show the gonococci. It would seem that the pathological processes instituted by the gonococcus may continue after the disappearance of the latter.

Symptoms. - The symptoms of chronic urethritis vary with the individual, the duration of the disease, and its 
location. In recent cases and in those undergoing active local treatment there is usually, in addition to the circumscribed pathological process, more or less congestion and catarrhal condition of the mucous membrane of the entire urethra, resulting in a mucous discharge which may be sufficient to stain the linen, or, if the prepuce be long, to keep its inner surface and that of the glans constantly moist; or there may be a subacute inflammation with a muco-purulent discharge.

In old cases in which most of the urethral mucous membrane is healthy and the disease is confined to one or more small areas, two or three drops of mucus or of mucopus collect in the urethra during the night and are seen at the meatus by the patient on rising in the morning. During the day, when the urethra is washed out more frequently by the passage of urine, the collection is usually only sufficient to cause slight moisture of the lips of the meatus or to slightly glue them together; or there may be no evidence of discharge during the day, and but an occasional agglutination of the lips of the meatus in the morning; or, finally, there may be no symptoms noticeable by the patient while he is living a regular life, though sexual indulgence or the use of alcohol or tobacco, or even cxcesses in eating or in exercising, may cause a prompt return of the discharge. These relapses are frequently considered new, mild infections, but they differ from the latter in that the symptoms appear at once, without any period of incubation, and subside in a few days under simple treatment. Sometimes the only evidence of disease is that found in the urine, which may be clouded with pus and mucus from the posterior urethra, or may be clear except for shreds composed of mucus, epithelium, and pus-cells. 
Subjective sensations are often entirely wanting. though the condition may persist for months or years. More frequently the patient experiences, while urinating, slight burning, pricking, or tingling sensations along the urethra or at the site of the lesions. In disease of the posterior urethra there may be a feeling of warmth, fulness or weight in the perineum, with possibly some increased frequency of urination, or even slight tenesmus; and if, as frequently happens, the inflammation invades the glands and the tissues of the prostate, there may be added all the distressing symptoms, both physical and mental, of chronic prostatitis.

If the process extends beneath the mucous membrane of the urethra, stricture may follow, with all its symptoms. If one or more follicles or glands of the urethra or the periurethral tissues are involved, the symptoms will depend upon the activity of the inflammation in these structures.

Pathology.-Regarding the pathology of chronic urethritis, Finger, who has made a histological study of a large number of cases, arrives at the following conclusions :

"I. Chronic urethritis is a focal process which runs its course as a chronic hyperplasia in the subepithelial connective tissue. Disease of the epithelium and glands is to be regarded in part as a complication, in part as a sequel.

"2. The foci of chronic blennorrhœa are localized preferably in the pendulous portion, the bulb, and the prostatic portion.

" 3. The membranous portion is relatively immune to the chronic process.

"4. In a series of cases the foci of chronic inflamma- 
tion in the pars anterior and posterior are situated superficially in the mucous and subepithelial connective tissue.

" 5. In another series of cases these foci extend by" continuity to the submucous tissue-in the pars anterior, to the periurethral and cavernous spongy tissue; in the pars posterior, to the prostate.

" 6 . This results in complicating focal processeschronic periurethritis in the pars anterior, prostatitis in the pars posterior.

"Hence arises the following classification of chronic urethritis :

"I. Chronic anterior urethritis: (a) Superficial anterior chronic urethritis; (b) deep anterior chronic urethritis (that is, plus chronic periurethritis).

"II. Chronic posterior urethritis: (a) Superficial chronic posterior urethritis; (b) deep chronic posterior urethritis (that is, plus chronic prostatitis).

"As a matter of course, mixed forms are frequentthat is, various foci in the pars anterior and posterior.

"The relation of gonococci to chronic urethritis is extremely obscure.... Their virulence is weakened by long proliferation upon the same soil for many generations. As proof may be cited the fact that chronic blennorrhœea is often conveyed as chronic, much more rarely as acute, blennorrhoea. The fact that each succeeding relapse is milder and shorter also indicates that the irritation of the papillary body by the gonococci gradually diminishes. The first relapses will always terminate by the removal of the gonococci to the surface, but the virulence may finally be diminished to such an extent that the acute purulent symptoms on renewed invasion of the papillary body no longer suffice to carry the gonococci to the surface. They will then remain in 
the papillary body, perhaps also in the follicles, and by their constant slight irritation give rise to the chronic proliferating processes in the mucous membrane. The conveyance of these enfeebled gonococci would explain the $a b$ initio chronic infection in women; and their proliferation in the deep layers enables us to understand the fact that gonococci may or may not be found in the secretion, the clap-shreds. But the chronic changes induced by the gonococci may develop further after the cocci have perished from any cause. This explains the fact that in certain chronic blennorrhœas we find the secretion and clap-shreds, but no gonococci.'

Diagnosis. - The symptoms of chronic urethritis are usually so evident that a diagnosis of urethritis is readily made. The difficulty lies in determining the seat and nature of the pathological process. The diagnosis between an exacerbation of a chronic urethritis and a recent mild infection is not difficult when it is remembered that the former appears in a few hours after exposure, without any period of incubation, is generally attended by no symptoms other than a trifling discharge, subsides readily under mild treatment, and has been preceded by other similar relapses of more or less recent date.

Chronic prostatitis may exist independently of urethritis, and may present symptoms identical with those of the same disorder when it complicates posterior urethritis. The diagnosis will depend upon the history and the absence of other evidences of urethral disease.

The adhesion of the lips of the meatus or the appearance of an occasional drop of mucus does not necessarily indicate urethritis. These symptoms may appear as a result of hypersecretion of mucus by a congested urethra. This condition is found in patients who are irritat- 
ing the mucus membrane of the urethra with needless injections or instrumentation. It is also found in persons who commit sexual excesses, natural or unnatural, or who indulge in ungratified sexual excitement. Under these circumstances the congestion and hypersecretion of mucus which always attend an erection become more or less persistent. Under the microscope such a discharge is seen to be composed of mucus and epithelial elements. The absence, on repeated examinations, of pus-cells and gonococci excludes urethritis.

The Infectiousness of Chronic Gonorrhar.-The discovery of characteristic gonococci in the secretion or shreds from the urethra at once determines the case to be one of chronic gonorrhcea. But in the majority of cases of chronic urethritis demonstration of the gonococci is not easy, since they are usually present in small numbers and associated with other micro-organisms. In a given case drops of pus and shreds may be examined for a number of days without discovering the gonococci, which a few days later may be found in considerable numbers. Negative findings are not conclusive, and so long as the secretion contains pus, the presence of gonococci should be suspected and sought for. In exacerbations of chronic urethritis the gonococci increase in numbers and can more readily be demonstrated; hence it may be justifiable, when repeated examinations give negative results, to cause an artificial inflammation of the urethra for the purposes of diagnosis. In anterior urethritis this object can be accomplished by irrigating ${ }^{1}$ the pars anterior a few times with a solution of bichloride in strength of I : 20,000 or I : 10,000 . In posterior urethritis a few drops of a $\mathrm{I}$ per cent. solution of nitrate of 'See Irrigation (index). 
silver may be placed in the deep urethra with a Keyes syringe. The increased (purulent) secretion which results will usually show gonococci if these be present. There are cases of urethritis in which artificial exacerbation of the disease and repeated examinations of the secretion fail to demonstrate the gonococci. The secretion in such cases is rarely anything more than mucus, and is the product of a catarrhal process in an overstimulated mucous membrane.

It is evident that with a chronic urethritis in the secretion of which gonococci are present but occasionally, a man may indulge in sexual intercourse repeatedly without infecting his partner, though he has no means of knowing at what time his discharge may become infectious. It is not safe, therefore, to allow a man with chronic urethritis to marry, or, if married, to resume marital intercourse, until during several weeks of frequent examinations the discharge shows no gonococci and the clinical symptoms point strongly to the presence of nothing more than a catarrhal discharge.

On this subject Finger says: "I permit a patient who is suffering from chronic blennorrhœa-that is, the morning drop or clap-shreds-to have marital intercourse only after I have convinced myself, by a two to four weeks' daily examination of the secretion or clap-shreds, that these contain only epithelium, and no pus-cells, and when, after irrigation of the urethra with a solution of silver nitrate or corrosive sublimate, and consequent suppuration, the secretion is entirely free from gonococci, and there is no further indication for the continuance of treatment. The conditions which I require are, accordingly, the absence of gonococci, pus-corpuscles, and periurethral complications." 
Noeggerath believes that if a man once have a urethral discharge containing gonococci, he never fully recovers, and that nine-tenths of the women married to men who have had gonorrhoea eventually develop pelvic inflammation due to infection by the gonococci. Such inflammations are usually subacute in their origin, and often date from the birth of the first child. On the other hand, Keyes, in his treatise of I 888 , says: "Care must be exercised in advising marriage, if the discharge be at all purulent and contain gonococci. No such pus can be pronounced free from contagious properties, although, practically, in my experience it has sometimes turned out to be so. In all cases of prolonged purulent gleet a lesion in the urethra (strictures, granulations) should be sought for and treated. If not found, and if no gonococci are present, marriage is proper, and not only not harmful, but even beneficial, in its effect upon the discharge."

Localization of Lesions.--In all cases of chronic urethritis it is necessary to determine the extent, location, and nature of the pathological process. In the majority of cases this is limited to small circumscribed areas, but in more recent cases, and in those giving a history of frequent relapses or continued local treatment, the entire mucous membrane may be the seat of a subacute inflammation, or at least of a chronic congestion. When a large portion of the urethra is thus involved, there will be a more abundant secretion, and the urine containing the washings of the urethra will be cloudy from the presence of pus or of mucus. Examination with instruments for the local lesion should be postponed until the more general disturbance has been removed by proper treatment. 
The first step in locating the seat of a chronic urethritis is to determine if it be in the anterior or the posterior urethra.

I. History and Sy'mptoms. - If there be a history of epididymitis, prostatitis, cystitis, vesiculitis, tenesmus, or other symptoms pointing to a former acute posterior urethritis, it is quite probable that in the posterior urethra will be found the lesion responsible for the chronic disorder. Subjective sensations are usually insignificant or wanting in chronic urethritis of the pars anterior, but if the pars posterior be involved there are usually ill-defined sensations and pains in the perineal region, and sensitiveness of this portion of the urethra on pressure upon the perineum or through the rectum; there may be frequency of urination, tenesmus, and other symptoms pointing to the presence of chronic prostatitis.

2. The Discharge. - If the discharge arises from some portion of the pendulous urethra, it will gravitate to the meatus, and appear there occasionally as a yellowish, milky, or transparent drop, or it may lightly glue together the lips of the meatus. When the process is situated in the bulb, the discharge, if small in amourt, may remain in situ until washed out by the urine. Discharges arising from the pars posterior will not appear at the meatus during the intervals of urination, but will remain in the prostatic portion or will pass backward into the bladder.

3. Examination of Urinc.-When the pathological process has become limited to circumscribed areas, neither pus nor mucus will accumulate sufficiently to appear as a discharge or to render the urine cloudy, but the urine may contain flakes or shreds composed 
of mucus, pus, and epithelium. The shreds may be transparent, delicate, narrow threads, often very long and branched. These threads are composed chiefly of mucus and epithelium, show a tendency to float in the urine, and in general indicate superficial and milder lesions of the urethra. Other shreds are shorter, firmer, and opaque, and contain a greater number of pus-cells. Such shreds sink rapidly to the bottom and indicate a more serious condition. A third type of shreds is sometimes found in the form of short, firm, commalike plugs or flocculi. These particles come from the excretory ducts of the various glands and follicles of the urethra that may be involved in the process. The character of the shreds gives some clue to the nature and intensity of the urethral disorder, but does not give reliable information regarding the location of the lesion. If, however, the shreds contain spermatozoa, or if they are of the comma-like variety and are present in the second portion of the urine, they come from the prostatic urethra.

The urine should be examined by the two-glass method, though this test is of less value than in acute urethritis, since the small amount of pus formed in the posterior urethra will be removed in the act of urinating before sufficient has accumulated to pass back into the bladder, and during the day, while the urine is passed at frequent intervals, that in the second glass will be clear and free from shreds. But if the urine be retained several hours until the prostatic urethra is well dilated, forming practically a part of the bladder, the pus and the shreds will mix with the urine in the bladder and appear in the second glass. Hence it is important that the patient bring his morning urine passed in two bottles, 
and that he again urinate in two glasses at the time of his visit to the physician. Another condition may be present, even when the urine in the bladder is clear, to cause cloudiness and shreds in the second glass: if the prostatic glands are inflamed, pus and comma-like plugs may be pressed out by the contraction of the muscle at the close of urination, appearing with, or just after, the last drops of urine.

The two-glass test may be modified by first cleansing (imgating) the anterior urethra with some simple aseptic or mildly antiseptic solution. A 6 per cent. solution of sodium chloride, or bichloride of mercury in the strength of I : 50,000, or boric acid, may be used. The reservoir holding the fluid should be but two or three feet above the level of the penis, and should be connected, by means of rubber tubing, with a short glass or hardrubber tube. The latter may be inserted into the urethra about an inch, and should not be large enough in diameter to completely fill the meatus, since space must be left by the side of the tube for the solution to escape. The fluid may then be allowed to flow until the pars anterior has been entirely cleansed of all pus, mucus, and shreds. If the patient now urinate in two glasses, the first glass will contain the washings of only the pars posterior; consequently, if the urine in both glasses be clear, it is evident that the disease is limited to the pars anterior; but if the urine in the first glass contain pus or shreds, while that in the second glass is clear, it is safe to make a diagnosis of posterior urethritis. If the urine in both glasses contain pus and mucus, inflammation of the bladder or of the kidneys is probably present. In this case other examinations should be made, and especially of urine that has been 
retained in the bladder but a short time. If the second glass at any time shows clear urine, cystitis may be excluded.

The reservoir should not be placed at too great an elevation, and the lips of the meatus should not be held against the tube to interfere with the free outflow, or the pressure upon the compressor urethre muscle will be sufficient to cause it to relax, and the fluid will pass on into the bladder, carrying with it pus and shreds from the pars anterior. The object of the test will thus be defeated, since the urine in the bladder-and therefore that in both glasses-will contain the washings of the

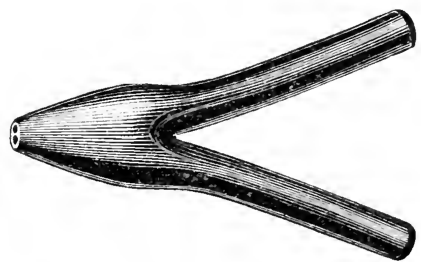

Fig. 17.-Kiefer's urethral irrigation nozzle (Tiemaun).

pars anterior. A Keifer nozzle (Fig. I7) or a double recurrent catheter may be used, but is not essential. Instead of the short tube a soft catheter may be employed. The tip should be introduced no further than the bulb of the urethra.

A simpler method of locating the origin of shreds in the urine is found in completely filling the anterior urethra by means of a gonorrhœal syringe with a solution of methylene-blue or other stain. This solution is left in the urethra for one or two minutes and is then allowed to escape. The patient should now urinate in two glasses. The first portion of urine may thus contain shreds from all parts of the urethra, but 
those from the pars anterior will be blue, while those from the pars posterior will be unstained.

4. Examination with Sounds and Bougics.-In a large proportion of cases of chronic urethritis stricture in some form is present, and in an instrumental examination of the urethra is the first thing to be searched for. A detailed description of instruments and methods used for this purpose is given in the discussion of Stricture, and is not repeated here.

When no stricture can be found, other forms of local lesions that may be present can often be located accurately by means of the steel sound or the rubber bougie. It is always well to begin with a blunt steel sound of the largest size that will easily pass the meatus. This larger sound, warmed and oiled, causes less pain than smaller ones, and will often answer every purpose. The sensations of the patient on the first passage of any sound should not be relied upon for the location of diseased areas, as the entire mucous membrane is often so sensitive that the patient, usually nervous and apprehensive in this his first experience with a sound, cannot tell definitely at what points the passage of the instrument causes him the most pain. In most cases all decided pains disappear when the sound ceases to move, and it should be held quietly in the urethra for from one to five minutes until the sensitiveness of the membrane is lessened and the fears of the patient are overcome, when it should be withdrawn gently and at once reintroduced, gently and slowly. Its second introduction will cause the patient comparatively little uneasiness except when the tip of the instrument comes in contact with areas of disease (inflamed follicles, granular patches, or superficial ulcers). At these points he will probably experience the 
sensation of burning or sharp, sticking pains. With the sound held in the urethra with one hand, the fingers of the other hand may explore the pendulous portion and accurately locate areas of thickening and sensitive points.

In disease of the pars posterior or of the prostate gland passage of the sound through the deep urethra may be accompanied by great pain and violent tenesmus. If relief does not quickly follow when the sound is held still, it should at once be withdrawn from this part of the urethra. In neurasthenics and in cases of urethral hyperæsthesia the first attempts to sound the urethra may be very painful, the muscular fibres of the urethra contracting about the instrument, forming spasmodic strictures which interfere with the passage of the sound. In these cases patience, gentleness, and repeated examinations are often required before the limited areas of disease can be located or before the sound can be passed fully into the bladder.

In less sensitive urethras, when the blunt sound fails to accurately locate the urethral lesions, the bulbous bougie or the urethrometer may be used. The largest sized bulbous bougie that can readily be introduced is oiled and passed to the deep urethra. It is then slowly withdrawn, and as the shoulders of the bulb come in contact with granulating and other sensitive areas the patient experiences sharp pains or sticking sensations. If on repeated examinations the patient complains of pain at the same points, the lesions are thus located. ${ }^{1}$

5. Endoscopic Examination.-Some few conditions of the urethra, such as tumors, polypi, granular patches,

${ }^{1}$ For a description of sounds and bougies, and the technique of their use, see Stricture. 
and ulcerations, are best recognized and treated by means of the endoscope. This instrument has a somewhat restricted field of usefulness, since its successful employment calls for much experience and practice on the part of the operator, and the introduction of the straight endoscopic tubes is attended by much more pain and irritation of the urethra than is caused by the passage of sounds. The endoscope should never be used during acute, or even subacute, stages of urethritis, for fear of aggravating the existing inflammation. It is of service chiefly in those cases of chronic urethritis in which other methods of diagnosis and treatment have proved insufficient. It is always well to postpone the use of the endoscope in any case until the sensitiveness of the urethra has been tested and lessened by the use of sounds.

(a) Discription of Instrumcuts.-Since Desormeaux first made practical use of the endoscope in 1853 , numerous modifications of his instrument have appeared, as well as some entirely new devices for exposing to view the mucous membrane of the urethra. The method recommended by Gruenfeld, and the one most frequently employed, is the simplest, the endoscopic tubes being separate from the illuminating apparatus. Gruenfeld's original tubes have been modified by Steuer, and again by

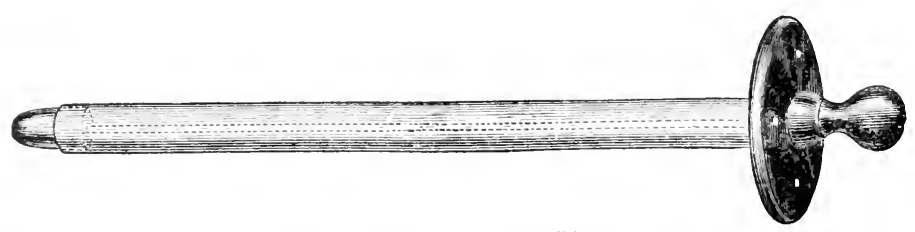

F1(i. 18.-Klotz's endoscope ('Tiemann).

Klotz (Fig. I 8). Either of the two modifications is probably better than the original tube, since the flat disk 
prevents painful distention of the meatus when the tube is forced back upon the glans, thus shortening the penis and allowing inspection of the entire urethra by a tube much shorter than the urethra itself. Tubes should be of metal or of hard rubber. In many respects the Klotz tube of coin-silver is best, since it is light, easily cleaned, and has thin walls, which allow a wider bore for the same size of tube.

The tubes needed will vary in diameter from 16 to 30 , French scale, and in length from three to five and a half inches. As a rule, a tube of the largest diameter that will pass the meatus should be used, in order to give the best illumination and the largest view possible. In the pendulous urethra specula (Figs. I9 and 20) may be used instead of tubes. They have an advantage

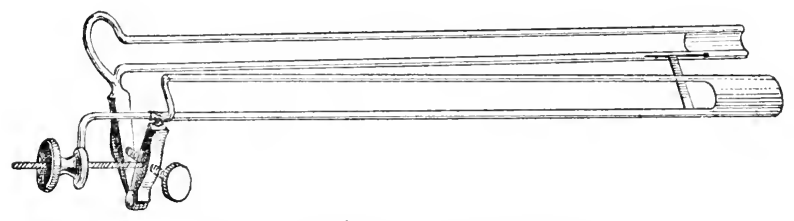

Fig. Ig.-Urethral speculum (Tiemann).

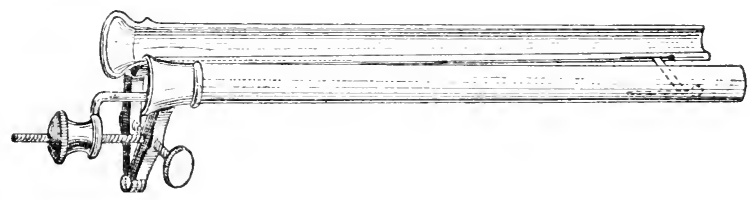

lig. 20,-Urethral speculum (Tiemann).

over the latter in displaying larger areas at a time, but the pressure produced by the sides of the instrument causes more or less anæmia of the mucous membrane, and consequently modifies its appearance.

Illumination is obtained by means of an ordinary mir- 
ror such as is used in examinations of the larynx. This may be fastened to a handle or, better, to a head-band. The source of light, in order of desirability, may be direct sunlight, bright diffused daylight, electric or gas light, or a kerosene lamp. If an artificial light is used, it should be mounted on a freely movable and adjustable bracket. The addition of a condenser will improve the illumination. An excellent apparatus is that devised by Dr. F. Tilden Brown (Fig. 2I).

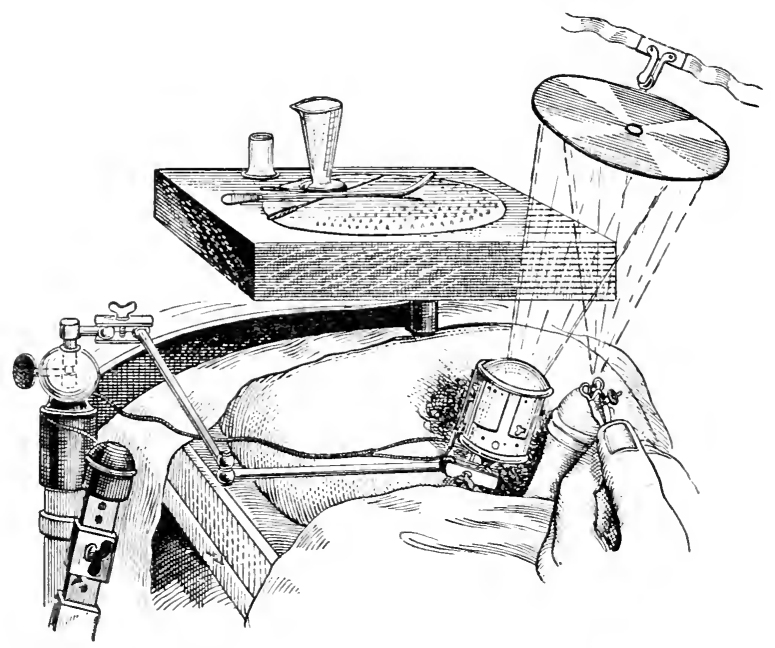

Fig. 21.-Bruwn's method of illuminating the urethra.

In another type of endoscope the tube is directly connected with an electric illuminating apparatus. The Leiter electro-endoscope, used and recommended by Finger, has been modified by several operators in America. The Otis electro-urethroscope (Fig. 22) is probably as serviceable as any endoscope of this type. It has the 
great advantage of being but one-sixth as heavy as Leiter's instrument.

(b) Mcthod of Examination.-For endoscopic examination of the urethra the patient should be placed on a table or an operating-chair that will bring the genitals on a level with the eyes of the operator, who sits in front. If a separate reflector is used, the rays of light should come from a little to one side of the patient, and should so strike the reflector that the best possible illumination of the urethra may be obtained. Cotton, tampons, and

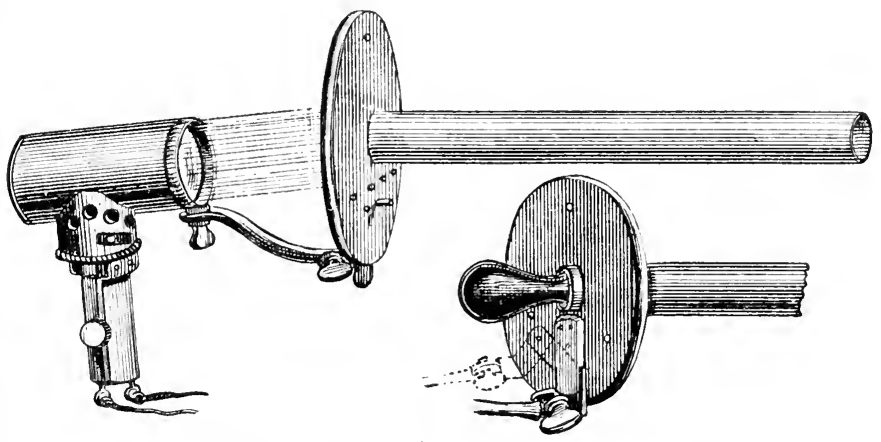

Fig. 22 -W. K. Otus's "perfected" urethroscope (Tiemann).

tampon-carriers (in the form of long wires or thin strips of wood, that can be thrown away after using once) should be within easy reach of the operator. With the penis at an angle of from $90^{\circ}$ to $130^{\circ}$ with the abdomen, the warmed and well-oiled tube, with its proper obturator, is directed along the upper wall of the urethra to the bulb, where it meets with resistance. The tube is now in position to begin examination of the pars anterior. If the pars posterior is to be examined, the proximal (ocular) end of the instrument is depressed anci gently pushed on until the visceral end enters the neck of the 
bladder, when partial removal of the obturator will allow some escape of urine if the bladder be moderately full, and the position of the tube is thus easily demonstrated. The tube is then withdrawn slightly, to the prostatic urethra, and the examination is begun. The passage of the straight instrument through this portion of the urethra is painful, and is also difficult of execution-sometimes impossible. In some men it will be necessary to depress the ocular end of the tube but to a horizontal line, while in others it must be carried much lower and considerable force must be employed. As a rule, it is not wise to attempt an endoscopic examination of the pars posterior during the first visit of a patient or before the pars anterior has been inspected carefully.

In making an endoscopic examination the tube should be inserted to the deepest part to be examined, and then slowly withdrawn, the operator cleaning and inspecting each portion of the mucous membrane as it comes in view at the distal end of the tube.

(c) Appearance of the Normal Urethra-To use the endoscope successfully the operator must be familiar with the appearances of the different portions of the normal urethra. Such knowledge can come only through much experience, and cannot be gained solely from written lescriptions, or even from plates. In the normal condition the urethra is not a tube with a definite calibre, but is a closed valve, the walls being in contact and lying in longitudinal folds. The introduction of the endoscopic tube separates the walls and smooths out the folds. A short distance from the end of the tube, however, the walls again come together in the form of a funnel, the folds radiating to a central point or a short line which has much the appearance of a 
sphincter and is called the "central figure." As the tube is slowly withdrawn the funnel follows, but if the tube be pushed backward, or if the one employed be too small for a given urethra, the folds of mucous membrane will fall together directly at the end of the tube, or will even project into it.

In the posterior portion of the prostatic urethra the tunnel is short and the surface is smooth or but slightly ridged and of a dark-red color. As the tube is withdrawn the membrane becomes paler, and a flat or rounded protuberance appears at the lower edge of the tube. This protuberance gradually increases in size until it occupies about three-fourths of the field of vision, and the funnel above appears in the form of a crescent. The protuberance is formed by the colliculus seminalis, on the summit of which, in favorable cases, may be seen the opening of the utricle. On further withdrawing the tube the colliculus gradually disappears, but its prolongation may remain in the field until the bulb is reached. On either side of the colliculus is a deep furrow. The tube may pass through one of these furrows, so that the colliculus is not seen at all or appears at one side. The picture obtained in the prostatic urethra is a complicated one, and differs greatly in different individuals.

In the membranous urethra the mucous membrane is paler in color than in the prostatic portion, and the funnel is short and regular, the "central figure" being a point. In passing to the bulbous portion the picture may change gradually and but slightly, though more frequently the funnel becomes shorter and the folds much larger. The latter may push into the tube in the shape of two external ridges which touch in the centre and 
give the central figure the form of a vertical line or fissure. The contractions of the bulbo-cavernosi and ischio-cavernosi muscles may render it difficult to keep the tube in the bulb. This object can be accomplished by the use of force or by elevating the ocular end of the instrument as in the removal of a sound.

In the pendulous urethra the funnel is again regular, the folds uniting in a central figure in the form of a horizontal slit. The color of the membrane is pink or pale red. Along the upper wall may be seen small, pin-pointsized depressions. These are the lacunæ Morgagni. In the glans the central figure is triangular, except in the fossa navicularis, where it is vertical. The membrane loses some of its red color and adds a bluish tint. At the meatus it is of almost a slate color.

(d) Appearance of the Urethra in Disease.-The shape and size of the funnel will be modified variously, depending upon the nature and extent of the infiltration and thickening present in the urethral walls. If the mucous membrane is œedematous, the natural folds will be increased in size and will come together nearer the end of the tube, forming a short, narrow funnel; or if this swelling be soft and considerable, the membrane will bulge into the end of the tube. In this condition the folds and the funnel re-form rapidly after moving the tube. If there be firmer and deeper infiltration of the tissues than in the condition just described, the walls of the urethra will not come together so readily, the funnel is longer and larger and more rigid, and the changes occur slowly. In either form of infiltration and swelling, if the process be unilateral, unequal, or irregular, the funnel will be unsymmetrical, the central figure will be variously distorted, and irregular folds may bulge into the tube. 
While pathological changes in the deeper tissues can thus be recosnized, the endoscope is chiefly valuable in demonstrating and treating lesions on the surface of the mucous membrane. The color of small or even large areas may be changed from the normal pink to a bright red or to some of the darker, duller shades of bluish and brownish red or purple; or, on the contrary, it may be almost white. The normal dull lustre may be lost, and the surface may appear smooth and shining or even glistening, or rough, dull, and cloudy. Losses of epithelium give the surface a finely stippled appearance. Areas of granulation are of frequent occurrence and are readily recognized. Morgagni's lacunæ are often involved in chronic urethritis, and appear as circumscribed reddened and swollen areas, or as sharply defined pinhead-sized or larger pits or depressions. Small areas of superficial ulceration may be found. These areas are very sensitive, may appear depressed, and lack the smoothness and lustre of the normal membrane. Occasionally tumors may be present in the urethra and be the cause of slight persistent discharge or disturbances in urination. These tumors are recognized by the endoscope in the form of smooth polypi or more frequently as small warts or papillomatous growths. The latter are usually situated near the meatus.

The above-described pathological changes are found chiefly in the bulbous and pendulous portions of the urethra. In the pars posterior they are found less frequently and are not so well understood. Endoscopic examination of this region is not often called for, though it is occasionally of great service in both diagnosis and treatment. The straight tube does not readily enter the prostatic urethra without the application of some force, 
and the resulting hemorrhage not infrequently is sufficient to prevent an accurate inspection of these parts.

Treatment of Chronic Urethritis.-It is difficult to lay down definite rules for the management of chronic urethritis, since so much depends on the individual, his habits and surroundings, and on the duration, character, and previous treatment of his disease. Frequently the condition of the patient, more than that of his urethra, should be considered. In cachectic individuals local treatment of the urethritis may accomplish little as compared with properly directed constitutional treatment. In cases of simple anæmia, debility, or exhaustion, rest and proper tonics will often be more effective in causing the disappearance of a urethral discharge than will any amount of local treatment. In men who have been violating the laws of sexual and general hygiene, changing the habits of living to conform with these laws may make other treatment unnecessary, and until such changes are made local treatment will do little, if any, good.

The hygiene of chronic urethritis is practically that of the acute disease, except that greater freedom is allowed the patient in matters of diet and exercise. Tobacco, alcohol, and rich or highly seasoned foods should be interdicted; sexual excitement and unrest should be avoided. For the unmarried man absolute continence, both mental and physical, is the only course. In married men moderate, unstimulated sexual relations are permissible, or even beneficial, in cases of slight, persistent discharges which the physician is satisfied are non-infectious. In the case of the unmarried man who has been tormenting both mind and body in his ceaseless efforts to remove the last traces of a catarrhal discharge from the urethra and to prepare himself for matrimony, and whose morbid mental 
condition interferes with sexual hygiene by keeping the organs in a state of unrest, marriage is the best remedy. Keyes says: "A regular, moderate exercise of the sexual organs tends surely to keep down congestion and to allow that rest which is most important in effecting a cure." It is needless to add that such exercise of the sexual organs cannot be found outside of the married state; and, furthermore, the mental and moral influence upon such a man of his marriage to a pure-minded woman is a large-frequently the most importantfactor in effecting his recovery. In such cases the physician will not, of course, recommend marriage until he is satisfied that the urethral discharge is non-infectious and that the man is resolutely endeavoring to live according to the laws of sexual hygiene.

The use in the urethra of strong injections, of bulbous sounds, of dilating instruments, or of the endoscope necessarily irritates the otherwise healthy portions of the mucous membrane, causing temporary inflammation of these areas. Even mild injections and the blunt steel sound are slightly irritating to the normal mucous membrane, and their use is followed by some hypersecretion of mucus. Hence the folly of continuing local treatment indefinitely in hope of removing the last drop of mucus which appears at the meatus, and hence the impossibility of making an accurate diagnosis in a case of urethritis that is being treated locally.

In undertaking the management of a case of chronic urethritis that has been under more or less constant local treatment, it is always advisable to give the urethra a rest for several weeks before again beginning topical applications. This rest gives the mucous membrane a chance to recover from the irritation produced by local 
interference, and allows the surgeon at the end of this time to determine with greater accuracy the nature of the organic lesions that may be present. It also happens that the same local treatment which was ineffective when pursued constantly will, after such a rest, be followed by prompt and beneficial results. In cases in which no organic lesions are present, cessation of local treatment for a few weeks may result in complete disappearance of the urethritis. Such cases are more numerous than the majority of practitioners are willing to believe. Many of the so-called "incurable" cases of chronic urethritis, which refuse to yield after months or years of treatment, need only rest and hygiene to bring about their recovery. In stopping all local treatment it may be well at first to give small doses of sandalwood or of cubebs. The urine should be kept unirritating at all times.

For purposes of treatment all cases of chronic urethritis may roughly be divided into two classes. The first class includes those cases in which a considerable portion or all of the urethral mucous membrane is involved to a greater or lesser degree. This condition is found in cases following a recent gonorrhœe, in relapses and exacerbations of chronic urethritis, in urethras subjected to constant or excessive local treatment, and in the chronic urethritis of men who are cachectic or who are not living hygienically. The amount of secretion in these cases may be considerable, and may vary from a mere hypersecretion of mucus to a more or less purulent discharge resulting from a true inflammation of the membrane. If posterior urethritis be present, the second portion of the urine will be cloudy. The second class includes those forms of urethritis in which the larger portion of the urethral mucous membrane has returned 
to its normal condition, the pathological process being limited to one or more circumscribed areas. The secretion in these cases is slight, and may not be apparent except as shreds in the urine. It is evident that a case of the second may temporarily be transformed into one of the first class, as a result of sexual or other excesses or of active local treatment.

Treatment of Cases of the First Class.-In these cases the discharge, the pus in the urine, or the subjective symptoms show that a considerable portion of the urethral mucous membrane is inflamed, or at least irritated and congested, and that all instrumentation of the urethra should be avoided. The treatment should correspond with that given for the late stages of gonorrhœea. Internally, sandalwood and cubebs give good results, though copaiba is indicated if the symptoms become at all acute. In posterior urethritis boric acid is of special value, and may be given in addition to one or more of the above-named remedies. Local treatment should be limited to the use of injections or irrigations.

The injections used may be those recommended for the declining stages of gonorrhœa, though it may be necessary gradually to increase their strength. Many other preparations are recommended, and sometimes prove serviceable in the treatment of chronic urethritis. Among them are nitrate of silver, sulphate of copper, and chloride of zinc. Each of these may be used in strengths varying from one-fourth of a grain to a grain in an ounce of distilled water. Alcohol or glycerin, or both, may be added to any of these preparations, the quantity added being small at first and gradually being increased if it does not irritate the urethra. Instead of alcohol an astringent wine may be used. The practitioner is again 
warned against the folly of using too many injections. He will obtain the best results if he limits himself to two or three preparations with which he has become thoroughly familiar. He can easily vary their strength and frequency of application to suit the needs of each case. Strong injections should not be used until weaker ones, after faithful trial, fail to do good. The solutions used should always be weak at first, and if necessary gradually be increased in strength, and injections strong enough to produce decided burning or smarting sensations in the urethra should not be used.

It should be remembered that often the last drop of mucus will not disappear from the meatus until after the injection has been stopped and the mucous membrane has had time to recover from the stimulating effects of local treatment. If after using an injection for two or three weeks, the discharge is reduced to a drop or two, the injection should be used with gradually dininishing frequency for a week or two and then be stopped, and the patient should be allowed a fortnight without local treatment. If the slight discharge or the shreds in the urine persist, the case is, so far as the anterior urethra is concerned, one of the second class, and is ready for examination and treatment with instruments.

Injections made with a gonorrhoal syringe will, of course, reach only the anterior urethra. If posterior urethritis be present in such degree that the second glass of urine is clouded with pus, the inflammation should be reduced as far as possible before beginning the use of sounds and instruments. This result can usually be accomplished by means of internal treatment, aided, in some cases, by dccp irrigation.

A number of methods of irrigating the deep urethra have 
been advocated, and several instruments have been devised for the purpose, but all the apparatus necessary is a short glass or rubber tube-or, if preferred, a soft catheter-connected by means of rubber tubing with the reservoir containing the solution to be used. The reservoir, filled with the warmed solution, is held about two feet above the level of the penis, and, after allowing the liquid to flow long enough to expel the air from the tubing, the nozzle is inserted for a short distance within the meatus and is held there loosely, permitting a free outflow of the solution. The anterior urethra is thus cleansed, and pus or shreds that have been present in this portion will not be carried into the pars posterior during the subsequent steps of the operation. When the solution coming from the urethra is clear and free from shreds, the lips of the meatus may be gently closed upon the tube and the reservoir slowly and gradually elevated until the pressure thus acquired overcomes the compressor urethræ muscle, and the fluid passes over the mucous membrane of the deep urethra into the bladder.

Instead of a short tube a soft catheter may be used. After washing out the anterior urethra the tip of the catheter may be passed to the membranous or the beginning of the prostatic urethra; the fluid will then fill the deep urethra and pass into the bladder. The point is known to have entered the membranous portion by the yielding of the compressor urethræ muscle, which can generally be recognized by the operator, or by the fact that the fluid no longer escapes from the meatus; or the catheter may be passed into the bladder and slowly withdrawn until the urine ceases to flow, and the tip is thus known to be in the deepest portion of the prostatic urethra. The catheter should be lubricated with glycerin, 
since oil or vaseline would coat the mucous membrane and interfere with the action of the solution.

Finger has devised an ingenious apparatus by means of which he varies the pressure of the liquid in the urethra without varying the elevation of the reservoir. The apparatus is practically a very large barrel-syringe fastened vertically against the wall. The lower end is provided with a rubber tube, a yard or more in length, terminating in a short pear-shaped nozzle fitted with a stopcock. The upper end of the barrel is simply a loose-fitting cover perforated by the piston-rod. To obtain a minimum pressure both cover and piston may readily be removed; to obtain greater pressure the piston is allowed to rest on the surface of the liquid in the barrel, and weights are placed on a disk fastened for this purpose to the upper end of the piston-rod. Ultzmann's instrument is an excellent one, but with the metal catheter which he employs there is greater danger of damaging the inflamed mucous membrane than with the soft catheter.

Of the many solutions used for irrigation of the deep urethra, the following are among the best: Nitrate of silver (I : 20,000 to 1 : 500 of distilled water), bichloride of mercury ( $\mathrm{I}: \$ 0,000$ to $\mathrm{I}: \mathrm{IO}, 000)$, sulphate or acetate of zinc ( $\mathrm{I}: \mathrm{IO0O}$ to $\mathrm{I}: \mathrm{IOO}$ ), and permanganate of potash ( $\mathrm{I}: 20,000$ to $\mathrm{I}: \mathrm{I000}$ ). Ultzmann's method was as follows: There was prepared a solution containing I part each of crude alum, zinc sulphate, and carbolic acid in 500 parts of water. For the first irrigation this solution was diluted with three times its bulk of warm water. If well borne, the strength was gradually increased until at the end of three or four days or a week the solution was used in full strength. 
This preparation was then changed for a solution of permanganate of potash, I : 20,000, gradually increased in strength up to I : IOOO. Lastly, a solution of nitrate of silver, I : 2000, was substituted, and was gradually increased in strength to I : Iooo. Irrigations were given daily.

As with injections, weaker solutions should be tried first, and the strength gradually be increased when necessary and when the resulting irritation of the urethra is slight. They may be used at first about once in three days, but when the urethra becomes accustomed to the process and shows a milder reaction after it, they may be given every other day or even daily. At each irrigation about 4 ounces of the warmed solution should be used in the posterior urethra, after the anterior urethra has been cleansed thoroughly. The bladder should be about half full, that the urine may dilute the solution sufficiently to prevent injury to the bladder-walls. It is desirable to have the patient retain his urine for some time after the irrigation, that the medicament may be left as long as possible in contact with the mucous membrane of the urethra.

In case both anterior and posterior urethritis are present, and irrigation is practised every second or third day, the patient may use an injection once or twice a day. If after two or three weeks of such treatment the subjective symptoms disappear, the discharge is reduced to a drop or two of mucus, and the urine is clear but for a few shreds, the irrigations and injections should be given at gradually increasing intervals for a week or more, and then be stopped entirely. A week or two of rest from local treatment may remove these last traces of the disorder; if, however, 
they persist, the case may be considered onc of the second class, and treatment with instruments is then proper.

Triatment of Cases of the Sccond Class.-In these cases the subjective symptoms, the discharge from the meatus, and the cloudiness of the urine are slight but persistent, and are usually due to local lesions. In a large proportion of cases stricture in some form or degree will be found, and is the most important and first lesion to be treated. In the absence of stricture there may be congested or infiltrated and thickened patches of mucous membrane or of submucous tissue. There may be inflamed follicles and lacunæ, or small areas of granulations, or superficial losses of tissue. When these conditions exist, they should be sought for and located by means of the steel sound, the bulbous bougie, or the urethrometer. In some cases of the torpid and persistent type no local lesions can be found, but there may be, instead, an atonic and mild catarrhal condition of a considerable portion of the membrane.

In almost all cases of chronic urethritis of the second class the most effective local treatment is found in the proper use of the steel sound. The dilation of the urethra by the full-sized steel sound opens and smooths out the folds and lacunae of the mucous membrane, thus freeing them of retained secretions which the stream of urine does not reach. The pressure exerted by the sound empties inflamed follicles of their contents and stimulates the process of absorption in the areas of congestion and thickening, while the effect of the cold steel upon granulating patches and upon an atonic, catarrhal condition of the mucous membrane is stimulating and often beneficial. The best instrument to use is a 
smooth, blunt steel sound of the largest size that will pass the meatus without stretching it. It is not necessary, as a rule, to cut the meatus unless the latter be abnormally small. If this be the case, the narrowing is usually due to a thin band of tissue at the lower end of the aperture; behind the band is a small pouch that can be detected by the point of a probe introduced into the urethra for half an inch and drawn forward along the floor. This condition may be the sole cause of the persistence of a discharge, since it interferes with the free drainage of the urethra. In this case all that is necessary is simple incision of the thin band of tissue, followed by the use of sounds for a few days to keep the cut edges from reuniting.

Any incision of the meatus should be made downward in the median line, and not upward, and should be done slowly and with great care, not with a single stroke. Since the meatus is often normally the narrowest point of the urethra, it is sometimes necessary to enlarge it in order to introduce larger instruments for the treatment of the deeper parts. But this little operation, simple as it is, leaves the meatus in a condition not natural to it, and therefore cannot be entirely harmless. The physician who treats many obstinate cases of chronic urethritis will see a proportion of them in which the lips of a freely cut meatus gape widely and expose a considerable portion of urethral membrane which under normal conditions would be covered and protected. In this condition may be found the cause of not a few persistent urethral discharges. The surgeon who recognizes these facts will not look upon the mutilation of the meatus as a simple and harmless procedure, to be adopted as a matter of conl- 
venience, but will reserve the operation for those cases in which the meatus is abnormaily small, or in which the condition of deeper portions of the urethra necessitates the use of instruments too large to pass the normal meatus.

Before introducing the sound it should be oiled, but not warmed. It should be introduced with great gentleness, allowed to remain from a few seconds to fifteen minutes, and as gently withdrawn. If the lesions to be treated are in the posterior urethra, the tip of the sound will be carried on into the bladder. It is rarely necessary to use a sound in this region more frequently than once in three days. If the lesions are limited to the pars anterior, the sound shouid not be passed further than necessary to affect the lesions, and a short, blunt sound is often more convenient than a curved one. Usually there is little gained in passing sounds oftener than once in three days, though they may be used more frequently in the pars anterior.

The use of the cold sound is especially valuable in disease of the pars posterior complicated by chronic prostatitis, prostatorrhœa, or sexual neurasthenia. In these cases the sound may be introduced as often as every second day, providing the irritation or reaction resulting from its passage subsides within a few hours; and when the urethra tolerates well the presence of the sound, it may be held in position from a few seconds to fifteen minutes before withdrawing. In place of the ordinary sound the cold sound of Vinternitz (Fig. 23) may be used. This instrument is a metal catheter closed at its vesical end and divided into two channels by a longitudinal septum. Just within the tip the two channels communicate, while 
externally they connect in a fork-shaped end with two rubber tubes. Water injected into one tube flows out of the other after passing through the entire length of

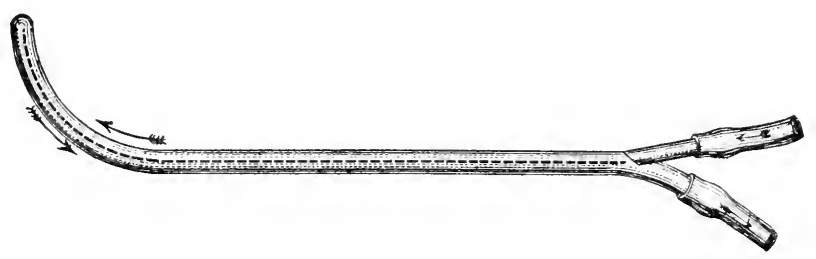

FIG. 23.-Winternitz's psychrophor (cooling sound).

the catheter twice. If the end of one tube be immersed in water and suction be made upon the other end, the water will flow through the catheter as in a siphon. It is well to begin with water at the temperature of the room, and to cool it gradually until eventually ice-water may be used. The application should last from five to fifteen minutes, and may be given about every second day.

Another method of treating disease of the pars posterior, highly recommended by Keyes and others, lies

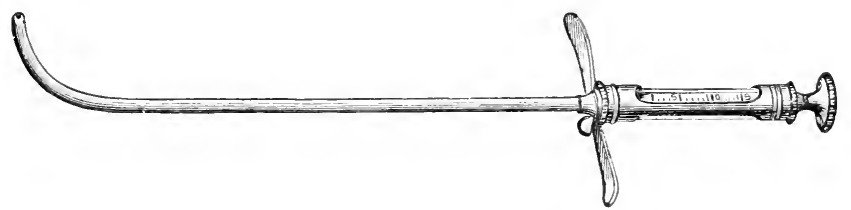

FIG. 24,-Keyes-Ultzmann syringe (Tiemann).

in the use of deep injections. The best instrument for the purpose is Keyes' modification of Ultzmann's deep urethral injector (Fig. 24). The tube of the catheter is capillary in size and open at the end. Before introducing the instrument the catheter is completely filled with 
the liquid, so that for every drop forced out of the barrel of the syringe an equal drop escapes from the tip. The lesion to be treated having been located, the tip of the syringe is carried to this point, and from 3 to 10 minims of the solution are deposited upon it. The best preparation for this use is a solution of nitrate of silver varying in strength from I grain to $\frac{1}{2} \mathrm{drachm}$ (in rare cases gr. xlv) to the ounce. This cauterizing or "etching" of the deep urethra is followed by a more or less violent reaction. The desire to urinate is felt almost immediately, and for a few hours tenesmus and other painful symptoms may be present. The application should not be repeated oftener than two or three times a week, and never before the irritating effects of the previous application have entirely disappeared. Solutions of this strength should not be injected into any portion of the anterior urethra, hence the operator must know that the tip of the catheter has at least entered the membranous portion. With the finger in the rectum, exact location of the tip should not be difficult to one familiar with the use of sounds. If in any case the operator is in doubt, he can satisfy himself by detaching the syringe from the catheter and using the latter in the manner described for deep irrigation : urine escapes, if the bladder be full, when the tip of the catheter enters the neck of the bladder.

Finger and others recommend the introduction of lanolin instead of watery solutions, and Tommasoli devised a syringe for the purpose. The piston within the catheter is on a flexible rod marked in decigrams, so that the amount of the application can be regulated with accuracy: One decigram of the following solution may be placed in the deep urethra at each treatment : 
R. Argent. nitrat.,

Lanolini,

gr. $x v-3 j$;

Ol. olivar.,

酐 ;

3iss.-M.

Sulphate of copper or creolin may be substituted for the nitrate of silver in this ointment. Regarding this form of medication Finger says: "The lanolin ointments possess the advantage of adhering intimately to the mucous membrane. If fluids, gelatin, or cacao-butter bougies are introduced, they are washed out of the urethra by the first micturition. On the contrary, the contracting urethral walls compress the lanolin ointment after the injection and press it into the mucous membrane. Micturition evacuates only small particles of the ointment; these particles are found in the urine even thirty-six hours after injection. Even pollutions do not remove all the ointment from the urethra. It therefore forms a real urethral bandage, and its protracted action and gradual absorption have a more favorable effect than the ephemerally acting solutions. In addition, as Professor Liebreich kindly informs me, lanolin is an aseptic substance."

The deep injection of an aqueous solution or a lanolin ointment immediately after using the cold sound is sometimes followed by excellent results. Finger recommends this combined treatment in old foci of infiltration in the pars posterior and in the bulb. For this purpose he uses the following ointment:

R. Potass. iodid.,

Iodin. puri,

Lanolini,

3 iss ;

Ol. olivar.,

gr. xv ;

亏ิiij;

3iss--M. 
Other methods of medicating the deep urethra have been tried. Among those still in use are soluble bougies, ointments introduced on grooved sounds, and the injection of finely divided solids suspended in a sticky fluid. These methods are not so serviceable as those already given, and are rapidly falling into disfavor.

Finally, by means of the endoscope granulating patches, superficial ulcers, areas of congestion, inflamed follicles, and foreign growths in the urethra may be brought into view, and applications may be made directly to them. The most useful preparation is nitrate of silver in solution varying from $\mathrm{I}$ to 20 per cent., though it may occasionally be used much stronger. Sulphate of copper may be used in the same manner. Lugol's solution and other preparations of iodine, as well as carbolic acid in varying strengths, alone or combined with iodine and glycerin, are serviceable at times. Any of the above drugs may be applied in the form of lanolin ointment, and iodoform, iodol, aristol, or other powders may be used. For the application of solutions or ointments nothing is better than bits of cotton twisted on the ends of wires or on thin strips of rood such as may be obtained from match-factories. Ultzmann invented a brush apparatus for the purpose. The handle of the brush can readily be adjusted so that the brush will reach only that portion of membrane that projects beyond the rim of the endoscopic tube. After the use of strong solutions the surface should be dried of any surplus, to prevent its reaching other portions of the membrane; to lessen pain, iodoform in powder or in ointment may be applied. Applications may be made from once in three or four day's to once in a week or two, depending on the 
strength of the solutions used, the sensitiveness of the patient, and the amount of irritation that follows. When the condition begins to improve, the intervals between treatments should gradually be lengthened.

In general, with reference to local treatment of urethritis, it should be remembered that the use of an injection or an instrument in the urethra is followed by more or less reaction and irritation. The reaction may appear in the form of an increased discharge from the meatus, in case the pars anterior is alone affected, or in frequency of micturition and tenesmus when the pars posterior also has been treated. The symptoms usually appear at once, increase for a few hours, and then rapidly subside. Irrigation, deep injection, or instrumentation of the urethra should not be repeated until all evidences of a reaction from the previous treatment have been absent for twentyfour hours. Rough treatment of the urethra is never permissible. All instruments should be used with great care and gentleness. Mild methods and preparations should always be used in beginning the management of any case. If these methods prove insufficient, more energetic measures may gradually be adopted. During local treatment the sensitiveness of the urethra becomes dulied, and good results follow the employment of remedies which, if used at first, would produce violent inflammation.

As a rule, the first micturition which follows a local treatment of the urethra is attended by more or less smarting and burning, and, when possible, should be delayed for a number of hours, for when urination follows too closely the use of instruments, the much-to-be-desired rest of the recently treated parts is prevented, and often the amount of irritation and reaction is increased; after local applications the immediate passage of urine removes 
some of the remedy and interferes with its action. In consequence, local treatment of the urethra should usually be preceded by evacuation of the bladder. The exceptions to this rule are found in irrigation of the deep urethra, for which the bladder should contain half a pint or more of urine, and occasionally in beginning the use of deep injections, when the immediate passage of urine may be desired to lessen the action of a remedy whose effect threatens to be too severe.

If improvement follows a certain treatment, it should be given with gradually diminishing frequency, and should finally be suspended. Its best effects will not be apparent until after the urethra has rested for a couple of weeks or more. If a given treatment fails to do good, it is usually best to follow it with a period of rest before trying anything else.

Finally, while studying and making intelligent application of local measures the successful physician will not fail to keep a constant watch over his patient's general health, habits, and surroundings.

Prognosis.-It is evident that $m$ chronic urethritis a guarded prognosis is necessary, and many factors must be considered.

In the tubercular, cachectic, or anamic, the future of the urethritis depends largely on the future health of the individual.

In a man who is violating the laws of sexual and general hygiene the future course of urethritis will depend chiefly upon the promptness and completeness with which he changes his mode of living to conform to these laws.

Recent cases that have received little treatment recover more promptly and certainly than do older cases. Old 
cases that have been subjected to more or less constant and severe forms of treatment are among the most intractable. Such urethras can never return fully to their virgin condition, and such portions as have been destroyed by cutting or cauterizing can never be replaced by normal tissue. It may therefore be impossible to remove entirely the few shreds in the urine, the drop of mucus at the meatus, or the sensitiveness of the prostatic urethra that necessitates slight increase over the normal in the frequency of urination, though in all other respects recovery may be complete.

Disease of the pars anterior can usually be cured, while lesions that persist cause no serious symptoms, if exception be made of the extensive and deforming cicatrices which sometimes follow ill-advised operations.

Disease of the pars posterior is less accessible to treatment, and if complicated by prostatitis and neurasthenic symptoms the prognosis is less farorable. Some patients for weeks or months-even for years-after all evidences of organic lesions have disappeared will complain of vague, ill-defined, or even neuralgic pains and other uncomfortable sensations in the perineum, in the testicles, or in the end of the penis, together with a frequent desire to urinate, hyperæsthesia of the urethra, and irritability of the sexual organs. These symptoms usually vary with the general nervous condition of the patient, and with time, patience, and proper management (chiefly hygienic) most of the cases recover.

If stricture be the cause of a urethritis, the prognosis of the latter will depend on the nature and location of the former.

The contagious element in most, if not in all, cases of chronic urethritis may be removed. 


\section{STRICTURE OF THE URETHRA.}

STRICTURe of the urethra has been defined commonly as an unnatural narrowing or constriction of some portion of the urethral canal. As the urethral walls are usually in apposition, and the urethra is a canal only when distended with fluids or instruments, stricture has been defined as a loss of dilatability of the urethra. These definitions include a number of conditions, which, for the sake of clearness, are given brief mention before beginning the consideration of the subject in handnamely, true organic stricture.

The calibre of the urethra may be narrowed, even to the point of complete obstruction, by pressure from without of a periurethral abscess, cyst, or other tumor, or of an inflamed or hypertrophied prostate; or by the presence within the urethra of polypi or other growths; but in these conditions the narrowing is secondary to other diseases, and, to avoid confusion of terms, should not be called "stricture."

The swelling of the mucous membrane in acute inflammation of the urethra may diminish the size of the canal sufficiently to interfere greatly with the passage of urine, but this condition, unless complicating a preexisting organic stricture, or unless associated with prostatic disease or urethral spasm, is never sufficient to cause complete retention, and, moreover, is transitory. The term "inflammatory stricture," which has been 
applied to this condition, is confusing and should be dropped.

Spasmodic stricture is a term applied to the spasmodic contraction of the urethral muscles that frequently occurs during instrumentation of the urethra, and which not uncommonly results from other local or reflex irritation or from psychical causes. In the majority of healthy urethras the passage of a bulbous bougie for the first time will induce a contraction of the urethal muscles sufficient to impede the progress of the instrument for a few seconds. Spasmodic contraction of the compressor urethræ and the "cut-off" muscles, or the failure to inhibit such contraction, makes it impossible for some men to urinate in the presence of others. In such a case the urethra and bladder may be entirely normal, and the cause of the failure is purely mental, for if the patient withdraw to a private closet, or if a catheter be passed beyond the contracted muscle, the urine flows freely.

While urethral spasm may thus occur in apparently healthy individuals with normal urethras, its production is usually due to some local or general pathological condition. Predisposing causes are found in a sensitive or irritable condition of the nervous system, in any disturbed mental state, in cachexia and debility, and in a rheumatic or gouty diathesis. It is easily provoked in the intemperate, and especially in those whose sexual hygiene is faulty. In some individuals there is a local hyperæsthesia of the urethral mucous membrane for which no sufficient cause is found, but in whom introduction of a sound always produces urethral spasm.

The exciting causes are found in any direct irritation, congestion, or inflammation of the urethra; in the reflex irritation due to disease of or operation upon any por- 
tion of the genito-urinary tract, rectum, or anus; in irritation reflected from more distant parts of the body; and in psychical disturbances. The use of instruments in the urethra or operations upon any portion of it may be followed by spasm of the deep urethra and retention of urine. Such spasmodic stricture may persist for several days. A similar condition frequently accompanies the congestion of the deep urethra resulting from alcoholic or sexual excesses or from exposure to cold. Occasionally such congestion and spasm are produced by the internal use of cantharides, turpentine, and other drugs. Reflex spasm of the deep urethra may follow operations upon, or disease of, any portion of the genito-urinary tract, or may result from inflamed hemorrhoids and other sources of irritation in the rectum. It is reported to have followed operations upon more distant parts of the body, and to have been produced by a number of other causes, including strongly concentrated urine, malaria, neuralgia, abscess of the lumbar vertebra (Keyes), necrosis of the coccy $x$ (Emmet), etc.

In most cases of urethral spasm, however, the cause lies in a congested or granular patch of mucous membrane or in an organic stricture situated in the bulbcus or bulbo-membranous portion of the urethra. Spasmodic stricture may be produced by local disease in other portions of the urethra, if such lesions be irritated in any manner. The importance of a small meatus or of a stricture of large calibre in the anterior urethra as a cause of spasm of the deep urethra has unquestionably been over-estimated. Spasmodic stricture due to a congenitally narrow meatus or to strictures of large calibre in the anterior urethra, uncomplicated by inflammation or other pathological changes, is certainly very rare. 
Spasmodic stricture, which is usually situated in the membranous urethra, is due to the contraction of the compressor urethre and accelerator urine muscles and of the voluntary perineal muscles which make up the "cut-off" muscles of Cruveilhier; but it occurs in less pronounced form in the anterior urethra when the unstriped muscular fibres of the urethral wall contract about a foreign body-as an instrument-or about an irritated area of disease.

The spasm of the urethral muscles that occurs during the passage of a steel sound is usually readily overcome by pressing the tip of the sound quietly and steadily against the contracted muscles for a few seconds. At the beginning of the membranous urethra, especially in a nervous or sensitive man whose urethra is being explored for the first time, the contraction of the muscles may be so firm and persistent that firm and steady but gentle pressure of the point of the sound will be resisted for several minutes before relaxation occurs and the instrument can pass. In such a case the relaxation is often sudden and can be felt by the hand holding the sound, the tip of which passes the obstruction with a slight jumping or jerking movement. As a rule, the largest blunt steel sound that a urethra can easily accommodate will overcome spasm better than a smaller one, and will often succeed when bulbous sounds or finer rubber bougies fail to pass. Hence, in the first examination of any urethra large sounds should be used. By beginning with smaller instruments, and especially with a bulbous sound, a diagnosis of organic stricture may be made when there is present nothing more than urethral spasm, which is readily overcome by the large blunt sound. 
In general, spasmodic stricture occurs suddenly and is paroxysmal, the stream of urine being normal in size except during the urethral spasm. If organic stricture is also present-and this is very frequently the caseits calibre will, of course, determine the usual size of the stream. Otis lays much stress upon the occasional occurrence of spasmodic stricture which may persist for years and in every way simulate organic stricture, even to resisting the passage of instruments in skilled hands. As every surgeon should make it a rule, before beginning a cutting operation on the deep urethra, to try to pass a sound when the patient is under an anæsthetic and the muscles are relaxed, these cases should always be recognized in time to prevent a needless operation. In the exceptional cases which simulate organic stricture in yielding only to gradual dilatation, careful watching will sooner or later reveal the true condition, while the removal of the cause of the spasm will be followed by the disappearance of the supposed stricture.

The treatment of spasmodic stricture lies in removing the cause when this can be discovered. Sexual and general hygiene and the general health of the patient should be properly regulated, a bland urine should be assured, and all sources of direct or reflex irritation, such as organic stricture and other lesions of the urethra or of other portions of the genito-urinary tract, should be removed. If retention occurs, it can usually be overcome by allowing the patient to recline in a hot bath until the muscles relax and the urine passes in the water. If this method fails, an opiate in full doses should be given, and, if necessary, a soft catheter may be passed after filling the urethra with warm oil; this operation is rendered easier if done while the patient reclines in the 
bath. In extreme cases the production of full anæsthesia may be required to cause relaxation of the spasm.

Congenital stricture is rare and is limited to the meatus and the quarter of an inch of urethra immedi-. ately posterior to it. The size of the normal meatus varies greatly in different individuals, and it is impossible to name a standard below which a meatus should be considered abnormally small. Keyes says: "An individual with an average sized penis and urethra whose meatus will only take No. Io (French) has stricture (congenital) of the meatus, although he may never suffer any inconvenience therefrom." The opening may be no larger than a pin-head, and yet may cause the individual no inconvenience. Such a condition is in reality a slight deformity, and calls for no treatment. It is better to limit the term "stricture" to narrowings that are associated with pathological changes or that interfere with the normal functions of the urethra.

If the normal urethra be distended to its greatest limit by means of the Otis urethrometer or of large bulbous bougies, in the large majority of cases the pendulous portion is found to be less distensible at some points than at others. These may be considered points of narrowing of the fully dilated urethra, but they are often inappropriately termed "strictures of large calibre," and as such are cut for the purpose of relieving deep urethral spasm and other functional genito-urinary disturbances for which the true causes are not apparent. These points of narrowing vary greatly in size, and are found chiefly within the first three inches from the meatus. As a result of many investigations both upon living and dead bodies, in which these points of contraction have been found in urethras otherwise 
normal, the large majority of modern surgeons believe them to be physiological. They do not produce disturbances of the genito-urinary functions, and they do not interfere with the normal distention of the urethra during urination, or with the passage of ordinary sized instruments. Some practitioners are alone in working on the theory that the pendulous urethra should be a tube of uniform calibre-or, rather, of uniform distensibility-the diameter of which should bear a fixed relation to the circumference of the flaccid penis. Other parts of the body are not constructed on such mathematical principles, and in the face of evidence to the contrary it is difficult to see why the penis should be considered an exception.

In chronic urethritis these points of physiological narrowing in the pendulous urethra, as well as narrowings of the urethra due to chronic congestion and infiltration of smaller or larger areas of the mucous membrane and the submucous tissues, have been subjects of much dispute. Some practitioners call such narrowings "strictures of large calibre," and operate upon them for the sole purpose of relieving the urethral discharge. The majority of surgeons, however, do not consider such narrowings strictures, but treat them as cases of chronic urethritis. There are undoubtedly cases in which the question becomes a relative one, for the majority of strictures are preceded by chronic urethritis, with congestion, infiltration, and thickening of portions of the mucous membrane and the submucous tissues. Just when the formation of connective tissue begins in such areas, and just when contraction of such tissue is sufficient to interfere with the normal calibre of the urethra, are often difficult questions to decide. It is best to class these nar- 
rowings with stricture only when there is periurethral or submucous deposit which has begun to contract and to diminish the lumen of the canal, producing a distinct contraction, or when symptoms of stricture (frequency of urination, dribbling of urine at the close of the act, gleet, etc.) are present. In a doubtful case, and especially if the point of narrowing is covered by an inflamed and thickened mucous membrane, it is better to postpone a diagnosis of stricture until the proper treatment for chronic urethritis-preferably the steel sound -has been given a faithful trial.

The question as to what constitutes the normal calibre of any given urethra is considered more fully in the section on diagnosis, but, as a rule, if the penis be of average size and the urethra allows a No. 22 to 26 (French) steel sound to pass easily, the presence of stricture may be excluded. Practitioners who find and cut numerous " strictures of large calibre" in the pendulous urethra claim that, though these narrowings be normal, in chronic urethritis they tend to increase the friction of urine and the irritation and inflammation at these points, and therefore to favor a deposit of plastic material and the ultimate formation of a true stricture. It is undoubtedly true that such a result follows in a small number of cases, and that it could be prevented by early cutting, followed by the use of the sound; but, on the other hand, it should be remembered that most of these cases recover under treatment (with the steel sound) given for chronic urethritis, and that cutting operations on the urethra, even in the most skilful hands, are attended by a mortality of from 2 to 5 per cent. 


\section{GRGANIC STRICTURE OF THE URETHRA.}

Definition.-Organic stricture of the urethra may be defined as a pathological connective-tissue growth in the submucous and periurethral tissues, interfering with the normal calibre and the normal functions of the urethra. Dr. Bryson ${ }^{1}$ calls attention to the fact that this connective-tissue growth constituting stricture results from continued pathological activity in the periurethral tissues and shows a distinct tendency to contract toward the axis of the canal. He proposes the name "chronic contracting periurethritis" by which to designate the "stricture disease."

Stricture is most frequently found in men between the ages of twenty and forty-five. It is uncommon in women.

Varieties of Stricture.-With reference to form, strictures are classed as (I) linear, (2) annular, and (3) tortuOus.

( I) Linear stricture is usually a cord-like band, such as would be produced by tying a cord about the urethra.

(2) Anmular stricture is a constriction such as would be formed by tying about the urethra a piece of tape not more than a quarter of an inch wide (Fig. 25).

(3) Tortuous or irregular stricture includes every stricture that is wider than an annular stricture. It may be so extensive as to include the greater portion of the pendulous urethra. The calibre of this form of stricture usually varies in different parts, rendering the channel tortuous and irregular, hence the name (Fig. 26).

Aside from the three forms of stricture described above, obstruction in the urethra may exist in the form

1 Gumal of Curuneus und Genito-urinary. Diseases, Aug., I889. 
of a thin membrane or diaphragm completely occluding the canal but for an opening situated centraliy or eccentrically; or a crescentic or other shaped band or septum may project from a portion of the urethral wall; or there may be a number of fine bands crossing the urethra

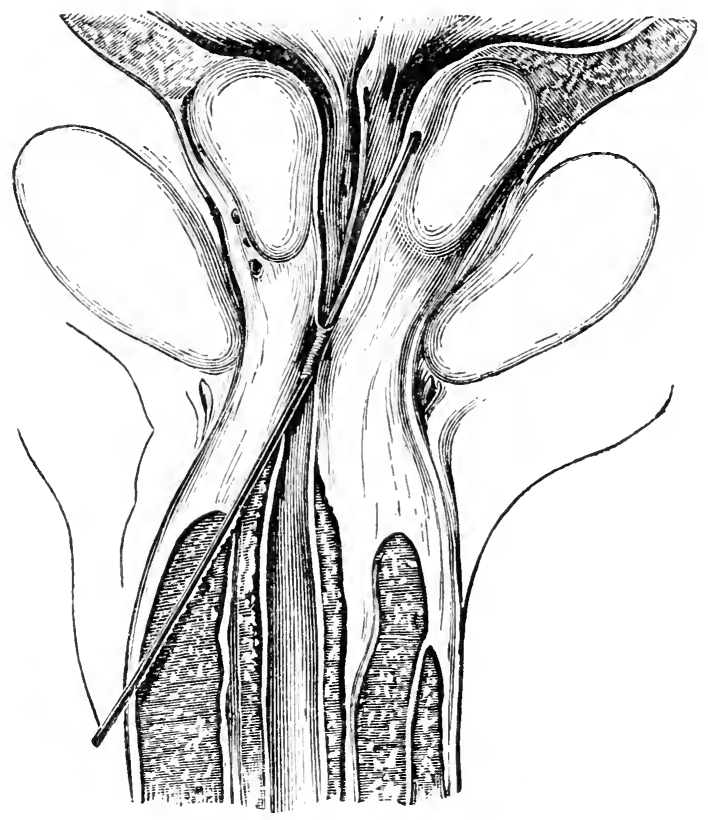

Fic 25-Annular stricture (Dittel).

obliquely or transversely, or so situated as to form flaps and valves. These finer bands, flaps, and valves are often the result of faulty instrumentation of the urethra.

According to the amount of contraction present, strictures are usually divided into strictures of small calibre, which will only permit the passage of an instrument smaller than No. I 5 (French), and strictures of large cali- 


\section{SIPHITIS A.TD THE IE.VEREAL DISEASES.}

bre, which will allow the passage of larger instruments. The division is an arbitrary one, but it is of practical value, since, as a rule, soft (flexible) instruments are to be preferred for all sizes below No. I5. The calibre of a stricture may become so narrowed that it will admit a

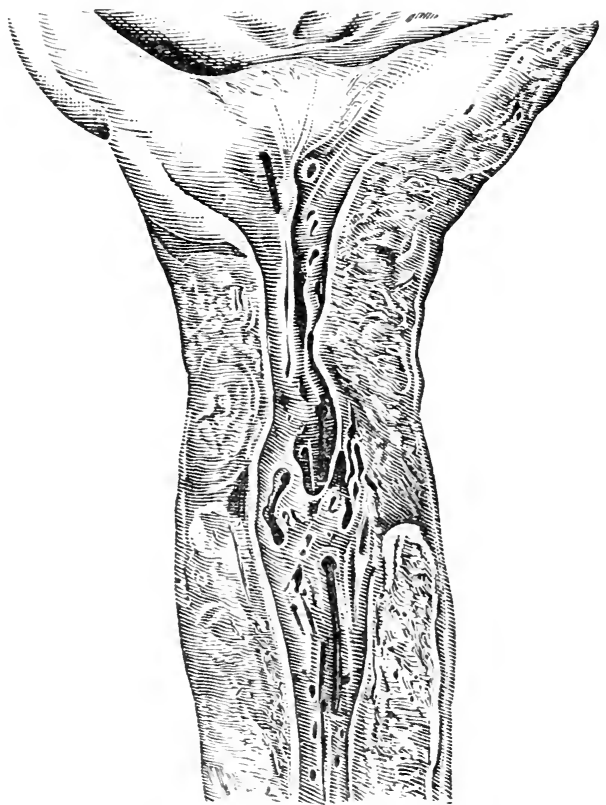

Fic. 2 h. -Tortuous stricture, showing dilated foliteles, lacunæ, and false passages (1) ittel).

fine probe with difficulty, but complete occlusion of the canal occurs only after traumatism or after some other outlet for the urine has been provided, as in the formation of fistulae.

According to their behavior with instruments, strictures are called imitubli, when they are very sensitive and easily inflamed, or resilient, when they are elastic 
and after being dilated contract rapidly to their former, or even a smaller, calibre.

Number of Strictures.-Strictures usually occur singly, but a urethra may contain several strictures. Thompson has never seen more than four in a single urethra. Other observers have reported larger numbers. When a number of strictures are found in a single urethra, careful examination will usually determine them to be irregular contractions of one stricture. Multiple "strictures of large calibre" in the pendulous urethra are frequently reported by those who do not recognize the existence in this region of points of physiological narrowing.

Location of Strictures.-The majority of strictures of the urethra are found in the region which includes the membranous and bulbous portions. The most common seat is the bulbous urethra. Stricture is frequently found within two and a half inches of the meatus, and is occasionally located in deeper portions of the pendulous urethra. Stricture does not occur in the prostatic portion. The majority of strictures occurring in the first two and a half inches of the urethra are found at the meatus or at the posterior limit of the fossa navicularis. The reason for the frequency of stricture in this region and in the bulb undoubtedly lies in the tendency of urethritis to become chronic in these parts - the result, probably, of their great vascularity and dependent position. The membranous urethra is more subject to traumatism than are the other portions, and is therefore the most frequent seat of stricture from this cause.

Otis, working on the theory that the pendulous urethra should be a tube of uniform dilatability, finds the largest 
number of strictures in this portion of the urethra, placing the majority within an inch and a half of the meatus.

Etiology.-Stricture of the urethra is probably always preceded by inflammation or traumatism of the mucous membrane, which is thus damaged sufficiently to allow the escape of smaller or larger quantities of urine into the submucous tissues. Any form of urethritis may be followed by stricture, but such records as have been collected indicate that at least 75 per cent. of all cases of stricture owe their origin to gonorrhaea. The more prolonged the inflammation, the greater the probability that stricture will follow; and chronic urethritis which persists for months or years results in stricture in a large proportion of cases. If during a urethritis the inflammation extends to the periurethral tissues, the danger of stricture is greatly increased. The "breaking" of chordee during gonorrhcea is certain to be followed by stricture of the worst type, since its origin is traumatic. Injections in gonorrhoea, when properly used, are not capable of producing stricture, but the careless use of a syringe with a long or rough nozzle can easily damage the mucous membrane near the meatus, and thus be the cause of stricture. This may be one of the reasons for the frequent occurrence of stricture in this region. If an injection (such as those used in attempts to abort gonorrhoea) be strong enough to excite an artificial urethritis or to destroy a portion of the nucous membrane, it will undoubtedly be followed by stricture. The formation of stricture as a result of urethral inflammation requires a number of months at least. Thompson reported, of 164 cases of stricture attributable to gonorrhoea, Io that appeared soon after the attack, 7 I within a year after its occur- 
rence, $6_{3}$ in from three to eight years, and 20 in from eight to twenty-five years. Guyon and other observers have found that stricture appears from one to ten or fifteen years after gonorrhœe. When it occurs very early, it is probably due to traumatism.

Traumatic stricture is most frequently found in the membranous urethra, where it follows blows upon the perineum, such as would be produced by a kick in this region or by a fall astride any hard substance such as a fence, a wheel, the tongue of a wagon, etc. If such an injury be violent and the injury to the urethra be considerable, it will immediately be followed by hemorrhage, and probably by retention due to inflammation and cedena of the urethral tissues. When the acute inflammation subsides, repair of the tissues begins, resulting in a cicatricial formation which ultimately contracts and produces stricture. In such cases the contraction is comparatively rapid and usually produces symptoms of stricture within a few months, though if the injury be limited to a portion of the urethral circumference cicatricial contraction may be slow and for several years may produce no evidence of stricture. If the first injury be slight, it may pass unnoticed and be forgotten until symptoms of stricture appear some years afterward.

In other portions of the urethra traumatic stricture may result from lacerations of the mucous membrane caused by the rough or prolonged use of instruments, or by the presence of other foreign bodies, such as sharp calculi, or from the breaking of chordee, or from other injury to the penis. Stricture may follow urethral chancre or other ulceration, or any process which destroys the mucous membrane of the part. It is 
highly improbable that stricture ever results solely from masturbation, excessive coition, or prolonged erections, although, after stricture has begun to form, its further development is encouraged by any source of irritation, of congestion, or of inflammation of the urethra. Stricture also forms more readily in tuberculous or syphilitic individuals and in those of a rheumatic or gouty diathesis.

Pathology.-Formation of Stricture.-According to Harrison's conclusions, ${ }^{1}$ which have been largely endorsed by other observers, the urethral epithelium is so damaged at one or more points, as a result of inflammation or injury, that it permits the escape of minute quantities of urine into the submucous tissues. To prevent urine soaking further into the tissues, inflammatory exudation is excited, and barriers of plastic lymph are thrown out opposite the places where leakage takes place. Such lymph ultimately organizes to form splints of connective tissue, evidently for the purpose of strengthening the urethral wall and preventing further leaking of the urine. But this connective tissue is apparently influenced in its growth by the presence of minute quantities of urine, and differs from similar tissue resulting from inflammatory exudates in other parts of the body in that it is denser and shows a more decided tendency to contract These characteristics are most marked in traumatic stricture, in which the mucous membrane is lacerated and the urine escapes in larger quantities. In these cases the cicatrix is dense and contracted, and produces stricture of the worst type. Such stricture is frequently resilient.

The Lesion in Stricture.--The connectire-tissue growth ${ }^{1}$ Lettsomian Lictures, is88. 
constituting stricture may be limited to a very narrow line (linear stricture) in the submucous tissues, but it is usually formed in irregular masses in the submucous and periurethral tissues. It may include large portions of the corpus spongiosum and may obliterate its spaces (blood-cavities). The active development of the stricture-formation continues beneath the mucous membrane proper, which is thus pushed into the urethra in front of the new growth, and may show but little change in thickness or character even when the underlying stricture is bulky and nearly obliterates the urethral canal, though it may be thinned, atrophied, inflamed, ulcerated, or included in a cicatricial formation. In recent cases the tissue forming the stricture is small in amount and is comparatively soft and yielding. This tissue, however, usually grows in density and in amount and continues to contract. In old cases, when due to gonorrhoea, the urethra may be surrounded at the site of the stricture by irregular areas of knobbed or corded, firm masses that can readily be felt by the fingers on the outside of the penis as they follow the course of the urethra. The opening of the stricture is rarely situated centrally, but is most frequently found near the roof, as the stricture-formation is usually most abundant on the floor. The opening may be but slightly smaller than the normal calibre of the urethra, or it may become so small as to be almost impermeable. Complete occlusion is, however, rare except after severe injury to the urethra or after fistulae have formed to provide an escape for the urine.

The rapidity with which stricture contracts and the density and extent of the connective tissue forming a stricture are dependent upon several factors. Strictures 
due to mechanical or chemical violence (traumatic stricture) contract earlier and are denser than those due to inflammation. The amount and shape of the new formation in traumatic stricture depends largely upon the nature and site of the injury, and the stricture may be linear, annular, or irregular. Strictures due to gonorrhœa are often irregular in shape and lumpy. Cachexia and individual tendencies influence the development of stricture, which seems to form readily, extensively, and irregularly in the tuberculous or syphilitic or in those of gouty or rheumatic diathesis. The habits of an individual whose urethra contains a forming stricture also play an important part; a faulty sexual hygiene, or anything that causes congestion of the urethra, will undoubtedly hasten the formation of stricture, and tend to make the latter dense and contractile.

Changes in the Urethra.-As the stricture contracts and forms an obstruction to the free outflow of urine, each act of urination is accompanied by a dilatation of the urethra immediately back of the stricture. The frequent stretching of this part of the urethra is slight at first, but it gradually increases until the walls at this point lose their tone, become thinned, and a permanent pouch or sac is formed. This pouch retains constantly a drop or two of urine, which is decomposed by the mucus and acts as an irritant to the mucous membrane. Thus it happens that the urethral membrane immediately back of a stricture is usually inflamed and produces a gleety discharge. The mouths of the ducts and follicles enlarge, and as the stricture contracts it increases during urination the pressure upon the urethral walls back of it. The mucous membrane in this situation becomes more and more thinned and atrophied, and may 
be pushed out between the bands of muscular fibres to form sacculi or pockets in which a few drops of urine are retained and decomposed, thus adding to the inflammation of the urethral wall, and therefore to the danger of ulceration. Such sacculi may have their origin in distended ducts or follicles. In severe cases ulcerations follow, allowing of the escape of a few drops of urine into the periurethral tissues. There are thus formed abscesses which open externally and result in urinary fistula. Occasionally some portion of the thinned urethral wall gives way, and urine in considerable quantity escapes into the surrounding tissues, producing the serious accident known as "extravasation of urine."

Resulting Changes in Bladder and Kidncy's.-Early in stricture the congestion may extend to the neck of the bladder, causing vesical irritation with frequent micturition; or cystitis may result. Later, as the stricture contracts and produces greater obstruction to the outflow of urine, the detrusor urinæ muscle, as a result of its efforts to empty the bladder, becomes hypertrophied and thickened, and bands of muscular tissue project into the bladder in ridges. The contraction of the hypertrophied muscles in the bladder-walls diminishes the size of the bladder-cavity and may eventually almost obliterate it, the muscles undergoing fibroid changes which render them incapable of distention. During the violent contractions of the bladder the weaker portions of the wall between the muscular ridges are pushed outward and stretched until finally they form sacculi or pouches which may have but a small opening connecting them with the proper bladder-cavity. These sacculi, having no muscular fibres in their walls, cannot empty themselves; they therefore retain the decomposing urine and 
furnish a favorite site for the formation of calculi. Rarely, as a result of rapid formation of stricture and over-distention of the bladder, the bladder-walls, instead of becoming hypertrophied, are thinned and atrophied. Cystitis results in most cases in which decomposing urine is allowed to remain in the bladder.

The pressure of the urine, especially during the act of micturition, extends backward through the ureters, which become dilated and hypertrophied, to the pelvis and to the calices of the kidneys, producing hydronephrosis. As a result of inflammation extending from the bladder through the ureters to the kidney, or more frequently as the result of microbic infection, there may follow pyelitis, pyelonephritis, or, rarely, abscess of the kidney and perinephritis.

Symptoms and Results of Stricture.-The symptoms produced directly by stricture are not so marked as are those resulting from secondary implication of other parts of the genito-urinary system. As these secondary symptoms or results of stricture may be first both in appearance and in importance, and as they are closely associated with such symptoms as may be due directly to the stricture-growth, it is best to consider them together.

Stricture of large calibre may exist for months or even for years without producing symptoms of importance, though when the contracture of the urethra begins to interfere with the normal function of the canal, its presence is usually manifested by a series of characteristic disturbances.

Increased frequency of micturition is among the earliest symptoms of stricture, though it may be so slight at first as to pass unnoticed, or may be entirely absent. As a 
rule, the desire to urinate gradually becomes more frequent, until in old cases the patient may find it necessary to empty the bladder every few minutes. The frequency with which the bladder is evacuated in a day does not, however, necessarily indicate the degree of contraction of the stricture, but is largely dependent upon the temperament of the individual and upon the presence or absence of cystitis and other complications. In the beginning this symptom may be due solely to irritation reflected from the site of the stricture to the bladder, and later to irritation resulting from the increased efforts of the bladder to overcome the urethral obstruction and to empty itself. In many cases congestion of the vesical neck undoubtedly occurs early, and, as the urethral inflammation back of the stricture increases, it extends at least to the deep urethra or to the neck of the bladder, resulting in a greatly increased frequency of micturition due directly to the posterior urethritis or cystitis.

In tight stricture of long standing, as a result of which the bladder-walls have hypertrophied and so reduced the size of the cavity that it will hold but a small quantity of urine, the demands to urinate may be so frequent as to amount almost to incontinence. The same clinical condition is found with an atonic and constantly distended bladder which has lost its power of complete contraction. Very late in the history of these cases the patient may be unable to retain his urine for even a short period, and it constantly dribbles from the urethra. In the first class of cases, in which the bladder-cavity is nearly obliterated, this condition constitutes true (and hopeless) incontinence, and should carefully be distinguished from the mere overflow of a distended and atonic bladder. In the latter case the use of a catheter 
will reveal the presence in the bladder of a large quantity of residual urine. A smaller amount of residual urine may be found in the majority of cases of organic stricture. In these two forms of bladder disease resulting from stricture the frequency of micturition and the incontinence are worse during the day, due to the fact that when the patient assumes the upright position the urine in the bladder produces pressure upon the vesical neck, or, in case the latter is relaxed, upon the stricture and the dilated urethra back of it; these same symptoms, when due to prostatic disease, are usually most pronounced at night.

Dribbling after urination is another early symptom of stricture. In a normal urethra the close of urination is followed by a wave of contraction of the muscular fibres surrounding the urethra, expelling the last drops of urine from the canal. This action is probably aided by a corresponding wave of blood sent through the corpus spongiosum by the contraction of the accelerator urinæ muscle. The submucous deposit in stricture prevents, by its mere presence, the accurate closure of the urethra, and later, by invading the muscle-fibres themselves and by obliterating the meshes of the corpus spongiosum, may yet further interfere with the closure of the canal. In consequence, the last drops of urine are retained behind the stricture, and do not escape until later, by force of gravity, when the penis is dependent.

Crethral discharge, which, appearing as gleet or as sinreds in the urine, is present in most cases of stricture, is produced by the urethral inflammation back of the constriction. In the forming stage of stricture the discharge may be furnished by the inflamed, granulated, or otherwise damaged urethral membrane beneath which 
stricture is forming. Opinions vary as to the constancy and importance of this symptom in its relation to stricture. Dr. White finds about 50 per cent. of strictures accompanied by a gleety discharge from the meatus, while a large majority of all others show shreds in the urine. Some observers maintain that the presence, for any considerable period, of a gleety discharge or of shreds and pus-corpuscles in the urine is sufficient evidence of stricture, and advise, in such cases, operation upon any points of narrowing, however slight, that can be detected in the urethra. That such a view is extreme and erroneous is evident, since many cases of chronic urethritis that have persisted for months or years recover completely under proper hygienic management and without local treatment. The amount of urethral discharge in stricture varies with the general condition of the patient and his habits of living. As in chronic urethritis, congestion or irritation of the urethra from any cause will increase the amount of the discharge. In stricture of large calibre the history and symptoms of the disease may differ in no particular from those of chronic urethritis.

Retention of urine may occur early in stricture if the mucous membrane covering it becomes inflamed and swollen as a result of gonorrhœal infection, alcoholic or sexual excesses, cold, or other causes of urethral irritation and congestion. Such retention, coming on suddenly after a debauch, may be the first evidence of stricture recognized by the patient. This form of retention, due chiefly to urethral spasm and to swelling of the mucous membrane, rarely lasts more than a few hours, and is readily relieved by hot baths, an opiate, or the introduction of a soft catheter. In an unobserving or dis- 


\section{SIPHILIS AND THE VENEREAL DISEASES.}

sipated patient other symptoms of stricture may pass unnoticed for months or even for a few years, and he may suffer from repeated attacks of sudden retention before applying for treatment. As the stricture contracts and diminishes the calibre of the urethra, less and less swelling of the mucous membrane is required to produce retention, which consequently is more easily provoked, occurs more frequently, is more persistent, and is relieved with greater difficulty. In old cases sudden retention may necessitate the performance of perineal section under unfavorable circumstances.

The stream of urinc becomes noticeably smaller only after the calibre of the urethra has been diminished considerably, as in health the size of the stream is much less than the urethral calibre (estimated by the size of the sound it will readily admit). As the stricture contracts, and especially if there be atony of the vesical walls, the patient finds he is unable to throw the stream of urine as far from his body as formerly, and more time is required to empty the bladder. In old and tight strictures the contraction may be so great that the urine passes in drops only. The stream may be so modified in shape that it is twisted, forked, peculiarly curved, or divided into several small streams; but as its form depends chiefly on the shape of the meatus, and as it varies greatly in health and as a result of conditions other than stricture, these modifications are of little importance.

The scuurl functions of the urethra may be impaired early in stricture, but usually they are not disturbed until the obstruction is sufficient to prevent the forcible ejaculation through the urethra of the semen, which then dribbles from the meatus after subsidence of the erection, or, in case of very tight stricture, is forced back into the 
bladder, to be discharged with the next flow of urine. The patient consequently is sterile. Ejaculation may be followed by pain in the urethra just back of the stricture, or in the bulb or the perineum, and the semen may be mixed with blood. If some of the meshes of the corpus spongiosum, and possibly of the corpora cavernosa, are occluded by the stricture-growth, the free flow of blood through these bodies is prevented, and erections are painful or are so imperfect as to render the patient impotent. In old cases sexual desire is diminished or entirely absent, but in recent cases the slight inflammation back of the stricture may serve to stimulate and irritate the sexual organs.

Local pains varying greatly in character occur at the site of the stricture, in the glans penis, testicles, cord, perineum, rectum, bladder, and even in more remote parts of the body. In stricture of small calibre there is usually some vesical tenesmus during the entire act of urination. While many of the pains are undoubtedly reflex in character, many of them are due to the presence of posterior urethritis, cystitis, or to other complications of stricture.

The urine usually contains some pus, and shreds composed of epithelium and pus-cells, but it is otherwise normal unless cystitis be present, when it has the characteristics of urine in cystitis from other causes. Keyes reports several cases of stricture in which hematuria was the only symptom.

Constitutional symptoms are not directly produced by stricture until late in the disease, when the almost constant, painful attempts to force urine through the narrow opening may allow the individual no rest, and may result in complete exhaustion, and, if unrelieved, in death. 
Stricture rarely reaches this stage, however, without producing cystitis and other results and complications of stricture; these secondary disorders present their own characteristic symptoms and may result fatally. Among the chief complications and results of stricture are inflammatory and other disorders of the bladder, ureters, and kidneys; urinary calculus; epididymitis; prostatitis ; perineal abscess; urinary extravasation and fistula; hemorrhoids (from pressure on the veins during straining efforts to empty the bladder); and disturbance or obliteration of the sexual functions.

Mental disturbance, as in other diseases of the genitourinary organs, is often extreme and difficult to overcome. Sexual hypochondriacs who are suffering from ill-defined pains and sensations or from other functional disturbances due to faulty sexual hygiene not infrequently imagine themselves the subjects of stricture, and are often reluctant to accept any other explanation of their sensations or fancied disorder.

Fistula and extraiasation are immediate results of severe forms of stricture. When ulceration occurs in some portion of the urethra-usually one of the sacculi or distended follicles--back of a stricture, and a few drops of urine escape into the surrounding tissues, abscess follows. Such an abscess may open again into the urethra and produce an internal blind fistula; but it usually opens externally, and, retaining its connection with the urethra, produces urinary fistula. A blind internal fistula may persist as such for some time, and may be felt as a hard lump in the periurethral tissue, but it usually inflames and fills with pus, which eventually finds an external outlet. Instead of forming a single fistula, pus in the periurethral tissues may burrow slowly in several 
directions and discharge through a number of external openings in the perineum, scrotum, body of the penis, thighs, groins, or nates. Civiale reported such a case in which the urine afterward escaped through fifty-two external openings. Usually, however, one fistula forms at a time and serves as an outlet for the urine. The walls of such a fistula are soft at first, but, influenced by contact with urine, they gradually undergo changes similar to those in stricture-formation, become hard and callous, and contract until the channel becomes too small to allow the escape of urine. A new abscess forms, and terminates in a new fistula which pursues a career similar to the one preceding, and the process may thus be continued indefinitely. Instead of opening on the surface, a fistula may find an exit in the rectum.

If a sufficient portion of the urethral mucous niembrane back of a stricture is destroyed or gives way at one time to allow a large quantity of urine to escape into the surrounding tissues, extravasation of urine follows. This unusual and serious complication of stricture is described by Keyes as follows:

"In infiltration the urine may take any one of five directions :

"I. It may, when small in quantity, get out of the urethra, but not penetrate Buck's fascia, in which case it may long remain confined to one spot in the perineum as a hard rounded swelling, like the blind internal fistula already described.

" 2 . It may find its way rapidly through the meshes of the corpus spongiosum, and cause gangrene of that body, with sloughing of the glans penis, preceded by coldness and the appearance of a black spot upon the glans. 
"3. It may burrow inside of Buck's fascia, but outside of the corpus spongiosum, forming a fistula opening behind the glans penis near its root, a hard ridge marking the course of the fistula within Buck's fascia.

" 4. It may escape behind the triangular ligament into the cavity of the pelvis.

"5. It may escape outside of the common fascia of the penis, in front of the triangular ligament, in which case it rapidly distends the perineum, the scrotum, and the connective subcutaneous tissue of the penis, and mounts up over the abdomen, and may also, more rarely, perforate the deeper layer of the superficial perineal fascia, and descend upon the thighs.

"When extensive infiltration of this sort occurs, all the parts affected becomes cedematous; gases form in the connective tissue, causing emphysema and making the tissues crackle when pressed by the finger. Dark spots soon appear, indicating gangrene, and extensive portions of tissue nay slough unless relief be promptly afforded.

"The constitutional symptoms are those of shock. A chill usually occurs, followed by great depression, a cold clammy skin, feeble, quick, irregular pulse, hurried respiration, furred tongue, complete anorexia, symptoms of septicæmia, and death.

"When urine escapes behind the triangular ligament - which it does more rarely-it infiltrates deeply around the prostate and rectum well back in the perineum, around the bladder and up behind the pubes, forming abscesses in the cellular tissue of the hypogastrium, or perhaps deep pelvic abscesses."

Keyes reports his own experiments and quotes those of Mengel to show that normal urine injected in small 
quantities into healthy tissues is absorbed without injuring them, and believes that if urine is evacuated by operation as soon as it has extravasated, serious gangrene may often be averted. If the urine be decomposed before its escape, as is often the case, or if infectious matter from the urethra be carried into the tissues with the urine, gangrene is certain to follow.

In very rare instances some portion of the bladder, instead of the urethra, may rupture. This accident is followed by an extravasation of urine that almost invariably terminates fatally.

Fistula and extravasation occur only in old and neglected cases of stricture, and are almost never found outside of dispensary and hospital practice.

\section{InStrumentation of THE UREThra.}

Before attempting the use of instruments in the urethra, the student should become thoroughly familiar with the anatomy of the urethra and the perineum and with the landmarks of these regions. The following characteristics of the urethra should also be borne in mind:

The meatus, as a rule, is the narrowest point in a normal urethra, while the membranous portion is nearly as narrow. In addition to these two points of decided narrowing in the urethra, the pendulous portion may contain one or more points of slight constriction, usually situated in the second or third inch from the meatus, which points are recognized only when the urethra is fully dilated. The urethra also contains three decided enlargements. The first is the fossa navicularis, in the roof of which, about half an inch from the meatus, is a mucous flap forming the lacuna magna, which often 
catches the points of fine instruments that have been improperly directed, in this region, to the upper wall. The second enlargement is in the bulb, which, of all portions of the urethra, is the most distensible and elastic, and therefore most liable to damage from the unskilful use of steel instruments. The third enlargement is in the prostatic portion (Fig. 27).

The pendulous urethra is freely movable, and its curvature depends on the position of the penis, but the portion of the urethra extending from a little in front

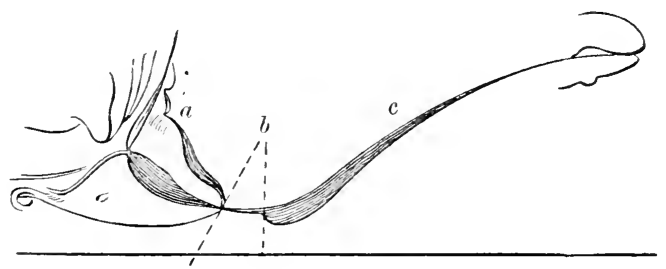

FIG. 27.- The prostatic (a), membranous (b), and spongy portions (c) of the normal urethra (Thompson).

of the triangular ligament to the neck of the bladder has normally a fixed curve. This portion of the urethra, and especially its floor, is not wholly immovable, and usually the curre may be nearly obliterated by the forcible introduction of straight instruments. Such a procedure is always painful, and usually is attended by danger of rupturing the urethra. It is of great importance, therefore, that all inflexible instruments intended for use in the deep urethra should be made with a curve corresponding closely with the fixed (subpubic) curve of this portion of the urethra. In the large majority of cases this curve is an arc of a circle three and onefourth inches in diameter, the cord of this arc being two 
and three-fourths inches long (Fig. 28). An instrument made with a curve of this shape and length (generally known as "the Thompson curve") will pass readily through the normal deep urethra, while an instrument with any other curve will pass with difficulty and is

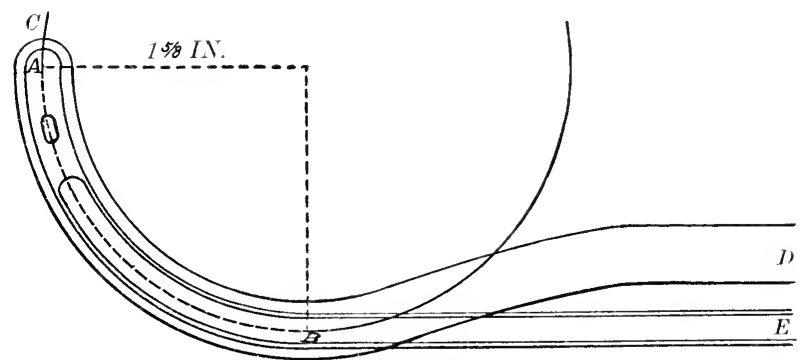

FIG. 28. $-A B E$ shows the proper curve (reduced in size) for unyielding male urethral instruments. $C B D$ shows an improper curve (Tiemann).

liable to injure the urethra. In old men and in those in whom the prostate is enlarged or the bladder is distended the curve may be a little longer than the one described above.

Guyon called attention to the fact that the fixed curve is found in the roof only of the urethra, since the floor is elastic, extensible, and soft, yields readily before instruments, which it therefore cannot support or guide, is lacerated or ruptured with comparative ease, and is less regular in formation than the upper wall. The latter is shorter, less movable, more regular and constant in its curve, smoother and firmer, less easily damaged, less vascular, and less intimately connected with important structures than the lower wall. The practical deduction is obvious: in passing instruments through the urethra, after the fossa navicularis is 
passed the point should be kept closely against the upper wall.

Cathetirism or Sounding of the Urethra.-The steel sound plays a most important part in the treatment of urethral disease, and the ability to introduce it properly and skilfully is one of the first requirements. The requisite skill cannot be acquired without practice, and the student should avail himself of every opportunity for passing the sound or the catheter, both in dead and in living subjects. No amount of experience and practice, however, will justify the rough or forcible passage of a sound or a catheter; the operation should invariably be performed with the greatest gentleness and patience. The beginner should make it a rule never to use any force. It is always best to know how to pass the sound with either hand, but in the following description, as a matter of convenience, the use of the right hand is implied:

The patient, with his thighs separated, should lie on his back on a firm table or couch. He should be made as comfortable as possible, both physically and mentally, in order to secure complete relaxation of all the muscles. To this end his fears should be removed by assuring him that if the instrumentation proves painful it will be stopped at once. The surgeon, standing at the patient's left, gently holds the penis just back of the corona with the left hand, while in the right hand he holds the sound, which has been properly cleaned, warmed, and anointed. With the sound held lightly, the shaft parallel with the patient's groin and near the skin, and with the handle well depressed (Fig. 29), the tip of the instrument is inserted in the meatus, and the penis is gently slipped up over the sound, which may be allowed to drop into the ure- 
thra by its own weight. The penis should gently be put on the stretch to efface the folds of the urethral

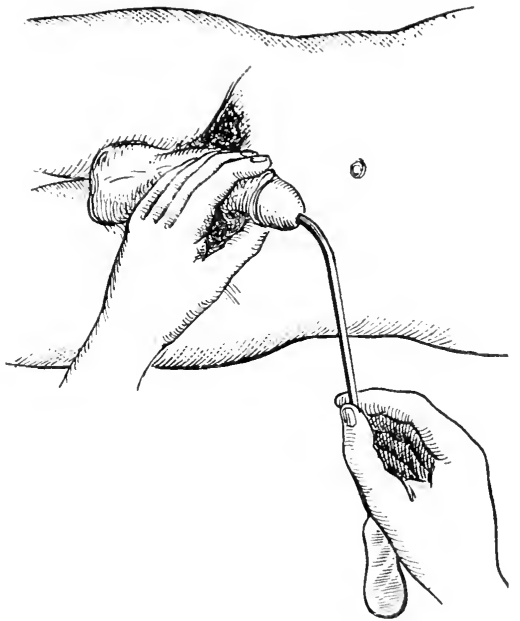

Fig. 29.-Sounding of the urethra (Keyes).

mucous membrane and to enable the point of the sound to gravitate as far as possible toward the bulb. With

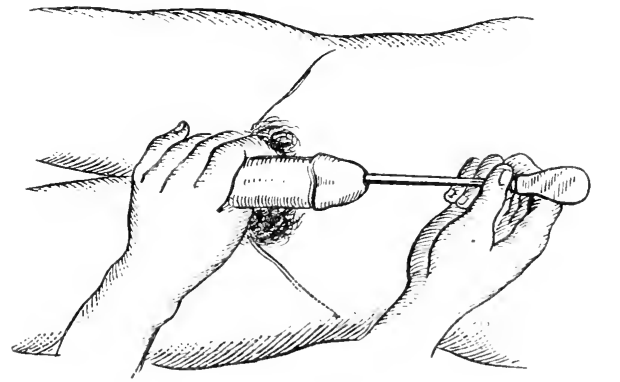

FIG 30.-Sounding of the urethra (Keyes).

the left hand still holding the penis, the handle of the instrument is steadily carried over the surface of the 
abdomen to the exact median line of the body (Fig. 30). Without elevating the handle of the sound, the latter is then gently pushed toward the feet of the patient, while the left hand gathers up the scrotum, testicles, and penis and makes slight upward traction upon them. The point of the sound should be followed closely throughout by the little finger of the left hand, and when the point has passed to the perineum and the curve of the instrument can be felt back of the scrotum, the penis and the tes-
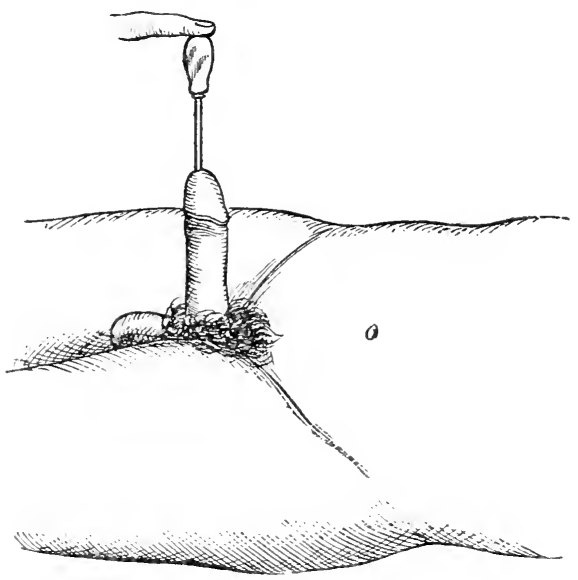

FIG. $3^{x}$-Sounding of the urethra (Keyes).

ticles are dropped and the fingers of the left hand are placed on the perineum, where they give support to the lower wall of the urethra and keep the point of the instrument well against the upper wall. Up to this time the handle of the sound has been held constantly close to the abdominal wall. The handle should now be lifted and gently carried toward the feet, thus describing an arc of a circle exactly in the median line of the body. When the handle of the sound has reached and 
passed the perpendicular (Fig. 3I), the left hand leaves the perineum and supports the handle of the sound, while the right hand presses upon the pubes and relaxes the triangular ligament, allowing the sound by its own weight to slip through the membranous and prostatic portions of the urethra into the bladder (Fig. 32). That the sound has entered the bladder may be demonstrated by partially rotating the handle, showing that the tip of the instrument is free.

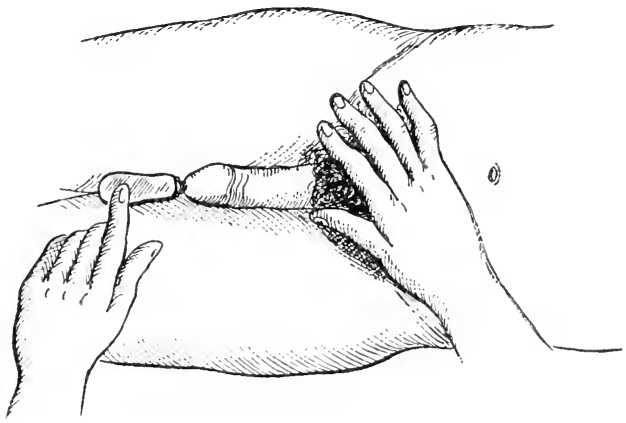

FIG. 32.-Sounding of the urethra (Keyes).

To remove the sound the handle is carried through motions exactly the reverse of those pursued during its introduction. Neither force nor traction is necessary.

Foree is required for the introduction of a sound in exceptional cases only, and should never be used by any but the expert. The sound should be held lightly between the tips of the thumb and the fingers, as a firmer grasp would interfere with the sense of touch, which is of great importance, and would increase the danger of unconsciously using force. It should never be forgotten that the sound is a lever of the first class, the tip forming its short and the handle its long arm, and that the 
pressure produced by the point upon the urethral wall is many times greater than that exerted by the hand of the operator upon the handle. Even in a normal urethra the progress of the sound may be arrested by urethral spasm, which occurs most frequently at the beginning of the membranous urethra. The gripping of the sound by the muscle-fibres produces a sensation readily recognized by the experienced hand holding the instrument. If the sound be held quietly against the contracted muscles, they soon relax and allow the instrument to pass. Coaxing rather than forcing is the rule at all times when using an instrument in the urethra.

The tip of the sound should always be kept steadily in the median line, and all irregular, jerking, or wabbling movements should be avoided, as these produce unnecessary motions of the tip in the urethra, and the resulting disturbance of the urethral walls is the most frequent cause of pain in this operation. A support for the surgeon's elbow is usually serviceable.

Beginners experience the most difficulty when the tip of the sound is passing from the large, distensible, and movable bulbous urethra to the narrower membranous portion. If the handle be raised from the abdomen too soon, the tip catches above in the subpubic ligament; while if the tip is not held well up against the upper wall by the fingers on the perineum, it may bury itself in the loose and movable floor below the orifice. Figure 33 show's the bulbous urethra greatly distended by the tip of a sound that has been allowed to fall and turn over. When the tip of the sound is arrested at this point, the curve of the instrument bulges out in the perineum as the shaft is depressed between the thighs, and if the handle be released, it springs back toward the perpendicular. 
In such a case the sound should be withdrawn an inch or two and reintroduced, care being taken not to raise the handle too soon and to keep the tip against the upper

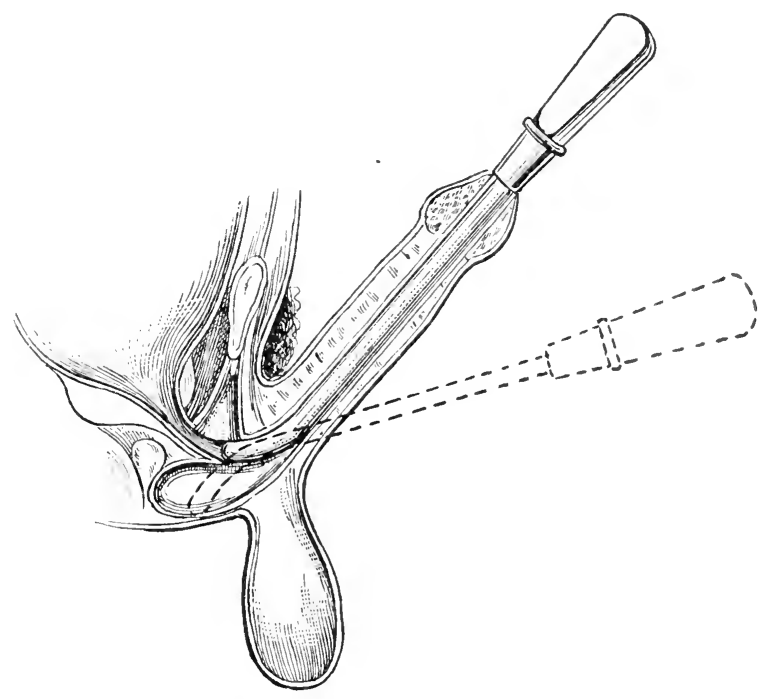

FIG. 33.-Relative positions of triangular ligament and bulb of urethra (diagrammatic, from (ulver and Hayden).

wall. A few gentle manœuvres should succeed in passing the instrument into the bladder, unless there be stricture or unless the sound is too large for the urethra.

While decided pain should not be produced by the passage of a sound or a catheter as described above, even a normal urethra shows some resentment at the introduction of a foreign body. Slight pricking, stinging, or tickling sensations are usually felt by the patient as the tip of the sound comes in contact with the mucous membrane, and as the instrument dilates the membranous and prostatic portions the desire to urinate may become 
so strong that the patient declares he cannot retain his urine another moment. In sensitive patients the operation may produce nausea or even complete syncope. These disturbances are usually most marked in a young man having his urethra explored for the first time, since the sensitiveness of the urethral mucous membrane is rapidly lessened as a result of repeated instrumentation. The first micturition following the use of an instrument in the urethra is usually attended by some smarting or burning. Urethral (urinary) fever, prostatitis, or epididymitis occasionally results even when every precaution is supposed to have been taken; but these results are frequentiy due to forcible or careless instrumentation of the urethra, or to the use of instruments that are not clean and aseptic.

Instruments.-Of all the instruments used in the treatment of stricture, the sted sound is the most important. Steel sounds for use in the deep urethra measure about nine inches from the tip to the junction of the shaft and the handle, and are blunt or conical. Blunt sounds are of a uniform diameter throughout their entire length; conical sounds are several sizes (from 3 to 7 sizes French) smaller at the tip than in the shaft, the conicity extending from the tip to about the beginning of the curve. The blunt sound is chiefly valuable for exploration and for the treatment of urethral lesions other than stricture. The slightly conical sound is the most generally useful and the most economical, as fewer sizes are needed. All steel sounds for use in the deep urethra should possess the curve already described, should be polished perfectly smooth, should be nickle-plated, and should have the size of the shaft plainly stamped on the handle.

The scale used for determining the size of urethral in- 
struments varies greatly in different countries and, unfortunately, with different instrument-makers. The French scale furnishes a definitely fixed standard, and is that to which all numbers in these pages refer. By this scale the number on a sound indicates its circumference in millimeters. No. I is one millimeter, No. 2 two millimeters, and so on through the entire scale. The divisions on this scale are so small that it is rarely necessary to have sounds representing all the numbers, especially in conical and in flexible instruments. In making a set every other number may be omitted, so that ten conical steel sounds ranging from $\mathrm{I} 5$ to 33 in sizes (the tip of each being three or four sizes smaller than the shaft) will meet the requirements of all but exceptional cases. As manufacturers of instruments do not yet use a uniform scale, and as most flexible instruments are neither accurately nor plainly marked, the surgeon should own an accurate scale-plate (Fig. 34), on the two faces of which are marked French, English, and American scales, as well as inches and millimeters.

Short sted sounds, made straight or with a very short curve at the tip, are convenient for use in the anterior ure-

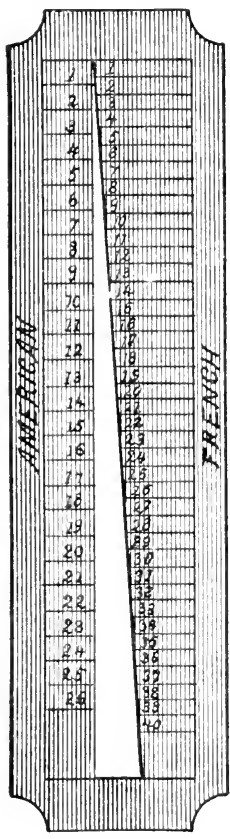

Fír. 34--Handerson's gauge (Tiemann). thra; they are, however, not necessary, since the long sound need be passed into the urethra no further than is desired.

The bulbous bougie (bougie à boulc) is the instrument 
most used for determining definitely the location and calibre of strictures (Fig. 35). The head should be short,

FIG. 35.-Bulbous bougie (Tiemann).

and the shoulder should join the much smaller shaft at almost a right angle. These instruments made of metal are to be preferred when large sizes are to be used in the anterior urethra only, but for general use the flexible gum bougics à boulc are better. Otis's urethrometer

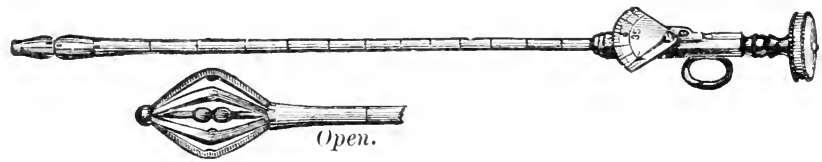

FIG. 36.-Utis's urethrometer (Tiemann).

(Fig. 36 is intended to serve the purpose of bulbous bougies of different sizes. After introduction into the urethra the bulb may be made larger or smaller as desired, and the size is indicated on the scale at the handle. A soft-rubber cap covers the bulb, to keep the wires from tearing the mucous membrane. This instrument is convenient when it works well and is carefully manipulated, but it usually causes more pain than do the bulbous bougies, and even in careful hands the rubber cap is liable to be torn or to be left in the urethra.

Silier cathetirs should correspond in shape and size with the blunt stcel sounds. They are of occasional service in the large sizes, and should be introduced in the same manner as the steel sound.

Flexible bougics (Fig. 3\%) are necessary in the treatment of stricture of small calibre; in the larger sizes 
they are often valuable, especially for beginners. As a rule, even the expert should use flexible bougies for all sizes below 15 or 18 , in order to avoid the danger of

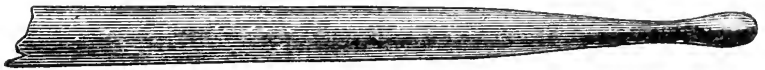

FIG. 37.-Olivary gum bougie (Tiemann).

making a false passage. Of these bougies, the conical are the best and most serviceable, though for the larger sizes the olive-tip bougie, if connected with the shaft by a slender, perfectly flexible neck, is preferable. They are made in all sizes from the filiform up.

Flixible catheters are made of the same size and shape as bougies. Some of these catheters are furnished with a metal stylet which stiffens them during introduction. This stylet is of only occasional value. The Mercier catheter (Fig. 38 ) is an excellent instrument in difficult

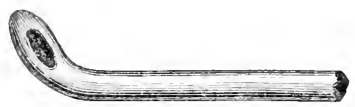

FIG. 38.-Mercier elbowed catheter (Tiemann).

cases, and especially if the prostate be enlarged. The tip should not be too stiff, as it often is in those of English manufacture.

FIG. 39.-Gouley's whalebone bougies (Tiemann).

Filiform bougics (Fig. 39) are necessary in the treatment of all strictures of very small calibre-tight strictures. The best filiforms are made of whalebone with fine bulbous 
tips. By placing them in hot water for a few minutes the ends may be so bent or twisted that the point will enter an eccentric opening in a stricture which a straight instrument would be unable to penetrate. If the two ends of a filiform bougie are thus bent in the same direction, when the tip engages in the stricture the outer end will serve as an index to the exact location of the opening, which can thus be found with much less difficulty at the next sitting. A filiform bougie, when it has been passed through a small and difficult opening in a stricture, may be used as a guide for a larger instrument. For this purpose the bougie should be at least eighteen inches long. Dr. E. A. Banks has devised a whalebone bougie which is filiform at its tip and throughout its first two or three inches, and then increases in size to form a larger shaft which can be pushed on into the stricture to dilate it. Filiforms are also made with caps on the outer end that can be screwed on to larger instruments which may thus be conducted through the stricture. These bougies are dangerous, as the caps may become loose and the filiform bougie may be left in the stricture or in the bladder; besides, they offer no advantage orer either of the two preceding methods. Whalebone bougies frequently become cracked, rough, or frayed as a result of keeping and handling; consequently it is necessary to inspect each bougie (as should be done with all soft instruments) just before using, to make sure that it is sound and smooth.

Tunnelled sounds and catluctors (Fig. 40) are so made that they can be threaded over a filiform bougie and thus be guided safely into the bladder. They should have a short curve. and the short tunnel should be large enough to allow the guide to slip through it easily, with 
edges smooth and rounded to prevent cutting the guide. To use the tunnelled sound safely and to advantage, at least five or six inches of a long filiform bougie should be passed into the bladder. The tunnelled sound is

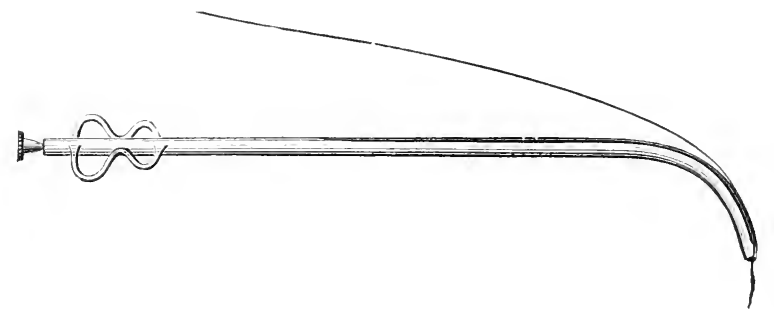

FIG. 40.-Gouley's catheter-staff (Tiemann).

then threaded over the guide and slipped down to the point of the stricture. Here it is gently pressed forward through the stricture as the guide is slowly and gently drawn out. If the guide becomes fast in the larger instrument, both guide and sound should be withdrawn until the former is again freely movable. Failure to observe these precautions may result in a false passage or in cutting the guide in two and leaving one end of it in the stricture.

Introduction of Flexible Instrumcnts.-In the use of soft and flexible instruments slight pressure or force is allowable, though some of the rubber bougies are stiff enough to do damage if carelessly used. The position of the penis during the introduction of flexible instruments is not a matter of great importance except for the small-sized instruments, for which the penis should be held parallel with the thighs and should be put lightly on the stretch, to make the canal as straight as possible and to efface the folds of mucous membrane in which the fine points of small instruments are frequently caught. 
For the introduction of filiform bougies the urethra should first be filled with warm, slightly benzoinated or carbolated olive oil. The points of these fine instruments are often caught, even in the healthy portion of the urethra, by folds of mucous membrane or in the sinuses of Morgagni. When this accident occurs the bougie is withdrawn an inch or more; the tip, bent to form an angle with the shaft, is then rotated in another direction and again advanced. These manœuvres are repeated gently, and, if necessary, many times, until the tip engages in the orifice of the stricture. To determine whether a bougie has entered the opening of a stricture or a blind pocket, it should be slightly withdrawn and advanced: if it be engaged in a stricture, the grip of the latter upon the instrument can be felt plainly by the hand of the operator.

In old and tortuous strictures, with many pockets, lacunæ, and partial false passages (Fig. 26) ready to entangle the point of a filiform bougie, it may be impossible to find the opening with a single instrument. In such a case a filiform bougie is passed until it is caught, when it is held in place and another bougie is passed by its side. In this way the urethra may be filled with a number of these fine instruments, each of which should repeatedly be withdrawn slightly, partially rotated, and again advanced, until one of them engages in the orifice of the stricture and passes into the bladder. The successful performance of the above-described procedure calls for a steady hand, a sensitive and delicate touch, a large amount of patience, and the greatest gentleness in every movement, lest the mucous membrane be damaged by much manipulation. The search may sometimes be continued for 
an hour or more, but the production of hemorrhage or of marked irritation at once renders further attempts useless until the urethra has had a rest of at least twenty-four hours.

Relatio' Value of Sted and of Flexible Instruments.The polished steel sound, of proper curve, in the hands of an expert, can be introduced into the urethra with less discomfort than can any other instrument, but it should not be used for sizes below 15 or 18 , for fear of making a false passage. For the smaller sizes the flexible instruments are safer and better. They may often be used in the larger sizes to advantage. The chief objections to them are that they produce more irritation in the urethra, and therefore more frequently provoke urethral spasm, and that they are less rapidly effective in dilating stricture than the steel sound when the latter is used with sufficient skill and care. They are, moreover, more difficult to clean and less durable. On the other hand, they are not capable of doing so much damage when used by one not specially trained in the proper introduction of the steel sound. The best flexible instruments have a foundation of woven linen covered with a smooth elastic composition. When new and in cool weather, the black French instruments are softer and better than the English, but they do not last so long; in hot weather they often become too limp to be of any use and are easily spoiled, as softening of their outer coating allows them to stick together and become rough. The English (yellow) bougies are firmer and more durable, and, if the softer ones be selected, are the best. If too stiff, they can be made more flexible by placing them in hot water for a few minutes just before using. 
All flexible instruments sooner or later wear out or become hard and brittle; consequently they should always be examined just before using, to make sure that they are sound and smooth. This remark is especially true of the soft catheters, made without the woren foundation, which every general practitioner is supposed to carry in his case. It happens occasionally that the tip of one of these old instruments breaks off and is left in the urethra. Fortunately, in the majority of cases, if left alone, the constriction about the piece relaxes after a few hours, and the fragment is washed out during the next urination.

Care of Instruments.-The principles of antiseptic surgery should be followed strictly in the care and use of all urethral instruments. Failure to observe these principles too often results in urethral fever, urethritis, cystitis, epididymitis, prostatitis, abscess, and other disagreeable and dangerous complications. All instruments, immediately after use, should be washed thoroughly with soap and warm water. Antiseptic solutions have little effect upon an instrument covered with oil or vaseline and smeared with pus, blood, or other matter. Moreover, if rubber instruments are allowed to remain corered with oil, vaseline, or fat, they soon become rough and useless. Steel instruments are best sterilized by boiling or by placing them for twenty minutes or half an hour in a steam-sterilizer. Before use they may be placed for a few minutes in a 5 per cent. carbolic-acid solution. Rubber and whalebone instruments should be placed, before use, in a solution of bichloride of mercury, I : IOoO. Refined vaseline, cosmoline, and albolene are the best lubricants for general use, since they do not become rancid. When desired, carbolic acid ( 2 per cent.) or 
boric acid (3j to $\tilde{3} \mathrm{j})$ may be added. As a rule, instruments should be warmed before use in the urethra. This precaution renders their introduction less irritating to the mucous membrane, and the production of spasm less frequent. In the absence of warm water, steel instruments may quickly be warmed by rubbing them rapidly through a towel held in the hand.

Steel instruments should be kept in cases made to fit them, as they are otherwise easily nicked or scratched. The slightest defect, such as a scratch or a rust-spot, in the surface of an instrument may cause urethral irritation, and may, moreover, serve as the lodging-point of infectious matter. Such an instrument should always be laid aside until properly repaired. Rubber instruments should be kept between layers of cotton or in narrow pasteboard trays lined with paper that is thrown away when soiled. In hot weather they should be dusted with finely powdered talc or otherwise separated, to keep them from adhering-an accident that almost invariably roughens and ruins the instrument.

Cutting instruments and the methods of their use are described in connection with the operations for which they are intended. For the benefit of the beginner, a summary of all other instruments necessary in the diagnosis and treatment of stricture is here given. The French scale is used, and in forming sets of instruments every other (odd or even) number may be omitted.

Ten conical steel sounds, Nos. I5 to 33; a corresponding set of short steel sounds, for use in the anterior urethra (convenient, but not necessary); two or three silver catheters, Nos I 8 to 24 ; a set of bulbous bougies, Nos. 7 to 33 (the larger sizes may be metallic if pre- 
ferred); a set of French or English conical, flexible bougies, Nos. 5 to 20 , or to 33 if desired; half a dozen soft catheters, Nos. 6 to 20 ; as many Mercier catheters, and one or two of larger size; half a dozen short and three or four long whalebone filiform bougies; a set of tumnelled conical steel sounds with short curve, Nos. 6 to I 8 ; two or three tunnelled (not conical) catheters, Nos. 6 to IO; a Banks whalebone bougie; and an accurate gauge. If the surgeon is within easy reach of surgical supplies the number of soft catheters should be reduced, as these instruments often deteriorate and become useless after a few months.

Diagnosis.-The instruments of precision furnished the surgeon render the diagnosis of stricture comparatively easy. The chief difficulty at present lies in distinguishing between beginning strictures of large calibre and normal points of narrowing in the urethral wall.

The indications for exploring the urethra are found in the presence of one or more of the symptoms of stricture, already described, appearing a number of months or years after urethritis (usually prolonged gonorrhœe) or urethral injury. Needless instrumentation of the urethra should always be avoided. Though the proper exploration of the urethra is usually attended by no worse results than some discomfort or pain to the patient and some smarting or burning in the wrethra at the next micturition, it happens occasionally that the apparently careful and skilful passage of a steel sound through a normal urethra is followed by syncope, shock, urethral fever, epididymitis, prostatitis, etc. If the urethra be diseased, if force be used, if the operator be careless or unskilled, or if his instruments be rough or soiled, these and other yet more unfortunate results of urethral instru- 
mentation occur not infrequently. Instruments should not be used in the urethra if there be a decided discharge from the meatus, or other evidences of urethral inflammation or irritation.

Exploration of the urethra for the first time should be begun by the introduction of a blunt steel sound of the largest size that will pass the meatus without stretching the latter sufficiently to cause pain or to produce the characteristic evidence of an over-stretched meatusnamely, a narrow white (anæmic) line at the edge of the orifice and in contact with the instrument. As a rule, if such an instrument pass easily into the bladder, stricture is absent. The exceptions to this rule are considered later. If the instrument meets with obstruction in the urethra and fails to pass, a series of blunt sounds gradually decreasing in size should be used until one is passed. Below size I5, soft instruments should be used. When an instrument has been passed, it should be left in position (unless it is producing pain or irritation) long enough for the fingers to explore the pendulous urethra for external evidences of stricture, which may be found in the form of circular or irregular bands of thickening and induration. In this way valuable information may be gained, and further instrumentation for purposes of diagnosis is often rendered unnecessary.

In many cases, however, accurate location and measurement of stricture is best accomplished by the use of bulbous bougies. An instrument with a bulb corresponding in size to the sound that has passed the stricture is properly prepared and gently inserted down to the anterior face of the stricture, where it is arrested. If the bulb does not pass the stricture, a smaller instrument is selected and passed into the bladder. As it is 
withdrawn it is again arrested as its shoulder reaches the posterior surface of the stricture. If the distance of the meatus from the end of the handle be measured and noted when the bulb is arrested, both in entering and in withdrawing, not only the location and calibre but also the approximate thickness of the stricture may be determined. In those unusual cases in which stricture is multiple there is no difficulty in locating and measuring each, if the anterior stricture is of larger calibre than the deeper one. If the reverse is true the Otis urethrometer may be used, though the diagnosis of the deeper stricture is of less practical value, since it does not call for treatment until the stricture in front of it has been dilated.

Several sources of error should always be considered in the use of bulbous instruments. They produce more irritation, and therefore more frequently provoke urethral spasm, than do blunt or conical sounds, and, moreover, they are less effective than the latter in overcoming spasm or in detecting the characteristics which distinguish it from organic stricture. In withdrawing the instrument the shoulder of the bulb, in passing from the prostatic to the membranous urethra, frequently catches on the posterior layer of the triangular ligament and produces a sensation closely resembling that produced by stricture. In the pendulous urethra the shoulder of the bulb may be arrested at one or more points of normal narrowing frequently found in the first three inches from the meatus. The danger of error in diagnosis is lessened if the bulbs be used in connection with the steel sound as above described.

The reasons for beginning with a full-sized steel sound are several: it produces less irritation than any other instrument; often it will easily enter the bladder when 
bulbous sounds or smaller instruments fail to pass because of the spasm excited or because of the finer points being caught in some of the folds or sinuses of the urethra; its ready passage may save much unnecessary instrumentation and irritation of the urethra; the introduction of one or more steel sounds in a urethra lessens for a time the sensitiveness of the nucous membrane, so that other instruments which follow produce less irritation and spasm than if used first.

As a rule, the first sitting should be made as short as possible, since strictures-and individuals also-vary greatly in the amount of instrumentation they will endure without unfavorable results. If the introduction of the first sound proves very irritating, as may happen in nervous men, it is often wise to postpone further attempts for a day or two, even though the bladder has not been entered. After locating and measuring the stricture, unless there be urgent symptoms demanding immediate attention, no attempt at dilatation should be made for two or three days, until the irritation which will probably result from the examination has subsided, and the surgeon is given a chance to gain some idea of how sensitive the patient and his stricture are to instrumentation. The patient should be warned that the next urination will probably be attended by some discomfort, and that he may notice a slight discharge from the meatus, or an aggravation of an existing one, for a few days. If indicated, an alkali or one of the balsams may be ordered, while small doses of quinine may be given to lessen the danger of urethral fever.

The general health and habits of the patient should be investigated fully, and necessary corrections should be made. Of particular importance is a careful exami- 
nation of the urine. If the latter contains pus, and especially if it is decomposed and indicates the presence of cystitis, the patient should at once be put upon boric acid or salol in Io-grain doses four times a day, to lessen the danger of urethral fever and other complications. The amount of residual urine in the bladder should be ascertained by passing a catheter immediately after the patient has urinated.

The accurate diagnosis of strictures of large calibre may require cutting of the meatus before instruments of sufficient size can be introduced into the urethra. Until recently many surgeons, acting under the impression that the operation was simple and harmless, have cut the meatus frequently and freely, simply as a matter of convenience in searching for possible stricture of large calibre. There is no doubt that the operation is often followed by harmful results. The gaping lips of a freely cut meatus are frequently the seat of a persistent gleety discharge, while the operation may greatly lessen the power of the individual to eject urine and semen from his urethra, so that dribbling of urine after micturition is not uncommon in these cases. If the meatus is abnormally small, and especially if it is rendered so by a thin band of tissue at the lower commissure, meatotomy should be performed to allow the entrance of a full-sized sound. The free division of the meatus, however, to allow the introduction of very large instruments is not warranted unless there be other and more urgent symptoms of stricture than a gleety discharge, or unless the latter has persisted for a year or more notwithstanding skilful and faithful treatment, including the best general and hygienic management. In such a case it is possible that a forming stricture, of a 
calibre as yet too large to interfere with the passage of an instrument of ordinary size, is responsible for the discharge, and, after other means of treatment have been tried, exploration of the urethra with instruments larger than will pass the normal meatus is proper.

In such an examination the question at once arises, What constitutes the normal calibre of the urethra, and what size of instrument should pass unobstructed into the bladder? On this point authorities differ, and it is evident that an exact answer to this question cannot be given, since the urethra is not a tube of uniform diameter, but is a closed canal or valve having normal points of narrowing. Dr. Otis maintains that the calibre of the urethra bears a constant relation to the circumference of the flaccid penis. But that such a relation is only approximate is evident when one considers the great variations in the size of the organ under the influence of heat, cold, mental state, etc. Dr. Otis's scale, which is much too large, is as follows: When the circumference of the flaccid penis is 3 inches, the urethra should receive an instrument of the size $30 ; 3 \frac{1}{4}$ inches, size $32 ; 3 \frac{1}{2}$ inches, size $34 ; 3 \frac{3}{4}$ inches, size $36 ; 4$ inches, size $38 ; 4 \frac{1}{t}$ to $4 \frac{1}{2}$ inches, size 40 . These sizes do not indicate the normal calibre of the urethra, but the limit to which, according to Dr. Otis, the urethra can safely be distended. For purposes of diagnosis and treatment the majority of modern surgeons adopt a scale from four to eight sizes smaller than those named above; they also recognize normal variations in the pendulous urethra.

Many surgeons who adopt the larger scale find and treat a great many so-called "strictures of large calibre" that are really nothing more than simple, normal contractions of the urethra. This unnecessary stretching 
and cutting of the urethra not infrequently results in permanent and even distressing deformity.

The diagnosis of stricture should, then, be reserved for a distinct contraction of the urethra, accompanied by a gleety discharge, frequent micturition, dribbling of urine after urination, or other symptoms of stricture.

Prostatic hypertrophy may lead to a careless diagnosis of stricture of the deep urethra, but in the former disorder the obstruction to the sound is situated more than six and a half inches from the meatus; the handle of the sound must be depressed considerably more than usual before the tip enters the bladder; a rectal examination reveals the enlarged prostate; the patient usually is over fifty years of age; and there is a history of gradually increasing frequency of micturition, most marked at night.

Treatment.-The directions already given for instrumentation of the urethra are not repeated here, although their observance forms a necessary and most important part of the treatment of stricture. It should never be forgotten by the operator that the exercise of gentleness, caution, and patience is not only less dangerous but far more effective in the treatment of stricture (with the exception of those cases in which a cutting operation is required) than is the employment of force.

Dilatation.-In the large majority of strictures, either of the deep or of the pendulous urethra, that are not complicated by retention or by other urgent symptoms, gradual dilatation is the most efficient and safest method of treatment. By this method the stricture is dilated during successive sittings by a series of sounds gradually increasing in size. The principles given for the introduction of the steel sound should be carefully studied 
and applied. Each sound or bougie should be cleaned, sterilized, warmed, and lubricated before it is used in the urethra. To further lessen the danger of urethral fever during treatment, the patient should take Io or I 5 grains of boric acid or of salol four times a day for thirty-six or forty-eight hours before and for an equal time after each sitting. The general health of the patient should be maintained, and he should fully understand that the success of the treatment, as well as freedom from distressing complications which delay progress, will depend largely upon his living simply and hygienically. Tobacco, alcohol, and other stimulants, sexual indulgence, and severe exercise are especially harmful. Frequent examinations of the urine, and especially of the residual urine, are necessary to keep the surgeon informed regarding the condition of the bladder and of the kidneys.

In strictures of large calibre the dilatation is best accomplished by means of conical steel sounds. There is selected a sound of the same size as the exploring instrument that entered the stricture during the examination of two or three days previous; this sound is gently passed through the stricture. If it occasions no distress, it should be allowed to remain in situ for five or ten minutes; it is then withdrawn gently and the next larger size is used in the same manner. If the stricture is in the pendulous urethra, a short sound should be used, or the long sound should be arrested before entering the bladder, to avoid unnecessary irritation. Usually the stricture may thus be dilated two or three numbers (French scale) at each sitting; but occasionally the same sound must be passed at several successive sittings before a larger one can be employed. This is a matter that must be decided in each case by the surgeon 
in attendance, after watching the effect of instrumentation upon the stricture and upon the individual. Dilatation should be stopped for that day whenever it has been carried far enough to produce a drop or two of blood or to cause decided pain or irritation. When attempts at dilatation are followed by marked urethral irritation, with possibly slight pain in the testicles, and especially if a suggestion of chill or fever has resulted, the surgeon will content himself with slower progress. There is nearly always a temptation to use a little force and haste, but such attempts are usually followed by urethritis, epididymitis, or urethral fever, necessitating the suspension of further dilatation until the new complication is removed. The conical steel sound is not only a powerful lever of the first class, but it is also a wedge, and few surgeons realize, until they have produced a false passage or met with other misfortune, how much force very slight pressure upon the handle may cause the point of such a sound to exert in the (usually damaged) urethra. Slight force may occasionally be necessary in the use of blunt instruments, but if a conical steel sound, properly held against a stricture for a few seconds, rufuses to pass by its own weight, it should be withdrawn and a smaller size be substituted.

At each sitting the dilatation should be begun by the introduction of a sound one or two sizes smaller than the largest sound used at the previous visit. It is always best to have the patient urinate in the surgeon's presence before passing the sounds, that the size of the stream and the condition of the urine may be tested, and that the first urination after the operation may be postponed for a few hours.

Valuable information bearing on the treatment of 
stricture may be obtained by watching the phenomena following the introduction of a sound into a stricture which is thus stretched slightly. When the instrument first enters, it is more or less tightly grasped, so that some force may be necessary to remove it, but if allowed to remain in situ a few seconds or minutes, the stricture relaxes and the sound moves through it easily; the spasmodic element has been overcome and the stricture has been stretched mechanically. As a rule, the stream of urine is increased in size for a short time-possibly twenty-four hours-before congestion, or even inflammation, in and about the stricture-growth follows; the resulting swelling narrows the calibre of the urethra and therefore the size of the stream. At this time there is usually an increase in the discharge from the meatus, with other symptoms indicating irritation or inflammation of the urethra. At the end of three or four days absorption begins; the calibre of the stricture, and hence the size of the stream, enlarges: the urethral discharge and other symptoms improve. This improvement continues from a day or two to a week before contraction again begins.

It is evident to one who stops to consider the significance of the above-described phenomena that after stretching a stricture the next attempt at dilatation should be postponed at least beyond the stage of contraction and irritation ; indeed, the best results are obtained when such instrumentation is delayed until just before the stricture again begins to contract. When the same instrument that was used a day or two before to dilate a stricture is reintroduced during the resulting stage of congestion, it passes with more difficulty and produces much more pain and irritation than when passed the first 
time. Unless the operator appreciates the situation, he will probably conclude that he has an irritable stricture to deal with. A great many irritable strictures are undoubtedly produced in just this way, as the result of too frequent and injudicious instrumentation. The commonest mistakes in the treatment of stricture are of this charater, and beginners especially are inclined to make the intervals between visits for dilatation altogether too short. The results of such over-treatment are slow progress if any, an irritable stricture, a constantly congested and inflamed urethra, and not infrequently more serious complications. In different cases the proper interval may vary from three to ten days, depending upon the character of the stricture, the amount of dilatation accomplished each time, the amount and nature of the reaction, and the general health and habits of the individual. The decision must be based on a careful study of each case, and especially of the phenomena described above. As a rule, the most rapid progress and the best results in dilatation of stricture, when steel instruments are used, are obtained by sittings with intervals averaging from five to seven days.

The treatment should be continued steadily through sereral weeks until the stricture has been dilated to the normal calibre of the urethra. ${ }^{1}$ By this time the symptoms will usually have disappeared, with the exception of traces of the gleety discharge, which will probably disappear as instrumentation is suspended.

The treatment must not end here, however, if it is to result in any permanent benefit, for if left alone the great majority of strictures will at once begin to re-contract.

1 The question of what constitutes the normal calibre of the urethra is considered in connection with diagnosis. 
The patient should clearly understand that unless the calibre of the urethra be maintained by the occasional use of a sound, contraction of the stricture will follow, and his treatment will prove of no permanent value.

To prevent re-contraction a full-sized sound should be passed with sufficient frequency. At first this procedure should be done once in a week or ten days, then once in two weeks, the intervals thus being lengthened gradually until the sound is no longer needed or until it is discovered how long the interval may be made before the stricture begins to contract. A few months of such treatment will render further instrumentation unnecessary in some cases, but usually the introduction of the sound must be repeated, at intervals varying from a week to three months, for several years, or even indefinitely.

With a little instruction the patient should, as a rule, learn to pass the full-sized sound upon himself, thus obviating the necessity of occasional visits to the surgeon during long periods of time, and also lessening the probability of neglect. The patient should be impressed especially with the necessity of keeping his sound clean and aseptic. If such a course of dilatation be faithfully carried out, many strictures disappear entirely, while others, with a little care on the part of the patient, are kept under control and rendered harmless.

That absorption of the stricture-growth during a course of gradual dilatation actually occurs can often be demonstrated by watching the progress of a case in which the growth can be felt by the fingers on the outside and its gradual disappearance be noted.

In strictures of small calibre uncomplicated by retention or other urgent symptoms, gradual dilatation is 
carried on as above described, except that flexible instruments should be used until the stricture is dilated sufficiently to receive a No. I 5 or i 8 sound. The great danger of making a false passage in using a conical steel sound smaller than No. is camnot be too strongly impressed upon those who have never had an opportunity to observe how easily this unfortunate accident can occur. Though with steel sounds progress is more rapid and discomfort to the patient is less, yet for all sizes below No. I5 or is soft instruments should be used.

Since less dilatation is usually accomplished at each sitting with the flexible bougies than with sounds, the resulting congestion and irritation are less and subside more rapidly, so that the intervals between sittings should be shorter. The proper interval varies from two to five days, and should be determined in each case in the same manner as when sounds are used.

In some cases of old stricture, though the patient be able to pass a small stream of urine, great difficulty is experienced in introducing even the finest instrument. The opening is usually eccentric and may possibly be covered with a flap of mucous membrane; or the canal through the stricture is tortuous and presents many small pockets, open follicles, or false passages (Fig. 26). Such strictures are often termed "impassable," but so long as a stricture has a calibre sufficient to allow the escape of urine, it is possible to enter it with filiform bougies if these be used with the proper skill, patience, and perseverance. The methods of using filiform bougies have already been described in detail.

It sometimes happens that a number of prolonged sittings at intervals of a day or two are necessary before 
an instrument can be made to enter the stricture; but these attempts should not be given up unless there be retention or other urgent symptoms demanding immediate relief, or unless the use of instruments is followed by urcthral fever or by other unfavorable result. Sometimes the end of the bougie enters the stricture far enough to be grasped, but will pass no further. In such a case the bougie should be left in this position for ten or fifteen minutes, when it probably can be introduced still further. If it refuses to pass further at the end of half an hour, it may be tied in place and left for twenty-four hours, at the end of which time, if it will not enter the bladder, it will at least have dilated the anterior portion of the stricture, which will consequently be more readily entered by other instruments, some of which will eventually pass to the bladder.

When a filiform bougie has passed a stricture after several unsuccessful attempts, the next step in treatment depends on a number of conditions. If the previous manipulations have caused little urethral irritation, if there are no symptoms demanding immediate interference, and especially if the operator has located the opening of the stricture, so that he can find it readily at the next visit, the bougie should be left in place for from fifteen minutes to an hour and then be removed. Retention due to swelling of the nucous membrane nay follow, but it rarely lasts for more than a few hours, and it can usually be relieved by a hot bath and, if necessary, by an opiate. At the end of forty-eight hours a large bougie can usually be passed, and treatment by gradual dilatation is then fairly begun. If the stricture is somewhat irritable, is complicated by partial or complete retention, and has been entered with great difficulty, 
and especially if the urethra be sensitive to instrumentation, the best method of treatment is by continuous dilatation.

Contimuous dilatation is accomplished as follows: The first filiform bougie that passes should be tied in and allowed to remain for twenty-four hours. After a few hours the stricture dilates and allows the urine to pass beside the bougie, so that retention rarely occurs. At the end of twenty-four hours the dilatation is usually considerable, and the filiform may be replaced by a bougie a size or two larger, which may be tied in for another twenty-four hours. Successive sizes may thus be used for a few days until the stricture will admit a No. 8 or No. IO bougie. Further dilatation is best accomplished by the gradual method. The chief objection to the employment of continuous dilatation is that it occasionally results in cystitis or produces urethral fever. The danger can be lessened greatly by using all antiseptic precautions, including irrigation of the urethra and the bladder, and by giving full doses of boric acid throughout the treatment. The new bougie or catheter substituted each day should be one or two sizes smaller than the largest that can be introduced. An instrument that completely fills the stricture produces more pressure and increases the danger of complications without producing more rapid dilatation than one a size or two smaller. Very small bougies, however, may easily be washed out of the urethra by the urine. The outer end of the bougie should be tied with two or three pieces of soft cotton twine to the pubic hairs. If these pieces of twine are also fastened to a ring (large enough to permit erection) of narrow tape encircling the penis an inch or two from the glans, the bougie will be held more 
securely. Unless some means be employed to secure the bougie, it may slip out of the urethra, or it may escape backward into the bladder and necessitate an operation for its removal.

Continuous dilatation should not be employed in patients who do not tolerate well the presence of a bougie in the urethra, nor in patients who will not remain in bed during the treatment. It should be substituted by some other method if it is attended by decided urethral fever. Slight fever and chills are not sufficient reason for suspending treatment, but such cases require careful and constant watching.

When continuous dilatation is not applicable to a given case, the first filiform bougie passed may be used as a guide for the introduction of a tunnelled sound or a catheter. The method has already been described in detail. If the attempt is successful, gradual dilatation may follow. If the tunnelled sound cannot be introduced, and if continuous dilatation is not permissible, urethrotomy must be performed.

Dilatation, as previously described, is the most effective as well as the safest method of treatment in most cases of stricture; but there are not infrequently encountered cases which refuse to yield to this method, and which may be rendered worse by attempting to continue it. Treatment by internal urethrotomy when the stricture is located in the pendulous urethra, and by external urethrotomy or by the combined method for stricture of the deep urethra, is necessary in the following classes: I. Most of the true strictures of the meatus. 2. Cicatricial and traumatic strictures which refuse to yield to either gradual or continuous dilatation. 3. Resilient strictures which rapidly re-contract after dilatation. 
4. Strictures in which attempts at dilatation are followed by marked urethral fever and chills. 5. Strictures complicated by abscess, fistulæ, extravasation, or other conditions which render a perineal section necessary, the stricture being divided at the same operation. 6. Strictures with which retention is complete or of long standing, or with which the general health is involved and immediate relief is necessary. 7. Some strictures complicated by an enlarged prostate. 8 . Strictures in individuals who cannot give the time necessary for treatment by dilatation.

Many operators cut all strictures of the pendulous urethra, but the majority of surgeons of the present day cut such strictures only when they refuse to yield to dilatation. The cutting operation must be followed by the regular use of the sound if the results are to be permanent, and internal urethrotomy is attended not only by some danger to life, but also, when the operation is extensive, by danger of subsequent deformity of the penis, rendering erections imperfect or painful and interfering with the expulsive power of the urethra, so that micturitition is followed by dribbling of urine. Many of the "strictures of large calibre" which have been reported as cured by internal urethrotomy alone were undoubtedly mere normal contractions of the urethra. Cutting should be reserved for those strictures to which dilatation is not applicable.

Meatotomy.-Strictures at the meatus or just within it do not yield to dilatation, which in this part of the urethra is painful and irritating. Such strictures should be cut. Congenital narrowing of the meatus does not call for operation unless interfering with the normal functions of the urethra and productive of symptoms, or 
unless it is necessary to introduce large instruments for treatment of the deeper portions of the urethra. Meatotomy may be performed with a straight bistoury or with a probe-pointed tenotome with a convex edge. The incisions should be made slowly and carefully, upon the floor and in the median line, until the tissue forming the stricture has been divided completely. In case of congenital narrowing the opening, in order to allow for some contraction, should be made slightly wider than it is ex. pected to remain. When hemorrhage occurs, it can be controlled by pressure, and, if necessary, the glans may be compressed continuously by wrapping around it several times a narrow strip of rubber plaster. The plaster will have to be removed with each act of micturition, but the patient can easily re-apply it. Beginning the second day after the operation, a full-sized sound should be inserted once a day until healing is complete ; reunion of cut surfaces is thus prevented. Treatment of the deeper portions should, if possible, be suspended during this period. Meatotomy rarely necessitates confining the patient to bed.

Internal Urethrotomy.-This operation should be limited to strictures within four inches of the meatus. For strictures of the bulbous and membranous portions external urethrotomy or Harrison's combined internal and external operation is much safer than the internal operation. No surgeon should perform internal urethrotomy of the deep urethra unless he is prepared to follow it, if necessary, with the external operation.

When possible, all cutting operations upon the urethra should be preceded for two or three days by the internal administration of boric acid in from 10- to 20grain doses four times a day. Such a course sterilizes 
the urine and greatly lessens the danger of urethral fever and other complications. The operation should be immediately preceded by irrigation of the urethra with a saturated solution of boric acid or a I : I0,000 solution of bichloride of mercury. In case of cystitis the bladder also should be irrigated. All instruments used should be absolutely clean and sterile. For several days previous to the operation instrumentation of the urethra should be avoided, to the end that urethral irritation may be reduced to a minimum. Strictures within two inches of the meatus, requiring but slight cutting, may be operated upon without confining the patient to bed, but for deeper seated strictures and for those requiring extensive cutting the patient should be prepared carefully as for any other surgical operation, and should remain in bed for two or three days after its performance.

For internal urethrotomy but three of the many instruments recommended for the purpose need be mentioned. In the pendulous urethra all incisions should be above, in the roof, and in the median line. If the stricture will admit a No. 5 (French) bougie, a Civiale urethrotome, or Gross's modification of the instrument ( Fig. 4I), which

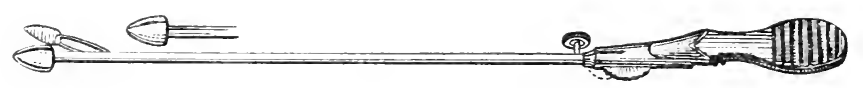

FIG. 4I.-Gross's modification of Civiale's urethrotome.

has an acorn-shaped head, is the simplest instrument. The bulb is passed through the stricture, the blade is exposed by a mechanism in the handle, and the instrument is withdrawn sufficiently to cut through the stricture from behind forward. The blade is then sheathed and the instrument is withcirawn from the urethra. A steel sound should then be passed through the cut stricture, 
but not to the bladder, in order to ascertain if the stricture be divided completely. If the division is not complete, the urethrotome may again be introduced and the stricture be divided thoroughly. If the cutting has been at all extensive, it is often best to tie a full-sized soft catheter in the urethra for the first twenty-four or forty-eight hours. Hemorrhage, which is not often severe, may be controlled by winding a strip of rubber plaster about the glans, as recommended after meatotomy. Twentyfour or forty-eight hours after the operation a full-sized steel sound should be passed through the stricture. This procedure is repeated daily for three or four days, and then a few times at intervals of three or four days. To render the result permanent, it is usually necessary to continue the occasional use of the sound in the same manner as after treatment by dilatation.

When strictures of a calibre larger than No. is or 20 require cutting, the Otis urethrotome (Fig. 42) is prob-

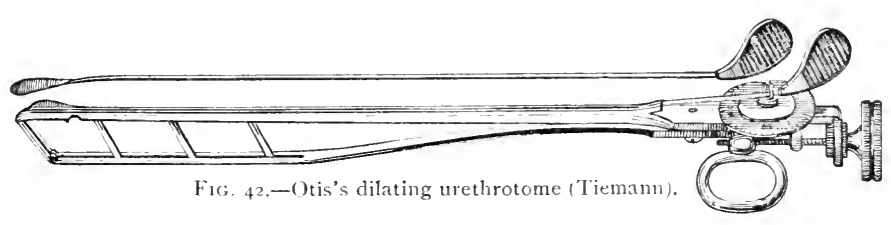

ably the best instrument in use. The closed instrument is introduced into the urethra until the point occupied by the knife is half an inch behind the stricture. By means of a screw in the handle the parallel blades are separated until the stricture is put fully on the stretch; the blade is then withdrawn, cutting the stricture from behind forward. The cutting should be followed by the use of sounds as above described.

The Maisonneuve urethrotome, formerly used for in- 
ternal urethrotomy in the deep urethra, is probably the best instrument for this purpose, since it can be attached to a filiform guide and thus be conducted through a stricture; but this operation is one to be avoided if possible, unless it be followed by perineal puncture and drainage as recomnended by Harrison in his combined operation. Some of the modifications are superior to the original instrument in having protecting

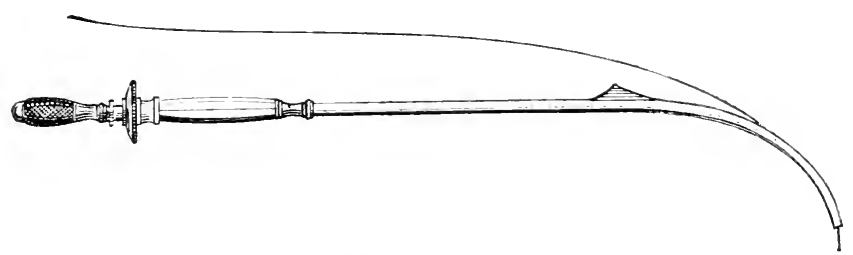

FIG. 4j.-Teevan's modification of Maisonneuve's urethrotome with guide (Tiemann).

sheaths for the knife, which can thus be made to cut the stricture-tissue without damaging the rest of the urethra, and in being fitted with a wire stylet, the removal of which allows the escape of urine if the instrument has properly entered the bladder, the danger of cutting a false passage being thus obviated (Fig. 43). The instrument is of occasional service in the anterior urethra, since, by means of its guide, it can be inserted into a stricture too narrow to admit the smallest Civiale urethrotome, and the stricture may then be divided partially from before backward. For this purpose the knife should run in a groove in the upper surface, in order to make the incision in the roof of the urethra.

The indications for internal urethrotomy have already been considered. This operation should be performed when the stricture is situated within four inches of the meatus and cannot be properly treated by dilatation. 
Extcrnal urethrotomy" (cxternal perincal urethrotomy, perincal siction) becomes necessary when stricture of the bulbous or membranous urethra is not amenable to treatment by dilatation, or when complications necessitate the immediate division of stricture. The operation should always be preceded by an attempt, while the patient is under ether, to pass a full-sized steel sound, in order that the possible mistake of cutting a mere spasmodic stricture may be avoided. If a filiform bougie can be passed, it is used as a guide in what is known as "Syme's operation " or in the combined method of Harrison; but when a filiform bougie cannot be made to enter the bladder, the much more difficult procedure of external perineal urethrotomy without a guide (generally known as "Wheelhouse's operation") must be performed.

These operations, fully described in all text-books on surgery, and therefore requiring no description in these pages, should be undertaken by none but skilled surgeons.

Divulsion of stricture in the deep urethra is practised by some surgeons when the patient will not consent to a urethrotomy, but it is a dangerous procedure. Rarely the method may be of advantage in the pendulous urethra, for the purpose of dilating a tight stricture sufficiently to admit a Civiale urethrotome. For this purpose a Thompson rapid dilator should be used, since it can be threaded on a filiform guide.

Other methods have been recommended and employed at various times for the treatment of stricture, but they are inferior to those described.

Complications of Stricture.-Retention.--When retention occurs suddenly, it is largely due to an added inflammation or irritation of the urethra, and can usually 


\section{SYPHILIS AND THE VENEREAL DISEASES.}

be relieved promptly by the use of the hot bath or by the soft catheter in the manner described for the relief of retention in gonorrhcea. If the patient is seen before the bladder is much distended, an opiate every hour may overcome spasm and give relief. A filiform bougie can often be passed when attempts with a catheter fail; if the bougie be left in the stricture for a few hours, the

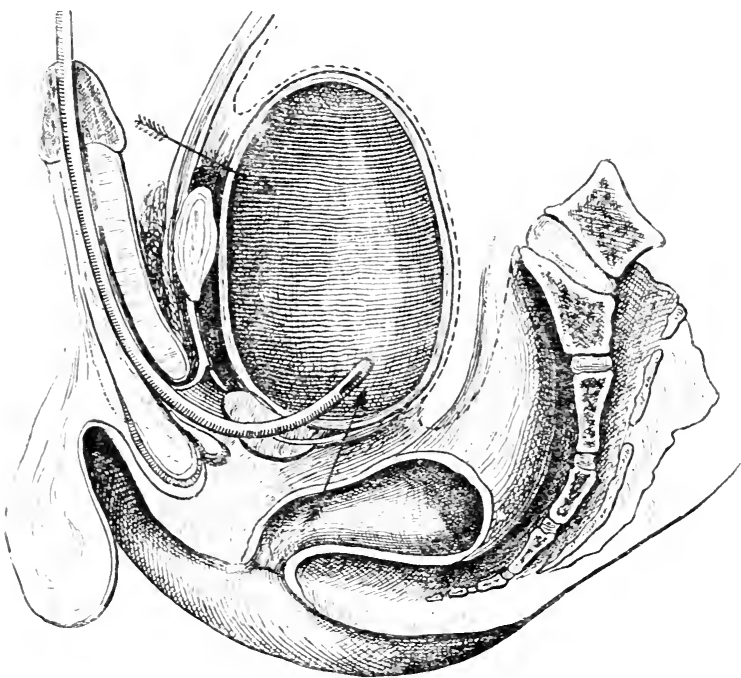

FIG. 44.-Suprapubic and rectal routes for the relief of retention of urine (redrawn from Holden).

stricture will dilate and allow the urine to escape. When other measures fail, an aspirator may be used. Puncture of the bladder with a trocar through the rectum is permissible in case an aspirator cannot be obtained (Fig. 44).

In old cases of tight stricture with a history of repeated attacks of retention or of gradually increasing 
difficulty in emptying the bladder, and when the stream of urine is very small at best, retention is a more serious matter. In these cases the hot bath, an opiate, and even an anæsthetic, may be tried, but these measures are frequently ineffective. If possible, a filiform bougie should be passed through the stricture, which may then be treated by continuous dilatation or by external urethrotomy. If a bougie cannot be passed, it is proper to relieve the bladder by aspiration for several days, if by so doing the instrument can eventually be made to enter the bladder. In case all such attempts fail, external urethrotomy without a guide becomes necessary. To determine the best method of proceeding the surgeon should consider carefully the character of the stricture, the experiences of the patient during previous attacks of retention, and all other conditions peculiar to the case.

Falsc Passages. - When false passages exist in connection with stricture, they should be recognized, located, and avoided during treatment. Such passages are usually small and serve to entrap the ends of fine instruments. If the directions already given for instrumentation of the urethra, and especially those for the use of filiform bougies, be followed carefully, danger of dilating a false passage will be avoided. The walls of old false passages that have been kept open sometimes undergo changes similar to those of stricture, and therefore will grip an instrument after the manner of a stricture. The diagnosis in these cases is difficult, and must be made with great care.

False passages are produced most frequently by the use of small steel instruments, usually during an attempt to pass the instrument from the bulbous to the mem- 
branous urethra. The urethral walls in front of a simple stricture and in the course of a tortuous stricture are often so thinned and softened that they may easily be penetrated by a small steel sound, especially if force be used. When a surgeon has been unfortunate enough to make a false passage, he should recognize the fact, or he may continue and dilate the opening instead of the stricture. The perforation of the urethral wall by the point of an instrument gives the hand of the operator a sensation very different from that produced when the mstrument passes through a strictured point in the urethra. The instrument is obstructed in its movements, but is not gripped as when it has entered a stricture. The direction of the handle shows that the point is not in the median line, and if the handle be depressed, it cannot be rotated as when the point is in the bladder. The finger on the perineum or in the rectum will probably distinguish the point of the instrument. On withdrawing the latter there is usually considerable hemorrhage.

The treatment of a recent false passage consists of rest, boric acid internally, hygiene, and the avoidance, if possible, of all instrumentation of the urethra for two or three weeks. The damaged tissue usually becomes inflamed, causing a discharge of blood and pus for a few days, but under favorable circumstances the wound heals in two or three weeks. Urethral fever, abscess, fistula, or even extravasation, may result.

Urethral Ficer (Urinary Fo're). - This much-dreaded complication of stricture usually finds its exciting cause in instrumentation of the urethra. In some instances the urethral fever may be due chiefly to shock or to reflex influences, but in most cases it is undoubtedly the 
result of septic infection. The urethra back of a stricture usually contains micro-organisms and their toxines capable of rapid absorption if the mucous membrane be even slightly cut, torn, or abraded, though there are evidences of such absorption in but a small minority of operations on stricture. Those cases in which nausea, syncope, or a chill occurs immediately after the insertion of an instrument into the urethra are undoubtedly due to nervous influence, and may result from the skilful passage of a smooth sound which has produced no damage to the mucous membrane.

Some patients are peculiarly susceptible to chills and fever, which in a few individuals follow every attempt at urethral instrumentation. This susceptibility may suddenly develop during the treatment of a stricture, or it may as suddenly disappear. Occasionally this complication is one of the symptoms of a stricture, and clisappears when the latter is properly treated. It occurs rarely after operations on the meatus, but it increases in frequency with the depth of the injury in the urethra, being most frequent after divulsion or internal urethrotomy of the deep urethra. In cases of old stricture, especially if complicated by bladder or kidney clisease, the danger of a fatal termination is greatly increased.

The symptoms usually appear within twenty-four hours after instrumentation, frequently following the first urination. In typical cases there is a sharp chill, lasting from a few seconds to several hours, followed by fever of irregular duration, ranging from $100^{\circ}$ to $106^{\circ} \mathrm{F}$., and terminating in more or less profuse perspiration. The patient may be well in twenty-four hours, or a feeling of lassitude and malaise may remain for a few days. In very mild cases slight chills may be the only symp- 
toms noticed by the patient. In severe cases the chill is sudden and violent and is attended by great prostration. The skin is cold and livid, and there may be vomiting and profuse diarrhœea. Suppression of urine, uremia, and death may occur within twenty-four or forty-eight hours. In yet other cases slight chills and mild fever may be followed by all the symptoms of septicæmia or of pyæmia, with a fatal termination.

The first chill may be followed by others without further exciting cause, and the fever may continue in an intermittent or remittent form. In these cases the symptoms do not conform in type and character to the first attack, but vary greatly. Finally, the fever may become chronic, and may simulate malaria except that the symptoms are more irregular and the disturbance of digestion and the impairment of nutrition are more marked. The persistent forms usually occur in connection with disease of the bladder and the kidneys.

The treatment is chiefly prophylactic. The directions already given for urethral instrumentation, including antiseptic precautions and urethral hygiene, should be followed carefully. Of special value in this respect is the use of boric acid in doses of from 10 to 20 grains four times a day, its administration being begun forty-eight hours before operating and being continued for several days. With some patients a chill may be prevented by a prolonged milk diet or by the use of morphine and pilocarpine just before operating. There is no specific treatment for urethral fever after its development. The patient should be put to bed, and free perspiration should be encouraged by the use of blankets, hot-water bottles, hot drinks, and in some cases by the administration of jaborandi. Further treatment is purely symptomatic. 
Quinine and other allied preparations are of little, if any, benefit.

Fistula.-If fistulæ are small, they frequently close when the stricture is dilated. If they are larger and remain open, they should be treated on surgical principles.

Alscess has been considered in connection with Periuretluitis. When complicating stricture of the deep urethra, external perineal urethrotomy is usually the best treatment.

Extrazasation of urine, if at all extensive, calls for prompt surgical treatment to secure free drainage and to prevent abscess, gangrene, and extensive sloughing of tissue. When the quantity of extravasated urine is slight, involving a small circumscribed region, is not enlarging, and is not interfering with micturition, incisions are not necessary. In such cases the treatment is directed mainly to the patient and to the stricture. Absorption of the extravasated fluid may be encouraged by rest and by the application of hot fomentations. 


\section{GONORRHCEA IN WOMEN.}

GoNorrheed in women has not been studied so long or so carefully as has the same disease in men. There is great diversity of opinion with reference to the frequency of its occurrence, its relation to other forms of inflammation of the organs involved, and the site of inoculation. Bumm and some other observers believe that gonococci never penetrate the vaginal epithelium, and that when found in a vaginal discharge they come from the cervix or body of the uterus. Other observers find that a vaginitis frequently is the first evidence of gonorrhœal infection. It is certain that in the acute gonorrhœa of girls and young women vaginitis is usually the most prominent symptom. In older women, and especially in those who have borne children, the ragina is less easily inflamed, and the process is most marked in the endometrium of the neck and body of the uterus or in the urethra. In children infected as the result of criminal violence or by contaminated towels or other media, vulvitis or vulvo-vaginitis most commonly results. Practically there is little difference whether the site of infection be the vulva, the urethra, the vagina, or the uterine neck, since in the great majority of acute cases, excepting those of vulvitis in young children, the inflammation extends eventually to all of these regions, and also to the uterus, the Fallopian tubes, the ovaries, and the peritoneum. In the chronic forms the disease is most fre- 
quent in the vaginal portion of the neck of the uterus, in the pelvic organs, in the urethra, and in the glands of Bartholin.

In acute gonorrhœa of women the etiology, the modes of infection, the period of incubation, the development of symptoms, and the pathological changes are similar to those belonging to the disease in men, though the symptoms (except those of pelvic inflammation) are usually less severe and of shorter duration.

The disease, however, has a yet greater tendency than in men to become chronic. The extent of surface involved and the inaccessibility of portions of it favor the continuation of the process. Furthermore, Noeggerath and others have demonstrated that many cases of chronic gonorrhceal inflammation of the pelvic organs in women develop insidiously, and are never preceded by the acute form of the disease. Such cases are found frequently in young married women whose husbands, though supposing themselves sound, had never fully recovered from an old gonorrhoea or a chronic gleet. A large percentage of the subacute and chronic pelvic disorders for which women consult the gynecologist originate in gonorrhœal infection. Another important feature of the disorder in women is its tendency to remain latent for long periods during which no evidences of the disease are apparent even on careful examination. Such a latent and unsuspected gonorrhoea may be aroused to activity by slight causes, and may prove a source of infection.

Diagnosis.-The symptoms of acute gonorrhœa in women are those of vaginitis, urethritis, vulvitis, Bartholinitis, and endometritis resulting from other causes. Frequently salpingitis, ovaritis, and peritonitis are also present. Full descriptions of these disorders are found in 
the text-books on gynecology. Brief consideration is here given merely to the main points in a differential diagnosis between the gonorrhoeal and the non-gonorrhoeal forms of inflammation.

General Charactiristics.-In general, gonorrhceal inflammation is more severe in type than other forms; it usually begins with slight symptoms, which rapidly increase in intensity for a few days, remain stationary for about a week, and then decline; it rarely remains limited to any one organ, but usually extends to several ; it shows a decided tendency to persist and become chronic; there is often a history of exposure to infection, followed by a period of incubation of from three to five or more days ; and the discharges show the presence of gonococci.

Vaginitis.-This condition is present in most acute cases of gonorrhœe, though whether any single attack be a true gonorrhœal infection or merely a severe catarrhal inflammation induced by irritating discharges from the uterine neck or from the urethra it is difficult to decide. Microscopical examination of the vaginal secretion is unsatisfactory, since the vagina contains many micro-organisms, including diplococci, which often cannot be differentiated from gonococci. The diagnosis must be based chiefly upon the presence or absence of the general characteristics of gonorrhoal inflammation, including the involvement of other organs. This form of vaginitis, which usually lasts for three or four weeks, shows a marked tendency to relapse with succeeding menstruations and other sources of local irritation, and to persist either as diffuse chronic vaginitis or in localized patches of congested, swollen, and eroded mucous membrane.

Urethritis.-This condition is probably present in the 
majority of cases of gonorrhoea, and, as it does not often occur from other causes, except those which are traumatic, its demonstration furnishes fairly good evidence of gonorrhoal infection. Finger thinks urethritis is present in practically all cases of recent infection, but many observers find it much less frequent. It is usually a mild affection, and, while it is not infrequently followed by cystitis, it rarely results in disease of the kidneys, as in men. The subjective symptoms may be so slight as to pass unnoticed, or there may be decided burning and smarting of the sensitive swollen membrane, with frequent and painful micturition, but the inflammation is very rarely so intense as in gonorrhoea in men.

The orifice of the urethra is red and swollen, and the congested mucous membrane may protrude. With a finger in the vagina the urethra is felt as a firm, tender cord, and if the patient has not urinated for several hours pus may be squeezed out of the urethra. If such pus, unmixed with secretions from the vagina or the vulva, contains gonococci, the diagnosis is unmistakable. The acute symptoms rarely last for more than two or three weeks, but they are frequently followed by a chronic urethritis which is often overlooked.

Chronic urethritis in women presents no subjective sensations, and is recognized only by careful examination. If the orifice be cleaned carefully and pressure be made upon the urethra from behind forward when the patient has not urinated for several hours, there can usually be expressed a drop of muco-pus containing gonococci. The endoscope may often be used to advantage. The many follicles of the urethra may be involved, thus favoring the continuation of the disease. Five or six large follicles near the orifice are of especial importance, and 
should be examined carefully, as the inflammation may be limited to them. Chronic urethritis in women is more easily cured than in men, but is quite commonly unrecognized and untreated.

I'ulizitis.-In adults vulvitis has not yet been demonstrated to be gonorrhceal in character. The inflammation simply results from contact of the surfaces with irritating discharges from the vagina and the urethra. This condition in women corresponds with balanitis in men. In children, however, gonorrhœal inflammation of the vulva has been demonstrated, though these cases have no characteristic features that will serve to distinguish them from vulvitis due to other causes.

Bartholinitis. - Inflammation of the vulvo-vaginal glands finds its most frequent cause in gonorrhœa, and when due to such infection usually runs a rapid course and terminates in suppuration. An acute infection is rarely, if ever, limited to the gland.

Chronic inflammation of these glands not infrequently complicates chronic gonorrhcea, and may survive as the sole relic of the original disease. The affected gland is usually recognized as a firm, painless nodule; its duct is dilated and reddened. Pressure on the gland usually causes the escape of a mucous or muco-purulent discharge which may contain gonococci and may prove highly infectious.

Inflammation of the Uterus and its Appendages.-Endometritis of the uterine neck occurs in most cases of acute gonorrhœa, and in a large percentage of cases the disease extends to the tubes, the ovaries, and the peritoneum. The origin of the inflammation in these organs cannot be determined by the symptoms alone, but is recognized by the presence of other evidences of gonorrhea and by 
the history. That the gonococcus is an active factor in the production of these pelvic inflammations is apparent from the fact that this micro-organism has been found in the pus of pyosalpinx and in the epithelium and connective tissue of the Fallopian tubes.

In chronic gonorrhœa the inflammation almost always involves the pelvic organs, and is one of the most frequent causes of sterility and of chronic invalidism in women.

The frequency with which a chronic gonorrhœal inflammation may remain latent and confined to one or more follicles of the urethra, to one of the vulvo-vaginal glands, or to the cervix or body of the uterus readily explains why a man may be infected from coitus with a woman who shows no signs of the disease, and also why he may have intercourse with her many times before coming in contact with the gonorrhœal virus.

Treatment.-Hygienic treatment, which is always of great importance, is practically that of gonorrhoea in men. Rest, a light diet, diluent drinks, and frequent washing of the external genitals should be secured. If urethritis is present, and micturition is painful, alkalies and the balsams should be given. All discharges should be caught on pads of cotton held in position by a bandage, and these pads should be burned when soiled. At first, if the vaginitis be severe, local treatment must be postponed until the parts are less sensitive. In the mean time rest and the application of cold or heat, as is most grateful, to the perineal and pubic regions is of value. As soon as the patient can tolerate it, the vagina should be irrigated thoroughly twice daily with hot solutions of boric acid. The external parts should be kept clean, dusted with a simple powder, and covered with thin 
layers of cotton or lint to prevent contact of surfaces. These measures will obviate the danger of vulvitis and will add to the patient's comfort. If vulvitis occur, slightly astringent lotions or powders may be used in addition.

As the inflammation subsides it is sometimes well to irrigate with a solution containing I per cent. of nitrate of silver or I to 2 per cent. of permanganate of potassium instead of boric acid; or somewhat stronger solutions may be applied with a brush or a cotton swab. The cervical canal should be kept clean and should receive a daily application of a solution of nitrate of silver (3ss-3j ad $\mathrm{f} \tilde{\mathrm{j}} \mathrm{j})$. Following these astringent applications the vagina may be tamponed with cotton soaked in iodoform glycerin, borated glycerin, or glycerite of tannin.

If the urethritis tend to become chronic, injections such as those recommended for use in subacute gonorrhœa in men may be used, the bladder always being moderately full. Later, solutions of nitrate of silver in gradually increasing strength may be applied through an endoscopic tube. If the follicles are involved, they should be destroyed with the fine point of a Paquelin cautery, or with caustic or acid.

Acute Bartholinitis should be treated by rest and by hot local applications. If suppuration occur, the abscess should be opened and treated on surgical principles. In chronic Bartholinitis the gland should be enucleated or be destroyed by the cautery.

The treatment of gonorrhœal inflammation of the uterus and its appendages should be left to the skilled gynecologist. 


\section{N D E X.}

AbSENCE of subjective sensations in syphilis, 76

Accidents and injuries in syphilis, $7 \mathbf{I}$

"Acclimatization," $37 \mathbf{I}$

Acquired syphilis, 27 infantile, $28_{3}$

Adenitis, gonorrhneal, 460

Adenopathy of syphilis, 32

syphilitic, primary, $3 \mathbf{I} 9$

Alcohol in syphilis, 227

Alopecia, syphilitic, treatment, 265

with structural changes, 134

without obvious structural change, I 33

syphilitica, diagnosis, I 35

Ano-rectal syphiloma, 177

Antiquity of venereal diseases, 20

Anus in hereditary syphilis, 223 syphilis of, 175

Aponeuroses, syphilis of, 167

Ardor urinæ, 376, 406

Arterio-sclerosis, 170

Atrophic tubercular syphiloderm, I 3

Atrophy of optic nerve, 202

“BAD DISOR DER," 27

Balanitis, 46, 33 I

diagnosis, 321,332

etiology, 33 I

symptoms, $33 \mathrm{I}$

treatment, 333

Balano-posthitis, 33I

Bartholinitis, gonorrhœal, 604

Bastard gonorrhcea, $372,3^{8} \mathrm{I}$ diagnostic table, 387

Bathing, 228

Benignant syphilis, 6o, 6I

Bestiality, $35^{6}$

Blennorrhagia, 360
Blennorrhagie, 360

Blennorrhœa, 360

Blood in syphilis, $2 \mathrm{~S}$

Blood-vessels, syphilis of, 170

"Blue-ball," 309

Bones in hereditary syphilis, 216, 223

in syphilis, treatment, 270

syphilis of, 159

Boubon, 309

Bougie à boule, 563

Bougies, bulbous, 563

flexible, 564

soft, 564

Brain, syphilis of, $\mathbf{1} 88$

Bronchi in hereditary syphilis, 22 I syphilis of, I 58

Bubo, etiology, 3 I 2 gonorrhœal, 460 of chancre, diagnosis, $3 \mathbf{1 9}$ of chancroid, 309 syphilitic, 32 treatment, 56

Bulbous bougies, 563

Bursæ, syphilis of, I 66

CACHexia, syphilitic, 70

Calcareous lesions of hereditary syphilis, 2 I I

Calibre of the urethra, 577

Care of instruments, 570

Catarrh of middle ear, 204

Catheterism of the urethra, $55^{6}$

Catheters, silver, 564 soft, 565

Central recurrent retinitis, 201

Cerebro-spinal syphilss, 192 -

Chancre, 30 deeply ulcerating, 36 diagnosis, 45,316 
Chancre duration of, 43 extra-genital, 40 hard, 30 Hunterian, 36 infecting, 30 in women, 39 location, 39

" mixed," 36, 305 pathological anatomy, 51 soft, 37 superficially ulcerating, 36 termination of, 43

treatment, $5 \mathbf{I}$

urethral, 39

vaginal, 40

Chancre mou, 293

Chancre syphilitique, 30

Chancrelle, 36, 293

Chancres, induration of, $+\mathbf{I}$

number, $4 \mathrm{I}$

of the syphilized, 38

portent of, 43

within the nares, 153

Chancroid, 36, 45, 293

adenopathy, 309

and paraphimosis, 306

and phagedena, 307

and phimosis, 306

auto-inoculability of secretion, 303 complications, 305

diagnosis, 314,3 I 6

duration, 300

erosive lesion, $29 \mathrm{~S}$

etiology, 294

extra-genital, 305

gangrene, 309

incubation, 301

induration, absent, 302

lesions, 297

location, 304

lymphadenitis, 309

lymphangitis, 309

number of, 299

pustular lesion, 297

size, 300

subjective sensations, 302

treatment, 322

variations, 299

with vegetations, 305

Chancroidal bubo, 309

diagnosis, 3 I
Chancroidal bubo, etiology, 312 treatment, $32 \mathrm{~S}$

Chaudifisse, 360

Chemical causes of urethritis, 370

Chordee, 377

treatment, 407

Chorio-retinitis, 200

Cicatrices from gummata, 124

Ciliary body, syphilis of, 199

Clap, 360

Classification of syphilodermata, $\delta \mathbf{I}$

Cold sound in urethritis, 5 I 8

Colles's law, 206, 207

Color in syphilodermata, 75

Color-test for shreds in urine, 497

Complications of urethritis, 417

Condensing osteitis, 162

Condyloma, 47, IOI

Condylumata lata, Ior

Congenital syphilis, 205

Conjunctiva, syphilis of, 196

Conjunctivitis, gonorrhceal, 470

diagnosis, 473

etiology, 47 I

pathology, 473

prognosis, 479

symptoms, $47 \mathrm{I}$

treatment, 474

Continuous immersion in chancroids, 326

Copaiba-rash, $40 \mathrm{I}$

"Copper color," 75

Cord, syphilis of, 191

Cornea, syphilis of, 196

Corneous syphiloderm, 99

Corona veneris, 9 I

Corymbiform syphiloderm, 94

Cowperitis, 459

Cranial bones in hereditary syphilis, 223

meninges, syphilis of, 188

Crystalline lens, syphilis of, 200

Curve of the urethra. 554

Cystitis, gonorrhoeal, 450

causes, 450

diagnosis, 452

symptoms, $45 \mathrm{I}$

treatment, 454

DACTYLITIS, syphilitic, 163

hereditary, 2 I 8 
“ Defective memory," 349

Délire des grandeurs, 193

Dementia paralytica, 192, 193

Diagnosis of chancre, 3 I 6 of chancroid, $3 \mathbf{1} 6$

Iisinclination for society, 349

Dreams, 349

"Dry " tubercular syphiloderm, I I 3

Ducrey, micro-organism of, in chancroid, 313

EAR in hereditary syphilis, 226 in syphilis, treatment, 276 syphilis of, 203

Eburnation, 162

Eczema of genital region, diagnosis, 321

Einfacher Schanker, 293

Endarteritis obliterans, 170

Endoscope in urethritis, 499

Endoscopic urethral instruments and methods, 500

Epididymis, syphilis of, ISI

Epididymitis, acute, 422

symptoms, 427

treatment, 436

chronic, symptoms, 427

treatment, 436

diagnosis, 428

etiology, 422

frequency, 422

prognosis, 436

relapses, 426

strapping the testicle in, 433

symptoms, 423

syphilitic, treatment, 273

treatment, 428

Epithelioma, genital, 48

of genital region, 32 I

Erosion, 33

superficial, 33

Erotomania, 356

Erythematous syphiloderm, 85

Esthiomène, 176, 308

of Huguier, I $S_{4}$

Evolution of syphilis after chancre, 66

of syphilodermata, 77

Excursions of syphilis, 57

Extravasation of urine due to stricture, 550, 599
Eye in hereditary syphilis, 225

in syphilis, treatment, 275

syphilis of, 194

Eyelids, syphilis of, 195

FAILURE of erection, 353

Family, the, and syphilis, 284

Fever, syphilitic, 68

urethral, 562

Fibrosis of kidney, I 86

Filiform bougies, 565

introduction of, 568

Finger, syphilitic, 163

Fistula, 599

of the urethra due to stricture, 550

Flexible and steel instruments com.

$$
\text { bougies, } 564
$$

Formative osteitis, I6 I

Fractures in bone-syphilis, heredi-

$$
\text { tary, } 217
$$

Frequency of micturition in stricture, 544

Fumigation with mercury, 243

Galloping syphilis, 59

Gangrene and chancroid, 309

Gastro-intestinal tract, syphilis of, 173

Gauge, Handerson's, 563

General considerations, etc., relative to syphilodermata, 79

Genital organs in hereditary syphilis, 215

Genito-urinary organs, syphilis of, I $\mathbf{I}$

in men, $\mathbf{I} \mathbf{r}$

in women, $1 \delta_{3}$

Glands, lymphatic, 69

Gleet, $360,48_{3}$

due to stricture, 546

Glossite tonsurante, 147

Gomme sirofuleuse, 125

Gonococcus, 362

characters, 365

culture of, 366

etiological factor in gonorrhea, cause of, 362

in chronic urethritis, 486, 489, $49 \mathrm{I}$ preparation of specimen, 362,388 value in diagnosis, 366,388

Gonorrhée, 360 
Gonorroë, 360

Gonorrhcea, acute, 360

bastard, $372,3 \mathrm{~S}_{1}, 387$

cause of pelvic inflammation in women, 492

chordee in, 377

chronic, $4 \mathrm{~S}_{3}$

complications, 4I 7

adenitis, 460

conjunctivitis, 470

Cowperitis, 459

cystitis, $45^{\circ}$

epididymitis, 422

folliculitis, 456

infection of mouth and rectum, $4 S \mathrm{I}$

lymphangitis, 460

ophthalmia neonatorum, 479

periurethritis, 457

posterior urethritis, 417

prostatitis, 437

pyelitis, 454

rheumatism, $46 \mathbf{1}$

vesiculitis, 448

definition, 360

diagnosis, $3 S_{3}$

differential, 385

examination of patient, $3 S_{3}$

diagnostic table, 387

diagnostic value of gonococcus, 366

differential diagnosis, 385

epididymitis from, 422

examination of discharge, 388

folliculitis in, 456

in women, 600

diagnosis, 60 I

treatment, 605

unrecognized, 605

lymphangitis in, 375

microscopical examination of discharge, $38 \mathrm{~S}$

most venereal of diseases, $36 \mathrm{I}$

pathology, $3 \mathrm{~S}_{\mathbf{I}}$

prognosis, 414

relapse, 379

sexual hygiene, 397

symptoms, constitutional, $37 \mathrm{~S}$

increasing stage, 375

prodromal stage, 375

stage of decline, 378

stage of incubation, 374
Gonorrhea, symptoms, stationary stage, $37 \mathrm{~S}$

variations in, 379

table of differential diagnosis, 387

treatment, 3 S9

abortive, 39 I

by stages, 405

dressing for penis, 398

general conditions, 390

hygiene, 395

beverages, 396

diet, 396

dressings, 397

rest, 396

sexual hygiene, 397

tobacco, 397

injections, 409

in early stages, 393

intermal, 400

irrigation in early stages, 392

of chordee, 407

of retention of urine, 407

of sexual irritation, 407

prophylaxis, 389

suspensory bandagres, 399

unrecognized, 373

Gonorrheal conjunctivitis, 470

rheumatic ophthalmia, 467,474

rheumatism, 461

diagnosis, 468

etiology, 46 I

of burse, 466

of symovial sheaths, 465

ophthalmic symptons, 466

pathology, 467

progmosis, 470

symptoms, 462

treatment, 469

varieties, 463

Gram's method of staining the gonococcus, 364

"Ground-glass" comea, 226

Gumma, I 2 I

Gummata of mouth, 149

of rectum, 177

treatment, 272

Gummatous fibrosis of lung, 172 iritis, 198

syphiloderm, I 2 I

diagnosis, 125

pathology, 127 
HAIr, syphilitic affections of, 133

Hairs in hereditary syphilis, 215

Handerson's gange, $56_{3}$

Hard chancre, 30

Hartes Geschioiur, 30

Heart, aneurysm of, 169

syphilis of, I 68

Herpes progenitalis, 46, 344 diagnosis, 320, 345

symptoms, 344

treatment, 346

History of venereal diseases, 20

Hunterian chancre, 36

Husband, infected, $2 \mathrm{~S}_{5}$

Hutchinson's teeth, 2 I9

Hydrargyrism, 250

Hygiene of syphilis, 227

of urethra, 395

Hyperostoses of tibia, $2 \mathbf{I} 7$

Hypochondriasis, 347

treatment, $35 \mathrm{~s}$

IIypodermatic injection of mercury, 245

Imaunity against syphilitic infection, 30

“Impotence," 349, 355

Incubation of chancre, first, $3 \mathbf{I}$ second, 69 of chancroid, 301

Indurated papule, 35

Infantile syphilis, acquired, $2 \mathrm{~S}_{3}$

Infecting chancre, 30

Infection of husband, 285

of physicians, 19

of wife, 285

Infectious wrethritis, 360

Infiltration of urine, 550

Inherited syphilis, 205

Initial lesion, 30

sclerosis, 30 diagnosis, $3 \mathbf{I} 6$

Injection of chancroidal buboes, 329 of deep urethra, 519

of mercury, hypodermatic, 245

Injections in gonorrhea, 409

in urethritis, 5 I I

Injuries and accidents in syphilis, 7 I

Insoluble salts of mercury for injection, 249

Instrumentation of the urethra, 553
Instrumentation of the urethra, indications for, 572

urethral spasm in, 529

with flexible instruments, 567

Instruments, care of, 570

list of those neederl, 57 I

urethral, 562

Insurance of the infecterl, 289

Internal urethrotomy, 5 S9

Interstitial keratitis, I 96

Intestinal tract in hereditary syphilis, 222

Introduction, 17

Inunction of mercury, $23 \mathrm{~S}$

Iodides in hereditary syphilis, 279

Iodine and its compounds, 252 compounds, toxic effects, 257

Iris, syphilis of, 197

Iritis, gummatous, i 98

serous, igs

syphilitic, treatment, 275

Irrigation of urethra, $496,5=2$

Jornts, syphulis of, 165

Keratitis, interstitial, 196

Keyes-Ultzmann syringe, 5 I 9

Kichey, syphilis of, I 5

Kidneys in hereditary syphilis, 232

Kiefer's urethral irrigation nozzle, 497

Krankheit der Französen. 27

LABYRINTH in syphilis, 205

Labyrinthitis of hereditary syphilis, 226

I achrymal gland, syphilis of, 194

Languitt's, ISo, is

Larynx in hereditary syphilis, 221 syphilis of, 155

prognosis, I $5 \mathrm{~S}$

Lassar paste, $2+3$

Latent gonorrhea in women, 605

Legal sanction of prostitution, 290

Leptomeningitis, 224

Lesions of chancroid, 297

I.encokeratosis, lingual, 147

Leucoma bucce, I +7

Leucoplasia of the month, I47

diagnosis, 150

patholog: 152 
Lichen planus, genital, 49

Life-assurance societies and syphilis, 289

"I ightning" symptoms, 64

Lipoma, I 26

"Listerine," 267

Liver in liereditary syphilis, 222 syphilis of, 173

Loss of memory, 354

"Losses at stool," $35 \mathrm{I}$

"Lost manhood," 349, 354

Lues venerea, 27

Lung, gummatous fibrosis of, $\mathbf{1} 7 \mathbf{2}$

Lungs in hereditary syphilis, 22 I syphilis of, I 7 I

Lung-syphilis, diagnosis of, $\mathbf{I} 7 \mathbf{2}$

Lupoma, I 25

Lupus of the rulva, 176

Lustseuche, 27

L, mpharlenitis and chancroid, 309

Lym hangitis and chancroid, 309

in gow rrhoea, 378

Lymphatic gian $\mathrm{h}$ ? in hereditary syphils, ? I5

Macular syphilis of mouth, I +3

syphiloderm, anatomy, Sy diagnosis, 89

syphilodermata, 82

Malignant sypliilis, 62,63

Mammalia, sexual relations of, $3+7$

Marriage after syphilis, 287

Masochism, 356

Masturbation, 349

“McI)ade formula," 256

Neatotomy, 517,588

indications for, 576

results of, 576

Mechanical causes of urethritis, 369

Melanotic whitlow, I64

Nemlrana tympani, 204

Meninges, syphilis of, $\mathbf{I} 9 \mathbf{I}$

Nental states due to syphilis, $\mathbf{1 9 2}$

Nercier catheter, 565

Mercurial inunctions in hereditary syphilis, $28 \mathrm{O}$ pains, 250

Nercury ly fumigation, 243

l,y injection, 245

by inunction, $23 \mathrm{~S}$

in syphilis, 232
Nercury, toxic effects of, 250

Method of examining patients, 22

Miliary papules, 92 pustular syphiloderm, 105

Mixed chancre, $3^{6}, 3^{\circ} 5$

". Mixed treatment," 259

Modes of infection in syphilis, 29

Moist papule, IoO, I44 diagnosis, $\mathrm{IO}_{3}$

wart, 47 , IO I

Nolluscum epitheliale, 48

Monti's formula, 279

Morbus gallicus, 27

Mouth, gummata of, I 49

leucoplasia of, $\mathbf{I}+7$

macular syphilis of, $\mathbf{I} 43$

smoker's patches of, 147

syphilis of, I 42

Mucous membranes in hereditary syphilis, 2 I 4

patches, IOO, I 45

plaques. 100

tubercle, It4

Multiformity in syphilis, 76

Multiple cerebro-spinal syphilis, I 92

IIuscie volitantes, 354

Muscles, syphilis of, 167

Nyositis, I67

progressive ossifying, I $6 \mathrm{~S}$

Narr, changes in, I 39

atrophic, I 39

hypertrophic, I 39

in tissues surrounding, 137

separation of, I 40

syphilis of, diagnosis, I4 I

syphilitic affections of, 136

Nails in hereditary syphilis, 215 syphilitic, treatment, 270

Nisal passages in hereditary syphilis, 219

syphilis of, 152

Naso-pharynx in syphilis, treatment, 2,6

Nervous syphilis, treatment, 273 system, syphilis of, 187

Night losses, 349

Nocturnal emissions, 349 pains, 160

Nudes, I6

Non-infecting chancre, 293 
Non-infectious urethritis, 369 etiology, 369

Nummular syphiloderm, 94

OCLAR appendages, syphilis of, I94 muscles, syphilis of, $2 \mathrm{O} 2$

Oculo-motor paralyses, I90

Oculo-motorius, paralysis of, I 90

(Fophagui in hereditary syphilis, 222

(Onychauxis, syphilitic, I 39

()nychia syphilitica, I39

()phthalnia, gonorrhaal rhemmatic, 467,474

neonatorum, 479

(Optic nerve, atrophy of, 202 syphilis of, 201

()rbit, syphilis of, 203

()rchitis, syphilitic, I $\$ 2$

() steitis, condensing, 162 formative, 162 rarefying, $16 \mathbf{I}$

() tis's urethrometer, 564

()zæna, 153

Psix in bone-syphilis, 160

Palmar syphiloderm, 96 diagnosis, 99

Panaris, 163

Pancreas, syphilis of, 175

Papillitis, 20I

Papular syphiloderm, diagnosis, 93 prognosis, 93

Papule chancre, 35

Papules, 90 dry, scaling, 35

dry, 92

lenticular, 93

miliary, 92

moist, 100

Papulo-squamous syphiloderm, 95

Paraphimosis, $33 \mathrm{~S}$

and chancroid, 306

reduction of, 340

symptoms, 338

treatment, 339

Paronychia, 136

syphilitica, I 37

Patches, mucous, roo

Pederasty, 356

"Pemphigun, syphilitic," 2 I I
I'erineal section, 593

Periodicity in seminal hosses, $35^{\circ}$

Periostitis, syphilitic, treatment. 27 I

Peripheral nerves, syphilis of, I93

Phagedena and chancroid. 307

Pharynx in hereslitary syphilis, 220 in syphilis, treatment, 260 syphilis of, $15+$

Phimosis, 334

and chancroid, 306

treatment, $32 \mathrm{~S}$

diagnosis, 336

symptoms, 335

treatment, 336

Physical examination of patients, 23

Physician, duty of, respecting syph. ilis in the family. 284

Physiology of generative organs, $3+7$

l'igmentary sy] hiloderm, 82

Placenta, syphilis of, 209

Plantar syphiloderm, 96

Plaque muquiuse, It

Plaques, mucous, roo

Plaques muqueuses, 100

Pnemmonia, syphilitic, 172

Pointed wart, IOz

Pollutions, $35 \mathrm{I}$

Polymorphism in syphilis, $7^{6}$

Posterior urethritis, 417

Pox, 27

Precautions required by physicians, 19

Precocious syphilis, 59

Pregnancy in syphilis, treatment, 277

Premature ejaculation of semen, 353

Primary syphilis, $5^{\&}$

Progressive ossifying myositis, I68

Proliferating syphilitic rectitis, I so

I'rostatitis, acute, 437

abscess in, 440

causes, 437

constitutional disturbances in, 439

liffuse, +38

follicular, $43^{S}$

parenchymatous, $43^{8}$

treatment, 44 I

chronic, $4+3$

follicular, $4+3$

parenchymatous, $4+4$

prognosis, $44 \mathrm{~S}$ 
Prostatitis. chronic, symptoms, 443 treatinent, +46

Prostatorrhoea, $4+3$

Prostitution and syphilis, 290 regulation of, by law, 290

Pseudo-gonorthora, 368

Pseudo paralysis of hereditary syphilis, 217

Psoriasis lingure, 147

of genital regions, 50

diagnosis. $32 \mathrm{I}$

syphilitic, 96

Purpuric syphiloderm, ss

Pustular syphiloderm,

\section{I I}

miliary, 105

pathology, I 12

syphilodermata, 104

Pustulo-crustaceous syphilodern, ro8

Pustulo-ulcerative syphiloderm, Io8

Pyelitis from gonorrhcea, 454

$$
\begin{aligned}
& \text { diagnosis, } 455 \\
& \text { smptoms, } 454 \\
& \text { treatment, } 455
\end{aligned}
$$

RADEZYGE, 27

Rarefring osteitis. I6I

Raw-ham color, 75

Rectal stricture, syphilitic, treatment, 272

Rectite proliférante suphilitique. I 80

Rectitis, proliferating, syphilitic, Iso

Rectun, gummata of, 177

in hereditary syphilis, 22.3

in syphilis, treatment. 272

syphilis of, 175

Relapses in gonorrhea, 379

Resolutive tubercular syphiloderm, I I 3

Respiratory tract, syphilis of, $\mathbf{1} 52$

Retention of urine due to stricture, 593

in stricture, 547

treatment. 407

Retina, syphilis of, 200

Retinitis, central recurrent, 20I

Rheumatism, gomorhceal, $46 \mathrm{I}$

Rhinitis, syphilitic, 153

Roseola syphilitica, 85

Rupia, 109
"SABRE-Blade DEForMITY" in he-

Sadism, 356 reditary syphilis, 2 I 6

Safeguard against syphilis, 292

Salivation, 250

Sapphism, 356

Satyriasis, 356

Scabies of genital region, diagnosi. 321

Scale for urethral instruments, 562 American, 562

English, 562

French, 562

Scaly patches of mouth, 147

Sihanker. 30

Sihleimfluss, 360

Sciatica, syphilitic, 193

Sclerotic, syphilis of, 197

Secondary syphilis, 58

Serpiginous syphiloderm, I2S diagnosis, I 30

Sexual debility, 349 indulgence after infection, 289 after syphilitic infection, 25 psychopathy, 356 weakness, 353

Sheath, tendimous, syphilis of, $\mathbf{I} 66$

Sifilide, 27

Sifilis, 27

Silver catheters, 564

simple chancre, 293

Situation of syphilodermata, 77

Skin in syphilis, 73

Smolier's patches, I.t7

"snuffles," 214

Society and syphilis, $2 S_{4}$

Soft chancre, 37,293

Soluble salts of mercury for injection, 247

Sounding the urethra, 556

Sounds, steel, 562

spermatic cord, syphilis of, I $\mathbf{I}$

Spermatozoa in urine, 352

Spizen Warzen, rol

Spleen in hereditary syphilis, 223 syphilis of, 175

Stages of syphilis, $5 \mathrm{~S}$

stains for the gonococcus, $3^{6} 3$

Steel and flexible instruments com. pared, 569

sounds, 562 
Steel sounds, short, $5^{6} 3$

Stomach in hereditary syphilis, 222

Strapping the testicle, method of, 433

Stricture, annular, 534

complications, 593

constitutional symptoms, 549

continuous dilatation, 586

definition, 534

diagnosis, 572

discharge of, 546

divulsion, 593

duration of treatment, 582

extravasation in, 550, 599

false passage in, 595

filiform loougies in, $56 \mathrm{~S}$

fistula, $55^{\circ}$

frequency of sittings, 5 So

gradual dilatation, 578

irritable, $53^{6}$

linear, 534

of large calibre, $5.35,579$

of small calibre, $535,58_{3}$

of the rectum, syphilitic. $17 \%$ treatment, 272

of the urethra, 526

changes in bladder and kirlneys, 453

in the urethra, 542

congenital, $53 \mathrm{I}$

etiology, $53^{8}$

lesion in, $54^{\circ}$

location, 537

number, 537

opening in, 54 I

organic, 534

pathology, 540

resilient, 536

results, 544

retention in, 593

sexual disturbances, 549

spasmodic, 527

symptoms, 544

time required for development, 538

tortuous, 5.34

traumatic, 539

urethral fever in, 596

varieties, 534

Suspensory bandlages, 399

Symmetry in syphilodermata, 75

Synonyms of syphilis, 27 syphilides, 73

syphilis, accidents and injuries in, $7 \mathrm{I}$

acquired, 27

infantile, $2 S_{j}$

and marriage, 286

and prostitution, 290

benignant, 60, $6 \mathbf{I}$

cerebro-spinal, I02

congenitai, 205

etiology, 27

evolution of, in stages, 57

gall sping, 59

germ of, 28

hereditary, 205

anus in, 223

bones in, 216

bronchi in, 221

cranial bones in, 223

cutaneous lesions in, 211

liagnosis, $2 \mathbf{I} 2$

ear in, 226

etiology, 205

eye in, 225

genital organs in, 215

glands in, 2 I 5

hairs in, 2 I 5

intestinal tract in, 222

larynx in, 221

liver in, 222

lungs in, 221

lymphatics in, 2 I 5

mucous membranes in, 215

nails in, 215

nasal passages in, 2 I 9

nervon system in, 223

cesopluagus in, 222

pathology, $2 \mathbf{1 0}$

pharynx in, 220

rectum in, 223

"sabre-blade" deformity in, 216

skin-lesions in, 2 I 1

bullous, 213

hemorrhagic, 215

macular, 212

papular, 2 I 2

tubercular, 2 I 5

spleen in, 223

stomach in, 222

symptoms, 210

teeth in, 220 
Syphilis, hereditary, trichea in, 22 I treatment, 277 inunctions in, $2 S_{1}$

hygiene, 227

inherited, 205

in relation with the family, $2 S_{4}$

with society, 284

malignant, 62, 63

of bones, $\mathbf{I} 59$

diagnosis, 163

pathology, I $6 \mathbf{1}$

of bony walls of orbit, $2 \mathrm{O}_{3}$

of brain, $\mathbf{1} 88$

of brunchi, 158

of ciliary body, I99

of cranial meninges, I 88

of crystalline lens, 200

of ear, 203

of epididymis, I $\$_{\mathbf{I}}$

of eye, 194

of evelids, 195

of gastro-intestinal tract, 173

of kidney, 185

of labyrinth, 205

of liver, 173

of ocular appendages, 194

of ocular muscles, 202

of optic nerve, $20 \mathrm{I}$

of placenta, 209

of retina, 200

of the anus, 175

of the aponeurises, 167

of the blood-versels, 170

of the bones, treatment, 270

of the bronchi, $15^{8}$

of the bur-ie, I 66

of the choroid, 200

of the cord, $19 \mathbf{I}$

of the car, treatment, 276

of the eye, treatment, 275

of the fingers, $16_{3}$

of the genito-urinary organs, I $\$$ in men, I $s_{\mathbf{I}}$

in women, I $S_{3}$

of the heart, 168

of the iris, 197

of the joints, 165

diagnosis, 166

patholugy, I 65

of the larynx, 155

diagno-i- 157
Syphilis of the larynx, prognosis, I 58

of the lungs, I 7 I

of the meninges, I9I

of the mouth, 142

treatment, 266

of the muscles, 167

of the nails, treatment, 270

of the nervous system, $1 S_{7}$

of the nose, treatment, 268

of the pancreas, 175

of the peripheral nerves, 193

of the pharymx, I 54 treatment, 260

of the rectum, I 75

of the respiratory tract, 152

of the sclerotic, 197

of the skin, 73

of the spermatic cord, I 81

of the spleen, 175

of the tendons and tendinous sheaths, I 66

of the testes, 182

of the third generation, 208

of the tongue, 142

of the trachea, 158

of the vagina, 184

of the vitreous humor, 200

precocious, 59

prophylaxis of, 292

tardy, 59

treatment, 227

Syphilitic affections of the hair, I 33 of the nail, $1_{3} 6$

alopecia with structural changes, I 34

without obvious structural

I)ubo, $3 \mathbf{I 9}$ change, I 33

cachexia, 70

chancre, $3^{\circ}$

dactylitis, 163

erythema. 85

fever, 68

lesions, treatment, 260

lupus, 82

mothers, 206

(n) ychanxis, I 39

patiaric, 163

papuler, 90

pemphigus, 2 I I

!neumonia. 172 
Syphilitic psoriasis, 82, 96

roseola, 85

sciatica, 193

snuffles, 214

stricture of rectum, 177

tubercles, 112

urethritis, 368

Syphiloderm, atrophic, I I 3

corneous, 99

corymbiform, 94

dry, II 3

gummatous, I 2 I

miliary, pustular, I05

nummular, 94

purpuric, 88

pustulo-crustaceous, Io8

pustulo-ulcerative, Io8

resolutive tubercular, $\mathbf{I}_{3}$

serpiginous, 128

tubercular, I I 2

ulcerative tubercular, I I 5

vegetating, I $3 \mathbf{I}$

Syphiloderma, 73

palmar, 96

plantar, 96

Syphiloclermata, characteristics of, 75

classification, $8 \mathbf{I}$

general features of, 74

macular, 82

pigmentary, 82

pustular, 104

Syphilophobia, 356

TABEs and syphilis, 19I

Table of diagnostic distinctions between chancroid, chancre, etc., $3 \mathbf{I} 6$

Tardy syphilis, 59

Teeth in hereditary syphilis, 220

Tendons, syphilis of, I 66

Tertiary syphilis, $5^{8}$

Tobacco, 228

Tongue, syphilis of, $\mathbf{I}_{42}$

Trachea in hereditary syphilis, 22 I syphilis of, 158

Treatment, external, 231

internal, 23I

of chancroidal bubo, 328

of chancroids with phimosis, 328

of complications of chancroid, 327

of hereditary syphilis, 277
Treatment of nervous syphilis, 273 of rectal stricture in syphilis, 272 of rectum in syphilis, 272 of syphilis, 227

during pregnancy, 277

" expectant," 232

" interrupted," 232

of the bones, 270

of the ear, 276

of the nose, 268

of the skin, $26 \mathbf{I}$

systemic, $23 \mathrm{I}$

time for beginuing, 229

time required for, $23 \mathbf{I}$

"tonic," 231

of syphilitic alopecia, 265

of syphilitic epididymitis, 273

of syphilitic iritis, 275

of syphilitic lesions, 260

of viscera in syphilis, $27 \mathbf{I}$

Tribadism, 356

Tripter, 360

Tripper fadin, 495

Tubercles, syphilitic, I I 2

Tubercular syphiloderm, I 12

diagnosis, I 8

ulcerative syphiloderm, I I 5

Tuberculous urethritis, $3^{68}$

Tunnelled sounds and catheters, 566

Two-glass method, 495

test, 4I9

Tympanum, 204

UlCerative tubercular syphiloderm, I 15

Ulcer-chancre. 35

Unrecoynized gonorrhea, 373

Unreine'r Fluss, 360

Urethral calibre, 577

chancres, 39

curve, 554

fever, 596

folliculitis, 456

hygiene, 395

Urethritis, acute, 360

causes other than gonorrhcal, 369

etiology, 360

treatment, general considerations, 390

of different forms, 390 
Urethritis, chronic, $4 S_{3}$

diagnosis, 490

etiology, $4 \mathrm{~S}_{3}$

infectiousness of, 49 I

localization of lesions, 493

pathology, 488

prognosis, 524

symptoms, $4 \$ 6$

treatment, $5 \circ 8$

urine in, 494

complications, 4 I 7

diagnostic table, $3 \mathrm{~S}_{7}$

endoscope in, 499

hygiene of, $395,484,508$

infectious, 360

in women, 602

non-infectious, $369,38 \mathbf{I}, 3 \delta_{7}$

pathology, $38 \mathbf{I}$

posterior acute, 4 I 7

diagnosis, 419

etiology, $4 \mathbf{I} 7$

symptoms, 4 I 8

treatment, 420

syphilitic, 368

tuberculusa, $36 \mathrm{~S}$

urine in, 494

shreds, color-test for, 497

two-glass method, 495

Urethrometer, 564

Otis's, 564

Urethrotome, Civiale's, 590

Maisonneuve's, 59 I

Otis's, 59 I

Urethrotomy, external, 593

indications for, $5 \delta_{7}$

internal, ${ }_{5} \mathrm{~S} 9$
Urinary fever, 596

Urine in gonorrhœa: copaiba reaction, 402

in urethritis, two-glass test, 4 I 9

VAGINA syphilis of, I $S_{4}$

Vaginitis, gonorrhoeal, 602

Vapor for the nostrils in syphilis 269

Vaporization of mercury, 244

Vegetating syphiloderm, I3 I

Vegetations with chancroid, 305

Venereal warts, 47 , IOI, 342 treatment, 343

lérole, 27

Verruca acuminata, 47, IOI

Vesiculitis, 448 symptoms, 448 treatment, 449

Veterans of syphilis, 287

Virulenter Bubo, 309

Virus, syphilitic, $2 \mathrm{~S}$

Virus-carriers, 29

Viscera in syphilis, treatment, 27 I

Vitreous humor, syphilis of, 200

Vulvitis, gonorhceal, 604

“WART-CURE," 263

Warts, moist, 47 , IOI pointed, 102 venereal, 47 , IоI, 342 treatment, 343

Weicher Sihanker, 293

Wife, infected, $2 S_{5}$

Wilkinson's salve, 262

W'internitz's psychrophor, 518 

$t$
1 

UNIVERSITY OF CALIFORNIA LIBRARY

\section{Los Angeles}

This book is DLE on the last date stamped below. 


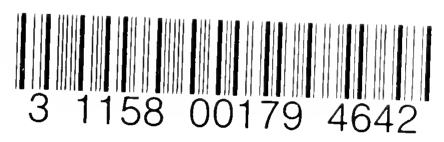


